\title{
The DUX4
}

\section{cytotoxic cascade, \\ and CRISPR \\ mitigation \\ methods}

Ator Ashoti 
The work described in this thesis was performed at the Hubrecht institute for Develeopmental Biology and Stem Cell Research (The Royal Netherlands Academy of Arts and Sciences, KNAW) within the framework of the $\mathrm{PhD}$ programme Regenerative Medicine (RM), which is part of the Utrecht Graduate school of Life Sciences (Utrecht University).

Cover: “Lamassu” by Batul Ashoti

Layout and design by Ator Ashoti and Batul Ashoti

Printed by Ridderprint: www.ridderprint.nl

ISBN: 978-94-6416-638-5

Copyright $\odot 2021$ by Ator Ashoti. All right reserved. No part of this book may be reproduced, stored in a retrieval system or transmitted in any form or by any means, without priot permission of the author. 


\section{The DUX4 cytotoxic cascade, and CRISPR mitigation methods}

De DUX4-cytotoxische cascade, en de CRISPR-mitigatie methoden (Met een samenvatting in het Nederlands)

\section{Proefschrift}

ter verkrijging van de graad van doctor aan de

Universiteit Utrecht op gezag van de

rector magnificus, prof.dr. H.R.B.M. Kummeling, ingevolge het besluit van het college voor promoties in het openbaar te verdedigen op

donderdag 17 juni 2021

des middags te 12.15 uur

door

\section{Ator Rafael Odisho Ashoti}

geboren op 25 november 1989

te Dihok, Irak 
Promotoren:

Prof. dr. W.L. de Laat

Prof. dr. N. Geijsen 


\section{Table of Content}

- Chapter 1

The Mystery that is FSHD

- Chapter 2

Generation of a cellular model to dissect early molecular events leading to DUX4-induced toxicity

- Chapter 3

DUX4 induces a homogeneous sequence of molecular changes, culminating in cellular apoptosis

- Chapter 4

A genome-wide CRISPR/Cas phenotypic screen for modulators of DUX4 cytotoxicity reveals screen complications

- Chapter 5

CRISPR-mediated functional silencing of DUX4

- Chapter 6

General discussion

- Addendum

Nederlandse samenvatting

204

Acknowledgements

Curriculum Vitae

List of publications 


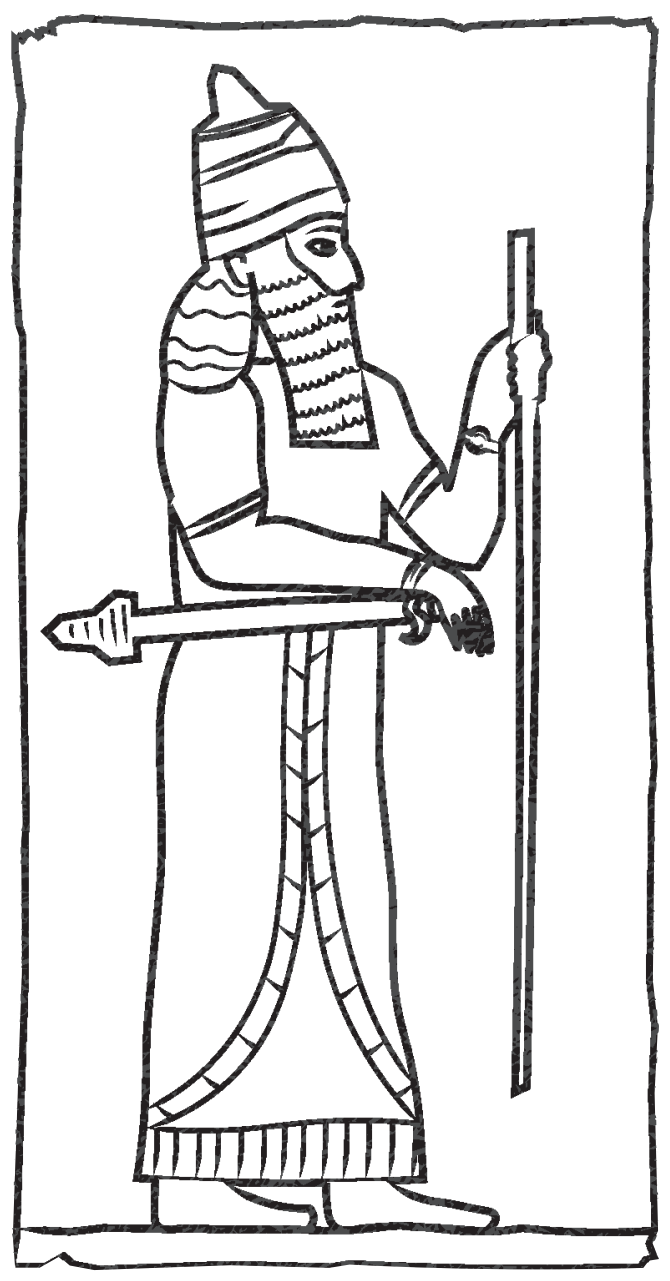

Illustration based on a stone carving on display at the Louvre

6 


\section{Chapter 1 \\ The Mystery that is FSHD}

Ator Ashoti \& Niels Geijsen 


\section{FSHD: A brief history}

Facioscapulohumeral muscular dystrophy (FSHD) is one of the most prevalent genetic muscular disorders ${ }^{1}$. The disorder was documented for the first time over a 136 years ago in 1884 by Landouzy and Dejerine ${ }^{2}$ and was further investigated in the 1900s, with a large familial study published in 1950. This large study of 1249 descendants included physical examination of 240 individuals and data gathering of deceased family members to create a pedigree. Of the 1249 descendants, 159 had the familial anomaly. Due to the large size of the individual family groups, with some of them being of a polygamous nature, the frequency of the disease was much higher within this family tree than in the general population. This gave the authors a unique chance for a large case study, where the pattern of inheritance and clinical features were documented and described ${ }^{3}$. FSHD is presently described as a hereditary autosomal dominant trait, however, $10-30 \%$ of the cases originate sporadically by de novo mutations ${ }^{4-8}$. Many of these sporadic cases are somatic mosaic, which likely originate from mitotic repeat rearrangement ${ }^{9-15}$.

FSHD patients experience muscle atrophy in a asymmetric fashion, starting in the facial muscles and muscles of the shoulder blades, and slowly progresses to the muscles in the upper arms, areas of the truck and in some cases the muscles in the lower extremities ${ }^{3,16}$. The prognosis for FSHD is compared to other muscular dystrophies one of the best, as it generally progresses at a slow pace and rarely effects cardiac output, with the majority of the cases having a normal life expectancy ${ }^{16,17}$. However, the psychological and psychosocial impact can be severe, as the facial muscles that show human emotion are the first to be affected.

Through microsatellite linkage analysis it was determined in 1990 that the origin of FSHD lies on chromosome $4^{18,19}$. This was quickly narrowed down to the subtelomeric region on the q-arm (4q35), often referred to as the 4q35-ter or 4qter ${ }^{20,21}$. In 1992 it was established that FSHD was linked to a $3.2 \mathrm{~kb}$ repeated structure in the 4qter which was named a D4Z4 macrosatellite repeat sequence ${ }^{22}$. A contraction of these repeated sequences was linked to the development of FSHD. The number of repeats can range between 1-100, arranged in a head to tail orientation. Healthy individuals generally possess between 11-100 repeats,

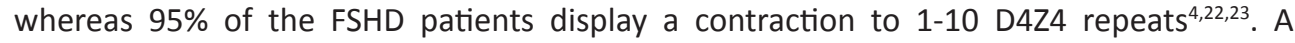
homeobox-containing gene was a likely suspect driving FSHD pathogenesis, since two homeobox sequences were identified within the $3.2 \mathrm{~kb}$ repeat ${ }^{4,22}$.

While these repeated structures were studied more in depth, assays for detecting D4Z4 sequences became more specific, using southern blot analysis and in-situ hybridization. It became apparent that the D4Z4 repeats were not restricted to the 4qter, but were also found at multiple other loci in the human genome. D4Z4 copies were found on chromosomes 1 , $3,9,13,14,15,20,21,22$ and $Y$, and as a similar tandem repeat structure on the q-arm of chromosome 10 (10qter, 10q26) ${ }^{23-25}$. This repeat array on chromosome 10q26 shares $98 \%$ in sequence homology to chromosome $4 \mathrm{q} 35^{26}$, yet the 10 qter shows no association with FSHD ${ }^{26-28}$. Fortunately for diagnostic purposes, these two highly similar tandem repeat arrays could still be discriminated from one another due to a specific Blnl (also known as AvrlI) restriction enzyme recognition site on each D4Z4 repeat located on chromosome $10 q 26^{29}$.

At the same time, it was also discovered that the double homeodomains found in the D4Z4 repeat units were contained in an open reading frame (ORF). The authors assumed it to be unlikely that the ORF would code for a functional protein, since no transcript of this ORF or any other sequences found at or around the D4Z4 repeated array had been identified ${ }^{23,24}$. 
Additionally, hybrid repeat arrays (chromosome 10 repeats found on chromosome 4 and vice versa) found in healthy individuals contributed to the idea that these repeat sequences do not encoding for a functional protein, as they believed that this rearrangement would disrupt the FSHD-related gene ${ }^{28}$. A different scenario was therefore proposed, in which FSHD was caused by a position effect due to the large deletions on the 4qter, and deletion of a critical number of D4Z4 repeat units could affect the expression of genes located in close proximity of this truncated repeated array $23,24,28$.

Hewitt et al. hypothesized that if the ORF would produce a functional protein, it would either be a large polymorphic gene encoding multiple homeodomains, or only one copy would be responsible for the production of a functional protein ${ }^{23}$. This last assumption, as we now know, proved to be true. A minimum of one D4Z4 repeat, containing an ORF that was later identified as double homeobox 4 (DUX4) ${ }^{30}$, is necessary for the development of FSHD ${ }^{31}$. This gene is a pioneer transcription factor ${ }^{32,33}$, that is normally expressed during early embryonic development (4-cell stage) ${ }^{34,35}$ and in the thymus ${ }^{36}$ and testis ${ }^{37}$. The first evidence supporting the involvement of DUX4 in FSHD was published in 2007, by Kowaljow et al. ${ }^{38}$ and Dixit et al. ${ }^{39}$. The authors showed an upregulation of DUX4 in FSHD muscle biopsies compared to biopsies of healthy controls ${ }^{38,39}$, and the pro-apoptotic feature of DUX $4^{38}$. This result was quickly corroborated by the group of Stephan Tapscott, indeed showing an upregulation of DUX4 expression in FSHD-derived muscle cells, together with many other sense and antisense RNA transcripts, novel mRNAs and other RNA fragments that are encoded within the D4Z4 repeat array. They furthermore confirmed the hypothesis of Hewitt at al. that one copy of the DUX4 ORF is involved with pathophysiology of FSHD, as they showed that a polyadenylated DUX4 transcript comes from the most distal (most telomeric) D4Z4 repeat ${ }^{40}$.

\section{Genetic background and DUX4 expression}

To uncover why a contracted repeat array on chromosome 10 is not associated with FSHD, differences in telomeric structures between the 10qter and the disease-linked 4qter were studied. Both chromosomes contain a sequence directly adjacent to the most distal D4Z4 unit, called the pLAM sequence. This sequence was previously used for the characterization of rearranged D4Z4 fragments, through the use of the pLAM probe ${ }^{22}$. Both chromosomes also possess an inverted D4Z4 repeat $42 \mathrm{~kb}$ upstream of the main repeat array. However, the inverted repeat on chromosome 10 misses a portion, which also happens to be the breakpoint in the $4 \mathrm{q}$ and $10 \mathrm{q}$ proximal homology. Downstream of this breakpoint the two chromosomes share a high degree of sequence homology (Fig. 1). In the process of uncovering the differences between the 4qter and 10qter, the authors also found two variants of the $4 q$ ter: one containing a pLAM sequence $(4 q A)$, and the other not $(4 q B)^{41}$ (Fig. 1). These two variants can be found almost equally frequent in the population, yet only $4 q A$ is associated with FSHD ${ }^{42-44}$. When the role of DUX4 in the development of FSHD was established, with the discovery of a stable polyadenylated DUX4 transcript in FSHDaffected muscle cells ${ }^{39,40}$, it did not take long to connect the missing piece as to why only $4 \mathrm{qA}$ is linked to the development of FSHD. About a year after, a genetic model for FSHD was published, where the authors identified an ATTAAA polyadenylation signal (PolyA) in the pLAM region on the $4 q A$ allele. Since the $4 q B$ allele lacks the pLAM sequence, DUX4 transcripts from this allele are not polyadenylated, which is necessary to stabilize the DUX4 transcript. While a pLAM sequence is present in the most distal 10qter repeat, a single nucleotide polymorphism (ATCAAA) ${ }^{45}$ at this locus disrupts the poly-adenylation sequence (Fig. 1). Without this essential polyA sequence, the DUX4 protein cannot be stably expressed. 

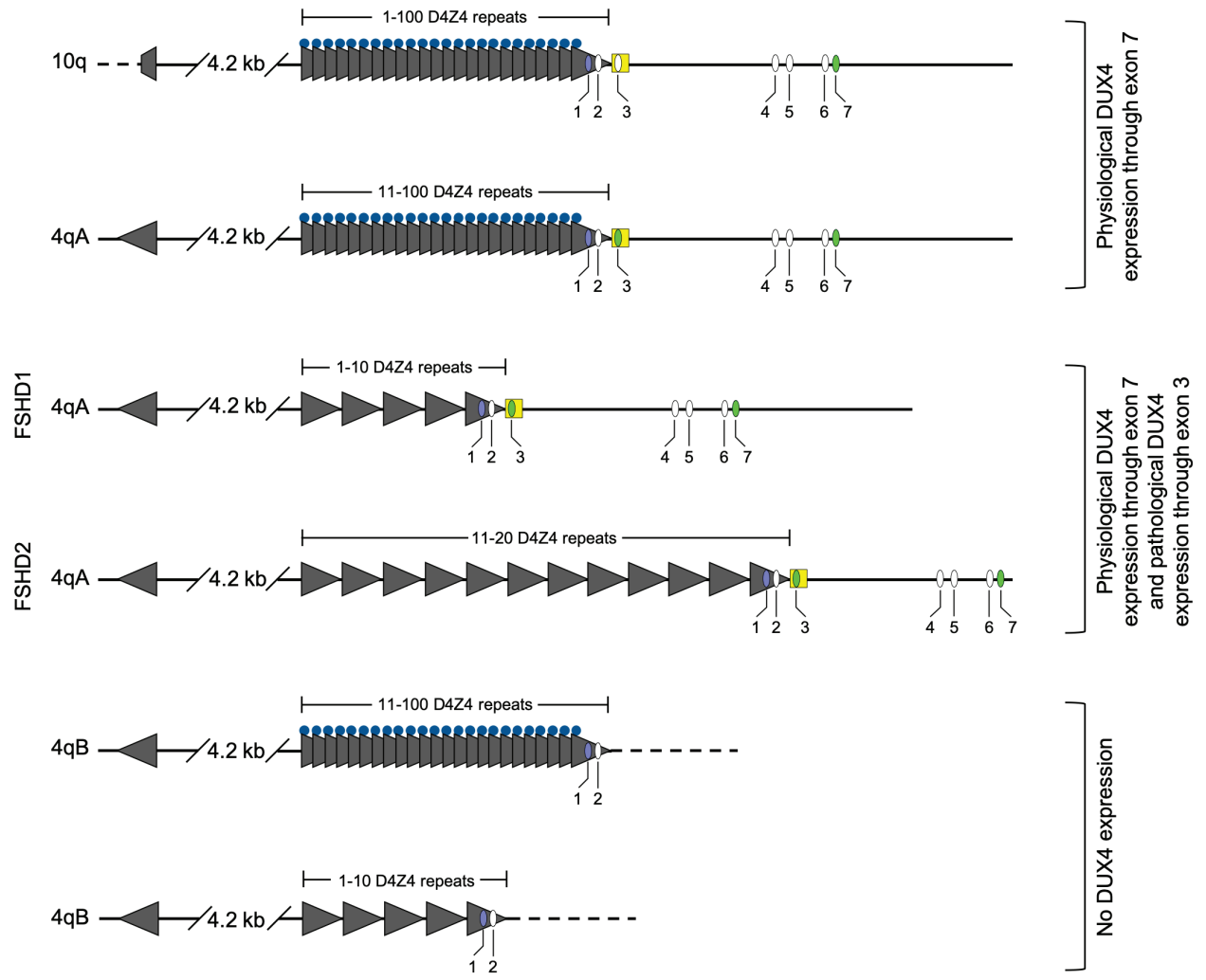

\begin{tabular}{llll}
\hline Inverted D4Z4 repeat & $\square$ pLAM & Methylation & 0 DUX4 open reading frame \\
D4Z4 repeat & - Shared homology with 4qA & 0 DUX4 exon & 0 DUX4 exon with polyA \\
Inverted partial D4Z4 repeat & -- No homology with 4qA &
\end{tabular}

Figure 1. Overview of the D4Z4 tandem repeat arrays on chromosome 4 and $\mathbf{1 0}$. Schematic representation of the organization of the D4Z4 tandem repeats arrays and the DUX4 gene in healthy and FSHD affected individuals, and the shared sequence homology between the 4qter and 10qter, deduced from van Geel et al. ${ }^{41}$, Lemmers et al. ${ }^{42}$, Lemmers et al. $^{45}$, and Snider et $\mathrm{al}^{37}$. Physiological DUX4 expression occurs through the use of the polyadenylation signal in exon 7, which can be found on chromosome 10 and 4qA. Pathological DUX4 expression occurs on a 4qA allele through the use of the polyadenylation signal on exon 3, and after loss of methylation at the 4 q35 loci. DUX4 is not expressed on a $4 \mathrm{qB}$ allele, due to a lack of a polyadenylation signal.

Specific haplotypes of the 4qter were identified, based on subtle and consistent sequence variations in the $D 4 Z 4$ repeated array, and its flanking regions ${ }^{45-47}$. Among the most common haplotypes (4A161, 4B163 and 4A166), only contractions on 4A161 are pathogenic, due to the fact that this haplotype contains a poly-adenylation signal in exon 3 , which stabilizes the DUX4 transcript ${ }^{45-47}$.

The DUX4 transcription factor is physiologically expressed during early embryonic development, as well as in the adult testis ${ }^{37}$ and thymus ${ }^{36}$. Stabilization of the physiological transcript is regulated through a different polyA sequence than the one found in exon 3 in the 4qA genetic background (Fig. 1). Downstream of the most distal D4Z4 unit lie 4 
more additional exons, exon 4 to 7 . Exon 7 contains a polyA sequence which appears to be more tightly regulated, and is the one used for the physiological expression of DUX4 during development an in mature tissues such as the testis and thymus. It therefore appears that the polyA sequence in exon 3 is pathological, as this transcript is only found in FSHD affected muscle cells ${ }^{37}$.

\section{FSHD2}

The contracted D4Z4 repeat array on chromosome $4 q 35$ was only found in $90-95 \%$ of all FSHD cases (FSHD1) $)^{10,48}$. This suggested the existence of a second locus or event linked to the disorder. These FHSD2 patients with normal a D4Z4 repeat length are clinically indistinguishable from FSHD1 patients that carry a contracted D4Z4 repeat array ${ }^{49-52}$. A common feature between FSHD1 and FSHD2 is the presence of a permissive, hypomethylated $4 q A$ allele ${ }^{50,51}$. In FSHD1 patients, the contraction of D4Z4 repeat array itself causes the loss of methylation and repressive chromatin, which leads to the permissive state of the D4Z4 array. ${ }^{22,23,49}$. While the D4Z4 region in FSHD2 patients is not contracted, all D4Z4 repeat arrays in FSHD2 subjects are hypomethylated, which includes both the $4 q$ and $10 q$ alleles. This is in contrast to FSHD1 patients, in which only the contracted repeat array is hypomethylated ${ }^{49-52}$. The fact that all D4Z4 repeat arrays are hypomethylated in FSHD2 subjects implies the loss of a gene responsible for the methylation of these loci. Indeed, many FSHD2 patients possessed a heterozygous mutation in Structural Maintenance of Chromosome Flexible Hinge Domain Containing gene $1(\mathrm{SMCHD} 1)^{53}$, a gene known for its role in $\mathrm{X}$ inactivation through the hypermethylation of $\mathrm{CpG}$ islets ${ }^{54-56}$. The loss of SMCHD1 co-segregates with the hypomethylated status of the D4Z4 repeat array, and even heterozygous loss of SMCHD1 can thus cause the hypomethylated state on the D4Z4 arrays in patients diagnosed with FSHD2 ${ }^{53,57}$.

In recent years, DNA Methyltransferase 2 Beta (DNMT3B) has also been identified in rare cases of FSHD2 ${ }^{58}$. This gene is involved in de novo methylation during early embryonic development and likely plays a role in the hypermethylation and inactivation of the D4Z4 array as well ${ }^{56,59-61}$.

Notably, even though the D4Z4 repeat array in FSHD2 patients is not considered contracted $(<10)$, the number of D4Z4 repeat units in most FSHD2 patients is lower (11-16) than most healthy individuals $(11-100)^{52,62-65}$. This suggests that haploinsufficiency of SMCHD1 is on its own not sufficient to fully derepress the permissive D4Z4 array, unless the number of D4Z4 repeats drops below a certain threshold (Fig. 1).

Thus, both FSHD1 and FSHD2 are caused by the inheritance of at least two dominant traits, a FSHD-permissive 4qA allele, and hypomethylated D4Z4 repeat array caused through either a contraction event or a mutated modifier gene. These events lead to the misexpression of DUX4 and subsequently the development of FSHD ${ }^{45,53}$.

\section{D4Z4 contractions in FSHD}

As described above, hypomethylation of the D4Z4 array can be caused in two ways: the contraction of the D4Z4 repeated array, or a mutation in a chromatin modifier gene (e.g. SMCHD1) necessary to establish and/or maintain the hypermethylated status of this locus. There are several hypotheses about how a contraction of the D4Z4 array leads to a more relaxed chromatin, which subsequently initiates the transcription of the DUX4 gene. The D4Z4 array is often described as heterochromatin as it has some similar features: its proximity to telomeres, an unusually high GC content, the presence hhspm3 and LSau repeats that are 
predominantly found in heterochromatin regions within the human genome ${ }^{23,24}$, and the abundance of $\mathrm{H} 3 \mathrm{~K} 9 \mathrm{me} 3$ and $\mathrm{H} 3 \mathrm{~K} 27 \mathrm{me} 3$ marks $^{36,66}$. The loss of some of the heterochromatin signature may lead to local chromatin relaxation, allowing the transcription of genes within the area. However, it is argued that the D4Z4 array is missing an important feature of heterochromatin, as the $\mathrm{H} 4$ acetylation levels at the D4Z4 array are not low enough to be classified as heterochromatin, and corresponds more to that of unexpressed euchromatin ${ }^{67}$. One study has shown that a contracted D4Z4 array enables the binding of CCCTC-binding factor (CTCF) and A-type Lamins to the contracted array, which could change the spatial positioning of the 4qter in the nuclear envelope. They hypothesize that a normal length D4Z4 array keeps the 4qter in a repressive compartment, and that binding of CTCF and A-type lamins to a contracted D4Z4 array positions the 4qter in a more permissive compartment at the nuclear envelope ${ }^{68}$. Other chromatin-binding proteins that bind to the D4Z4 repeat array and influence the expression of nearby genes have also been identified. These proteins: YY1, HMGB2, nucleolin and EZH2, are either part of, or are associated with the polycomb group $(P c G)^{69,70}$. PcG complexes are known for their repressive effects on gene expression by adding repressive histone modification marks to nucleosomal histones ${ }^{71}$. The lowered occupancy of these proteins at the D4Z4 repeated array of FSHD-affected muscle cells leads to a reduction of these repressive marks, like the repressive histone mark $\mathrm{H} 3 \mathrm{~K} 27 \mathrm{me} 3^{70}$.

It appears that the loss of a piece of chromatin at the 4qter carrying essential repressive features, including DNA methylation and binding motifs for repressive proteins, causes major epigenetic dysregulation upon their loss, which leads to an open locus that is permissive for transcription.

The mechanism of the D4Z4 contraction is a topic of discussion as well. Due to the telomeric location of the D4Z4 repeats, rearrangements of this region during either meiosis or mitosis are likely to occur. With FSHD1, the contraction of the D4Z4 repeated array primarily occurs during mitotic cell division in early embryonic development ${ }^{14,45,72}$. D4Z4 rearrangement can occur through either intrachromosomal or interchromosomal rearrangements, with interchromosomal rearrangements appearing to be the more common event ${ }^{14,72}$. Partners for interchromosomal rearrangement in FSHD1 can be sister chromatids, or chromosome 10 , as this D4Z4 repeat array shares high sequence homology with the repeat array on the $4 q$ ter $^{26,41}$. Interchromosomal rearrangement with the sister chromatid as a partner seems to be a logical option, as this plays a major role in double-stranded break repair in mammalian cells $^{73}$, however, as of yet no FSHD1 cases caused by this type of rearrangement have been identified. The more likely course of events is therefore mitotic interchromosomal rearrangements between the 4qter and $10 \mathrm{qter}^{72}$, as several of these types rearrangements have been identified ${ }^{14,48,72,74}$. See figure two for a schematic overview.

\section{FSHD: a muscle-specific disorder}

FSHD is described as a muscle disorder, because it mainly effects muscle tissue. Other tissues are either less severely affected, have a lower impact on the patient's quality of life, or are rare occurrences only effecting a small percentage of patients. These symptoms include mild to moderate retinal pathologies, high-tone hearing loss, and in rare cases, that are predominantly early onset, patients can suffer from intellectual disabilities and epilepsy ${ }^{5,75-78}$. Skeletal muscle is the most affected tissue, likely due to the cellular structure and other muscle-specific characteristics. Muscle fibers are long multinucleated structures, some reaching $\sim 20 \mathrm{~cm}^{79}$, containing dozens of myonuclei per $\mathrm{mm}$ of fiber. 


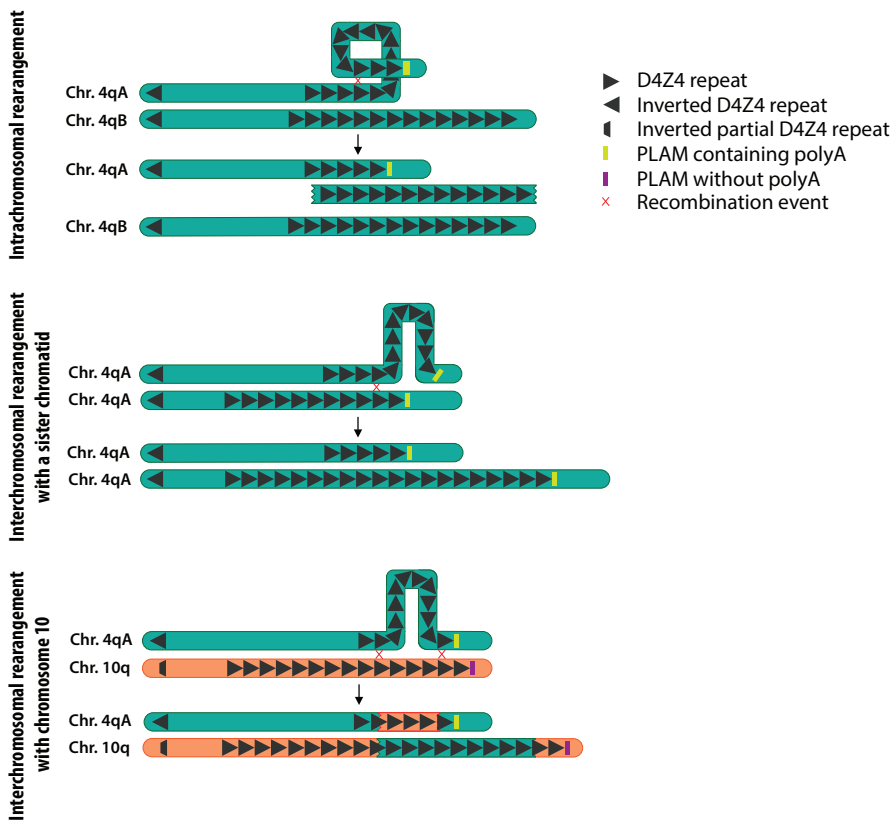

Figure 2. Schematic representation of chromosomal rearrangement events between D4Z4 arrays on chromosome 4 and 10. Top panel: Intrachromosomal rearrangement between D4Z4 repeats on chromosome 4. Middle panel: Interchromosomal rearrangement between D4Z4 repeat arrays on chromosome 4 sister chromatids. Bottom panel: Interchromosomal rearrangement between D4Z4 repeat arrays on chromosome 4 and chromosome 10.

Thus, depending on the size of the fiber, many will contain hundreds or thousands of nuclei ${ }^{80-82}$. Considering that FSHD is caused by a burst of DUX4 expression in approximately $1 / 200$ to $1 / 1000$ nuclei ${ }^{37,83,84}$, many affected muscle fibers of FSHD patients will likely contain one or more of these nuclei. These bursts of DUX4 expressions are rare, with the translation of the DUX4 transcript occurring in the cytoplasm of the myofiber. Both DUX4 transcript and protein can diffuse to other parts of the myofiber, forming a gradient ${ }^{84-86}$.

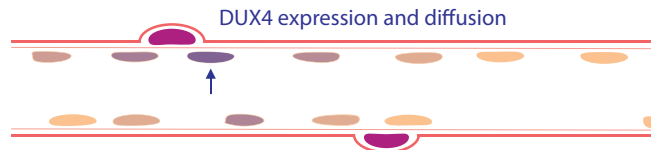

DUX4 downstream TF expression and diffusion
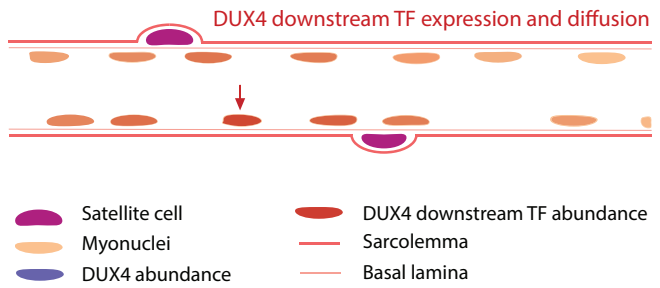

Figure 3. Depiction of the diffusion of DUX4 protein and DUX4 regulated transcription factors within a myofiber. Panels demonstrate a section of a myofiber containing multiple nuclei. Top panel: A DUX4 burst expression occurs in one nucleus (blue arrow), which diffuses and is taken up in the surrounding nuclei. As DUX4 diffuses away from the DUX4 expressing nuclei, it forms a gradient in the surrounding area, which is also reflected in the surrounding nuclei. Bottom panel: an example of surrounding nuclei taking in small amounts of DUX4 protein, which induced the expression of another transcription factor that forms its own gradient (red arrow). 
As DUX4 is a transcription factor, it possesses NLS signals ${ }^{87}$, enabling DUX4 protein to enter surrounding nuclei, activating and continuing gene expression changes that are ultimately cytotoxic. DUX4 diffusion into surrounding nuclei is also made evident due to the presence of DUX4 protein in more myonuclei than the DUX4 transcript. Detection of the DUX4 protein ranged between 0.5 to $16.5 \%$ of counted myonuclei in primary FSHD cells ${ }^{88}$, whereas the transcript is found between 0.1 to $0.5 \%{ }^{37,83,84}$. Other transcription factors whose expression is regulated by DUX4 can in turn also diffuse along the length of the myofiber's, entering surrounding nuclei and continuing the cytotoxicity (Fig. 3). Therefore, due to the multinucleated nature of muscle tissue, the toxic effects caused by aberrant DUX4 expression are amplified. This, in combination with the low turnover of skeletal muscle cells ${ }^{89}$, makes the muscle tissue more prone to manifest visible symptoms.

The cellular structure of muscle is not the only factor that makes muscle more prone for the development of FSHD symptoms. Two muscle-specific enhancers have been identified, located upstream of the D4Z4 repeats, that are able to control expression of genes in their surroundings, including DUX $4^{90}$. These enhancers possess binding motifs for (myogenic) transcription factors, but also binding motifs for CTCF proteins. CTCF can also bind to the contracted D4Z4 array ${ }^{68}$, which could subsequently facilitate the looping of the enhancers to the DUX4 promotor and lead to gene activation. This looping is less likely to occur with a normal-sized D4Z4 array (11-100), as the chromatin is more compacted, containing more repressive motifs, thereby preventing binding of CTCF to the D4Z4 array. In contrast, a contracted D4Z4 array lowers the competition between the DUX4 promotors contained in the D4Z4 units, to bind to the enhancers. This increases the odds of the enhancers associating with the most proximal D4Z4 repeat unit, which in a 4qA genetic background is connected to a polyA sequence (Fig. 4).

\section{Development and Severity}

The severity of FHSD1 is inversely correlated to the length of the D4Z4 repeat array on a permissive haplotype ${ }^{7,91,92}$. In mosaic FSHD1 patients the severity also depends on the timing of de novo rearrangement during embryonic development, which determines the number and types of tissues that contain affected cells, as well as the proportion of affected cells. As de novo mitotic D4Z4 rearrangement is a common reoccurrence and leads to mosaicism, gametes of a mosaic FSHD1 carrier/patient can be made up of cells containing a contracted 4qter, and cells with normal-sized 4qters. This frequently leads to offspring with FSHD1, that are more severely affected than the parent, since they carry the mutation in all their cells $s^{9-15}$.

In FSHD2 patients, the type of mutation in the disease-causing modifier genes can influence the disease severity. FSHD2 patients can be affected more or less, due to the impact of the mutations on the activity of the modifiers, like SMCHD1 and DNMT3B 57,62,65,93.

It should be noted that FSHD1 and FSHD2 are not mutually exclusive. There are patients possessing both defects (FSHD1+2), which often exacerbates the disease development, progression and overall severity. FSHD is therefore considered a disease continuum rather than a disease with specific subclasses, as many factors (known and unknown) influence the development and progression of FSHD when occurring in a permissive genetic background ${ }^{57,58,62,65,93,94}$. 


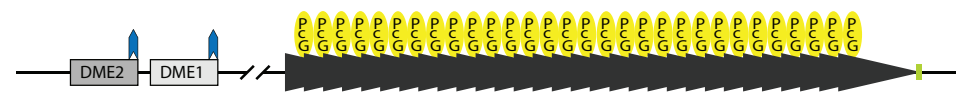

Contracted D4Z4 repeat array
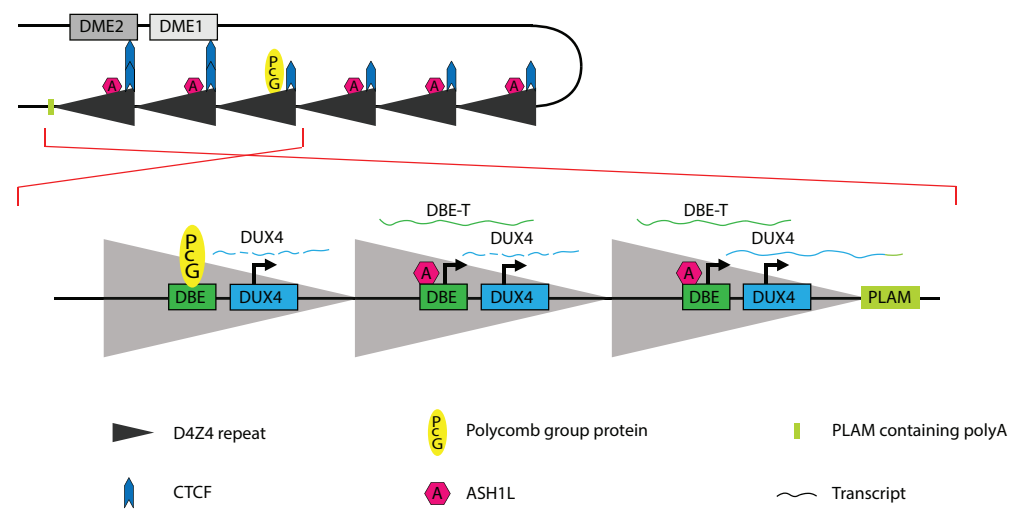

Figure 4. Model of derepression of the D4Z4 array and subsequent expression of DUX4 and DBE-T in muscle cells. Top panel: a normal sized D4Z4 array, carrying repressive Polycomb group (PcG) marks, in a condensed state. Depicted upstream of the D4Z4 array lie DUX4 myogenic enhancer 1 and 2 (DME1 and DME2), bound by CTCF. Lower panel: a contracted D4Z4 array, in a more relaxed state. Relaxation of this area creates openings for CTCF to bind to D4Z4 units. D4Z4-CTCF bind to the CTCFs bound to DME1 and DME2 through looping. Relaxation of the D4Z4 array leads to the expression of DBE-T. DBE-T recruits ASH1L. ASH1L counteracts PcG repression, and promotes further relaxation and expression of surrounding genes. This allows transcription of DUX4, with stable DUX4 transcript being expressed from the most distal D4Z4 unit, attached to the polyA containing PLAM.

In addition to the genetic factors described above (D4Z4 array length, presence of a poly-A signal in exon 3, and the status of SMCHD1 and DNMT3B), several other factors influence the onset and severity of the FSHD phenotype:

- Number of permissive alleles

The number of permissive alleles also influences certain aspects of FSHD, including the age of onset, disease progression and severity. In FSHD1, if both 4q35 alleles are contracted and permissive (4qA), DUX4 expression can occur on both alleles, increasing both the likelihood of DUX4 burst expression and potentially the level of DUX4 expression in myotubes. With FSHD2 or FSHD1+2, all D4Z4 repeat arrays will be hypomethylated, thus enabling transcription on both permissive alleles ${ }^{93,95}$.

\section{- Telomere length}

Another factor that can play an important role in the development of FSHD is the telomeric length on chromosome 4. Telomere length can influence the expression of genes relatively close to the telomere (up to $\sim 10 \mathrm{mb}$ upstream of the telomere) through telomere looping ${ }^{96,97}$. As the telomeres shorten, telomere looping is diminished, and previous areas of the chromosome that had been in close proximity to the heterochromatin signature of the telomere ends, have lost that repressive connection and are therefore more prone for transcription ${ }^{97}$. Shorter telomeres have shown to be inversely proportional with DUX4 expression, as myoblasts with shorter telomeres have higher DUX4 expression. The 
involvement of the telomeres in the development of FSHD can explain the late age of onset seen in many patients, as significant shortening of the telomeres would be required to contribute to the derepression of the D4Z4 array ${ }^{98}$.

\section{- $\quad$ Non-coding RNAs}

The D4Z4 repeated array contains not only the DUX4 gene, but many other transcriptional start sites, in both the sense and anti-sense direction, suggesting it can give rise to other transcripts such as long non-coding RNAs (IncRNA), or small non-coding RNAs such as small interfering RNA (siRNA), micro RNA (miRNA), or piwi-interacting RNA (piRNA) ${ }^{40,99,100}$. Indeed, many other transcripts have been identified in FSHD-affected cells, that map back to D4Z4 region ${ }^{40,99,100}$. These ncRNAs are hypothesized to influence DUX4 expression. Some antisense RNA fragments are thought to silence the D4Z4 array ${ }^{99}$, whereas a specific IncRNA in the sense orientation has been shown to further induce DUX4 expression. This IncRNA lies upstream of the DUX4 ORF within each D4Z4 unit, and is known to be a D4Z4-binding element (DBE) for the Polycomb group (PcG) proteins ${ }^{69,70}$. A transcript of DBE (DBE-T) has been discovered in FSHD-affected muscle, which aids the de-repression of the D4Z4 array further ${ }^{100}$. As DBE is normally bound by PcG proteins, a contraction of the D4Z4 array will lead to the loss of repressive binding motifs, diminishing PcG occupancy, resulting in chromatin relaxation ${ }^{69,70}$. This relaxation can be enough for the expression of the DBE-T, which in turn can recruit the Trithorax group protein ASH1L to the D4Z4 array (Fig. 4). ASH1L is a transcriptional activator that can counteracts the PcG repression ${ }^{101-104}$, therefore further derepressing the D4Z4 array. Furthermore, as ASH1L de-represses the locus further, it promotes the expression of DBE-T, continuing the de-repression of the D4Z4 array as positive feedback loop ${ }^{100}$.

With FSHD2, the genetic defect in a chromatin-modifier gene leads to the loss of CpG methylation at the D4Z4 array, resulting in a more permissive chromatin. It would therefore stand to reason that this too could be enough to facilitate the transcription of DBE-T, which again would cause further depression of the D4Z4 region, subsequently leading to the expression of DUX4.

\section{Animal models}

Animal models are widely used to study human diseases in a more physiological context. Since DUX4 is more primate-specific, finding a suitable model is challenging. In addition, modeling FSHD in other species is challenged by the wide clinical variability, the high potency of DUX4 cytotoxicity when overexpressed, and its stochastic expression in FSHDaffected tissue. Although DUX4 is not conserved in most of the conventional animal models, many of the downstream genes and pathways are. Several animal models have thus been generated to study the effect of FSHD candidate genes, primarily based on (induced) ectopic DUX4 expression. FSHD models have been created in Xenopus ${ }^{105}$, zebrafish ${ }^{106-108}$, and even Drosophila ${ }^{109}$. Some of these models recapitulate aspects of FSHD ${ }^{107,108}$, however, the general effect of expressing DUX4 in these models is embryonic lethality, caused by major cellular loss that is not muscle-specific, and much more severe than what is seen in humans. As there is no DUX4 ortholog found in any of these species, it is not surprising these models respond differently to ectopic DUX4 expression. However, zebrafish models did point out an interesting possibility of a potential developmental role of DUX4 in causing FHSD in later life ${ }^{104,105}$. Conditional expression of human DUX4 in developing zebrafish embryos resulted 
in an asymmetric degenerative effect in the adult zebrafish, after they initially appeared to function normally. If there is indeed a developmental origin of FSHD, this too can be taken into account when considering treatment options or developing therapeutic interventions. Mice do have a Dux gene that shares some sequence and functional homology with its human DUX4 ortholog, allowing them to bind each other's binding motifs and activate a number of the same set of genes ${ }^{110}$. The Dux gene in mice likely evolved independently from the same ancestor retrogene as DUX4, known as DUXC, a gene that is now lost in both species ${ }^{111-114}$. Although DUX4 and Dux are similar, a Dux mouse model will likely have problems determining the underlying mechanism, as not all genes and pathways are conserved between species. Additionally, due to the differences between human DUX4 and mouse Dux, the model is a less ideal candidate for preclinical testing of therapeutic applications that target DUX4 transcript or protein. Yet multiple different mouse models have been generated. Mouse models which ectopically express DUX4 show a high variability of disease manifestation ${ }^{106,115-118}$. Some mouse models were not, or very mildly affected, displaying only an eye phenotype ${ }^{115}$. Others display a more evident phenotype, that is not limited to skeletal muscle and is rather severe ${ }^{106,116}$, much more so than in human FSHD patients. Recently two DUX4-inducible mouse models have been generated that recapitulate mild, moderate and severe forms of FSHD through the titration of an inducing agent (doxycycline or tamoxifen). These models show more similarities in disease manifestation and even recapitulate some of the underlying molecular mechanisms, such as DUX4 expression in sporadic nuclei similar to the burst expression seen in FSHD patients, and in the differentially expressed downstream genes and pathways ${ }^{117-120}$. The DUX4 animal models mentioned here can be helpful for research and drug-screening purposes. However, ectopic expression of the human-specific DUX4 gene in animal model systems can lead to unspecific effects. Moreover, as with the Dux mouse model, these model systems are not suitable to study mechanisms activated by DUX4 expression, as they lack the appropriate human genetic context. While many genes and pathways that are linked to DUX4 expression are conserved in these model systems, this does not necessarily apply for their regulatory regions. The DUX4 binding motif found in the regulatory regions of human genes could be missing in animal models. Indeed, FSHD-related gene 1 (FRG1) is a downstream target gene of DUX4 in human cells ${ }^{121}$ and the gene itself is conserved in mice, but is not activated after ectopic human DUX4 expression in murine cells $^{115}$. In humans, FRG1 contains a functional intronic DUX4 binding site, but this DUX4 binding motif is absent in the Frg1 murine counterpart ${ }^{121}$. It is therefore difficult to predict if a drug that is successful in an animal model, would act the same in humans. Moreover, we do not possess a complete understanding of the underlying mechanisms of FSHD, and could therefore be missing important players, that might again not be recapitulated in other species. These models could however be useful when developing treatments that target DUX4 transcript or protein directly.

A relatively novel approach to allow the study of human-specific disorders, is the use of xenograft models. Immunodeficient mice are engrafted with human primary tissue or human cells, to produce human tissue in a physiological animal system. These models have already been successful in cancer research, where antitumor treatments were identified, that then moved into clinical trials where similar effects were observed ${ }^{122-124}$. These types of models have also been generated for the purpose of studying FSHD and its underlying molecular mechanisms, by engrafting or growing muscle tissue derived from FSHD patients in the animal model. The human muscle tissue in these mice was shown to be vascularized, innervated and functional ${ }^{125-127}$. Naturally, these models too have their limitations: such as 
the variability of engraftment in mice, the fact that a whole-body assessment is not possible, and the need for the use of immunodeficient animals to avoid human tissue rejection, which can potentially effect disease manifestation. These models will however enable researchers to test a broad range of therapeutics that could affect pathways acting upstream or downstream of DUX4 activation, and therefore hold great promise and value in finding a working therapeutic treatment.

\section{Final remarks}

Since both the DUX4 transcript and protein are notoriously difficult to detect, and DUX4 derepression in FSHD patients is caused by many underlying factors, the main cause of FSHD remained elusive for more than 100 years. This chapter has given an overview of the work and discoveries that have led to the unmasking of the main, but not sole, culprit of FSHD. It demonstrates that this muscular dystrophy is not as simple as one mutation in one gene, but requires a combination of genetic and epigenetic factors or events for the disease to manifest. Factors such as:

- The length of the D4Z4 repeat array

- A genetic defect in a chromatin-modifier gene or other FSHD-related genes (both known and unknown)

- The type of mutation in FSHD-related genes involved in FSHD pathogenesis

- Telomere length

- Heterozygosity or homozygosity for the 4qA-permissive alleles

- The 'degree' of mosaicism if FSHD is not familial and originated de novo

- Genetic variations in FSHD-linked enhancers

- The expression and abundance of ncRNAs such as small ncRNA and long ncRNA

All these variations in the population can influence FSHD penetrance, age of onset, disease progression and overall severity. It is therefore not surprising that there is such a large variability between FSHD patients, or even between closely related family members. Patients can range from asymptomatic carriers to being wheelchair-dependent and even requiring ventilation ${ }^{13,128}$.

There are still many unknowns regarding the molecular mechanisms of the disease, including which transcription factors, co-factors and or kinases are involved in the expression and activation of DUX4. Some transcription factors are suspected to be involved, due to the presence of binding motifs in the myogenic enhancers identified by Himeda et $\mathrm{al}^{90}$. These enhancers contain E-box motifs that can be bound by basic helix-loop-helix (bHLH) factors such as MyoD and Myogenin, and homeodomain motifs that can bind homeodomaincontaining genes such as the PAX family of transcription factors ${ }^{90}$. The presence of these binding motifs in enhancer and promotor regions is not sufficient evidence of their involvement in the expression of DUX4, but does make them justified suspects. One study identified Bromodomain-containing 4 (BRD4), a member of the bromodomain and extra terminal domain (BET) family of proteins, to be involved in the activation of DUX4, and demonstrated that BET inhibitors decreased DUX4 expression in FSHD patient-derived myoblasts ${ }^{129}$. BET inhibitors are therefore interesting candidates for future clinical trials. 


\section{Scope and outline of this thesis}

Many of the underlying mechanism of FSHD remain unclear, which hampers the development of effective methods for therapeutic intervention. Work will therefore continue to either clarify these unknown areas of the molecular mechanism, or to modulate DUX4 directly. The work described in this thesis was done with this goal in mind. We generated a versatile human in vitro model and applied this cell model to analyze the sequential occurrence of events following expression of DUX4 through RNA sequencing (Chapter $\mathbf{2}$ and $\mathbf{3}$ ). In attempts to find novel key players that mediate the cytotoxic effects downstream of DUX4; we used our in vitro model to perform a (genome-wide) CRISPR/Cas9 knockout screen (Chapter 2 and 4); and as the cell model also contains the genomic sequence of the first three exons of the DUX4 gene (including the pathological polyA sequence in exon 3), it was used to directly target the DUX4 transgene with genome-editing tools (Chapter 5 ) in order to find new and safe ways of knocking-out DUX4. The last chapter integrates the findings of this thesis with current and potential future perspectives of the field (Chapter 6). 


\section{References}

1. Deenen, J. C. W. et al. Population-based incidence and prevalence of facioscapulohumeral dystrophy. Neurology 83, 1056-1059 (2014).

2. Landouzy, L. \& Dejerine, J. De la myopathie atrophique progressive (Myopathie héréditaire débutant, dans l'enfance, par la face, sans altération du système nerveux). C R Acad Sci 98, 53-55 (1884).

3. Tyler, F. H. \& Stephens, F. E. Studies in disorders of muscle. II Clinical manifestations and inheritance of facioscapulohumeral dystrophy in a large family. Ann. Intern. Med. 32, 640660 (1950).

4. Wijmenga, C. et al. Chromosome 4q DNA rearrangements associated with facioscapulohumeral muscular dystrophy. Nat. Genet. 2, 26-30 (1992).

5. Padberg, G. W. et al. Facioscapulohumeral muscular dystrophy in the dutch population. Muscle Nerve 18, S81-S84 (1995).

6. Zatz, M. et al. High proportion of new mutations and possible anticipation in Brazilian facioscapulohumeral muscular dystrophy families. Am. J. Hum. Genet. 56, 99-105 (1995).

7. Tawil, R. et al. Evidence for anticipation and association of deletion size with severity in facioscapulohumeral muscular systrophy. Ann. Neurol. 39, 744-748 (1996).

8. Lunt, P. W. 44th ENMC International Workshop: Facioscapulohumeral muscular dystrophy: Molecular studies, 19-21 July 1996, Naarden, The Netherlands. Neuromuscul. Disord. 8, 126-130 (1998).

9. Upadhyaya, M. et al. Germinal mosaicism in facioscapulohumeral muscular dystrophy (FSHD). Muscle Nerve 18, S45-S49 (1995).

10. Bakker, E. et al. Diagnostic, predictive, and prenatal testing for facioscapulohumeral muscular dystrophy: Diagnostic approach for sporadic and familial cases. J. Med. Genet. 33, 29-35 (1996).

11. Köhler, J., Rupilius, B., Otto, M., Bathke, K. \& Koch, M. C. Germline mosaicism in 4q35 facioscapulohumeral muscular dystrophy (FSHD1A) occurring predominantly in oogenesis. Hum. Genet. 98, 485-490 (1996).

12. Zatz, M. et al. The facioscapulohumeral muscular dystrophy (FSHD1) gene affects males more severely and more frequently than females. Am. J. Med. Genet. 77, 155-161 (1998).

13. Galluzzi, G. et al. Molecular analysis of $4 \mathrm{q} 35$ rearrangements in facioscapulohumeral muscular dystrophy (FSHD): Application to family studies for a correct genetic advice and a reliable prenatal diagnosis of the disease. Neuromuscul. Disord. 9, 190-198 (1999).

14. Van Der Maarel, S. M. et al. De novo facioscapulohumeral muscular dystrophy: Frequent somatic mosaicism, sex-dependent phenotype, and the role of mitotic transchromosomal repeat interaction between chromosomes 4 and 10. Am. J. Hum. Genet. 66, 26-35 (2000).

15. Lemmers, R. J. L. F. et al. Somatic mosaicism in FSHD often goes undetected. Ann. Neurol. 55, 845-850 (2004).

16. Tawil, R. \& Van Der Maarel, S. M. Facioscapulohumeral muscular dystrophy. Muscle and Nerve 34, 1-15 (2006).

17. van Dijk, G. P. et al. High prevalence of incomplete right bundle branch block in facioscapulohumeral muscular dystrophy without cardiac symptoms. Funct. Neurol. 29, 159-165 (2014).

18. Wijmenga, C. et al. Location of facioscapulohumeral muscular dystrophy gene on chromosome 4. Lancet 336, 651-653 (1990).

19. Upadhyaya, M. et al. DNA marker applicable to presymptomatic and prenatal diagnosis of facioscapulohumeral disease. Lancet 336, 1320-1321 (1990).

20. Wijmenga, C. et al. Mapping of facioscapulohumeral muscular dystrophy gene to chromosome 4q35-qter by multipoint linkage analysis and in situ hybridization. Genomics $\mathbf{9}$, 570-575 (1991).

21. Sarfarazi, M. et al. Regional mapping of facioscapulohumeral muscular dystrophy gene on 4q35: Combined analysis of an international consortium. Am. J. Hum. Genet. 51, 396-403 (1992).

22. Deutekom, J. C. T. va. et al. FSHD associated DNA rearrangements are due to deletions of integral copies of a $3.2 \mathrm{~kb}$ tandemly repeated unit. Hum. Mol. Genet. 2, 2037-2042 (1993).

23. Hewitt, J. E. et al. Analysis of the tandem repeat locus D4Z4 associated with 
facioscapulohumeral muscular dystropothhy. Hum. Mol. Genet. 3, 1287-1295 (1994).

24. Winokur, S. T. et al. The DNA rearrangement associated with facioscapulohumeral muscular dystrophy involves a heterochromatin-associated repetitive element: Implications for a role of chromatin structure in the pathogenesis of the disease. Chromosom. Res. 2, 225-234 (1994).

25. Deidda, G. et al. Physical mapping evidence for a duplicated region on chromosome 10qter showing high homology with the facioscapulohumeral muscular dystrophy locus on chromosome 4qter. European Journal of Human Genetics vol. 3 155-167 (1995).

26. Cacurri, S. et al. Sequence homology between 4qter and 10qter loci facilitates the instability of subtelomeric Kpnl repeat units implicated in facioscapulohumeral muscular dystrophy. Am. J. Hum. Genet. 63, 181-190 (1998).

27. Bakker, E. et al. The FSHD-linked locus D4F104S1 (p13E-11) ON 4q35 has a homologue on 10qter. Muscle Nerve 18, S39-S44 (1995).

28. Lemmers, R. et al. Inter- and intrachromosomal sub-telomeric rearrangements on 4q35: implications for facioscapulohumeral muscular dystrophy (FSHD) aetiology and diagnosis. Hum. Mol. Genet. 7, 1207-1214 (1998).

29. Deidda, G., Cacurri, S., Piazzo, N. \& Felicetti, L. Direct detection of $4 q 35$ rearrangements implicated in facioscapulohumeral muscular dystrophy (FSHD). J. Med. Genet. 33, 361-365 (1996).

30. Gabriëls, J. et al. Nucleotide sequence of the partially deleted D4Z4 locus in a patient with FSHD identifies a putative gene within each 3.3 kb element. Gene 236, 25-32 (1999).

31. Tupler, R. et al. Monosomy of distal $4 \mathrm{q}$ does not cause facioscapulohumeral muscular dystrophy. J. Med. Genet. 33, 366-370 (1996).

32. Choi, S. H. et al. DUX4 recruits p300/CBP through its C-terminus and induces global H3K27 acetylation changes. Nucleic Acids Res. 44, 5161-5173 (2016).

33. Vuoristo, S. et al. DUX4 regulates oocyte to embryo transition in human. Biorxiv (2019) doi:http://dx.doi.org/10.1101/732289.

34. Whiddon, J. L., Langford, A. T., Wong, C. J., Zhong, J. W. \& Tapscott, S. J. Conservation and innovation in the DUX4-family gene network. Nat. Genet. 49, 935-940 (2017).

35. Hendrickson, P. G. et al. Conserved roles of mouse DUX and human DUX4 in activating cleavage-stage genes and MERVL/HERVL retrotransposons. Nat. Genet. 49, 925-934 (2017).

36. Das, S. \& Chadwick, B. P. Influence of repressive histone and DNA methylation upon D4Z4 transcription in non-myogenic cells. PLoS One 11, 1-26 (2016).

37. Snider, L. et al. Facioscapulohumeral dystrophy: Incomplete suppression of a retrotransposed gene. PLoS Genet. 6, 1-14 (2010).

38. Kowaljow, V. et al. The DUX4 gene at the FSHD1A locus encodes a pro-apoptotic protein. Neuromuscul. Disord. 17, 611-623 (2007).

39. Dixit, M. et al. DUX4, a candidate gene of facioscapulohumeral muscular dystrophy, encodes a transcriptional activator of PITX1. Proc. Natl. Acad. Sci. U. S. A. 104, 18157-18162 (2007).

40. Snider, L. et al. RNA transcripts, miRNA-sized fragments and proteins produced from D4Z4 units: New candidates for the pathophysiology of facioscapulohumeral dystrophy. Hum. Mol. Genet. 18, 2414-2430 (2009).

41. Van Geel, M. et al. Genomic analysis of human chromosome $10 q$ and $4 q$ telomeres suggests a common origin. Genomics 79, 210-217 (2002).

42. Lemmers, R. J. L. F. et al. Facioscapulohumeral muscular dystrophy is uniquely associated with one of the two variants of the 4q subtelomere. Nat. Genet. 32, 235-236 (2002).

43. Lemmers, R. J. F. L. et al. Contractions of D4Z4 on $4 \mathrm{qB}$ subtelomeres do not cause facioscapulohumeral muscular dystrophy. Am. J. Hum. Genet. 75, 1124-1130 (2004).

44. Thomas, N. S. T. et al. A large patient study confirming that facioscapulohumeral muscular dystrophy (FSHD) disease expression is almost exclusively associated with an FSHD locus located on a 4qA-defined 4qter subtelomere. J. Med. Genet. 44, 215-218 (2007).

45. Lemmers, R. J. L. F. et al. A unifying genetic model for facioscapulohumeral muscular dystrophy. Science (80-. ). 329, 1650-1653 (2010).

46. Lemmers, R. J. L. F. et al. Specific sequence variations within the $4 q 35$ region are associated with facioscapulohumeral muscular dystrophy. Am. J. Hum. Genet. 81, 884-894 (2007).

47. Lemmers, R. J. L. F. et al. Worldwide Population Analysis of the $4 \mathrm{q}$ and $10 \mathrm{q}$ Subtelomeres Identifies Only Four Discrete Interchromosomal Sequence Transfers in Human Evolution. 
Am. J. Hum. Genet. 86, 364-377 (2010).

48. van Deutekom, J. Evidence for subtelomeric exchange of $3.3 \mathrm{~kb}$ tandemly repeated units between chromosomes $4 q 35$ and 10q26: implications for genetic counselling and etiology of FSHD1. Hum. Mol. Genet. 5, 1997-2003 (1996).

49. Van Overveld, P. G. M. et al. Hypomethylation of D4Z4 in 4q-linked and non-4q-linked facioscapulohumeral muscular dystrophy. Nat. Genet. 35, 315-317 (2003).

50. De Greef, J. C. et al. Hypomethylation is restricted to the D4Z4 repeat array in phenotypic FSHD. Neurology 69, 1018-1026 (2007).

51. de Greef, J. C. et al. Common epigenetic changes of D4Z4 in contraction-dependent and contraction-independent FSHD. Hum. Mutat. 30, 1449-1459 (2009).

52. de Greef, J. et al. Clinical features of facioscapulohumeral muscular dystrophy 2. Neurology 75, 1548-1554 (2010).

53. Lemmers, R. J. L. F. et al. Digenic inheritance of an SMCHD1 mutation and an FSHDpermissive D4Z4 allele causes facioscapulohumeral muscular dystrophy type 2. Nat. Genet. 44, 1370-1374 (2012).

54. Blewitt, M. E. et al. An N-ethyl-N-nitrosourea screen for genes involved in variegation in the mouse. Proc. Natl. Acad. Sci. U. S. A. 102, 7629-7634 (2005).

55. Blewitt, M. E. et al. SmcHD1, containing a structural-maintenance-of-chromosomes hinge domain, has a critical role in X inactivation. Nat. Genet. 40, 663-669 (2008).

56. Gendrel, A. V. et al. Smchd1-Dependent and-Independent Pathways Determine Developmental Dynamics of CpG Island Methylation on the Inactive X Chromosome. Dev. Cell 23, 265-279 (2012).

57. Larsen, M. et al. Diagnostic approach for FSHD revisited: SMCHD1 mutations cause FSHD2 and act as modifiers of disease severity in FSHD1. Eur. J. Hum. Genet. 23, 808-816 (2015).

58. Van Den Boogaard, M. L. et al. Mutations in DNMT3B Modify Epigenetic Repression of the D4Z4 Repeat and the Penetrance of Facioscapulohumeral Dystrophy. Am. J. Hum. Genet. 98, 1020-1029 (2016).

59. Okano, M., Xie, S. \& Li, E. Cloning and characterization of a family of novel mammalian DNA ( cytosine-5) methyltransferases Non-invasive sexing of preimplantation stage mammalian embryos. Nat. Am. Inc. 19, 219-220 (1998).

60. Okano, M., Bell, D. W., Haber, D. A. \& Li, E. DNA methyltransferases Dnmt3a and Dnmt3b are essential for de novo methylation and mammalian development. Cell 99, 247-257 (1999).

61. Auclair, G., Guibert, S., Bender, A. \& Weber, M. Ontogeny of CpG island methylation and specificity of DNMT3 methyltransferases during embryonic development in the mouse. Genome Biol. 15, 545 (2014).

62. Lemmers, R. J. L. F. et al. Inter-individual differences in CpG methylation at D4Z4 correlate with clinical variability in FSHD1 and FSHD2. Hum. Mol. Genet. 24, 659-669 (2015).

63. Lemmers, R. J. L. F. et al. Hemizygosity for SMCHD1 in Facioscapulohumeral Muscular Dystrophy Type 2: Consequences for 18p Deletion Syndrome. Hum. Mutat. 36, 679-683 (2015).

64. Balog, J. et al. Monosomy $18 p$ is a risk factor for facioscapulohumeral dystrophy. J. Med. Genet. 55, 469-478 (2018).

65. Sacconi, S. et al. FSHD1 and FSHD2 form a disease continuum. Neurology 92, E2273-E2285 (2019).

66. Zeng, W. et al. Specific loss of histone H3 lysine 9 trimethylation and HP1Y/cohesin binding at D4Z4 repeats is associated with facioscapulohumeral dystrophy (FSHD). PLoS Genet. 5, (2009).

67. Jiang, G. et al. Testing the position-effect variegation hypothesis for facioscapulohumeral muscular dystrophy by analysis of histone modification and gene expression in subtelomeric 4q. Hum. Mol. Genet. 12, 2909-2921 (2003).

68. Ottaviani, A. et al. The D4Z4 macrosatellite repeat acts as a CTCF and A-type laminsdependent insulator in Facio-Scapulo-Humeral dystrophy. PLoS Genet. 5, (2009).

69. Gabellini, D., Green, M. R. \& Tupler, R. Inappropriate gene activation in FSHD: A repressor complex binds a chromosomal repeat deleted in dystrophic muscle. Cell 110, 339-348 (2002).

70. Bodega, B. et al. Remodeling of the chromatin structure of the facioscapulohumeral muscular dystrophy (FSHD) locus and upregulation of FSHD-related gene 1 (FRG1) 
expression during human myogenic differentiation. BMC Biol. 7, 41 (2009).

71. Golbabapour, S. et al. Gene silencing and polycomb group proteins: An overview of their structure, mechanisms and phylogenetics. Omi. A J. Integr. Biol. 17, 283-296 (2013).

72. Lemmers, R. J. L. F. et al. Mechanism and timing of mitotic rearrangements in the subtelomeric D4Z4 repeat involved in facioscapulohumeral muscular dystrophy. Am. J. Hum. Genet. 75, 44-53 (2004).

73. Johnson, R. D. \& Jasin, M. Sister chromatid gene conversion is a prominent double-strand break repair pathway in mammalian cells. EMBO J. 19, 3398-3407 (2000).

74. Overveld, P. G. M. v. Interchromosomal repeat array interactions between chromosomes 4 and 10: a model for subtelomeric plasticity. Hum. Mol. Genet. 9, 2879-2884 (2000).

75. Padberg, G. W. et al. On the significance of retinal vascular disease and hearing loss in facioscapulohumeral muscular dystrophy. Muscle Nerve 18, S73-S80 (1995).

76. Funakoshi, M., Goto, K. \& Arahata, K. Epilepsy and mental retardation in a subset of early onset 4q35- facioscapulohumeral muscular dystrophy. Neurology 50, 1791-1794 (1998).

77. Trevisan, C. P. et al. Facioscapulohumeral muscular dystrophy: Hearing loss and other atypical features of patients with large 4q35 deletions. Eur. J. Neurol. 15, 1353-1358 (2008).

78. Goselink, R. J. M. et al. Ophthalmological findings in facioscapulohumeral dystrophy. Brain Commun. 1, 1-9 (2019).

79. Heron, M. I. \& Richmond, F. J. R. In-series fiber architecture in long human muscles. J. Morphol. 216, 35-45 (1993).

80. Kawano, F. et al. Essential role of satellite cells in the growth of rat soleus muscle fibers. Am. J. Physiol. - Cell Physiol. 295, 458-467 (2008).

81. van der Meer, S. F. T., Jaspers, R. T. \& Degens, H. Is the myonuclear domain size fixed? J. Musculoskelet. Neuronal Interact. 11, 286-297 (2011).

82. Kramer, I. F. et al. Extensive Type II Muscle Fiber Atrophy in Elderly Female Hip Fracture Patients. Journals Gerontol. - Ser. A Biol. Sci. Med. Sci. 72, 1369-1375 (2017).

83. Jones, T. I. et al. Facioscapulohumeral muscular dystrophy family studies of DUX4 expression: Evidence for disease modifiers and a quantitative model of pathogenesis. Hum. Mol. Genet. 21, 4419-4430 (2012).

84. Tassin, A. et al. DUX4 expression in FSHD muscle cells: How could such a rare protein cause a myopathy? J. Cell. Mol. Med. 17, 76-89 (2013).

85. Ferreboeuf, M. et al. Nuclear protein spreading: Implication for pathophysiology of neuromuscular diseases. Hum. Mol. Genet. 23, 4125-4133 (2014).

86. Rickard, A. M., Petek, L. M. \& Miller, D. G. Endogenous DUX4 expression in FSHD myotubes is sufficient to cause cell death and disrupts RNA splicing and cell migration pathways. Hum. Mol. Genet. 24, 5901-5914 (2015).

87. Corona, E. D., Jacquelin, D., Gatica, L. \& Rosa, A. L. Multiple Protein Domains Contribute to Nuclear Import and Cell Toxicity of DUX4, a Candidate Pathogenic Protein for Facioscapulohumeral Muscular Dystrophy. PLoS One 8, 1-11 (2013).

88. Block, G. J. et al. Wnt/ $\beta$-catenin signaling suppresses DUX4 expression and prevents apoptosis of FSHD muscle cells. Hum. Mol. Genet. 22, 4661-4672 (2013).

89. Claydon, A. J., Thom, M. D., Hurst, J. L. \& Beynon, R. J. Protein turnover: Measurement of proteome dynamics by whole animal metabolic labelling with stable isotope labelled amino acids. Proteomics 12, 1194-1206 (2012).

90. Himeda, C. L. et al. Myogenic Enhancers Regulate Expression of the Facioscapulohumeral Muscular Dystrophy-Associated DUX4 Gene. Mol. Cell. Biol. 34, 1942-1955 (2014).

91. Lunt, P. W. et al. Correlation between fragment size at D4F104S1 and age at onset or at wheelchair use, with a possible generational effect, accounts for much phenotypic variation in 4q35-facioscapulohumeral muscular dystrophy (FSHD). Hum. Mol. Genet. 4, 951-958 (1995).

92. Lunt, P. W. et al. Phenotypic-genotypic correlation will assist genetic counseling in 4q35facioscapulohumeral muscular dystrophy. Muscle Nerve 18, S103-S109 (1995).

93. Lemmers, R. J. et al. Deep characterization of a common D4Z4 variant identifies biallelic DUX4 expression as a modifier for disease penetrance in FSHD2. Eur. J. Hum. Genet. 26, 94-106 (2018).

94. Sacconi, S. et al. The FSHD2 gene SMCHD1 Is a modifier of disease severity in families affected by FSHD1. Am. J. Hum. Genet. 93, 744-751 (2013). 
95. Renard, D. et al. Inflammatory facioscapulohumeral muscular dystrophy type 2 in $18 p$ deletion syndrome. Am. J. Med. Genet. Part A 176, 1760-1763 (2018).

96. Wright, W. E. \& Shay, J. W. Telomere positional effects and the regulation of cellular senescence. Trends Genet. 8, 193-197 (1992).

97. Robin, J. D. et al. Telomere position effect: Regulation of gene expression with progressive telomere shortening over long distances. Genes Dev. 28, 2464-2476 (2014).

98. Stadler, G. et al. Telomere position effect regulates DUX4 in human facioscapulohumeral muscular dystrophy. Nat. Struct. Mol. Biol. 20, 671-678 (2013).

99. Block, G. J. et al. Asymmetric bidirectional transcription from the FSHD-causing D4Z4 array modulates DUx4 production. PLoS One 7, e35532 (2012).

100. Cabianca, D. S. et al. A long ncRNA links copy number variation to a polycomb/trithorax epigenetic switch in fshd muscular dystrophy. Cell 149, 819-831 (2012).

101. Klymenko, T. \& Jürg, M. The histone methyltransferases Trithorax and Ash1 prevent transcriptional silencing by Polycomb group proteins. EMBO Rep. 5, 373-377 (2004).

102. Schmitges, F. W. et al. Histone Methylation by PRC2 Is Inhibited by Active Chromatin Marks. Mol. Cell 42, 330-341 (2011).

103. Yuan, W. et al. H3K36 methylation antagonizes PRC2-mediated H3K27 methylation. J. Biol. Chem. 286, 7983-7989 (2011).

104. Miyazaki, H. et al. Ash1l Methylates Lys36 of Histone H3 Independently of Transcriptional Elongation to Counteract Polycomb Silencing. PLoS Genet. 9, (2013).

105. Wuebbles, R. D., Long, S. W., Hanel, M. L. \& Jones, P. L. Testing the effects of FSHD candidate gene expression in vertebrate muscle development. Int. J. Clin. Exp. Pathol. 3, 386-400 (2010).

106. Wallace, L. M. et al. DUX4, a candidate gene for facioscapulohumeral muscular dystrophy, causes p53-dependent myopathy in vivo. Ann. Neurol. 69, 540-552 (2011).

107. Mitsuhashi, H., Mitsuhashi, S., Lynn-jones, T., Kawahara, G. \& Kunkel, L. M. Expression of DUX4 in zebrafish development recapitulates facioscapulohumeral muscular dystrophy. Hum. Mol. Genet. 22, 568-577 (2013).

108. Pakula, A. et al. Transgenic zebrafish model of DUX4 misexpression reveals a developmental role in FSHD pathogenesis. Hum. Mol. Genet. 28, 320-331 (2019).

109. Jones, T. I., Parilla, M. \& Jones, P. L. Transgenic drosophila for investigating dux4 and frg1, two genes associated with facioscapulohumeral muscular dystrophy (fshd). PLoS One 11, (2016).

110. Eidahl, J. O. et al. Mouse Dux is myotoxic and shares partial functional homology with its human paralog DUX4. Hum. Mol. Genet. 25, ddw287 (2016).

111. Winokur, S. T., Ulla, B., Vargas, J. C., Wasmuth, J. J. \& Altherr, M. R. The evolutionary distribution and structural organization of the homeobox-containing repeat D4Z4 indicates a functional role for the ancestral copy in the FSHD region. Hum. Mol. Genet. 6, 502-502 (1997).

112. Clark, L. N., Koehler, U., Ward, D. C., Wienberg, J. \& Hewitt, J. E. Analysis of the organisation and localisation of the FSHD-associated tandem array in primates: Implications for the origin and evolution of the $3.3 \mathrm{~kb}$ repeat family. Chromosoma 105, 180-189 (1996).

113. Clapp, J. et al. Evolutionary conservation of a coding function for D4Z4, the tandem DNA repeat mutated in facioscapulohumeral muscular dystrophy. Am. J. Hum. Genet. 81, 264279 (2007).

114. Leidenroth, A. et al. Evolution of DUX gene macrosatellites in placental mammals. Chromosoma 121, 489-497 (2012).

115. Krom, Y. D. et al. Intrinsic Epigenetic Regulation of the D4Z4 Macrosatellite Repeat in a Transgenic Mouse Model for FSHD. PLoS Genet. 9, (2013).

116. Dandapat, A. et al. Dominant Lethal Pathologies in Male Mice Engineered to Contain an X-Linked DUX4 Transgene. Cell Rep. 8, 1484-1496 (2014).

117. Bosnakovski, D. et al. Muscle pathology from stochastic low level DUX4 expression in an FSHD mouse model. Nat. Commun. 8, 1-9 (2017).

118. Jones, T. \& Jones, P. L. A cre-inducible DUX4 transgenic mouse model for investigating facioscapulohumeral muscular dystrophy. PLOS ONE vol. 13 (2018).

119. Bosnakovski, D. et al. Transcriptional and cytopathological hallmarks of FSHD in chronic DUX4-expressing mice. J. Clin. Invest. 130, 2465-2477 (2020). 
120. Jones, T. I. et al. Transgenic mice expressing tunable levels of DUX4 develop characteristic facioscapulohumeral muscular dystrophy-like pathophysiology ranging in severity. Skelet. Muscle 10, 1-28 (2020).

121. Ferri, G., Huichalaf, C. H., Caccia, R. \& Gabellini, D. Direct interplay between two candidate genes in FSHD muscular dystrophy. Hum. Mol. Genet. 24, 1256-1266 (2015).

122. Adams, J. Development of the Proteasome Inhibitor PS-341. Oncologist 7, 9-16 (2002).

123. Baselga, J. Phase I and II clinical trials of trastuzumab.pdf. Ann. Oncol. 12, S49-S55 (2001).

124. Pegram, M. \& Ngo, D. Application and potential limitations of animal models utilized in the development of trastuzumab (Herceptin ${ }^{\circledast}$ ): A case study. Adv. Drug Deliv. Rev. 58, 723-734 (2006).

125. Zhang, Y. et al. Humanskeletal musclexenograft as anewpreclinical model for muscle disorders. Hum. Mol. Genet. 23, 3180-3188 (2014).

126. Mueller, A. L. et al. Muscle xenografts reproduce key molecular features of facioscapulohumeral muscular dystrophy. Exp. Neurol. 320, 113011 (2019).

127. Sakellariou, P. et al. Neuromuscular electrical stimulation promotes development in mice of mature human muscle from immortalized human myoblasts. Skelet. Muscle 6, 1-14 (2016).

128. Tawil, R., van der Maarel, S. M. \& Tapscott, S. J. Facioscapulohumeral dystrophy: The path to consensus on pathophysiology. Skelet. Muscle 4, 1-15 (2014).

129. Campbell, A. E. et al. BET bromodomain inhibitors and agonists of the beta-2 adrenergic receptor identified in screens for compounds that inhibit DUX4 expression in FSHD muscle cells. Skelet. Muscle 7, 1-18 (2017). 


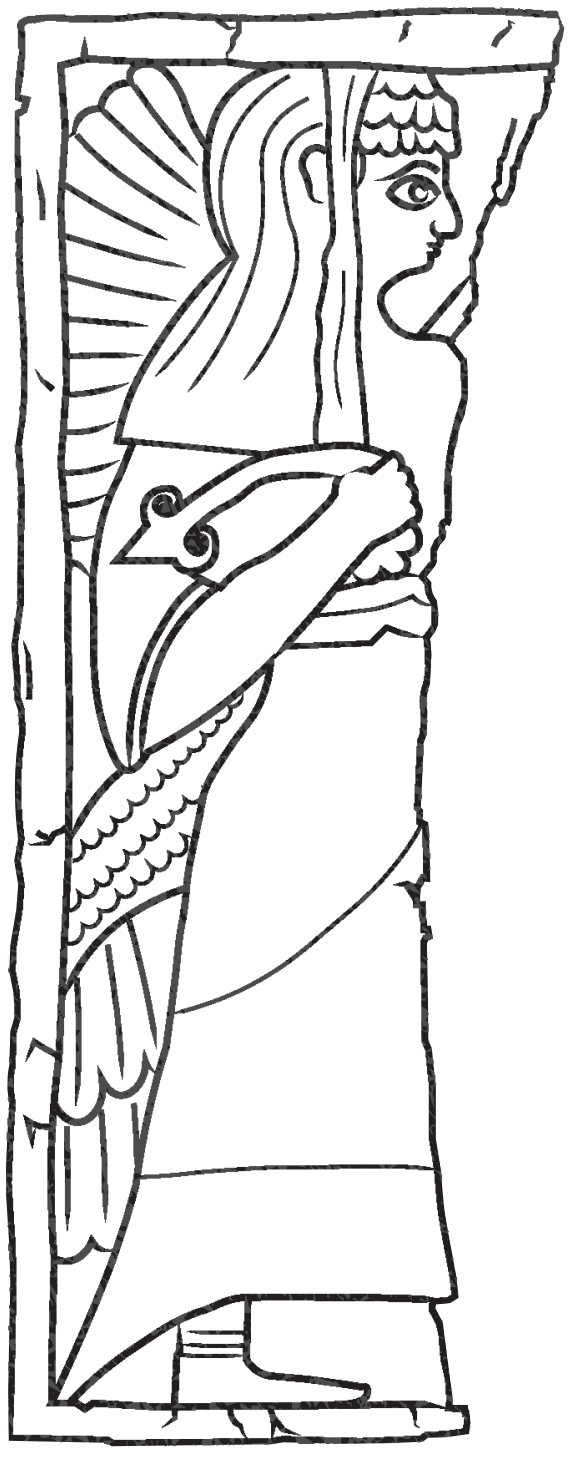

Illustration based on a stone carving on display at the MET 


\section{Chapter 2 \\ Generation of a cellular model to dissect early molecular events leading to DUX4- induced toxicity}

Ator Ashoti, Mirna Baak, Menno Creyghton, Niels Geijsen

Parts of this chapter are available in an adapted form on bioRxiv

Ashoti, A. et al. A genome-wide CRISPR/Cas phenotypic screen for modulators of DUX4 cytotoxicity reveals screen complications. bioRxiv (2020).

doi:10.1101/2020.07.27.223420 


\begin{abstract}
Facioscapulohumeral muscular dystrophy (FSHD) is a complex disease that can be caused by several genetic and epigenetic factors. One such factor is the failure to epigenetically silence the sub-telomeric region of chromosome 4, causing misexpression of the Double homeobox 4 (DUX4) gene. Expression of DUX4 is normally tightly regulated and restricted to the thymus, testis and early cleavage stage embryos. Aberrant expression of DUX4 in skeletal muscle underlies the pathogenesis of FSHD. To gain insight into the pathophysiology of FSHD, we aimed to identify the downstream targets leading to DUX4-induced cytotoxicity and assess if manipulation of downstream targets could potentially mitigate DUX4 cytotoxicity. We developed a cell line that, upon inducible DUX4 expression, recapitulates the FSHD transcriptional signature and ultimately undergoes apoptotic cell death. We also developed a small-scale screening assay to knockout DUX4 target genes that were expressed early after DUX4 induction, in order to test their ability to modulate DUX4-induced cytotoxicity. Thus, we have developed a robust system to investigate the molecular and cellular events that follow DUX4 expression and are causal to the emergence of FSHD.
\end{abstract}




\section{Introduction}

Muscular dystrophies are a group of genetic disorders characterized by progressive loss of muscle strength and muscle degeneration. These diseases often have few treatment options, if any, and current therapies primarily focus on symptom relief, not resolving the underlying cause. Treatments consist of a combination of physical therapy and anti-inflammatory agents aimed at preserving muscle function as much as possible. Facioscapulohumeral muscular dystrophy (FHSD) is one of the most prevalent forms of muscular dystrophy worldwide ${ }^{1}$. FSHD first manifests in muscle groups of the face, effecting speech and facial expression. Patients lose the ability to express emotion which hampers their ability to engage in social interactions. FSHD progresses sequentially and in an asymmetric fashion from the face, the shoulders, upper arms, trunk and the lower extremities ${ }^{2}$. Due to the consequences of muscle weakening in the face, the disorder can affect the patient's societal interactions and can cause significant emotional stress.

A unified model for the underlying genetics of FSHD, published in 2010, demonstrated that a permissive chromosomal background, together with epigenetic de-repression of the D4Z4 locus results in the (mis)expression of the transcription factor double homeobox 4 $(D U X 4)^{3}$ and is the main cause for the development of FSHD. DUX4 is a so-called pioneer transcription factor ${ }^{4,5}$, capable of regulating its target genes independent of their chromatin state. The network of genes activated by pioneer factors is therefore less affected by cellular identity. Indeed, Jones and colleagues have demonstrated that DUX4 activates the same downstream target genes in B lymphocytes as were previously identified in skeletal muscle myoblasts ${ }^{6,7}$.

To explore the molecular mechanisms that trigger DUX4-mediated cytotoxicity, and to explore potential ways to mitigate this toxicity, we generated a cell line in which DUX4 expression can be induced by the addition of doxycycline. We introduced the DUX4 intronexon structure (exons 1-3) involved in FSHD pathogenesis into the adherent KBM7 cell line 8- $^{8-}$ ${ }^{17}$, under control of a doxycycline-inducible promotor, and identified a clone that displayed robust DUX4-dependent cell death upon addition of doxycycline. Using this cell line, we determined the temporal molecular events that are triggered upon doxycycline-mediated DUX4 induction and demonstrate that the transcriptome changes induced by DUX4 in our inducible system are highly similar to those previously identified in myoblasts from FSHD patients ${ }^{18-20}$.

To test the feasibility of using this system to screen for factors that could mitigate DUX4 cytotoxicity, and at the same time test the role of a small list of genes downstream of the DUX4 transcription factor, we developed a small-scale CRISPR/Cas9 screening assay. Genes that were significantly upregulated after inducing DUX4 expression for 4.5 hours were considered early targets of DUX4. We hypothesize that targeting early-induced DUX4 target genes could interfere with the induction of the DUX4 cytotoxic transcriptional network thereby delaying or even abrogating DUX4-induced cell death. Early transcription and cofactors are of particular interest because of their potential role in perpetuating the toxic cascade of events. Our small-scale CRIPSR/Cas9 screen allowed us to test this hypothesis, in a fast and cost-effective manner. 


\section{Results}

\section{Generation and validation of a DUX4 inducible cell line}

To perform large screens, cells should preferably be highly proliferative, be easily transfectable and display a robust and screenable phenotype. An adherent clone of the KBM7 cell-line possess most of these characteristics and has already been used extensively in a wide variety of functional screens ${ }^{8-17}$. The cells were initially near-haploid ${ }^{8,21}$, but subsequently rediploidized ${ }^{22,23}$. These adherent diploid KBM7 cells were used for the generation of our FSHD cell model.

Low levels of DUX4 expression can efficiently induce apoptosis ${ }^{19,24,25}$, which interfered with the generation of our FSHD cell model. To circumvent premature DUX4 toxicity, caused by the leaky expression of the Tet-On system ${ }^{26,27}$, we inserted a LoxP-DsRed-LoxP-stopcassette (LSL) in between the Tet-operator and the DUX4 transgene (Fig. 1A). The DUX4 transgene element itself consisted of the first three exons (starting with the translational start site) and the two introns of the DUX4 gene, including the polyA sequence. This is the same sequence found in the most common pathology-associated haplotype, 4A161 ${ }^{28}$. This construct was introduced in the adherent KBM7 cells in combination with a constitutive rtTA expression construct. After stable integration of the construct, the stop-cassette was removed using CRE recombinase, placing DUX4 under the control of the Tet operator (Fig. 1A). Eighty monoclonal lines were derived by single cell flow-cytometry sorting, of which one displayed tight doxycycline-dependent DUX4 induction and robust cell death upon doxycycline addition (Fig. 1B). A monoclonal cell line was derived from this positive clone, which we named the 'DUX4 Inducible Expression' (DIE) cell line.

To further characterize the DIE cells, we determined the sites of integration of the rTTA/ BlastR and DUX4/PuroR transgenes. Targeted locus amplification (TLA) ${ }^{29}$ was performed and confirmed single integration sites for both the rtTA and DUX4 transgenes (Fig. 1C). The DUX4 cassette integrated into the $\mathrm{p}$-arm of chromosome 19 within the MAST1 gene, and the rtTA cassette integrated into the MGAT4B gene, which is located at the end of chromosome $5 q$. To further analyze the functional effect of DUX4 induction, DIE cells were stained with AnnexinV-Alexa Fluor 488 and propidium iodine (PI) (Fig. S1). As shown in the supplementary videos, DIE cells stained positive for the apoptotic marker AnnexinV during 12 hours of doxycycline exposure. To show that the apoptotic phenotype was dependent on induction of the DUX4 transgene, we knocked out the DUX4 transgene using CRISPR/Cas9. To target the DUX4 transgene, 2 independent guide RNAs (gRNAs) were used, one targeting the C-terminal domain of the DUX4 open reading frame (ORF) and the other close to the polyA tail of DUX4. RT-qPCR and Western blot (WB) analysis of the DIE and the DIE-DUX4 knockout (DIE-KO) cells demonstrated successful knockout of the DUX4 transgene at RNA and protein levels (Fig. 1D). In addition, CRISPR/Cas9 targeting of the DUX4 transgene successfully rescued the DIE cells from apoptosis upon doxycycline administration, demonstrating that apoptosis upon doxycycline induction in the DIE cell line is mediated by DUX4 (Fig. 1E). DUX4 induction in the DIE cells also resulted in induction of its known downstream target genes (LEUTX, ZSCAN4, PRAMFE1 and ZNF217) (Fig. 1F), demonstrating that inducing DUX4 expression induces downstream target genes that are also activated by endogenous DUX4. 

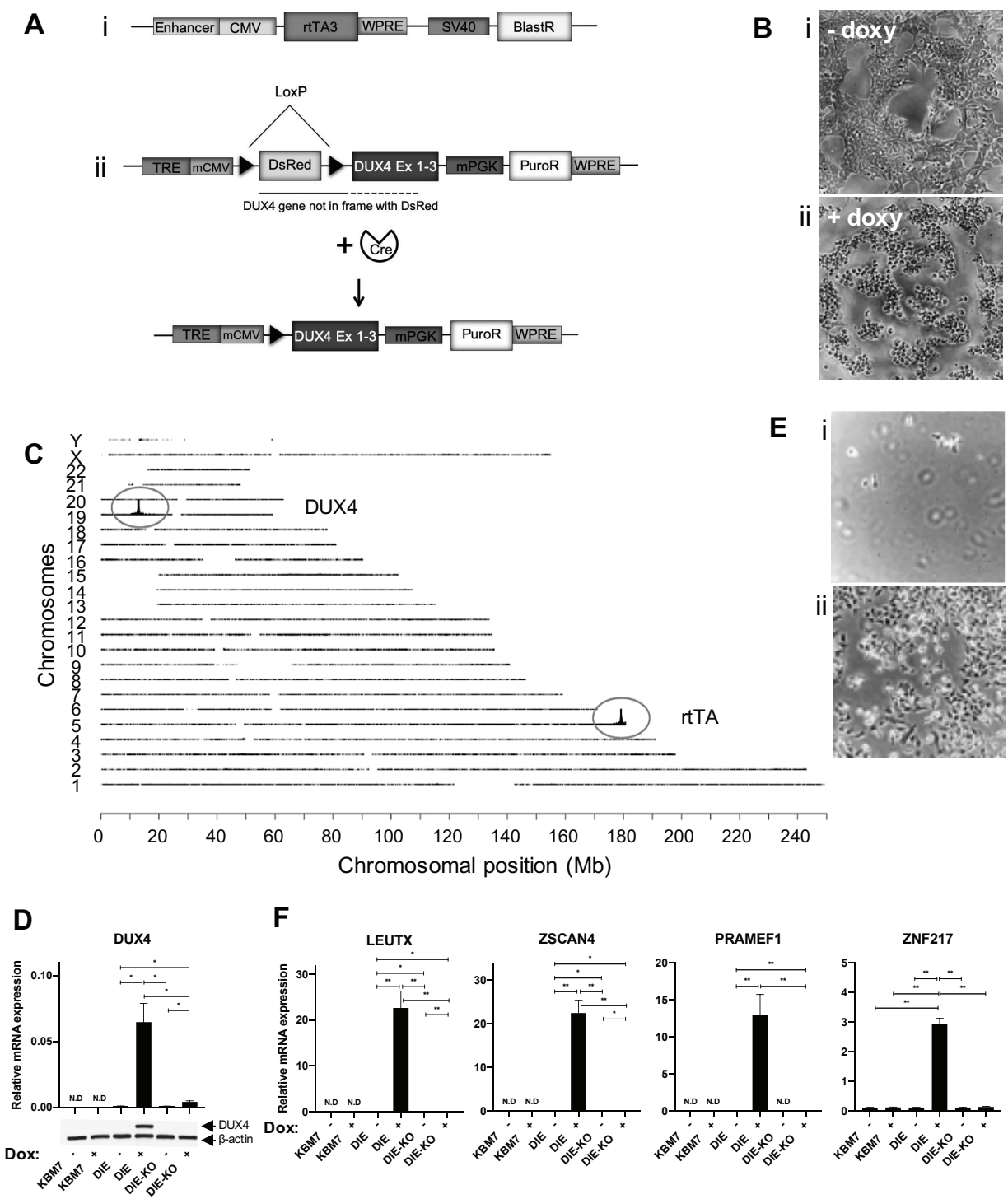

Figure 1. Creation and validation of the DIE cell line. (A) Schematic representation of the i) rtTA construct, ii) the inducible LSL-DUX4 cassettes, and the inducible DUX4 cassette upon removal of the LSL through CRE recombinase. (B) Phase contrast images of DIE cells i) without doxycycline exposure and ii) with $24 \mathrm{~h}$ of doxycycline exposure. (C) Schematic representation of transgene integration sites within human genome, by TLA analysis. The inducible DUX4 cassette maps back to the p-arm of chromosome 19, and the rtTA transgene maps back to the end of the q-arm of chromosome 5. (D) Expression of DUX4 mRNA and protein in the parental KBM7 cells, DIE and DIE-KO cells with or without doxycycline admission, as detected by qRT-PCR (top panel) and western blot analysis (bottom panel), with $\beta$-actin serving as a loading control in the western blot. (E) Phase contrast images of doxycycline exposed DIE cells which were transduced with either i) only Cas9 protein, or ii) Cas9 protein and DUX4 sgRNAs. Dead cells were removed by a DPBS wash to expose the surviving population. (F) mRNA expression of known downstream targets of DUX4 in KBM7, DIE and DIE-KO cells with or without doxycycline admission, as measured by qRT-PCR. The statistical significance in all qRT-PCR data was determined by a two-tailed Student t-test. LSL: LoxPDsRed-stop-LoxP, KO: Knock-out, N.D: not detected. 


\section{DUX4 gene expression signature in DIE cells}

Next we analyzed the downstream transcriptional changes that were induced by DUX4 in the DIE cells by RNA sequencing. We compared 4 induced and uninduced technical replicates of two lines; the DIE line, and the DIE-KO line. As shown in Fig. 2A, DUX4 induction resulted in progressive temporal changes in gene expression. Figure $2 \mathrm{~B}$ shows the magnitude of the combined transcriptional changes induced by DUX4 at different time intervals and schematically emphasizes both the increasing number of differentially transcribed genes as well as the speed at which these transcriptional changes occur over time. Indeed, DUX4 induction results in a profound and progressive increase in the number of differentially expressed genes; with 64 differentially expressed transcripts at 4.5 hours post DUX4 induction and 467 differentially expressed transcripts at 8.5 hours after induction (Fig. 2B and $2 \mathrm{C}$ ). The number of induced genes is greater than those with reduced expression levels as can be seen in Fig. 2B. Differential expression analysis reflects this, demonstrating more differentially upregulated genes in both induced DIE samples compared to uninduced DIE sample [Padj value $\leq 0.01$, absolute Log2foldchange $\geq 1$ ] (Fig. 2B and D, Supplementary Table S1 and S2). Most differentially expressed genes are shared between the two induced samples (Fig. 2C). Among the upregulated genes are well known downstream targets of DUX4, including LEUTX, ZSCAN4, PRAMEF1 and ZNF217 (Fig. 2E). We next used Enrichr ${ }^{30,31}$ to search for other similar studies that show similarities in their transcriptome. Based on Enrichr entries, the upregulated genes in induced DIE cells are linked to DUX4 activity $[$-Log10 (P-value) $=100.3]$, as are the downregulated genes $[-$ Log10(P-value) $=3.8]$ (Fig. $2 \mathrm{~F}$ and Tables S3-4). It shows high similarity between data from our study and one other DUX4 study that has been entered into the Enrichr database (GSE33799) ${ }^{18}$. We next compared the list of differentially expressed genes (DIE_8.5h) with 4 other studies that have previously identified the DUX4 transcriptional network in myoblast models or patients derived muscle biopsies (Geng, Rickard, Jagannathan and Heuvel) ${ }^{18-20,32}$ and observed a high percentage of overlap between datasets. $72 \%$ of the upregulated genes and $52.8 \%$ of the downregulated genes overlap with at least one of 4 datasets (Fig. 2G and Table S5 and S6). In addition, overlapping percentages mentioned here are likely an underrepresentation, due to the presence of gene families containing paralogs and pseudogenes in either reference genome databases, which can lead to multi-mapped or ambiguous reads ${ }^{33}$. To conclude, data shown here strongly suggest that in our DIE cell system, DUX4 induces transcriptional changes similar to those found in myoblasts from FSHD patients.

Figure 2. RNA-sequencing data revealing differentially expressed genes upon DUX4 expression. (A) Heatmap showing differentially expressed genes between samples, with gene clusters (color coded) on y-axis, and samples on the x-axis. (B) Gene density plot demonstrating the effects of DUX4 activation on the transcriptome of the DIE cell line. DUX4 activation results in an increase of differentially expressed genes compared to uninduced DIE cells, as indicated by the bell shape widening and shortening. (C) Venn diagram showing the overlap and the number of differentially expressed genes at $4.5 \mathrm{~h}$ and $8.5 \mathrm{~h}$ of doxycycline induction (Adjusted P-value $\leq 0.01$, and absolute Log2FC $\geq 1$ ). (D) Scatter plots of gene expression (RPM: reads per million) of induced DIE cells versus uninduced DIE cells. Left two panels demonstrate uninduced DIE cells (DIE_Oh) on the x-axis versus uninduced or induced DIE-KO samples (KO_Oh and KO_8.5h) on the $\mathrm{y}$-axis. Right two panels compare the uninduced DIE cells with induced DIE samples (4.5h and $8.5 \mathrm{~h})$. Green and red dots represent the differentially expressed genes with an Adjusted P-value $\leq 0.01$, and absolute $\log 2 \mathrm{FC} \geq 1$. Green dots represent upregulated genes, and the red dots represent downregulated genes. (E) Count plots showing unique molecular identifier (UMI) and between sample normalized transcript counts of 4 known DUX4 targets genes: LEUTX, ZSCAN4, PRAMEF1 and ZNF217, in uninduced and induced DIE and DIE-KO cells. Every sample shows 4 dots, representative of the 4 technical replicates. (F) Transcription factor (TF) perturbations analysis identifying transcription factors that are linked to the i) upregulation and ii) downregulation of the differentially expressed genes found in this study. Activation: OE or ACTIVATION. 
Inhibition: KO, KD, SIRNA, SHRNA, INACTIVATION, or INHIBITION. (G) Quintuple Venn diagram comparing DUX4 i) upregulated and ii) downregulated genes found in this study (Ashoti) to those found in previous transcriptomic studies (Geng with P-value $\leq 0.01$, FDR $\leq 0.05$, abs L2FC $\geq 1$; Rickard with Padj value of $<0.005$ and abs $L 2 F C>2$; Jagannathan with P-value $\leq 0.01$, FDR $\leq 0.05$, abs L2FC $\geq 1$, Heuvel with P-value $\leq 0.005, F D R \leq 0.05$, abs $L 2 F C \geq 1$ ). See supplementary material Tables 5 and 6.
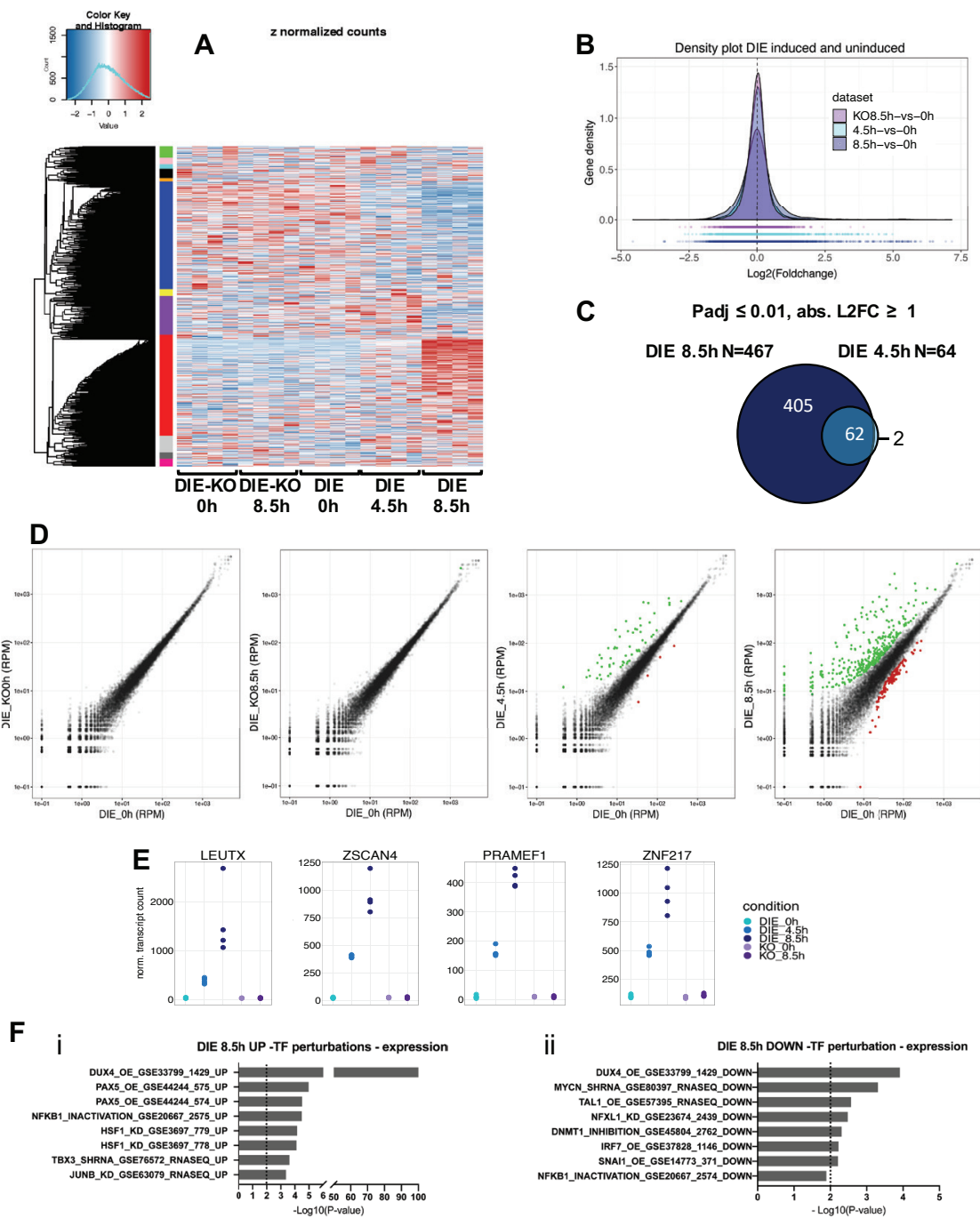

G

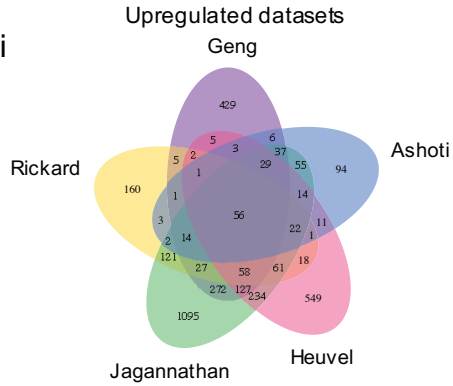

ii

Downregulated datasets

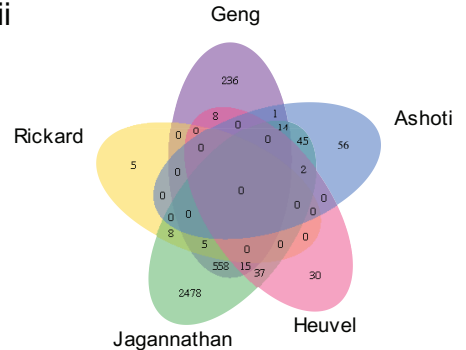




\section{DUX4 induces an early embryonic transcription factor network}

We noticed that the list of early DUX4-affected genes contains a relatively high number of transcription- and co-factors (19 out of 64 differentially-expressed genes, Table 1), more than could be expected based on random distribution, since transcription factors and co factors comprise only between $11-13.5 \%$ of all protein coding genes in the human genome. DUX4 is a pioneer factor that is normally expressed during early, preimplantation embryonic development ${ }^{34,35}$. Figure $3 \mathrm{~A}$ displays the expression of DUX4 in oocytes, zygotes and cleavage-stage embryos as well as later stages of pre-implantation development. As shown, DUX4 peaks at the 4-cell stage, and quickly wanes thereafter. When analyzing the DUX4 target genes identified in our transcriptome analysis, we noticed that many of them are also specifically expressed in preimplantation embryos ${ }^{36} .57$ out of 60 genes which were upregulated after $4.5 \mathrm{hrs}$ of DUX4 expression, overlapped with the single-cell sequencing (SCS) dataset of pre-implantation embryo development ${ }^{36}$ and are specifically expressed at distinct, early stages of embryonic development (Fig. 3B and S2). Moreover, the expression of 43 DUX4-induced genes increase or peak at the 8-cell stage, which suggest and corroborates that DUX4 induction at the 4-cell stage is regulating the expression of many of these early genes. Figure $\mathrm{S} 3$ also validates that this increase or peak in expression is linked to DUX4 induction and is not due to a general increase in transcription around the 8-cell stage, as common housekeeping genes demonstrate a different expression pattern throughout development.

Table 1. DUX4 differentially expressed transcription- and co-factors

\begin{tabular}{|l|l|l|}
\hline \multicolumn{2}{|l|}{ Fene } & \multicolumn{1}{l|}{ Expression peak Stage } \\
\hline ZSCAN4 & transcription & 8-cell \\
\hline ZNF217 & transcription & 8-cell, Morulae, Epiblast \\
\hline ZNF296 & transcription & 8-cell \\
\hline LEUTX & transcription & 8-cell \\
\hline ZNF622 & transcription & Morulae \\
\hline ZNF574 & transcription & 4-cell \\
\hline DUXA & transcription & 8-cell, Morulae \\
\hline HOXB2 & transcription & 8-cell, Primitive-endoderm \\
\hline SNAI1 & transcription & 8-cell \\
\hline ZNF705A & transcription & 8-cell \\
\hline OSR2 & transcription & Oocyte, 4-cell, Morulae \\
\hline CCNA1 & co & 8-cell \\
\hline HSPA1A & co & Oocyte, 4-cell \\
\hline GTF2F1 & co & Oocyte to Morulae \\
\hline HSPA1B & co & 4-cell \\
\hline MED26 & co & 8-cell \\
\hline ID1* & transcription & hESC \\
\hline ID3* & transcription & hESC \\
\hline HES7* & transcription & Morulae \\
\hline SIgnificant & Thegulated factors in \\
\hline
\end{tabular}

* Significant down regulated factors in induced DIE cells 
A

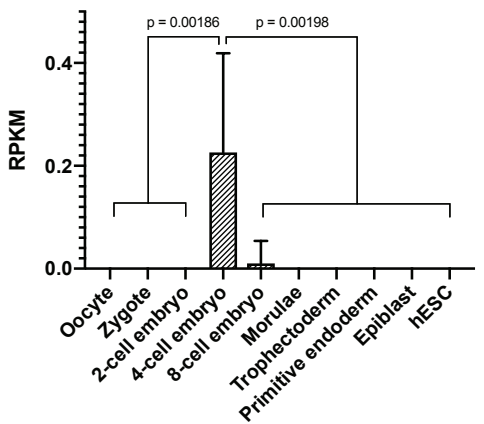

B Stacked expression DUX4 target genes

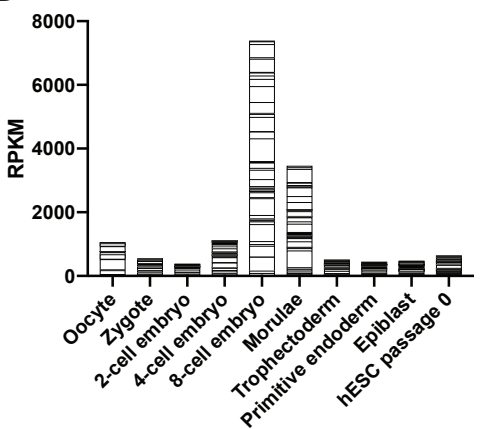

Figure 3. Expression of DUX4 and DUX4 early target genes during pre-implantation development. (A) DUX4 expression in preimplantation embryos. DUX4 expression is significantly upregulated at the 4-cell embryonic stage. Single cell RNA-seq data from preimplantation embryo's is from Yan et al ${ }^{36}$. Statistical significance was determined by a two-tailed Student t-test. (B) A graph demonstrating the stacked expression of 54 DUX4 activated target genes identified by RNA-seq, in reads per kilobase per million (RPKM). The genes KHDC1L, DPPA3 and RGS2 were excluded due to disproportional high expression, which would otherwise skew the data (see Fig. S2C). hESC: human embryonic stem cells.

\section{Screening for factors which modulate DUX4 cytotoxicity}

To test if any of these differentially upregulated genes contribute to DUX4-induced cytotoxicity, we developed a small-scale CRIPSR/Cas9 screening assay to quickly, efficiently and cost-effectively screen up to a few hundred sgRNAs. To set up this screening platform, a CRISPR/Cas9 reporter line was used for an easy and quantifiable read out to track the effectiveness of screening conditions. The reporter line consists of a constitutively expressed out of frame non-fluorescent dTomato gene, with an AAVSI target site directly upstream of the dTomato coding sequence (Fig. 4). Targeting the AAVSI sequence with CRISPR/Cas9 induces a frame shift, restoring the reading frame between the ATG start and the dTomato transgene, thereby activating dTomato fluorescence in the target cells ${ }^{37}$. AAVSI sgRNA was generated by in-vitro transcription from a dsDNA template that was created by annealing two ssDNA oligomers and filling in the overhangs by PCR (Fig 5A\&B). The dsDNA template was then used to generate the sgRNA by in-vitro transcription from the T7 RNA-polymerase promotor. Since the sgRNA mixture containing both PCR and IVT components was to be directly transduced into the reporter cells together with Cas9 protein (Fig. 5C), we tested different ratios of PCR and IVT mixtures, to identify which mixture minimally impacted growth and survival of the cells upon transduction (Table 2). Figure 5D shows the toxic effect of the indicated mixtures on adherent KBM7 reporter cells. Cell growth and survival could be further enhanced, by reducing the overall osmolarity of the transduction mixture (Figure 5D, bottom panel). Quantification of dTomato expression by FACS analysis confirmed these results, demonstrating improved survival and efficiencies when the PCR mixture was diluted more. Reduction of the osmolarity of the transduction mixture and reduction of the transduction time, from 45 to 35 minutes further enhanced cell survival (Figure 5E). The optimized experimental setup was subsequently used to screen the 60 upregulated genes found during RNA seq analysis. Short $57 \mathrm{nt}$ oligomers with gene specific spacer sequences were used to generate 3 different sgRNA per gene (Table S7). The spacer sequences were designed using the GPP sgRNA designer too ${ }^{38,39}$. Guides targeting the DUX4 transgene were used as positive controls. While the screen worked technically, based on cell survival seen in 
the positive controls, no increased viability was observed in the other targeted DIE samples (Data not shown). These results imply that knocking-out any of these 60 "early" genes individually was insufficient to mitigate DUX4 cytotoxicity.

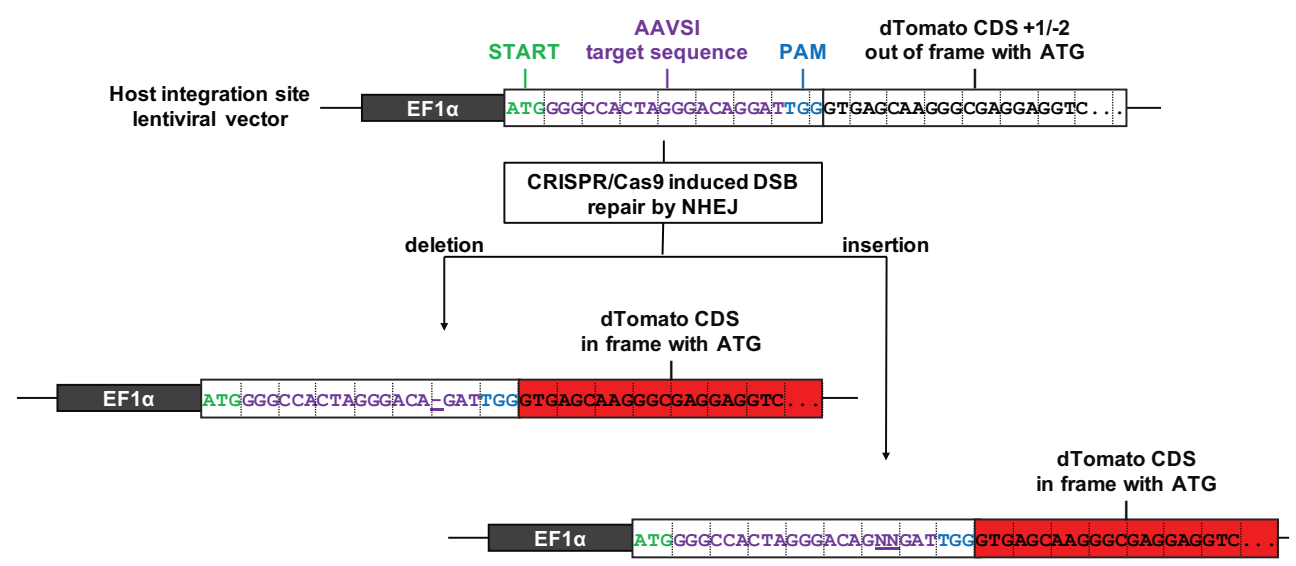

Figure 4. Schematic representation of the CRISPR/Cas9 reporter line. The stable integrated lentiviral vector contains an elongation factor-1 alpha (EF1 $\alpha$ ) promoter, which regulates the expression of the dTomato gene. The out of frame dTomato sequence contains an AAVSI targeting sequence right between the start codon (ATG), and the dTomato coding sequence (CDS). Due to the presence of one additional nucleotide or the absence of 2 nucleotides (+1/-2) in the AAVSI sequence, the last nucleotide of the AAVSI sequence forms a codon with the first two nucleotides of dTomato CDS, putting the dTomato out of frame. Cas 9 targeting of the AAVSI sequence can cause indels of different sizes, which can put the dTomato gene back in frame.

Table 2. IV-RT reactions with different amount of PCR mixture

\begin{tabular}{|l|c|c|c|c|}
\cline { 2 - 5 } \multicolumn{1}{c|}{} & \multicolumn{4}{c|}{ PCR reaction dilutions } \\
\hline Nuclease free water & $\mathbf{1 . 5 x}$ & 10 & 20 & 25 \\
\hline T7 10x reaction buffer & 0 & 5 & 5 & 5 \\
\hline $25 \mathrm{mM}$ NTP mix & 5 & 10 & 10 & 10 \\
\hline $5 \mu \mathrm{M}$ T7 & 10 & 5 & 5 & 5 \\
\hline Template DNA (PCR mix) & 30 & 20 & 10 & 5 \\
\hline Total volume (in ul) & 50 & 50 & 50 & 50 \\
\hline
\end{tabular}

Figure 5. Set up of a fast and efficient CRISPR/Cas9 small-scale screen. A and B) A schematic representation of the generation of the dsDNA template used for the production of sgRNAs. A) The 57nt single stranded (ss) DNA oligo containing a T7 promotor sequence, a guanine nucleotide, a spacer sequence, and a short piece of the tracr sequence, is annealed to a 76nt ssDNA oligomer containing the full tracr sequence. B) A PCR reaction fills up the $5^{\prime}$ overhangs, generating a double stranded DNA template for in-vitro transcription (IVT). C) A schematic representation of the CRISPR/Cas9 iTOP transduction procedure. The dsDNA template and the sgRNA are made in a consecutive manner in the same tube/container. The PCR/IVT mixture is subsequently supplemented with transduction media and protein to produce the transduction solution. The transduction solution is added to cells. After the 35-45 min transduction period, the size of the cells will be temporarily reduced due to the loss of water. D) Bright field and fluorescent microscopy images of the KBM7 dTomato reporter cells transduced with Cas9 and sgRNA. The top panels show the controls. The positive controls are the dTomato reporter cells transduced with 
Cas9 and purified sgRNA. The negative control are the same reporter cells transduced with Cas 9 only. Panels below show the reporter cells which were transduced with purified Cas9 and various conditions of unpurified IVT sgRNA. E) FACs analysis showing the amount of living cells and percentage of dTomato positive cells of each of the tested condition.

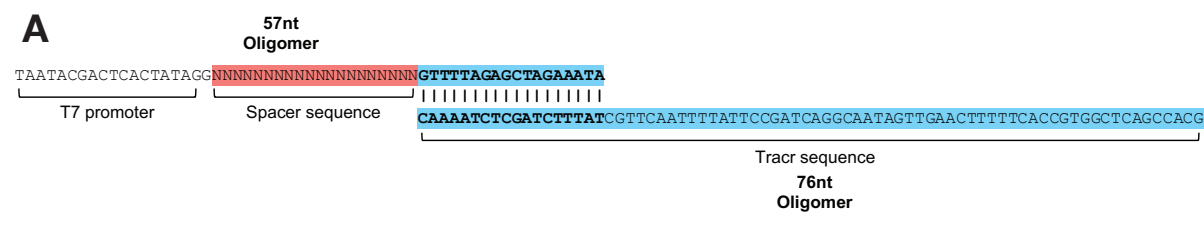

B
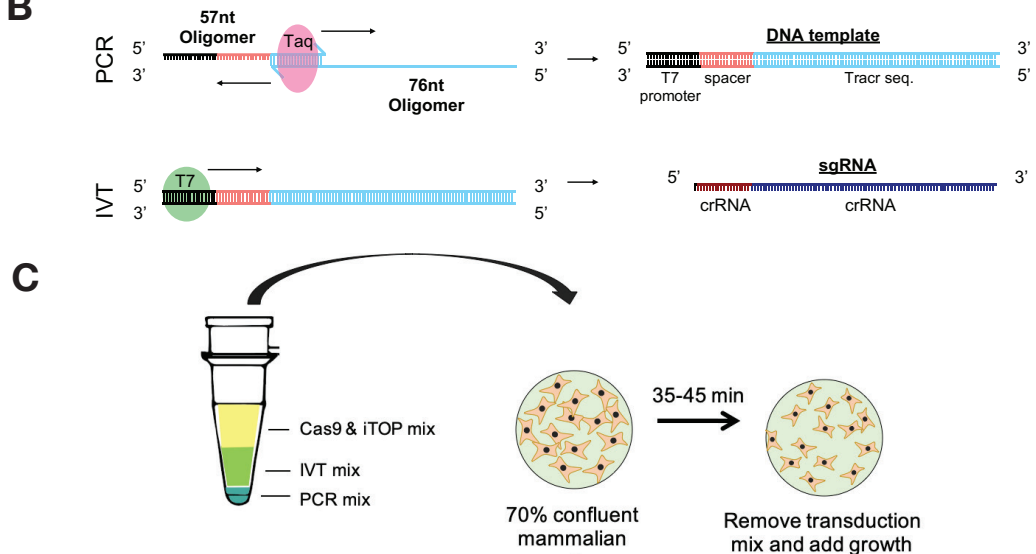
D
cells
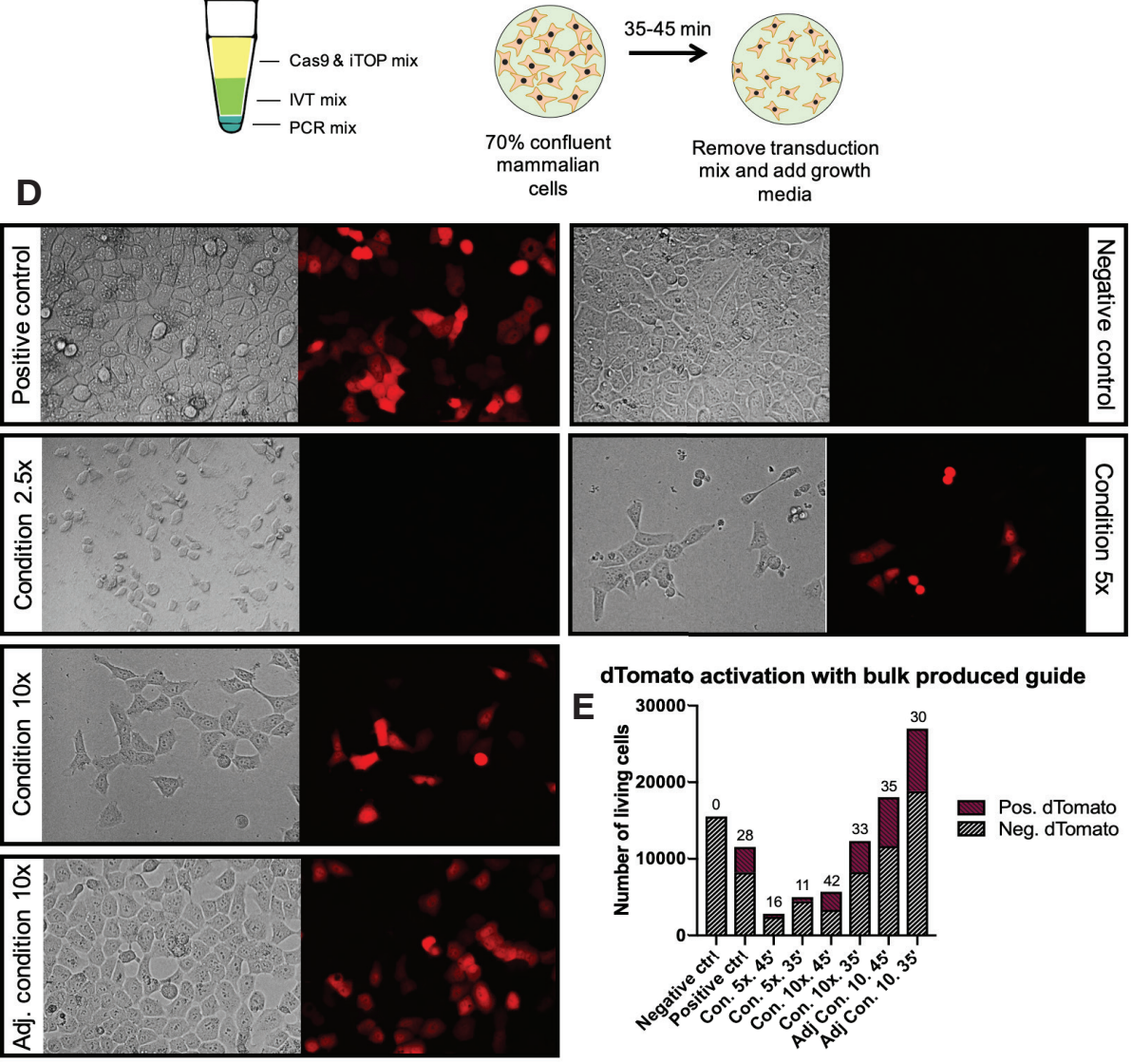


\section{Discussion}

The lack of effective treatment for FSHD is, among other things, due to the complex nature of FSHD and incomplete understanding of the underlying molecular mechanism that initiates upon DUX4 expression and the sequential chain of events that ensues. To uncover the cytotoxic mechanisms induced by DUX4, we generated a DUX4-inducible cell line (DIE cell line). Since even sporadic, intermittent and low DUX4 expression has been shown to be sufficient to cause profound muscle degeneration ${ }^{19,24,25}$, the potent cytotoxic effect of DUX4 is evident. This toxicity proved to be a big hurdle when generating the inducible DUX4-expressing line, ultimately resulting in a single clone out of 80 that displayed tight, DUX4-mediated apoptosis in a doxycycline-dependent manner. Many known DUX4 target genes are induced upon DUX4 induction in these DIE cells, despite their myeloid leukemia cellular background, supporting DUX4's role as a pioneer factor. Furthermore, many induced target genes encode transcription factors and cofactors, which in turn can activate their transcriptional program, potentially continuing and exacerbating the cytotoxic cascade.

With our inducible in vitro model for DUX4 cytotoxicity, we set out to investigate the underlying mechanism by which DUX4 expression leads to cell death. We developed and employed a small-scale CRIPSR/Cas9 screening assay. This assay allowed us to quickly and cost-effectively screen 183 sgRNAs, targeting 61 genes, including DUX4 itself (Table S7). Because the guides are screened individually, it allowed us to directly assess the effect of individual genes downstream of DUX4. The developed screening method has the advantage that it does not involve any cloning steps, or the generation of a viral library. Combined, these properties make this screening tool fast, effective and cost-efficient. The small-scale screening assay was deployed to screen 60 early DUX4 targets, to test the hypothesis that knock-out of early genes holds more potential for modulating DUX4 toxicity, as interference would occur early in the DUX4 induced cascade. However, other than knocking out DUX4 itself, none of the other 60 targeted genes showed an effect on the DUX4 induced cytotoxicity. These results are indicative of the complex nature of FSHD, which is most likely mediated by more than one gene, acting up and/or downstream of DUX4.

This cell model did however demonstrate the effectiveness of these types of screening assays, which has provided us another tool in the toolkit for future small-scale screening purposes, or follow up experiments.

Here, we established a system that will allow us to identify cellular events and gene expression over time. This inducible system allows us to simultaneously control the amount of DUX4 expression, the timing of DUX4 induction and the length of the induction time. We have shown that the downstream transcriptional changes that follow DUX4 expression in the DIE cell line, greatly overlap with reported data of FSHD myoblasts, demonstrating that the DIE cell system recapitulates the molecular events underlying the disease. Given the complex nature of FSHD, this system enables us to obtain a more thorough insight into the temporal sequence of events that occur downstream of DUX4, which is the focus in chapter 3. This versatile model can furthermore be used to develop targeting strategies aimed at the DUX4 gene, as this system contains the genomic sequence (three exons and two introns) of DUX4, which is the focus in chapter 5 . Ultimately, this system will allow us to develop targeting strategies and/or identify molecular events that are relevant for the pathogenesis of FSHD. 


\section{Methods}

\section{Cloning and generating the DIE cell line}

To generate the inducible DsRed/DUX4 system, the third generation lenti-viral plasmid pRRLsincPPT-wpre ${ }^{40}$ was used as the backbone. The linearized viral backbone was created by restriction digestion using $\mathrm{Hpal}$ and Sall (NEB). All inserts were generated with PCR amplification using phusion DNA polymerase (Fischer Scientific). Insert were created with $15 \mathrm{bp}$ adapter sequences, matching the backbone or neighboring fragments, for in-fusion cloning (Clonetech). The first fragment consisted of cPPT/CTS-TRE-mCMV sequences, and the second fragment contained the LoxP-DsRed-LoxP (LSL) sequence. After inserting these two fragments into the pRRLsincPPT-wpre backbone, this newly cloned construct was transformed into chemically competent Stbl3 Escherichia coli (E.coli). The plasmid was isolated and purified from the Stbl3 cells using the HiPure plasmid kits from Invitrogen (Fischer scientific). This TRE-LSL plasmid was then digested with Xbal and EcoRI (NEB) after which the remaining three inserts: DUX4 (exon1-3), mPGK and PuroR-WPRE, were cloned downstream from the LoxP-DsRed-LoxP in similar fashion.

The DIE cell line was obtained by transducing diploid KBM7 cells with lentiviral particles containing the inducible DsRed/DUX4 cassette mentioned above. 2 days after lentiviral transduction, transfected cells were selected with puromycin. After establishing a stable line by puromycin selection, lentiviral particles containing CMV-rtTA3-BlastR were added to the DsRed/DUX4 containing KBM7 cells. Positively transfected cells were subsequently selected with blasticidin, and FACs sorted for DsRed expression after exposure to doxycycline. The pLenti CMV rtTA3 Blast (w756-1) plasmid was a gift from Eric Campeau (Addgene plasmid \#26429, http://www.addgene.org/browse/article/3669/).

\section{Cell culturing}

The KBM7 cells that were used to create the DIE line were near-haploid ${ }^{8,21}$. Haploids cells are however unstable and do not remain haploid (reviewed in Yilmaz et al.) ${ }^{41}$ and rediploidize ${ }^{22,23}$. KBM7 cells were cultured in IMDM media (Fischer Scientific) and 10\% FBS. The DIE cells were cultured in IMDM media with $10 \%$ Tet system-approved FBS (Clontech), supplemented with $5 \mu \mathrm{g} / \mathrm{ml}$ Puromycin and $6 \mu \mathrm{g} / \mathrm{ml}$ Blasticidin. For transduction experiments, well from a 96-wells plate were coated with Matrigel coated wells (Matrigel in PBS 1:250). 15.000 cells were seeded on top of the coated wells and incubated overnight $(\mathrm{O} / \mathrm{N})$ at $5 \% \mathrm{CO}_{2}$, and $37^{\circ} \mathrm{C}$, until $70-80 \%$ confluency was reached.

\section{Doxycycline titration curve}

200.000 cells were seeded into wells of a 24 -wells plates and kept at $5 \% \mathrm{CO}_{2}$, and $37^{\circ} \mathrm{C}$ until they reached a confluency of $90-100 \%$. Different concentrations of doxycycline were added to the vertical lanes $(100 \mathrm{ng} / \mathrm{ml}, 250 \mathrm{ng} / \mathrm{ml}, 500 \mathrm{ng} / \mathrm{ml}, 750 \mathrm{ng} / \mathrm{ml}, 1000 \mathrm{ng} / \mathrm{ml}$ ), with the horizontal lanes experiencing different exposure times (48h, 36h, 24h, 12h). After the doxycycline exposure, wells were washed with DPBS and were given a recovery period of 96. Surviving cells were subsequently stained using Giemsa modified staining solution (See paragraph viability staining).

\section{Viability staining}

Tissue culture (TC) plates containing cultured cells were washed with DPBS, and fixed with 
$100 \%$ Methanol for 10 minutes. Giemsa stain modified solution (Sigma) was subsequently added for 45 minutes, after which it was removed and the wells were washed with demineralized water.

\section{Protein extraction and Western blot}

DIE cells were harvested by trypsinization and lysed in RIPA buffer. Total protein concentrations were determined using a Pierce BCA protein assay kit (Fischer Scientific). 20ug protein was denatured using $4 x$ Laemmli sample buffer (Bio-rad) with $10 \%$ BME (Sigma), and boiled for 5 minutes. Samples were run on a 15\% SDS-polyacrylamide gel and transferred to a PVDF membrane (Merck). Membranes were blocked for an hour using $5 \%$ BSA in TBST, and were subsequently incubated overnight with anti-DUX4 antibody [E5-5] (Abcam, ab124699) in blocking solution (5\% BSA in TBST), at $4^{\circ} \mathrm{C}$. Membranes were than incubated for an hour with Secondary goat anti-rabbit-HRP antibody (Santa Cruz, sc-2004), and primary rabbit mAb $\beta$-Actin HRP conjugated antibody (Cell signaling, 5125s) in blocking buffer. Chemiluminescent signal was detected using GE ImageQuant LAS 4000 imager, using Pierce ECL Plus Western Blotting substrate (Fischer Scientific).

\section{RNA extraction and RT-qPCR}

Cultured cells were rinsed with DPBS just prior to the additional of TRIzol reagent (Thermo Scientific). Total RNA samples were subsequently extracted by addition chloroform, and isopropanol precipitation, and finally treated with RNase free DNase I (Promega). Reverse transcription was performed using the Superscript III kit (Invitrogen) and random primers (Promega), generating CDNA. Quantitative PCR was then initiated using IQ SYBR Green Supermix (Bio-Rad 1708880), 50 ng of cDNA, and the following gene-specific primers:

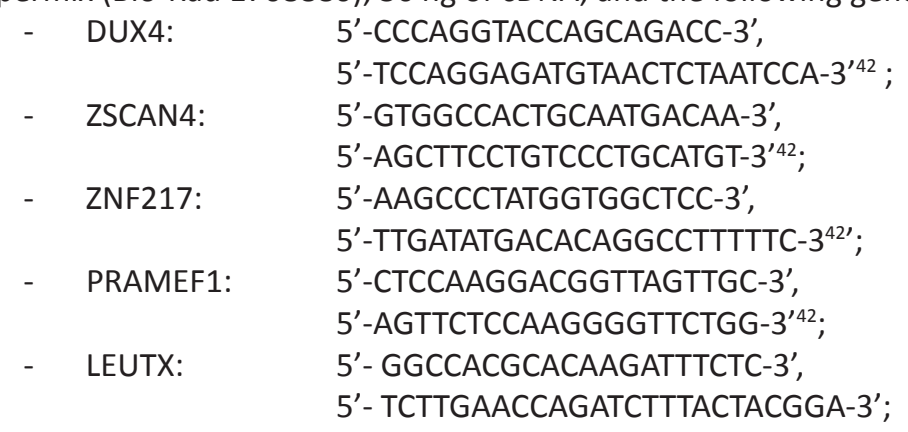

Data were normalized to HPRT expression by using the following primer pair: 5'CCTGGCGTCGTGATTAGTGA-3', 5' - CGAGCAAGACGTTCAGTCCT-3'43.

\section{Live imaging}

DIE cells were seeded into an 8-chamber coverslip slide (Ibidi) 24-36 hours prior to imaging. Growth media was supplemented with 1:50 Annexin5-Alexa Fluor 488, 1:100 Propidium lodide and $1 \mathrm{ug} / \mathrm{ml}$ doxycycline, and imaged for $12 \mathrm{~h}$ with a Confocal Zeiss LSM 700 microscope at $37^{\circ} \mathrm{C}$ and $\% \mathrm{CO}_{2}$.

\section{RNAseq sample preparation and sequencing}

Cultured cells were rinsed with DPBS just prior to the additional of TRIzol reagent (Thermo Scientific). Total RNA samples were subsequently extracted by addition chloroform, and 
isopropanol precipitation. The library prep was performed using CEL-seq1 primers ${ }^{44}$ and the Life technologies Ambion kit $(\mathrm{AM} 1751)^{45}$, and were processed using CEL-seq2 protocol ${ }^{46}$. Samples were sequenced using Illumina Nextseq 500, 2x75 kit, high output. Four technical replicates per samples were send for sequencing, and were sequenced to an average of 600.000 reads per replicate (combined read count of 2.4 million reads per sample). Differential expression analysis was done using the DESeq2 package ${ }^{47}$.

\section{Small-scale CRISPR-Cas9 screen by iTOP}

Large quantities of 180 sgRNAs were generated by producing a dsDNA template for each sgRNA. For this, a short ssDNA fragment containing the gene-specific spacer sequence was annealed to a longer ssDNA fragment containing the complete complementary sequence of the spCas9 tracr. The short ssDNA piece contains a T7 promotor, an additional guanine nucleotide, the 20nt spacer sequence, and the first 19nt of the spCas9 tracr sequence: $5^{\prime}$ TAATACGACTCACTATAGG-20nt-GTTTTAGAGCTAGAAATAG-3'. This short ssDNA fragment was annealed to a longer ssDNA piece containing the complete complementary sequence of the spCas9 tracr:5'-GTTTTAGAGCTAGAAATAGCAAGTTAAAATAAGGCTAGTCCGTTATCAACTT GAAAAAGTGGCACCGAGTCGGTGC-'3. The shorter variable pieces of ssDNA were supplied in a 96-well plate. The longer ssDNA fragment containing the anti-sense tracr sequence was mixed in a 1:1 ration to all wells containing the variable short ssDNA Oligomer. The two fragments are able to anneal to each other due to 19nt tracr sequence that is complementary between the two ssDNA fragments, which serves as a primed region for amplification. Taq DNA polymerase (Thermo scientific) was used to fill up the ss overhangs, thereby creating a dsDNA template for IV-RT reaction. IV-RT kit used for the generation of sgRNA was supplied by NTRANS technologies. The total volume of the PCR reaction did not exceed $5 \mu \mathrm{l}$, and was diluted $10 \mathrm{x}$ in the IV-RT mixture, that consisted of nuclease free water, 1x T7 RNA polymerase reaction buffer, 5nM of each NTP (Jenabioscience), and 500nM of T7 RNA polymerase. The IV-RT reaction mixture was incubated overnight (12-15h) at $37^{\circ} \mathrm{C}$. Residual DNA was removed by the addition of $2 \mathrm{U}$ Turbo DNAse (Fischer scientific) per sample, and incubation at 37 $\mathrm{C}$ for $30 \mathrm{~min}$. The DNAse was inactivated by an incubation step of $65 \square$ C for $10 \mathrm{~min}$. Cas9 protein (in 5x Transduction buffer) and adjusted CRISPR/Cas9 transduction media was added to the newly synthesized sgRNA in appropriate volumes (See Table 3 for the composition of the adjusted CRSPR-Cas9 media). Half of the transduction mixture was added to $70-80 \%$ confluent cells, that were plated out a day before on Matrigel coated 96-wells plates. The cells were exposed to the transduction mixture for $40-45 \mathrm{~min}$, after which the mix was removed and normal growth media was added gently, completely filling up the well to dilute out remaining transduction mixture. After a recovery period of $72-96$ hours, $1000 \mathrm{ng} / \mathrm{ml}$ of doxycycline was added for $24 \mathrm{~h}$. The doxycycline media was subsequently removed, the wells were washed with DPBS to remove the majority of dead/ floating cells, normal growth media was added to the cells and all plates were then placed back into the $5 \% \mathrm{CO}_{2}$, and $37^{\circ} \mathrm{C}$ incubator for 2-4 days. This allowed the remaining cells that have started the apoptotic process to perish, or let surviving cells grow out and therefore become more visible.

\section{Flowcytometry sorting (FACS) and analysis}

dTomato reporter cells were trypsinized using $0.25 \%$ Trypsin-EDTA, then resuspended in iMDM media with $10 \%$ FBS and DAPI nuclear stain. Cells were subsequently strained using Cell-strainer capped tubes (Falcon) and analyzed using the BD FACSCanto II. 


\section{Data Resources}

Data containing the bulk RNA sequencing samples in quadruplicate are available from the GEO data base, accession number: GSE154649.

Table 3. Adjusted CRISPR-Cas9 transduction media (5 ml)

\begin{tabular}{|l|l|}
\hline Compound & amount \\
\hline GABA & $208 \mathrm{mg}$ \\
\hline $5 \mathrm{M} \mathrm{NaCl}$ & $550 \mathrm{ul}$ \\
\hline $100 x$ Glutamine & $75 \mathrm{ul}$ \\
\hline $100 x$ non-essential amino acids & $75 \mathrm{ul}$ \\
\hline $100 x$ N2 supplement & $75 \mathrm{ul}$ \\
\hline $50 x$ B27 supplement & $150 \mathrm{ul}$ \\
\hline Opti-MEM & $3780 \mathrm{ul}$ \\
\hline $100 u g / m l ~ E G F$ & $5 u l$ \\
\hline $100 \mathrm{ug} / \mathrm{ml} \mathrm{bFGF}$ & $10 \mathrm{ul}$ \\
\hline MiliQ & $98 \mathrm{ul}$ \\
\hline
\end{tabular}

\section{References}

1. Deenen, J. C. W. et al. Population-based incidence and prevalence of facioscapulohumeral dystrophy. Neurology 83, 1056-1059 (2014).

2. Tawil, R. \& Van Der Maarel, S. M. Facioscapulohumeral muscular dystrophy. Muscle and Nerve 34, 1-15 (2006).

3. Lemmers, R. J. L. F. et al. A unifying genetic model for facioscapulohumeral muscular dystrophy. Science 329, 1650-1653 (2010).

4. Choi, S. H. et al. DUX4 recruits p300/CBP through its C-terminus and induces global H3K27 acetylation changes. Nucleic Acids Res. 44, 5161-5173 (2016).

5. Vuoristo, S. et al. DUX4 regulates oocyte to embryo transition in human. Biorxiv (2019) doi:http://dx.doi.org/10.1101/732289.

6. Jones, T. I., Himeda, C. L., Perez, D. P. \& Jones, P. L. Large family cohorts of lymphoblastoid cells provide a new cellular model for investigating facioscapulohumeral muscular dystrophy. Neuromuscul. Disord. 27, 221-238 (2017).

7. Banerji, C. R. S., Zammit, P. S. \& Panamarova, M. Lymphocytes contribute to DUX4 target genes in 1 FSHD muscle biopsies. Biorxiv (2019) doi:http://dx.doi.org/10.1101/717652.

8. Mulherkar, N. et al. Ebola Virus entry requires the cholesterol transporter Niemann-Pick C1. Nature 477, 340-343 (2012).

9. Papatheodoroua, P. et al. Lipolysis-stimulated lipoprotein receptor (LSR) is the host receptor for the binary toxin Clostridium difficile transferase (CDT). Proc. Natl. Acad. Sci. U. S. A. 108, 16422-16427 (2011).

10. Jae, L. T. et al. Deciphering the Glycosylome of Dystroglycanopathies Using Haploid Screens for Lassa Virus Entry. 340, 479-483 (2014).

11. Jae, L. T. et al. Lassa virus entry requires a trigger-induced receptor switch. Science $\mathbf{3 4 4}$, 1506-1510 (2014).

12. Blomen, V. A. et al. Gene essentiality and synthetic lethality in haploid human cells. Science 350, 1092-6 (2015).

13. Rong, Y. et al. Genome-wide screening of genes required for glycosylphosphatidylinositol biosynthesis. PLoS One 10, 1-18 (2015).

14. Wang, T. et al. Identification and characterization of essential genes in the human genome. Science 350, 1096-1101 (2015).

15. Gerhards, N. M. et al. Haploid genetic screens identify genetic vulnerabilities to 
microtubule-targeting agents. Mol. Oncol. 12, 953-971 (2018).

16. Luteijn, R. D. et al. A Genome-Wide Haploid Genetic Screen Identifies Heparan SulfateAssociated Genes and the Macropinocytosis Modulator TMED10 as Factors Supporting Vaccinia Virus Infection. J. Virol. 93, (2019).

17. Mezzadra, R. et al. SLFN11 can sensitize tumor cells towards IFN- - -mediated T cell killing. PLoS One 14, 1-16 (2019).

18. Geng, L. N. et al. DUX4 Activates Germline Genes, Retroelements, and Immune Mediators: Implications for Facioscapulohumeral Dystrophy. Dev. Cell 22, 38-51 (2012).

19. Rickard, A. M., Petek, L. M. \& Miller, D. G. Endogenous DUX4 expression in FSHD myotubes is sufficient to cause cell death and disrupts RNA splicing and cell migration pathways. Hum. Mol. Genet. 24, 5901-5914 (2015).

20. Van Den Heuvel, A. et al. Single-cell RNA sequencing in facioscapulohumeral muscular dystrophy disease etiology and development. Hum. Mol. Genet. 28, 1064-1075 (2019).

21. Andersson, B. S. et al. Ph-positive chronic myeloid leukemia with near-haploid conversion in vivo and establishment of a continuously growing cell line with similar cytogenetic pattern. Cancer Genet. Cytogenet. 24, 335-343 (1987).

22. Carette, J. E. et al. Generation of iPSCs from cultured human malignant cells. Blood 115, 4039-4042 (2010).

23. Olbrich, T. et al. A Chemical Screen Identifies Compounds Capable of Selecting for Haploidy in Mammalian Cells. Cell Rep. 28, 597-604.e4 (2019).

24. Snider, L. et al. Facioscapulohumeral dystrophy: Incomplete suppression of a retrotransposed gene. PLoS Genet. 6, 1-14 (2010).

25. Bosnakovski, D. et al. Muscle pathology from stochastic low level DUX4 expression in an FSHD mouse model. Nat. Commun. 8, 1-9 (2017).

26. Agha-Mohammadi, S. et al. Second-generation tetracycline-regulatable promoter: Repositioned tet operator elements optimize transactivator synergy while shorter minimal promoter offers tight basal leakiness. J. Gene Med. 6, 817-828 (2004).

27. Urlinger, S. et al. Exploring the sequence space for tetracycline-dependent transcriptional activators: Novel mutations yield expanded range and sensitivity. Proc. Natl. Acad. Sci. U.S. A. 97, 7963-7968 (2000).

28. Lemmers, R. J. et al. Deep characterization of a common D4Z4 variant identifies biallelic DUX4 expression as a modifier for disease penetrance in FSHD2. Eur. J. Hum. Genet. 26, 94-106 (2018).

29. De Vree, P. J. P. et al. Targeted sequencing by proximity ligation for comprehensive variant detection and local haplotyping. Nat. Biotechnol. 32, 1019-1025 (2014).

30. Chen, E. Y. et al. Enrichr: Interactive and collaborative HTML5 gene list enrichment analysis tool. BMC Bioinformatics 14, (2013).

31. Kuleshov, M. V. et al. Enrichr: a comprehensive gene set enrichment analysis web server 2016 update. Nucleic Acids Res. 44, W90-W97 (2016).

32. Jagannathan, $\mathrm{S}$. et al. Model systems of DUX4 expression recapitulate the transcriptional profile of FSHD cells. Hum. Mol. Genet. 25, ddw271 (2016).

33. Robert, C. \& Watson, M. Errors in RNA-Seq quantification affect genes of relevance to human disease. Genome Biol. 16, 1-16 (2015).

34. Hendrickson, P. G. et al. Conserved roles of mouse DUX and human DUX4 in activating cleavage-stage genes and MERVL/HERVL retrotransposons. Nat. Genet. 49, 925-934 (2017).

35. Whiddon, J. L., Langford, A. T., Wong, C. J., Zhong, J. W. \& Tapscott, S. J. Conservation and innovation in the DUX4-family gene network. Nat. Genet. 49, 935-940 (2017).

36. Yan, L. et al. Single-cell RNA-Seq profiling of human preimplantation embryos and embryonic stem cells. Nat. Struct. Mol. Biol. 20, 1131-1139 (2013).

37. D'Astolfo, D. S. et al. Efficient intracellular delivery of native proteins. Cell 161, 674-690 (2015).

38. Doench, J. G. et al. Optimized sgRNA design to maximize activity and minimize off-target effects of CRISPR-Cas9. Nat. Biotechnol. 34, 184-191 (2016).

39. Sanson, K. R. et al. Optimized libraries for CRISPR-Cas9 genetic screens with multiple modalities. Nat. Commun. 9, 1-15 (2018).

40. Dull, T. et al. A third-generation lentivirus vector with a conditional packaging system. J. Virol. 72, 8463-71 (1998). 
41. Yilmaz, A., Peretz, M., Sagi, I. \& Benvenisty, N. Haploid Human Embryonic Stem Cells: Half the Genome, Double the Value. Cell Stem Cell 19, 569-572 (2016).

42. Ferreboeuf, M. et al. DUX4 and DUX4 downstream target genes are expressed in fetal FSHD muscles. Hum. Mol. Genet. 23, 171-181 (2014).

43. van Attekum, M., Terpstra, S., Reinen, E., Kater, A. \& Eldering, E. Macrophage-mediated chronic lymphocytic leukemia cell survival is independent of APRIL signaling. Cell Death Discov. 2, (2016).

44. Hashimshony, T., Wagner, F., Sher, N. \& Yanai, I. CEL-Seq: Single-Cell RNA-Seq by Multiplexed Linear Amplification. Cell Rep. 2, 666-673 (2012).

45. Grün, D. et al. Single-cell messenger RNA sequencing reveals rare intestinal cell types. Nature 525, 251-255 (2015).

46. Hashimshony, T. et al. CEL-Seq2: Sensitive highly-multiplexed single-cell RNA-Seq. Genome Biol. 17, 1-7 (2016).

47. Love, M. I., Huber, W. \& Anders, S. Moderated estimation of fold change and dispersion for RNA-seq data with DESeq2. Genome Biol. 15, 1-21 (2014). 


\section{Supplementary movie legend}

Movie 1. Related to Figure 1. Adherent KBM7 cells in growth media supplemented with doxycycline and AnnexinV-Alexa Fluor 488 conjugated antibody. Live imaging was done using a confocal Zeiss LSM 700 microscope.

Movie 2. Related to Figure 1. DIE cells in growth media supplemented with AnnexinVAlexa Fluor 488 conjugated antibody. Live imaging was done using a confocal Zeiss LSM 700 microscope.

Movie 3. Related to Figure 1. DIE cells in growth media supplemented with doxycycline and AnnexinV-Alexa Fluor 488 conjugated antibody. Live imaging was done using a confocal Zeiss LSM 700 microscope.

\section{Supplementary Material}
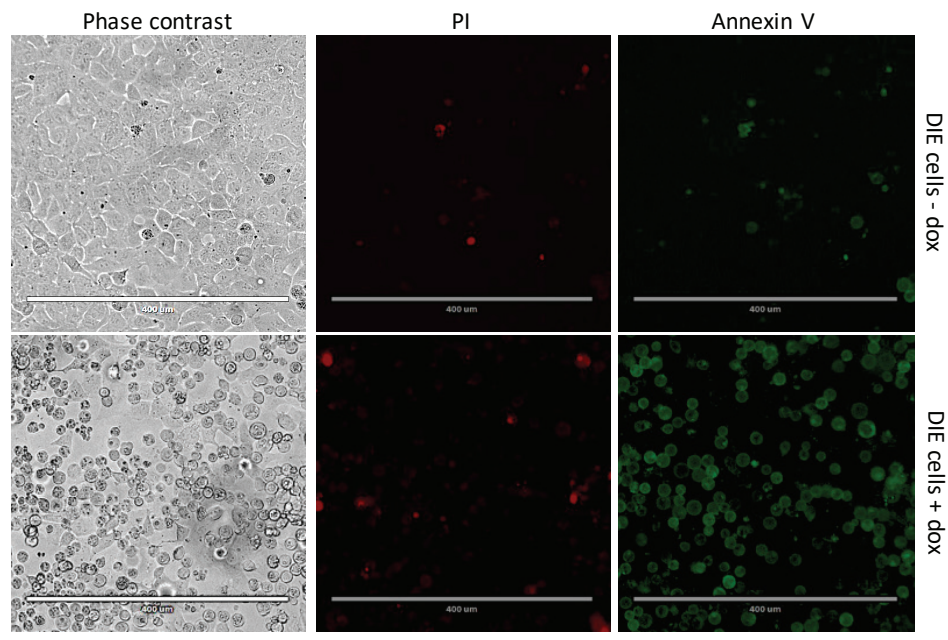

Figure S1. Living and dying DIE cells. Uninduced (top panel) and doxycycline-induced (bottom panel) DIE cells, stained with Propidium lodide (PI) (middle panel) and AnnexinV FITC (right panel), with a phase contrast image in the left panel. DIE cells in the bottom panel are stained positive for AnnexinV, with no increasing PI signal compared to uninduced DIE cells (top panel). 
A
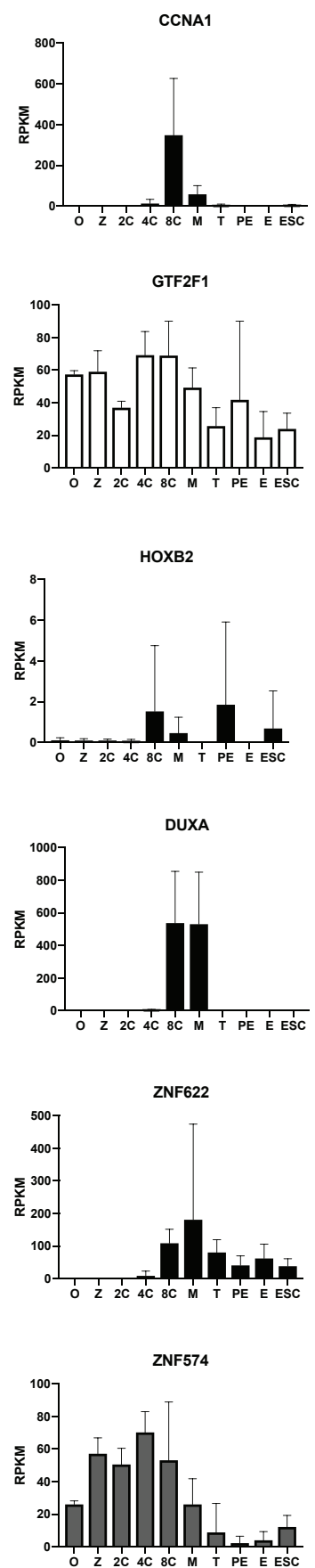
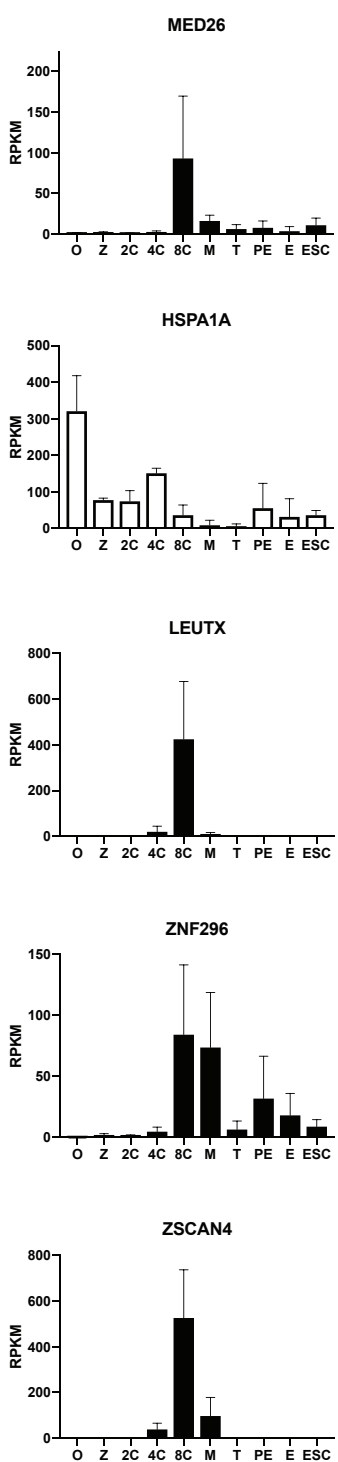

OSR2

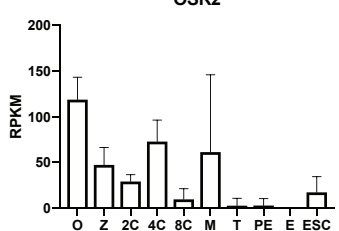

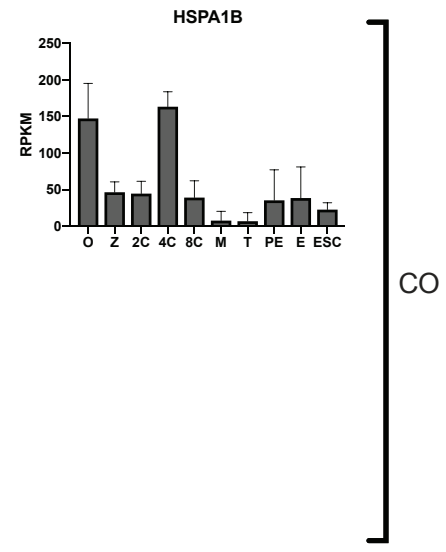
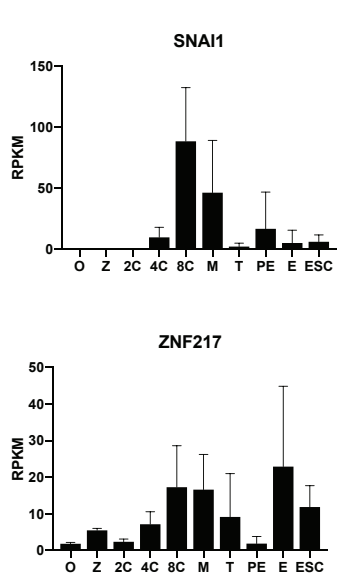

TF

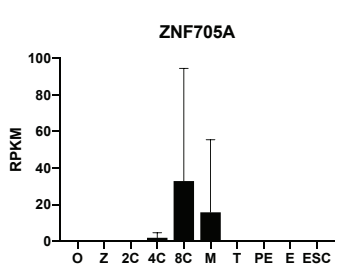

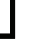


B
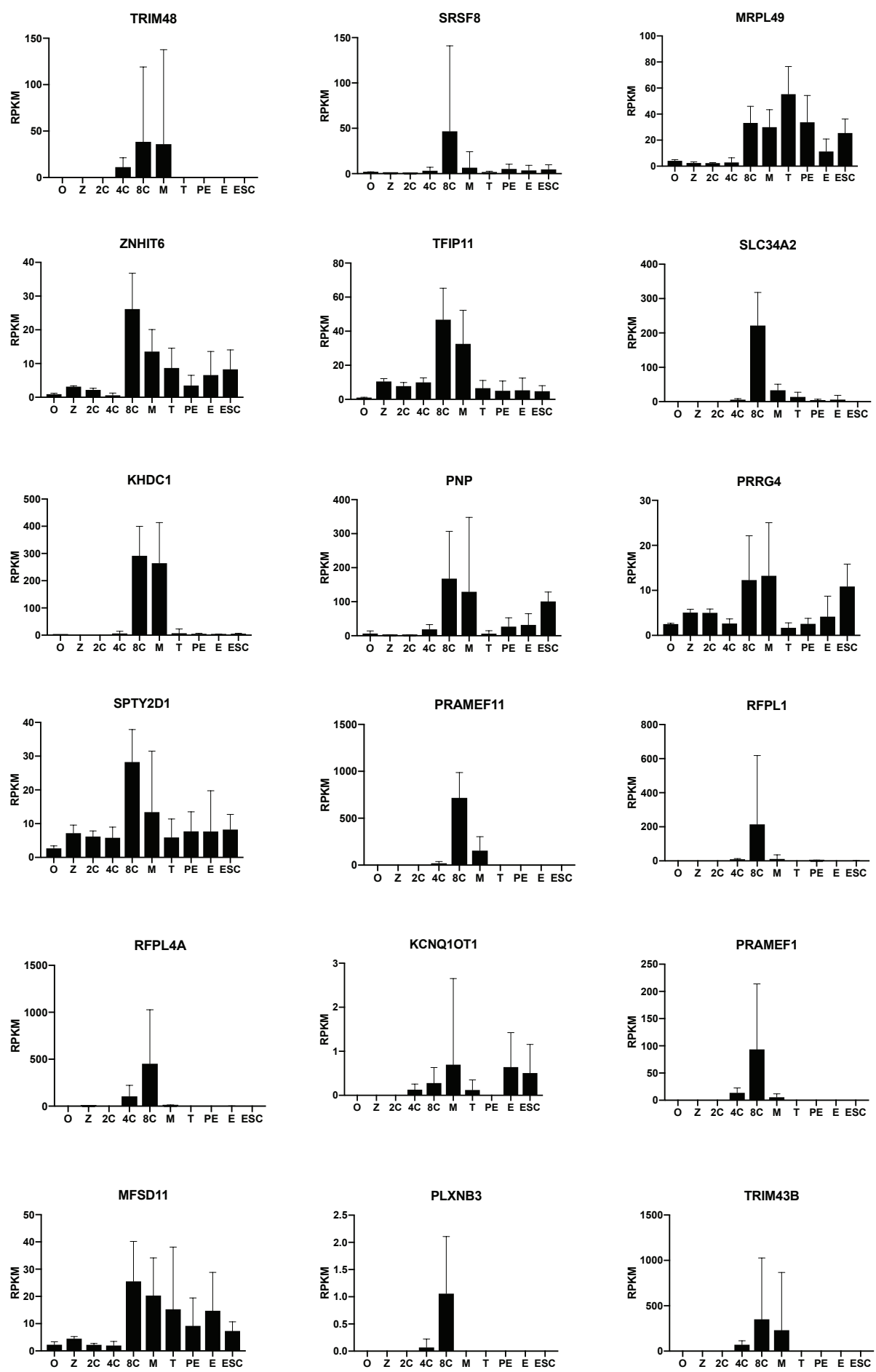
B (continued)
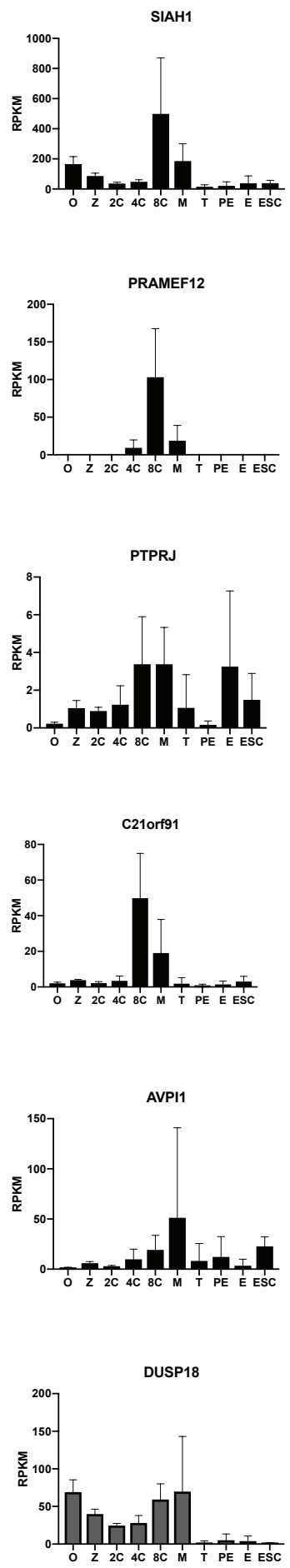
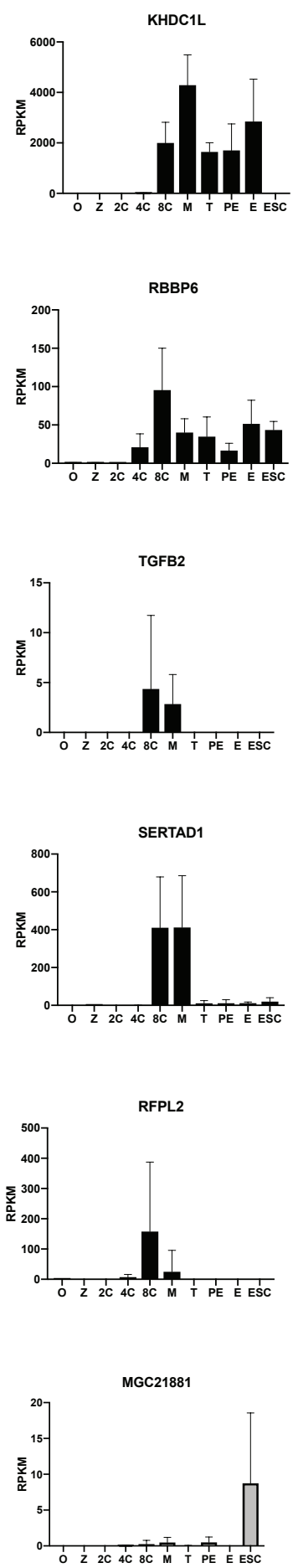
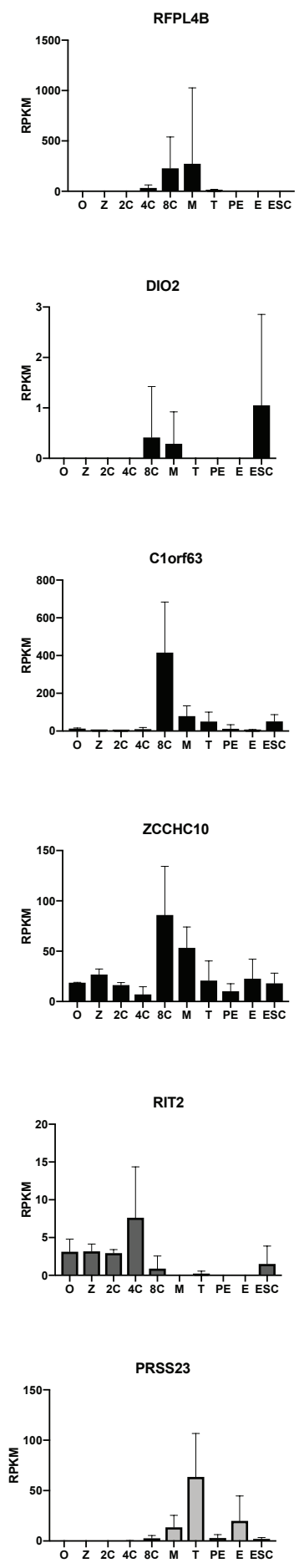
B (continued)
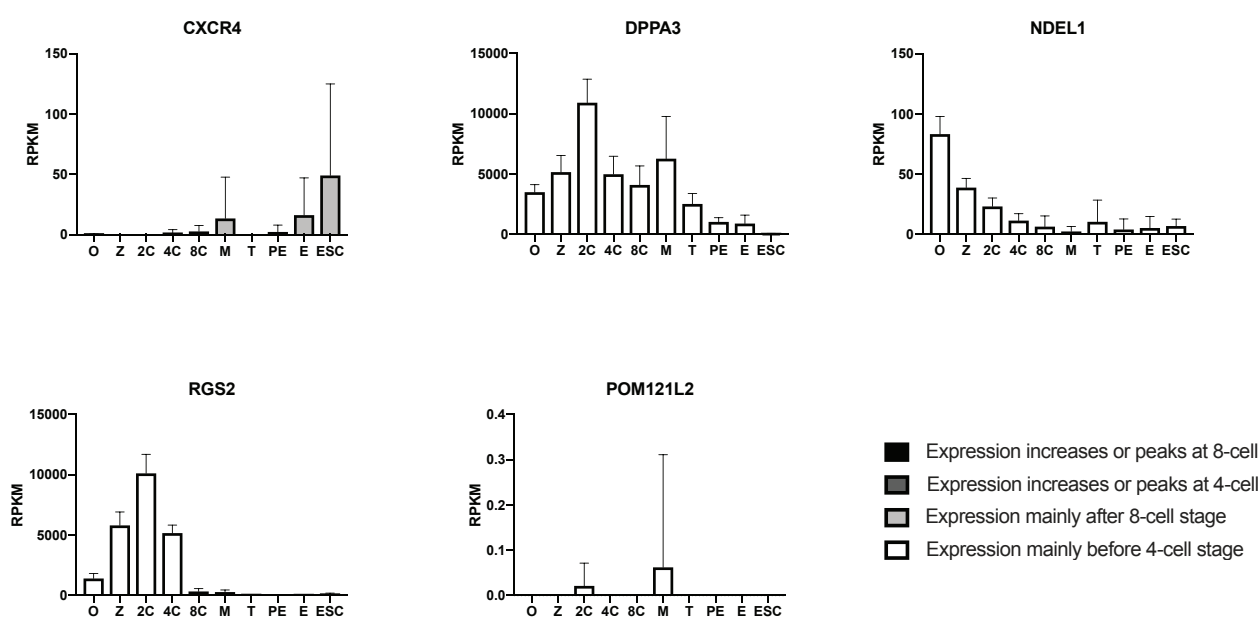

C

Stacked expression DUX4 target genes

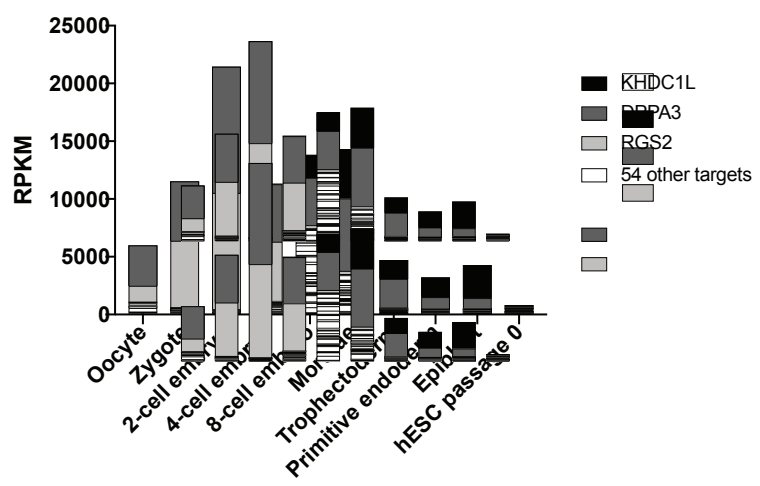

Figure S2. Expression of DUX4-induced early genes during embryonic development. (A) DUX4 induced cofactors (CO) and transcription-factors (TF) during the early stages of embryonic development in reads per kilobase per million (RPKM) mapped reads. (B) Expression of the other DUX4 induced early genes in RPKM. (C) Stacked expression of all 57 DUX4 induced early genes. KHDC1L, DPPA3, and RGS2 expression is disproportionally greater than de other 54 genes, and are individually color coded and annotated. O: Oocyte, Z: Zygote, 2C: 2-cell embryo, 4C: 4-cell embryo, 8C: 8-cell embryo, M: Morulae, T: Trophectoderm, PE: Primitive endoderm, E: Epiblast, hESC: human embryonic stem cells. Single cell RNA-seq data from preimplantation embryo's is from Yan et al. ${ }^{36}$. 
A
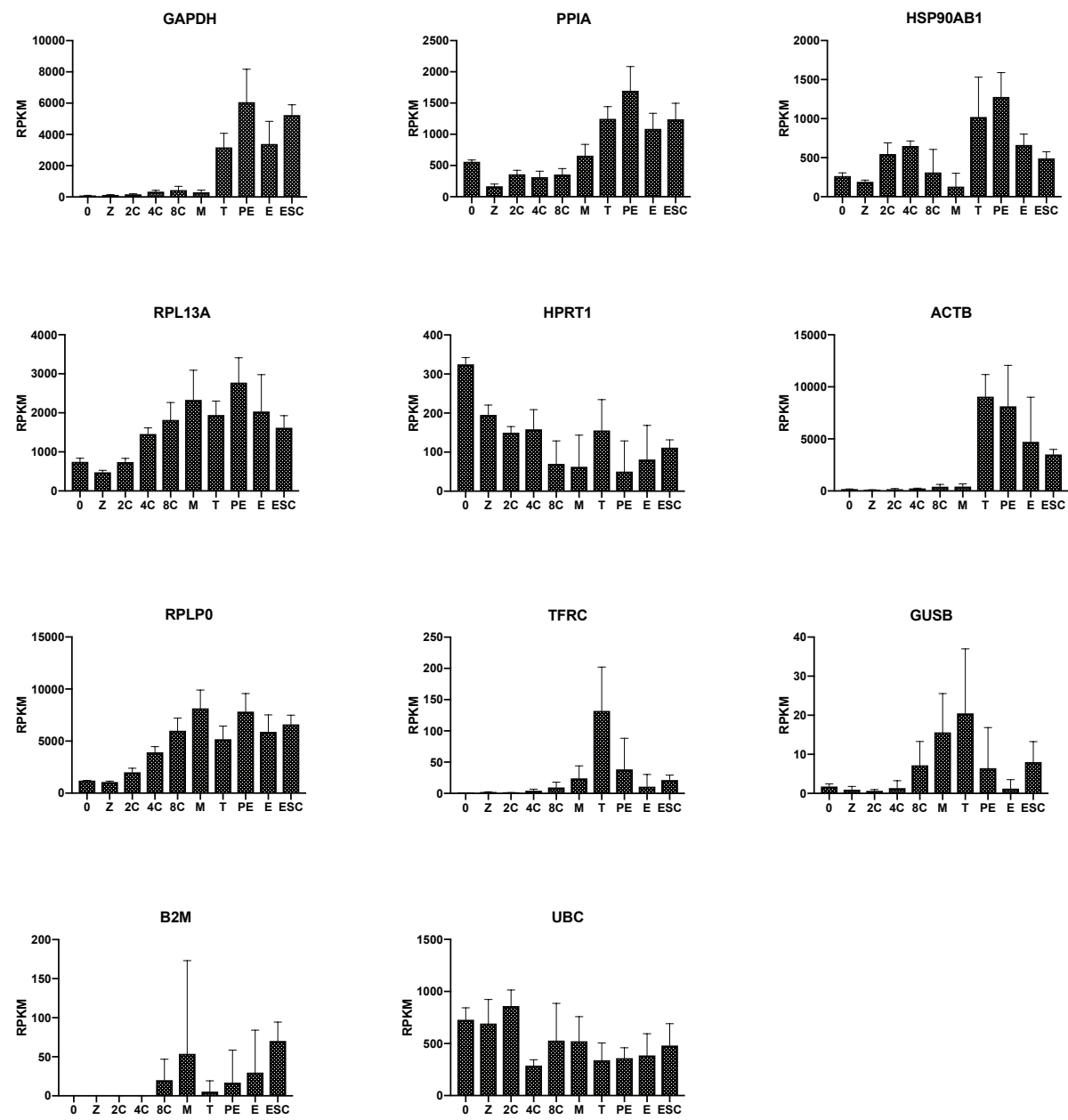

\section{B Stacked expression household genes}

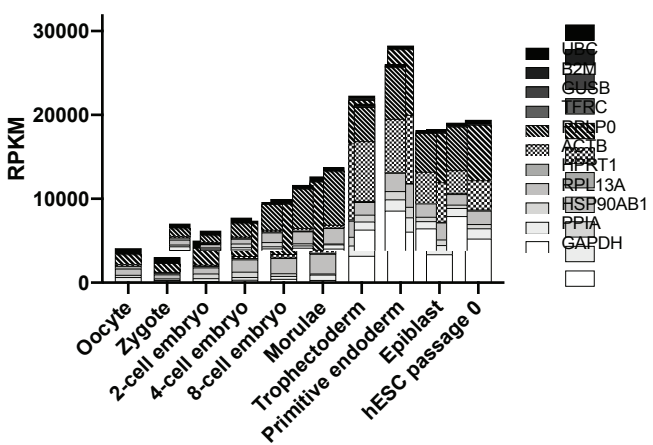

Figure S3. Expression housekeeping genes in preimplantation embryos. (A) Expression in RPKM mapped reads of 11 housekeeping genes in the early stages of embryonic development. (B) Stacked Expression of the housekeeping genes at same stages of early embryonic development, in RPKM. O: Oocyte, Z: Zygote, 2C: 2-cell embryo, 4C: 4-cell embryo, 8C: 8-cell embryo, M: Morulae, T: Trophectoderm, PE: Primitive endoderm, E: Epiblast, hESC: human embryonic stem cells. Single cell RNA-seq data from preimplantation embryo's is from Yan et al. ${ }^{36}$ 
Table S1: Differentially expressed genes after $4.5 \mathrm{~h}$ of doxycycline induction

* Adjusted $p$ value $\leq 0.01$, absolute $\log 2 \mathrm{FC} \geq 1$

\begin{tabular}{|c|c|c|c|c|c|c|}
\hline Gene.Symbol & baseMean & $\log 2 \mathrm{FC}$ & IfcSE & stat & pvalue & padj \\
\hline ZSCAN4 & 285.62 & 4.08 & 0.18 & 23.25 & $1.51 \mathrm{E}-119$ & $1.26 \mathrm{E}-115$ \\
\hline LINC00633 & 137.90 & 3.26 & 0.18 & 18.02 & $1.43 \mathrm{E}-72$ & $5.96 \mathrm{E}-69$ \\
\hline ZNF217 & 358.21 & 2.26 & 0.13 & 17.44 & $4.24 \mathrm{E}-68$ & $1.18 \mathrm{E}-64$ \\
\hline SRSF8 & 133.28 & 2.41 & 0.14 & 17.29 & $5.57 \mathrm{E}-67$ & $1.16 \mathrm{E}-63$ \\
\hline PRAMEF1 & 120.48 & 4.25 & 0.25 & 17.10 & $1.40 \mathrm{E}-65$ & $2.34 \mathrm{E}-62$ \\
\hline RBBP6 & 221.34 & 1.90 & 0.12 & 15.39 & $2.03 E-53$ & $2.83 E-50$ \\
\hline PNP & 260.82 & 1.76 & 0.12 & 14.69 & $8.01 \mathrm{E}-49$ & $9.54 \mathrm{E}-46$ \\
\hline ZNF296 & 105.83 & 2.73 & 0.19 & 14.28 & $2.94 \mathrm{E}-46$ & $3.06 \mathrm{E}-43$ \\
\hline SIAH1 & 89.75 & 2.93 & 0.21 & 14.12 & $2.74 \mathrm{E}-45$ & $2.53 E-42$ \\
\hline TRIM51 & 68.72 & 3.39 & 0.25 & 13.68 & $1.42 \mathrm{E}-42$ & $1.18 \mathrm{E}-39$ \\
\hline RFPL4B & 87.89 & 4.47 & 0.33 & 13.50 & $1.60 \mathrm{E}-41$ & $1.22 \mathrm{E}-38$ \\
\hline KHDC1L & 131.92 & 2.86 & 0.22 & 13.08 & $4.20 \mathrm{E}-39$ & $2.92 \mathrm{E}-36$ \\
\hline CCNA1 & 208.50 & 2.17 & 0.17 & 12.64 & $1.27 \mathrm{E}-36$ & $7.58 \mathrm{E}-34$ \\
\hline TFIP11 & 83.33 & 2.73 & 0.22 & 12.64 & $1.26 \mathrm{E}-36$ & $7.58 E-34$ \\
\hline PRAMEF12 & 63.82 & 3.69 & 0.30 & 12.32 & $7.12 \mathrm{E}-35$ & $3.96 E-32$ \\
\hline LEUTX & 415.52 & 3.62 & 0.30 & 12.02 & $2.83 E-33$ & $1.48 \mathrm{E}-30$ \\
\hline ZNF622 & 66.31 & 2.26 & 0.19 & 11.78 & $5.22 \mathrm{E}-32$ & $2.56 \mathrm{E}-29$ \\
\hline HSPA1A & 281.27 & 1.03 & 0.11 & 9.60 & $7.76 \mathrm{E}-22$ & $3.59 \mathrm{E}-19$ \\
\hline RFPL1 & 47.03 & 3.86 & 0.41 & 9.48 & $2.57 \mathrm{E}-21$ & 1.13E-18 \\
\hline PTPRJ & 33.57 & 3.15 & 0.34 & 9.28 & $1.70 \mathrm{E}-20$ & $7.09 \mathrm{E}-18$ \\
\hline RFPL2 & 60.64 & 3.32 & 0.36 & 9.22 & $2.94 \mathrm{E}-20$ & 1.17E-17 \\
\hline RFPL4A & 51.75 & 3.34 & 0.36 & 9.17 & $4.87 \mathrm{E}-20$ & $1.85 \mathrm{E}-17$ \\
\hline ID1 & 100.44 & -1.47 & 0.16 & -8.95 & $3.40 \mathrm{E}-19$ & 1.23E-16 \\
\hline SLC34A2 & 57.57 & 2.19 & 0.25 & 8.61 & $7.45 \mathrm{E}-18$ & $2.59 \mathrm{E}-15$ \\
\hline TRIM43B & 23.75 & 4.10 & 0.52 & 7.88 & $3.25 \mathrm{E}-15$ & $1.08 \mathrm{E}-12$ \\
\hline GTF2F1 & 58.72 & 1.54 & 0.20 & 7.70 & $1.32 \mathrm{E}-14$ & 4.24E-12 \\
\hline TRIM48 & 29.06 & 2.95 & 0.40 & 7.36 & $1.81 \mathrm{E}-13$ & $5.58 \mathrm{E}-11$ \\
\hline DIO2 & 26.35 & 3.73 & 0.51 & 7.35 & $2.04 \mathrm{E}-13$ & $6.07 E-11$ \\
\hline MRPL49 & 94.37 & 1.06 & 0.15 & 6.90 & $5.27 \mathrm{E}-12$ & 1.46E-09 \\
\hline LOC441081 & 33.58 & 3.75 & 0.55 & 6.83 & $8.78 \mathrm{E}-12$ & 2.36E-09 \\
\hline ZNF574 & 73.36 & 1.03 & 0.16 & 6.32 & $2.62 \mathrm{E}-10$ & $6.83 E-08$ \\
\hline C1orf63 & 31.18 & 1.87 & 0.30 & 6.17 & $6.75 \mathrm{E}-10$ & $1.71 \mathrm{E}-07$ \\
\hline DUXA & 29.80 & 2.60 & 0.42 & 6.14 & $8.50 \mathrm{E}-10$ & 2.08E-07 \\
\hline HSPA1B & 50.54 & 1.06 & 0.17 & 6.07 & $1.31 \mathrm{E}-09$ & 3.13E-07 \\
\hline C21orf91 & 17.50 & 2.36 & 0.40 & 5.94 & 2.93E-09 & $6.78 \mathrm{E}-07$ \\
\hline DUSP18 & 10.48 & 3.14 & 0.53 & 5.90 & 3.74E-09 & $8.21 \mathrm{E}-07$ \\
\hline
\end{tabular}


Table S1 continued

\begin{tabular}{|c|c|c|c|c|c|c|}
\hline Gene.Symbol & baseMean & $\log 2 F C$ & IfcSE & stat & pvalue & padj \\
\hline ZNHIT6 & 60.07 & 1.14 & 0.19 & 5.90 & 3.71E-09 & $8.21 \mathrm{E}-07$ \\
\hline DPPA3 & 32.41 & 2.47 & 0.42 & 5.83 & 5.49E-09 & 1.17E-06 \\
\hline AVPI1 & 32.14 & 1.35 & 0.24 & 5.73 & $1.01 \mathrm{E}-08$ & 2.06E-06 \\
\hline CXCR4 & 15.51 & 3.89 & 0.70 & 5.58 & $2.44 \mathrm{E}-08$ & 4.84E-06 \\
\hline ID3 & 42.15 & -1.10 & 0.20 & -5.55 & $2.90 \mathrm{E}-08$ & 5.63E-06 \\
\hline NDEL1 & 34.42 & 1.19 & 0.23 & 5.22 & $1.82 \mathrm{E}-07$ & 3.30E-05 \\
\hline HOXB2 & 28.18 & 1.42 & 0.28 & 5.16 & $2.45 \mathrm{E}-07$ & 4.34E-05 \\
\hline MGC21881 & 36.17 & 1.22 & 0.24 & 5.07 & $3.89 \mathrm{E}-07$ & 6.75E-05 \\
\hline MFSD11 & 20.82 & 1.71 & 0.34 & 5.07 & 4.07E-07 & $6.92 \mathrm{E}-05$ \\
\hline PLXNB3 & 61.80 & 1.58 & 0.31 & 5.06 & 4.27E-07 & 7.12E-05 \\
\hline SNAI1 & 12.65 & 3.47 & 0.71 & 4.88 & $1.05 \mathrm{E}-06$ & $1.68 \mathrm{E}-04$ \\
\hline KHDC1 & 18.95 & 2.69 & 0.56 & 4.84 & $1.28 \mathrm{E}-06$ & $2.02 \mathrm{E}-04$ \\
\hline C20orf112 & 9.87 & -2.37 & 0.50 & -4.77 & $1.85 \mathrm{E}-06$ & 2.86E-04 \\
\hline PRAMEF11 & 8.00 & 3.71 & 0.79 & 4.67 & $3.04 \mathrm{E}-06$ & 4.53E-04 \\
\hline HES7 & 21.19 & -1.34 & 0.29 & -4.56 & 5.09E-06 & 7.30E-04 \\
\hline TMEM254-AS1 & 44.46 & 1.51 & 0.34 & 4.44 & $9.12 \mathrm{E}-06$ & $1.23 \mathrm{E}-03$ \\
\hline PRRG4 & 8.04 & 2.68 & 0.61 & 4.41 & $1.06 \mathrm{E}-05$ & 1.37E-03 \\
\hline SPTY2D1 & 20.68 & 1.48 & 0.34 & 4.40 & 1.07E-05 & $1.38 \mathrm{E}-03$ \\
\hline RIT2 & 8.91 & 4.75 & 1.08 & 4.38 & $1.16 \mathrm{E}-05$ & 1.47E-03 \\
\hline ZNF705A & 6.03 & 4.75 & 1.09 & 4.34 & $1.40 \mathrm{E}-05$ & $1.74 \mathrm{E}-03$ \\
\hline KCNQ10T1 & 29.91 & 1.05 & 0.25 & 4.20 & 2.67E-05 & 3.05E-03 \\
\hline SERTAD1 & 17.17 & 1.28 & 0.31 & 4.14 & $3.54 \mathrm{E}-05$ & 3.99E-03 \\
\hline TGFB2 & 13.62 & 1.94 & 0.47 & 4.13 & 3.59E-05 & 4.00E-03 \\
\hline RGS2 & 23.97 & 1.18 & 0.29 & 4.03 & 5.61E-05 & 6.00E-03 \\
\hline MED26 & 8.95 & 1.87 & 0.47 & 4.01 & $6.10 \mathrm{E}-05$ & $6.36 \mathrm{E}-03$ \\
\hline OSR2 & 7.12 & 2.32 & 0.58 & 4.01 & $6.08 \mathrm{E}-05$ & $6.36 \mathrm{E}-03$ \\
\hline PRSS23 & 8.83 & 1.91 & 0.48 & 3.94 & $8.02 \mathrm{E}-05$ & 8.16E-03 \\
\hline ZCCHC10 & 18.53 & 1.22 & 0.31 & 3.88 & $1.03 \mathrm{E}-04$ & $9.83 \mathrm{E}-03$ \\
\hline PRSS23 & 7.14 & 1.91 & 0.49 & 3.92 & 8.80E-05 & $9.46 \mathrm{E}-03$ \\
\hline
\end{tabular}

Table S2: Differentially expressed genes after $8.5 \mathrm{~h}$ of doxycycline induction

* Adjusted $p$ value $\leq 0.01$, absolute $\log 2 \mathrm{FC} \geq 1$ )

\begin{tabular}{|lrrrrrr|}
\hline Gene.Symbol & baseMean & log2FC & IfcSE & stat & pvalue & padj \\
ZSCAN4 & 285.62 & 5.33 & 0.17 & 30.85 & $5.93 \mathrm{E}-209$ & $5.72 \mathrm{E}-205$ \\
CCNA1 & 208.50 & 4.55 & 0.16 & 28.08 & $1.79 \mathrm{E}-173$ & $8.65 \mathrm{E}-170$ \\
LINC00633 & 137.90 & 4.56 & 0.17 & 26.11 & $3.04 \mathrm{E}-150$ & $9.76 \mathrm{E}-147$ \\
ZNF217 & 358.21 & 3.30 & 0.13 & 26.07 & $8.67 \mathrm{E}-150$ & $2.09 \mathrm{E}-146$
\end{tabular}


Table S2 continued

\begin{tabular}{|c|c|c|c|c|c|c|}
\hline Gene.Symbol & baseMean & $\log 2 \mathrm{FC}$ & IfcSE & stat & pvalue & padj \\
\hline KHDC1L & 131.92 & 5.34 & 0.21 & 25.98 & 8.35E-149 & $1.61 \mathrm{E}-145$ \\
\hline PNP & 260.82 & 2.75 & 0.12 & 23.74 & $1.35 \mathrm{E}-124$ & $2.18 \mathrm{E}-121$ \\
\hline PRAMEF1 & 120.48 & 5.59 & 0.24 & 22.96 & $1.09 \mathrm{E}-116$ & $1.51 \mathrm{E}-113$ \\
\hline ZNF296 & 105.83 & 4.07 & 0.18 & 22.27 & 7.75E-110 & $9.35 \mathrm{E}-107$ \\
\hline SIAH1 & 89.75 & 4.29 & 0.20 & 21.65 & $5.98 \mathrm{E}-104$ & $6.41 \mathrm{E}-101$ \\
\hline SRSF8 & 133.28 & 2.83 & 0.14 & 20.76 & $9.70 E-96$ & $9.36 \mathrm{E}-93$ \\
\hline TPRX1 & 61.43 & 4.67 & 0.24 & 19.39 & $8.53 E-84$ & $7.48 \mathrm{E}-81$ \\
\hline TFIP11 & 83.33 & 4.00 & 0.21 & 19.25 & $1.29 E-82$ & $1.04 \mathrm{E}-79$ \\
\hline PLXNB3 & 61.80 & 5.13 & 0.27 & 18.99 & $2.08 \mathrm{E}-80$ & $1.54 \mathrm{E}-77$ \\
\hline LEUTX & 415.52 & 5.66 & 0.30 & 18.94 & $5.56 \mathrm{E}-80$ & 3.83E-77 \\
\hline TRIM51 & 68.72 & 4.51 & 0.24 & 18.79 & 8.39E-79 & $5.40 E-76$ \\
\hline RFPL4B & 87.89 & 6.04 & 0.33 & 18.55 & 8.01E-77 & $4.83 E-74$ \\
\hline SLC34A2 & 57.57 & 4.25 & 0.23 & 18.16 & $1.14 \mathrm{E}-73$ & $6.46 \mathrm{E}-71$ \\
\hline PRAMEF12 & 63.82 & 5.21 & 0.29 & 17.95 & $4.58 \mathrm{E}-72$ & $2.46 \mathrm{E}-69$ \\
\hline NXF1 & 107.66 & 2.18 & 0.12 & 17.61 & $1.96 \mathrm{E}-69$ & $9.94 \mathrm{E}-67$ \\
\hline RBBP6 & 221.34 & 2.11 & 0.12 & 17.34 & $2.45 E-67$ & $1.18 \mathrm{E}-64$ \\
\hline RFPL2 & 60.64 & 5.79 & 0.34 & 16.86 & $9.15 E-64$ & 4.20E-61 \\
\hline ZNF622 & 66.31 & 3.09 & 0.18 & 16.83 & $1.48 \mathrm{E}-63$ & 6.47E-61 \\
\hline PTP4A1 & 160.48 & 1.87 & 0.11 & 16.55 & $1.53 \mathrm{E}-61$ & $6.41 E-59$ \\
\hline HNRNPF & 350.21 & 1.97 & 0.12 & 16.45 & 7.99E-61 & $3.21 \mathrm{E}-58$ \\
\hline TMEM254-AS1 & 44.46 & 4.83 & 0.29 & 16.42 & $1.27 \mathrm{E}-60$ & $4.91 \mathrm{E}-58$ \\
\hline RFPL4A & 51.75 & 5.44 & 0.35 & 15.54 & $1.86 \mathrm{E}-54$ & $6.90 E-52$ \\
\hline ZNHIT6 & 60.07 & 2.69 & 0.17 & 15.44 & $8.64 \mathrm{E}-54$ & 3.09E-51 \\
\hline GTF2F1 & 58.72 & 2.85 & 0.18 & 15.42 & $1.18 \mathrm{E}-53$ & 4.06E-51 \\
\hline RFPL1 & 47.03 & 5.85 & 0.39 & 14.85 & $6.90 \mathrm{E}-50$ & $2.30 \mathrm{E}-47$ \\
\hline MRPL49 & 94.37 & 2.04 & 0.14 & 14.30 & $2.15 E-46$ & $6.92 \mathrm{E}-44$ \\
\hline DPPA3 & 32.41 & 5.33 & 0.39 & 13.70 & $9.53 E-43$ & $2.96 \mathrm{E}-40$ \\
\hline RYBP & 47.53 & 2.43 & 0.18 & 13.28 & $3.04 \mathrm{E}-40$ & $9.17 \mathrm{E}-38$ \\
\hline DUXA & 29.80 & 5.12 & 0.39 & 13.05 & $6.10 E-39$ & $1.78 \mathrm{E}-36$ \\
\hline PTPRJ & 33.57 & 4.27 & 0.33 & 13.05 & $6.31 E-39$ & $1.79 \mathrm{E}-36$ \\
\hline TRIM48 & 29.06 & 4.92 & 0.38 & 12.96 & $2.11 E-38$ & $5.83 E-36$ \\
\hline EXOSC10 & 86.08 & 1.82 & 0.14 & 12.95 & $2.41 E-38$ & $6.45 E-36$ \\
\hline TFAP2C & 52.23 & 2.15 & 0.17 & 12.82 & $1.20 E-37$ & $3.14 \mathrm{E}-35$ \\
\hline C1orf63 & 31.18 & 3.52 & 0.28 & 12.64 & $1.34 \mathrm{E}-36$ & $3.40 \mathrm{E}-34$ \\
\hline ANXA5 & 256.82 & 1.09 & 0.09 & 12.21 & $2.64 \mathrm{E}-34$ & $6.53 E-32$ \\
\hline ALYREF & 220.09 & 1.27 & 0.11 & 12.12 & $8.47 E-34$ & $2.04 \mathrm{E}-31$ \\
\hline LOC441081 & 33.58 & 6.37 & 0.53 & 12.06 & $1.71 E-33$ & $4.03 E-31$ \\
\hline ZNF574 & 73.36 & 1.83 & 0.15 & 12.03 & $2.43 E-33$ & 5.59E-31 \\
\hline
\end{tabular}


Table S2 continued

\begin{tabular}{|c|c|c|c|c|c|c|}
\hline Gene.Symbol & baseMean & $\log 2 F C$ & IfcSE & stat & pvalue & padj \\
\hline INO80C & 80.41 & 1.65 & 0.14 & 11.96 & $5.50 E-33$ & $1.23 \mathrm{E}-30$ \\
\hline LINC00493 & 63.11 & 1.96 & 0.17 & 11.68 & $1.59 \mathrm{E}-31$ & $3.48 \mathrm{E}-29$ \\
\hline MGC21881 & 36.17 & 2.50 & 0.22 & 11.43 & $3.11 \mathrm{E}-30$ & $6.66 \mathrm{E}-28$ \\
\hline DIO2 & 26.35 & 5.54 & 0.49 & 11.26 & $1.97 E-29$ & 4.13E-27 \\
\hline ID1 & 100.44 & -1.79 & 0.17 & -10.79 & $3.95 E-27$ & $8.12 \mathrm{E}-25$ \\
\hline TRIM43B & 23.75 & 5.45 & 0.51 & 10.75 & $6.21 \mathrm{E}-27$ & $1.25 \mathrm{E}-24$ \\
\hline ALPPL2 & 19.14 & 3.30 & 0.31 & 10.72 & $8.59 E-27$ & 1.69E-24 \\
\hline AVPI1 & 32.14 & 2.30 & 0.22 & 10.56 & 4.47E-26 & $8.62 \mathrm{E}-24$ \\
\hline KHDC1 & 18.95 & 5.35 & 0.52 & 10.38 & $3.18 \mathrm{E}-25$ & $6.02 E-23$ \\
\hline RNF11 & 45.92 & 1.85 & 0.18 & 10.32 & $5.56 \mathrm{E}-25$ & $1.03 \mathrm{E}-22$ \\
\hline SPTY2D1 & 20.68 & 3.10 & 0.30 & 10.25 & $1.20 \mathrm{E}-24$ & $2.18 \mathrm{E}-22$ \\
\hline HOXB2 & 28.18 & 2.60 & 0.25 & 10.23 & $1.43 E-24$ & $2.56 \mathrm{E}-22$ \\
\hline SNUPN & 140.64 & 2.47 & 0.24 & 10.17 & $2.63 E-24$ & $4.61 \mathrm{E}-22$ \\
\hline LOC100216545 & 25.93 & 2.52 & 0.25 & 10.12 & 4.33E-24 & 7.47E-22 \\
\hline RGS2 & 23.97 & 2.66 & 0.26 & 10.12 & $4.52 E-24$ & $7.66 \mathrm{E}-22$ \\
\hline CCNJ & 28.13 & 2.35 & 0.24 & 9.95 & $2.42 \mathrm{E}-23$ & $4.03 E-21$ \\
\hline NDEL1 & 34.42 & 2.08 & 0.21 & 9.84 & $7.23 \mathrm{E}-23$ & $1.18 \mathrm{E}-20$ \\
\hline TCEB3 & 44.12 & 1.83 & 0.19 & 9.81 & $9.79 E-23$ & 1.57E-20 \\
\hline PNN & 80.38 & 1.33 & 0.14 & 9.74 & $2.05 E-22$ & $3.25 \mathrm{E}-20$ \\
\hline MFSD11 & 20.82 & 3.00 & 0.31 & 9.64 & $5.54 \mathrm{E}-22$ & 8.63E-20 \\
\hline ADPGK & 32.75 & 2.10 & 0.22 & 9.56 & $1.13 \mathrm{E}-21$ & $1.73 \mathrm{E}-19$ \\
\hline RFK & 42.75 & 1.85 & 0.19 & 9.50 & $2.08 \mathrm{E}-21$ & 3.14E-19 \\
\hline C21orf91 & 17.50 & 3.54 & 0.37 & 9.48 & $2.62 \mathrm{E}-21$ & $3.88 \mathrm{E}-19$ \\
\hline KDM5B & 27.91 & 2.22 & 0.24 & 9.42 & 4.63E-21 & 6.76E-19 \\
\hline MMRN2 & 13.16 & 4.38 & 0.47 & 9.35 & $8.68 \mathrm{E}-21$ & $1.25 \mathrm{E}-18$ \\
\hline ODC1 & 132.72 & 1.04 & 0.11 & 9.25 & $2.21 \mathrm{E}-20$ & $3.14 \mathrm{E}-18$ \\
\hline ID3 & 42.15 & -2.13 & 0.23 & -9.20 & $3.60 \mathrm{E}-20$ & 5.04E-18 \\
\hline DDX10 & 73.50 & 1.28 & 0.14 & 9.17 & 4.55E-20 & $6.27 \mathrm{E}-18$ \\
\hline ARL4D & 37.76 & 1.85 & 0.20 & 9.08 & $1.06 \mathrm{E}-19$ & $1.44 \mathrm{E}-17$ \\
\hline DYX1C1 & 33.45 & 1.85 & 0.21 & 8.99 & 2.39E-19 & 3.20E-17 \\
\hline TGFB2 & 13.62 & 3.81 & 0.43 & 8.96 & 3.31E-19 & $4.38 \mathrm{E}-17$ \\
\hline C8orf33 & 76.19 & 1.35 & 0.15 & 8.85 & 8.44E-19 & $1.09 \mathrm{E}-16$ \\
\hline CDC42EP1 & 122.65 & -1.18 & 0.13 & -8.84 & $9.56 \mathrm{E}-19$ & $1.21 \mathrm{E}-16$ \\
\hline ELOF1 & 115.88 & 1.01 & 0.12 & 8.79 & $1.54 \mathrm{E}-18$ & $1.93 \mathrm{E}-16$ \\
\hline LOC284551 & 12.05 & 4.12 & 0.47 & 8.77 & $1.75 \mathrm{E}-18$ & 2.17E-16 \\
\hline SLC2A3 & 11.49 & 3.76 & 0.43 & 8.71 & $3.08 \mathrm{E}-18$ & $3.76 \mathrm{E}-16$ \\
\hline TRAPPC6B & 29.92 & 1.88 & 0.22 & 8.67 & 4.17E-18 & $5.04 \mathrm{E}-16$ \\
\hline CXCR4 & 15.51 & 5.83 & 0.68 & 8.62 & $6.51 \mathrm{E}-18$ & 7.75E-16 \\
\hline
\end{tabular}


Table S2 continued

\begin{tabular}{|c|c|c|c|c|c|c|}
\hline Gene.Symbol & baseMean & $\log 2 F C$ & IfcSE & stat & pvalue & padj \\
\hline SUPT6H & 50.14 & 1.46 & 0.17 & 8.59 & $8.94 \mathrm{E}-18$ & $1.05 \mathrm{E}-15$ \\
\hline TTC23 & 22.59 & 2.39 & 0.28 & 8.54 & $1.32 \mathrm{E}-17$ & $1.53 \mathrm{E}-15$ \\
\hline ST6GAL1 & 70.93 & -1.36 & 0.16 & -8.45 & $3.00 \mathrm{E}-17$ & $3.45 E-15$ \\
\hline LOC100188947 & 21.78 & 2.26 & 0.27 & 8.44 & $3.15 E-17$ & $3.58 \mathrm{E}-15$ \\
\hline ZRANB2 & 93.18 & 1.13 & 0.14 & 8.33 & 8.33E-17 & $9.35 E-15$ \\
\hline C1D & 39.32 & 1.63 & 0.20 & 8.31 & $9.75 \mathrm{E}-17$ & $1.08 \mathrm{E}-14$ \\
\hline PPP1R18 & 77.02 & -1.38 & 0.17 & -8.26 & $1.42 \mathrm{E}-16$ & $1.56 \mathrm{E}-14$ \\
\hline SNAI1 & 12.65 & 5.54 & 0.68 & 8.13 & $4.34 \mathrm{E}-16$ & 4.60E-14 \\
\hline RBM25 & 98.59 & 1.13 & 0.14 & 8.04 & $9.11 \mathrm{E}-16$ & $9.45 E-14$ \\
\hline CWC15 & 79.29 & 1.21 & 0.15 & 7.97 & $1.62 \mathrm{E}-15$ & $1.66 \mathrm{E}-13$ \\
\hline RHOBTB1 & 14.62 & 2.61 & 0.33 & 7.92 & $2.33 \mathrm{E}-15$ & $2.36 \mathrm{E}-13$ \\
\hline YPEL5 & 25.81 & 1.86 & 0.24 & 7.90 & 2.77E-15 & $2.79 \mathrm{E}-13$ \\
\hline CLK1 & 19.40 & 2.30 & 0.30 & 7.79 & $6.54 \mathrm{E}-15$ & $6.44 \mathrm{E}-13$ \\
\hline PSMD9 & 98.75 & 1.05 & 0.13 & 7.77 & $7.59 \mathrm{E}-15$ & 7.40E-13 \\
\hline DBR1 & 14.99 & 2.55 & 0.33 & 7.63 & 2.31E-14 & $2.23 \mathrm{E}-12$ \\
\hline ZSCAN5A & 15.28 & 2.53 & 0.33 & 7.60 & 2.87E-14 & $2.72 \mathrm{E}-12$ \\
\hline ACAP2 & 18.44 & 2.06 & 0.27 & 7.60 & $2.95 \mathrm{E}-14$ & 2.77E-12 \\
\hline YTHDC1 & 49.90 & 1.35 & 0.18 & 7.54 & $4.66 \mathrm{E}-14$ & 4.33E-12 \\
\hline ALG13 & 36.76 & 1.44 & 0.19 & 7.47 & $7.89 \mathrm{E}-14$ & 7.25E-12 \\
\hline ATF3 & 13.69 & 2.77 & 0.37 & 7.47 & $8.12 \mathrm{E}-14$ & 7.39E-12 \\
\hline PNRC1 & 13.55 & 2.49 & 0.33 & 7.43 & $1.08 \mathrm{E}-13$ & $9.70 \mathrm{E}-12$ \\
\hline SHC1 & 54.31 & 1.20 & 0.16 & 7.42 & $1.20 \mathrm{E}-13$ & 1.07E-11 \\
\hline MEX3A & 74.86 & -1.25 & 0.17 & -7.30 & $2.90 \mathrm{E}-13$ & $2.54 \mathrm{E}-11$ \\
\hline PANX2 & 19.32 & 2.09 & 0.29 & 7.26 & $3.78 \mathrm{E}-13$ & $3.28 \mathrm{E}-11$ \\
\hline ALDH9A1 & 46.05 & 1.27 & 0.18 & 7.21 & $5.52 \mathrm{E}-13$ & 4.75E-11 \\
\hline KIAA1551 & 14.82 & 2.31 & 0.32 & 7.20 & $5.84 \mathrm{E}-13$ & $4.98 \mathrm{E}-11$ \\
\hline SERTAD1 & 17.17 & 2.04 & 0.29 & 7.09 & $1.37 \mathrm{E}-12$ & $1.16 \mathrm{E}-10$ \\
\hline GLUL & 57.83 & 1.11 & 0.16 & 7.09 & $1.39 \mathrm{E}-12$ & 1.17E-10 \\
\hline SIRT1 & 26.62 & 1.60 & 0.23 & 7.02 & $2.19 \mathrm{E}-12$ & $1.82 \mathrm{E}-10$ \\
\hline SAMD8 & 8.41 & 4.23 & 0.60 & 7.01 & $2.30 \mathrm{E}-12$ & $1.90 \mathrm{E}-10$ \\
\hline DYNC2H1 & 18.84 & 2.03 & 0.29 & 6.99 & $2.66 \mathrm{E}-12$ & 2.17E-10 \\
\hline DUSP18 & 10.48 & 3.61 & 0.52 & 6.94 & 3.97E-12 & $3.22 \mathrm{E}-10$ \\
\hline BIRC2 & 22.55 & 1.72 & 0.25 & 6.91 & $4.80 \mathrm{E}-12$ & $3.86 \mathrm{E}-10$ \\
\hline MELK & 43.85 & 1.32 & 0.19 & 6.89 & $5.59 \mathrm{E}-12$ & 4.46E-10 \\
\hline EFNB1 & 39.53 & -1.48 & 0.22 & -6.85 & $7.48 \mathrm{E}-12$ & 5.87E-10 \\
\hline RIT2 & 8.91 & 7.17 & 1.05 & 6.83 & $8.58 \mathrm{E}-12$ & 6.63E-10 \\
\hline MPHOSPH8 & 50.27 & 1.19 & 0.18 & 6.66 & $2.69 \mathrm{E}-11$ & 2.04E-09 \\
\hline C20orf203 & 6.74 & 4.32 & 0.65 & 6.61 & $3.97 \mathrm{E}-11$ & 3.00E-09 \\
\hline
\end{tabular}


Table S2 continued

\begin{tabular}{|c|c|c|c|c|c|c|}
\hline Gene.Symbol & baseMean & $\log 2 F C$ & IfcSE & stat & pvalue & padj \\
\hline ZCCHC10 & 18.53 & 1.92 & 0.29 & 6.57 & $5.13 E-11$ & $3.81 E-09$ \\
\hline SHISA3 & 73.52 & -1.00 & 0.15 & -6.54 & $6.12 \mathrm{E}-11$ & 4.51E-09 \\
\hline PRRG4 & 8.04 & 3.77 & 0.58 & 6.52 & $7.03 E-11$ & 5.10E-09 \\
\hline SAPCD2 & 70.23 & -1.15 & 0.18 & -6.50 & $7.84 \mathrm{E}-11$ & 5.64E-09 \\
\hline HHLA2 & 36.67 & 1.25 & 0.19 & 6.50 & $8.26 \mathrm{E}-11$ & 5.86E-09 \\
\hline ZBTB24 & 17.44 & 1.81 & 0.28 & 6.50 & $8.21 \mathrm{E}-11$ & 5.86E-09 \\
\hline FAM58A & 46.70 & 1.13 & 0.17 & 6.49 & $8.34 \mathrm{E}-11$ & 5.87E-09 \\
\hline PRAMEF11 & 8.00 & 5.01 & 0.77 & 6.49 & $8.61 \mathrm{E}-11$ & $6.02 \mathrm{E}-09$ \\
\hline LOC100507557 & 4.20 & 6.94 & 1.08 & 6.43 & $1.28 \mathrm{E}-10$ & 8.90E-09 \\
\hline PRSS23 & 8.83 & 2.91 & 0.45 & 6.43 & $1.29 \mathrm{E}-10$ & 8.90E-09 \\
\hline MAD2L1BP & 17.98 & 1.69 & 0.26 & 6.42 & $1.36 \mathrm{E}-10$ & $9.34 \mathrm{E}-09$ \\
\hline MCM9 & 9.33 & 2.71 & 0.42 & 6.40 & $1.53 \mathrm{E}-10$ & $1.03 \mathrm{E}-08$ \\
\hline PRELP & 14.09 & 2.10 & 0.33 & 6.37 & $1.91 \mathrm{E}-10$ & $1.28 \mathrm{E}-08$ \\
\hline TRIM23 & 9.52 & 2.51 & 0.40 & 6.34 & $2.24 \mathrm{E}-10$ & $1.49 \mathrm{E}-08$ \\
\hline IER5 & 19.30 & 1.77 & 0.28 & 6.33 & $2.52 \mathrm{E}-10$ & 1.67E-08 \\
\hline PIM1 & 14.23 & 1.87 & 0.30 & 6.31 & $2.76 \mathrm{E}-10$ & $1.81 \mathrm{E}-08$ \\
\hline GLIS2 & 31.39 & -1.59 & 0.25 & -6.29 & $3.26 \mathrm{E}-10$ & 2.10E-08 \\
\hline NAT8L & 48.61 & -1.30 & 0.21 & -6.29 & $3.27 \mathrm{E}-10$ & $2.10 \mathrm{E}-08$ \\
\hline NKIRAS1 & 34.49 & 1.33 & 0.21 & 6.25 & 4.23E-10 & $2.71 \mathrm{E}-08$ \\
\hline NRDE2 & 8.26 & 3.17 & 0.51 & 6.22 & $4.96 \mathrm{E}-10$ & $3.11 \mathrm{E}-08$ \\
\hline HDAC9 & 39.67 & -1.34 & 0.22 & -6.21 & $5.27 \mathrm{E}-10$ & $3.28 \mathrm{E}-08$ \\
\hline TIPARP & 15.01 & 1.88 & 0.31 & 6.12 & $9.12 \mathrm{E}-10$ & $5.54 \mathrm{E}-08$ \\
\hline NUDT10 & 16.60 & 1.76 & 0.29 & 6.11 & $9.94 \mathrm{E}-10$ & 5.99E-08 \\
\hline SOX12 & 30.90 & -1.48 & 0.24 & -6.11 & $1.02 \mathrm{E}-09$ & $6.11 \mathrm{E}-08$ \\
\hline ZNF705A & 6.03 & 6.47 & 1.06 & 6.08 & 1.20E-09 & 7.14E-08 \\
\hline TC2N & 9.35 & 2.24 & 0.37 & 6.07 & $1.30 \mathrm{E}-09$ & $7.68 \mathrm{E}-08$ \\
\hline $\mathrm{ZC} 3 \mathrm{H} 4$ & 47.94 & -1.16 & 0.19 & -6.06 & $1.35 \mathrm{E}-09$ & 7.92E-08 \\
\hline C2orf69 & 46.21 & 1.14 & 0.19 & 6.06 & $1.40 \mathrm{E}-09$ & 8.19E-08 \\
\hline PHF23 & 29.92 & -1.51 & 0.25 & -6.04 & $1.59 \mathrm{E}-09$ & $9.11 \mathrm{E}-08$ \\
\hline ART3 & 5.63 & 4.71 & 0.78 & 6.01 & $1.88 \mathrm{E}-09$ & $1.08 \mathrm{E}-07$ \\
\hline KLHL15 & 9.45 & 2.41 & 0.41 & 5.94 & 2.77E-09 & $1.56 \mathrm{E}-07$ \\
\hline MBD3L2 & 3.72 & 6.46 & 1.09 & 5.93 & 3.03E-09 & $1.69 \mathrm{E}-07$ \\
\hline PRAMEF5 & 5.05 & 3.99 & 0.68 & 5.91 & $3.42 \mathrm{E}-09$ & $1.87 \mathrm{E}-07$ \\
\hline ACSL3 & 23.34 & 1.42 & 0.24 & 5.90 & 3.71E-09 & 2.01E-07 \\
\hline LOC152217 & 426.72 & 1.19 & 0.20 & 5.89 & 3.79E-09 & 2.05E-07 \\
\hline SOGA1 & 25.04 & -1.72 & 0.29 & -5.86 & $4.58 \mathrm{E}-09$ & $2.41 \mathrm{E}-07$ \\
\hline ELL2 & 17.64 & 1.62 & 0.28 & 5.85 & 4.77E-09 & 2.50E-07 \\
\hline AHCYL2 & 7.69 & 2.49 & 0.43 & 5.79 & 7.16E-09 & $3.71 \mathrm{E}-07$ \\
\hline
\end{tabular}


Table S2 continued

\begin{tabular}{|c|c|c|c|c|c|c|}
\hline Gene.Symbol & baseMean & $\log 2 F C$ & IfcSE & stat & pvalue & padj \\
\hline HES7 & 21.19 & -1.74 & 0.30 & -5.76 & 8.39E-09 & 4.33E-07 \\
\hline SGCG & 3.11 & 6.35 & 1.10 & 5.75 & $8.82 \mathrm{E}-09$ & 4.53E-07 \\
\hline PELI2 & 8.26 & 2.34 & 0.41 & 5.75 & 8.93E-09 & 4.56E-07 \\
\hline NRBF2 & 11.97 & 2.06 & 0.36 & 5.74 & $9.64 \mathrm{E}-09$ & 4.89E-07 \\
\hline PRDM7 & 7.39 & 3.00 & 0.52 & 5.73 & $9.80 \mathrm{E}-09$ & 4.95E-07 \\
\hline EPB41L2 & 40.95 & 1.09 & 0.19 & 5.72 & $1.05 \mathrm{E}-08$ & $5.25 \mathrm{E}-07$ \\
\hline ISOC1 & 15.26 & 1.70 & 0.30 & 5.72 & $1.05 \mathrm{E}-08$ & $5.25 \mathrm{E}-07$ \\
\hline PLEKHG3 & 30.00 & -1.52 & 0.27 & -5.70 & $1.19 \mathrm{E}-08$ & $5.88 \mathrm{E}-07$ \\
\hline OXR1 & 16.65 & 1.74 & 0.31 & 5.69 & $1.29 \mathrm{E}-08$ & $6.35 \mathrm{E}-07$ \\
\hline ZNF10 & 11.15 & 2.02 & 0.36 & 5.66 & $1.49 \mathrm{E}-08$ & 7.31E-07 \\
\hline MTAP & 24.72 & 1.32 & 0.23 & 5.63 & 1.77E-08 & $8.56 \mathrm{E}-07$ \\
\hline GOLGB1 & 18.99 & 1.54 & 0.27 & 5.63 & $1.78 \mathrm{E}-08$ & 8.61E-07 \\
\hline LINC00652 & 5.43 & 3.38 & 0.60 & 5.59 & $2.28 \mathrm{E}-08$ & $1.09 \mathrm{E}-06$ \\
\hline KIN & 23.66 & 1.33 & 0.24 & 5.58 & $2.42 \mathrm{E}-08$ & 1.15E-06 \\
\hline SIX5 & 28.74 & -1.43 & 0.26 & -5.57 & $2.56 \mathrm{E}-08$ & $1.20 \mathrm{E}-06$ \\
\hline PAPOLG & 27.90 & 1.30 & 0.23 & 5.56 & $2.67 \mathrm{E}-08$ & $1.25 \mathrm{E}-06$ \\
\hline FAM155B & 22.83 & -1.74 & 0.31 & -5.54 & $3.01 \mathrm{E}-08$ & $1.40 \mathrm{E}-06$ \\
\hline RNF213 & 23.65 & 1.37 & 0.25 & 5.47 & $4.53 \mathrm{E}-08$ & $2.06 \mathrm{E}-06$ \\
\hline TESK2 & 5.55 & 3.44 & 0.63 & 5.46 & 4.63E-08 & $2.10 \mathrm{E}-06$ \\
\hline OSR2 & 7.12 & 3.02 & 0.55 & 5.46 & $4.81 \mathrm{E}-08$ & 2.17E-06 \\
\hline KITLG & 9.18 & 2.21 & 0.41 & 5.45 & $5.10 \mathrm{E}-08$ & $2.29 \mathrm{E}-06$ \\
\hline STIL & 15.20 & 1.67 & 0.31 & 5.43 & $5.72 \mathrm{E}-08$ & $2.53 \mathrm{E}-06$ \\
\hline KDM5A & 20.14 & 1.38 & 0.25 & 5.41 & $6.28 \mathrm{E}-08$ & 2.77E-06 \\
\hline PPP1R9B & 23.55 & -1.61 & 0.30 & -5.40 & $6.66 \mathrm{E}-08$ & $2.92 \mathrm{E}-06$ \\
\hline SNIP1 & 16.23 & 1.56 & 0.29 & 5.40 & $6.71 \mathrm{E}-08$ & $2.93 \mathrm{E}-06$ \\
\hline KIAA0040 & 7.19 & 2.30 & 0.43 & 5.36 & $8.50 \mathrm{E}-08$ & $3.68 \mathrm{E}-06$ \\
\hline RARG & 20.40 & -1.60 & 0.30 & -5.36 & $8.54 \mathrm{E}-08$ & $3.68 \mathrm{E}-06$ \\
\hline JUN & 6.88 & 2.53 & 0.47 & 5.35 & $8.94 \mathrm{E}-08$ & $3.82 \mathrm{E}-06$ \\
\hline CASP6 & 14.88 & 1.69 & 0.32 & 5.33 & $1.00 \mathrm{E}-07$ & 4.25E-06 \\
\hline ZSCAN5B & 3.42 & 5.80 & 1.09 & 5.32 & $1.04 \mathrm{E}-07$ & $4.38 \mathrm{E}-06$ \\
\hline FAM57A & 48.24 & -1.04 & 0.20 & -5.30 & $1.14 \mathrm{E}-07$ & 4.76E-06 \\
\hline NR2F2 & 22.61 & -1.51 & 0.29 & -5.30 & 1.17E-07 & 4.87E-06 \\
\hline NR2F6 & 41.02 & -1.11 & 0.21 & -5.30 & $1.18 \mathrm{E}-07$ & 4.87E-06 \\
\hline SRPK3 & 3.80 & 4.27 & 0.81 & 5.29 & $1.22 \mathrm{E}-07$ & $5.04 \mathrm{E}-06$ \\
\hline SGK1 & 5.78 & 2.96 & 0.56 & 5.29 & $1.23 \mathrm{E}-07$ & 5.07E-06 \\
\hline DAB2 & 7.59 & 2.17 & 0.41 & 5.28 & $1.32 \mathrm{E}-07$ & $5.38 \mathrm{E}-06$ \\
\hline LOC256021 & 4.84 & 5.58 & 1.06 & 5.27 & $1.36 \mathrm{E}-07$ & 5.56E-06 \\
\hline N4BP2L2 & 19.05 & 1.42 & 0.27 & 5.27 & $1.38 \mathrm{E}-07$ & 5.61E-06 \\
\hline
\end{tabular}


Table S2 continued

\begin{tabular}{|c|c|c|c|c|c|c|}
\hline Gene.Symbol & baseMean & $\log 2 F C$ & IfcSE & stat & pvalue & padj \\
\hline ITPRIPL2 & 29.13 & -1.32 & 0.25 & -5.27 & $1.40 \mathrm{E}-07$ & $5.64 \mathrm{E}-06$ \\
\hline MED15 & 24.18 & 1.34 & 0.25 & 5.26 & $1.41 \mathrm{E}-07$ & 5.66E-06 \\
\hline BCAS2 & 36.36 & 1.02 & 0.19 & 5.25 & $1.50 \mathrm{E}-07$ & 5.99E-06 \\
\hline NFYA & 8.17 & 2.33 & 0.44 & 5.25 & $1.52 \mathrm{E}-07$ & 6.07E-06 \\
\hline RAB11FIP1 & 37.80 & 1.06 & 0.20 & 5.22 & $1.80 \mathrm{E}-07$ & 7.10E-06 \\
\hline TRIM35 & 25.50 & 1.28 & 0.24 & 5.22 & $1.84 \mathrm{E}-07$ & 7.20E-06 \\
\hline GABPB1-AS1 & 19.09 & 1.40 & 0.27 & 5.21 & $1.87 \mathrm{E}-07$ & 7.32E-06 \\
\hline ATXN1L & 10.29 & 2.02 & 0.39 & 5.21 & $1.88 \mathrm{E}-07$ & 7.32E-06 \\
\hline RILPL1 & 24.29 & -1.38 & 0.27 & -5.19 & $2.06 \mathrm{E}-07$ & 7.95E-06 \\
\hline GNG11 & 10.13 & 2.19 & 0.42 & 5.17 & $2.35 \mathrm{E}-07$ & 8.99E-06 \\
\hline LOC400027 & 28.50 & 1.10 & 0.21 & 5.13 & $2.89 \mathrm{E}-07$ & 1.10E-05 \\
\hline ARL4C & 44.75 & -1.01 & 0.20 & -5.12 & $3.06 \mathrm{E}-07$ & 1.15E-05 \\
\hline ASH1L-AS1 & 8.76 & 2.06 & 0.40 & 5.09 & $3.53 E-07$ & $1.32 \mathrm{E}-05$ \\
\hline TOPORS & 17.01 & 1.42 & 0.28 & 5.06 & 4.09E-07 & $1.52 \mathrm{E}-05$ \\
\hline SLC35E4 & 8.94 & 2.16 & 0.43 & 5.06 & 4.19E-07 & $1.54 \mathrm{E}-05$ \\
\hline ITGB8 & 4.94 & 3.29 & 0.65 & 5.05 & 4.37E-07 & 1.60E-05 \\
\hline SNX33 & 30.35 & -1.23 & 0.24 & -5.05 & 4.41E-07 & $1.61 \mathrm{E}-05$ \\
\hline ATG14 & 18.38 & 1.38 & 0.27 & 5.04 & $4.66 \mathrm{E}-07$ & 1.70E-05 \\
\hline LGALS3 & 4.93 & 3.45 & 0.69 & 5.03 & 4.92E-07 & $1.78 \mathrm{E}-05$ \\
\hline KDM4E & 2.61 & 5.66 & 1.13 & 5.02 & $5.23 \mathrm{E}-07$ & $1.88 \mathrm{E}-05$ \\
\hline MIDN & 26.26 & -1.26 & 0.25 & -5.01 & $5.56 \mathrm{E}-07$ & 1.99E-05 \\
\hline IRX5 & 18.35 & 1.32 & 0.26 & 5.00 & 5.87E-07 & $2.10 \mathrm{E}-05$ \\
\hline C3 & 2.78 & 5.50 & 1.10 & 4.99 & 5.97E-07 & $2.12 \mathrm{E}-05$ \\
\hline TRIM47 & 26.57 & -1.28 & 0.26 & -4.99 & 5.99E-07 & $2.13 \mathrm{E}-05$ \\
\hline B3GNT2 & 4.55 & 3.42 & 0.69 & 4.99 & $6.06 \mathrm{E}-07$ & $2.13 \mathrm{E}-05$ \\
\hline PHOX2B & 2.39 & 5.67 & 1.14 & 4.99 & $6.06 \mathrm{E}-07$ & $2.13 \mathrm{E}-05$ \\
\hline TGIF2 & 21.56 & -1.41 & 0.28 & -4.98 & $6.20 \mathrm{E}-07$ & $2.18 \mathrm{E}-05$ \\
\hline ASF1A & 31.54 & 1.01 & 0.20 & 4.97 & $6.56 \mathrm{E}-07$ & $2.29 \mathrm{E}-05$ \\
\hline IGDCC3 & 18.29 & -1.55 & 0.31 & -4.97 & $6.78 \mathrm{E}-07$ & $2.36 \mathrm{E}-05$ \\
\hline NARG2 & 22.32 & 1.29 & 0.26 & 4.96 & 7.05E-07 & 2.45E-05 \\
\hline GSC & 4.24 & 3.70 & 0.75 & 4.95 & $7.43 \mathrm{E}-07$ & 2.57E-05 \\
\hline DDN & 18.40 & -1.59 & 0.32 & -4.92 & $8.50 \mathrm{E}-07$ & 2.91E-05 \\
\hline ZNF280A & 3.27 & 4.05 & 0.82 & 4.92 & 8.47E-07 & 2.91E-05 \\
\hline ACSM2A & 2.86 & 5.41 & 1.10 & 4.92 & $8.62 \mathrm{E}-07$ & $2.94 \mathrm{E}-05$ \\
\hline TGS1 & 28.36 & 1.07 & 0.22 & 4.92 & 8.70E-07 & 2.96E-05 \\
\hline SETD1B & 30.15 & -1.33 & 0.27 & -4.91 & $9.22 \mathrm{E}-07$ & $3.08 \mathrm{E}-05$ \\
\hline HARS2 & 18.47 & 1.32 & 0.27 & 4.90 & $9.41 \mathrm{E}-07$ & 3.13E-05 \\
\hline KIAA2018 & 5.02 & 2.77 & 0.57 & 4.90 & $9.58 \mathrm{E}-07$ & 3.18E-05 \\
\hline
\end{tabular}


Table S2 continued

\begin{tabular}{|c|c|c|c|c|c|c|}
\hline Gene.Symbol & baseMean & $\log 2 F C$ & IfcSE & stat & pvalue & padj \\
\hline CLP1 & 29.80 & 1.04 & 0.21 & 4.90 & $9.79 E-07$ & $3.23 \mathrm{E}-05$ \\
\hline STK17B & 10.16 & 1.79 & 0.37 & 4.89 & $9.93 \mathrm{E}-07$ & 3.27E-05 \\
\hline PTCSC3 & 4.01 & 5.20 & 1.06 & 4.88 & $1.06 \mathrm{E}-06$ & $3.45 \mathrm{E}-05$ \\
\hline FOSL1 & 17.87 & -1.72 & 0.35 & -4.87 & $1.10 \mathrm{E}-06$ & 3.57E-05 \\
\hline LOC644656 & 7.63 & 2.11 & 0.43 & 4.87 & $1.14 \mathrm{E}-06$ & 3.71E-05 \\
\hline MED26 & 8.95 & 2.19 & 0.45 & 4.86 & $1.15 \mathrm{E}-06$ & $3.72 \mathrm{E}-05$ \\
\hline STX6 & 24.08 & 1.11 & 0.23 & 4.85 & $1.21 \mathrm{E}-06$ & $3.88 \mathrm{E}-05$ \\
\hline HOXA11 & 19.29 & -1.46 & 0.30 & -4.85 & $1.25 \mathrm{E}-06$ & $3.98 \mathrm{E}-05$ \\
\hline C16orf55 & 13.90 & 1.41 & 0.29 & 4.84 & $1.29 \mathrm{E}-06$ & 4.10E-05 \\
\hline UTP23 & 21.58 & 1.24 & 0.26 & 4.84 & $1.29 \mathrm{E}-06$ & 4.10E-05 \\
\hline FAM195A & 34.44 & -1.03 & 0.21 & -4.82 & $1.41 \mathrm{E}-06$ & 4.46E-05 \\
\hline ATF7IP & 27.84 & 1.09 & 0.23 & 4.82 & $1.41 \mathrm{E}-06$ & 4.46E-05 \\
\hline OTUD3 & 18.71 & 1.39 & 0.29 & 4.81 & $1.52 \mathrm{E}-06$ & 4.75E-05 \\
\hline MBD3L5 & 3.30 & 4.45 & 0.93 & 4.80 & $1.55 \mathrm{E}-06$ & 4.85E-05 \\
\hline DNTT & 1.66 & 5.58 & 1.17 & 4.79 & $1.69 \mathrm{E}-06$ & $5.24 \mathrm{E}-05$ \\
\hline C12orf50 & 3.42 & 5.14 & 1.08 & 4.78 & $1.73 \mathrm{E}-06$ & 5.33E-05 \\
\hline GADD45A & 5.73 & 2.41 & 0.50 & 4.78 & $1.74 \mathrm{E}-06$ & 5.35E-05 \\
\hline EFNA2 & 10.19 & -2.02 & 0.42 & -4.78 & $1.75 \mathrm{E}-06$ & 5.37E-05 \\
\hline RBM5 & 31.71 & 1.03 & 0.22 & 4.76 & $1.89 \mathrm{E}-06$ & 5.77E-05 \\
\hline BAMBI & 7.96 & 2.02 & 0.42 & 4.76 & $1.90 \mathrm{E}-06$ & $5.78 \mathrm{E}-05$ \\
\hline PANX1 & 9.53 & 1.70 & 0.36 & 4.76 & $1.91 \mathrm{E}-06$ & 5.81E-05 \\
\hline DLC1 & 7.47 & 2.15 & 0.45 & 4.75 & $2.06 \mathrm{E}-06$ & 6.17E-05 \\
\hline KCNQ10T1 & 29.91 & 1.15 & 0.24 & 4.74 & $2.18 \mathrm{E}-06$ & $6.49 \mathrm{E}-05$ \\
\hline SCAPER & 7.02 & 2.17 & 0.46 & 4.73 & $2.28 \mathrm{E}-06$ & $6.78 \mathrm{E}-05$ \\
\hline SIKE1 & 26.60 & 1.13 & 0.24 & 4.71 & $2.45 \mathrm{E}-06$ & 7.23E-05 \\
\hline E2F2 & 24.80 & -1.32 & 0.28 & -4.71 & $2.54 \mathrm{E}-06$ & 7.45E-05 \\
\hline CITED4 & 34.75 & -1.03 & 0.22 & -4.70 & $2.62 \mathrm{E}-06$ & 7.66E-05 \\
\hline ZIM3 & 1.49 & 5.53 & 1.18 & 4.69 & $2.70 \mathrm{E}-06$ & $7.88 \mathrm{E}-05$ \\
\hline BEND4 & 31.73 & -1.05 & 0.22 & -4.69 & $2.75 \mathrm{E}-06$ & 7.99E-05 \\
\hline NAA35 & 11.43 & 1.65 & 0.35 & 4.69 & $2.78 \mathrm{E}-06$ & 8.04E-05 \\
\hline FBXL12 & 26.06 & 1.10 & 0.23 & 4.69 & $2.80 \mathrm{E}-06$ & 8.05E-05 \\
\hline SAMD10 & 21.23 & -1.42 & 0.30 & -4.68 & $2.80 \mathrm{E}-06$ & 8.05E-05 \\
\hline WHAMM & 20.80 & 1.26 & 0.27 & 4.68 & $2.91 \mathrm{E}-06$ & 8.33E-05 \\
\hline BIK & 26.05 & 1.06 & 0.23 & 4.67 & $3.02 \mathrm{E}-06$ & 8.61E-05 \\
\hline BHLHE22 & 3.50 & 5.13 & 1.10 & 4.66 & $3.18 \mathrm{E}-06$ & $9.06 \mathrm{E}-05$ \\
\hline FAM78A & 7.27 & -2.91 & 0.63 & -4.65 & $3.29 \mathrm{E}-06$ & $9.35 \mathrm{E}-05$ \\
\hline SOX13 & 21.63 & -1.38 & 0.30 & -4.65 & 3.33E-06 & 9.39E-05 \\
\hline SCG3 & 3.48 & 5.00 & 1.08 & 4.65 & 3.39E-06 & $9.52 \mathrm{E}-05$ \\
\hline
\end{tabular}


Table S2 continued

\begin{tabular}{|c|c|c|c|c|c|c|}
\hline Gene.Symbol & baseMean & $\log 2 F C$ & IfcSE & stat & pvalue & padj \\
\hline TMED7 & 15.33 & 1.31 & 0.28 & 4.64 & 3.57E-06 & $1.00 \mathrm{E}-04$ \\
\hline ORAI1 & 36.53 & -1.07 & 0.23 & -4.62 & 3.84E-06 & 1.07E-04 \\
\hline TAF4B & 2.89 & 5.12 & 1.11 & 4.62 & $3.85 \mathrm{E}-06$ & 1.07E-04 \\
\hline TMEM185A & 51.31 & 1.20 & 0.26 & 4.61 & 3.97E-06 & $1.10 \mathrm{E}-04$ \\
\hline ZNF776 & 26.87 & 1.03 & 0.22 & 4.61 & 4.07E-06 & $1.12 \mathrm{E}-04$ \\
\hline GRAMD1C & 3.26 & 4.96 & 1.08 & 4.61 & 4.12E-06 & $1.13 \mathrm{E}-04$ \\
\hline FBXO33 & 17.67 & 1.32 & 0.29 & 4.60 & 4.31E-06 & 1.17E-04 \\
\hline USP38 & 11.59 & 1.63 & 0.36 & 4.58 & $4.62 \mathrm{E}-06$ & $1.25 \mathrm{E}-04$ \\
\hline PLSCR1 & 8.81 & 1.89 & 0.41 & 4.58 & 4.74E-06 & 1.27E-04 \\
\hline BTG1 & 11.90 & 1.54 & 0.34 & 4.57 & $4.84 \mathrm{E}-06$ & $1.29 \mathrm{E}-04$ \\
\hline DUSP16 & 19.08 & -1.35 & 0.29 & -4.57 & 4.99E-06 & $1.33 \mathrm{E}-04$ \\
\hline MKRN9P & 1.59 & 5.36 & 1.17 & 4.56 & $5.00 \mathrm{E}-06$ & $1.33 \mathrm{E}-04$ \\
\hline HEXIM1 & 9.97 & 1.72 & 0.38 & 4.56 & $5.10 \mathrm{E}-06$ & $1.35 \mathrm{E}-04$ \\
\hline FERMT2 & 18.50 & 1.24 & 0.27 & 4.55 & $5.25 \mathrm{E}-06$ & $1.39 \mathrm{E}-04$ \\
\hline LOC401557 & 1.45 & 5.36 & 1.18 & 4.55 & $5.43 E-06$ & $1.43 \mathrm{E}-04$ \\
\hline C15orf60 & 3.57 & 4.91 & 1.08 & 4.53 & $5.84 \mathrm{E}-06$ & $1.53 \mathrm{E}-04$ \\
\hline NT5DC3 & 11.48 & 1.49 & 0.33 & 4.53 & $5.87 \mathrm{E}-06$ & $1.53 \mathrm{E}-04$ \\
\hline KLF17 & 2.77 & 4.90 & 1.08 & 4.52 & $6.12 \mathrm{E}-06$ & $1.59 \mathrm{E}-04$ \\
\hline CCNL1 & 8.85 & 1.83 & 0.41 & 4.50 & 6.67E-06 & $1.73 \mathrm{E}-04$ \\
\hline VEPH1 & 1.41 & 5.28 & 1.17 & 4.50 & 6.79E-06 & $1.75 \mathrm{E}-04$ \\
\hline TGIF1 & 39.96 & -1.03 & 0.23 & -4.50 & $6.91 \mathrm{E}-06$ & $1.78 \mathrm{E}-04$ \\
\hline GUSBP1 & 10.33 & 1.60 & 0.36 & 4.48 & $7.56 \mathrm{E}-06$ & $1.94 \mathrm{E}-04$ \\
\hline CEBPB & 15.31 & -1.68 & 0.38 & -4.47 & 7.71E-06 & 1.97E-04 \\
\hline ARRDC3 & 24.43 & 1.02 & 0.23 & 4.47 & 7.83E-06 & $2.00 \mathrm{E}-04$ \\
\hline LAMTOR3 & 21.08 & 1.23 & 0.28 & 4.47 & 7.93E-06 & $2.02 \mathrm{E}-04$ \\
\hline C12orf43 & 18.14 & 1.22 & 0.27 & 4.46 & $8.12 \mathrm{E}-06$ & $2.05 E-04$ \\
\hline TRIM49 & 1.54 & 5.23 & 1.17 & 4.46 & $8.12 \mathrm{E}-06$ & 2.05E-04 \\
\hline RWDD1 & 33.11 & 1.04 & 0.23 & 4.46 & $8.16 \mathrm{E}-06$ & 2.05E-04 \\
\hline ZNF134 & 7.86 & 1.85 & 0.42 & 4.45 & $8.58 \mathrm{E}-06$ & $2.16 \mathrm{E}-04$ \\
\hline LDB2 & 30.13 & -1.10 & 0.25 & -4.45 & 8.75E-06 & $2.19 \mathrm{E}-04$ \\
\hline EN2 & 19.03 & -1.47 & 0.33 & -4.44 & $9.14 \mathrm{E}-06$ & 2.27E-04 \\
\hline SRSF5 & 19.21 & 1.14 & 0.26 & 4.43 & $9.64 \mathrm{E}-06$ & $2.38 \mathrm{E}-04$ \\
\hline CDO1 & 12.71 & 1.57 & 0.35 & 4.42 & $9.74 \mathrm{E}-06$ & $2.40 \mathrm{E}-04$ \\
\hline MCMDC2 & 1.33 & 5.23 & 1.18 & 4.42 & $9.72 \mathrm{E}-06$ & $2.40 \mathrm{E}-04$ \\
\hline PLCL2 & 20.31 & -1.31 & 0.30 & -4.42 & $9.83 \mathrm{E}-06$ & 2.41E-04 \\
\hline ATXN2 & 30.87 & -1.06 & 0.24 & -4.42 & $9.90 \mathrm{E}-06$ & $2.42 \mathrm{E}-04$ \\
\hline PLK2 & 4.67 & 2.65 & 0.60 & 4.42 & $1.01 \mathrm{E}-05$ & $2.46 \mathrm{E}-04$ \\
\hline CCDC174 & 13.46 & 1.43 & 0.32 & 4.41 & $1.01 \mathrm{E}-05$ & 2.47E-04 \\
\hline
\end{tabular}


Table S2 continued

\begin{tabular}{|c|c|c|c|c|c|c|}
\hline Gene.Symbol & baseMean & $\log 2 F C$ & IfcSE & stat & pvalue & padj \\
\hline RADIL & 17.71 & 1.26 & 0.29 & 4.41 & $1.03 \mathrm{E}-05$ & $2.49 \mathrm{E}-04$ \\
\hline CNOT8 & 10.23 & 1.54 & 0.35 & 4.41 & $1.04 \mathrm{E}-05$ & $2.51 \mathrm{E}-04$ \\
\hline ANK3 & 15.26 & 1.34 & 0.30 & 4.40 & $1.06 \mathrm{E}-05$ & $2.53 \mathrm{E}-04$ \\
\hline GRPEL2 & 24.82 & 1.08 & 0.25 & 4.40 & $1.07 E-05$ & $2.56 \mathrm{E}-04$ \\
\hline H19 & 25.80 & -1.26 & 0.29 & -4.38 & 1.17E-05 & 2.79E-04 \\
\hline LRFN1 & 17.04 & -1.45 & 0.33 & -4.37 & $1.22 \mathrm{E}-05$ & 2.87E-04 \\
\hline CROT & 12.10 & 1.49 & 0.34 & 4.37 & $1.23 \mathrm{E}-05$ & $2.90 \mathrm{E}-04$ \\
\hline LOC285540 & 2.67 & 4.77 & 1.09 & 4.37 & $1.24 \mathrm{E}-05$ & 2.91E-04 \\
\hline TAL1 & 9.88 & -1.88 & 0.43 & -4.37 & $1.26 \mathrm{E}-05$ & $2.95 \mathrm{E}-04$ \\
\hline LOC100130557 & 4.38 & 2.48 & 0.57 & 4.37 & $1.26 \mathrm{E}-05$ & 2.95E-04 \\
\hline DNAJC3 & 12.24 & 1.38 & 0.32 & 4.36 & $1.28 \mathrm{E}-05$ & 2.99E-04 \\
\hline SH3KBP1 & 24.89 & 1.09 & 0.25 & 4.34 & $1.44 \mathrm{E}-05$ & $3.35 \mathrm{E}-04$ \\
\hline BTG2 & 5.47 & -3.42 & 0.79 & -4.32 & $1.58 \mathrm{E}-05$ & $3.64 \mathrm{E}-04$ \\
\hline HIPK1 & 9.69 & 1.62 & 0.37 & 4.31 & $1.62 \mathrm{E}-05$ & $3.73 E-04$ \\
\hline GNA14 & 2.27 & 4.91 & 1.14 & 4.30 & $1.68 \mathrm{E}-05$ & $3.82 \mathrm{E}-04$ \\
\hline PPIL3 & 11.84 & 1.52 & 0.35 & 4.30 & $1.73 \mathrm{E}-05$ & 3.91E-04 \\
\hline TLX1 & 21.58 & -1.21 & 0.28 & -4.30 & $1.73 \mathrm{E}-05$ & 3.91E-04 \\
\hline TNS3 & 28.97 & -1.08 & 0.25 & -4.29 & 1.75E-05 & $3.94 \mathrm{E}-04$ \\
\hline ILF3-AS1 & 18.45 & 1.16 & 0.27 & 4.29 & $1.76 \mathrm{E}-05$ & $3.96 \mathrm{E}-04$ \\
\hline GPBAR1 & 1.89 & 4.87 & 1.14 & 4.28 & $1.84 \mathrm{E}-05$ & 4.13E-04 \\
\hline C6orf147 & 6.80 & 1.96 & 0.46 & 4.28 & 1.87E-05 & 4.17E-04 \\
\hline LINC00310 & 2.50 & 3.62 & 0.85 & 4.27 & $1.94 \mathrm{E}-05$ & $4.32 \mathrm{E}-04$ \\
\hline CASP10 & 24.94 & 1.03 & 0.24 & 4.27 & $1.99 \mathrm{E}-05$ & 4.41E-04 \\
\hline AJUBA & 18.32 & -1.34 & 0.31 & -4.27 & 2.00E-05 & $4.42 \mathrm{E}-04$ \\
\hline SALL2 & 17.01 & -1.41 & 0.33 & -4.25 & $2.16 \mathrm{E}-05$ & 4.73E-04 \\
\hline NBPF3 & 25.18 & -1.08 & 0.25 & -4.24 & 2.19E-05 & 4.78E-04 \\
\hline NINJ1 & 28.87 & -1.03 & 0.24 & -4.23 & $2.30 \mathrm{E}-05$ & 5.00E-04 \\
\hline KAT6B & 6.89 & 1.94 & 0.46 & 4.21 & $2.55 \mathrm{E}-05$ & 5.47E-04 \\
\hline $\mathrm{C} 2 \mathrm{CD} 4 \mathrm{~B}$ & 1.86 & 4.81 & 1.14 & 4.21 & 2.57E-05 & 5.51E-04 \\
\hline OTX1 & 13.06 & 1.45 & 0.35 & 4.20 & 2.69E-05 & 5.75E-04 \\
\hline RGMA & 7.97 & -2.21 & 0.53 & -4.19 & $2.74 \mathrm{E}-05$ & $5.80 \mathrm{E}-04$ \\
\hline SLU7 & 19.24 & 1.17 & 0.28 & 4.19 & 2.79E-05 & 5.89E-04 \\
\hline FAM124A & 14.42 & -1.52 & 0.36 & -4.18 & 2.97E-05 & $6.26 \mathrm{E}-04$ \\
\hline BCL9 & 25.23 & -1.05 & 0.25 & -4.17 & $3.02 \mathrm{E}-05$ & $6.34 \mathrm{E}-04$ \\
\hline TMUB1 & 17.58 & -1.27 & 0.30 & -4.17 & $3.06 \mathrm{E}-05$ & $6.38 \mathrm{E}-04$ \\
\hline HCG27 & 3.93 & 2.80 & 0.68 & 4.13 & 3.57E-05 & 7.36E-04 \\
\hline TEFM & 7.31 & 1.82 & 0.44 & 4.13 & $3.65 \mathrm{E}-05$ & 7.52E-04 \\
\hline IMPACT & 22.03 & 1.03 & 0.25 & 4.13 & $3.68 \mathrm{E}-05$ & 7.56E-04 \\
\hline
\end{tabular}


Table S2 continued

\begin{tabular}{|c|c|c|c|c|c|c|}
\hline Gene.Symbol & baseMean & $\log 2 F C$ & IfcSE & stat & pvalue & padj \\
\hline KIAA0317 & 20.97 & 1.12 & 0.27 & 4.12 & 3.73E-05 & 7.63E-04 \\
\hline TRIM36 & 4.65 & 2.26 & 0.55 & 4.12 & $3.81 \mathrm{E}-05$ & 7.77E-04 \\
\hline TRIM24 & 42.40 & -1.24 & 0.30 & -4.11 & $3.90 \mathrm{E}-05$ & 7.94E-04 \\
\hline ELOVL1 & 17.94 & -1.23 & 0.30 & -4.11 & $3.96 \mathrm{E}-05$ & 8.05E-04 \\
\hline NAV1 & 15.80 & -1.46 & 0.36 & -4.10 & 4.11E-05 & 8.32E-04 \\
\hline CELSR2 & 19.58 & -1.18 & 0.29 & -4.09 & $4.28 \mathrm{E}-05$ & 8.63E-04 \\
\hline MLLT6 & 30.21 & -1.01 & 0.25 & -4.09 & 4.39E-05 & 8.81E-04 \\
\hline ZNF256 & 5.92 & 1.80 & 0.44 & 4.08 & 4.59E-05 & 9.19E-04 \\
\hline LOC256880 & 1.72 & 4.69 & 1.15 & 4.07 & 4.65E-05 & $9.28 \mathrm{E}-04$ \\
\hline STK11 & 21.09 & -1.15 & 0.28 & -4.07 & 4.64E-05 & $9.28 \mathrm{E}-04$ \\
\hline PHF15 & 9.73 & -1.98 & 0.49 & -4.07 & 4.78E-05 & $9.50 \mathrm{E}-04$ \\
\hline ANKRD34A & 3.06 & -4.57 & 1.12 & -4.06 & 4.89E-05 & $9.65 \mathrm{E}-04$ \\
\hline PDGFRA & 13.39 & 1.28 & 0.31 & 4.06 & $4.90 \mathrm{E}-05$ & $9.65 \mathrm{E}-04$ \\
\hline ZNF789 & 5.98 & 1.96 & 0.48 & 4.06 & $4.90 \mathrm{E}-05$ & $9.65 \mathrm{E}-04$ \\
\hline KIAA1210 & 1.07 & 4.97 & 1.23 & 4.05 & 5.11E-05 & $1.00 \mathrm{E}-03$ \\
\hline TLE3 & 24.43 & -1.01 & 0.25 & -4.04 & $5.24 \mathrm{E}-05$ & $1.02 \mathrm{E}-03$ \\
\hline SHB & 12.39 & -1.76 & 0.43 & -4.04 & $5.26 \mathrm{E}-05$ & $1.02 \mathrm{E}-03$ \\
\hline RYK & 12.63 & 1.35 & 0.33 & 4.04 & $5.40 \mathrm{E}-05$ & $1.05 \mathrm{E}-03$ \\
\hline KIAA0907 & 11.57 & 1.36 & 0.34 & 4.03 & $5.46 \mathrm{E}-05$ & $1.05 \mathrm{E}-03$ \\
\hline SERTAD3 & 6.58 & -2.25 & 0.56 & -4.03 & 5.67E-05 & 1.09E-03 \\
\hline ZSCAN16 & 16.55 & -1.30 & 0.32 & -4.03 & 5.67E-05 & 1.09E-03 \\
\hline KIAA0020 & 16.54 & 1.21 & 0.30 & 4.02 & 5.71E-05 & 1.09E-03 \\
\hline ELF4 & 30.76 & -1.06 & 0.26 & -4.02 & 5.76E-05 & 1.10E-03 \\
\hline DMRTA2 & 16.89 & -1.24 & 0.31 & -4.00 & 6.31E-05 & $1.20 \mathrm{E}-03$ \\
\hline DHRS3 & 17.09 & -1.24 & 0.31 & -3.99 & $6.58 \mathrm{E}-05$ & $1.24 \mathrm{E}-03$ \\
\hline NOG & 10.34 & -1.62 & 0.41 & -3.98 & $6.76 \mathrm{E}-05$ & $1.27 \mathrm{E}-03$ \\
\hline FAM174B & 11.87 & -1.60 & 0.40 & -3.97 & 7.33E-05 & 1.37E-03 \\
\hline C20orf112 & 9.87 & -1.57 & 0.40 & -3.96 & $7.34 \mathrm{E}-05$ & 1.37E-03 \\
\hline DIS3 & 20.01 & 1.05 & 0.26 & 3.96 & 7.51E-05 & $1.39 \mathrm{E}-03$ \\
\hline SPSB4 & 9.01 & -1.61 & 0.41 & -3.95 & 7.87E-05 & $1.46 \mathrm{E}-03$ \\
\hline BBS4 & 12.40 & 1.40 & 0.36 & 3.94 & $8.16 \mathrm{E}-05$ & $1.50 \mathrm{E}-03$ \\
\hline SUSD2 & 2.24 & 3.81 & 0.97 & 3.93 & $8.40 \mathrm{E}-05$ & $1.54 \mathrm{E}-03$ \\
\hline FADD & 23.05 & -1.02 & 0.26 & -3.92 & 8.71E-05 & $1.59 \mathrm{E}-03$ \\
\hline CDKN1A & 8.39 & 1.60 & 0.41 & 3.92 & $8.78 \mathrm{E}-05$ & 1.60E-03 \\
\hline ZMAT3 & 7.03 & 1.95 & 0.50 & 3.91 & $9.24 \mathrm{E}-05$ & 1.67E-03 \\
\hline NPHP3 & 4.54 & 2.27 & 0.58 & 3.91 & $9.31 \mathrm{E}-05$ & $1.68 \mathrm{E}-03$ \\
\hline SFTPB & 3.91 & 2.60 & 0.67 & 3.89 & $9.89 \mathrm{E}-05$ & 1.77E-03 \\
\hline CITED2 & 3.17 & 2.98 & 0.77 & 3.87 & 1.07E-04 & $1.90 \mathrm{E}-03$ \\
\hline
\end{tabular}


Table S2 continued

\begin{tabular}{|c|c|c|c|c|c|c|}
\hline Gene.Symbol & baseMean & $\log 2 F C$ & IfcSE & stat & pvalue & padj \\
\hline IQCE & 5.12 & -2.84 & 0.73 & -3.87 & $1.08 \mathrm{E}-04$ & $1.91 \mathrm{E}-03$ \\
\hline $\mathrm{BBC} 3$ & 7.02 & -2.07 & 0.54 & -3.87 & $1.10 \mathrm{E}-04$ & $1.94 \mathrm{E}-03$ \\
\hline TTC14 & 8.96 & 1.44 & 0.37 & 3.86 & $1.13 \mathrm{E}-04$ & 1.97E-03 \\
\hline CTH & 9.14 & 1.42 & 0.37 & 3.86 & $1.14 \mathrm{E}-04$ & 1.99E-03 \\
\hline RTN4R & 13.12 & -1.51 & 0.39 & -3.84 & $1.22 \mathrm{E}-04$ & 2.11E-03 \\
\hline SAMD5 & 8.57 & 1.52 & 0.40 & 3.84 & $1.24 \mathrm{E}-04$ & $2.14 \mathrm{E}-03$ \\
\hline STK31 & 1.32 & 4.54 & 1.18 & 3.84 & $1.24 \mathrm{E}-04$ & $2.14 \mathrm{E}-03$ \\
\hline LOC220729 & 6.73 & 1.58 & 0.41 & 3.83 & $1.29 \mathrm{E}-04$ & $2.21 \mathrm{E}-03$ \\
\hline EPC1 & 20.23 & -1.12 & 0.29 & -3.81 & $1.38 \mathrm{E}-04$ & $2.34 \mathrm{E}-03$ \\
\hline DDX20 & 15.53 & 1.08 & 0.28 & 3.81 & $1.39 \mathrm{E}-04$ & $2.35 \mathrm{E}-03$ \\
\hline ERF & 10.51 & -1.72 & 0.46 & -3.79 & $1.52 \mathrm{E}-04$ & $2.55 \mathrm{E}-03$ \\
\hline NAB2 & 5.07 & -3.42 & 0.91 & -3.77 & $1.62 \mathrm{E}-04$ & $2.69 \mathrm{E}-03$ \\
\hline TRIM54 & 19.77 & -1.07 & 0.28 & -3.76 & $1.67 \mathrm{E}-04$ & $2.76 \mathrm{E}-03$ \\
\hline PLEKHO1 & 11.55 & -1.43 & 0.38 & -3.73 & $1.90 \mathrm{E}-04$ & 3.09E-03 \\
\hline DDIT4 & 5.07 & -3.38 & 0.91 & -3.73 & $1.90 \mathrm{E}-04$ & 3.09E-03 \\
\hline EPN2 & 16.45 & 1.05 & 0.28 & 3.71 & $2.10 \mathrm{E}-04$ & $3.36 \mathrm{E}-03$ \\
\hline SH3GL2 & 2.78 & 3.54 & 0.96 & 3.70 & $2.14 \mathrm{E}-04$ & $3.41 \mathrm{E}-03$ \\
\hline RLF & 9.26 & 1.47 & 0.40 & 3.69 & $2.22 \mathrm{E}-04$ & $3.54 \mathrm{E}-03$ \\
\hline ARHGAP42 & 3.30 & 2.56 & 0.69 & 3.69 & $2.24 \mathrm{E}-04$ & $3.56 \mathrm{E}-03$ \\
\hline CHKA & 20.89 & -1.04 & 0.28 & -3.68 & $2.29 \mathrm{E}-04$ & $3.62 \mathrm{E}-03$ \\
\hline C9orf66 & 1.87 & 4.30 & 1.17 & 3.68 & $2.35 \mathrm{E}-04$ & 3.70E-03 \\
\hline FAM120C & 13.23 & 1.14 & 0.31 & 3.68 & $2.36 \mathrm{E}-04$ & $3.71 E-03$ \\
\hline SIPA1L2 & 12.33 & 1.23 & 0.33 & 3.67 & 2.39E-04 & $3.75 \mathrm{E}-03$ \\
\hline SETD5-AS1 & 12.66 & 1.20 & 0.33 & 3.67 & $2.41 \mathrm{E}-04$ & 3.77E-03 \\
\hline TNFRSF10D & 8.68 & 1.46 & 0.40 & 3.67 & $2.42 \mathrm{E}-04$ & $3.78 \mathrm{E}-03$ \\
\hline BCOR & 14.20 & -1.16 & 0.32 & -3.67 & $2.47 \mathrm{E}-04$ & $3.83 \mathrm{E}-03$ \\
\hline DDX58 & 5.49 & 1.91 & 0.52 & 3.65 & $2.59 \mathrm{E}-04$ & 4.00E-03 \\
\hline CDKN2B & 3.51 & 2.38 & 0.65 & 3.65 & 2.61E-04 & 4.03E-03 \\
\hline THOC5 & 11.96 & 1.17 & 0.32 & 3.65 & $2.62 \mathrm{E}-04$ & 4.03E-03 \\
\hline РPР3СС & 10.16 & -1.72 & 0.47 & -3.65 & $2.65 \mathrm{E}-04$ & 4.07E-03 \\
\hline CHST3 & 9.81 & -1.69 & 0.46 & -3.64 & $2.69 \mathrm{E}-04$ & 4.12E-03 \\
\hline FAM189A2 & 2.33 & 2.90 & 0.80 & 3.64 & $2.70 \mathrm{E}-04$ & 4.13E-03 \\
\hline GIT1 & 23.57 & -1.02 & 0.28 & -3.64 & $2.70 \mathrm{E}-04$ & 4.13E-03 \\
\hline LINC00115 & 5.14 & 1.82 & 0.50 & 3.64 & $2.72 \mathrm{E}-04$ & 4.14E-03 \\
\hline PDPK1 & 14.78 & 1.18 & 0.32 & 3.64 & $2.75 \mathrm{E}-04$ & 4.19E-03 \\
\hline CNNM4 & 8.09 & 1.39 & 0.38 & 3.63 & $2.80 \mathrm{E}-04$ & 4.24E-03 \\
\hline SGMS1 & 8.31 & 1.47 & 0.40 & 3.63 & $2.80 \mathrm{E}-04$ & $4.24 \mathrm{E}-03$ \\
\hline ARL14EP & 9.59 & 1.36 & 0.38 & 3.62 & $2.94 \mathrm{E}-04$ & 4.40E-03 \\
\hline
\end{tabular}


Table S2 continued

\begin{tabular}{|c|c|c|c|c|c|c|}
\hline Gene.Symbol & baseMean & $\log 2 \mathrm{FC}$ & IfcSE & stat & pvalue & padj \\
\hline CD97 & 11.12 & -1.46 & 0.40 & -3.61 & $3.02 \mathrm{E}-04$ & 4.49E-03 \\
\hline C5orf44 & 8.94 & 1.38 & 0.38 & 3.61 & 3.09E-04 & 4.57E-03 \\
\hline PDSS1 & 2.33 & 3.10 & 0.86 & 3.61 & $3.10 \mathrm{E}-04$ & 4.57E-03 \\
\hline IRX3 & 11.61 & -1.40 & 0.39 & -3.60 & $3.16 \mathrm{E}-04$ & 4.63E-03 \\
\hline TMEM189 & 12.62 & -1.33 & 0.37 & -3.60 & $3.18 \mathrm{E}-04$ & 4.65E-03 \\
\hline DKFZP434I0714 & 6.90 & 1.60 & 0.45 & 3.60 & $3.23 \mathrm{E}-04$ & 4.70E-03 \\
\hline MTF1 & 10.79 & 1.25 & 0.35 & 3.60 & $3.23 E-04$ & 4.70E-03 \\
\hline TRIM43 & 1.95 & 4.07 & 1.13 & 3.60 & $3.23 \mathrm{E}-04$ & 4.70E-03 \\
\hline GSE1 & 19.25 & -1.09 & 0.30 & -3.59 & $3.26 \mathrm{E}-04$ & 4.72E-03 \\
\hline GLMN & 5.35 & 1.87 & 0.52 & 3.58 & $3.45 \mathrm{E}-04$ & $4.98 \mathrm{E}-03$ \\
\hline CENPI & 4.92 & 1.83 & 0.51 & 3.57 & $3.53 \mathrm{E}-04$ & 5.07E-03 \\
\hline SYNJ1 & 3.94 & 2.08 & 0.58 & 3.57 & $3.56 \mathrm{E}-04$ & 5.10E-03 \\
\hline SGSH & 8.51 & 1.42 & 0.40 & 3.57 & $3.58 \mathrm{E}-04$ & 5.12E-03 \\
\hline RBM7 & 4.21 & 2.06 & 0.58 & 3.57 & $3.59 \mathrm{E}-04$ & $5.12 \mathrm{E}-03$ \\
\hline TBX5 & 13.12 & -1.29 & 0.36 & -3.56 & $3.65 \mathrm{E}-04$ & 5.19E-03 \\
\hline ZBTB10 & 4.24 & 2.00 & 0.56 & 3.55 & $3.82 \mathrm{E}-04$ & 5.41E-03 \\
\hline TP53BP2 & 12.27 & 1.16 & 0.33 & 3.55 & $3.90 \mathrm{E}-04$ & 5.51E-03 \\
\hline PACRGL & 5.03 & 1.69 & 0.48 & 3.53 & 4.14E-04 & $5.78 \mathrm{E}-03$ \\
\hline TMTC1 & 3.73 & 2.30 & 0.65 & 3.53 & 4.19E-04 & 5.82E-03 \\
\hline KIAA1429 & 12.70 & 1.16 & 0.33 & 3.52 & 4.27E-04 & 5.93E-03 \\
\hline DSEL & 8.39 & 1.46 & 0.42 & 3.51 & $4.52 \mathrm{E}-04$ & $6.20 \mathrm{E}-03$ \\
\hline PTCH1 & 8.24 & -1.55 & 0.44 & -3.50 & 4.62E-04 & $6.33 \mathrm{E}-03$ \\
\hline LMO4 & 15.74 & 1.10 & 0.32 & 3.50 & 4.72E-04 & $6.42 \mathrm{E}-03$ \\
\hline ZNF48 & 15.07 & -1.18 & 0.34 & -3.48 & 4.93E-04 & 6.67E-03 \\
\hline TPRN & 13.03 & -1.23 & 0.35 & -3.48 & $4.95 \mathrm{E}-04$ & $6.68 \mathrm{E}-03$ \\
\hline CCDC85B & 11.03 & -1.29 & 0.37 & -3.48 & 4.99E-04 & $6.72 \mathrm{E}-03$ \\
\hline TIGD5 & 9.69 & -1.63 & 0.47 & -3.48 & $5.04 \mathrm{E}-04$ & 6.77E-03 \\
\hline INSR & 19.55 & -1.03 & 0.30 & -3.47 & $5.12 \mathrm{E}-04$ & $6.86 \mathrm{E}-03$ \\
\hline ZNF473 & 13.03 & 1.07 & 0.31 & 3.47 & $5.12 \mathrm{E}-04$ & $6.86 \mathrm{E}-03$ \\
\hline ZNF827 & 17.64 & 1.00 & 0.29 & 3.47 & $5.27 \mathrm{E}-04$ & 7.00E-03 \\
\hline LOC100505659 & 2.12 & 3.08 & 0.89 & 3.47 & $5.29 \mathrm{E}-04$ & 7.02E-03 \\
\hline MT-ND2 & 2153.05 & 1.18 & 0.34 & 3.46 & $5.33 \mathrm{E}-04$ & 7.03E-03 \\
\hline SFT2D2 & 7.82 & 1.44 & 0.41 & 3.46 & $5.33 \mathrm{E}-04$ & 7.03E-03 \\
\hline WDR89 & 15.53 & -1.15 & 0.33 & -3.46 & $5.34 \mathrm{E}-04$ & 7.04E-03 \\
\hline ZNF674-AS1 & 13.75 & 1.10 & 0.32 & 3.45 & 5.57E-04 & 7.30E-03 \\
\hline MED31 & 6.70 & 1.52 & 0.44 & 3.45 & $5.63 \mathrm{E}-04$ & 7.37E-03 \\
\hline FAM188A & 9.60 & 1.32 & 0.38 & 3.43 & 5.93E-04 & $7.68 \mathrm{E}-03$ \\
\hline LOC100507373 & 2.63 & 2.60 & 0.76 & 3.43 & 5.93E-04 & $7.68 \mathrm{E}-03$ \\
\hline
\end{tabular}


Table S2 continued

\begin{tabular}{|lrrrrrr|}
\hline Gene.Symbol & baseMean & log2FC & IfcSE & stat & pvalue & padj \\
CETN3 & 9.38 & 1.34 & 0.39 & 3.43 & $6.15 \mathrm{E}-04$ & $7.91 \mathrm{E}-03$ \\
ST3GAL1 & 11.35 & -1.43 & 0.42 & -3.42 & $6.31 \mathrm{E}-04$ & $8.09 \mathrm{E}-03$ \\
GAS2L1 & 16.34 & -1.13 & 0.33 & -3.41 & $6.59 \mathrm{E}-04$ & $8.38 \mathrm{E}-03$ \\
LEF1 & 12.74 & -1.40 & 0.41 & -3.40 & $6.63 \mathrm{E}-04$ & $8.40 \mathrm{E}-03$ \\
MAN1A1 & 4.19 & 1.96 & 0.58 & 3.40 & $6.72 \mathrm{E}-04$ & $8.50 \mathrm{E}-03$ \\
JAG2 & 15.56 & -1.10 & 0.33 & -3.37 & $7.50 \mathrm{E}-04$ & $9.31 \mathrm{E}-03$ \\
LRFN4 & 13.80 & -1.11 & 0.33 & -3.35 & $8.05 \mathrm{E}-04$ & $9.92 \mathrm{E}-03$ \\
\hline
\end{tabular}


Table S3: TF perturbations followed by expression

Filtered for: HUMAN, upregulated genes (UP), P-value $\leq 0.01$

Input: differentially upregulated genes (358) in DIE_8.5h dataset

\begin{tabular}{|c|c|c|c|c|c|}
\hline Term & Overlap & P.value & $\begin{array}{r}\text { Adj. } \\
\text { P.value }\end{array}$ & $\begin{array}{l}\text { Odds } \\
\text { Ratio }\end{array}$ & $\begin{array}{r}\text { Comb. } \\
\text { Score }\end{array}$ \\
\hline DUX4_OE_GSE33799_CREEDSID_GENE_1429 & $113 / 451$ & $5.1 \mathrm{E}-101$ & $9.9 \mathrm{E}-98$ & 14.36 & 3315.88 \\
\hline PAX5_OE_GSE44244_CREEDSID_GENE_575 & $21 / 407$ & 1.1E-05 & 4.2E-03 & 2.96 & 33.84 \\
\hline PAX5_OE_GSE44244_CREEDSID_GENE_574 & $24 / 541$ & 3.1E-05 & $1.0 \mathrm{E}-02$ & 2.54 & 26.41 \\
\hline NFKB1_INACTIVATION_GSE20667_CREEDSID_GENE_2575 & $17 / 309$ & 3.3E-05 & $9.3 \mathrm{E}-03$ & 3.15 & 32.50 \\
\hline HSF1_KD_GSE3697_CREEDSID_GENE_779 & $17 / 328$ & 7.0E-05 & 1.7E-02 & 2.97 & 28.42 \\
\hline HSF1_KD_GSE3697_CREEDSID_GENE_778 & $17 / 331$ & $7.8 \mathrm{E}-05$ & 1.7E-02 & 2.94 & 27.83 \\
\hline TBX3_SHRNA_HFF_GSE76572_RNASEQ & $17 / 364$ & $2.4 \mathrm{E}-04$ & $4.8 \mathrm{E}-02$ & 2.68 & 22.27 \\
\hline JUNB_KD_FORESKIN_GSE63079_RNASEQ & $15 / 313$ & 4.2E-04 & $6.4 \mathrm{E}-02$ & 2.75 & 21.31 \\
\hline TP63_KD_GSE20286_CREEDSID_GENE_2453 & $15 / 334$ & 8.3E-04 & $9.6 \mathrm{E}-02$ & 2.57 & 18.25 \\
\hline MYB_KD_GSE49286_CREEDSID_GENE_1842 & $13 / 268$ & $9.1 \mathrm{E}-04$ & $9.9 \mathrm{E}-02$ & 2.78 & 19.47 \\
\hline KLF9_OE_GBM1A_GSE62212_RNASEQ & $17 / 411$ & $9.6 \mathrm{E}-04$ & $9.9 \mathrm{E}-02$ & 2.37 & 16.47 \\
\hline LEF1_KD_GSE42637_CREEDSID_GENE_1775 & $15 / 351$ & $1.4 \mathrm{E}-03$ & $1.3 \mathrm{E}-01$ & 2.45 & 16.15 \\
\hline GATA2_OE_HESC_GSE57395_RNASEQ & $19 / 505$ & $1.5 \mathrm{E}-03$ & $1.3 \mathrm{E}-01$ & 2.17 & 14.02 \\
\hline NFKB1_INACTIVATION_GSE20667_CREEDSID_GENE_2577 & $11 / 220$ & 1.7E-03 & $1.5 \mathrm{E}-01$ & 2.87 & 18.20 \\
\hline MYB_KD_GSE49286_CREEDSID_GENE_1835 & $14 / 333$ & 2.3E-03 & $1.5 \mathrm{E}-01$ & 2.41 & 14.67 \\
\hline SETDB1_KO_HELA_GSE86813_RNASEQ & $9 / 170$ & $3.1 \mathrm{E}-03$ & 2.0E-01 & 3.03 & 17.56 \\
\hline MYC_OE_MCF7_GSE101738_RNASEQ & $16 / 441$ & 4.9E-03 & $2.2 \mathrm{E}-01$ & 2.08 & 11.06 \\
\hline FLI1_KD_GSE27524_CREEDSID_GENE_1612 & $12 / 289$ & $5.0 \mathrm{E}-03$ & $2.2 \mathrm{E}-01$ & 2.38 & 12.60 \\
\hline MAF_OE_MACROPHAGE_GSE98368_RNASEQ & $19 / 567$ & 5.3E-03 & 2.3E-01 & 1.92 & 10.05 \\
\hline FLI1_KD_GSE27524_CREEDSID_GENE_1595 & $12 / 293$ & $5.6 \mathrm{E}-03$ & $2.3 \mathrm{E}-01$ & 2.35 & 12.17 \\
\hline SREBF2_KD_GSE50588_CREEDSID_GENE_2823 & $12 / 294$ & 5.7E-03 & 2.3E-01 & 2.34 & 12.07 \\
\hline FLI1_KD_GSE27524_CREEDSID_GENE_1611 & $12 / 297$ & $6.2 \mathrm{E}-03$ & $2.4 \mathrm{E}-01$ & 2.32 & 11.77 \\
\hline FLI1_KD_GSE27524_CREEDSID_GENE_1596 & $13 / 335$ & 6.3E-03 & $2.4 \mathrm{E}-01$ & 2.22 & 11.29 \\
\hline NFKB1_INACTIVATION_GSE20667_CREEDSID_GENE_2574 & $11 / 262$ & $6.5 \mathrm{E}-03$ & 2.3E-01 & 2.41 & 12.10 \\
\hline HSF1_KD_GSE3697_CREEDSID_GENE_783 & $11 / 263$ & 6.7E-03 & 2.3E-01 & 2.40 & 11.99 \\
\hline IRX6_SIRNA_BT549_GSE79586_RNASEQ & $16 / 469$ & 8.6E-03 & $2.5 \mathrm{E}-01$ & 1.96 & 9.30 \\
\hline
\end{tabular}


Table S4: TF perturbations followed by expression

Filtered for: HUMAN, downregulated genes (DOWN), P-value $\leq 0.01$ Input: differentially downregulated genes (125) in DIE_8.5h dataset

\begin{tabular}{|lrrrrr}
\hline Term & Overlap & P.value & $\begin{array}{r}\text { Adj. } \\
\text { P.value }\end{array}$ & $\begin{array}{r}\text { Odds } \\
\text { Ratio }\end{array}$ & $\begin{array}{r}\text { Comb. } \\
\text { Score }\end{array}$ \\
DUX4_OE_GSE33799_CREEDSID_GENE_1429 & $6 / 130$ & $1.22 \mathrm{E}-04$ & 0.027 & 7.823 & 70.489 \\
MYCN_SHRNA_IMR575_GSE80397_12HR_RNASEQ & $10 / 472$ & $4.89 \mathrm{E}-04$ & 0.064 & 3.591 & 27.372 \\
TAL1_OE_HESC_GSE57395_RNASEQ & $3 / 47$ & $2.69 \mathrm{E}-03$ & 0.175 & 10.819 & 64.039 \\
NFXL1_KD_GSE23674_CREEDSID_GENE_2439 & $7 / 327$ & $3.29 \mathrm{E}-03$ & 0.208 & 3.628 & 20.746 \\
DNMT1_INHIBITION_GSE45804_CREEDSID_GENE_2762 & $6 / 265$ & $4.90 \mathrm{E}-03$ & 0.234 & 3.838 & 20.411 \\
IRF7_OE_GSE37828_CREEDSID_GENE_1146 & $5 / 195$ & $6.03 \mathrm{E}-03$ & 0.251 & 4.346 & 22.215 \\
SNAI1_OE_GSE14773_CREEDSID_GENE_371 & $6 / 278$ & $6.16 \mathrm{E}-03$ & 0.246 & 3.658 & 18.620 \\
\hline
\end{tabular}


Table S5: Shared differentially upregulated genes between $8.5 \mathrm{~h}$ induced DIE cells and other datasets

\begin{tabular}{|c|c|c|c|c|c|c|}
\hline & & & & & & \\
\hline & & & & & Jaggana & \\
\hline Gene & Geng & Rickard & Heuvel & iDUX4 & enDUX4 & vDUX4 \\
\hline ACAP2 & yes & no & no & yes & yes & yeS \\
\hline ACSL3 & no & no & no & yes & no & yes \\
\hline ACSM2A & no & no & no & no & no & no \\
\hline ADPGK & yes & yes & yes & yes & yes & yes \\
\hline AHCYL2 & no & no & no & yes & no & no \\
\hline ALDH9A1 & no & no & no & no & no & no \\
\hline ALG13 & yes & no & yes & no & no & no \\
\hline ALPPL2 & no & yes & yes & yes & yes & yes \\
\hline ALYREF & no & yeS & yes & yes & yes & yes \\
\hline ANK3 & no & no & no & no & no & no \\
\hline ANXA5 & no & no & no & no & no & no \\
\hline ARHGAP42 & no & no & yes & yes & yes & yes \\
\hline ARL14EP & no & no & no & no & no & no \\
\hline ARL4D & no & no & no & yeS & no & yes \\
\hline ARRDC3 & no & no & no & yes & no & yes \\
\hline ART3 & yes & yes & yes & yeS & yes & yes \\
\hline ASF1A & no & no & no & no & no & no \\
\hline ASH1L-AS1 & no & no & yes & no & no & no \\
\hline ATF3 & yes & no & no & yes & no & yes \\
\hline ATF7IP & no & no & no & yes & yes & yes \\
\hline ATG14 & no & no & no & yes & yes & yes \\
\hline ATXN1L & yes & no & no & yes & yes & yes \\
\hline AVPI1 & yes & yes & yes & yes & yes & yes \\
\hline B3GNT2 & yes & no & yes & yes & yes & yes \\
\hline BAMBI & yes & yes & no & yes & yes & yes \\
\hline $\mathrm{BBs} 4$ & no & no & no & no & no & no \\
\hline BCAS2 & no & no & yes & yes & yes & yes \\
\hline BHLHE22 & yes & no & no & yes & no & yes \\
\hline BIK & no & yes & yes & yes & no & no \\
\hline BIRC2 & no & no & yes & nO & no & no \\
\hline в TG1 & no & no & no & yes & no & no \\
\hline C12orf43 & yes & no & yes & no & no & no \\
\hline C12orf50 & yes & yes & yes & yes & yes & yes \\
\hline C15orf60 & yes & no & no & yes & no & no \\
\hline C16orf55 & no & no & no & no & yes & yes \\
\hline
\end{tabular}


Table S5 continued

\begin{tabular}{|c|c|c|c|c|c|c|}
\hline & & & \multirow[b]{3}{*}{ Heuvel } & \multirow{2}{*}{\multicolumn{3}{|c|}{ Jagganathan }} \\
\hline & & & & & & \\
\hline Gene & Geng & Rickard & & iDUX4 & enDUX4 & vDUX4 \\
\hline C1D & no & no & no & no & no & yes \\
\hline C1orf63 & yes & no & no & yes & yes & yes \\
\hline C20orf203 & no & yes & no & no & no & no \\
\hline C21orf91 & yes & no & yes & yes & yes & yes \\
\hline $\mathrm{C} 2 \mathrm{CD} 4 \mathrm{~B}$ & no & no & no & no & no & no \\
\hline C2orf69 & no & no & yes & no & no & yes \\
\hline C3 & no & no & no & no & no & no \\
\hline C5orf44 & no & no & no & no & no & no \\
\hline C6orf147 & no & no & no & no & no & no \\
\hline C8orf33 & yes & yes & yes & yes & yes & yes \\
\hline C9orf66 & no & no & no & no & no & no \\
\hline CASP10 & no & no & no & no & no & no \\
\hline CASP6 & yes & no & no & no & yes & yes \\
\hline CCDC174 & no & no & yes & no & no & no \\
\hline CCNA1 & yes & yes & yes & yes & yes & yes \\
\hline CCNJ & yes & no & no & yes & no & yes \\
\hline CCNL1 & no & no & yes & yes & no & yes \\
\hline CDKN1A & yes & no & no & no & no & no \\
\hline CDKN2B & no & no & no & no & no & no \\
\hline CDO1 & yes & no & no & no & no & yes \\
\hline CENPI & no & no & no & no & no & no \\
\hline CETN3 & no & no & no & no & no & no \\
\hline CITED2 & no & no & no & no & no & no \\
\hline CLK1 & yes & no & yes & yes & yes & yes \\
\hline CLP1 & yes & no & yes & yes & yes & yes \\
\hline CNNM4 & yes & yes & yes & yes & yes & yes \\
\hline CNOT8 & no & no & no & no & no & no \\
\hline CROT & no & no & no & yes & no & yes \\
\hline CTH & yes & no & no & yes & no & yes \\
\hline CWC15 & yes & yes & yes & no & no & no \\
\hline CXCR4 & yes & yes & no & yes & yes & yes \\
\hline DAB2 & no & no & no & no & no & no \\
\hline DBR1 & yes & yes & yes & yes & yes & yes \\
\hline DDX10 & no & no & no & yes & yes & yes \\
\hline DDX20 & no & no & no & yes & yes & yes \\
\hline DDX58 & no & no & no & no & no & no \\
\hline DIO2 & no & no & yes & yes & yes & no \\
\hline
\end{tabular}


Table S5 continued

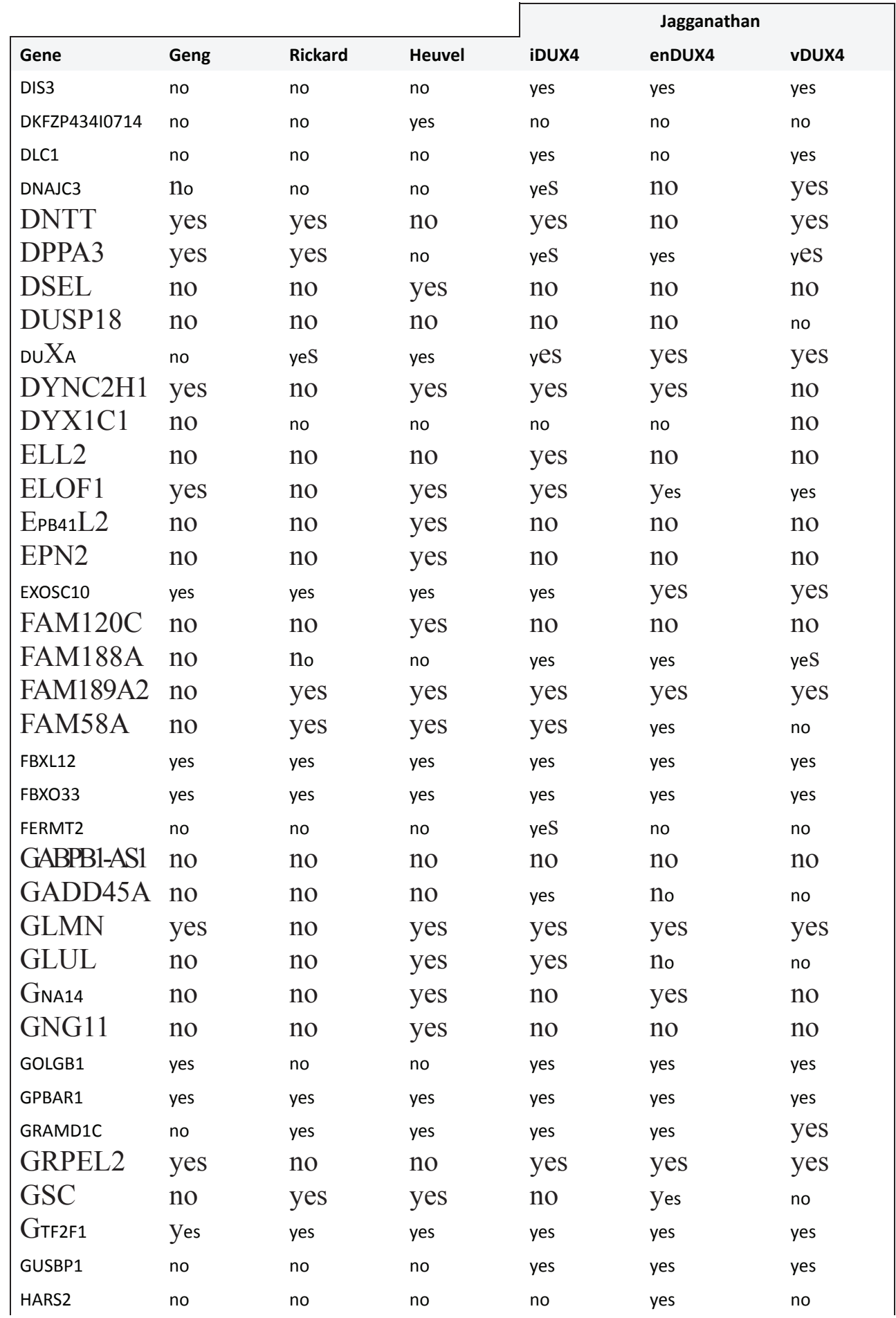


Table S5 continued

\begin{tabular}{|c|c|c|c|c|c|c|}
\hline & & & & & Jaggana & \\
\hline Gene & Geng & Rickard & Heuvel & iDUX4 & enDUX4 & vDUX4 \\
\hline HCG27 & no & no & no & no & no & no \\
\hline HEXIM1 & no & no & no & yes & no & yes \\
\hline HHLA2 & no & no & no & no & no & no \\
\hline HIPK1 & no & no & no & no & no & yes \\
\hline HNRNPF & yes & yes & yes & yes & yes & yes \\
\hline HOXB2 & yes & no & no & yes & yes & yes \\
\hline IER5 & no & no & no & no & no & no \\
\hline ILF3-AS1 & no & no & no & no & no & no \\
\hline IMPACT & no & no & no & no & no & no \\
\hline INO80C & yes & yes & yes & yes & yes & yes \\
\hline IRX5 & yes & no & no & yes & yes & no \\
\hline ISOC1 & yes & no & yes & yes & no & yes \\
\hline ITGB8 & no & no & no & no & no & no \\
\hline JUN & no & no & no & yes & no & no \\
\hline KAT6B & no & no & no & no & no & no \\
\hline KCNQ10T1 & no & no & no & no & no & no \\
\hline KDM4E & no & yes & yes & yes & yes & yes \\
\hline KDM5A & no & yes & yes & yes & yes & yes \\
\hline KDM5B & yes & no & yes & yes & yes & yes \\
\hline KHDC1 & no & yes & yes & yes & yes & no \\
\hline KHDC1L & yes & yes & yes & yes & yes & yes \\
\hline KIAA0020 & yes & no & no & yes & yes & yes \\
\hline KIAA0040 & no & yes & yes & yes & yes & yes \\
\hline KIAA0317 & no & no & no & no & yes & yes \\
\hline KIAA0907 & no & no & no & yes & no & no \\
\hline KIAA1210 & no & no & no & no & no & no \\
\hline KIAA1429 & yes & no & no & no & yes & yes \\
\hline KIAA1551 & no & yes & no & yes & yes & yes \\
\hline KIAA2018 & no & no & no & no & no & yes \\
\hline KIN & no & no & no & yes & no & yes \\
\hline KITLG & yes & no & no & yes & no & no \\
\hline KLF17 & yes & yes & yes & yes & yes & yes \\
\hline KLHL15 & yes & yes & yes & yes & yes & yes \\
\hline LAMTOR3 & no & no & no & no & no & no \\
\hline LEUTX & no & yes & yes & yes & yes & yes \\
\hline LGALS3 & no & no & no & no & no & no \\
\hline LINC00115 & no & no & no & no & no & no \\
\hline
\end{tabular}


Table S5 continued

\begin{tabular}{|c|c|c|c|c|c|c|}
\hline & & & & & & \\
\hline Gene & Geng & Rickard & Heuvel & iDUX4 & $\begin{array}{l}\text { Jaggana } \\
\text { enDUX4 }\end{array}$ & vDUX4 \\
\hline LINC00310 & no & no & no & no & no & no \\
\hline LINC00493 & no & no & no & no & no & no \\
\hline LINC00633 & no & no & no & no & no & no \\
\hline LINC00652 & no & no & no & no & no & no \\
\hline LMO4 & yes & no & no & yes & no & yes \\
\hline LOC100130557 & no & no & no & no & no & no \\
\hline LOC100188947 & no & no & no & no & nO & no \\
\hline LOC100216545 & no & no & no & no & no & no \\
\hline LOCl00505659 & no & no & no & no & no & no \\
\hline LOC100507373 & no & no & nO & no & no & no \\
\hline LOC100507557 & no & no & no & no & no & no \\
\hline LOC152217 & no & no & no & nO & no & no \\
\hline LoC220729 & no & no & no & no & no & no \\
\hline LOC256021 & no & no & no & no & no & no \\
\hline LOC256880 & no & nO & no & no & no & no \\
\hline LOC284551 & no & no & no & no & no & no \\
\hline LOC285540 & no & no & no & nO & no & no \\
\hline LoC400027 & no & no & no & no & no & no \\
\hline LOC401557 & no & no & no & no & no & no \\
\hline LOC441081 & yes & yes & no & no & no & no \\
\hline LOC644656 & no & no & no & no & no & no \\
\hline MAD2L1BP & yes & no & yes & yes & yes & yes \\
\hline MAN1A1 & no & no & no & yes & no & yes \\
\hline MBD3L2 & yes & yes & yes & Yes & yes & yes \\
\hline MBD3L5 & no & yes & yes & yes & yes & yes \\
\hline MCM9 & no & no & no & yes & no & no \\
\hline MCMDC2 & no & no & no & no & no & no \\
\hline MED15 & yes & no & no & no & no & no \\
\hline MED26 & yes & yes & Yes & yes & yes & yes \\
\hline MED31 & yes & no & yes & yes & yes & yes \\
\hline MELK & yes & yes & yes & no & yes & no \\
\hline MFSD 11 & no & no & no & yes & yes & yes \\
\hline MGC21881 & no & no & no & no & no & no \\
\hline MKRN9P & no & yes & no & no & no & no \\
\hline MMRN2 & no & no & no & no & no & no \\
\hline MPHOSPH8 & no & no & yes & no & yes & yes \\
\hline MRPL49 & no & no & yes & yes & yes & yes \\
\hline
\end{tabular}


Table S5 continued

\begin{tabular}{|c|c|c|c|c|c|c|}
\hline & & & \multirow[b]{2}{*}{ Heuvel } & \multicolumn{3}{|c|}{ Jagganathan } \\
\hline Gene & Geng & Rickard & & iDUX4 & $\begin{array}{l}\text { Jaggana } \\
\text { enDUX4 }\end{array}$ & vDUX4 \\
\hline MT-ND2 & no & no & no & no & no & no \\
\hline MTAP & yes & no & no & no & no & no \\
\hline MTF1 & no & no & no & yes & yes & yes \\
\hline N4BP2L2 & no & no & no & no & no & no \\
\hline NAA35 & no & no & no & no & yes & yes \\
\hline NARG2 & yes & no & no & no & no & no \\
\hline NDEL1 & yes & no & no & yes & no & yes \\
\hline NFYA & yes & no & yes & yes & yes & yes \\
\hline NKIRAS1 & yes & no & yes & yes & yes & yes \\
\hline NPHP3 & no & no & no & no & no & no \\
\hline NRBF2 & yes & no & yes & yes & yes & yes \\
\hline NRDE2 & no & yes & yes & yes & yes & yes \\
\hline NT5DC3 & yes & no & yes & yes & yes & yes \\
\hline NUDT10 & no & yes & no & no & yes & no \\
\hline NXF1 & yes & yes & yes & yes & yes & yes \\
\hline ODC1 & yes & yes & yes & yes & yes & yes \\
\hline OSR2 & yes & no & no & yes & no & yes \\
\hline OTUD3 & no & no & no & yes & yes & yes \\
\hline OTX1 & no & no & no & no & no & no \\
\hline OXR1 & yes & no & yes & yes & no & yes \\
\hline PACRGL & no & no & no & yes & no & no \\
\hline PANX1 & no & no & no & yes & no & no \\
\hline PANX2 & yes & yes & yes & yes & yes & yes \\
\hline PAPOLG & no & no & no & yes & yes & yes \\
\hline PDGFRA & no & no & no & yes & no & yes \\
\hline PDPK1 & no & no & yes & no & yes & no \\
\hline PDSS1 & yes & yes & no & yes & yes & yes \\
\hline PELI2 & yes & no & no & yes & no & yes \\
\hline PHOX2B & no & no & no & no & no & no \\
\hline PIM1 & yes & no & yes & yes & no & yes \\
\hline PLK2 & no & no & no & yes & no & no \\
\hline PLSCR1 & no & no & no & no & no & no \\
\hline PLXNB3 & no & no & no & no & no & no \\
\hline PNN & yes & no & no & yes & no & yes \\
\hline PNP & no & yes & yes & yes & yes & yes \\
\hline PNRC1 & no & no & no & yes & no & no \\
\hline PPIL3 & no & no & no & no & yes & no \\
\hline
\end{tabular}


Table S5 continued

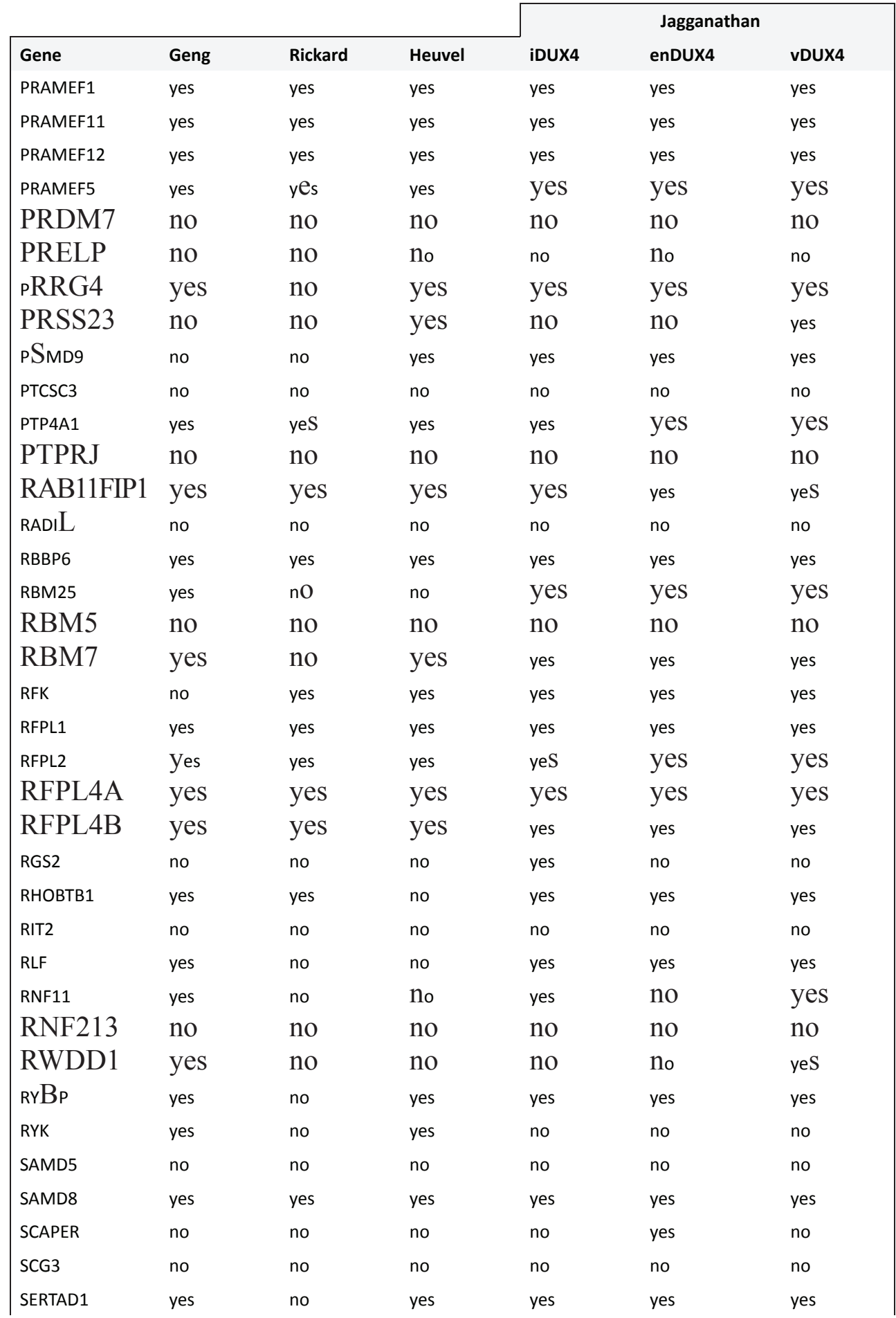


Table S5 continued

\begin{tabular}{|c|c|c|c|c|c|c|}
\hline & & & & & Jaggana & \\
\hline Gene & Geng & Rickard & Heuvel & iDUX4 & enDUX4 & vDUX4 \\
\hline SETD5-AS1 & no & no & no & no & no & no \\
\hline SFT2D2 & yes & no & yes & yes & yes & yes \\
\hline SFTPB & no & no & no & no & no & no \\
\hline SGCG & yes & no & no & yes & no & yes \\
\hline SGK1 & no & yes & yes & yes & yes & yes \\
\hline SGMS1 & no & no & no & no & no & no \\
\hline SGSH & yes & no & no & no & no & no \\
\hline SH3GL2 & yes & yes & no & yes & no & no \\
\hline SH3KBP1 & no & no & no & no & no & no \\
\hline SHC1 & no & no & no & no & no & no \\
\hline SIAH1 & yes & yes & yes & yes & yes & yes \\
\hline SIKE1 & no & no & no & no & no & no \\
\hline SIPA1L2 & no & no & no & yes & yes & no \\
\hline SIRT1 & yes & no & yes & yes & yes & yes \\
\hline SLC2A3 & yes & yes & yes & yes & yes & yes \\
\hline SLC34A2 & yes & yes & yes & yes & yes & yes \\
\hline SLC35E4 & no & no & yes & no & yes & no \\
\hline SLU7 & yes & no & no & yes & yes & yes \\
\hline SNAI1 & yes & yes & yes & yes & yes & yes \\
\hline SNIP1 & yes & no & no & yes & yes & yes \\
\hline SNUPN & no & no & no & no & yes & no \\
\hline SPTY2D1 & yes & yes & yes & yes & yes & yes \\
\hline SRPK3 & no & no & no & no & no & no \\
\hline SRSF5 & no & no & no & no & no & no \\
\hline SRSF8 & no & no & yes & yes & yes & yes \\
\hline STIL & yes & yes & no & yes & yes & yes \\
\hline STK17B & no & no & no & no & no & no \\
\hline STK31 & no & no & no & no & no & no \\
\hline STX6 & yes & no & no & yes & yes & yes \\
\hline SUPT6H & yes & no & yes & yes & yes & yes \\
\hline SUSD2 & no & no & no & no & no & no \\
\hline SYNJ1 & yes & yes & yes & yes & yes & yes \\
\hline TAF4B & yes & yes & no & yes & yes & yes \\
\hline TC2N & yes & yes & yes & yes & yes & yes \\
\hline TCEB3 & yes & no & yes & yes & yes & yes \\
\hline TEFM & no & no & no & yes & yes & yes \\
\hline TESK2 & yes & yes & no & yes & yes & yes \\
\hline
\end{tabular}


Table S5 continued

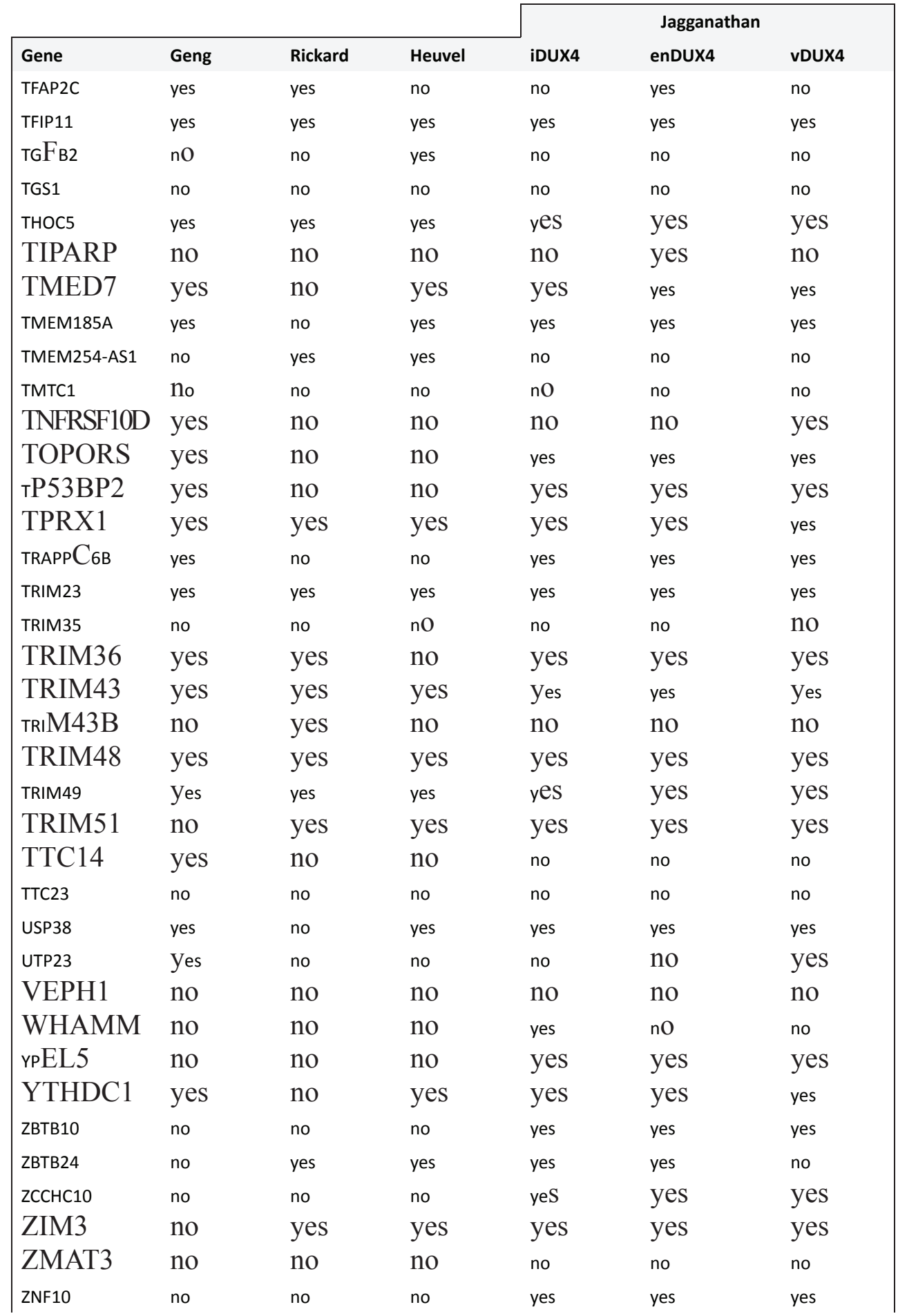


Table S5 continued

\begin{tabular}{|c|c|c|c|c|c|c|}
\hline & & & \multirow{2}{*}{\multicolumn{3}{|c|}{ Jagganathan }} \\
\hline & & & & & & \\
\hline Gene & Geng & Rickard & Heuvel & iDUX4 & enDUX4 & vDUX4 \\
\hline ZNF134 & no & no & no & yes & yes & yes \\
\hline ZNF217 & yes & yes & yes & yes & yes & yes \\
\hline ZNF256 & yes & no & no & yes & yes & no \\
\hline ZNF280A & yes & yes & no & yes & yes & yes \\
\hline ZNF296 & yes & yes & yes & yes & yes & yes \\
\hline ZNF473 & no & no & no & yes & yes & no \\
\hline ZNF574 & yes & yes & yes & yes & yes & yes \\
\hline ZNF622 & yes & no & no & yes & yes & yes \\
\hline ZNF674-AS1 & no & no & yes & no & no & no \\
\hline ZNF705A & yes & yes & yes & yes & yes & yes \\
\hline ZNF776 & no & no & no & no & yes & no \\
\hline ZNF789 & no & no & no & no & no & no \\
\hline ZNF827 & no & no & no & no & no & no \\
\hline ZNHIT6 & yes & yes & yes & yes & yes & yes \\
\hline ZRANB2 & no & no & no & no & no & no \\
\hline ZSCAN4 & yes & yes & yes & yes & yes & yes \\
\hline ZSCAN5A & yes & yes & no & yes & yes & no \\
\hline ZSCAN5B & no & yes & yes & yes & yes & yes \\
\hline
\end{tabular}

Table S6: Shared differentially downregulated genes between $8.5 \mathrm{~h}$ induced DIE cells and other datasets

\begin{tabular}{|lllllll|}
\hline Gene & & & & \multicolumn{3}{c}{ Jagganathan } \\
AJUBA & Geng & Rickard & Heuvel & iDUX4 & enDUX4 & vDUX4 \\
ANKRD34A & no & no & yes & no & no & no \\
ARL4C & no & no & no & no & no & no \\
ATXN2 & no & no & no & no & no & no \\
BBC3 & no & no & no & no & no & no \\
BCL9 & no & no & no & no & yes & no \\
BCOR & no & no & no & no & no & no \\
BEND4 & no & no & no & no & no & no \\
BTG2 & yes & no & yes & no & yes & no \\
C20orf112 & no & no & no & no & no & no \\
CCDC85B & no & no & yes & no & yes & no \\
CD97 & no & no & yes & no & yes & no
\end{tabular}


Table S6 continued

\begin{tabular}{|c|c|c|c|c|c|c|}
\hline & & & & & \\
\hline & & & & \multicolumn{3}{|c|}{ Jagganathan } \\
\hline Gene & Geng & Rickard & Heuvel & iDUX4 & enDUX4 & vDUX4 \\
\hline CDC42EP1 & no & no & yes & no & yes & yes \\
\hline CEBPB & yes & no & yes & yes & yes & no \\
\hline CELSR2 & no & no & no & no & no & no \\
\hline CHKA & no & no & no & no & no & no \\
\hline CHST3 & no & no & yes & yes & no & no \\
\hline CITED4 & no & no & no & no & no & no \\
\hline DDIT4 & yes & no & yes & yes & yes & no \\
\hline DDN & no & no & no & no & no & no \\
\hline DHRS3 & no & no & no & no & no & no \\
\hline DMRTA2 & no & no & no & no & no & no \\
\hline DUSP16 & no & no & no & no & no & no \\
\hline $\mathrm{E} 2 \mathrm{~F} 2$ & no & no & no & no & no & no \\
\hline EFNA2 & no & no & no & no & no & no \\
\hline EFNB1 & no & no & no & yes & no & no \\
\hline ELF4 & no & no & no & no & yes & no \\
\hline ELOVL1 & no & no & yes & no & yes & no \\
\hline EN2 & no & no & no & no & no & no \\
\hline EPC1 & no & no & no & no & no & no \\
\hline ERF & no & no & yes & no & no & no \\
\hline FADD & no & no & yes & no & no & no \\
\hline FAM124A & no & no & no & no & no & no \\
\hline FAM155B & no & no & no & no & no & no \\
\hline FAM174B & yes & no & no & no & no & no \\
\hline FAM195A & no & no & no & no & no & no \\
\hline FAM57A & yes & no & yes & yes & no & no \\
\hline FAM78A & no & no & no & yes & no & no \\
\hline FOSL1 & no & no & yes & no & no & no \\
\hline GAS2L1 & no & no & yes & no & no & no \\
\hline GIT1 & no & no & yes & no & no & no \\
\hline GLIS2 & no & no & yes & yes & yes & no \\
\hline GSE1 & no & no & yes & no & no & no \\
\hline H19 & no & no & no & no & no & no \\
\hline HDAC9 & no & no & no & no & no & no \\
\hline HES7 & no & no & no & no & no & no \\
\hline HOXA11 & no & no & yes & no & yes & no \\
\hline ID1 & yes & no & yes & no & no & no \\
\hline ID3 & yes & no & yes & yes & no & no \\
\hline
\end{tabular}


Table S6 continued

\begin{tabular}{|c|c|c|c|c|c|c|}
\hline & & & \multirow[b]{3}{*}{ Heuvel } & \multirow{2}{*}{\multicolumn{3}{|c|}{ Jagganathan }} \\
\hline & & & & & & \\
\hline Gene & Geng & Rickard & & iDUX4 & enDUX4 & vDUX4 \\
\hline IGDCC3 & no & no & no & no & no & no \\
\hline INSR & no & no & no & no & no & no \\
\hline IQCE & no & no & yes & no & yes & no \\
\hline IRX3 & no & no & yes & no & no & no \\
\hline ITPRIPL2 & no & no & yes & yes & no & no \\
\hline JAG2 & no & no & no & no & no & no \\
\hline LDB2 & no & no & no & no & no & no \\
\hline LEF1 & no & no & no & no & no & no \\
\hline LRFN1 & no & no & no & no & no & no \\
\hline LRFN4 & no & no & yes & no & no & no \\
\hline MEX3A & no & no & no & no & no & no \\
\hline MIDN & no & no & no & no & no & no \\
\hline MLLT6 & no & no & yes & no & yes & no \\
\hline NAB2 & no & no & yes & no & no & no \\
\hline NAT8L & no & no & no & no & no & no \\
\hline NAV1 & no & no & yes & yes & yes & no \\
\hline NBPF3 & no & no & no & no & no & no \\
\hline NINJ1 & yes & no & yes & yes & yes & no \\
\hline NOG & no & no & yes & no & no & no \\
\hline NR2F2 & no & no & yes & yes & no & no \\
\hline NR2F6 & no & no & no & no & yes & no \\
\hline ORAI1 & no & no & yes & no & no & no \\
\hline PHF15 & yes & no & yes & yes & yes & no \\
\hline PHF23 & no & no & no & no & no & no \\
\hline PLCL2 & no & no & no & yes & no & no \\
\hline PLEKHG3 & no & no & yes & no & no & no \\
\hline PLEKHO1 & no & no & yes & no & yes & no \\
\hline PPP1R18 & no & no & yes & yes & yes & no \\
\hline PPP1R9B & no & no & yes & yes & yes & no \\
\hline РРРЗСС & yes & no & yes & no & yes & no \\
\hline PTCH1 & no & no & no & no & no & no \\
\hline RARG & no & no & yes & yes & yes & no \\
\hline RGMA & no & no & no & no & no & no \\
\hline RILPL1 & yes & no & yes & no & no & no \\
\hline RTN4R & no & no & no & no & no & no \\
\hline SALL2 & no & no & yes & no & no & no \\
\hline SAMD10 & no & no & no & no & no & no \\
\hline
\end{tabular}


Table S6 continued

\begin{tabular}{|c|c|c|c|c|c|c|}
\hline \multirow[b]{2}{*}{ Gene } & \multirow[b]{2}{*}{ Geng } & \multirow[b]{2}{*}{ Rickard } & \multirow[b]{2}{*}{ Heuvel } & \multicolumn{3}{|c|}{ Jagganathan } \\
\hline & & & & iDUX4 & enDUX4 & vDUX4 \\
\hline SAPCD2 & no & no & yes & no & no & no \\
\hline SERTAD3 & no & no & no & no & no & no \\
\hline SETD1B & no & no & no & no & yes & no \\
\hline SHB & no & no & no & no & no & no \\
\hline SHISA3 & no & no & no & no & no & no \\
\hline SIX5 & yes & no & yes & no & yes & no \\
\hline SNX33 & no & no & yes & yes & yes & no \\
\hline SOGA1 & no & no & yes & no & no & no \\
\hline SOX12 & no & no & yes & no & yes & no \\
\hline SOX13 & no & no & no & yes & no & no \\
\hline SPSB4 & no & no & no & no & no & no \\
\hline ST3GAL1 & no & no & no & no & no & no \\
\hline ST6GAL1 & no & no & no & no & no & no \\
\hline STK11 & no & no & no & no & yes & no \\
\hline TAL1 & no & no & no & no & no & no \\
\hline TBX5 & no & no & no & no & no & no \\
\hline TGIF1 & no & no & no & yes & yes & yes \\
\hline TGIF2 & no & no & no & no & no & no \\
\hline TIGD5 & yes & no & yes & no & no & no \\
\hline TLE3 & no & no & yes & yes & no & no \\
\hline TLX1 & no & no & no & no & no & no \\
\hline TMEM189 & no & no & no & no & yes & no \\
\hline TMUB1 & no & no & no & no & no & no \\
\hline TNS3 & yes & no & yes & yes & yes & no \\
\hline TPRN & no & no & no & no & no & no \\
\hline TRIM24 & no & no & no & no & no & no \\
\hline TRIM47 & yes & no & no & yes & yes & no \\
\hline TRIM54 & no & no & no & no & no & no \\
\hline WDR89 & no & no & no & no & no & no \\
\hline $\mathrm{ZC} 3 \mathrm{H} 4$ & no & no & yes & no & no & no \\
\hline ZNF48 & no & no & no & no & no & no \\
\hline ZSCAN16 & no & no & no & no & no & no \\
\hline
\end{tabular}


Table S7. Gene specifc spacers targeting the 4.5h upregulated genes

\begin{tabular}{|c|c|c|c|}
\hline Gene & spacer 1 & spacer 2 & spacer 3 \\
\hline ZSCAN4 & ACAGCAATAATTCATATGCA & CCATCACCATAGGGACACCT & AACCCTGTACTCACTAAGGC \\
\hline ZNF217 & САAАATCTCACCCTGAAACG & GGACACATAATGGCAAATCG & AACATGTCTTAATGCAACAC \\
\hline ETDB & GTCATAAAGCAACTCTAGGG & TGACATCGACCTGTCAAGAT & TCAGTAAAACAGCACAACCG \\
\hline SRSF8 & CGGACGAAAGCGAAGCCCCG & CGCTACAGGGAATCTCGCTA & ACAGCCGATCTCCTTACAGC \\
\hline PRAMEF1 & ACTGGAGGTGTTCCAGCCCG & GAGTCTGGAAGTGTCTCCTG & AGACAGCAGAGGACCGTCCA \\
\hline RBBP6 & AAGTCGAACTGAACCAGCGA & GATATCATCGATCTAGGTCA & TGAGACACAACAATTCATCT \\
\hline PNP & AGTGGTCAGAACCCTCTCAG & CCGGTCGTAGGCATCAGACA & TTGCCAGTACCTGTACTTCG \\
\hline SIAH1 & AAGTTGCGAATGGATCCCAA & CAGAAGACGCATATTTACAG & TAAGTCCATTACAACCCTAC \\
\hline ZNF296 & CTGTGGCAAACAGTTCACAG & GGCCGCTGCCACTTGCACGG & GTGAGCGCATGTGCACTTTG \\
\hline TRIM51 & CTGAATGCAAGAAGACAACG & АСTCACCTCTGAATCCACTG & AAATCTCAGAAATCTGAACA \\
\hline RFPL4B & CGTGCAGACTTTGCTCAAGT & TCTGCTTTGATTGCATCCAG & GAGGTGAAGTCATGGTCCCT \\
\hline KHDC1L & TGTGCAGCTCAATGCAGCGA & GGGAACGAGTGCTCTCAGCA & TCTTCCATGTGGAACACCAT \\
\hline CCNA1 & ACAAACTCGTCTACTTCAGG & AGATGAATCTACCAGCATAG & AGGCATGCGCACGATTCTGG \\
\hline TFIP11 & CAAATACTCTTACAAGACCG & GGGTGCACATTATTCCTGTG & CATCGTCTATCCACTCATGA \\
\hline LEUTX & CAGAAGGCTCACGTAGATCA & CCAGGATGAAACCCTCGCAG & TTGGGAACAGACCTTTACTA \\
\hline PRAMEF12 & TAGATTACATGACTTCATCA & AAGGTCTAGGATCACCATCA & CATTACGACTGTCTCCAAGG \\
\hline ZNF622 & GTGTTGGCAAGATTTGCTTG & GTTCTCGTAGGCGTTGAAAG & TGATGAAGAATTGGAATGTG \\
\hline HSPA1A & GGTGCTGGACAAGTGTCAAG & AАTCTACCTCCTCAATGGTG & GTCAGGCCCCACCATTGAGG \\
\hline RFPL1 & TCTGTGTGATGCACCCACTT & GACAGCGCATCCACACTCCA & CAAAGTAGATCCTCCCCATG \\
\hline RFPL4A & AGTGGATATGACGTTCGATG & AGGAATCTGTGAACCGACAG & CCTGAGGAGTTTCCGAAGTG \\
\hline PTPRJ & TTACTGTTGTGCATCAACCA & CTATACCTACAAGATACATG & ATGGGTCCACAGGTCCCACG \\
\hline RFPL2 & CСАСАССCTCTAACCTGATG & GCAGTGAATTAATGCACTTG & TGTGGGGAAGGGGCACACGA \\
\hline SLC34A2 & TATGATCTCGAGGTAATGGG & GCTGACAACGATGGACGTTG & GGGTGTAACTCACCAATCAG \\
\hline DIO2 & CCTGTTTGTAGGCATCGAGG & GGTGGAAGAGTTCTCCTCAG & AGCCGCTCCAAGTCCACTCG \\
\hline TRIM43B & GGAAATGTGTCATAAACCAG & CATATCCCTACAGGGCGATG & AGAGGCAGCTGAGGAAGACC \\
\hline GTF2F1 & GAGGTGGACTACATGTCAGA & GTTCAACCGCAAGCTTCGGG & ACAAAGTCAACTTTGCTACG \\
\hline TRIM48 & GCAAATGTGTGGCATTCACA & GAACCCTTCAAAGAACCCAG & AATCTCGCAAGTCTTCCAGA \\
\hline MRPL49 & GTCTACAAGGACATCACGCA & GAACATTATCCTACCCCTAG & ATGTTCCGGGCTACGCTGCG \\
\hline LOC441081 & GGATCTGAGTTGGAGAACAG & TACCCCAGCTCTAAGCGATG & AAATTGAAGACGGAATCACC \\
\hline ZNF574 & CCAGCCGATGCACAAAACGT & AGGGCCCGGAGGAACAACAG & TGTGGAGCACTCATACCGAA \\
\hline HSPA1B & GGTTCTGGACAAGTGTCAAG & GTCAGGCCCTACCATTGAGG & TGGGTCAGGCCCTACCATTG \\
\hline HSPA1A/B & CAAGGTGCAGAAGCTGCTGC & CGGCTGATTGGCCGCAAGTT & CAAGGGCAAGATCAGCGAGG \\
\hline DUXA & TCAGTTACACACTCTCATCA & AGATGGTAAAAACAAATCAT & TTACTTTGAAACTCCACACC \\
\hline DUSP18 & TGCTGACCATATCCACAGCG & CACTGAGACATTGATGACCA & GAGTTAGGGGAGTCAGCCAC \\
\hline C21orf91 & GTGTGCAAGAGATCAGACTT & GGTACTTGGGAGTCAAACTG & GTGGCCTCATAGTCACAACC \\
\hline
\end{tabular}


Table S7 continued

\begin{tabular}{|c|c|c|c|}
\hline Gene & spacer 1 & spacer 2 & spacer 3 \\
\hline ZNHIT6 & CCTCCGATAAATCACCCGGT & CAGGAATATCGCATACAACG & GAGGTGAAGGATGAGAACGC \\
\hline DPPA3 & TTAATCCAACCTACATCCCA & TTGAGATACATGTTACTCGG & TGTAGGAGCAGCAGTCCTCA \\
\hline AVPI1 & CAAGCCCTGTTTCAACGCAG & GATCATCTGGGAATGTGCAG & GGCTGCAGTGGTGTAGCGAG \\
\hline CXCR4 & CAACCACCCACAAGTCATTG & TGACATGGACTGCCTTGCAT & CAGGACAGGATGACAATACC \\
\hline HOXB2 & GCAAGGCCGCGATCTCGACG & CGACCCTGCCGAGGAACCCG & ACCGGCGCATGAAGCACAAG \\
\hline GLIDR & CAGGCTCAAGAGCAACAAGG & CTTCAGTTAAAGACTACCAG & GCCACGACTCAGATCTACAC \\
\hline MFSD11 & TGCCCAGAACAATCTGACAA & TAACGGTGATTAGCCTTGTG & GATTTCCAAAGAACAAGCTG \\
\hline NDEL1 & AAGATGATTTAAGTCAGACT & CTCCGTTCCTTTGCCAACAG & GTTGGAGGCACAATTAGTAC \\
\hline PLXNB3 & GCAGTCGTACAGGATCACTA & GCCCTCTCTCTACCGCACGT & CCGGAGACTCTGCTACACGG \\
\hline SNAI1 & GGGACTCTCCTGGAGCCGAA & GGCTTCGGATGTGCATCTTG & GCTGACCTCCCTGTCAGATG \\
\hline KHDC1 & CACATACCTTCGCTGCATTG & TGACTGTAGTCGGACCACAC & GACTCCTATCATCATGCTCG \\
\hline PRAMEF11 & AGTTCTACAAACACAGTCAA & AAGTTCTACAAACACAGTCA & GCTTCTGAAGATTCCTCAAG \\
\hline TGFB2 & AGAAAACTATAAAGTCCACT & TAGGGTCTGTAGAAAGTGGG & AACAGCATCAGTTACATCGA \\
\hline SPTY2D1 & ACCACTGACTGTCCGCCTAG & CACAGGGCCAGAACTAACTG & ACTCTGCGTGATTGTACTCG \\
\hline TMEM254-AS1 & GAAGAGGAAGTGTAAAACCG & ATGCTGATCTACATCGACAA & ATGTGAAAGGCGCCCCCTAG \\
\hline RIT2 & ATGCGGGAGCAGTACATGCG & GAGTACAAGGTGGTAATGCT & AGAAGATGCTTATAAGACCC \\
\hline PRRG4 & TTTGGACCTCTTGCGCAATG & GATCAAATCTATTATACAGA & CAGCAATTAATCCAGTCAGA \\
\hline ZNF705A & CAGCGTTTGTTAACTCACAG & AAGGCGAAAGCAATTAGTAT & AATTTGTTTATGTGGTTTAG \\
\hline KCNQ10T1 & TAGACCAAAAGCTCCCAACG & CAGTTATTGAAACCTCTACG & GTATCCATGTGCAACCAATG \\
\hline SERTAD1 & GAGGTCAAAGAGGGAGCTAG & GCCACCAGGCCGTAGCATCG & СTCTGGCAGTCGACTCCTGG \\
\hline MED26 & GGTTGTAGGAAACACGACTT & GCGGTCGCACGGCGTTGACG & CAGAGCTTGTATGCACCCAA \\
\hline OSR2 & CTTAGGCGGATCCTCTTGCG & AGGGGAAGCGCGCGTCCACG & TGGGATACCCCAGCGTCCAG \\
\hline ZCCHC10 & CATTGGACTTATGAATGCAC & CCATGCATCGGCTAATAGCC & ACAGATTATTATTGCAACAA \\
\hline RGS2 & TCAACACGACTGCAGACCCA & TTGTAAGAAGTAGCTCAAAC & ACTCCTGGGAAGCCCAAAAC \\
\hline PRSS23 & GGAACCCAGAAGCTTCGAGT & GCTAAATTGAGGGTAGACTG & AAACGCACCCATGTGCCCAA \\
\hline DUX4 & TGCCAGCGCGGAGCTCCTGG & GCTCCGCGCTGGCAGCTGGG & GGCAGGCGGCCTGTGCAGCG \\
\hline
\end{tabular}




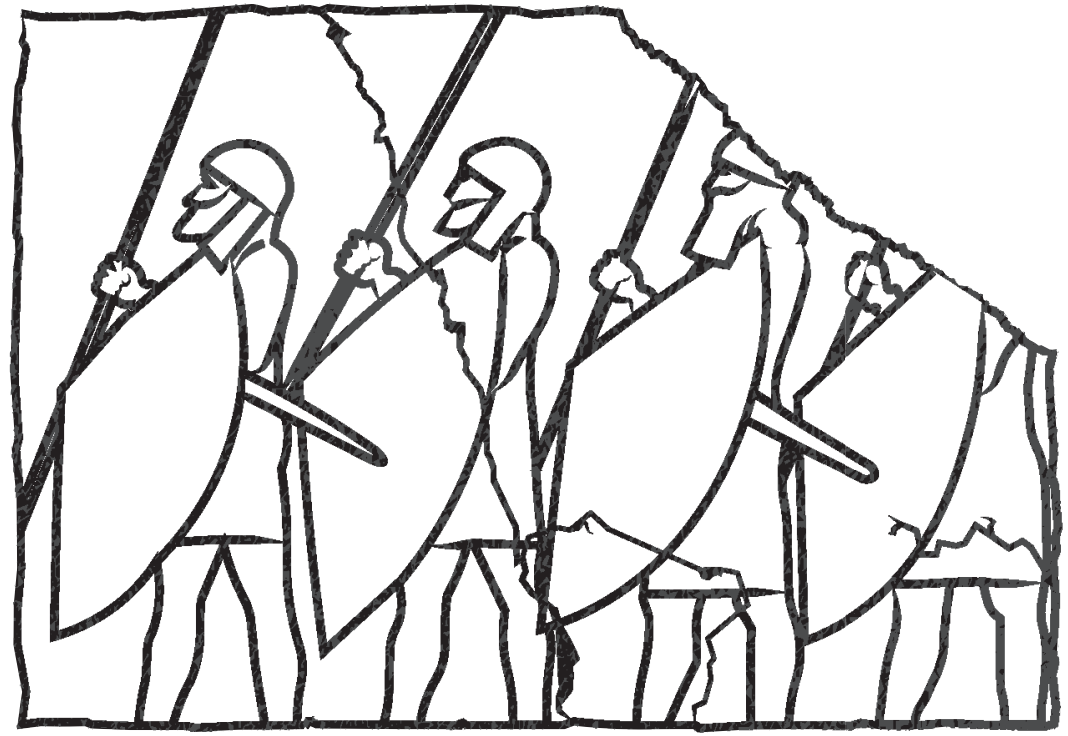

Illustration based on a stone carving on display at the Pergamon 


\section{Chapter 3 \\ DUX4 induces a homogeneous sequence of molecular changes, culminating in cellular apoptosis}

Ator Ashoti, Anna Alemany, Fanny Sage and Niels Geijsen

This chapter is available in an adapted form on bioRxiv

Ashoti, A., Alemany, A., Sage, F. \& Geijsen, N. DUX4 induces a homogeneous sequence of molecular changes, culminating in the activation of a stem-celllike transcriptional network and induction of apoptosis in somatic cells. bioRxiv (2021). doi.org/10.1101/2021.05.04.442407 


\section{Abstract}

Facioscapulohumeral muscular dystrophy (FSHD) is a muscle degenerative disease that disproportionally affects the muscles of the face, shoulder girdle and upper arms. FSHD is caused by the ectopic expression of Double Homeobox 4 (DUX4), which has been derepressed due to aberrant genetic and/or epigenetic events. The expression of DUX4 in FSHD-affected tissue is low, with both transcript and protein proven difficult to detect. Yet when mis-expressed, this low expression can have great implications, which is evident in patients suffering from FSHD. This suggest that there might be more of these elusive genes, perhaps regulated by DUX4 itself, that can have great implications in the development of FSHD, but that have remained elusive due to stringent parameters set in transcriptional studies. Given that the earlier the intervention in the DUX4 induced cytotoxic cascade, the greater the impact on disease development and progression, we focused on finding subtle but robust changes in gene expression patterns early after DUX4 induction, by single cell RNA sequencing. 


\section{Introduction}

Facioscapulohumeral muscular dystrophy (FSHD) is the third most prevalent muscular dystrophy worldwide ${ }^{1}$. The disease is autosomal dominant, caused by a gain-of-function event, which leads to the ectopic expression of Double Homeobox 4 (DUX4) ${ }^{2-4}$ in affected skeletal muscle, primarily in the face, shoulders and upper arms. DUX4 is a transcription factor normally expressed during early embryonic development ${ }^{5,6}$, in the adult testis ${ }^{7}$ and in the thymus ${ }^{8}$. It induces the expression of a network of genes involved in many different cellular processes, including embryonic development ${ }^{7,9,10}$, RNA processing ${ }^{9,11,12}$, protein homeostasis ${ }^{12,13}$, germline development ${ }^{7,9,10}$, stress response $\mathrm{e}^{12,14}$, and cell adhesion and migration ${ }^{11,15}$, among many others. Expression of DUX4 is stochastic and low, yet potent enough to induce apoptosis in muscle tissue ${ }^{7,11,16,17}$. Which genes and pathways play a defined role in inducing apoptosis downstream of DUX4 is not yet known.

Like many other muscular dystrophies, there is no effective treatment for FSHD. Many interconnected genetic and epigenetic events play a role, but at its core, the aberrant expression of DUX4 is the causal event that leads to muscle deterioration in FSHD patients. Genes involved in the expression and activation of DUX4, and genes that directly contribute to DUX4-induced cytotoxicity remain largely unknown. DUX4 is stochastically expressed in a burst-like fashion in only around $0.1-0.5 \%$ of myonuclei $i^{7,17,18}$. Therefore, identifying key players, such as downstream key transcription factors, by performing RNA transcriptomics on primary material might fall short. Genes might be missed, especially ones that are as lowly expressed as DUX4 is in muscle fibers ${ }^{7,11,16}$. This is evident in a study of Heuvel et al. ${ }^{19}$, where muscle tissue derived from 4 FSHD patients was analyzed by single-cell RNA sequencing. Out of the 5133 cells that were collected and analyzed from these patients, only 23 cells were classified as DUX4-affected. Cells were considered DUX4-affected if 5 or more DUX4 biomarker genes (out of a list of 67 biomarker genes ${ }^{20}$ ) were differentially expressed compared to healthy control samples. This reinforces the idea that potential key players might be missed due to the low number of DUX4 affected cells not reaching a critical number needed to detect DUX4-induced transcriptional changes, and/or due to stringency in the analysis. This explains in part why there is little known about the underlying mechanism induced by DUX4 that leads these cells to apoptosis.

We have generated a transgenic cell line, in which DUX4 expression can be induced through the addition of doxycycline ${ }^{21}$. These so called DUX4-inducible expression (DIE) cells allow for precise titration and timing of the DUX4 response. The response in the DIE cells is robust, as $99-100 \%$ of the induced cells enter apoptosis ${ }^{21}$. Using this line, we interrogated whether DUX4 induction led to the induction of defined and orderly molecular changes, or whether it induced a stochastic disruption of gene expression networks before ultimately triggering apoptosis. In order to address this question, we performed single-cell RNA sequencing (SCS) on induced DIE cells, as early as 2 hours after induction. By mapping the early molecular changes that follow DUX4 activation at high temporal resolution, we demonstrate that DUX4 induction homogeneously triggers a series of sequential molecular changes that ultimately lead to apoptosis. 


\section{Results}

\section{Single cell analysis induced DIE cells}

We previously demonstrated that the transcriptomic changes induced by DUX4 in DIE cells are very similar to those reported in FSHD-patient cells and other cellular models ${ }^{21}$. A robust DUX4 expression profile could be seen after only 4.5 hours of induction. However, the manner in which DUX4 expression leads to apoptosis, and what sort of paths are taken is not yet understood. Does DUX4 initiate a defined sequence of transcriptional events every time, or does it initiate a stochastic response that causes a disproportional amount of disruption in the cells that will eventually lead to cell death? To explore this question, we decided to analyze at single-cell resolution the transcriptional changes that occur shortly after DUX4 induction. Our inducible system allows us to examine the immediate effect of DUX4 induction, and track the changes overtime. Due to the robust induction of DUX4, $>99 \%$ of the DIE cells enter apoptosis withing 48hours of DUX4 induction (Chapter 5), these changes can be tracked at a high resolution. This type of data would be difficult to attain with primary material, due to the low frequency of DUX4 expression, that occurs in a burst like fashion in a small subset of myonuclei. Furthermore, this inducible system allowed us to time the induction of DUX4, creating a clear timeline trajectory, which is not possible when working with primary material. SCS, will also allow us to detect subtle and perhaps rare early transcriptional changes in specific cell populations that could otherwise be drowned out and missed in bulk RNA sequencing. DIE cells were induced for 2, 3, 4 and 6 hours with doxycycline before sampling and processing for SCS. By reducing the dimensionality using t-Distributed Stochastic Neighbor Embedding (t-SNE) mapping, we were able to have a 2-D visualization of the cell clustering ${ }^{22,23}$. Each point in the t-SNE map is a cell of which its position is determined based on its transcriptome. Cells with a similar transcriptome are more likely to cluster together than cells with large variations in their transcriptomes. Generally, the larger the differences in transcriptomics between cells, the further apart they will be in a dimensionality-reduced map. Our results show separate embedding of uninduced cells (Oh) and $6 \mathrm{~h}$-induced cells, but mixed populations of the intermediate states ( $2 \mathrm{~h}$, $3 \mathrm{~h}$ and $4 \mathrm{~h}$ ) (Fig. $1 \mathrm{~A})$. The cells do however orientate themselves on the $y$-axis, from the uninduced cells at the top, to the maximum of $6 \mathrm{~h}$-induced cells at the bottom (Fig. 1B). This is evident when the expression of known DUX4 target genes were projected onto the t-SNE map (Fig. 1C). LEUTX, PRAMEF1 and ZSCAN4 are genes that have previously shown to increase in expression in FSHD models or FSHD-affected muscle cells $s^{9,11,19,24}$. This can indeed also be seen in Fig. 1C, where the expression of these genes is significantly upregulated in $6 \mathrm{~h}$-induced cells. This also holds true for genes that are downregulated upon DUX4 expression, such as ID $1^{9,19}$. Figure $1 \mathrm{C}$ also shows that as DUX4 induction persists, the expression of ID1 decreases significantly. As the cells are organized on the $y$-axis, we manually divided the vertical axis of the t-SNE into 10 clusters of equal size (Fig. 1D). The mean induction state was calculated for each cluster by considering all cells and their time of induction (Table 1). To avoid confusion, the mean induction state of each cluster will from here on be referred to as the experimental induction times used. Clusters 1 and 2 will therefore be referred to as $0 \mathrm{~h}, 3 \& 4$ as $2 \mathrm{~h}, 5 \& 6$ as $3 \mathrm{~h}, 7 \& 8$ as $4 \mathrm{~h}$ and $9 \& 10$ as $6 \mathrm{~h}$. Using this type of clustering, differential gene expression analysis was performed to identify differentially expressed genes between the uninduced cell clusters (clusters $1 \& 2$ ) and the induced cell clusters, as schematically indicated in figure 1D (right). 
A

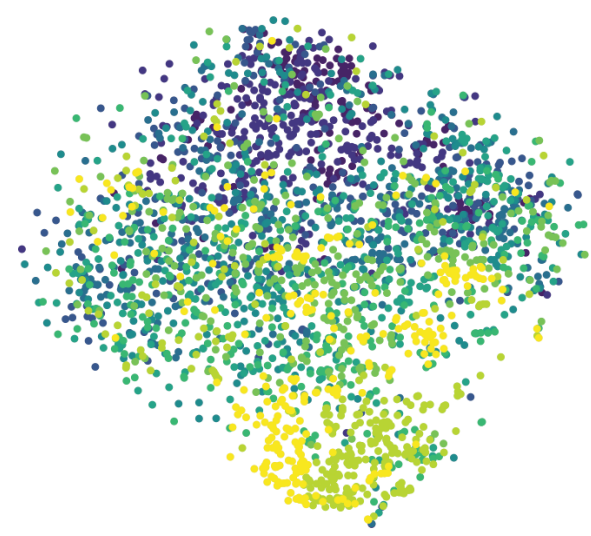

B
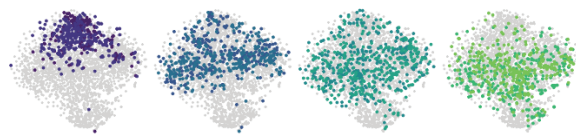

- Oh.A

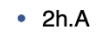

- 3h.A

- $4 h . A$

- 2h.B

- 3h.B

- $4 h . B$

\section{Induction states}
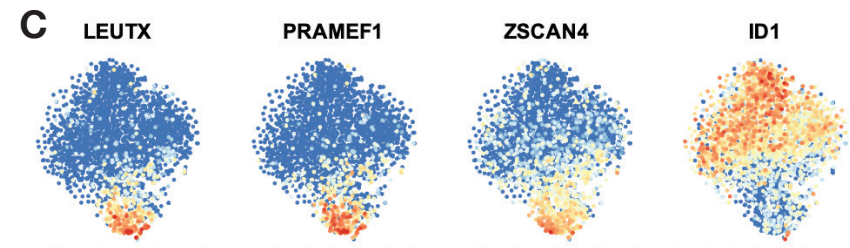

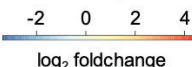

\begin{tabular}{cccccc}
-3 & -2 & -1 & 0 & 1 & 2 \\
\hline & $\begin{array}{lll}1 & 1 & 1\end{array}$ \\
\hline $\log _{2}$ foldchange
\end{tabular}

$\begin{array}{ccccc}-2 & 0 & 2 & 4 & 6\end{array}$

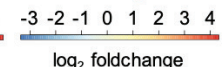

D
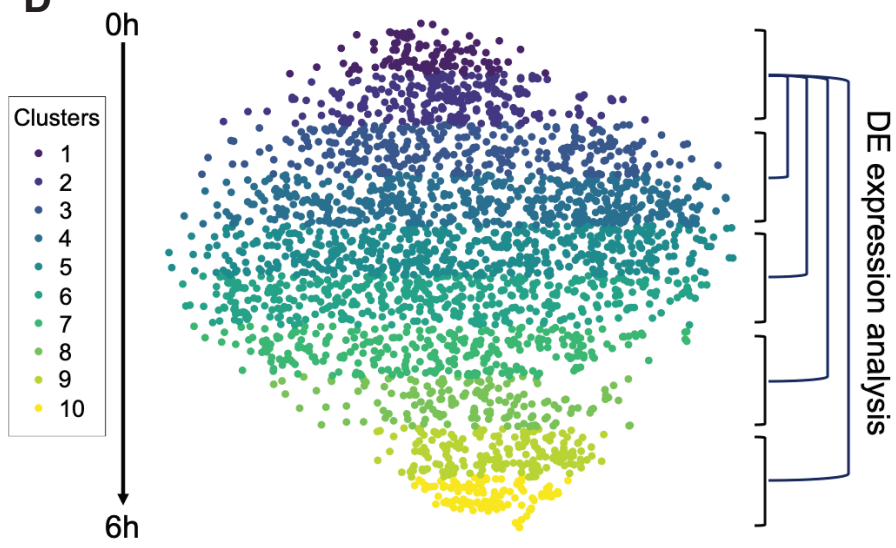

Figure 1. SCS data of DIE cells analyzed with RacelD. A) Induced and uninduced DIE single cell data represented in a t-distributed stochastic neighbour embedding (t-SNE) map. Each point represents a single cell, with the induction time of the cells indicated by color. B) Individual t-SNE maps for each of the induction time. Each sample is indicated in a different color. Induction states are shown from left to right, starting with the two $O$ h replicates. $A$ and $B$ annotations indicate the two replicates. C) t-SNE maps highlighting the expression of DUX4 marker genes. The fold change in gene expression is shown on a $\log _{2}$ scale as a linear color scale. D) Clustering of the cells in the t-SNE map based on the $y$-axis coordinates. Clusters are numbered (1-10) and colorcoded. Clusters 1 and 2 contain the most uninduced DIE cells, and will be used as the control situation for differential gene expression (DE) analysis with the induced clusters $(3 \& 4,5 \& 6$, $7 \& 8$, and 9\&10). 
Table 1. Cell make up of t-SNE clusters

\begin{tabular}{|c|c|c|c|c|c|c|c|c|c|c|c|c|}
\hline cluster & $\begin{array}{l}\text { Oh } \\
\text { A }\end{array}$ & $\begin{array}{l}\text { Oh } \\
\text { B }\end{array}$ & $\begin{array}{l}2 \mathrm{~h} \\
\mathrm{~A}\end{array}$ & $\begin{array}{l}2 \mathrm{~h} \\
B\end{array}$ & $\begin{array}{l}3 \mathrm{~h} \\
\mathrm{~A}\end{array}$ & $\begin{array}{l}3 \mathrm{~h} \\
\mathrm{~B}\end{array}$ & $\begin{array}{l}4 \mathrm{~h} \\
\mathrm{~A}\end{array}$ & $\begin{array}{l}4 \mathrm{~h} \\
B\end{array}$ & $\begin{array}{l}6 \mathrm{~h} \\
\mathrm{~A}\end{array}$ & $\begin{array}{l}6 \mathrm{~h} \\
B\end{array}$ & $\begin{array}{l}\text { cells/ } \\
\text { cl. }\end{array}$ & $\begin{array}{l}\text { mean } \\
\text { state }\end{array}$ \\
\hline cl.1 & 33 & 23 & 15 & 10 & 10 & 5 & 0 & 4 & 5 & 1 & 106 & 1.39 \\
\hline cl. 2 & 61 & 77 & 22 & 17 & 25 & 14 & 7 & 11 & 6 & 1 & 241 & 1.28 \\
\hline cl.3 & 38 & 106 & 48 & 29 & 30 & 23 & 9 & 17 & 11 & 1 & 312 & 1.57 \\
\hline cl.4 & 34 & 57 & 83 & 71 & 69 & 58 & 38 & 49 & 18 & 29 & 506 & 2.61 \\
\hline cl.5 & 10 & 20 & 54 & 108 & 58 & 51 & 69 & 90 & 15 & 40 & 515 & 3.14 \\
\hline cl.6 & 1 & 3 & 33 & 41 & 58 & 65 & 64 & 69 & 23 & 48 & 405 & 3.64 \\
\hline cl.7 & 0 & 0 & 12 & 14 & 33 & 41 & 76 & 37 & 31 & 39 & 283 & 4.05 \\
\hline cl.8 & 0 & 0 & 2 & 1 & 15 & 14 & 20 & 11 & 52 & 42 & 157 & 4.97 \\
\hline cl.9 & 0 & 1 & 0 & 0 & 2 & 6 & 25 & 5 & 84 & 61 & 184 & 5.51 \\
\hline cl.10 & 1 & 0 & 1 & 1 & 1 & 2 & 3 & 1 & 71 & 33 & 114 & 5.73 \\
\hline
\end{tabular}

\section{Differential gene expression analysis}

In order to detect subtle but significant changes in expression, differentially expressed genes were filtered for an adjusted $p$-value ( $P$ adj) of $\left\langle 10^{* *}-6\right.$, and a $\log _{2}$ (foldchange) $(\log 2 \mathrm{FC}$ ) of $\rangle$ 0.5 and $<-0.5$ (Tables S1-4). This analysis demonstrated that as the induction time increases, so does the number of differentially expressed genes, with a core group of differentially expressed genes being shared between induction states, (Fig. 2A, Table 2 and Tables S1-4). This suggests that a deterministic chain of events is induced early on, if not immediately after DUX4 expression. Interestingly, even though DUX4 expression itself was not detected in the induced DIE cells, a DUX4 expression profile is readily detected only $3 \mathrm{~h}$ post DUX4 induction, as can be seen when expression data is entered through the Enrichr database ${ }^{25,26}$ (Fig. 2B). This DUX4 profile even becomes more apparent as induction time increases. The Enricher database can match the entered lists of genes with previously entered studies, matching our gene lists with one other study from Geng et al. ${ }^{9}$ in which DUX4 had been overexpressed in human primary myoblasts. Such an early induced DUX4 expression profile has (to the best of our knowledge) not been seen before, with other studies measuring the effects of DUX4 $6 \mathrm{~h}^{27}$ or $14 \mathrm{~h}^{24}$ post induction, or $24-36 \mathrm{~h}$ post lentiviral transfection ${ }^{9,24}$. As no other study has examined the effects of DUX4 at such early time points, our datasets uniquely show the earliest DUX4 affected genes. Furthermore, previously identified DUX4 affected genes such as RFPL4B, GOLGB1, ZNF296, SRSF8, ID1 and ID3 $3^{9,11,19,24}$, could too be classified as potential early marker genes, as they have been identified as being differentially expressed after a mere $3 \mathrm{~h}$ of DUX4 induction.

Remarkably, a high percentage (29-36\%) of upregulated transcripts encode for transcription factors and cofactors, in addition to a number of differentially expressed kinases (Table 2, Table S5), which was also observed in previous bulk-seq experiments ${ }^{21}$. This suggests that DUX4 induces a network of downstream transcription factors that in turn induces a cascade of secondary transcriptional events, ultimately leading to apoptosis. Since the expression of transcription factors can be low, many additional factors might fall under the detection limit 
A

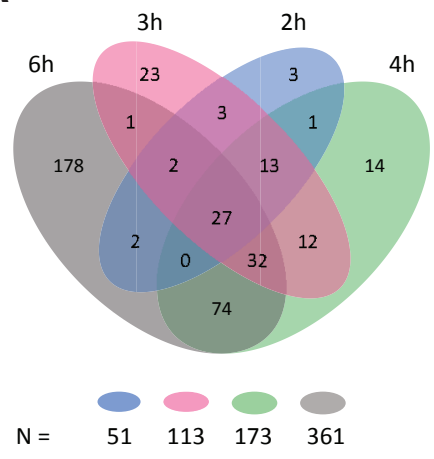

B Significant DUX4 expression profile

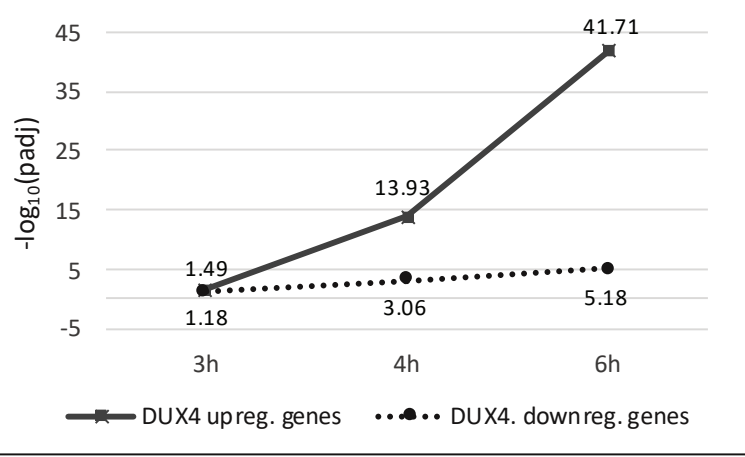

Figure 2. DUX4 differential gene expression profile between induction states. A) Venn diagram demonstrating the number of shared differentially expressed genes between different induction times. Total number of differentially expressed genes in each state is shown below the graph, in the same color-coding. B) A graph demonstrating the increase in significance of the upregulated and downregulated expression profile of DUX4 in induced DIE cells, according to entries in the Enrichr database. Induction samples (each containing two biological replicates) are plotted on the $x$-axis, and the $y$-axis displays the negative $\log _{10}$ of the adjusted $p$-value (padj), of the detected DUX4 expression profile.

in single-cell sequencing data. Enrichr ${ }^{25,26}$ was therefore used to analyze our datasets for the presence of signature gene expression profiles, or "transcriptional footprints", that are indicative of the activity of specific transcription factors. Analysis of our DIE cell data using Enrichr yielded a list of potential transcription factors that can explain the observed changes in gene expression (Table S6). Some of the identified profiles did indeed match differentially expressed transcription factors in the induced DIE cells (SOX3, NR2F2, ZNF217 and OTX2). In addition, the Enrichr algorithm detected several other transcription factor profiles of transcription factors which themselves are not found to be differentially expressed in our dataset (Table S6). However, a number of these "transcriptional footprints" were found in all 4 timepoints examined (LIN28, SOX5, ZIC3, JUNB, KLF10, MEIS2, MYCN, PITX, SETB1, ZEB2, ZNF503, MYB, WT1, NR2F2), suggesting that these factors are induced early after DUX4 induction and persist with continued DUX4 expression. Of particular interest is the identification of several transcription factors which are represented in both the upregulated as well as the downregulated gene set (e.g. ZIC3, JUNB, KLF10, MEIS2, MYCN and SETB1). As both upregulated and downregulated genes corresponded to the activity status of these transcription factors, it does strongly suggest their role in the DUX4-induced cytotoxic cascade, as opposed to only finding a one-sided effect.

In summary, we have found that the induction of DUX4 promotes changes in the transcriptional landscape of DIE cells as early as 2 hours post doxycycline administration. Many differentially expressed genes found in the early data sets maintain their differentially expressed status with continued DUX4 expression. This corroborates the notion that DUX4 initiates a clear progressive cascade of events, and does not stochastically and/or randomly affects genes and pathways. This is further corroborated by our finding that transcription factors and co-factors are overrepresented in the list of differentially expressed genes and account for approximately $\sim 33 \%$ of the differentially upregulated genes, whereas they only comprise $11-13.5 \%$ of the human genome. This indeed suggests that DUX4 activates a coherent network of transcriptional regulators that together initiate a new cellular program 
that ultimately leads to cell death. Lastly the presence of transcription factors could also be deduced from the detection of their transcriptional footprint, even when the expression of the individual factors themselves were not always detected. This is a common shortcoming of single cell sequencing, where the detection of low abundant transcripts can be missed.

Table 2: Summary of differentially expressed genes found in induced DIE cells at different induction times.

\begin{tabular}{|r|rrrr|}
\hline Induction state & $2 \mathrm{~h}$ & $3 \mathrm{~h}$ & $4 \mathrm{~h}$ & $6 \mathrm{~h}$ \\
\hline Upregulated genes & 43 & 77 & 133 & 248 \\
\hline Downregulated genes & 8 & 36 & 40 & 68 \\
\hline Differentially expressed genes total & 51 & 113 & 173 & 361 \\
\hline Transcription- and co-factors & 12 & 28 & 44 & 81 \\
\hline Kinases & 3 & 3 & 6 & 10 \\
\hline
\end{tabular}

\section{Gene ontology}

To identify which biological processes are affected by the temporal changes in gene expression in the induced DIE cells, the differentially upregulated and downregulated genes were analyzed using the PANTHER (Protein ANalysis $\underline{\text { TH}}$ rough Evolutionary Relationships) algorithm. PANTHER is an online tool that classifies proteins (and their corresponding genes) based on their family or subfamily, their molecular function, and their involvement in any biological processes and pathways, to facilitate high throughput analysis of datasets ${ }^{28-30}$. The biological processes that were identified are shown in Fig. 3A and Table S7. Gene ontology (GO) terms were assigned a general "umbrella" term. Table S7 shows the full list of GO terms.

As shown, DUX4-induction initially triggered the activation of an early developmental program and processes involved in the cell cycle and proliferation. Three hours after induction, genes involved in developmental processes are less prominent, with the majority of processes now being involved in cell cycle and RNA processing. At $6 \mathrm{~h}$ of induction, the first apoptotic processes were identified. GO terms identified with the downregulated genes appeared more incoherent than the GO terms detected with the upregulated gene sets. This could be due to the nature of DUX4 being more of a transcriptional activator, rather than a repressor $^{10}$. GO terms found with the downregulated gene stets might thus reflect the loss of cell identity, consistent with the idea that DUX4 initiates an early embryonic transcriptional program. Nonetheless, processes involved in programmed cell death were also found upon analyzing the downregulated genes after $6 \mathrm{~h}$ of DUX4 induction (Fig. 3B). Furthermore, at 3h post DUX4-induction, downregulated genes demonstrate changes in cellular respiration and energy production. These processes contribute to oxidative stress, a common occurrence in FSHD-affected cells that is likely involved in DUX4-induced apoptosis ${ }^{31-33}$.

The temporal identification of altered biological processes revealed a sequential path that is activated upon DUX4 induction. This path starts by activating developmental processes, and subsequently many other processes involved in RNA processing, protein production and regulation, cellular respiration, kinase activity, eventually leading to the induction of apoptosis 


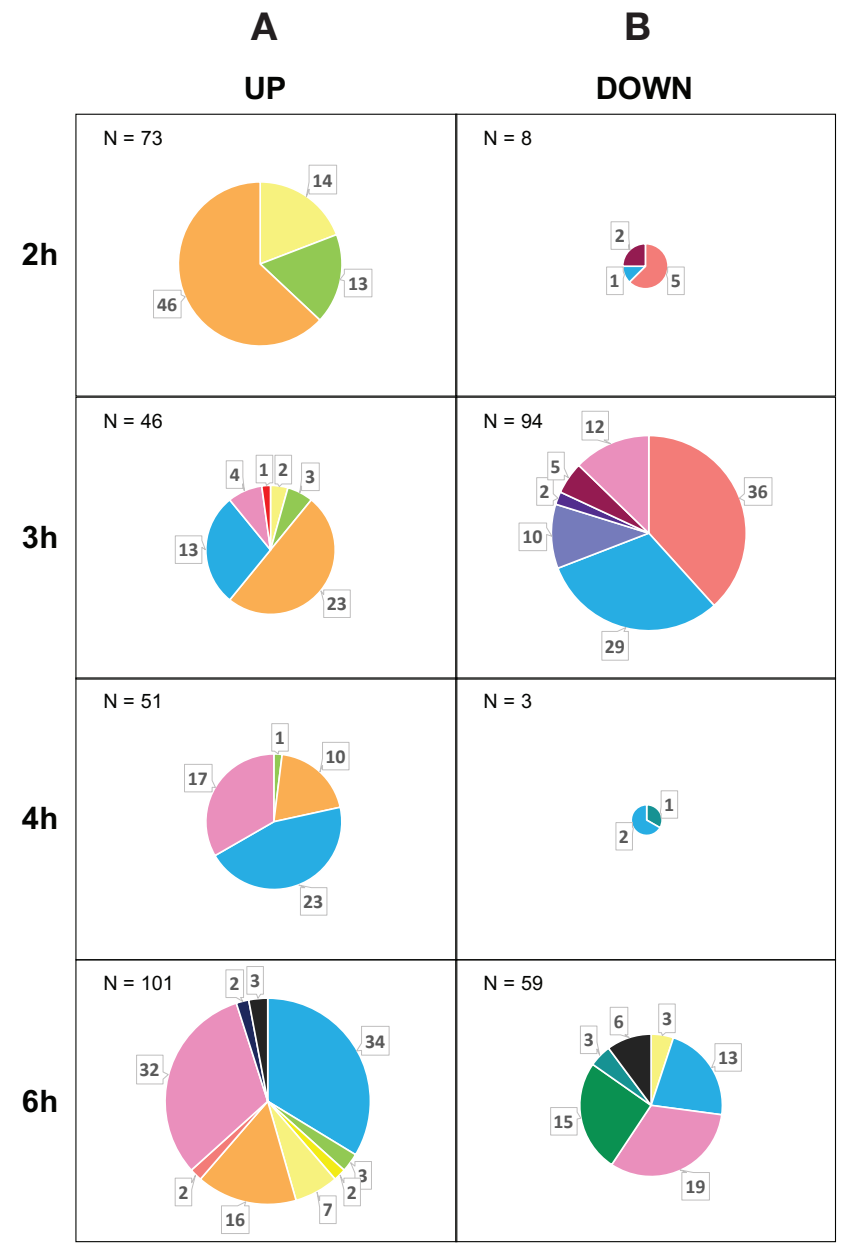

Protein production and regulation

$\square$ RNA production and processing

$\square$ System development

Stemness and stemcell maintenance

$\square$ Embryonic development

$\square \quad$ Cell cycle and division

General cellular processes

Cellular resipiration and energy production

Figure 3. Gene ontology reveals DUX4-induced paths. Gene ontology performed on gene lists of differentially A) upregulated and B) downregulated genes from the 4 induction states. Only biological processes with an FDR $<0.05$, and a raw $p$ value of $<10^{* *-3}$ are included. Biological processes are color-coded based on their general "umbrella" term. The total number of detected biological processes is indicated in the top left corner of each diagram, with the number of biological processes per umbrella term annotated in the pie chart. See table S7 for the full list of biological processes per induction state. 


\section{$\underline{\text { StemID }}$}

The observation that DUX4 initially activates an early embryonic transcriptional program was interesting and suggests that DUX4 temporarily converts cells toward a developmentally immature state. This notion is corroborated by StemID, an algorithm that uses transcriptome entropy to identify stem cells within a cell population ${ }^{34}$. Determining transcriptome diversity in single cells is done by using Shannon's entropy ${ }^{35}$, which measures disorder in high-dimensional systems. The entropy value of a given cell type indicates the degree of transcriptomic promiscuity. As pluripotent stem cells have the option of differentiation in any cell type, a wide number of signaling pathways need to remain active, which is reflected as high transcriptome entropy. As these cells become more committed to a specific cell fate, the number of active pathways decrease to a few specific pathways needed to maintain their cell identity, which in turn leads to a decrease in transcriptome entropy ${ }^{34,36,37}$. When a lineage trajectory is projected onto the t-SNE map, it becomes clear by the color indication of the vertices that transcriptome entropy peaks in cluster 4 (Fig. 4A). The barplot in Fig. 4B clearly shows the increase in transcriptome entropy, until it peaks in cluster 4 , which has an induction state of around $2 \mathrm{~h}$. Transcriptome entropy then slowly decreased with increasing induction times. This is thus corroborating gene ontology results that showed the induction of a more embryonic developmental state in $\sim 2 h$ induced DIE cells (clusters $3 \& 4$ ).

\section{A}

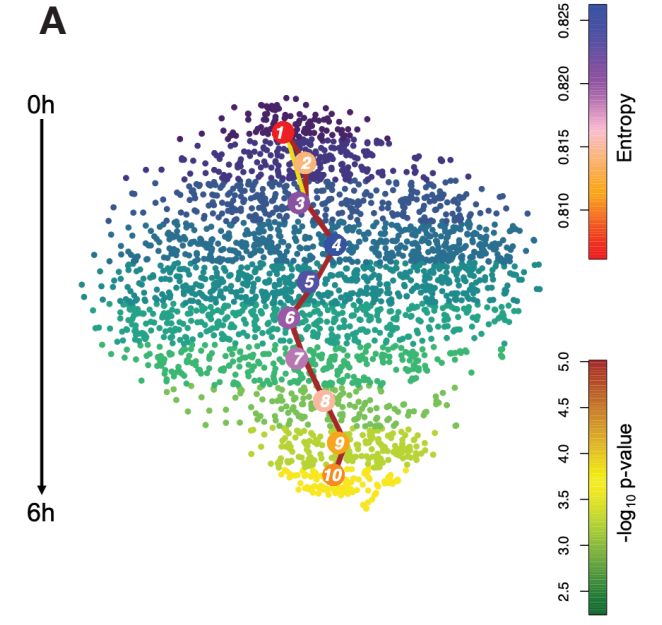

B

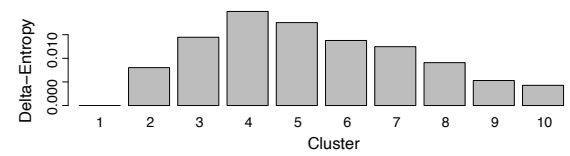

Figure 4. StemID analysis identified a stem cell state in single cell clusters. A) Inferred lineage tree superimposed onto the t-SNE map. The level of entropy of a cluster is indicated by the color of the vertices. The link color between vertices represents the $-\log _{10}$ value, with only the significant links being shown $(p<0.01)$. B) Barplot showing the deltaentropy score per cluster. The delta entropy was calculated by subtracting the lowest entropy score across all cells from the median transcriptome entropy of each cluster.

\section{Pseudotime analysis with FatelD}

StemID revealed a transient trajectory of stemness in induced DIE clusters, which peaked at cluster 4 and was subsequently followed by a gradual decrease of stemness at later time points, when further transcriptome changes reflect profound changes in metabolism and RNA processing. To follow up on this observation, pseudotime analysis was employed to further define temporal stages of transcriptional states, using FatelD ${ }^{38}$. By doing so, we were able to identify stage-specific co-expression patterns across this vertical trajectory based on previous t-SNE clustering (Fig. 1D). Expression patterns of known DUX4 target genes show a gradual increase (LEUTX, ZSCAN4, ZNF217, and PRAMFE1), or decrease in expression (ID1 and ID3) as DUX4 expression persisted (Fig. 5A), which is in line with earlier observations seen above (Fig. 1A) and previous observations in other studies ${ }^{9,11,19,24}$. These genes were 

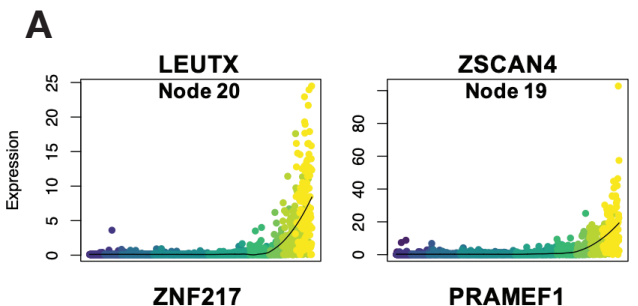

B
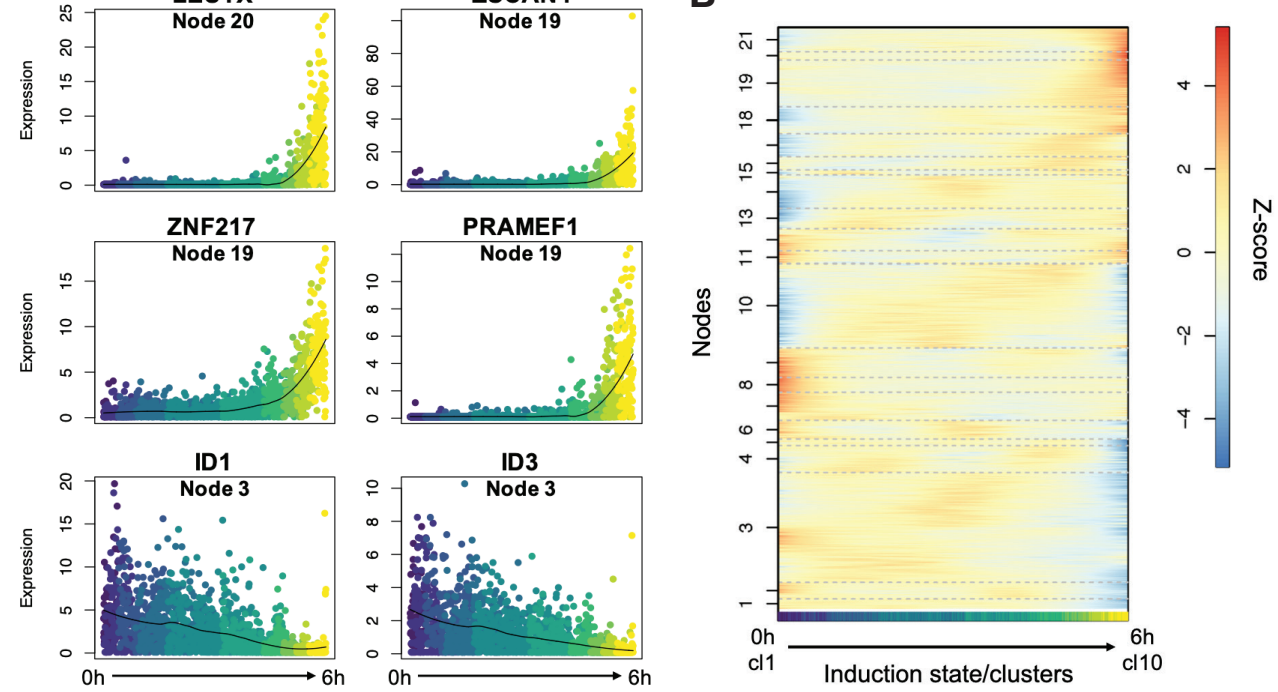

Figure 5. Gene expression patterns in the induced DIE cell trajectory by FatelD pseudotime analysis. A) Expression patterns of 6 known DUX4 marker genes following the DIE cell induced trajectory (Oh-6h induction). The nodes in which these genes are contained are annotated below the gene name. B) Self-organizing heatmap of z-score transformed pseudotime expression profiles across the DIE cell induced trajectory (0h-6h induction), based on the t-SNE map. Cells are represented on the $x$-axis, and the genes are organized in nodes that are represented on the $y$-axis. Genes with a similar expression pattern are clustered in nodes, with a color indication representing gene expression, based on their transformed z-score.

present in gene nodes that showed a gradual increase or decrease in gene expression (e.g. nodes $18-21$ or $1-3$ respectively) (Fig. $5 B$ ). Moreover, dynamic gene expression patterns were identified in other gene nodes, such as oscillating expression patterns during the 6 hours of DUX4 induction (e.g. 4, 6, 10 and 17) (Fig. 5B and S2). This suggests the activation of a very dynamic underlying process, upon DUX4 induction, in which some genes are induced and inhibited multiple times in a relatively short time frame.

Analysis of the differentially expressed genes in the oscillating nodes did not yield a clear answer as to why these particular sets of genes vary in their expression during DUX4 induction. Of the nodes that demonstrated clear oscillating patterns $(4,6,10,12,17)$, node 4 and 6 did not contain differentially expressed genes, node 12 contained one differentially downregulated gene (COX7A2), node 10 contained 27 differentially upregulated genes, and node 17 contained 17 differentially upregulated genes (Table 3). Using the STRING database ${ }^{39,40}$, we were able to determine that the differentially expressed genes from node 10 are primarily involved in developmental process, system development, and cell cycle and division, with many genes interconnecting and involved in all three processes. STRING is a database of known and predicted protein-protein interactions (both direct and indirect), that allowed us to visualize the types of associations between genes (Fig. 6A). The biological processes identified with STRING are similar to those identified using the PANTHER algorithm (Fig. 3A). The expression pattern in node 10 therefore fits previous 
$\mathrm{GO}$ analyses, demonstrating a temporal increase in the number of developmental genes and processes, peaking at around 2-3h (Fig. 5B and 6B). Differentially upregulated genes in node 17 did not show to be part of any significantly affected biological processes, nor a clear coherent core network could be seen between the genes as was found in node 10.
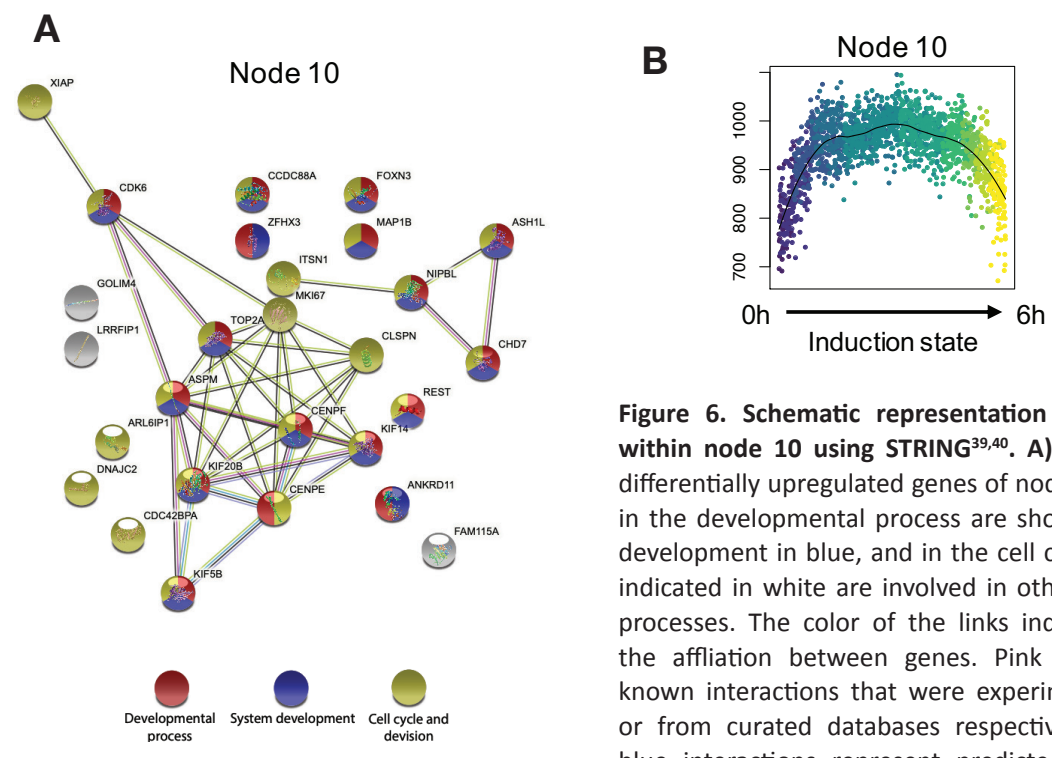

Figure 6. Schematic representation of gene affiliations within node 10 using STRING ${ }^{39,40}$. A) Affiliations between differentially upregulated genes of node 10 . Genes involved in the developmental process are shown in red, in system development in blue, and in the cell cycle in yellow. Genes indicated in white are involved in other general molecular processes. The color of the links indicates the nature of the affliation between genes. Pink and cyan represent known interactions that were experimentally determined, or from curated databases respectively. Green, red and blue interactions represent predicted interactions based on gene neighborhood, gene fusions and gene occurrence respectively. Yellow, black and purple interactions are based on textmining, co-expression, and protein homology respectively. Images were adapted from STRING-derived interaction networks. B) Expression pattern graph of node 10 genes, following the DIE cell induced time trajectory (0h-6h induction).

\section{Discussion}

Although ectopic activation of the transcription factor DUX4 has been identified as the main culprit of FSHD this past 10 years $^{2-4}$, the exact mechanism by which DUX4 expression initiates muscle fiber degeneration remains elusive. A thorough understanding of the temporal molecular changes that are brought about by DUX4 is essential for the identification of potential targets to modulate DUX4 cytotoxicity. To gain knowledge of the molecular mechanism of FSHD, many researchers have studied the transcriptomics of FSHD models or FSHD-affected primary cells, ${ }^{9,11,19,24}$. Yet due to the broad range of gene regulation and the low and stochastic expression of the disease-causing gene (DUX4) ${ }^{7,16-18}$, finding key players in the DUX4 induced cytotoxic cascade has been challenging. We therefore applied a novel transgenic cell model in which DUX4 expression can robustly be induced, allowing high-resolution temporal analysis of early transcriptional events following DUX4 induction at single-cell level. Our SCS data reveals how DUX4 induction lead to the activation of a transient early embryonic and stemness state, by activating a network of developmental factors. Early intervention in the DUX4-induced cytotoxic cascade will most likely have a greater impact on slowing down disease development and progression, than intervening at a later stage. By doing so we aimed to identify perhaps subtle and early changes, that can ideally be tracked further along the cytotoxic cascade, with potential implications in the 
progressive cytotoxic cascade.

Our analysis of the single-cell transcriptional changes following DUX4 induction suggests that DUX4 initiates a non-random consecutive chain of events that is exacerbated as time progresses. In addition, analysis of gene expression profiles using Enrichr revealed that transcriptional signatures of transcription factors, most of which were not detected during the induction periods, are already prevalent 2 hours post-induction and indeed became more profound as induction time progresses. As such, we have uncovered a number of transcription factors that might play a role in triggering subsequent molecular changes that ultimately contribute to DUX4 -mediated cytotoxicity.

At a high resolution, we were able to show the early events that occur only a few hours after DUX4 induction. Gene ontology analysis of the differentially upregulated genes at different time points after DUX4 induction corroborates our conclusion that DUX4 induces a non-random, consecutive sequence of events. Our results suggest an initial activation of developmental processes that lead cells to an increased stemness state only 2 hours after DUX4 induction. Next, additional biological processes such as RNA processing, and protein production and regulation were activated, eventually leading to the activation of apoptotic processes 6 hours post DUX4 induction.

The analysis performed in this study revealed which genes started diverging in their expression during the first few hours of DUX4 induction. At these early timepoints, some transcriptional changes were subtle, but as these changes in expression remained or even intensified with increased induction times, we believe them to be significant. More attention should be focused towards some of these subtle changes in expression at these early postinduction timepoints, that could normally be missed due to too stringent filtering. DUX4 itself proves that the smallest changes in expression can cause major consequences. This factor was not identified in our transcriptomic analysis, concurrent with its low transcriptional and abundance levels in muscle tissue of FSHD patients ${ }^{3,7,17,18}$. More attention should thus be directed towards genes that leave behind a detectable "footprint" in the transcriptome of affected cells, again with DUX4 as prime example, as classification of most cells that are DUX4-affected is based on the detection of DUX4 marker genes, and not the detection of DUX4 itself. These elusive genes could have great implications in the pathophysiology of FSHD, and could therefore hold promise for its treatment.

\section{Methods}

\section{Cell culturing and seeding}

DIE cells were cultured in growth medium consisting of IMDM basal medium with $10 \%$ Tet system-approved FBS (Clontech) and 55 MM 2-mercaptoethanol, supplemented with $5 \mu \mathrm{g} / \mathrm{ml}$ Puromycin and $6 \mu \mathrm{g} / \mathrm{ml}$ Blasticidin.

\section{Sample preparation and SORT-seq}

DIE cells were grown in 48-wells plates, until a $~ 90 \%$ confluency was reached. Cells were exposed to $1 \mu \mathrm{g} / \mathrm{ml}$ doxycycline for $2,3,4$, and 6 hours. Doxycycline-exposed and untreated DIE cells were rinsed with DPBS after which $0.25 \%$ Trypsin-EDTA (Thermo Scientific) was added. Trypsin was immediately removed after it had covered the complete surface. Cell 
were incubated for 1 minute at $5 \% \mathrm{CO}_{2}$ and $37^{\circ} \mathrm{C}$, after which the trypsin was deactivated by adding IMDM media supplemented with $10 \%$ Tet-system approved FBS and DAPI nuclear stain. Trypsinized DIE cells were resuspended in the media and then strained using Cellstrainer capped tubes (Falcon). Cells were stored on ice until FACS sorting. Viable DAPI negative cells were sorted into 384 hard shell plates (Biorad) with $5 \mu$ l of vapor-lock (QIAGEN) containing 100-200 nl RT primers, dNTPs, and synthetic mRNA Spike-Ins, using the FACSJazz (BD biosciences). The plates were immediately spun down and stored a $-80^{\circ} \mathrm{C}$. Cells were processed as described in Muraro et al. ${ }^{41}$, using the CEL-seq2-bases ScRNA-seq. Samples were sequenced using Illumina Nextseq 500, 2x75 kit, high output. Two biological replicates per samples were sent for sequencing. Initial normalization and mapping were done as described by Muraro et $\mathrm{al}^{41}$.

\section{Data analysis}

Illumina sequencing-generated paired-end reads were aligned, mapped, and normalized as previously described ${ }^{41}$. For single cell analysis, cells with a minimum of 6000 transcripts were considered, and data normalization was performed by downsampling transcript counts to 6000 for all cells (Fig. S1). Initial analysis revealed a batch affect between the two uninduced biological replicates. One sample showed signs of additional metabolic stress. The top 187 diverging genes ( $\operatorname{padj}<10^{* *}-7$ ) between the two uninduced biological replicates were removed from all data to account for any source of metabolic stress. Dimensionality reduction of cells was done using RacelD ${ }^{23}$, after which the clusters were manually determined by dividing the y-axis of the tSNE map in 10 clusters of equal size. Differential expression of genes between cell clusters were identified as described by Muraro et $\mathrm{al}^{41}$, based on a previous publication of Anders and Huber ${ }^{42}$. Pseudotime analysis was performed using StemID ${ }^{34}$ and FatelD ${ }^{38}$.

\section{Data Resources}

RNA sequencing data is available in the GEO data base, accession number: GSE156154.

\section{References}

1. Deenen, J. C. W. et al. Population-based incidence and prevalence of facioscapulohumeral dystrophy. Neurology 83, 1056-1059 (2014).

2. Kowaljow, V. et al. The DUX4 gene at the FSHD1A locus encodes a pro-apoptotic protein. Neuromuscul. Disord. 17, 611-623 (2007).

3. Dixit, M. et al. DUX4, a candidate gene of facioscapulohumeral muscular dystrophy, encodes a transcriptional activator of PITX1. Proc. Natl. Acad. Sci. U. S. A. 104, 18157-18162 (2007).

4. Snider, L. et al. RNA transcripts, miRNA-sized fragments and proteins produced from D4Z4 units: New candidates for the pathophysiology of facioscapulohumeral dystrophy. Hum. Mol. Genet. 18, 2414-2430 (2009).

5. Whiddon, J. L., Langford, A. T., Wong, C. J., Zhong, J. W. \& Tapscott, S. J. Conservation and innovation in the DUX4-family gene network. Nat. Genet. 49, 935-940 (2017).

6. Hendrickson, P. G. et al. Conserved roles of mouse DUX and human DUX4 in activating cleavage-stage genes and MERVL/HERVL retrotransposons. Nat. Genet. 49, 925-934 (2017).

7. Snider, L. et al. Facioscapulohumeral dystrophy: Incomplete suppression of a retrotransposed gene. PLoS Genet. 6, 1-14 (2010).

8. Das, S. \& Chadwick, B. P. Influence of repressive histone and DNA methylation upon D4Z4 transcription in non-myogenic cells. PLoS One 11, 1-26 (2016).

9. Geng, L. N. et al. DUX4 Activates Germline Genes, Retroelements, and Immune Mediators: Implications for Facioscapulohumeral Dystrophy. Dev. Cell 22, 38-51 (2012). 
10. Knopp, P. et al. DUX4 induces a transcriptome more characteristic of a less-differentiated cell state and inhibits myogenesis. J. Cell Sci. 129, 3816-3831 (2016).

11. Rickard, A. M., Petek, L. M. \& Miller, D. G. Endogenous DUX4 expression in FSHD myotubes is sufficient to cause cell death and disrupts RNA splicing and cell migration pathways. Hum. Mol. Genet. 24, 5901-5914 (2015).

12. Jagannathan, S., Ogata, Y., Gafken, P. R., Tapscott, S. J. \& Bradley, R. K. Quantitative proteomics reveals key roles for post-transcriptional gene regulation in the molecular pathology of facioscapulohumeral muscular dystrophy. Elife 8, 1-16 (2019).

13. Homma, S., Beermann, M. Lou, Boyce, F. M. \& Miller, J. B. Expression of FSHD-related DUX4FL alters proteostasis and induces TDP-43 aggregation. Ann. Clin. Transl. Neurol. 2, 151-166 (2015).

14. Winokur, S. T. et al. Facioscapulohumeral muscular dystrophy (FSHD) myoblasts demonstrate increased susceptibility to oxidative stress. Neuromuscul. Disord. 13, 322-333 (2003).

15. Bosnakovski, D. et al. Transcriptional and cytopathological hallmarks of FSHD in chronic DUX4-expressing mice. J. Clin. Invest. 130, 2465-2477 (2020).

16. Bosnakovski, D. et al. Muscle pathology from stochastic low level DUX4 expression in an FSHD mouse model. Nat. Commun. 8, 1-9 (2017).

17. Tassin, A. et al. DUX4 expression in FSHD muscle cells: How could such a rare protein cause a myopathy? J. Cell. Mol. Med. 17, 76-89 (2013).

18. Jones, T. I. et al. Facioscapulohumeral muscular dystrophy family studies of DUX4 expression: Evidence for disease modifiers and a quantitative model of pathogenesis. Hum. Mol. Genet. 21, 4419-4430 (2012).

19. Van Den Heuvel, A. et al. Single-cell RNA sequencing in facioscapulohumeral muscular dystrophy disease etiology and development. Hum. Mol. Genet. 28, 1064-1075 (2019).

20. Yao, Z. et al. DUX4-induced gene expression is the major molecular signature in FSHD skeletal muscle. Hum. Mol. Genet. 23, 5342-5352 (2014).

21. Ashoti, A. et al. A genome-wide CRISPR/Cas phenotypic screen for modulators of DUX4 cytotoxicity reveals screen complications. bioRxiv 2020.07.27.223420 (2020) doi:10.1101/2020.07.27.223420.

22. van der Maaten, L. \& Hinton, G. H. Visualizing Data using t-SNE. J. Mach. Learn. Res. 9, 2579-2605 (2008).

23. Grün, D. et al. Single-cell messenger RNA sequencing reveals rare intestinal cell types. Nature 525, 251-255 (2015).

24. Jagannathan, S. et al. Model systems of DUX4 expression recapitulate the transcriptional profile of FSHD cells. Hum. Mol. Genet. 25, ddw271 (2016).

25. Chen, E. Y. et al. Enrichr: Interactive and collaborative HTML5 gene list enrichment analysis tool. BMC Bioinformatics 14, (2013).

26. Kuleshov, M. V. et al. Enrichr: a comprehensive gene set enrichment analysis web server 2016 update. Nucleic Acids Res. 44, W90-W97 (2016).

27. Choi, S. H. et al. DUX4 recruits p300/CBP through its C-terminus and induces global H3K27 acetylation changes. Nucleic Acids Res. 44, 5161-5173 (2016).

28. Ashburner, M. et al. Gene Ontology: tool for the unification of biology. Nat. Genet. 25 25-29 (2000).

29. Carbon, S. et al. The Gene Ontology Resource: 20 years and still GOing strong. Nucleic Acids Res. 47, D330-D338 (2019).

30. Mi, H., Muruganujan, A., Ebert, D., Huang, X. \& Thomas, P. D. PANTHER version 14: More genomes, a new PANTHER GO-slim and improvements in enrichment analysis tools. Nucleic Acids Res. 47, D419-D426 (2019).

31. Tsumagari, K. et al. Gene expression during normal and FSHD myogenesis. BMC Med. Genomics 4, (2011).

32. Banerji, C. R. S. et al. $\beta$-catenin is central to DUX4-driven network rewiring in facioscapulohumeral muscular dystrophy. J. R. Soc. Interface 12, (2015).

33. Lek, A. et al. Applying genome-wide CRISPR-Cas9 screens for therapeutic discovery in facioscapulohumeral muscular dystrophy. Sci. Transl. Med. 12, 9-11 (2020).

34. Grün, D. et al. De Novo Prediction of Stem Cell Identity using Single-Cell Transcriptome Data. Cell Stem Cell 19, 266-277 (2016).

35. Shannon, C. E. A Mathematical Theory of Communication. Bell Syst. Tech. J. 27, 623-656 
(1948).

36. Macarthur, B. D. \& Lemischka, I. R. Statistical mechanics of pluripotency. Cell 154, 484-489 (2013).

37. Banerji, C. R. S. et al. Cellular network entropy as the energy potential in Waddington's differentiation landscape. Sci. Rep. 3, 25-27 (2013).

38. Herman, J. S., Sagar \& Grün, D. FatelD infers cell fate bias in multipotent progenitors from single-cell RNA-seq data. Nat. Methods 15, 379-386 (2018).

39. Snel, B., Lehmann, G., Bork, P. \& Huynen, M. A. String: A web-server to retrieve and display the repeatedly occurring neighbourhood of a gene. Nucleic Acids Res. 28, 3442-3444 (2000).

40. Szklarczyk, D. et al. STRING v11: Protein-protein association networks with increased coverage, supporting functional discovery in genome-wide experimental datasets. Nucleic Acids Res. 47, D607-D613 (2019).

41. Muraro, M. J. et al. A Single-Cell Transcriptome Atlas of the Human Pancreas. Cell Syst. 3, 385-394.e3 (2016).

42. Anders, S. et al. Differential expression analysis for sequence count data via mixtures of negative binomials. Adv. Environ. Biol. 7, 2803-2809 (2010).

\section{Supplementary data}

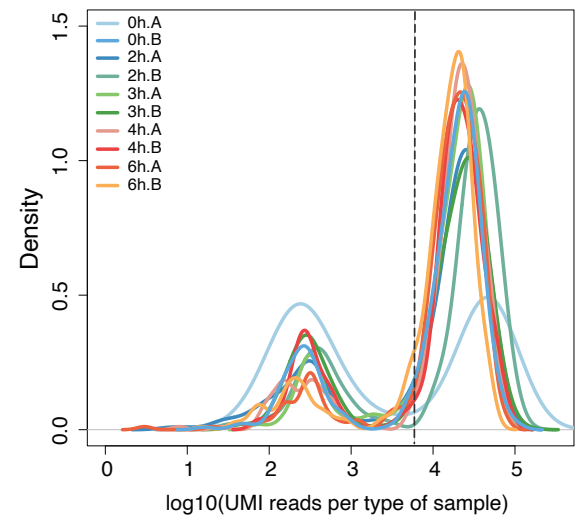

Figure S1. Density plot representing the total read count of all samples. Samples are color coded. An A or B annotation represents to which biological replicate the sample belongs. The intermittened line shows the cutoff of the number of UMI reads (6000) used to determine which cells to inlcude for the analysis. All cells with a normalized transript count above 6000 have been included in the RacelD analysis. 

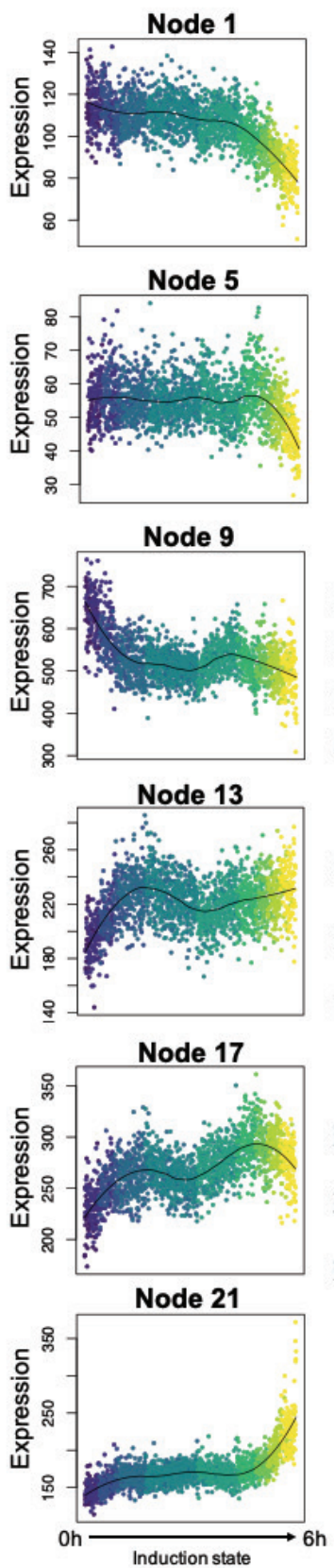

Node 2

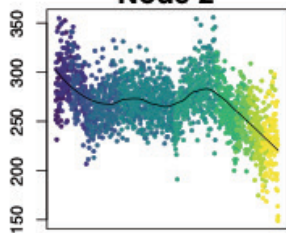

Node 6

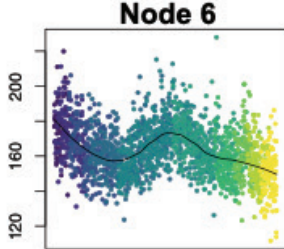

Node 10

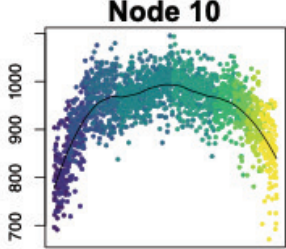

Node 14

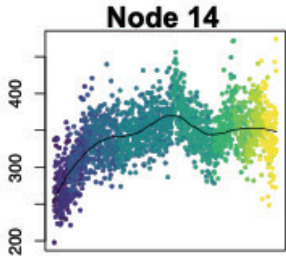

Node 18
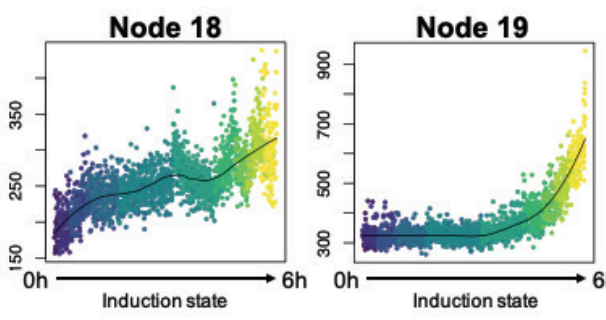

Node 3

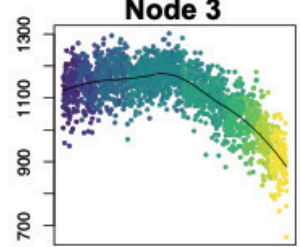

Node 7

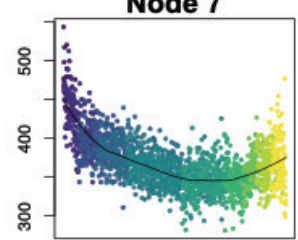

Node 11

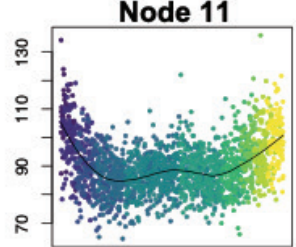

Node 15

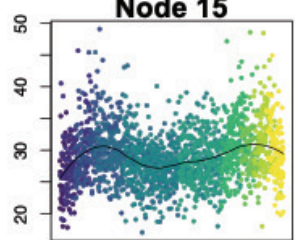

Node 19

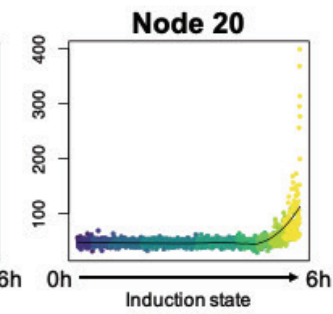

Figure S2. Gene expression patterns of gene nodes from pseudotime analysis. Dynamic gene expression patterns of all nodes of the self-organizing heat map of figure 5A. Each point represents a cell. The color of the point and its location on the $\mathrm{x}$-axis represents its induction state from uninduced ( $\mathrm{h}$ ) to $6 \mathrm{~h}$ induced. Normalized expression is plotted on the $y$-axis. The black line indicates a local regression. 
Table S1. Differentially expressed genes between uninduced (Oh, clusters $1 \& 2$ ) and $2 \mathrm{~h}$ induced DIE cells (clusters $3 \& 4$ ).

* Adjusted $\mathrm{p}$ value $<10 * *-6$, absolute $\log 2 \mathrm{FC}>0.5$

\begin{tabular}{|c|c|c|c|c|c|c|c|c|c|}
\hline Gene & $\begin{array}{l}\text { base } \\
\text { Mean }\end{array}$ & $\begin{array}{l}\text { base } \\
\text { MeanA }\end{array}$ & $\begin{array}{l}\text { base } \\
\text { Mean B }\end{array}$ & $\begin{array}{l}\text { fold } \\
\text { Change }\end{array}$ & $\begin{array}{l}\log 2 \\
\text { FC }\end{array}$ & pval & padj & $\begin{array}{l}\text { shared } \\
\text { in states }\end{array}$ & Node \\
\hline CHD2 & 0.68 & 0.52 & 0.83 & 1.61 & 0.68 & $4.4 \mathrm{E}-09$ & $3.6 \mathrm{E}-07$ & All 4 & 14 \\
\hline UQCR11 & 1.09 & 1.29 & 0.89 & 0.69 & -0.54 & $1.8 \mathrm{E}-09$ & $1.6 \mathrm{E}-07$ & All 4 & 8 \\
\hline MDN1 & 0.33 & 0.22 & 0.45 & 2.08 & 1.06 & $1.1 \mathrm{E}-09$ & $9.9 \mathrm{E}-08$ & All 4 & 14 \\
\hline SREK1 & 1.03 & 0.83 & 1.24 & 1.49 & 0.57 & $1.0 \mathrm{E}-09$ & $9.2 \mathrm{E}-08$ & All 4 & 17 \\
\hline PGM5P2 & 0.70 & 0.52 & 0.87 & 1.66 & 0.74 & $1.6 \mathrm{E}-10$ & $1.7 \mathrm{E}-08$ & All 4 & 18 \\
\hline BPTF & 1.26 & 1.02 & 1.50 & 1.46 & 0.55 & $6.5 \mathrm{E}-11$ & $7.3 \mathrm{E}-09$ & All 4 & 14 \\
\hline AKAP9 & 0.72 & 0.53 & 0.91 & 1.71 & 0.77 & $7.9 \mathrm{E}-12$ & $1.0 \mathrm{E}-09$ & All 4 & 14 \\
\hline PRR11 & 1.06 & 0.82 & 1.30 & 1.60 & 0.67 & $4.6 \mathrm{E}-13$ & $6.5 \mathrm{E}-11$ & All 4 & 13 \\
\hline NKTR & 1.36 & 1.07 & 1.64 & 1.53 & 0.61 & $1.2 \mathrm{E}-13$ & 1.7E-11 & All 4 & 14 \\
\hline SRRM1 & 2.02 & 1.66 & 2.38 & 1.43 & 0.52 & $5.9 \mathrm{E}-15$ & $9.9 \mathrm{E}-13$ & All 4 & 14 \\
\hline BDP1 & 0.77 & 0.53 & 1.00 & 1.87 & 0.90 & $2.5 \mathrm{E}-16$ & $4.6 \mathrm{E}-14$ & All 4 & 14 \\
\hline GOLGA4 & 1.21 & 0.90 & 1.52 & 1.69 & 0.76 & $4.9 \mathrm{E}-18$ & $9.8 \mathrm{E}-16$ & All 4 & 14 \\
\hline PRPF38B & 2.03 & 1.62 & 2.43 & 1.50 & 0.59 & $1.5 \mathrm{E}-18$ & $3.1 \mathrm{E}-16$ & All 4 & 18 \\
\hline TOP1 & 1.59 & 1.23 & 1.95 & 1.59 & 0.67 & $1.3 \mathrm{E}-18$ & $2.9 \mathrm{E}-16$ & All 4 & 14 \\
\hline MAB21L3 & 0.91 & 0.63 & 1.19 & 1.88 & 0.91 & $2.2 \mathrm{E}-19$ & $5.3 \mathrm{E}-17$ & All 4 & 18 \\
\hline USMG5 & 1.91 & 2.35 & 1.47 & 0.63 & -0.68 & 4.7E-24 & $1.5 \mathrm{E}-21$ & All 4 & 8 \\
\hline BRD4 & 1.55 & 1.13 & 1.97 & 1.75 & 0.80 & $2.1 \mathrm{E}-25$ & $7.3 \mathrm{E}-23$ & All 4 & 14 \\
\hline UGDH-AS1 & 1.38 & 0.98 & 1.78 & 1.82 & 0.87 & 4.7E-26 & $1.7 \mathrm{E}-23$ & All 4 & 18 \\
\hline ANKRD11 & 2.05 & 1.55 & 2.55 & 1.64 & 0.72 & $1.1 \mathrm{E}-26$ & $3.9 \mathrm{E}-24$ & All 4 & 10 \\
\hline $\begin{array}{l}\text { LOC } \\
100131257\end{array}$ & 2.17 & 1.63 & 2.70 & 1.65 & 0.72 & $5.8 \mathrm{E}-29$ & $2.4 \mathrm{E}-26$ & All 4 & 18 \\
\hline CENPE & 1.09 & 0.71 & 1.48 & 2.07 & 1.05 & $3.3 \mathrm{E}-29$ & $1.4 \mathrm{E}-26$ & All 4 & 10 \\
\hline ASPM & 1.41 & 0.91 & 1.91 & 2.09 & 1.07 & $6.3 E-38$ & $3.9 \mathrm{E}-35$ & All 4 & 10 \\
\hline SMC4 & 6.10 & 4.99 & 7.21 & 1.44 & 0.53 & $9.8 \mathrm{E}-44$ & $8.4 \mathrm{E}-41$ & All 4 & 14 \\
\hline RPL37A & 8.14 & 9.57 & 6.70 & 0.70 & -0.52 & $2.3 E-57$ & $2.5 \mathrm{E}-54$ & All 4 & 8 \\
\hline CENPF & 3.13 & 2.14 & 4.13 & 1.94 & 0.95 & $1.0 \mathrm{E}-67$ & $2.0 \mathrm{E}-64$ & All 4 & 10 \\
\hline RPS29 & 12.43 & 14.98 & 9.87 & 0.66 & -0.60 & $1.8 \mathrm{E}-117$ & $1.1 \mathrm{E}-113$ & All 4 & 8 \\
\hline KCNQ1OT1 & 7.48 & 5.20 & 9.75 & 1.87 & 0.91 & $6.7 \mathrm{E}-145$ & 8.0E-141 & All 4 & 18 \\
\hline TOP2A & 1.74 & 1.25 & 2.24 & 1.79 & 0.84 & $1.6 \mathrm{E}-30$ & $7.2 \mathrm{E}-28$ & $2 \mathrm{~h} \mid 6 \mathrm{~h}$ & 10 \\
\hline MKI67 & 2.57 & 1.96 & 3.19 & 1.63 & 0.71 & $1.8 \mathrm{E}-32$ & $9.3 \mathrm{E}-30$ & $2 \mathrm{~h} \mid 6 \mathrm{~h}$ & 10 \\
\hline FOXN3 & 1.18 & 0.97 & 1.40 & 1.44 & 0.53 & $1.2 \mathrm{E}-09$ & $1.1 \mathrm{E}-07$ & $2 \mathrm{~h} \mid 4 \mathrm{~h}$ & 10 \\
\hline HIST1H2BK & 0.78 & 0.95 & 0.61 & 0.64 & -0.64 & $1.2 \mathrm{E}-09$ & $1.1 \mathrm{E}-07$ & $2 \mathrm{~h}|3 \mathrm{~h}| 6 \mathrm{~h}$ & 19 \\
\hline KIF14 & 0.95 & 0.69 & 1.20 & 1.73 & 0.79 & $1.5 \mathrm{E}-15$ & $2.6 \mathrm{E}-13$ & $2 \mathrm{~h}|3 \mathrm{~h}| 6 \mathrm{~h}$ & 10 \\
\hline
\end{tabular}


Table S1 continued

\begin{tabular}{|c|c|c|c|c|c|c|c|c|c|}
\hline Gene & $\begin{array}{l}\text { base } \\
\text { Mean }\end{array}$ & $\begin{array}{l}\text { base } \\
\text { MeanA }\end{array}$ & $\begin{array}{l}\text { base } \\
\text { MeanB }\end{array}$ & $\begin{array}{l}\text { fold } \\
\text { Change }\end{array}$ & $\begin{array}{l}\log 2 \\
F C\end{array}$ & pval & padj & $\begin{array}{l}\text { shared } \\
\text { in states }\end{array}$ & Node \\
\hline GOLIM4 & 0.43 & 0.30 & 0.56 & 1.86 & 0.90 & $1.2 \mathrm{E}-09$ & $1.1 \mathrm{E}-07$ & $2 h|3 h| 4 h$ & 10 \\
\hline ANKRD12 & 0.81 & 0.63 & 0.99 & 1.58 & 0.66 & $6.8 \mathrm{E}-10$ & $6.4 \mathrm{E}-08$ & $2 \mathrm{~h}|3 \mathrm{~h}| 4 \mathrm{~h}$ & 13 \\
\hline CDC42BPA & 0.75 & 0.58 & 0.93 & 1.61 & 0.69 & $3.5 \mathrm{E}-10$ & $3.5 \mathrm{E}-08$ & $2 h|3 h| 4 h$ & 10 \\
\hline ROCK1 & 0.52 & 0.37 & 0.67 & 1.80 & 0.85 & $2.4 \mathrm{E}-10$ & $2.5 \mathrm{E}-08$ & $2 \mathrm{~h}|3 \mathrm{~h}| 4 \mathrm{~h}$ & 14 \\
\hline PIP5K1A & 0.32 & 0.44 & 0.21 & 0.48 & -1.06 & $1.4 \mathrm{E}-10$ & $1.5 \mathrm{E}-08$ & $2 \mathrm{~h}|3 \mathrm{~h}| 4 \mathrm{~h}$ & 8 \\
\hline CCDC88A & 0.65 & 0.47 & 0.82 & 1.73 & 0.79 & $3.6 \mathrm{E}-11$ & 4.2E-09 & $2 \mathrm{~h}|3 \mathrm{~h}| 4 \mathrm{~h}$ & 10 \\
\hline ITSN1 & 1.11 & 0.88 & 1.35 & 1.53 & 0.61 & $1.4 \mathrm{E}-11$ & 1.7E-09 & $2 \mathrm{~h}|3 \mathrm{~h}| 4 \mathrm{~h}$ & 10 \\
\hline NOP10 & 1.82 & 2.13 & 1.50 & 0.70 & -0.51 & $1.3 \mathrm{E}-13$ & $1.9 \mathrm{E}-11$ & $2 \mathrm{~h}|3 \mathrm{~h}| 4 \mathrm{~h}$ & 8 \\
\hline DNAJC2 & 1.17 & 0.89 & 1.44 & 1.62 & 0.70 & $3.4 \mathrm{E}-15$ & $5.8 \mathrm{E}-13$ & $2 h|3 h| 4 h$ & 10 \\
\hline KTN1 & 2.33 & 1.90 & 2.76 & 1.45 & 0.54 & $3.9 \mathrm{E}-18$ & 8.0E-16 & $2 h|3 h| 4 h$ & 14 \\
\hline ZFHX3 & 1.78 & 1.38 & 2.17 & 1.57 & 0.65 & $1.3 \mathrm{E}-19$ & $3.2 \mathrm{E}-17$ & $2 h|3 h| 4 h$ & 10 \\
\hline RPL39 & 8.54 & 10.09 & 6.99 & 0.69 & -0.53 & $2.1 \mathrm{E}-63$ & $2.5 \mathrm{E}-60$ & $2 h|3 h| 4 h$ & 8 \\
\hline MAP1B & 7.22 & 5.48 & 8.96 & 1.63 & 0.71 & $1.8 \mathrm{E}-88$ & $5.4 \mathrm{E}-85$ & $2 h|3 h| 4 h$ & 10 \\
\hline TAF3 & 0.39 & 0.27 & 0.50 & 1.87 & 0.91 & 5.9E-09 & $4.8 \mathrm{E}-07$ & $2 \mathrm{~h} \mid 3 \mathrm{~h}$ & 14 \\
\hline NIPBL & 0.83 & 0.66 & 1.01 & 1.53 & 0.62 & 3.3E-09 & $2.8 \mathrm{E}-07$ & $2 \mathrm{~h} \mid 3 \mathrm{~h}$ & 10 \\
\hline $\mathrm{CHD7}$ & 1.06 & 0.85 & 1.26 & 1.49 & 0.57 & $5.6 \mathrm{E}-10$ & $5.3 \mathrm{E}-08$ & $2 \mathrm{~h} \mid 3 \mathrm{~h}$ & 10 \\
\hline CDK6 & 0.63 & 0.48 & 0.78 & 1.62 & 0.70 & $8.8 \mathrm{E}-09$ & $6.9 \mathrm{E}-07$ & $2 \mathrm{~h}$ & 10 \\
\hline KIF2OB & 1.33 & 1.09 & 1.56 & 1.43 & 0.51 & 4.3E-10 & 4.2E-08 & $2 \mathrm{~h}$ & 10 \\
\hline KIF5B & 2.41 & 1.93 & 2.88 & 1.49 & 0.58 & $2.4 \mathrm{E}-21$ & 7.0E-19 & $2 \mathrm{~h}$ & 10 \\
\hline
\end{tabular}

Table S2. Differentially expressed genes between uninduced (Oh, clusters 1\&2) and $3 \mathrm{~h}$ induced DIE cells (clusters 5\&6).

* Adjusted $\mathrm{p}$ value $<10 * *-6$, absolute $\log 2 \mathrm{FC}>0.5$

\begin{tabular}{|c|c|c|c|c|c|c|c|c|c|}
\hline Gene & $\begin{array}{l}\text { base } \\
\text { Mean }\end{array}$ & $\begin{array}{l}\text { base } \\
\text { MeanA }\end{array}$ & $\begin{array}{l}\text { base } \\
\text { MeanB }\end{array}$ & $\begin{array}{l}\text { fold } \\
\text { Change }\end{array}$ & $\begin{array}{l}\log 2 \\
\text { FC }\end{array}$ & pval & padj & $\begin{array}{l}\text { shared } \\
\text { in states }\end{array}$ & Node \\
\hline RPL37A & 7.67 & 9.25 & 6.10 & 0.66 & -0.60 & $1.5 \mathrm{E}-75$ & $1.6 \mathrm{E}-72$ & All 4 & 8 \\
\hline UQCR11 & 1.02 & 1.25 & 0.79 & 0.63 & -0.66 & $1.6 \mathrm{E}-13$ & 1.7E-11 & All 4 & 8 \\
\hline RPS29 & 11.76 & 14.47 & 9.04 & 0.63 & -0.68 & $1.0 \mathrm{E}-145$ & $6.0 \mathrm{E}-142$ & All 4 & 8 \\
\hline USMG5 & 1.80 & 2.27 & 1.33 & 0.58 & -0.78 & $8.9 E-31$ & $2.6 \mathrm{E}-28$ & All 4 & 8 \\
\hline ANKRD11 & 2.16 & 1.50 & 2.81 & 1.87 & 0.90 & $5.6 \mathrm{E}-44$ & 2.7E-41 & All 4 & 10 \\
\hline ASPM & 1.24 & 0.88 & 1.60 & 1.81 & 0.86 & $7.8 \mathrm{E}-24$ & $1.5 \mathrm{E}-21$ & All 4 & 10 \\
\hline CENPF & 2.83 & 2.07 & 3.60 & 1.74 & 0.80 & $5.3 \mathrm{E}-46$ & $2.7 \mathrm{E}-43$ & All 4 & 10 \\
\hline CENPE & 0.92 & 0.69 & 1.14 & 1.65 & 0.72 & $2.3 \mathrm{E}-13$ & $2.2 \mathrm{E}-11$ & All 4 & 10 \\
\hline PRR11 & 1.00 & 0.79 & 1.21 & 1.53 & 0.61 & $5.6 \mathrm{E}-11$ & $4.3 \mathrm{E}-09$ & All 4 & 13 \\
\hline
\end{tabular}


Table S2 continued

\begin{tabular}{|c|c|c|c|c|c|c|c|c|c|}
\hline Gene & $\begin{array}{l}\text { base } \\
\text { Mean }\end{array}$ & $\begin{array}{l}\text { base } \\
\text { MeanA }\end{array}$ & $\begin{array}{l}\text { base } \\
\text { MeanB }\end{array}$ & $\begin{array}{l}\text { fold } \\
\text { Change }\end{array}$ & $\begin{array}{l}\log 2 \\
F C\end{array}$ & pval & padj & $\begin{array}{l}\text { shared } \\
\text { in states }\end{array}$ & Node \\
\hline MDN1 & 0.33 & 0.21 & 0.45 & 2.13 & 1.09 & $1.1 \mathrm{E}-10$ & 7.8E-09 & All 4 & 14 \\
\hline BDP1 & 0.76 & 0.52 & 0.99 & 1.91 & 0.93 & $2.5 \mathrm{E}-17$ & $3.6 \mathrm{E}-15$ & All 4 & 14 \\
\hline BRD4 & 1.56 & 1.09 & 2.03 & 1.86 & 0.89 & 8.9E-32 & 2.7E-29 & All 4 & 14 \\
\hline AKAP9 & 0.73 & 0.52 & 0.94 & 1.81 & 0.85 & $2.1 \mathrm{E}-14$ & $2.5 \mathrm{E}-12$ & All 4 & 14 \\
\hline NKTR & 1.46 & 1.04 & 1.87 & 1.80 & 0.85 & $3.9 E-27$ & $9.4 \mathrm{E}-25$ & All 4 & 14 \\
\hline $\mathrm{CHD} 2$ & 0.70 & 0.51 & 0.90 & 1.77 & 0.83 & $3.5 \mathrm{E}-13$ & 3.4E-11 & All 4 & 14 \\
\hline GOLGA4 & 1.21 & 0.87 & 1.55 & 1.77 & 0.83 & $8.4 \mathrm{E}-22$ & $1.5 \mathrm{E}-19$ & All 4 & 14 \\
\hline TOP1 & 1.58 & 1.19 & 1.97 & 1.66 & 0.73 & $3.6 \mathrm{E}-22$ & $6.5 \mathrm{E}-20$ & All 4 & 14 \\
\hline BPTF & 1.29 & 0.99 & 1.59 & 1.61 & 0.68 & $1.5 \mathrm{E}-16$ & $2.1 \mathrm{E}-14$ & All 4 & 14 \\
\hline SRRM1 & 2.08 & 1.61 & 2.55 & 1.58 & 0.66 & $1.8 \mathrm{E}-24$ & $3.6 \mathrm{E}-22$ & All 4 & 14 \\
\hline SMC4 & 5.84 & 4.82 & 6.86 & 1.42 & 0.51 & $6.8 \mathrm{E}-40$ & $3.0 E-37$ & All 4 & 14 \\
\hline SREK1 & 1.05 & 0.81 & 1.28 & 1.59 & 0.67 & $3.5 \mathrm{E}-13$ & $3.4 \mathrm{E}-11$ & All 4 & 17 \\
\hline KCNQ10T1 & 8.73 & 5.03 & 12.44 & 2.47 & 1.31 & $0.0 \mathrm{E}+00$ & $0.0 \mathrm{E}+00$ & All 4 & 18 \\
\hline UGDH-AS1 & 1.57 & 0.95 & 2.19 & 2.32 & 1.21 & $3.4 \mathrm{E}-54$ & 2.0E-51 & All 4 & 18 \\
\hline PGM5P2 & 0.80 & 0.51 & 1.10 & 2.17 & 1.12 & $6.7 \mathrm{E}-25$ & $1.5 \mathrm{E}-22$ & All 4 & 18 \\
\hline MAB21L3 & 0.96 & 0.61 & 1.31 & 2.15 & 1.10 & 8.1E-29 & $2.3 E-26$ & All 4 & 18 \\
\hline $\begin{array}{l}\text { LOC } \\
100131257\end{array}$ & 2.36 & 1.58 & 3.14 & 1.98 & 0.99 & $3.0 \mathrm{E}-56$ & $2.1 \mathrm{E}-53$ & All 4 & 18 \\
\hline PRPF38B & 2.07 & 1.57 & 2.58 & 1.65 & 0.72 & $2.8 \mathrm{E}-28$ & $7.2 \mathrm{E}-26$ & All 4 & 18 \\
\hline POLR2L & 2.15 & 2.53 & 1.76 & 0.70 & -0.52 & $2.3 \mathrm{E}-17$ & $3.4 \mathrm{E}-15$ & $3 \mathrm{~h} \mid 6 \mathrm{~h}$ & 8 \\
\hline ID1 & 3.17 & 4.19 & 2.14 & 0.51 & -0.97 & 6.7E-80 & 8.0E-77 & $3 h|4 h| 6 h$ & 3 \\
\hline ID3 & 1.54 & 2.09 & 0.98 & 0.47 & -1.09 & $2.0 \mathrm{E}-49$ & $1.2 \mathrm{E}-46$ & $3 h|4 h| 6 h$ & 3 \\
\hline FKBP10 & 0.41 & 0.52 & 0.29 & 0.55 & -0.86 & $2.2 \mathrm{E}-09$ & $1.3 \mathrm{E}-07$ & $3 h|4 h| 6 h$ & 7 \\
\hline COX7C & 4.86 & 5.69 & 4.02 & 0.71 & -0.50 & $1.1 \mathrm{E}-34$ & $4.0 \mathrm{E}-32$ & $3 h|4 h| 6 h$ & 8 \\
\hline PRDX4 & 1.43 & 1.70 & 1.15 & 0.68 & -0.56 & $1.2 \mathrm{E}-13$ & $1.2 \mathrm{E}-11$ & $3 h|4 h| 6 h$ & 8 \\
\hline APP & 2.32 & 2.86 & 1.78 & 0.63 & -0.68 & $3.3 E-30$ & $9.4 \mathrm{E}-28$ & $3 h|4 h| 6 h$ & 8 \\
\hline MAGOH & 0.71 & 0.87 & 0.55 & 0.64 & -0.66 & $8.4 \mathrm{E}-10$ & $5.4 \mathrm{E}-08$ & $3 h|4 h| 6 h$ & 9 \\
\hline ATRX & 2.10 & 1.66 & 2.55 & 1.54 & 0.62 & $8.6 \mathrm{E}-22$ & $1.5 \mathrm{E}-19$ & $3 h|4 h| 6 h$ & 14 \\
\hline CCAR1 & 3.46 & 2.75 & 4.18 & 1.52 & 0.61 & 1.7E-33 & $5.5 \mathrm{E}-31$ & $3 h|4 h| 6 h$ & 14 \\
\hline DMWD & 1.23 & 1.01 & 1.44 & 1.43 & 0.51 & $1.3 \mathrm{E}-09$ & 8.1E-08 & $3 h|4 h| 6 h$ & 14 \\
\hline SLC4A7 & 0.59 & 0.45 & 0.74 & 1.64 & 0.71 & $6.4 \mathrm{E}-09$ & $3.5 \mathrm{E}-07$ & $3 h|4 h| 6 h$ & 17 \\
\hline MLL5 & 0.67 & 0.52 & 0.83 & 1.60 & 0.68 & 4.5E-09 & $2.5 \mathrm{E}-07$ & $3 h|4 h| 6 h$ & 17 \\
\hline ZNF471 & 0.58 & 0.38 & 0.77 & 2.02 & 1.01 & $1.4 \mathrm{E}-15$ & $1.8 \mathrm{E}-13$ & $3 h|4 h| 6 h$ & 18 \\
\hline F5 & 0.42 & 0.28 & 0.55 & 1.95 & 0.96 & $1.5 \mathrm{E}-10$ & $1.0 \mathrm{E}-08$ & $3 h|4 h| 6 h$ & 18 \\
\hline TMEM212 & 0.46 & 0.32 & 0.60 & 1.87 & 0.90 & $1.8 \mathrm{E}-10$ & $1.3 \mathrm{E}-08$ & $3 h|4 h| 6 h$ & 18 \\
\hline CCDC144B & 0.46 & 0.33 & 0.59 & 1.80 & 0.85 & 2.0E-09 & $1.2 \mathrm{E}-07$ & $3 h|4 h| 6 h$ & 18 \\
\hline
\end{tabular}


Table S2 continued

\begin{tabular}{|c|c|c|c|c|c|c|c|c|c|}
\hline Gene & $\begin{array}{l}\text { base } \\
\text { Mean }\end{array}$ & $\begin{array}{l}\text { base } \\
\text { MeanA }\end{array}$ & $\begin{array}{l}\text { base } \\
\text { MeanB }\end{array}$ & $\begin{array}{l}\text { fold } \\
\text { Change }\end{array}$ & $\begin{array}{l}\log 2 \\
\text { FC }\end{array}$ & pval & padj & $\begin{array}{l}\text { shared } \\
\text { in states }\end{array}$ & Node \\
\hline TSIX & 0.44 & 0.32 & 0.56 & 1.77 & 0.82 & 8.6E-09 & $4.6 \mathrm{E}-07$ & $3 h|4 h| 6 h$ & 18 \\
\hline BOD1L1 & 0.77 & 0.57 & 0.96 & 1.68 & 0.75 & $4.9 \mathrm{E}-12$ & 4.3E-10 & $3 \mathrm{~h}|4 \mathrm{~h}| 6 \mathrm{~h}$ & 18 \\
\hline GOLGB1 & 0.95 & 0.71 & 1.19 & 1.67 & 0.74 & $1.8 \mathrm{E}-14$ & $2.3 \mathrm{E}-12$ & $3 \mathrm{~h}|4 \mathrm{~h}| 6 \mathrm{~h}$ & 18 \\
\hline LUC7L3 & 2.10 & 1.65 & 2.55 & 1.55 & 0.63 & $2.5 \mathrm{E}-22$ & 4.6E-20 & $3 \mathrm{~h}|4 \mathrm{~h}| 6 \mathrm{~h}$ & 18 \\
\hline RBM25 & 3.15 & 2.49 & 3.82 & 1.54 & 0.62 & $6.1 \mathrm{E}-32$ & $1.9 \mathrm{E}-29$ & $3 h|4 h| 6 h$ & 18 \\
\hline MPHOSPH8 & 0.83 & 0.66 & 1.00 & 1.53 & 0.61 & $3.4 \mathrm{E}-09$ & $1.9 \mathrm{E}-07$ & $3 h|4 h| 6 h$ & 18 \\
\hline GADD45A & 1.72 & 1.37 & 2.07 & 1.51 & 0.59 & 8.3E-17 & $1.2 \mathrm{E}-14$ & $3 h|4 h| 6 h$ & 18 \\
\hline PNISR & 1.94 & 1.58 & 2.31 & 1.47 & 0.55 & 1.7E-16 & $2.3 \mathrm{E}-14$ & $3 h|4 h| 6 h$ & 18 \\
\hline SLTM & 1.25 & 1.03 & 1.47 & 1.42 & 0.51 & 1.1E-09 & $6.8 \mathrm{E}-08$ & $3 h|4 h| 6 h$ & 18 \\
\hline RFPL4B & 0.46 & 0.26 & 0.65 & 2.49 & 1.31 & $2.2 \mathrm{E}-19$ & $3.5 \mathrm{E}-17$ & $3 h|4 h| 6 h$ & 19 \\
\hline ZNF296 & 0.86 & 0.60 & 1.12 & 1.87 & 0.90 & $2.2 \mathrm{E}-18$ & $3.3 \mathrm{E}-16$ & $3 h|4 h| 6 h$ & 19 \\
\hline RBBP6 & 3.70 & 2.59 & 4.81 & 1.86 & 0.89 & $1.2 \mathrm{E}-72$ & $1.0 \mathrm{E}-69$ & $3 h|4 h| 6 h$ & 19 \\
\hline SRSF8 & 2.42 & 1.77 & 3.08 & 1.74 & 0.80 & 3.7E-39 & $1.6 \mathrm{E}-36$ & $3 \mathrm{~h}|4 \mathrm{~h}| 6 \mathrm{~h}$ & 19 \\
\hline ZMAT3 & 0.89 & 0.69 & 1.09 & 1.58 & 0.66 & $3.4 \mathrm{E}-11$ & 2.6E-09 & $3 h|4 h| 6 h$ & 19 \\
\hline ZNF217 & 0.81 & 0.64 & 0.97 & 1.52 & 0.60 & $6.1 \mathrm{E}-09$ & 3.3E-07 & $3 h|4 h| 6 h$ & 19 \\
\hline PNN & 3.62 & 2.95 & 4.30 & 1.46 & 0.54 & $1.0 \mathrm{E}-28$ & $2.8 \mathrm{E}-26$ & $3 h|4 h| 6 h$ & 21 \\
\hline MT2A & 1.26 & 1.48 & 1.03 & 0.70 & -0.52 & $1.2 \mathrm{E}-10$ & 8.7E-09 & $3 \mathrm{~h} \mid 4 \mathrm{~h}$ & 7 \\
\hline SNRPD2 & 2.08 & 2.47 & 1.68 & 0.68 & -0.56 & $3.6 \mathrm{E}-19$ & 5.7E-17 & $3 h \mid 4 h$ & 7 \\
\hline TOMM7 & 1.48 & 1.79 & 1.17 & 0.65 & -0.61 & $1.3 \mathrm{E}-16$ & $1.8 \mathrm{E}-14$ & $3 h \mid 4 h$ & 7 \\
\hline TMEM258 & 1.26 & 1.50 & 1.03 & 0.68 & -0.55 & $1.1 \mathrm{E}-11$ & $9.5 \mathrm{E}-10$ & $3 h \mid 4 h$ & 8 \\
\hline ATP5O & 2.52 & 3.02 & 2.01 & 0.67 & -0.59 & $1.1 \mathrm{E}-24$ & $2.3 \mathrm{E}-22$ & $3 h \mid 4 h$ & 8 \\
\hline ATP5J2 & 3.47 & 4.21 & 2.74 & 0.65 & -0.62 & $6.0 \mathrm{E}-37$ & $2.4 \mathrm{E}-34$ & $3 \mathrm{~h} \mid 4 \mathrm{~h}$ & 8 \\
\hline CLSPN & 1.51 & 1.21 & 1.82 & 1.50 & 0.59 & $1.4 \mathrm{E}-14$ & $1.8 \mathrm{E}-12$ & $3 h \mid 4 h$ & 10 \\
\hline ZC3H13 & 0.52 & 0.39 & 0.65 & 1.66 & 0.74 & 2.0E-08 & 1.0E-06 & $3 h \mid 4 h$ & 14 \\
\hline CHD9 & 1.00 & 0.80 & 1.20 & 1.50 & 0.59 & 4.7E-10 & $3.2 \mathrm{E}-08$ & $3 h \mid 4 h$ & 14 \\
\hline REV3L & 0.99 & 0.80 & 1.19 & 1.49 & 0.58 & $5.9 \mathrm{E}-10$ & 3.9E-08 & $3 h \mid 4 h$ & 14 \\
\hline THOC2 & 1.52 & 1.23 & 1.80 & 1.46 & 0.55 & 4.0E-13 & $3.8 \mathrm{E}-11$ & $3 h \mid 4 h$ & 14 \\
\hline APH1A & 0.29 & 0.39 & 0.19 & 0.49 & -1.03 & $1.5 \mathrm{E}-09$ & $9.0 \mathrm{E}-08$ & $3 h \mid 4 h$ & NA \\
\hline PSMB3 & 1.10 & 1.30 & 0.90 & 0.70 & -0.52 & $1.4 \mathrm{E}-09$ & $8.2 \mathrm{E}-08$ & $3 h$ & 7 \\
\hline NDUFA11 & 1.34 & 1.58 & 1.10 & 0.70 & -0.53 & $2.1 \mathrm{E}-11$ & 1.7E-09 & $3 h$ & 7 \\
\hline LAMTOR5 & 0.94 & 1.13 & 0.76 & 0.68 & -0.56 & 1.7E-09 & $9.9 \mathrm{E}-08$ & $3 h$ & 7 \\
\hline UQCR10 & 1.16 & 1.40 & 0.92 & 0.66 & -0.60 & $1.1 \mathrm{E}-12$ & $9.9 \mathrm{E}-11$ & $3 h$ & 7 \\
\hline RPA3 & 0.51 & 0.63 & 0.39 & 0.61 & -0.71 & 1.7E-08 & 8.7E-07 & $3 h$ & 7 \\
\hline UBE2T & 1.10 & 1.29 & 0.91 & 0.71 & -0.51 & $3.9 \mathrm{E}-09$ & $2.2 \mathrm{E}-07$ & $3 h$ & 8 \\
\hline RPL31 & 5.01 & 5.90 & 4.13 & 0.70 & -0.51 & 4.7E-37 & $2.0 \mathrm{E}-34$ & $3 h$ & 8 \\
\hline
\end{tabular}


Table S2 continued

\begin{tabular}{|c|c|c|c|c|c|c|c|c|c|}
\hline Gene & $\begin{array}{l}\text { base } \\
\text { Mean }\end{array}$ & $\begin{array}{l}\text { base } \\
\text { MeanA }\end{array}$ & $\begin{array}{l}\text { base } \\
\text { MeanB }\end{array}$ & $\begin{array}{l}\text { fold } \\
\text { Change }\end{array}$ & $\begin{array}{l}\log 2 \\
\text { FC }\end{array}$ & pval & padj & $\begin{array}{l}\text { shared } \\
\text { in states }\end{array}$ & Node \\
\hline RPL37 & 14.22 & 16.72 & 11.72 & 0.70 & -0.51 & $6.6 \mathrm{E}-100$ & $2.0 \mathrm{E}-96$ & $3 \mathrm{~h}$ & 8 \\
\hline RPL28 & 7.98 & 9.43 & 6.54 & 0.69 & -0.53 & 4.6E-61 & 3.7E-58 & $3 \mathrm{~h}$ & 8 \\
\hline UBL5 & 2.88 & 3.41 & 2.35 & 0.69 & -0.54 & $7.8 \mathrm{E}-24$ & $1.5 \mathrm{E}-21$ & $3 h$ & 8 \\
\hline SNRPG & 2.82 & 3.35 & 2.28 & 0.68 & -0.56 & $8.4 \mathrm{E}-25$ & $1.8 \mathrm{E}-22$ & $3 \mathrm{~h}$ & 8 \\
\hline RPS28 & 6.63 & 7.93 & 5.33 & 0.67 & -0.57 & $7.8 \mathrm{E}-60$ & $5.8 \mathrm{E}-57$ & $3 h$ & 8 \\
\hline ASH1L & 0.75 & 0.56 & 0.94 & 1.67 & 0.74 & $9.8 \mathrm{E}-12$ & 8.3E-10 & $3 \mathrm{~h}$ & 10 \\
\hline XIAP & 0.82 & 0.64 & 1.00 & 1.55 & 0.63 & $9.1 \mathrm{E}-10$ & $5.8 \mathrm{E}-08$ & $3 \mathrm{~h}$ & 10 \\
\hline FAM115A & 0.88 & 0.71 & 1.05 & 1.48 & 0.56 & $1.9 \mathrm{E}-08$ & $9.5 \mathrm{E}-07$ & $3 \mathrm{~h}$ & 10 \\
\hline REST & 0.93 & 0.75 & 1.11 & 1.48 & 0.56 & $5.5 \mathrm{E}-09$ & $3.0 \mathrm{E}-07$ & $3 h$ & 10 \\
\hline COX7A2 & 2.82 & 3.36 & 2.29 & 0.68 & -0.55 & $7.9 E-25$ & 1.7E-22 & $3 \mathrm{~h}$ & 12 \\
\hline TNRC6B & 0.74 & 0.56 & 0.93 & 1.67 & 0.74 & $2.3 \mathrm{E}-11$ & $1.8 \mathrm{E}-09$ & $3 h$ & 14 \\
\hline TPR & 0.82 & 0.65 & 0.99 & 1.51 & 0.60 & 7.6E-09 & 4.1E-07 & $3 \mathrm{~h}$ & 14 \\
\hline SETD2 & 0.87 & 0.70 & 1.03 & 1.48 & 0.57 & $1.8 \mathrm{E}-08$ & $9.2 \mathrm{E}-07$ & $3 \mathrm{~h}$ & 14 \\
\hline ESF1 & 0.92 & 0.74 & 1.10 & 1.48 & 0.56 & 7.7E-09 & 4.1E-07 & $3 \mathrm{~h}$ & 14 \\
\hline LRRC58 & 1.01 & 0.82 & 1.20 & 1.46 & 0.54 & 5.0E-09 & $2.8 \mathrm{E}-07$ & $3 \mathrm{~h}$ & 14 \\
\hline WNK1 & 1.28 & 1.05 & 1.50 & 1.43 & 0.51 & 4.5E-10 & $3.1 \mathrm{E}-08$ & $3 \mathrm{~h}$ & 14 \\
\hline KIF14 & 0.86 & 0.67 & 1.04 & 1.54 & 0.62 & 1.0E-09 & 6.7E-08 & $2 h|3 h| 6 h$ & 10 \\
\hline HIST1H2BK & 0.74 & 0.92 & 0.57 & 0.63 & -0.68 & $9.8 \mathrm{E}-11$ & 7.2E-09 & $2 h|3 h| 6 h$ & 19 \\
\hline NOP10 & 1.70 & 2.06 & 1.33 & 0.65 & -0.63 & $9.6 \mathrm{E}-20$ & $1.6 \mathrm{E}-17$ & $2 h|3 h| 4 h$ & 8 \\
\hline RPL39 & 8.02 & 9.75 & 6.29 & 0.65 & -0.63 & $4.8 \mathrm{E}-87$ & 7.1E-84 & $2 h|3 h| 4 h$ & 8 \\
\hline PIP5K1A & 0.31 & 0.42 & 0.20 & 0.46 & -1.11 & $1.9 \mathrm{E}-11$ & $1.5 \mathrm{E}-09$ & $2 \mathrm{~h}|3 \mathrm{~h}| 4 \mathrm{~h}$ & 8 \\
\hline GOLIM4 & 0.44 & 0.29 & 0.58 & 1.99 & 0.99 & $1.1 \mathrm{E}-11$ & $9.5 \mathrm{E}-10$ & $2 \mathrm{~h}|3 \mathrm{~h}| 4 \mathrm{~h}$ & 10 \\
\hline CCDC88A & 0.65 & 0.46 & 0.84 & 1.83 & 0.87 & $2.1 \mathrm{E}-13$ & 2.1E-11 & $2 h|3 h| 4 h$ & 10 \\
\hline DNAJC2 & 1.15 & 0.86 & 1.44 & 1.67 & 0.74 & 2.7E-17 & $3.9 \mathrm{E}-15$ & $2 h|3 h| 4 h$ & 10 \\
\hline MAP1B & 7.04 & 5.30 & 8.79 & 1.66 & 0.73 & $4.0 E-94$ & $8.0 \mathrm{E}-91$ & $2 h|3 h| 4 h$ & 10 \\
\hline ITSN1 & 1.13 & 0.86 & 1.41 & 1.65 & 0.72 & $6.9 \mathrm{E}-16$ & $9.2 \mathrm{E}-14$ & $2 h|3 h| 4 h$ & 10 \\
\hline CDC42BPA & 0.73 & 0.56 & 0.91 & 1.62 & 0.70 & $2.6 \mathrm{E}-10$ & $1.8 \mathrm{E}-08$ & $2 h|3 h| 4 h$ & 10 \\
\hline ZFHX3 & 1.73 & 1.34 & 2.13 & 1.59 & 0.67 & $6.8 \mathrm{E}-21$ & $1.1 \mathrm{E}-18$ & $2 h|3 h| 4 h$ & 10 \\
\hline ANKRD12 & 0.81 & 0.61 & 1.00 & 1.65 & 0.73 & $7.2 \mathrm{E}-12$ & $6.2 \mathrm{E}-10$ & $2 h|3 h| 4 h$ & 13 \\
\hline ROCK1 & 0.54 & 0.36 & 0.72 & 1.98 & 0.99 & $4.2 \mathrm{E}-14$ & $4.8 \mathrm{E}-12$ & $2 h|3 h| 4 h$ & 14 \\
\hline KTN1 & 2.34 & 1.84 & 2.85 & 1.55 & 0.63 & $5.4 \mathrm{E}-25$ & $1.2 \mathrm{E}-22$ & $2 h|3 h| 4 h$ & 14 \\
\hline CHD7 & 1.04 & 0.82 & 1.26 & 1.54 & 0.62 & $1.3 \mathrm{E}-11$ & 1.1E-09 & $2 \mathrm{~h} \mid 3 \mathrm{~h}$ & 10 \\
\hline NIPBL & 0.80 & 0.64 & 0.97 & 1.52 & 0.60 & $1.2 \mathrm{E}-08$ & $6.4 \mathrm{E}-07$ & $2 \mathrm{~h} \mid 3 \mathrm{~h}$ & 10 \\
\hline TAF3 & 0.38 & 0.26 & 0.49 & 1.88 & 0.91 & $5.2 \mathrm{E}-09$ & $2.9 \mathrm{E}-07$ & $2 \mathrm{~h} \mid 3 \mathrm{~h}$ & 14 \\
\hline
\end{tabular}


Table S3. Differentially expressed genes between uninduced (Oh, clusters $1 \& 2$ ) and $4 \mathrm{~h}$ induced DIE cells (clusters 7\&8).

* Adjusted $\mathrm{p}$ value $<10 * *-6$, absolute $\log 2 \mathrm{FC}>0.5$

\begin{tabular}{|c|c|c|c|c|c|c|c|c|c|}
\hline Gene & $\begin{array}{l}\text { base } \\
\text { Mean }\end{array}$ & $\begin{array}{l}\text { base } \\
\text { MeanA }\end{array}$ & $\begin{array}{l}\text { base } \\
\text { MeaanB }\end{array}$ & $\begin{array}{l}\text { fold } \\
\text { Change }\end{array}$ & $\begin{array}{l}\log 2 \\
\text { FC }\end{array}$ & pval & padj & $\begin{array}{l}\text { shared } \\
\text { in states }\end{array}$ & Node \\
\hline RPL37A & 7.63 & 9.21 & 6.06 & 0.66 & -0.61 & $8.0 \mathrm{E}-58$ & $4.8 \mathrm{E}-55$ & All 4 & 8 \\
\hline RPS29 & 11.81 & 14.41 & 9.21 & 0.64 & -0.65 & 4.3E-96 & $5.2 \mathrm{E}-93$ & All 4 & 8 \\
\hline USMG5 & 1.84 & 2.27 & 1.42 & 0.63 & -0.67 & $3.5 \mathrm{E}-18$ & $4.8 \mathrm{E}-16$ & All 4 & 8 \\
\hline UQCR11 & 1.01 & 1.24 & 0.77 & 0.62 & -0.70 & $1.5 \mathrm{E}-11$ & $1.1 \mathrm{E}-09$ & All 4 & 8 \\
\hline ASPM & 1.25 & 0.88 & 1.61 & 1.83 & 0.87 & $1.2 \mathrm{E}-19$ & $1.8 \mathrm{E}-17$ & All 4 & 10 \\
\hline ANKRD11 & 2.03 & 1.50 & 2.57 & 1.72 & 0.78 & $9.2 \mathrm{E}-26$ & $2.0 \mathrm{E}-23$ & All 4 & 10 \\
\hline CENPE & 0.89 & 0.69 & 1.09 & 1.58 & 0.66 & $3.3 \mathrm{E}-09$ & $1.7 \mathrm{E}-07$ & All 4 & 10 \\
\hline CENPF & 2.61 & 2.06 & 3.16 & 1.53 & 0.62 & $3.3 \mathrm{E}-21$ & $5.7 \mathrm{E}-19$ & All 4 & 10 \\
\hline PRR11 & 1.00 & 0.79 & 1.21 & 1.53 & 0.62 & $5.1 \mathrm{E}-09$ & $2.6 \mathrm{E}-07$ & All 4 & 13 \\
\hline MDN1 & 0.34 & 0.21 & 0.47 & 2.25 & 1.17 & $2.9 \mathrm{E}-10$ & $1.8 \mathrm{E}-08$ & All 4 & 14 \\
\hline BDP1 & 0.78 & 0.52 & 1.04 & 2.00 & 1.00 & $3.9 \mathrm{E}-16$ & $4.3 \mathrm{E}-14$ & All 4 & 14 \\
\hline AKAP9 & 0.78 & 0.52 & 1.03 & 2.00 & 1.00 & $2.6 \mathrm{E}-16$ & $3.0 \mathrm{E}-14$ & All 4 & 14 \\
\hline NKTR & 1.47 & 1.04 & 1.91 & 1.84 & 0.88 & $1.4 \mathrm{E}-23$ & $2.7 \mathrm{E}-21$ & All 4 & 14 \\
\hline BRD4 & 1.54 & 1.09 & 1.98 & 1.82 & 0.87 & $1.1 \mathrm{E}-23$ & $2.1 \mathrm{E}-21$ & All 4 & 14 \\
\hline CHD2 & 0.69 & 0.50 & 0.88 & 1.75 & 0.81 & $4.4 \mathrm{E}-10$ & $2.6 \mathrm{E}-08$ & All 4 & 14 \\
\hline GOLGA4 & 1.18 & 0.87 & 1.50 & 1.72 & 0.78 & $1.2 \mathrm{E}-15$ & $1.3 \mathrm{E}-13$ & All 4 & 14 \\
\hline BPTF & 1.34 & 0.99 & 1.69 & 1.72 & 0.78 & 2.4E-17 & $3.1 \mathrm{E}-15$ & All 4 & 14 \\
\hline SRRM1 & 2.08 & 1.60 & 2.56 & 1.60 & 0.68 & 2.4E-20 & $4.0 \mathrm{E}-18$ & All 4 & 14 \\
\hline TOP1 & 1.54 & 1.19 & 1.89 & 1.59 & 0.67 & $3.2 \mathrm{E}-15$ & $3.3 \mathrm{E}-13$ & All 4 & 14 \\
\hline SMC4 & 5.84 & 4.80 & 6.89 & 1.43 & 0.52 & $3.9 \mathrm{E}-33$ & $1.2 \mathrm{E}-30$ & All 4 & 14 \\
\hline SREK1 & 1.10 & 0.80 & 1.39 & 1.72 & 0.79 & $9.8 \mathrm{E}-15$ & $9.6 \mathrm{E}-13$ & All 4 & 17 \\
\hline KCNQ1OT1 & 10.12 & 5.01 & 15.22 & 3.04 & 1.60 & $0.0 \mathrm{E}+00$ & $0.0 \mathrm{E}+00$ & All 4 & 18 \\
\hline UGDH-AS1 & 1.83 & 0.94 & 2.73 & 2.89 & 1.53 & $1.1 \mathrm{E}-76$ & $1.1 \mathrm{E}-73$ & All 4 & 18 \\
\hline PGM5P2 & 0.92 & 0.51 & 1.34 & 2.64 & 1.40 & $9.3 E-34$ & $3.0 \mathrm{E}-31$ & All 4 & 18 \\
\hline MAB21L3 & 1.11 & 0.61 & 1.60 & 2.63 & 1.39 & $5.6 \mathrm{E}-40$ & $2.2 \mathrm{E}-37$ & All 4 & 18 \\
\hline $\begin{array}{l}\text { LOC } \\
100131257\end{array}$ & 2.73 & 1.58 & 3.88 & 2.47 & 1.30 & $3.3 \mathrm{E}-85$ & $3.6 \mathrm{E}-82$ & All 4 & 18 \\
\hline PRPF38B & 2.24 & 1.56 & 2.92 & 1.87 & 0.90 & $1.3 \mathrm{E}-36$ & 4.6E-34 & All 4 & 18 \\
\hline SLC7A5 & 0.87 & 1.08 & 0.66 & 0.61 & -0.71 & 2.6E-10 & $1.6 \mathrm{E}-08$ & $4 h \mid 6 h$ & 1 \\
\hline FTL & 9.12 & 10.70 & 7.54 & 0.71 & -0.50 & $1.3 \mathrm{E}-48$ & $5.7 E-46$ & $4 \mathrm{~h} \mid 6 \mathrm{~h}$ & 2 \\
\hline UBE2S & 2.92 & 3.50 & 2.35 & 0.67 & -0.58 & $3.7 \mathrm{E}-21$ & $6.4 \mathrm{E}-19$ & $4 \mathrm{~h} \mid 6 \mathrm{~h}$ & 3 \\
\hline DYNLL1 & 6.98 & 8.57 & 5.39 & 0.63 & -0.67 & $1.9 \mathrm{E}-64$ & $1.3 \mathrm{E}-61$ & $4 \mathrm{~h} \mid 6 \mathrm{~h}$ & 3 \\
\hline SHISA3 & 1.55 & 1.92 & 1.18 & 0.61 & -0.71 & $5.3 \mathrm{E}-17$ & $6.6 \mathrm{E}-15$ & $4 \mathrm{~h} \mid 6 \mathrm{~h}$ & 3 \\
\hline
\end{tabular}


Table S3 continued

\begin{tabular}{|c|c|c|c|c|c|c|c|c|c|}
\hline Gene & $\begin{array}{l}\text { base } \\
\text { Mean }\end{array}$ & $\begin{array}{l}\text { base } \\
\text { MeanA }\end{array}$ & $\begin{array}{l}\text { base } \\
\text { MeanB }\end{array}$ & $\begin{array}{l}\text { fold } \\
\text { Change }\end{array}$ & $\begin{array}{l}\log 2 \\
\text { FC }\end{array}$ & pval & padj & $\begin{array}{l}\text { shared } \\
\text { in states }\end{array}$ & Node \\
\hline NEDD9 & 0.88 & 1.09 & 0.67 & 0.61 & -0.71 & $2.5 \mathrm{E}-10$ & $1.5 \mathrm{E}-08$ & $4 h \mid 6 h$ & 3 \\
\hline MIDN & 0.97 & 1.23 & 0.72 & 0.58 & -0.78 & $3.9 \mathrm{E}-13$ & 3.2E-11 & $4 h \mid 6 h$ & 3 \\
\hline CYR61 & 1.18 & 1.50 & 0.86 & 0.57 & -0.81 & 7.1E-17 & 8.7E-15 & $4 h \mid 6 h$ & 3 \\
\hline PCDH18 & 0.57 & 0.76 & 0.39 & 0.51 & -0.97 & 3.0E-12 & 2.4E-10 & $4 h \mid 6 h$ & 3 \\
\hline PIM1 & 0.38 & 0.50 & 0.25 & 0.51 & -0.98 & $2.2 \mathrm{E}-08$ & 9.9E-07 & $4 h \mid 6 h$ & 3 \\
\hline TGIF1 & 0.68 & 0.92 & 0.44 & 0.48 & -1.05 & $3.1 \mathrm{E}-16$ & $3.5 \mathrm{E}-14$ & $4 h \mid 6 h$ & 3 \\
\hline NOG & 0.31 & 0.43 & 0.20 & 0.47 & -1.08 & 1.9E-08 & 8.7E-07 & $4 h \mid 6 h$ & 3 \\
\hline NUAK2 & 0.36 & 0.50 & 0.23 & 0.46 & -1.12 & 4.7E-10 & 2.7E-08 & $4 h \mid 6 h$ & 3 \\
\hline HNRNPAO & 1.61 & 1.94 & 1.28 & 0.66 & -0.61 & $1.8 \mathrm{E}-13$ & $1.5 \mathrm{E}-11$ & $4 h \mid 6 h$ & 8 \\
\hline RPL23A & 10.89 & 12.91 & 8.87 & 0.69 & -0.54 & 7.1E-65 & $5.0 \mathrm{E}-62$ & $4 h \mid 6 h$ & 9 \\
\hline ISOC2 & 0.43 & 0.56 & 0.30 & 0.53 & -0.92 & $1.8 \mathrm{E}-08$ & 8.6E-07 & $4 h \mid 6 h$ & 9 \\
\hline RIF1 & 1.52 & 1.21 & 1.83 & 1.51 & 0.59 & 4.4E-12 & $3.4 \mathrm{E}-10$ & $4 h \mid 6 h$ & 13 \\
\hline GUSBP3 & 0.53 & 0.37 & 0.69 & 1.87 & 0.91 & 7.1E-10 & 4.0E-08 & $4 h \mid 6 h$ & 14 \\
\hline CUX1 & 0.97 & 0.77 & 1.18 & 1.53 & 0.61 & $1.2 \mathrm{E}-08$ & 5.7E-07 & $4 h \mid 6 h$ & 14 \\
\hline BBX & 1.48 & 1.19 & 1.76 & 1.48 & 0.56 & 7.7E-11 & $5.2 \mathrm{E}-09$ & $4 h \mid 6 h$ & 14 \\
\hline PPIG & 1.67 & 1.36 & 1.99 & 1.47 & 0.55 & $1.2 \mathrm{E}-11$ & $9.0 \mathrm{E}-10$ & $4 h \mid 6 h$ & 14 \\
\hline SYNE2 & 0.75 & 0.50 & 0.99 & 1.98 & 0.99 & $3.9 \mathrm{E}-15$ & $3.9 \mathrm{E}-13$ & $4 h \mid 6 h$ & 17 \\
\hline POLQ & 0.85 & 0.64 & 1.06 & 1.66 & 0.74 & $1.5 \mathrm{E}-10$ & 9.7E-09 & $4 h \mid 6 h$ & 17 \\
\hline SMC3 & 1.57 & 1.27 & 1.87 & 1.47 & 0.56 & 3.6E-11 & $2.5 \mathrm{E}-09$ & $4 h \mid 6 h$ & 17 \\
\hline TNRC6A & 1.61 & 1.32 & 1.91 & 1.45 & 0.54 & $1.1 \mathrm{E}-10$ & 7.3E-09 & $4 h \mid 6 h$ & 17 \\
\hline ODF2L & 0.55 & 0.38 & 0.72 & 1.91 & 0.93 & $1.0 \mathrm{E}-10$ & 6.7E-09 & $4 h \mid 6 h$ & 18 \\
\hline $\begin{array}{l}\text { GABPB1- } \\
\text { AS1 }\end{array}$ & 0.51 & 0.36 & 0.66 & 1.87 & 0.90 & 1.7E-09 & $9.3 \mathrm{E}-08$ & $4 h \mid 6 h$ & 18 \\
\hline PHACTR2 & 0.54 & 0.38 & 0.70 & 1.87 & 0.90 & 6.5E-10 & $3.6 \mathrm{E}-08$ & $4 h \mid 6 h$ & 18 \\
\hline NET1 & 1.09 & 0.83 & 1.35 & 1.63 & 0.71 & 3.6E-12 & $2.8 \mathrm{E}-10$ & $4 h \mid 6 h$ & 18 \\
\hline ZMYND8 & 0.99 & 0.76 & 1.21 & 1.60 & 0.67 & 2.9E-10 & $1.8 \mathrm{E}-08$ & $4 h \mid 6 h$ & 18 \\
\hline SLC25A36 & 1.43 & 1.16 & 1.70 & 1.47 & 0.56 & $2.4 \mathrm{E}-10$ & $1.4 \mathrm{E}-08$ & $4 h \mid 6 h$ & 18 \\
\hline SRSF11 & 4.36 & 3.54 & 5.18 & 1.46 & 0.55 & 7.0E-28 & $1.8 \mathrm{E}-25$ & $4 h \mid 6 h$ & 18 \\
\hline DPPA4 & 4.12 & 3.39 & 4.85 & 1.43 & 0.52 & $1.8 \mathrm{E}-23$ & $3.4 \mathrm{E}-21$ & $4 h \mid 6 h$ & 18 \\
\hline ZRANB2 & 2.49 & 2.05 & 2.93 & 1.43 & 0.52 & 9.3E-15 & $9.2 \mathrm{E}-13$ & $4 h \mid 6 h$ & 18 \\
\hline ZSCAN4 & 1.16 & 0.26 & 2.06 & 7.90 & 2.98 & 3.1E-131 & $\begin{array}{r}6.2 \mathrm{E}- \\
128\end{array}$ & $4 h \mid 6 h$ & 19 \\
\hline TRIM51 & 0.74 & 0.25 & 1.23 & 4.96 & 2.31 & $3.1 E-60$ & $2.0 \mathrm{E}-57$ & $4 h \mid 6 h$ & 19 \\
\hline PRAMEF1 & 0.27 & 0.11 & 0.44 & 3.80 & 1.93 & $9.3 \mathrm{E}-18$ & $1.3 \mathrm{E}-15$ & $4 \mathrm{~h} \mid 6 \mathrm{~h}$ & 19 \\
\hline PRRG4 & 0.37 & 0.17 & 0.58 & 3.36 & 1.75 & $6.1 \mathrm{E}-21$ & $1.1 \mathrm{E}-18$ & $4 h \mid 6 h$ & 19 \\
\hline PRAMEF12 & 0.27 & 0.13 & 0.41 & 3.14 & 1.65 & 3.0E-14 & $2.8 \mathrm{E}-12$ & $4 h \mid 6 h$ & 19 \\
\hline
\end{tabular}


Table S3 continued

\begin{tabular}{|c|c|c|c|c|c|c|c|c|c|}
\hline Gene & $\begin{array}{l}\text { base } \\
\text { Mean }\end{array}$ & $\begin{array}{l}\text { base } \\
\text { MeanA }\end{array}$ & $\begin{array}{l}\text { base } \\
\text { MeanB }\end{array}$ & $\begin{array}{l}\text { fold } \\
\text { Change }\end{array}$ & $\begin{array}{l}\log 2 \\
\text { FC }\end{array}$ & pval & padj & $\begin{array}{l}\text { shared } \\
\text { in states }\end{array}$ & Node \\
\hline SLC34A2 & 0.23 & 0.12 & 0.35 & 2.89 & 1.53 & $9.2 \mathrm{E}-11$ & $6.1 \mathrm{E}-09$ & $4 h \mid 6 h$ & 19 \\
\hline KDM4E & 0.20 & 0.10 & 0.29 & 2.75 & 1.46 & 7.4E-09 & 3.7E-07 & $4 h \mid 6 h$ & 19 \\
\hline GTF2F1 & 2.55 & 1.36 & 3.73 & 2.74 & 1.45 & $7.9 E-97$ & $1.1 \mathrm{E}-93$ & $4 h \mid 6 h$ & 19 \\
\hline ARID5B & 0.57 & 0.31 & 0.83 & 2.70 & 1.44 & $4.2 \mathrm{E}-22$ & 7.7E-20 & $4 h \mid 6 h$ & 19 \\
\hline PNP & 2.84 & 1.57 & 4.11 & 2.61 & 1.39 & $5.2 \mathrm{E}-99$ & $7.8 \mathrm{E}-96$ & $4 h \mid 6 h$ & 19 \\
\hline SPTY2D1 & 0.55 & 0.31 & 0.79 & 2.53 & 1.34 & $1.9 \mathrm{E}-19$ & $2.9 \mathrm{E}-17$ & $4 \mathrm{~h} \mid 6 \mathrm{~h}$ & 19 \\
\hline TFIP11 & 0.31 & 0.18 & 0.44 & 2.47 & 1.31 & 7.0E-11 & $4.8 \mathrm{E}-09$ & $4 h \mid 6 h$ & 19 \\
\hline ESRG & 0.45 & 0.27 & 0.63 & 2.36 & 1.24 & 2.7E-14 & $2.5 \mathrm{E}-12$ & $4 h \mid 6 h$ & 19 \\
\hline HOXB2 & 1.29 & 0.78 & 1.80 & 2.29 & 1.20 & $9.8 \mathrm{E}-36$ & $3.4 \mathrm{E}-33$ & $4 h \mid 6 h$ & 19 \\
\hline ZNF622 & 0.71 & 0.44 & 0.97 & 2.20 & 1.14 & $1.1 \mathrm{E}-18$ & $1.5 \mathrm{E}-16$ & $4 \mathrm{~h} \mid 6 \mathrm{~h}$ & 19 \\
\hline PDGFRA & 0.47 & 0.30 & 0.64 & 2.16 & 1.11 & 4.7E-12 & 3.6E-10 & $4 h \mid 6 h$ & 19 \\
\hline CDH10 & 0.76 & 0.50 & 1.01 & 2.01 & 1.00 & $4.8 \mathrm{E}-16$ & $5.2 \mathrm{E}-14$ & $4 \mathrm{~h} \mid 6 \mathrm{~h}$ & 19 \\
\hline DBR1 & 0.58 & 0.40 & 0.76 & 1.92 & 0.94 & 2.6E-11 & 1.9E-09 & $4 \mathrm{~h} \mid 6 \mathrm{~h}$ & 19 \\
\hline NFAT5 & 0.78 & 0.54 & 1.03 & 1.90 & 0.93 & $2.3 E-14$ & $2.2 \mathrm{E}-12$ & $4 \mathrm{~h} \mid 6 \mathrm{~h}$ & 19 \\
\hline ARHGEF26 & 1.07 & 0.74 & 1.39 & 1.88 & 0.91 & $2.2 \mathrm{E}-18$ & $3.2 \mathrm{E}-16$ & $4 \mathrm{~h} \mid 6 \mathrm{~h}$ & 19 \\
\hline EXOSC10 & 2.11 & 1.47 & 2.75 & 1.86 & 0.90 & $2.8 \mathrm{E}-34$ & $9.2 \mathrm{E}-32$ & $4 h \mid 6 h$ & 19 \\
\hline ZNF644 & 0.78 & 0.56 & 1.00 & 1.77 & 0.82 & $1.1 \mathrm{E}-11$ & $8.2 \mathrm{E}-10$ & $4 \mathrm{~h} \mid 6 \mathrm{~h}$ & 19 \\
\hline NXF1 & 1.38 & 1.04 & 1.73 & 1.67 & 0.74 & $1.6 \mathrm{E}-16$ & $1.9 \mathrm{E}-14$ & $4 h \mid 6 h$ & 19 \\
\hline SHC1 & 1.34 & 1.04 & 1.65 & 1.60 & 0.67 & $1.4 \mathrm{E}-13$ & $1.2 \mathrm{E}-11$ & $4 h \mid 6 h$ & 19 \\
\hline MRPL49 & 1.82 & 1.41 & 2.23 & 1.58 & 0.66 & $6.5 \mathrm{E}-17$ & 8.0E-15 & $4 h \mid 6 h$ & 19 \\
\hline PLK4 & 0.93 & 0.72 & 1.13 & 1.57 & 0.65 & 3.3E-09 & 1.7E-07 & $4 h \mid 6 h$ & 19 \\
\hline CCNL2 & 1.29 & 1.05 & 1.53 & 1.45 & 0.54 & 4.9E-09 & $2.5 \mathrm{E}-07$ & $4 h \mid 6 h$ & 19 \\
\hline CIRBP & 3.89 & 3.18 & 4.60 & 1.45 & 0.53 & $1.9 E-23$ & $3.5 E-21$ & $4 h \mid 6 h$ & 19 \\
\hline RFPL4A & 0.38 & 0.15 & 0.62 & 4.24 & 2.08 & $1.1 \mathrm{E}-27$ & $2.8 \mathrm{E}-25$ & $4 \mathrm{~h} \mid 6 \mathrm{~h}$ & 20 \\
\hline LEUTX & 0.30 & 0.14 & 0.45 & 3.35 & 1.74 & $1.4 \mathrm{E}-16$ & 1.7E-14 & $4 h \mid 6 h$ & 20 \\
\hline RICTOR & 0.64 & 0.39 & 0.88 & 2.25 & 1.17 & $1.3 \mathrm{E}-17$ & 1.7E-15 & $4 h \mid 6 h$ & 21 \\
\hline ITGB8 & 0.31 & 0.20 & 0.42 & 2.16 & 1.11 & $1.9 \mathrm{E}-08$ & 8.7E-07 & $4 h \mid 6 h$ & 21 \\
\hline ZNF827 & 0.48 & 0.31 & 0.64 & 2.06 & 1.04 & $3.2 \mathrm{E}-11$ & $2.2 \mathrm{E}-09$ & $4 \mathrm{~h} \mid 6 \mathrm{~h}$ & 21 \\
\hline ZSWIM6 & 0.48 & 0.34 & 0.62 & 1.84 & 0.88 & $2.1 \mathrm{E}-08$ & $9.5 \mathrm{E}-07$ & $4 \mathrm{~h} \mid 6 \mathrm{~h}$ & 21 \\
\hline BTAF1 & 0.66 & 0.49 & 0.83 & 1.70 & 0.76 & 7.0E-09 & $3.5 \mathrm{E}-07$ & $4 h \mid 6 h$ & 21 \\
\hline KIAA1551 & 0.75 & 0.56 & 0.94 & 1.69 & 0.76 & 1.1E-09 & $6.2 \mathrm{E}-08$ & $4 h \mid 6 h$ & 21 \\
\hline YTHDC1 & 1.29 & 0.98 & 1.60 & 1.63 & 0.71 & 2.7E-14 & $2.5 \mathrm{E}-12$ & $4 h \mid 6 h$ & 21 \\
\hline LRRC8B & 0.89 & 0.68 & 1.09 & 1.62 & 0.70 & 5.7E-10 & $3.2 \mathrm{E}-08$ & $4 \mathrm{~h} \mid 6 \mathrm{~h}$ & 21 \\
\hline PUM1 & 2.15 & 1.76 & 2.54 & 1.44 & 0.53 & $1.3 \mathrm{E}-13$ & $1.2 \mathrm{E}-11$ & $4 h \mid 6 h$ & 21 \\
\hline CTGF & 0.46 & 0.59 & 0.32 & 0.54 & -0.90 & 7.0E-09 & $3.5 \mathrm{E}-07$ & $4 \mathrm{~h}$ & 3 \\
\hline
\end{tabular}


Table S3 continued

\begin{tabular}{|c|c|c|c|c|c|c|c|c|c|}
\hline Gene & $\begin{array}{l}\text { base } \\
\text { Mean }\end{array}$ & $\begin{array}{l}\text { base } \\
\text { MeanA }\end{array}$ & $\begin{array}{l}\text { base } \\
\text { MeanB }\end{array}$ & $\begin{array}{l}\text { fold } \\
\text { Change }\end{array}$ & $\begin{array}{l}\log 2 \\
F C\end{array}$ & pval & padj & $\begin{array}{l}\text { shared } \\
\text { in states }\end{array}$ & Node \\
\hline CKS2 & 1.58 & 1.87 & 1.28 & 0.69 & -0.55 & $5.8 \mathrm{E}-11$ & 4.0E-09 & $4 \mathrm{~h}$ & 7 \\
\hline ID2 & 0.41 & 0.58 & 0.24 & 0.42 & -1.24 & $1.3 \mathrm{E}-13$ & $1.2 \mathrm{E}-11$ & $4 \mathrm{~h}$ & 7 \\
\hline LRRFIP1 & 1.62 & 1.27 & 1.96 & 1.55 & 0.63 & 3.3E-14 & 3.0E-12 & $4 \mathrm{~h}$ & 10 \\
\hline LIMS1 & 0.75 & 0.56 & 0.94 & 1.67 & 0.74 & 1.6E-09 & 8.7E-08 & $4 \mathrm{~h}$ & 14 \\
\hline LOC642236 & 0.36 & 0.22 & 0.50 & 2.25 & 1.17 & $2.2 \mathrm{E}-10$ & $1.4 \mathrm{E}-08$ & $4 \mathrm{~h}$ & 17 \\
\hline PCLO & 0.40 & 0.27 & 0.54 & 2.02 & 1.02 & 3.7E-09 & $1.9 \mathrm{E}-07$ & $4 \mathrm{~h}$ & 17 \\
\hline BAZ2B & 0.39 & 0.27 & 0.52 & 1.95 & 0.96 & $2.0 \mathrm{E}-08$ & $9.1 \mathrm{E}-07$ & $4 \mathrm{~h}$ & 17 \\
\hline ASCC3 & 0.81 & 0.62 & 0.99 & 1.60 & 0.68 & $9.2 \mathrm{E}-09$ & 4.5E-07 & $4 \mathrm{~h}$ & 17 \\
\hline HELLS & 0.81 & 0.62 & 0.99 & 1.58 & 0.66 & $1.8 \mathrm{E}-08$ & 8.5E-07 & $4 h$ & 17 \\
\hline PAXBP1 & 1.22 & 0.95 & 1.48 & 1.56 & 0.64 & $3.1 \mathrm{E}-11$ & 2.2E-09 & $4 h$ & 17 \\
\hline CDR2 & 0.99 & 0.78 & 1.20 & 1.53 & 0.61 & 8.7E-09 & $4.2 \mathrm{E}-07$ & $4 \mathrm{~h}$ & 17 \\
\hline GLI3 & 0.97 & 0.77 & 1.17 & 1.52 & 0.61 & $1.2 \mathrm{E}-08$ & 5.7E-07 & $4 h$ & 17 \\
\hline LRRN3 & 1.25 & 1.00 & 1.50 & 1.50 & 0.58 & $5.4 \mathrm{E}-10$ & $3.1 \mathrm{E}-08$ & $4 \mathrm{~h}$ & 17 \\
\hline ZFHX4 & 0.87 & 0.53 & 1.20 & 2.27 & 1.18 & $9.6 \mathrm{E}-24$ & $1.9 \mathrm{E}-21$ & $4 h$ & 21 \\
\hline ID3 & 1.26 & 2.09 & 0.44 & 0.21 & -2.26 & 4.0E-104 & $\begin{array}{r}6.9 \mathrm{E}- \\
101\end{array}$ & $3 h|4 h| 6 h$ & 3 \\
\hline ID1 & 2.42 & 4.18 & 0.67 & 0.16 & -2.65 & $8.8 \mathrm{E}-249$ & $\begin{array}{r}3.5 \mathrm{E}- \\
245\end{array}$ & $3 h|4 h| 6 h$ & 3 \\
\hline FKBP10 & 0.38 & 0.52 & 0.24 & 0.47 & -1.10 & $2.0 \mathrm{E}-10$ & $1.2 \mathrm{E}-08$ & $3 h|4 h| 6 h$ & 7 \\
\hline cox7C & 4.82 & 5.67 & 3.96 & 0.70 & -0.52 & $1.1 \mathrm{E}-27$ & 2.7E-25 & $3 h|4 h| 6 h$ & 8 \\
\hline PRDX4 & 1.43 & 1.69 & 1.17 & 0.69 & -0.53 & 1.0E-09 & $5.6 \mathrm{E}-08$ & $3 h|4 h| 6 h$ & 8 \\
\hline APP & 2.24 & 2.84 & 1.64 & 0.58 & -0.80 & $5.0 \mathrm{E}-30$ & $1.4 \mathrm{E}-27$ & $3 h|4 h| 6 h$ & 8 \\
\hline MAGOH & 0.68 & 0.87 & 0.49 & 0.57 & -0.82 & $1.8 \mathrm{E}-10$ & $1.1 \mathrm{E}-08$ & $3 h|4 h| 6 h$ & 9 \\
\hline ATRX & 2.19 & 1.65 & 2.72 & 1.65 & 0.72 & $8.9 E-24$ & $1.8 \mathrm{E}-21$ & $3 h|4 h| 6 h$ & 14 \\
\hline CCAR1 & 3.55 & 2.74 & 4.37 & 1.60 & 0.68 & $2.1 \mathrm{E}-33$ & $6.6 \mathrm{E}-31$ & $3 h|4 h| 6 h$ & 14 \\
\hline DMWD & 1.26 & 1.01 & 1.50 & 1.49 & 0.58 & 7.1E-10 & 4.0E-08 & $3 h|4 h| 6 h$ & 14 \\
\hline MLL5 & 0.83 & 0.52 & 1.15 & 2.23 & 1.15 & $3.2 \mathrm{E}-22$ & $6.0 \mathrm{E}-20$ & $3 h|4 h| 6 h$ & 17 \\
\hline SLC4A7 & 0.70 & 0.45 & 0.96 & 2.13 & 1.09 & 3.5E-17 & 4.4E-15 & $3 h|4 h| 6 h$ & 17 \\
\hline F5 & 0.50 & 0.28 & 0.72 & 2.52 & 1.34 & $8.4 \mathrm{E}-18$ & $1.1 \mathrm{E}-15$ & $3 h|4 h| 6 h$ & 18 \\
\hline ZNF471 & 0.65 & 0.38 & 0.92 & 2.43 & 1.28 & 6.7E-21 & $1.1 \mathrm{E}-18$ & $3 \mathrm{~h}|4 \mathrm{~h}| 6 \mathrm{~h}$ & 18 \\
\hline TMEM212 & 0.52 & 0.32 & 0.72 & 2.24 & 1.17 & $1.1 \mathrm{E}-14$ & $1.0 \mathrm{E}-12$ & $3 h|4 h| 6 h$ & 18 \\
\hline CCDC144B & 0.52 & 0.33 & 0.71 & 2.20 & 1.14 & $6.9 \mathrm{E}-14$ & $6.3 \mathrm{E}-12$ & $3 h|4 h| 6 h$ & 18 \\
\hline TSIX & 0.49 & 0.32 & 0.67 & 2.11 & 1.08 & 3.0E-12 & $2.4 \mathrm{E}-10$ & $3 h|4 h| 6 h$ & 18 \\
\hline GOLGB1 & 1.03 & 0.71 & 1.36 & 1.92 & 0.94 & 2.7E-19 & 4.1E-17 & $3 h|4 h| 6 h$ & 18 \\
\hline MPHOSPH8 & 0.91 & 0.65 & 1.16 & 1.77 & 0.83 & $1.1 \mathrm{E}-13$ & $9.6 \mathrm{E}-12$ & $3 h|4 h| 6 h$ & 18 \\
\hline BOD1L1 & 0.79 & 0.57 & 1.01 & 1.76 & 0.82 & 8.0E-12 & $6.1 \mathrm{E}-10$ & $3 h|4 h| 6 h$ & 18 \\
\hline
\end{tabular}


Table S3 continued

\begin{tabular}{|c|c|c|c|c|c|c|c|c|c|}
\hline Gene & $\begin{array}{l}\text { base } \\
\text { Mean }\end{array}$ & $\begin{array}{l}\text { base } \\
\text { MeanA }\end{array}$ & $\begin{array}{l}\text { base } \\
\text { MeanB }\end{array}$ & $\begin{array}{l}\text { fold } \\
\text { Change }\end{array}$ & $\begin{array}{l}\log 2 \\
\text { FC }\end{array}$ & pval & padj & $\begin{array}{l}\text { shared } \\
\text { in states }\end{array}$ & Node \\
\hline RBM25 & 3.42 & 2.48 & 4.36 & 1.76 & 0.82 & $1.5 \mathrm{E}-45$ & $6.2 \mathrm{E}-43$ & $3 h|4 h| 6 h$ & 18 \\
\hline LUC7L3 & 2.19 & 1.65 & 2.73 & 1.66 & 0.73 & $2.6 \mathrm{E}-24$ & $5.5 \mathrm{E}-22$ & $3 h|4 h| 6 h$ & 18 \\
\hline PNISR & 2.04 & 1.57 & 2.52 & 1.60 & 0.68 & 4.2E-20 & $6.9 \mathrm{E}-18$ & $3 h|4 h| 6 h$ & 18 \\
\hline GADD45A & 1.74 & 1.37 & 2.10 & 1.54 & 0.62 & 7.3E-15 & 7.3E-13 & $3 h|4 h| 6 h$ & 18 \\
\hline SLTM & 1.28 & 1.03 & 1.54 & 1.50 & 0.59 & $3.9 \mathrm{E}-10$ & $2.3 \mathrm{E}-08$ & $3 h|4 h| 6 h$ & 18 \\
\hline RFPL4B & 1.70 & 0.26 & 3.14 & 11.94 & 3.58 & $3.6 \mathrm{E}-234$ & $\begin{array}{r}8.5 \mathrm{E}- \\
231\end{array}$ & $3 h|4 h| 6 h$ & 19 \\
\hline RBBP6 & 7.77 & 2.58 & 12.96 & 5.02 & 2.33 & $0.0 \mathrm{E}+00$ & $0.0 \mathrm{E}+00$ & $3 h|4 h| 6 h$ & 19 \\
\hline SRSF8 & 4.16 & 1.77 & 6.55 & 3.71 & 1.89 & $5.4 \mathrm{E}-243$ & $\begin{array}{r}1.6 \mathrm{E}- \\
239\end{array}$ & $3 h|4 h| 6 h$ & 19 \\
\hline ZNF217 & 1.43 & 0.64 & 2.22 & 3.47 & 1.80 & $6.5 \mathrm{E}-78$ & $6.5 \mathrm{E}-75$ & $3 h|4 h| 6 h$ & 19 \\
\hline ZNF296 & 1.29 & 0.60 & 1.99 & 3.33 & 1.74 & 4.0E-67 & $3.0 \mathrm{E}-64$ & $3 h|4 h| 6 h$ & 19 \\
\hline ZMAT3 & 1.01 & 0.69 & 1.33 & 1.94 & 0.95 & 4.0E-19 & $6.0 \mathrm{E}-17$ & $3 h|4 h| 6 h$ & 19 \\
\hline PNN & 4.26 & 2.94 & 5.57 & 1.90 & 0.92 & 3.3E-71 & $2.6 \mathrm{E}-68$ & $3 h|4 h| 6 h$ & 21 \\
\hline MT2A & 1.25 & 1.47 & 1.03 & 0.70 & -0.52 & $2.2 \mathrm{E}-08$ & $9.8 \mathrm{E}-07$ & $3 h \mid 4 h$ & 7 \\
\hline SNRPD2 & 2.08 & 2.47 & 1.69 & 0.69 & -0.54 & 8.0E-14 & 7.2E-12 & $3 h \mid 4 h$ & 7 \\
\hline TOMM7 & 1.49 & 1.79 & 1.19 & 0.67 & -0.58 & $1.2 \mathrm{E}-11$ & $8.8 \mathrm{E}-10$ & $3 h \mid 4 h$ & 7 \\
\hline ATP5O & 2.53 & 3.01 & 2.04 & 0.68 & -0.56 & $1.9 \mathrm{E}-17$ & $2.4 \mathrm{E}-15$ & $3 \mathrm{~h} \mid 4 \mathrm{~h}$ & 8 \\
\hline TMEM258 & 1.25 & 1.49 & 1.01 & 0.68 & -0.57 & $1.4 \mathrm{E}-09$ & $7.5 \mathrm{E}-08$ & $3 \mathrm{~h} \mid 4 \mathrm{~h}$ & 8 \\
\hline ATP5J2 & 3.49 & 4.19 & 2.79 & 0.67 & -0.58 & 1.7E-25 & $3.6 \mathrm{E}-23$ & $3 \mathrm{~h} \mid 4 \mathrm{~h}$ & 8 \\
\hline CLSPN & 1.58 & 1.20 & 1.95 & 1.62 & 0.69 & $2.1 \mathrm{E}-16$ & $2.5 \mathrm{E}-14$ & $3 \mathrm{~h} \mid 4 \mathrm{~h}$ & 10 \\
\hline ZC3H13 & 0.55 & 0.39 & 0.70 & 1.80 & 0.85 & 4.4E-09 & $2.3 \mathrm{E}-07$ & $3 h \mid 4 h$ & 14 \\
\hline CHD9 & 1.04 & 0.80 & 1.28 & 1.60 & 0.68 & 8.2E-11 & 5.5E-09 & $3 h \mid 4 h$ & 14 \\
\hline REV3L & 1.00 & 0.79 & 1.20 & 1.52 & 0.60 & $1.2 \mathrm{E}-08$ & 5.7E-07 & $3 h \mid 4 h$ & 14 \\
\hline THOC2 & 1.51 & 1.23 & 1.79 & 1.46 & 0.55 & $1.9 \mathrm{E}-10$ & $1.1 \mathrm{E}-08$ & $3 \mathrm{~h} \mid 4 \mathrm{~h}$ & 14 \\
\hline APH1A & 0.28 & 0.39 & 0.18 & 0.46 & -1.12 & $1.6 \mathrm{E}-08$ & 7.7E-07 & $3 h \mid 4 h$ & NA \\
\hline FOXN3 & 1.17 & 0.93 & 1.40 & 1.50 & 0.59 & 2.0E-09 & $1.1 \mathrm{E}-07$ & $2 \mathrm{~h} \mid 4 \mathrm{~h}$ & 10 \\
\hline NOP10 & 1.74 & 2.06 & 1.42 & 0.69 & -0.54 & $1.1 \mathrm{E}-11$ & $8.2 \mathrm{E}-10$ & $2 \mathrm{~h}|3 \mathrm{~h}| 4 \mathrm{~h}$ & 8 \\
\hline RPL39 & 8.10 & 9.71 & 6.50 & 0.67 & -0.58 & $2.4 \mathrm{E}-56$ & $1.4 \mathrm{E}-53$ & $2 \mathrm{~h}|3 \mathrm{~h}| 4 \mathrm{~h}$ & 8 \\
\hline PIP5K1A & 0.30 & 0.42 & 0.18 & 0.43 & -1.21 & 4.6E-10 & 2.7E-08 & $2 h|3 h| 4 h$ & 8 \\
\hline GOLIM4 & 0.44 & 0.29 & 0.59 & 2.02 & 1.01 & $7.8 \mathrm{E}-10$ & $4.4 \mathrm{E}-08$ & $2 \mathrm{~h}|3 \mathrm{~h}| 4 \mathrm{~h}$ & 10 \\
\hline CCDC88A & 0.64 & 0.46 & 0.83 & 1.81 & 0.86 & $1.4 \mathrm{E}-10$ & $8.8 \mathrm{E}-09$ & $2 h|3 h| 4 h$ & 10 \\
\hline ITSN1 & 1.16 & 0.85 & 1.46 & 1.72 & 0.78 & $2.8 \mathrm{E}-15$ & $2.9 \mathrm{E}-13$ & $2 h|3 h| 4 h$ & 10 \\
\hline CDC42BPA & 0.73 & 0.56 & 0.91 & 1.63 & 0.71 & $1.0 \mathrm{E}-08$ & $4.9 \mathrm{E}-07$ & $2 h|3 h| 4 h$ & 10 \\
\hline ZFHX3 & 1.70 & 1.33 & 2.06 & 1.55 & 0.63 & 7.3E-15 & 7.3E-13 & $2 h|3 h| 4 h$ & 10 \\
\hline MAP1B & 6.64 & 5.28 & 8.01 & 1.52 & 0.60 & $3.2 \mathrm{E}-49$ & $1.5 \mathrm{E}-46$ & $2 h|3 h| 4 h$ & 10 \\
\hline
\end{tabular}


Table S3 continued

\begin{tabular}{|c|c|c|c|c|c|c|c|c|c|}
\hline Gene & $\begin{array}{l}\text { base } \\
\text { Mean }\end{array}$ & $\begin{array}{l}\text { base } \\
\text { MeanA }\end{array}$ & $\begin{array}{l}\text { base } \\
\text { MeanB }\end{array}$ & $\begin{array}{l}\text { fold } \\
\text { Change }\end{array}$ & $\begin{array}{l}\log 2 \\
\text { FC }\end{array}$ & pval & padj & $\begin{array}{l}\text { shared } \\
\text { in states }\end{array}$ & Node \\
\hline DNAJC2 & 1.08 & 0.86 & 1.30 & 1.52 & 0.60 & $3.2 \mathrm{E}-09$ & 1.7E-07 & $2 h|3 h| 4 h$ & 10 \\
\hline ANKRD12 & 0.85 & 0.61 & 1.09 & 1.81 & 0.85 & $1.8 \mathrm{E}-13$ & $1.5 \mathrm{E}-11$ & $2 h|3 h| 4 h$ & 13 \\
\hline ROCK1 & 0.54 & 0.36 & 0.72 & 1.99 & 0.99 & $1.5 \mathrm{E}-11$ & 1.1E-09 & $2 \mathrm{~h}|3 \mathrm{~h}| 4 \mathrm{~h}$ & 14 \\
\hline KTN1 & 2.33 & 1.83 & 2.84 & 1.55 & 0.63 & $8.4 \mathrm{E}-20$ & $1.4 \mathrm{E}-17$ & $2 \mathrm{~h}|3 \mathrm{~h}| 4 \mathrm{~h}$ & 14 \\
\hline
\end{tabular}

Table S4. Differentially expressed genes between uninduced (Oh, clusters 1\&2) and $6 \mathrm{~h}$ induced DIE cells (clusters 9\&10).

* Adjusted $p$ value $<10 * *-6$, absolute $\log 2 \mathrm{FC}>0.5$

\begin{tabular}{|c|c|c|c|c|c|c|c|c|c|}
\hline Gene & $\begin{array}{l}\text { base } \\
\text { Mean }\end{array}$ & $\begin{array}{l}\text { base } \\
\text { MeanA }\end{array}$ & $\begin{array}{l}\text { base } \\
\text { MeanB }\end{array}$ & $\begin{array}{l}\text { fold } \\
\text { Change }\end{array}$ & $\begin{array}{l}\log 2 \\
\text { FC }\end{array}$ & pval & padj & $\begin{array}{l}\text { shared } \\
\text { in states }\end{array}$ & Node \\
\hline UGDH-AS1 & 2.27 & 0.95 & 3.60 & 3.81 & 1.93 & $4.4 \mathrm{E}-119$ & $2.5 \mathrm{E}-116$ & All 4 & 18 \\
\hline KCNQ10T1 & 10.90 & 5.01 & 16.79 & 3.35 & 1.74 & 0 & 0 & All 4 & 18 \\
\hline $\begin{array}{l}\text { LOC } \\
100131257\end{array}$ & 3.28 & 1.58 & 4.99 & 3.16 & 1.66 & $1.7 \mathrm{E}-133$ & $1.2 \mathrm{E}-130$ & All 4 & 18 \\
\hline MAB21L3 & 1.21 & 0.61 & 1.82 & 2.98 & 1.58 & $2.4 \mathrm{E}-46$ & $6.6 \mathrm{E}-44$ & All 4 & 18 \\
\hline PGM5P2 & 0.93 & 0.51 & 1.36 & 2.69 & 1.43 & $2.5 \mathrm{E}-30$ & 3.7E-28 & All 4 & 18 \\
\hline AKAP9 & 0.89 & 0.52 & 1.26 & 2.43 & 1.28 & 4.6E-24 & $5.5 E-22$ & All 4 & 14 \\
\hline MDN1 & 0.35 & 0.21 & 0.49 & 2.30 & 1.20 & 2.1E-09 & 7.6E-08 & All 4 & 14 \\
\hline ASPM & 1.42 & 0.88 & 1.97 & 2.23 & 1.16 & $1.3 \mathrm{E}-31$ & 2.0E-29 & All 4 & 10 \\
\hline PRPF38B & 2.47 & 1.56 & 3.37 & 2.16 & 1.11 & $2.3 E-49$ & $6.4 \mathrm{E}-47$ & All 4 & 18 \\
\hline BDP1 & 0.79 & 0.52 & 1.06 & 2.04 & 1.03 & $9.8 \mathrm{E}-15$ & $6.6 \mathrm{E}-13$ & All 4 & 14 \\
\hline $\mathrm{CHD} 2$ & 0.75 & 0.50 & 1.00 & 1.98 & 0.99 & 3.7E-13 & $2.2 \mathrm{E}-11$ & All 4 & 14 \\
\hline SREK1 & 1.16 & 0.81 & 1.51 & 1.88 & 0.91 & $2.9 \mathrm{E}-17$ & $2.4 \mathrm{E}-15$ & All 4 & 17 \\
\hline BRD4 & 1.52 & 1.09 & 1.95 & 1.79 & 0.84 & 4.2E-19 & $3.9 E-17$ & All 4 & 14 \\
\hline CENPE & 0.96 & 0.69 & 1.23 & 1.78 & 0.83 & $2.2 \mathrm{E}-12$ & $1.2 \mathrm{E}-10$ & All 4 & 10 \\
\hline BPTF & 1.36 & 0.99 & 1.73 & 1.75 & 0.81 & $4.4 \mathrm{E}-16$ & 3.3E-14 & All 4 & 14 \\
\hline GOLGA4 & 1.18 & 0.87 & 1.49 & 1.71 & 0.77 & 4.4E-13 & $2.6 \mathrm{E}-11$ & All 4 & 14 \\
\hline PRR11 & 1.05 & 0.79 & 1.32 & 1.67 & 0.74 & 8.7E-11 & 3.8E-09 & All 4 & 13 \\
\hline NKTR & 1.36 & 1.04 & 1.69 & 1.63 & 0.70 & 1.0E-12 & $5.6 \mathrm{E}-11$ & All 4 & 14 \\
\hline SRRM1 & 2.10 & 1.60 & 2.60 & 1.62 & 0.70 & $2.1 \mathrm{E}-18$ & $1.9 \mathrm{E}-16$ & All 4 & 14 \\
\hline CENPF & 2.67 & 2.06 & 3.27 & 1.59 & 0.67 & 3.2E-21 & 3.3E-19 & All 4 & 10 \\
\hline TOP1 & 1.52 & 1.19 & 1.86 & 1.56 & 0.64 & $5.1 \mathrm{E}-12$ & 2.7E-10 & All 4 & 14 \\
\hline ANKRD11 & 1.88 & 1.50 & 2.27 & 1.51 & 0.60 & $1.2 \mathrm{E}-12$ & $6.8 \mathrm{E}-11$ & All 4 & 10 \\
\hline SMC4 & 5.84 & 4.81 & 6.88 & 1.43 & 0.52 & $1.2 \mathrm{E}-27$ & $1.6 \mathrm{E}-25$ & All 4 & 14 \\
\hline RPL37A & 7.68 & 9.22 & 6.14 & 0.67 & -0.59 & $1.8 \mathrm{E}-44$ & 4.7E-42 & All 4 & 8 \\
\hline
\end{tabular}


Table S4 continued

\begin{tabular}{|c|c|c|c|c|c|c|c|c|c|}
\hline Gene & $\begin{array}{l}\text { base } \\
\text { Mean }\end{array}$ & $\begin{array}{l}\text { base } \\
\text { MeanA }\end{array}$ & $\begin{array}{l}\text { base } \\
\text { MeanB }\end{array}$ & $\begin{array}{l}\text { fold } \\
\text { Change }\end{array}$ & $\begin{array}{l}\log 2 \\
\text { FC }\end{array}$ & pval & padj & $\begin{array}{l}\text { shared } \\
\text { in states }\end{array}$ & Node \\
\hline USMG5 & 1.87 & 2.27 & 1.48 & 0.65 & -0.62 & $1.9 \mathrm{E}-13$ & $1.2 \mathrm{E}-11$ & All 4 & 8 \\
\hline RPS29 & 11.82 & 14.43 & 9.21 & 0.64 & -0.65 & $3.9 E-68$ & 1.7E-65 & All 4 & 8 \\
\hline UQCR11 & 0.99 & 1.25 & 0.74 & 0.59 & -0.76 & $1.2 \mathrm{E}-10$ & $5.2 \mathrm{E}-09$ & All 4 & 8 \\
\hline CCNA1 & 1.88 & 0.22 & 3.54 & 16.37 & 4.03 & $3.9 E-256$ & $4.2 E-253$ & $6 h$ & 20 \\
\hline LOC441081 & 0.89 & 0.13 & 1.66 & 13.20 & 3.72 & $4.6 \mathrm{E}-113$ & $2.3 \mathrm{E}-110$ & $6 \mathrm{~h}$ & 20 \\
\hline RFPL1 & 0.53 & 0.11 & 0.95 & 8.90 & 3.15 & $3.7 \mathrm{E}-57$ & $1.2 \mathrm{E}-54$ & $6 \mathrm{~h}$ & 20 \\
\hline LINC00633 & 0.65 & 0.15 & 1.15 & 7.82 & 2.97 & $6.1 \mathrm{E}-64$ & $2.3 \mathrm{E}-61$ & $6 \mathrm{~h}$ & 19 \\
\hline KHDC1L & 0.45 & 0.11 & 0.79 & 7.38 & 2.88 & $4.8 \mathrm{E}-44$ & $1.2 \mathrm{E}-41$ & $6 \mathrm{~h}$ & 20 \\
\hline ALPPL2 & 0.44 & 0.11 & 0.78 & 7.26 & 2.86 & $5.6 \mathrm{E}-43$ & $1.3 \mathrm{E}-40$ & $6 \mathrm{~h}$ & 20 \\
\hline RFPL2 & 0.39 & 0.10 & 0.68 & 6.67 & 2.74 & $1.1 \mathrm{E}-35$ & $2.2 \mathrm{E}-33$ & $6 \mathrm{~h}$ & 20 \\
\hline SIAH1 & 1.40 & 0.37 & 2.44 & 6.67 & 2.74 & $5.3 \mathrm{E}-124$ & 3.3E-121 & $6 \mathrm{~h}$ & 20 \\
\hline PLXNB3 & 0.53 & 0.15 & 0.91 & 6.26 & 2.65 & $1.2 \mathrm{E}-45$ & $3.1 \mathrm{E}-43$ & $6 \mathrm{~h}$ & 20 \\
\hline ART3 & 0.32 & 0.11 & 0.53 & 4.95 & 2.31 & $5.9 E-24$ & $6.9 \mathrm{E}-22$ & $6 \mathrm{~h}$ & 19 \\
\hline SNAI1 & 0.38 & 0.14 & 0.63 & 4.66 & 2.22 & $3.5 E-26$ & 4.6E-24 & $6 \mathrm{~h}$ & 19 \\
\hline TRIM48 & 0.27 & 0.10 & 0.43 & 4.18 & 2.06 & 7.0E-17 & 5.6E-15 & $6 \mathrm{~h}$ & 20 \\
\hline RHOBTB1 & 0.49 & 0.20 & 0.78 & 3.89 & 1.96 & $2.0 \mathrm{E}-27$ & 2.7E-25 & $6 \mathrm{~h}$ & 19 \\
\hline SAMD8 & 0.60 & 0.25 & 0.96 & 3.84 & 1.94 & $3.1 E-33$ & 5.1E-31 & $6 \mathrm{~h}$ & 19 \\
\hline DUSP18 & 0.35 & 0.15 & 0.55 & 3.80 & 1.93 & $1.3 \mathrm{E}-19$ & $1.3 \mathrm{E}-17$ & $6 \mathrm{~h}$ & 19 \\
\hline C1orf63 & 0.77 & 0.32 & 1.22 & 3.79 & 1.92 & $3.1 \mathrm{E}-41$ & 7.0E-39 & $6 \mathrm{~h}$ & 19 \\
\hline EOMES & 0.25 & 0.11 & 0.39 & 3.58 & 1.84 & $1.5 \mathrm{E}-13$ & $9.7 E-12$ & $6 \mathrm{~h}$ & 19 \\
\hline MKRN9P & 0.23 & 0.10 & 0.35 & 3.49 & 1.80 & $9.1 \mathrm{E}-12$ & 4.6E-10 & $6 \mathrm{~h}$ & 19 \\
\hline MFSD11 & 0.49 & 0.22 & 0.77 & 3.44 & 1.78 & $5.1 \mathrm{E}-24$ & $6.0 \mathrm{E}-22$ & $6 \mathrm{~h}$ & 19 \\
\hline RIT2 & 0.24 & 0.11 & 0.37 & 3.40 & 1.77 & $2.6 \mathrm{E}-12$ & $1.4 \mathrm{E}-10$ & $6 \mathrm{~h}$ & 19 \\
\hline PHOX2B & 0.24 & 0.11 & 0.36 & 3.14 & 1.65 & 3.6E-11 & 1.7E-09 & $6 \mathrm{~h}$ & NA \\
\hline TRIM49B & 0.22 & 0.11 & 0.33 & 3.12 & 1.64 & 4.2E-10 & $1.6 \mathrm{E}-08$ & $6 \mathrm{~h}$ & 19 \\
\hline GRAMD1C & 0.27 & 0.14 & 0.41 & 3.02 & 1.59 & $1.1 \mathrm{E}-11$ & $5.6 \mathrm{E}-10$ & $6 \mathrm{~h}$ & 19 \\
\hline PANX2 & 0.69 & 0.34 & 1.03 & 3.02 & 1.59 & $1.9 \mathrm{E}-27$ & $2.6 \mathrm{E}-25$ & $6 \mathrm{~h}$ & 19 \\
\hline C3orf80 & 0.33 & 0.17 & 0.49 & 2.96 & 1.57 & $2.6 \mathrm{E}-13$ & $1.6 \mathrm{E}-11$ & $6 \mathrm{~h}$ & 19 \\
\hline ZNHIT6 & 1.33 & 0.67 & 1.98 & 2.96 & 1.57 & 7.0E-50 & $2.0 \mathrm{E}-47$ & $6 \mathrm{~h}$ & 19 \\
\hline BHLHE22 & 0.26 & 0.14 & 0.39 & 2.93 & 1.55 & $9.4 \mathrm{E}-11$ & 4.1E-09 & $6 \mathrm{~h}$ & 19 \\
\hline PRSS23 & 0.38 & 0.20 & 0.57 & 2.89 & 1.53 & 4.5E-15 & $3.2 \mathrm{E}-13$ & $6 \mathrm{~h}$ & 19 \\
\hline DPPA3 & 0.21 & 0.11 & 0.31 & 2.87 & 1.52 & $2.0 \mathrm{E}-08$ & $6.3 \mathrm{E}-07$ & $6 \mathrm{~h}$ & 19 \\
\hline NKIRAS1 & 0.42 & 0.22 & 0.63 & 2.87 & 1.52 & $5.8 \mathrm{E}-16$ & 4.3E-14 & $6 \mathrm{~h}$ & 19 \\
\hline $\begin{array}{l}\text { LOC } \\
100188947\end{array}$ & 1.95 & 1.03 & 2.88 & 2.80 & 1.49 & $3.2 E-66$ & $1.3 \mathrm{E}-63$ & $6 \mathrm{~h}$ & 19 \\
\hline
\end{tabular}


Table S4 continued

\begin{tabular}{|c|c|c|c|c|c|c|c|c|c|}
\hline Gene & $\begin{array}{l}\text { base } \\
\text { Mean }\end{array}$ & $\begin{array}{l}\text { base } \\
\text { MeanA }\end{array}$ & $\begin{array}{l}\text { base } \\
\text { MeanB }\end{array}$ & $\begin{array}{l}\text { fold } \\
\text { Change }\end{array}$ & $\begin{array}{l}\log 2 \\
F C\end{array}$ & pval & padj & $\begin{array}{l}\text { shared } \\
\text { in states }\end{array}$ & Node \\
\hline ADPGK & 1.82 & 0.97 & 2.67 & 2.76 & 1.47 & 1.7E-60 & $5.9 E-58$ & $6 \mathrm{~h}$ & 19 \\
\hline ZFHX4 & 0.99 & 0.53 & 1.44 & 2.72 & 1.45 & $9.0 \mathrm{E}-33$ & $1.4 \mathrm{E}-30$ & $6 \mathrm{~h}$ & 21 \\
\hline C9orf66 & 0.21 & 0.11 & 0.31 & 2.70 & 1.43 & 3.3E-08 & $9.8 \mathrm{E}-07$ & $6 \mathrm{~h}$ & 19 \\
\hline C1D & 0.74 & 0.40 & 1.08 & 2.67 & 1.41 & $3.8 \mathrm{E}-24$ & $4.5 \mathrm{E}-22$ & $6 \mathrm{~h}$ & 19 \\
\hline NDEL1 & 0.42 & 0.23 & 0.61 & 2.64 & 1.40 & $6.8 \mathrm{E}-14$ & 4.4E-12 & $6 \mathrm{~h}$ & 19 \\
\hline LRRK1 & 0.39 & 0.22 & 0.57 & 2.62 & 1.39 & $4.2 \mathrm{E}-13$ & $2.5 \mathrm{E}-11$ & $6 \mathrm{~h}$ & 21 \\
\hline ANK3 & 0.61 & 0.34 & 0.88 & 2.58 & 1.37 & 3.7E-19 & 3.5E-17 & $6 \mathrm{~h}$ & 19 \\
\hline DNM3 & 0.29 & 0.16 & 0.42 & 2.57 & 1.36 & $1.2 \mathrm{E}-09$ & $4.4 \mathrm{E}-08$ & $6 \mathrm{~h}$ & 19 \\
\hline OSR2 & 0.27 & 0.15 & 0.38 & 2.54 & 1.34 & 7.5E-09 & $2.5 \mathrm{E}-07$ & $6 \mathrm{~h}$ & 19 \\
\hline AVPI1 & 0.35 & 0.20 & 0.49 & 2.48 & 1.31 & 1.7E-10 & 7.0E-09 & $6 \mathrm{~h}$ & 19 \\
\hline CLK1 & 0.56 & 0.33 & 0.80 & 2.47 & 1.30 & $2.5 \mathrm{E}-16$ & $1.9 \mathrm{E}-14$ & $6 \mathrm{~h}$ & 19 \\
\hline MAST1 & 0.34 & 0.20 & 0.48 & 2.41 & 1.27 & 4.3E-10 & 1.7E-08 & $6 \mathrm{~h}$ & 19 \\
\hline STK17B & 0.31 & 0.18 & 0.43 & 2.39 & 1.26 & $6.6 \mathrm{E}-09$ & $2.2 \mathrm{E}-07$ & $6 \mathrm{~h}$ & 21 \\
\hline TAF4B & 0.40 & 0.24 & 0.57 & 2.38 & 1.25 & $4.0 \mathrm{E}-11$ & 1.9E-09 & $6 \mathrm{~h}$ & 21 \\
\hline PELI2 & 0.64 & 0.38 & 0.90 & 2.35 & 1.23 & $1.2 \mathrm{E}-16$ & $9.4 \mathrm{E}-15$ & $6 \mathrm{~h}$ & 21 \\
\hline EPHA4 & 0.31 & 0.19 & 0.44 & 2.34 & 1.23 & 6.6E-09 & $2.2 \mathrm{E}-07$ & $6 \mathrm{~h}$ & 19 \\
\hline HEXIM1 & 0.64 & 0.38 & 0.89 & 2.31 & 1.21 & $4.9 \mathrm{E}-16$ & 3.6E-14 & $6 \mathrm{~h}$ & 20 \\
\hline CNNM4 & 0.48 & 0.29 & 0.67 & 2.30 & 1.20 & $3.4 \mathrm{E}-12$ & $1.8 \mathrm{E}-10$ & $6 \mathrm{~h}$ & 19 \\
\hline PRELP & 0.40 & 0.24 & 0.55 & 2.26 & 1.18 & $3.9 \mathrm{E}-10$ & $1.5 \mathrm{E}-08$ & $6 \mathrm{~h}$ & 19 \\
\hline ZNF574 & 0.87 & 0.53 & 1.20 & 2.25 & 1.17 & $2.5 \mathrm{E}-20$ & $2.5 \mathrm{E}-18$ & $6 \mathrm{~h}$ & 19 \\
\hline ACAP2 & 0.51 & 0.32 & 0.71 & 2.24 & 1.17 & $1.3 \mathrm{E}-12$ & $7.4 \mathrm{E}-11$ & $6 \mathrm{~h}$ & 19 \\
\hline RGS2 & 0.65 & 0.40 & 0.89 & 2.24 & 1.17 & $1.6 \mathrm{E}-15$ & $1.1 \mathrm{E}-13$ & $6 \mathrm{~h}$ & 19 \\
\hline KIAA1217 & 0.44 & 0.27 & 0.61 & 2.24 & 1.16 & 7.1E-11 & $3.2 \mathrm{E}-09$ & $6 \mathrm{~h}$ & 21 \\
\hline DNAJC25 & 0.51 & 0.32 & 0.71 & 2.23 & 1.16 & 3.0E-12 & $1.6 \mathrm{E}-10$ & $6 \mathrm{~h}$ & 21 \\
\hline TPMT & 0.45 & 0.28 & 0.62 & 2.22 & 1.15 & 6.7E-11 & 3.0E-09 & $6 \mathrm{~h}$ & 19 \\
\hline NCOA7 & 0.36 & 0.23 & 0.50 & 2.21 & 1.15 & 7.5E-09 & $2.5 \mathrm{E}-07$ & $6 \mathrm{~h}$ & 19 \\
\hline SUPT6H & 1.21 & 0.76 & 1.67 & 2.21 & 1.15 & $8.6 \mathrm{E}-27$ & $1.1 \mathrm{E}-24$ & $6 \mathrm{~h}$ & 19 \\
\hline C21orf91 & 0.59 & 0.37 & 0.81 & 2.19 & 1.13 & $2.4 \mathrm{E}-13$ & 1.5E-11 & $6 \mathrm{~h}$ & 19 \\
\hline ALG13 & 0.87 & 0.55 & 1.20 & 2.19 & 1.13 & $3.3 \mathrm{E}-19$ & $3.1 \mathrm{E}-17$ & $6 \mathrm{~h}$ & 19 \\
\hline EPN2 & 0.35 & 0.22 & 0.48 & 2.19 & 1.13 & $1.9 \mathrm{E}-08$ & 5.9E-07 & $6 \mathrm{~h}$ & 19 \\
\hline MGC21881 & 0.45 & 0.28 & 0.61 & 2.19 & 1.13 & $1.8 \mathrm{E}-10$ & 7.5E-09 & $6 \mathrm{~h}$ & 19 \\
\hline USP3 & 0.88 & 0.56 & 1.21 & 2.16 & 1.11 & $6.8 \mathrm{E}-19$ & $6.2 \mathrm{E}-17$ & $6 \mathrm{~h}$ & 19 \\
\hline $\begin{array}{l}\text { RP1- } \\
177 G 6.2\end{array}$ & 0.44 & 0.28 & 0.60 & 2.16 & 1.11 & $3.6 \mathrm{E}-10$ & $1.4 \mathrm{E}-08$ & $6 \mathrm{~h}$ & 18 \\
\hline C5orf44 & 0.66 & 0.42 & 0.90 & 2.16 & 1.11 & $2.8 \mathrm{E}-14$ & $1.8 \mathrm{E}-12$ & $6 \mathrm{~h}$ & 19 \\
\hline ZNF480 & 0.85 & 0.54 & 1.16 & 2.15 & 1.10 & 7.6E-18 & $6.4 \mathrm{E}-16$ & $6 \mathrm{~h}$ & 21 \\
\hline
\end{tabular}


Table S4 continued

\begin{tabular}{|c|c|c|c|c|c|c|c|c|c|}
\hline Gene & $\begin{array}{l}\text { base } \\
\text { Mean }\end{array}$ & $\begin{array}{l}\text { base } \\
\text { MeanA }\end{array}$ & $\begin{array}{l}\text { base } \\
\text { MeanB }\end{array}$ & $\begin{array}{l}\text { fold } \\
\text { Change }\end{array}$ & $\begin{array}{l}\log 2 \\
\text { FC }\end{array}$ & pval & padj & $\begin{array}{l}\text { shared } \\
\text { in states }\end{array}$ & Node \\
\hline OXR1 & 0.47 & 0.30 & 0.63 & 2.13 & 1.09 & $3.5 \mathrm{E}-10$ & $1.4 \mathrm{E}-08$ & $6 \mathrm{~h}$ & 19 \\
\hline G2E3 & 0.76 & 0.49 & 1.04 & 2.12 & 1.09 & $6.9 \mathrm{E}-16$ & 5.0E-14 & $6 \mathrm{~h}$ & 21 \\
\hline $\begin{array}{l}\text { LOC } \\
100131067\end{array}$ & 0.41 & 0.27 & 0.56 & 2.10 & 1.07 & $1.0 \mathrm{E}-08$ & 3.3E-07 & $6 \mathrm{~h}$ & 21 \\
\hline SHQ1 & 0.47 & 0.31 & 0.64 & 2.09 & 1.06 & $6.2 \mathrm{E}-10$ & $2.4 \mathrm{E}-08$ & $6 \mathrm{~h}$ & 19 \\
\hline BRCA2 & 0.37 & 0.24 & 0.51 & 2.08 & 1.05 & $2.9 \mathrm{E}-08$ & 8.9E-07 & $6 \mathrm{~h}$ & 14 \\
\hline TIPARP & 0.97 & 0.63 & 1.31 & 2.07 & 1.05 & $1.1 \mathrm{E}-18$ & $9.5 \mathrm{E}-17$ & $6 \mathrm{~h}$ & 19 \\
\hline TFAP2C & 0.42 & 0.28 & 0.57 & 2.04 & 1.03 & $1.1 \mathrm{E}-08$ & $3.4 \mathrm{E}-07$ & $6 \mathrm{~h}$ & 19 \\
\hline STIL & 0.57 & 0.38 & 0.76 & 2.02 & 1.02 & $9.3 \mathrm{E}-11$ & 4.0E-09 & $6 \mathrm{~h}$ & 19 \\
\hline BIRC2 & 0.49 & 0.32 & 0.65 & 2.02 & 1.02 & $2.4 \mathrm{E}-09$ & 8.6E-08 & $6 \mathrm{~h}$ & 19 \\
\hline ZNF91 & 0.46 & 0.30 & 0.61 & 2.00 & 1.00 & 9.1E-09 & $3.0 \mathrm{E}-07$ & $6 \mathrm{~h}$ & 18 \\
\hline FBXO33 & 0.58 & 0.38 & 0.77 & 2.00 & 1.00 & $1.2 \mathrm{E}-10$ & 4.9E-09 & $6 \mathrm{~h}$ & 19 \\
\hline SCAPER & 0.48 & 0.32 & 0.64 & 2.00 & 1.00 & 4.4E-09 & $1.5 \mathrm{E}-07$ & $6 \mathrm{~h}$ & 18 \\
\hline EPM2AIP1 & 0.83 & 0.56 & 1.11 & 1.99 & 0.99 & $9.6 \mathrm{E}-15$ & $6.6 \mathrm{E}-13$ & $6 \mathrm{~h}$ & 19 \\
\hline LUZP1 & 0.51 & 0.34 & 0.67 & 1.98 & 0.99 & 1.9E-09 & 7.0E-08 & $6 \mathrm{~h}$ & 18 \\
\hline UFL1 & 0.64 & 0.43 & 0.85 & 1.98 & 0.98 & 1.7E-11 & 8.3E-10 & $6 \mathrm{~h}$ & 21 \\
\hline TOPORS & 0.71 & 0.48 & 0.94 & 1.95 & 0.96 & $5.2 \mathrm{E}-12$ & 2.7E-10 & $6 \mathrm{~h}$ & 21 \\
\hline TMEM185A & 0.52 & 0.36 & 0.69 & 1.94 & 0.95 & 3.7E-09 & $1.3 \mathrm{E}-07$ & $6 \mathrm{~h}$ & 19 \\
\hline CCNL1 & 0.78 & 0.53 & 1.02 & 1.92 & 0.94 & $9.6 \mathrm{E}-13$ & $5.4 \mathrm{E}-11$ & $6 \mathrm{~h}$ & 21 \\
\hline TCEB3 & 1.07 & 0.73 & 1.40 & 1.92 & 0.94 & 7.3E-17 & 5.7E-15 & $6 \mathrm{~h}$ & 20 \\
\hline MLL3 & 0.50 & 0.34 & 0.66 & 1.91 & 0.93 & 1.7E-08 & $5.3 \mathrm{E}-07$ & $6 \mathrm{~h}$ & 18 \\
\hline PNPLA8 & 0.55 & 0.38 & 0.72 & 1.90 & 0.93 & 4.6E-09 & $1.6 \mathrm{E}-07$ & $6 \mathrm{~h}$ & 19 \\
\hline $\mathrm{NOTCH} 2$ & 0.57 & 0.39 & 0.74 & 1.90 & 0.92 & 2.9E-09 & $1.0 \mathrm{E}-07$ & $6 \mathrm{~h}$ & 21 \\
\hline ELOF1 & 1.54 & 1.07 & 2.02 & 1.89 & 0.92 & $1.3 \mathrm{E}-22$ & $1.4 \mathrm{E}-20$ & $6 \mathrm{~h}$ & 19 \\
\hline ZNF281 & 0.66 & 0.46 & 0.87 & 1.88 & 0.91 & 1.7E-10 & 7.0E-09 & $6 \mathrm{~h}$ & 21 \\
\hline CASP6 & 0.67 & 0.47 & 0.88 & 1.87 & 0.90 & $2.5 \mathrm{E}-10$ & $1.0 \mathrm{E}-08$ & $6 \mathrm{~h}$ & 19 \\
\hline RPP14 & 1.24 & 0.87 & 1.62 & 1.86 & 0.90 & $8.2 \mathrm{E}-18$ & $6.8 \mathrm{E}-16$ & $6 \mathrm{~h}$ & 19 \\
\hline CLCN3 & 0.83 & 0.58 & 1.08 & 1.86 & 0.90 & 3.7E-12 & $1.9 \mathrm{E}-10$ & $6 \mathrm{~h}$ & 19 \\
\hline TRAPPC6B & 0.53 & 0.37 & 0.69 & 1.86 & 0.89 & $3.2 \mathrm{E}-08$ & $9.8 \mathrm{E}-07$ & $6 \mathrm{~h}$ & 21 \\
\hline RBM26 & 0.70 & 0.49 & 0.91 & 1.85 & 0.89 & $1.6 \mathrm{E}-10$ & $6.5 \mathrm{E}-09$ & $6 \mathrm{~h}$ & 21 \\
\hline MELK & 1.31 & 0.93 & 1.69 & 1.83 & 0.87 & $9.6 \mathrm{E}-18$ & 8.0E-16 & $6 \mathrm{~h}$ & 19 \\
\hline METTL8 & 0.66 & 0.47 & 0.86 & 1.82 & 0.87 & 1.7E-09 & $6.1 \mathrm{E}-08$ & $6 \mathrm{~h}$ & 21 \\
\hline RNF213 & 0.56 & 0.40 & 0.72 & 1.82 & 0.86 & $2.4 \mathrm{E}-08$ & 7.5E-07 & $6 \mathrm{~h}$ & 21 \\
\hline TNFRSF10D & 1.07 & 0.76 & 1.38 & 1.81 & 0.86 & 3.3E-14 & $2.1 \mathrm{E}-12$ & $6 \mathrm{~h}$ & 18 \\
\hline USP33 & 1.02 & 0.72 & 1.31 & 1.81 & 0.86 & $9.1 \mathrm{E}-14$ & $5.8 \mathrm{E}-12$ & $6 \mathrm{~h}$ & 18 \\
\hline KDM5B & 0.60 & 0.43 & 0.78 & 1.80 & 0.85 & $9.5 \mathrm{E}-09$ & $3.1 \mathrm{E}-07$ & $6 \mathrm{~h}$ & 21 \\
\hline
\end{tabular}


Table S4 continued

\begin{tabular}{|c|c|c|c|c|c|c|c|c|c|}
\hline Gene & $\begin{array}{l}\text { base } \\
\text { Mean }\end{array}$ & $\begin{array}{l}\text { base } \\
\text { MeanA }\end{array}$ & $\begin{array}{l}\text { base } \\
\text { MeanB }\end{array}$ & $\begin{array}{l}\text { fold } \\
\text { Change }\end{array}$ & $\begin{array}{l}\log 2 \\
\text { FC }\end{array}$ & pval & padj & $\begin{array}{l}\text { shared } \\
\text { in states }\end{array}$ & Node \\
\hline PTP4A1 & 3.78 & 2.70 & 4.87 & 1.80 & 0.85 & $3.8 \mathrm{E}-46$ & $1.0 \mathrm{E}-43$ & $6 \mathrm{~h}$ & 19 \\
\hline WAPAL & 0.78 & 0.56 & 1.01 & 1.79 & 0.84 & $2.0 \mathrm{E}-10$ & 8.3E-09 & $6 \mathrm{~h}$ & 21 \\
\hline KIAA0020 & 0.74 & 0.53 & 0.95 & 1.79 & 0.84 & $5.2 \mathrm{E}-10$ & $2.0 \mathrm{E}-08$ & $6 \mathrm{~h}$ & 18 \\
\hline SACM1L & 0.68 & 0.49 & 0.87 & 1.78 & 0.83 & 5.0E-09 & 1.7E-07 & $6 \mathrm{~h}$ & 19 \\
\hline PSME4 & 0.73 & 0.53 & 0.93 & 1.77 & 0.82 & $1.8 \mathrm{E}-09$ & $6.4 \mathrm{E}-08$ & $6 \mathrm{~h}$ & 18 \\
\hline STX16 & 0.92 & 0.67 & 1.17 & 1.76 & 0.82 & $1.2 \mathrm{E}-11$ & $6.0 \mathrm{E}-10$ & $6 \mathrm{~h}$ & 21 \\
\hline ZNF609 & 1.02 & 0.74 & 1.30 & 1.76 & 0.82 & $1.5 \mathrm{E}-12$ & 8.3E-11 & $6 \mathrm{~h}$ & 18 \\
\hline $\mathrm{ZHX1}$ & 0.68 & 0.49 & 0.86 & 1.76 & 0.81 & $6.4 \mathrm{E}-09$ & $2.2 \mathrm{E}-07$ & $6 \mathrm{~h}$ & 19 \\
\hline TBPL1 & 0.74 & 0.54 & 0.95 & 1.76 & 0.81 & $1.8 \mathrm{E}-09$ & 6.7E-08 & $6 \mathrm{~h}$ & 19 \\
\hline MT1X & 0.65 & 0.47 & 0.83 & 1.76 & 0.81 & $2.0 \mathrm{E}-08$ & $6.3 \mathrm{E}-07$ & $6 \mathrm{~h}$ & 20 \\
\hline ALDH9A1 & 0.94 & 0.68 & 1.19 & 1.74 & 0.80 & 3.1E-11 & $1.5 \mathrm{E}-09$ & $6 \mathrm{~h}$ & 19 \\
\hline RSRC2 & 1.83 & 1.34 & 2.33 & 1.74 & 0.80 & 1.7E-20 & 1.7E-18 & $6 \mathrm{~h}$ & 19 \\
\hline DEPDC1 & 0.71 & 0.52 & 0.90 & 1.72 & 0.78 & $2.0 \mathrm{E}-08$ & $6.1 \mathrm{E}-07$ & $6 \mathrm{~h}$ & 21 \\
\hline DIS3 & 0.79 & 0.59 & 1.00 & 1.71 & 0.78 & $2.2 \mathrm{E}-09$ & $7.8 \mathrm{E}-08$ & $6 \mathrm{~h}$ & 19 \\
\hline KDM5A & 0.72 & 0.54 & 0.91 & 1.69 & 0.76 & $2.6 \mathrm{E}-08$ & 8.0E-07 & $6 \mathrm{~h}$ & 21 \\
\hline HMGXB4 & 0.91 & 0.68 & 1.15 & 1.69 & 0.76 & $5.4 \mathrm{E}-10$ & 2.1E-08 & $6 \mathrm{~h}$ & 21 \\
\hline BRD8 & 0.82 & 0.61 & 1.03 & 1.69 & 0.76 & 5.0E-09 & 1.7E-07 & $6 \mathrm{~h}$ & 21 \\
\hline ZNF292 & 0.82 & 0.61 & 1.02 & 1.67 & 0.74 & $8.5 \mathrm{E}-09$ & $2.8 \mathrm{E}-07$ & $6 \mathrm{~h}$ & 14 \\
\hline SH3KBP1 & 0.75 & 0.56 & 0.94 & 1.67 & 0.74 & $2.9 \mathrm{E}-08$ & 8.7E-07 & $6 h$ & 21 \\
\hline MTF2 & 1.21 & 0.91 & 1.50 & 1.65 & 0.73 & $5.3 \mathrm{E}-12$ & 2.7E-10 & $6 \mathrm{~h}$ & 18 \\
\hline CTR9 & 0.87 & 0.66 & 1.08 & 1.63 & 0.71 & $1.5 \mathrm{E}-08$ & $4.8 \mathrm{E}-07$ & $6 \mathrm{~h}$ & 19 \\
\hline AAR2 & 0.91 & 0.69 & 1.13 & 1.63 & 0.71 & 4.4E-09 & $1.5 \mathrm{E}-07$ & $6 \mathrm{~h}$ & 21 \\
\hline SMARCAD1 & 1.13 & 0.86 & 1.39 & 1.63 & 0.70 & $1.1 \mathrm{E}-10$ & 4.6E-09 & $6 \mathrm{~h}$ & 19 \\
\hline LOC152217 & 1.25 & 0.96 & 1.53 & 1.60 & 0.68 & 5.3E-11 & $2.4 \mathrm{E}-09$ & $6 \mathrm{~h}$ & 18 \\
\hline ENAH & 4.17 & 3.21 & 5.13 & 1.60 & 0.68 & $3.0 \mathrm{E}-33$ & $5.1 \mathrm{E}-31$ & $6 \mathrm{~h}$ & 18 \\
\hline TERF2IP & 1.34 & 1.03 & 1.65 & 1.60 & 0.68 & $1.3 \mathrm{E}-11$ & $6.5 \mathrm{E}-10$ & $6 \mathrm{~h}$ & 19 \\
\hline TFE3 & 0.99 & 0.77 & 1.22 & 1.60 & 0.67 & 6.1E-09 & $2.1 \mathrm{E}-07$ & $6 \mathrm{~h}$ & 21 \\
\hline MCC & 1.19 & 0.92 & 1.46 & 1.58 & 0.66 & $3.5 \mathrm{E}-10$ & $1.4 \mathrm{E}-08$ & $6 \mathrm{~h}$ & 17 \\
\hline RBM5 & 1.23 & 0.95 & 1.50 & 1.57 & 0.65 & $4.2 \mathrm{E}-10$ & $1.7 \mathrm{E}-08$ & $6 \mathrm{~h}$ & 21 \\
\hline ARL6IP1 & 3.46 & 2.70 & 4.21 & 1.56 & 0.65 & $2.2 \mathrm{E}-25$ & $2.8 \mathrm{E}-23$ & $6 \mathrm{~h}$ & 10 \\
\hline TUG1 & 1.34 & 1.06 & 1.63 & 1.54 & 0.62 & 5.5E-10 & 2.1E-08 & $6 h$ & 18 \\
\hline KIF11 & 1.43 & 1.13 & 1.74 & 1.54 & 0.62 & $1.2 \mathrm{E}-10$ & 5.0E-09 & $6 \mathrm{~h}$ & 21 \\
\hline SOX2 & 1.31 & 1.03 & 1.58 & 1.53 & 0.61 & $9.6 \mathrm{E}-10$ & 3.7E-08 & $6 \mathrm{~h}$ & 19 \\
\hline ANXA5 & 8.57 & 6.88 & 10.26 & 1.49 & 0.58 & $5.1 \mathrm{E}-46$ & $1.4 \mathrm{E}-43$ & $6 \mathrm{~h}$ & 18 \\
\hline MARCH6 & 1.54 & 1.24 & 1.84 & 1.49 & 0.58 & $6.5 \mathrm{E}-10$ & $2.5 \mathrm{E}-08$ & $6 \mathrm{~h}$ & 19 \\
\hline
\end{tabular}


Table S4 continued

\begin{tabular}{|c|c|c|c|c|c|c|c|c|c|}
\hline Gene & $\begin{array}{l}\text { base } \\
\text { Mean }\end{array}$ & $\begin{array}{l}\text { base } \\
\text { MeanA }\end{array}$ & $\begin{array}{l}\text { base } \\
\text { MeanB }\end{array}$ & $\begin{array}{l}\text { fold } \\
\text { Change }\end{array}$ & $\begin{array}{l}\log 2 \\
\text { FC }\end{array}$ & pval & padj & $\begin{array}{l}\text { shared } \\
\text { in states }\end{array}$ & Node \\
\hline SEC61A1 & 1.50 & 1.21 & 1.79 & 1.48 & 0.57 & 2.0E-09 & 7.1E-08 & $6 \mathrm{~h}$ & 19 \\
\hline TOMM70A & 1.99 & 1.63 & 2.35 & 1.45 & 0.53 & 6.7E-11 & 3.0E-09 & $6 \mathrm{~h}$ & 21 \\
\hline OGT & 2.55 & 2.10 & 3.00 & 1.43 & 0.52 & $7.5 \mathrm{E}-13$ & 4.3E-11 & $6 \mathrm{~h}$ & 16 \\
\hline FTH1 & 8.48 & 9.95 & 7.02 & 0.71 & -0.50 & $6.1 \mathrm{E}-35$ & $1.1 \mathrm{E}-32$ & $6 \mathrm{~h}$ & 9 \\
\hline CCNB1 & 1.89 & 2.22 & 1.56 & 0.70 & -0.51 & 1.6E-09 & $6.0 \mathrm{E}-08$ & $6 \mathrm{~h}$ & 3 \\
\hline TKT & 2.10 & 2.47 & 1.73 & 0.70 & -0.51 & $1.8 \mathrm{E}-10$ & 7.5E-09 & $6 \mathrm{~h}$ & 1 \\
\hline NACA & 2.13 & 2.50 & 1.76 & 0.70 & -0.51 & $1.0 \mathrm{E}-10$ & 4.4E-09 & $6 \mathrm{~h}$ & 9 \\
\hline NQO1 & 2.18 & 2.58 & 1.77 & 0.69 & -0.54 & $5.2 \mathrm{E}-12$ & 2.7E-10 & $6 \mathrm{~h}$ & 1 \\
\hline MRPS34 & 1.63 & 1.95 & 1.32 & 0.67 & -0.57 & $3.5 \mathrm{E}-10$ & $1.4 \mathrm{E}-08$ & $6 \mathrm{~h}$ & 3 \\
\hline HSPH1 & 1.93 & 2.30 & 1.55 & 0.67 & -0.57 & 7.2E-12 & $3.6 \mathrm{E}-10$ & $6 \mathrm{~h}$ & 3 \\
\hline CHCHD10 & 1.41 & 1.69 & 1.13 & 0.67 & -0.58 & 2.6E-09 & $9.2 \mathrm{E}-08$ & $6 \mathrm{~h}$ & 3 \\
\hline DNAJA1 & 1.81 & 2.20 & 1.43 & 0.65 & -0.63 & $3.6 \mathrm{E}-13$ & 2.2E-11 & $6 \mathrm{~h}$ & 3 \\
\hline SLBP & 1.50 & 1.83 & 1.18 & 0.65 & -0.63 & 3.2E-11 & $1.5 \mathrm{E}-09$ & $6 \mathrm{~h}$ & 2 \\
\hline SNRPE & 2.65 & 3.22 & 2.08 & 0.65 & -0.63 & $9.6 \mathrm{E}-19$ & 8.7E-17 & $6 \mathrm{~h}$ & 9 \\
\hline PGAM1 & 1.12 & 1.37 & 0.88 & 0.64 & -0.64 & $5.8 \mathrm{E}-09$ & 2.0E-07 & $6 \mathrm{~h}$ & 2 \\
\hline TRIM24 & 1.04 & 1.26 & 0.81 & 0.64 & -0.64 & 1.7E-08 & $5.4 \mathrm{E}-07$ & $6 \mathrm{~h}$ & 3 \\
\hline MRPL34 & 1.17 & 1.45 & 0.90 & 0.62 & -0.68 & 1.7E-10 & 7.0E-09 & $6 \mathrm{~h}$ & 1 \\
\hline SOCS2 & 1.17 & 1.46 & 0.87 & 0.60 & -0.75 & $3.6 \mathrm{E}-12$ & $1.9 \mathrm{E}-10$ & $6 \mathrm{~h}$ & 1 \\
\hline TRIP10 & 1.30 & 1.63 & 0.97 & 0.60 & -0.75 & $2.6 \mathrm{E}-13$ & $1.6 \mathrm{E}-11$ & $6 \mathrm{~h}$ & 1 \\
\hline SAPCD2 & 0.81 & 1.02 & 0.60 & 0.59 & -0.77 & 3.5E-09 & $1.2 \mathrm{E}-07$ & $6 \mathrm{~h}$ & 3 \\
\hline OTX2 & 1.44 & 1.84 & 1.04 & 0.57 & -0.82 & $2.9 \mathrm{E}-17$ & $2.4 \mathrm{E}-15$ & $6 \mathrm{~h}$ & 3 \\
\hline CBX4 & 0.61 & 0.78 & 0.43 & 0.55 & -0.86 & $1.1 \mathrm{E}-08$ & 3.7E-07 & $6 \mathrm{~h}$ & 3 \\
\hline MEX3A & 1.13 & 1.46 & 0.80 & 0.54 & -0.88 & $1.8 \mathrm{E}-15$ & $1.3 \mathrm{E}-13$ & $6 \mathrm{~h}$ & 3 \\
\hline EFNB1 & 0.85 & 1.11 & 0.59 & 0.53 & -0.92 & $8.1 \mathrm{E}-13$ & 4.6E-11 & $6 \mathrm{~h}$ & 3 \\
\hline SH3BP4 & 0.83 & 1.09 & 0.57 & 0.52 & -0.94 & $5.6 \mathrm{E}-13$ & 3.2E-11 & $6 \mathrm{~h}$ & 3 \\
\hline PPP1R18 & 0.58 & 0.76 & 0.40 & 0.52 & -0.94 & 1.9E-09 & $6.9 \mathrm{E}-08$ & $6 \mathrm{~h}$ & 1 \\
\hline MSMO1 & 0.71 & 0.94 & 0.48 & 0.52 & -0.95 & $9.4 \mathrm{E}-12$ & 4.7E-10 & $6 \mathrm{~h}$ & 1 \\
\hline CDC42EP1 & 0.46 & 0.61 & 0.31 & 0.51 & -0.96 & 2.7E-08 & $8.4 \mathrm{E}-07$ & $6 \mathrm{~h}$ & 3 \\
\hline BCOR & 0.55 & 0.73 & 0.37 & 0.50 & -0.99 & 7.5E-10 & 2.9E-08 & $6 \mathrm{~h}$ & 3 \\
\hline CBX2 & 0.57 & 0.78 & 0.37 & 0.47 & -1.10 & $1.6 \mathrm{E}-12$ & 8.5E-11 & $6 \mathrm{~h}$ & 1 \\
\hline DUSP14 & 0.89 & 1.22 & 0.57 & 0.46 & -1.11 & $1.6 \mathrm{E}-18$ & $1.4 \mathrm{E}-16$ & $6 \mathrm{~h}$ & 1 \\
\hline IRF2BP2 & 0.49 & 0.67 & 0.31 & 0.46 & -1.12 & 8.9E-11 & $3.9 \mathrm{E}-09$ & $6 \mathrm{~h}$ & 3 \\
\hline TRAF4 & 0.34 & 0.47 & 0.21 & 0.45 & -1.14 & $3.0 \mathrm{E}-08$ & $9.0 \mathrm{E}-07$ & $6 \mathrm{~h}$ & 1 \\
\hline sox21 & 0.46 & 0.63 & 0.28 & 0.45 & -1.15 & $1.5 \mathrm{E}-10$ & $6.1 \mathrm{E}-09$ & $6 \mathrm{~h}$ & 3 \\
\hline AJUBA & 0.45 & 0.63 & 0.28 & 0.45 & -1.16 & $5.5 \mathrm{E}-11$ & $2.5 \mathrm{E}-09$ & $6 \mathrm{~h}$ & 3 \\
\hline
\end{tabular}


Table S4 continued

\begin{tabular}{|c|c|c|c|c|c|c|c|c|c|}
\hline Gene & $\begin{array}{l}\text { base } \\
\text { Mean }\end{array}$ & $\begin{array}{l}\text { base } \\
\text { MeanA }\end{array}$ & $\begin{array}{l}\text { base } \\
\text { MeanB }\end{array}$ & $\begin{array}{l}\text { fold } \\
\text { Change }\end{array}$ & $\begin{array}{l}\log 2 \\
F C\end{array}$ & pval & padj & $\begin{array}{l}\text { shared } \\
\text { in states }\end{array}$ & Node \\
\hline XBP1 & 0.44 & 0.61 & 0.27 & 0.44 & -1.18 & $8.8 \mathrm{E}-11$ & 3.9E-09 & $6 \mathrm{~h}$ & 1 \\
\hline GDF15 & 0.45 & 0.63 & 0.27 & 0.43 & -1.21 & $2.0 \mathrm{E}-11$ & $9.8 \mathrm{E}-10$ & $6 \mathrm{~h}$ & 3 \\
\hline BTG2 & 0.42 & 0.59 & 0.25 & 0.42 & -1.24 & $2.6 \mathrm{E}-11$ & $1.3 \mathrm{E}-09$ & $6 \mathrm{~h}$ & 3 \\
\hline PHLDA1 & 0.32 & 0.46 & 0.18 & 0.40 & -1.33 & $6.6 \mathrm{E}-10$ & $2.5 \mathrm{E}-08$ & $6 \mathrm{~h}$ & 3 \\
\hline NR2F2 & 1.04 & 1.52 & 0.56 & 0.37 & -1.45 & 7.7E-34 & $1.3 \mathrm{E}-31$ & $6 \mathrm{~h}$ & 3 \\
\hline ARL4C & 0.70 & 1.03 & 0.37 & 0.36 & -1.48 & $7.2 \mathrm{E}-24$ & $8.3 E-22$ & $6 \mathrm{~h}$ & 3 \\
\hline NAB2 & 0.26 & 0.39 & 0.13 & 0.32 & -1.64 & $1.3 \mathrm{E}-11$ & $6.6 \mathrm{E}-10$ & $6 \mathrm{~h}$ & 3 \\
\hline IRF2BPL & 0.30 & 0.46 & 0.15 & 0.32 & -1.66 & $4.2 \mathrm{E}-13$ & $2.5 \mathrm{E}-11$ & $6 \mathrm{~h}$ & 3 \\
\hline ZSCAN4 & 5.37 & 0.26 & 10.48 & 40.09 & 5.33 & 0 & 0 & $4 \mathrm{~h} \mid 6 \mathrm{~h}$ & 19 \\
\hline LEUTX & 2.31 & 0.14 & 4.48 & 33.08 & 5.05 & 0 & 0 & $4 \mathrm{~h} \mid 6 \mathrm{~h}$ & 20 \\
\hline RFPL4A & 1.97 & 0.15 & 3.79 & 26.02 & 4.70 & 4.2E-304 & 5.0E-301 & $4 \mathrm{~h} \mid 6 \mathrm{~h}$ & 20 \\
\hline PRAMEF1 & 1.47 & 0.11 & 2.83 & 24.70 & 4.63 & $2.6 \mathrm{E}-224$ & $2.4 \mathrm{E}-221$ & $4 h \mid 6 h$ & 19 \\
\hline PRAMEF12 & 1.01 & 0.13 & 1.89 & 14.44 & 3.85 & $2.1 \mathrm{E}-133$ & $1.4 \mathrm{E}-130$ & $4 \mathrm{~h} \mid 6 \mathrm{~h}$ & 19 \\
\hline SLC34A2 & 0.89 & 0.12 & 1.65 & 13.83 & 3.79 & $2.9 \mathrm{E}-114$ & $1.5 \mathrm{E}-111$ & $4 \mathrm{~h} \mid 6 \mathrm{~h}$ & 19 \\
\hline TRIM51 & 1.68 & 0.25 & 3.11 & 12.51 & 3.65 & 6.7E-208 & 5.7E-205 & $4 \mathrm{~h} \mid 6 \mathrm{~h}$ & 19 \\
\hline TFIP11 & 0.72 & 0.18 & 1.26 & 7.11 & 2.83 & $4.3 E-67$ & $1.8 \mathrm{E}-64$ & $4 \mathrm{~h} \mid 6 \mathrm{~h}$ & 19 \\
\hline KDM4E & 0.41 & 0.10 & 0.72 & 6.92 & 2.79 & $2.1 \mathrm{E}-38$ & $4.1 \mathrm{E}-36$ & $4 \mathrm{~h} \mid 6 \mathrm{~h}$ & 19 \\
\hline GTF2F1 & 4.95 & 1.37 & 8.54 & 6.25 & 2.64 & 0 & 0 & $4 \mathrm{~h} \mid 6 \mathrm{~h}$ & 19 \\
\hline PRRG4 & 0.55 & 0.17 & 0.93 & 5.43 & 2.44 & $5.8 \mathrm{E}-43$ & $1.4 \mathrm{E}-40$ & $4 h \mid 6 h$ & 19 \\
\hline PNP & 4.95 & 1.57 & 8.32 & 5.29 & 2.40 & 0 & 0 & $4 \mathrm{~h} \mid 6 \mathrm{~h}$ & 19 \\
\hline SPTY2D1 & 0.83 & 0.31 & 1.34 & 4.28 & 2.10 & $1.2 \mathrm{E}-50$ & 3.7E-48 & $4 \mathrm{~h} \mid 6 \mathrm{~h}$ & 19 \\
\hline ESRG & 0.69 & 0.27 & 1.11 & 4.14 & 2.05 & 3.7E-41 & 8.1E-39 & $4 \mathrm{~h} \mid 6 \mathrm{~h}$ & 19 \\
\hline ARID5B & 0.76 & 0.31 & 1.21 & 3.94 & 1.98 & $2.4 \mathrm{E}-42$ & $5.5 E-40$ & $4 \mathrm{~h} \mid 6 \mathrm{~h}$ & 19 \\
\hline ZNF622 & 1.09 & 0.44 & 1.74 & 3.92 & 1.97 & $1.3 \mathrm{E}-59$ & $4.6 \mathrm{E}-57$ & $4 \mathrm{~h} \mid 6 \mathrm{~h}$ & 19 \\
\hline DBR1 & 0.96 & 0.40 & 1.52 & 3.82 & 1.93 & 4.2E-51 & $1.3 \mathrm{E}-48$ & $4 \mathrm{~h} \mid 6 \mathrm{~h}$ & 19 \\
\hline NXF1 & 2.35 & 1.04 & 3.66 & 3.53 & 1.82 & $8.2 \mathrm{E}-112$ & 3.9E-109 & $4 \mathrm{~h} \mid 6 \mathrm{~h}$ & 19 \\
\hline HOXB2 & 1.71 & 0.79 & 2.63 & 3.35 & 1.74 & $2.8 \mathrm{E}-76$ & $1.2 \mathrm{E}-73$ & $4 \mathrm{~h} \mid 6 \mathrm{~h}$ & 19 \\
\hline EXOSC10 & 3.03 & 1.47 & 4.59 & 3.11 & 1.64 & $2.4 \mathrm{E}-120$ & $1.5 \mathrm{E}-117$ & $4 \mathrm{~h} \mid 6 \mathrm{~h}$ & 19 \\
\hline ITGB8 & 0.40 & 0.20 & 0.60 & 3.06 & 1.61 & $1.6 \mathrm{E}-16$ & $1.2 \mathrm{E}-14$ & $4 \mathrm{~h} \mid 6 \mathrm{~h}$ & 21 \\
\hline RICTOR & 0.78 & 0.39 & 1.17 & 2.99 & 1.58 & $1.3 \mathrm{E}-30$ & $1.9 \mathrm{E}-28$ & $4 \mathrm{~h} \mid 6 \mathrm{~h}$ & 21 \\
\hline PDGFRA & 0.58 & 0.30 & 0.86 & 2.90 & 1.54 & $9.9 \mathrm{E}-22$ & $1.1 \mathrm{E}-19$ & $4 \mathrm{~h} \mid 6 \mathrm{~h}$ & 19 \\
\hline $\mathrm{CDH} 10$ & 0.96 & 0.51 & 1.41 & 2.80 & 1.48 & $2.2 \mathrm{E}-33$ & $3.8 \mathrm{E}-31$ & $4 \mathrm{~h} \mid 6 \mathrm{~h}$ & 19 \\
\hline ZNF827 & 0.55 & 0.31 & 0.79 & 2.54 & 1.34 & $6.4 \mathrm{E}-17$ & 5.1E-15 & $4 \mathrm{~h} \mid 6 \mathrm{~h}$ & 21 \\
\hline MRPL49 & 2.41 & 1.42 & 3.40 & 2.40 & 1.27 & 2.7E-61 & $9.8 \mathrm{E}-59$ & $4 \mathrm{~h} \mid 6 \mathrm{~h}$ & 19 \\
\hline ZSWIM6 & 0.56 & 0.34 & 0.79 & 2.34 & 1.23 & $1.3 \mathrm{E}-14$ & $8.8 \mathrm{E}-13$ & $4 h \mid 6 h$ & 21 \\
\hline
\end{tabular}


Table S4 continued

\begin{tabular}{|c|c|c|c|c|c|c|c|c|c|}
\hline Gene & $\begin{array}{l}\text { base } \\
\text { Mean }\end{array}$ & $\begin{array}{l}\text { base } \\
\text { MeanA }\end{array}$ & $\begin{array}{l}\text { base } \\
\text { MeanB }\end{array}$ & $\begin{array}{l}\text { fold } \\
\text { Change }\end{array}$ & $\begin{array}{l}\log 2 \\
\text { FC }\end{array}$ & pval & padj & $\begin{array}{l}\text { shared } \\
\text { in states }\end{array}$ & Node \\
\hline ODF2L & 0.62 & 0.38 & 0.87 & 2.29 & 1.20 & $1.0 \mathrm{E}-15$ & $7.4 \mathrm{E}-14$ & $4 h \mid 6 h$ & 18 \\
\hline SHC1 & 1.70 & 1.04 & 2.36 & 2.28 & 1.19 & $5.3 \mathrm{E}-39$ & 1.1E-36 & $4 h \mid 6 h$ & 19 \\
\hline KIAA1551 & 0.91 & 0.56 & 1.27 & 2.28 & 1.19 & $1.0 \mathrm{E}-21$ & 1.1E-19 & $4 h \mid 6 h$ & 21 \\
\hline YTHDC1 & 1.58 & 0.98 & 2.18 & 2.23 & 1.15 & $1.3 \mathrm{E}-34$ & $2.2 \mathrm{E}-32$ & $4 h \mid 6 h$ & 21 \\
\hline $\begin{array}{l}\text { GABPB1- } \\
\text { AS1 }\end{array}$ & 0.57 & 0.36 & 0.79 & 2.22 & 1.15 & $1.4 \mathrm{E}-13$ & $8.6 \mathrm{E}-12$ & $4 h \mid 6 h$ & 18 \\
\hline SYNE2 & 0.81 & 0.50 & 1.11 & 2.22 & 1.15 & $2.8 \mathrm{E}-18$ & $2.4 \mathrm{E}-16$ & $4 h \mid 6 h$ & 17 \\
\hline LRRC8B & 1.08 & 0.68 & 1.49 & 2.20 & 1.14 & $1.3 \mathrm{E}-23$ & $1.5 \mathrm{E}-21$ & $4 h \mid 6 h$ & 21 \\
\hline BTAF1 & 0.77 & 0.49 & 1.06 & 2.18 & 1.12 & $5.2 \mathrm{E}-17$ & $4.2 \mathrm{E}-15$ & $4 h \mid 6 h$ & 21 \\
\hline ARHGEF26 & 1.17 & 0.74 & 1.61 & 2.17 & 1.12 & $1.1 \mathrm{E}-24$ & $1.4 \mathrm{E}-22$ & $4 h \mid 6 h$ & 19 \\
\hline NFAT5 & 0.85 & 0.54 & 1.16 & 2.14 & 1.10 & $6.6 \mathrm{E}-18$ & $5.6 \mathrm{E}-16$ & $4 h \mid 6 h$ & 19 \\
\hline ZNF644 & 0.86 & 0.57 & 1.16 & 2.05 & 1.03 & $3.6 \mathrm{E}-16$ & 2.7E-14 & $4 h \mid 6 h$ & 19 \\
\hline GUSBP3 & 0.53 & 0.37 & 0.70 & 1.89 & 0.92 & $1.3 \mathrm{E}-08$ & 4.1E-07 & $4 h \mid 6 h$ & 14 \\
\hline PUM1 & 2.53 & 1.76 & 3.29 & 1.87 & 0.90 & $8.2 \mathrm{E}-35$ & $1.5 \mathrm{E}-32$ & $4 h \mid 6 h$ & 21 \\
\hline PHACTR2 & 0.54 & 0.38 & 0.70 & 1.87 & 0.90 & 1.7E-08 & $5.4 \mathrm{E}-07$ & $4 h \mid 6 h$ & 18 \\
\hline PLK4 & 1.03 & 0.72 & 1.33 & 1.85 & 0.89 & $1.3 \mathrm{E}-14$ & $8.6 \mathrm{E}-13$ & $4 h \mid 6 h$ & 19 \\
\hline ZMYND8 & 1.05 & 0.76 & 1.35 & 1.77 & 0.82 & $3.4 \mathrm{E}-13$ & 2.0E-11 & $4 h \mid 6 h$ & 18 \\
\hline CCNL2 & 1.45 & 1.06 & 1.84 & 1.74 & 0.80 & $9.4 \mathrm{E}-17$ & 7.4E-15 & $4 h \mid 6 h$ & 19 \\
\hline NET1 & 1.10 & 0.83 & 1.38 & 1.67 & 0.74 & $1.5 \mathrm{E}-11$ & $7.4 \mathrm{E}-10$ & $4 h \mid 6 h$ & 18 \\
\hline CIRBP & 4.24 & 3.19 & 5.30 & 1.66 & 0.73 & 5.7E-39 & $1.2 \mathrm{E}-36$ & $4 h \mid 6 h$ & 19 \\
\hline POLQ & 0.85 & 0.64 & 1.06 & 1.65 & 0.73 & $1.2 \mathrm{E}-08$ & $3.8 \mathrm{E}-07$ & $4 h \mid 6 h$ & 17 \\
\hline ZRANB2 & 2.71 & 2.05 & 3.38 & 1.65 & 0.72 & $1.0 \mathrm{E}-24$ & $1.3 \mathrm{E}-22$ & $4 h \mid 6 h$ & 18 \\
\hline SRSF11 & 4.64 & 3.54 & 5.74 & 1.62 & 0.70 & $1.4 \mathrm{E}-38$ & $2.8 \mathrm{E}-36$ & $4 h \mid 6 h$ & 18 \\
\hline PPIG & 1.76 & 1.36 & 2.16 & 1.59 & 0.67 & $1.2 \mathrm{E}-14$ & $7.8 \mathrm{E}-13$ & $4 h \mid 6 h$ & 14 \\
\hline CUX1 & 1.00 & 0.77 & 1.22 & 1.59 & 0.67 & 7.9E-09 & $2.6 \mathrm{E}-07$ & $4 h \mid 6 h$ & 14 \\
\hline SLC25A36 & 1.47 & 1.16 & 1.79 & 1.54 & 0.62 & $5.8 \mathrm{E}-11$ & 2.7E-09 & $4 h \mid 6 h$ & 18 \\
\hline BBX & 1.51 & 1.19 & 1.82 & 1.53 & 0.61 & 7.3E-11 & $3.3 \mathrm{E}-09$ & $4 h \mid 6 h$ & 14 \\
\hline RIF1 & 1.53 & 1.22 & 1.85 & 1.52 & 0.61 & 8.9E-11 & 3.9E-09 & $4 h \mid 6 h$ & 13 \\
\hline SMC3 & 1.58 & 1.27 & 1.90 & 1.49 & 0.58 & $2.8 \mathrm{E}-10$ & $1.1 \mathrm{E}-08$ & $4 h \mid 6 h$ & 17 \\
\hline DPPA4 & 4.17 & 3.40 & 4.95 & 1.46 & 0.54 & $2.9 \mathrm{E}-22$ & 3.2E-20 & $4 h \mid 6 h$ & 18 \\
\hline TNRC6A & 1.62 & 1.32 & 1.92 & 1.46 & 0.54 & $1.8 \mathrm{E}-09$ & $6.4 \mathrm{E}-08$ & $4 h \mid 6 h$ & 17 \\
\hline FTL & 9.08 & 10.71 & 7.44 & 0.70 & -0.53 & $1.3 \mathrm{E}-39$ & $2.8 \mathrm{E}-37$ & $4 h \mid 6 h$ & 2 \\
\hline RPL23A & 10.78 & 12.92 & 8.63 & 0.67 & -0.58 & $4.6 \mathrm{E}-53$ & $1.5 \mathrm{E}-50$ & $4 h \mid 6 h$ & 9 \\
\hline UBE2S & 2.92 & 3.50 & 2.33 & 0.67 & -0.59 & 4.1E-18 & $3.5 \mathrm{E}-16$ & $4 h \mid 6 h$ & 3 \\
\hline HNRNPAO & 1.59 & 1.95 & 1.23 & 0.63 & -0.67 & $4.4 \mathrm{E}-13$ & 2.6E-11 & $4 \mathrm{~h} \mid 6 \mathrm{~h}$ & 8 \\
\hline DYNLL1 & 6.59 & 8.58 & 4.60 & 0.54 & -0.90 & $1.5 \mathrm{E}-86$ & 7.0E-84 & $4 h \mid 6 h$ & 3 \\
\hline
\end{tabular}


Table S4 continued

\begin{tabular}{|c|c|c|c|c|c|c|c|c|c|}
\hline Gene & $\begin{array}{l}\text { base } \\
\text { Mean }\end{array}$ & $\begin{array}{l}\text { base } \\
\text { MeanA }\end{array}$ & $\begin{array}{l}\text { base } \\
\text { MeanB }\end{array}$ & $\begin{array}{l}\text { fold } \\
\text { Change }\end{array}$ & $\begin{array}{l}\log 2 \\
F C\end{array}$ & pval & padj & $\begin{array}{l}\text { shared } \\
\text { in states }\end{array}$ & Node \\
\hline SLC7A5 & 0.83 & 1.08 & 0.57 & 0.53 & -0.92 & 1.6E-12 & 8.9E-11 & $4 h \mid 6 h$ & 1 \\
\hline PCDH18 & 0.57 & 0.76 & 0.37 & 0.48 & -1.05 & $4.2 \mathrm{E}-11$ & 2.0E-09 & $4 h \mid 6 h$ & 3 \\
\hline ISOC2 & 0.40 & 0.56 & 0.25 & 0.44 & -1.18 & $2.5 \mathrm{E}-10$ & $1.0 \mathrm{E}-08$ & $4 \mathrm{~h} \mid 6 \mathrm{~h}$ & 9 \\
\hline SHISA3 & 1.37 & 1.92 & 0.83 & 0.43 & -1.22 & 7.6E-33 & $1.2 \mathrm{E}-30$ & $4 h \mid 6 h$ & 3 \\
\hline TGIF1 & 0.64 & 0.92 & 0.35 & 0.38 & -1.39 & $1.3 \mathrm{E}-19$ & $1.3 \mathrm{E}-17$ & $4 \mathrm{~h} \mid 6 \mathrm{~h}$ & 3 \\
\hline PIM1 & 0.34 & 0.50 & 0.19 & 0.37 & -1.43 & $6.1 \mathrm{E}-12$ & $3.1 \mathrm{E}-10$ & $4 \mathrm{~h} \mid 6 \mathrm{~h}$ & 3 \\
\hline NEDD9 & 0.75 & 1.09 & 0.40 & 0.37 & -1.44 & $2.2 \mathrm{E}-24$ & 2.7E-22 & $4 \mathrm{~h} \mid 6 \mathrm{~h}$ & 3 \\
\hline MIDN & 0.83 & 1.23 & 0.43 & 0.35 & -1.51 & $2.4 \mathrm{E}-29$ & $3.4 \mathrm{E}-27$ & $4 \mathrm{~h} \mid 6 \mathrm{~h}$ & 3 \\
\hline CYR61 & 1.02 & 1.51 & 0.53 & 0.35 & -1.52 & $8.4 \mathrm{E}-36$ & $1.6 \mathrm{E}-33$ & $4 \mathrm{~h} \mid 6 \mathrm{~h}$ & 3 \\
\hline NUAK2 & 0.33 & 0.50 & 0.16 & 0.32 & -1.63 & 8.6E-14 & $5.5 \mathrm{E}-12$ & $4 \mathrm{~h} \mid 6 \mathrm{~h}$ & 3 \\
\hline NOG & 0.27 & 0.43 & 0.12 & 0.27 & -1.87 & $2.6 \mathrm{E}-14$ & 1.7E-12 & $4 h \mid 6 h$ & 3 \\
\hline POLR2L & 2.14 & 2.53 & 1.76 & 0.70 & -0.52 & 4.7E-11 & $2.2 \mathrm{E}-09$ & $3 \mathrm{~h} \mid 6 \mathrm{~h}$ & 8 \\
\hline RFPL4B & 6.91 & 0.26 & 13.56 & 51.62 & 5.69 & 0 & 0 & $3 h|4 h| 6 h$ & 19 \\
\hline ZNF217 & 3.16 & 0.64 & 5.68 & 8.89 & 3.15 & 0 & 0 & $3 h|4 h| 6 h$ & 19 \\
\hline RBBP6 & 12.63 & 2.58 & 22.68 & 8.78 & 3.13 & 0 & 0 & $3 \mathrm{~h}|4 \mathrm{~h}| 6 \mathrm{~h}$ & 19 \\
\hline SRSF8 & 6.06 & 1.77 & 10.35 & 5.86 & 2.55 & 0 & 0 & $3 h|4 h| 6 h$ & 19 \\
\hline ZNF296 & 2.03 & 0.60 & 3.46 & 5.79 & 2.53 & 4.3E-161 & $3.2 E-158$ & $3 \mathrm{~h}|4 \mathrm{~h}| 6 \mathrm{~h}$ & 19 \\
\hline F5 & 0.56 & 0.28 & 0.83 & 2.94 & 1.56 & $6.1 \mathrm{E}-22$ & $6.6 \mathrm{E}-20$ & $3 h|4 h| 6 h$ & 18 \\
\hline PNN & 5.76 & 2.94 & 8.57 & 2.92 & 1.54 & $2.5 \mathrm{E}-205$ & $2.0 \mathrm{E}-202$ & $3 h|4 h| 6 h$ & 21 \\
\hline ZMAT3 & 1.30 & 0.69 & 1.91 & 2.78 & 1.47 & 4.2E-44 & $1.1 \mathrm{E}-41$ & $3 h|4 h| 6 h$ & 19 \\
\hline CCDC144B & 0.60 & 0.33 & 0.87 & 2.67 & 1.42 & $1.3 \mathrm{E}-19$ & $1.2 \mathrm{E}-17$ & $3 h|4 h| 6 h$ & 18 \\
\hline TSIX & 0.58 & 0.32 & 0.84 & 2.64 & 1.40 & $6.6 \mathrm{E}-19$ & $6.1 \mathrm{E}-17$ & $3 h|4 h| 6 h$ & 18 \\
\hline ZNF471 & 0.69 & 0.38 & 0.99 & 2.60 & 1.38 & $1.6 \mathrm{E}-21$ & 1.7E-19 & $3 h|4 h| 6 h$ & 18 \\
\hline GOLGB1 & 1.24 & 0.71 & 1.77 & 2.51 & 1.33 & $7.3 \mathrm{E}-35$ & $1.3 \mathrm{E}-32$ & $3 \mathrm{~h}|4 \mathrm{~h}| 6 \mathrm{~h}$ & 18 \\
\hline TMEM212 & 0.54 & 0.32 & 0.76 & 2.37 & 1.24 & $9.6 \mathrm{E}-15$ & $6.6 \mathrm{E}-13$ & $3 h|4 h| 6 h$ & 18 \\
\hline MLL5 & 0.85 & 0.52 & 1.19 & 2.31 & 1.21 & 3.3E-21 & $3.4 \mathrm{E}-19$ & $3 h|4 h| 6 h$ & 17 \\
\hline MPHOSPH8 & 1.03 & 0.65 & 1.41 & 2.15 & 1.11 & $2.8 \mathrm{E}-21$ & $2.9 \mathrm{E}-19$ & $3 h|4 h| 6 h$ & 18 \\
\hline BoD1L1 & 0.90 & 0.57 & 1.22 & 2.13 & 1.09 & $2.6 \mathrm{E}-18$ & $2.2 \mathrm{E}-16$ & $3 h|4 h| 6 h$ & 18 \\
\hline GADD45A & 2.12 & 1.37 & 2.88 & 2.11 & 1.08 & $9.9 \mathrm{E}-41$ & $2.2 \mathrm{E}-38$ & $3 \mathrm{~h}|4 \mathrm{~h}| 6 \mathrm{~h}$ & 18 \\
\hline RBM25 & 3.77 & 2.48 & 5.06 & 2.04 & 1.03 & $3.4 \mathrm{E}-65$ & $1.3 \mathrm{E}-62$ & $3 h|4 h| 6 h$ & 18 \\
\hline SLC4A7 & 0.68 & 0.45 & 0.91 & 2.01 & 1.01 & $1.6 \mathrm{E}-12$ & 8.6E-11 & $3 h|4 h| 6 h$ & 17 \\
\hline PNISR & 2.24 & 1.57 & 2.90 & 1.85 & 0.89 & $4.9 \mathrm{E}-30$ & $7.2 \mathrm{E}-28$ & $3 \mathrm{~h}|4 \mathrm{~h}| 6 \mathrm{~h}$ & 18 \\
\hline LUC7L3 & 2.31 & 1.65 & 2.97 & 1.81 & 0.85 & $8.3 E-29$ & $1.2 \mathrm{E}-26$ & $3 h|4 h| 6 h$ & 18 \\
\hline SLTM & 1.35 & 1.03 & 1.68 & 1.64 & 0.71 & $8.0 \mathrm{E}-13$ & 4.6E-11 & $3 h|4 h| 6 h$ & 18 \\
\hline ATRX & 2.10 & 1.65 & 2.55 & 1.54 & 0.63 & 3.3E-15 & $2.3 \mathrm{E}-13$ & $3 h|4 h| 6 h$ & 14 \\
\hline
\end{tabular}


Table S4 continued

\begin{tabular}{|c|c|c|c|c|c|c|c|c|c|}
\hline Gene & $\begin{array}{l}\text { base } \\
\text { Mean }\end{array}$ & $\begin{array}{l}\text { base } \\
\text { MeanA }\end{array}$ & $\begin{array}{l}\text { base } \\
\text { MeanB }\end{array}$ & $\begin{array}{l}\text { fold } \\
\text { Change }\end{array}$ & $\begin{array}{l}\log 2 \\
F C\end{array}$ & pval & padj & $\begin{array}{l}\text { shared } \\
\text { in states }\end{array}$ & Node \\
\hline DMWD & 1.26 & 1.01 & 1.52 & 1.51 & 0.59 & 8.7E-09 & $2.9 \mathrm{E}-07$ & $3 h|4 h| 6 h$ & 14 \\
\hline CCAR1 & 3.42 & 2.74 & 4.09 & 1.50 & 0.58 & $1.0 \mathrm{E}-20$ & $1.1 \mathrm{E}-18$ & $3 h|4 h| 6 h$ & 14 \\
\hline cox7C & 4.83 & 5.68 & 3.99 & 0.70 & -0.51 & $2.2 \mathrm{E}-22$ & $2.4 \mathrm{E}-20$ & $3 h|4 h| 6 h$ & 8 \\
\hline PRDX4 & 1.43 & 1.70 & 1.17 & 0.69 & -0.54 & $2.8 \mathrm{E}-08$ & 8.5E-07 & $3 h|4 h| 6 h$ & 8 \\
\hline APP & 2.16 & 2.85 & 1.47 & 0.52 & -0.96 & 6.7E-33 & $1.1 \mathrm{E}-30$ & $3 \mathrm{~h}|4 \mathrm{~h}| 6 \mathrm{~h}$ & 8 \\
\hline MAGOH & 0.65 & 0.87 & 0.43 & 0.50 & -1.01 & $5.6 \mathrm{E}-12$ & $2.9 \mathrm{E}-10$ & $3 h|4 h| 6 h$ & 9 \\
\hline FKBP10 & 0.37 & 0.52 & 0.21 & 0.41 & -1.30 & $7.5 \mathrm{E}-11$ & 3.3E-09 & $3 h|4 h| 6 h$ & 7 \\
\hline ID1 & 2.32 & 4.18 & 0.45 & 0.11 & -3.21 & $2.2 \mathrm{E}-237$ & $2.2 \mathrm{E}-234$ & $3 \mathrm{~h}|4 \mathrm{~h}| 6 \mathrm{~h}$ & 3 \\
\hline ID3 & 1.16 & 2.09 & 0.23 & 0.11 & -3.21 & 8.9E-119 & 4.9E-116 & $3 h|4 h| 6 h$ & 3 \\
\hline TOP2A & 1.67 & 1.21 & 2.12 & 1.76 & 0.81 & $1.3 \mathrm{E}-19$ & $1.3 \mathrm{E}-17$ & $2 \mathrm{~h} \mid 6 \mathrm{~h}$ & 10 \\
\hline MKI67 & 2.31 & 1.89 & 2.74 & 1.45 & 0.54 & $1.4 \mathrm{E}-12$ & $7.9 \mathrm{E}-11$ & $2 \mathrm{~h} \mid 6 \mathrm{~h}$ & 10 \\
\hline KIF14 & 0.89 & 0.67 & 1.10 & 1.64 & 0.71 & $7.2 \mathrm{E}-09$ & $2.4 \mathrm{E}-07$ & $2 h|3 h| 6 h$ & 10 \\
\hline HIST1H2BK & 1.18 & 0.91 & 1.44 & 1.57 & 0.65 & $9.1 \mathrm{E}-10$ & $3.5 \mathrm{E}-08$ & $2 h|3 h| 6 h$ & 19 \\
\hline
\end{tabular}

Table S5. Differentially expressed transcription factors, cofactors and kinases between uninduced and induced DIE cells.

\begin{tabular}{|c|c|c|c|}
\hline Gene & DE & Induction states & Factor \\
\hline CDK6 & UP & $2 \mathrm{~h}$ & Kinase \\
\hline TAF3 & UP & $2 h \mid 3 h$ & Cofactor \\
\hline NIPBL & UP & $2 \mathrm{~h} \mid 3 \mathrm{~h}$ & Cofactor \\
\hline CHD7 & UP & $2 \mathrm{~h} \mid 3 \mathrm{~h}$ & Cofactor \\
\hline ROCK1 & UP & $2 \mathrm{~h}|3 \mathrm{~h}| 4 \mathrm{~h}$ & Kinase \\
\hline CDC42BPA & UP & $2 \mathrm{~h}|3 \mathrm{~h}| 4 \mathrm{~h}$ & Kinase \\
\hline CCDC88A & UP & $2 \mathrm{~h}|3 \mathrm{~h}| 4 \mathrm{~h}$ & Transcription factor \\
\hline DNAJC2 & UP & $2 \mathrm{~h}|3 \mathrm{~h}| 4 \mathrm{~h}$ & Transcription factor \\
\hline ZFHX3 & UP & $2 \mathrm{~h}|3 \mathrm{~h}| 4 \mathrm{~h}$ & Transcription factor \\
\hline CENPF & UP & All 4 & Cofactor \\
\hline BDP1 & UP & All 4 & Cofactor \\
\hline BRD4 & UP & All 4 & Cofactor \\
\hline TOP1 & UP & All 4 & Cofactor \\
\hline BPTF & UP & All 4 & Cofactor \\
\hline FOXN3 & UP & $2 \mathrm{~h} \mid 4 \mathrm{~h}$ & Transcription factor \\
\hline ASH1L & UP & $3 \mathrm{~h}$ & Cofactor \\
\hline XIAP & UP & $3 h$ & Cofactor \\
\hline TPR & UP & $3 \mathrm{~h}$ & Cofactor \\
\hline
\end{tabular}


Table S5 continued

\begin{tabular}{|c|c|c|c|}
\hline Gene & DE & Induction states & Factor \\
\hline SETD2 & UP & $3 h$ & Cofactor \\
\hline ESF1 & UP & $3 h$ & Cofactor \\
\hline WNK1 & UP & $3 h$ & Kinase \\
\hline REST & UP & $3 h$ & Transcription factor \\
\hline CHD9 & UP & $3 h \mid 4 h$ & Cofactor \\
\hline ATRX & UP & $3 h|4 h| 6 h$ & Cofactor \\
\hline MPHOSPH8 & UP & $3 h|4 h| 6 h$ & Cofactor \\
\hline CCAR1 & UP & $3 h|4 h| 6 h$ & Cofactor \\
\hline SLTM & UP & $3 h|4 h| 6 h$ & Cofactor \\
\hline ZNF471 & UP & $3 h|4 h| 6 h$ & Transcription factor \\
\hline ZNF296 & UP & $3 h|4 h| 6 h$ & Transcription factor \\
\hline ZNF217 & UP & $3 h|4 h| 6 h$ & Transcription factor \\
\hline ASCC3 & UP & $4 h$ & Cofactor \\
\hline HELLS & UP & $4 h$ & Cofactor \\
\hline ZFHX4 & UP & $4 h$ & Transcription factor \\
\hline BAZ2B & UP & $4 h$ & Transcription factor \\
\hline PAXBP1 & UP & $4 h$ & Transcription factor \\
\hline LRRFIP1 & UP & $4 \mathrm{~h}$ & Transcription factor \\
\hline GLI3 & UP & $4 h$ & Transcription factor \\
\hline KDM4E & UP & $4 \mathrm{~h} \mid 6 \mathrm{~h}$ & Cofactor \\
\hline GTF2F1 & UP & $4 h \mid 6 h$ & Cofactor \\
\hline BTAF1 & UP & $4 h \mid 6 h$ & Cofactor \\
\hline ZMYND8 & UP & $4 h \mid 6 h$ & Cofactor \\
\hline DPPA4 & UP & $4 h \mid 6 h$ & Cofactor \\
\hline PDGFRA & UP & $4 h \mid 6 h$ & Kinase \\
\hline PLK4 & UP & $4 h \mid 6 h$ & Kinase \\
\hline ZSCAN4 & UP & $4 h \mid 6 h$ & Transcription factor \\
\hline LEUTX & UP & $4 \mathrm{~h} \mid 6 \mathrm{~h}$ & Transcription factor \\
\hline ARID5B & UP & $4 h \mid 6 h$ & Transcription factor \\
\hline HOXB2 & UP & $4 h \mid 6 h$ & Transcription factor \\
\hline ZNF622 & UP & $4 h \mid 6 h$ & Transcription factor \\
\hline ZNF827 & UP & $4 h \mid 6 h$ & Transcription factor \\
\hline NFAT5 & UP & $4 h \mid 6 h$ & Transcription factor \\
\hline ZNF644 & UP & $4 h \mid 6 h$ & Transcription factor \\
\hline CUX1 & UP & $4 h \mid 6 h$ & Transcription factor \\
\hline
\end{tabular}


Table S5 continued

\begin{tabular}{|c|c|c|c|}
\hline Gene & $\mathrm{DE}$ & Induction states & Factor \\
\hline BBX & UP & $4 h \mid 6 h$ & Transcription factor \\
\hline CCNA1 & UP & $6 \mathrm{~h}$ & Cofactor \\
\hline C1D & UP & $6 \mathrm{~h}$ & Cofactor \\
\hline TAF4B & UP & $6 \mathrm{~h}$ & Cofactor \\
\hline HEXIM1 & UP & $6 \mathrm{~h}$ & Cofactor \\
\hline NCOA7 & UP & $6 \mathrm{~h}$ & Cofactor \\
\hline SUPT6H & UP & $6 \mathrm{~h}$ & Cofactor \\
\hline BRCA2 & UP & $6 \mathrm{~h}$ & Cofactor \\
\hline BIRC2 & UP & $6 \mathrm{~h}$ & Cofactor \\
\hline UFL1 & UP & $6 \mathrm{~h}$ & Cofactor \\
\hline TOPORS & UP & $6 \mathrm{~h}$ & Cofactor \\
\hline $\mathrm{NOTCH} 2$ & UP & $6 \mathrm{~h}$ & Cofactor \\
\hline ELOF1 & UP & $6 \mathrm{~h}$ & Cofactor \\
\hline KDM5B & UP & $6 \mathrm{~h}$ & Cofactor \\
\hline TBPL1 & UP & $6 \mathrm{~h}$ & Cofactor \\
\hline DEPDC1 & UP & $6 \mathrm{~h}$ & Cofactor \\
\hline KDM5A & UP & $6 \mathrm{~h}$ & Cofactor \\
\hline BRD8 & UP & $6 \mathrm{~h}$ & Cofactor \\
\hline MTF2 & UP & $6 \mathrm{~h}$ & Cofactor \\
\hline CTR9 & UP & $6 \mathrm{~h}$ & Cofactor \\
\hline SMARCAD1 & UP & $6 \mathrm{~h}$ & Cofactor \\
\hline TERF2IP & UP & $6 \mathrm{~h}$ & Cofactor \\
\hline OGT & UP & $6 \mathrm{~h}$ & Cofactor \\
\hline LRRK1 & UP & $6 \mathrm{~h}$ & Kinase \\
\hline CLK1 & UP & $6 \mathrm{~h}$ & Kinase \\
\hline MAST1 & UP & $6 \mathrm{~h}$ & Kinase \\
\hline STK17B & UP & $6 \mathrm{~h}$ & Kinase \\
\hline EPHA4 & UP & $6 \mathrm{~h}$ & Kinase \\
\hline MELK & UP & $6 \mathrm{~h}$ & Kinase \\
\hline SNAI1 & UP & $6 \mathrm{~h}$ & Transcription factor \\
\hline EOMES & UP & $6 \mathrm{~h}$ & Transcription factor \\
\hline $\mathrm{PHOX} 2 \mathrm{~B}$ & UP & $6 \mathrm{~h}$ & Transcription factor \\
\hline BHLHE22 & UP & $6 \mathrm{~h}$ & Transcription factor \\
\hline OSR2 & UP & $6 \mathrm{~h}$ & Transcription factor \\
\hline ZNF574 & UP & $6 \mathrm{~h}$ & Transcription factor \\
\hline
\end{tabular}


Table S5 continued

\begin{tabular}{|c|c|c|c|}
\hline Gene & DE & Induction states & Factor \\
\hline ZNF480 & UP & $6 h$ & Transcription factor \\
\hline TFAP2C & UP & $6 \mathrm{~h}$ & Transcription factor \\
\hline ZNF91 & UP & $6 \mathrm{~h}$ & Transcription factor \\
\hline ZNF281 & UP & $6 \mathrm{~h}$ & Transcription factor \\
\hline ZNF609 & UP & $6 \mathrm{~h}$ & Transcription factor \\
\hline ZHX1 & UP & $6 \mathrm{~h}$ & Transcription factor \\
\hline HMGXB4 & UP & $6 \mathrm{~h}$ & Transcription factor \\
\hline ZNF292 & UP & $6 \mathrm{~h}$ & Transcription factor \\
\hline TFE3 & UP & $6 \mathrm{~h}$ & Transcription factor \\
\hline SOX2 & UP & $6 \mathrm{~h}$ & Transcription factor \\
\hline ID1 & DOWN & $3 h|4 h| 6 h$ & Transcription factor \\
\hline ID3 & DOWN & $3 h|4 h| 6 h$ & Transcription factor \\
\hline ID2 & DOWN & $4 h$ & Transcription factor \\
\hline PIM1 & DOWN & $4 h \mid 6 h$ & Cofactor/Kinase \\
\hline NUAK2 & DOWN & $4 h \mid 6 h$ & Kinase \\
\hline TGIF1 & DOWN & $4 h \mid 6 h$ & Transcription factor \\
\hline NACA & DOWN & $6 \mathrm{~h}$ & Cofactor \\
\hline TRIM24 & DOWN & $6 \mathrm{~h}$ & Cofactor \\
\hline CBX4 & DOWN & $6 \mathrm{~h}$ & Cofactor \\
\hline BCOR & DOWN & $6 \mathrm{~h}$ & Cofactor \\
\hline $\mathrm{CBX} 2$ & DOWN & $6 \mathrm{~h}$ & Cofactor \\
\hline AJUBA & DOWN & $6 \mathrm{~h}$ & Cofactor \\
\hline NAB2 & DOWN & $6 \mathrm{~h}$ & Cofactor \\
\hline IRF2BPL & DOWN & $6 \mathrm{~h}$ & Cofactor \\
\hline OTX2 & DOWN & $6 \mathrm{~h}$ & Transcription factor \\
\hline SOX21 & DOWN & $6 \mathrm{~h}$ & Transcription factor \\
\hline XBP1 & DOWN & $6 \mathrm{~h}$ & Transcription factor \\
\hline NR2F2 & DOWN & $6 \mathrm{~h}$ & Transcription factor \\
\hline
\end{tabular}


Table S6. Enrichr detected expression profiles of transcription factor in induced DIE cells (Adjusted $p$ value $<0.001$ )

- Category A: The expression/actiavtion of the transcription factor can cause the upregulation of a set of genes found to be differentially expressed induced DIE cells.

- Category B: The inhibition/deactivation of the transcription factor can cause the upregulation of a set of genes found to be differentially expressed induced DIE cells.

- Category C: The expression/actiavtion of the transcription factor can cause the downregulation of a set of genes found to be differentially expressed induced DIE cells.

- Category D: The inhibition/deactivation of the transcription factor can cause the downregulation of a set of genes found to be differentially expressed induced DIE cells.

- Other: No clear annotation of the expression/activaty status of the transcription factors.

\begin{tabular}{|c|c|c|c|c|c|}
\hline TF & $\begin{array}{l}\text { Exp. Profile } \\
\text { found at }\end{array}$ & & TF & $\begin{array}{l}\text { Exp. Profile } \\
\text { found at }\end{array}$ & Category \\
\hline ADAR & $3 \mathrm{~h}$ & A & ATF3 & $4 \mathrm{~h}$ & D \\
\hline DUX4 & $4 \mathrm{~h} \mid 6 \mathrm{~h}$ & A & BNC2 & $3 \mathrm{~h} \mid 4 \mathrm{~h}$ & D \\
\hline HIF1A & $2 \mathrm{~h} \mid 6 \mathrm{~h}$ & A & CREB1 & $3 \mathrm{~h}$ & D \\
\hline LIN28 & All 4 & A & DOT1L & $3 \mathrm{~h}|4 \mathrm{~h}| 6 \mathrm{~h}$ & D \\
\hline MYC & $2 \mathrm{~h}|3 \mathrm{~h}| 6 \mathrm{~h}$ & A & E2F1 & $3 \mathrm{~h}$ & D \\
\hline PAX7 & $2 \mathrm{~h}$ & A & EHF & $6 \mathrm{~h}$ & D \\
\hline RBM10 & $2 \mathrm{~h}$ & A & ELF3 & $3 \mathrm{~h}$ & D \\
\hline SOX5 & All 4 & A & ELK1 & $3 \mathrm{~h}|4 \mathrm{~h}| 6 \mathrm{~h}$ & D \\
\hline ZIC3 & All 4 & A & EPAS1 & $3 \mathrm{~h} \mid 4 \mathrm{~h}$ & D \\
\hline AFF4 & $2 \mathrm{~h}|3 \mathrm{~h}| 4 \mathrm{~h}$ & B & ERG & $6 \mathrm{~h}$ & D \\
\hline ASCL1 & $2 \mathrm{~h}$ & B & ESR1 & $3 h|4 h| 6 h$ & D \\
\hline ATF4 & $2 \mathrm{~h}$ & B & EZH2 & $3 \mathrm{~h}|4 \mathrm{~h}| 6 \mathrm{~h}$ & D \\
\hline BNC2 & $2 \mathrm{~h} \mid 3 \mathrm{~h}$ & B & FOXA2 & $6 \mathrm{~h}$ & D \\
\hline ELF3 & $2 \mathrm{~h}$ & B & FOXM1 & $3 \mathrm{~h}|4 \mathrm{~h}| 6 \mathrm{~h}$ & D \\
\hline EZH2 & $2 \mathrm{~h}$ & B & FOXP1 & $3 h|4 h| 6 h$ & D \\
\hline FOXP1 & $6 \mathrm{~h}$ & B & HNF4A & $3 \mathrm{~h}|4 \mathrm{~h}| 6 \mathrm{~h}$ & D \\
\hline HOXA7 & $2 \mathrm{~h}$ & B & IRF4 & $3 \mathrm{~h}|4 \mathrm{~h}| 6 \mathrm{~h}$ & D \\
\hline HSF1 & $3 \mathrm{~h}|4 \mathrm{~h}| 6 \mathrm{~h}$ & B & JUN & $6 \mathrm{~h}$ & D \\
\hline JUNB & All 4 & B & JUNB & $6 \mathrm{~h}$ & D \\
\hline KLF10 & All 4 & B & JUND & $6 \mathrm{~h}$ & D \\
\hline MEIS2 & All 4 & B & KLF10 & $3 \mathrm{~h} \mid 6 \mathrm{~h}$ & D \\
\hline MYCN & All 4 & B & KLF2 & $3 \mathrm{~h}|4 \mathrm{~h}| 6 \mathrm{~h}$ & D \\
\hline NFKB1 & $6 \mathrm{~h}$ & B & MBD2 & $4 \mathrm{~h}$ & D \\
\hline PITX2 & All 4 & B & MBNL1 & $6 \mathrm{~h}$ & D \\
\hline PPARD & $2 \mathrm{~h} \mid 3 \mathrm{~h}$ & B & MECOM & $6 \mathrm{~h}$ & D \\
\hline
\end{tabular}


Table S6 continued

\begin{tabular}{|c|c|c|c|c|c|}
\hline TF & $\begin{array}{l}\text { Exp. Profile } \\
\text { found at }\end{array}$ & & TF & $\begin{array}{l}\text { Exp. Profile } \\
\text { found at }\end{array}$ & Category \\
\hline SETDB1 & All 4 & B & MEIS2 & $3 h$ & D \\
\hline Sox11 & $2 \mathrm{~h}$ & B & MITF & $4 h \mid 6 h$ & $\mathrm{D}$ \\
\hline STAT3 & $2 \mathrm{~h}$ & B & MYB & $6 \mathrm{~h}$ & D \\
\hline TP53 & $4 h \mid 6 h$ & B & MYC & $3 h|4 h| 6 h$ & D \\
\hline ZEB2 & All 4 & B & MYCN & $4 \mathrm{~h} \mid 6 \mathrm{~h}$ & D \\
\hline ZMAT4 & $2 h|3 h| 4 h$ & B & NANOG & $3 h|4 h| 6 h$ & D \\
\hline ZNF253 & $2 \mathrm{~h}|3 \mathrm{~h}| 4 \mathrm{~h}$ & B & NFKB1 & $3 h|4 h| 6 h$ & $\mathrm{D}$ \\
\hline ZNF503 & All 4 & B & NFXL1 & $3 h|4 h| 6 h$ & D \\
\hline ZNF750 & $2 \mathrm{~h} \mid 6 \mathrm{~h}$ & B & $\mathrm{NR} 2 \mathrm{~F} 2$ & All 4 & D \\
\hline ATF6 & $3 \mathrm{~h} \mid 6 \mathrm{~h}$ & C & OTX2 & $4 h$ & D \\
\hline DLX4 & $3 h|4 h| 6 h$ & C & PCGF2 & $3 h|4 h| 6 h$ & $\mathrm{D}$ \\
\hline DUX4 & $4 \mathrm{~h} \mid 6 \mathrm{~h}$ & C & POU5F1 & $4 h \mid 6 h$ & D \\
\hline E2F1 & $4 h \mid 6 h$ & C & PPARD & $3 h$ & D \\
\hline EHF & $3 h$ & C & RARA & $4 h \mid 6 h$ & D \\
\hline FOXP1 & $6 \mathrm{~h}$ & C & RELA & $3 h \mid 4 h$ & D \\
\hline FOXP2 & $3 h$ & C & SALL4 & $3 h$ & D \\
\hline FOXP3 & $3 h \mid 4 h$ & C & SETDB1 & $6 \mathrm{~h}$ & D \\
\hline GATA4 & $3 h|4 h| 6 h$ & C & SON & $3 h|4 h| 6 h$ & D \\
\hline GATA6 & $3 h$ & C & SOX11 & $3 h|4 h| 6 h$ & D \\
\hline HIF1A & $4 \mathrm{~h} \mid 6 \mathrm{~h}$ & C & SOX4 & $3 h|4 h| 6 h$ & D \\
\hline HNF1A & $3 \mathrm{~h}|4 \mathrm{~h}| 6 \mathrm{~h}$ & C & SP1 & $6 \mathrm{~h}$ & D \\
\hline HNF1B & $3 \mathrm{~h}$ & C & SP3 & $4 h$ & D \\
\hline HNF4G & $3 h$ & C & STAT3 & $6 \mathrm{~h}$ & D \\
\hline KLF4 & $3 h|4 h| 6 h$ & C & SUZ12 & $3 h|4 h| 6 h$ & D \\
\hline MYB & All 4 & C & TBX3 & $3 h|4 h| 6 h$ & D \\
\hline MYC & $3 h \mid 4 h$ & C & TCF21 & $6 \mathrm{~h}$ & D \\
\hline NANOG & $4 h$ & C & TCF4 & $3 h \mid 4 h$ & D \\
\hline NME2 & $6 \mathrm{~h}$ & C & TCF7L2 & $4 h \mid 6 h$ & D \\
\hline NR4A2 & $3 h|4 h| 6 h$ & C & TP53 & $3 h \mid 4 h$ & D \\
\hline OVOL1 & $3 h|4 h| 6 h$ & C & TP63 & $4 h \mid 6 h$ & D \\
\hline OVOL2 & $3 h|4 h| 6 h$ & C & TSHZ3 & $4 h \mid 6 h$ & D \\
\hline POU1F1 & $3 h|4 h| 6 h$ & C & YY1 & $3 h|4 h| 6 h$ & D \\
\hline RARA & $6 \mathrm{~h}$ & C & ZBTB48 & $4 h \mid 6 h$ & D \\
\hline RBM10 & $3 h|4 h| 6 h$ & C & ZNF395 & $4 \mathrm{~h}$ & D \\
\hline SOX17 & $3 h \mid 4 h$ & C & ZNF658 & $3 h$ & D \\
\hline
\end{tabular}


Table S6 continued

\begin{tabular}{|c|c|c|c|c|c|}
\hline TF & $\begin{array}{l}\text { Exp. Profile } \\
\text { found at }\end{array}$ & Category & TF & $\begin{array}{l}\text { Exp. Profile } \\
\text { found at }\end{array}$ & Category \\
\hline SOX2 & $3 h \mid 4 h$ & C & ZXDC & $6 \mathrm{~h}$ & D \\
\hline SOX7 & $3 h|4 h| 6 h$ & C & ESR1 & $3 \mathrm{~h}$ & Other \\
\hline WT1 & All 4 & C & FLI1 & $2 \mathrm{~h}|3 \mathrm{~h}| 4 \mathrm{~h}$ & Other \\
\hline ZIC3 & $3 \mathrm{~h}|4 \mathrm{~h}| 6 \mathrm{~h}$ & C & IKZF1 & $3 \mathrm{~h} \mid 4 \mathrm{~h}$ & Other \\
\hline ZNF217 & $4 \mathrm{~h} \mid 6 \mathrm{~h}$ & C & MECP2 & $3 h \mid 4 h$ & Other \\
\hline AFF4 & $4 h \mid 6 h$ & D & THRA & $3 \mathrm{~h}$ & Other \\
\hline$A R$ & $3 \mathrm{~h}|4 \mathrm{~h}| 6 \mathrm{~h}$ & D & THRB & $3 \mathrm{~h}$ & Other \\
\hline ARID2 & $4 h \mid 6 h$ & D & TP63 & $6 \mathrm{~h}$ & Other \\
\hline ARX & $3 h \mid 6 h$ & D & TWIST2 & $3 h|4 h| 6 h$ & Other \\
\hline
\end{tabular}


Tables S7. Gene ontology results of differentially expressed genes in DIE cells

- Analysis Type: PANTHER Overrepresentation Test (Released 20200728)

- Annotation Version and Release Date: GO Ontology database DOI: 10.5281/ zenodo.4033054 Released 2020-09-10

- Analyzed List: upload_1 (Homo sapiens)

- Reference List: Homo sapiens (all genes in database)

- Test Type: FISHER

- Correction: False Discovery Rate (FDR < 0.05)

\begin{tabular}{|c|c|c|c|c|c|c|c|}
\hline \multirow{2}{*}{$\begin{array}{l}\text { 2h UP } \\
\text { General term }\end{array}$} & \multirow[b]{2}{*}{ GO \# } & \multirow[b]{2}{*}{$\operatorname{in}_{\text {Ref }}$} & \multirow[b]{2}{*}{$\begin{array}{l}\text { in } \\
\text { data }\end{array}$} & \multirow[b]{2}{*}{$\begin{array}{l}\text { exp- } \\
\text { ected }\end{array}$} & \multirow[b]{2}{*}{$\begin{array}{l}\text { fold } \\
\text { Enr. }\end{array}$} & \multirow[b]{2}{*}{$\begin{array}{l}\text { raw } \\
\text { P-value }\end{array}$} & \multirow[b]{2}{*}{ FDR } \\
\hline & & & & & & & \\
\hline Embryonic development & GO:0045927 & 270 & 5 & 0.53 & 9.42 & 1.9E-04 & 4.5E-02 \\
\hline Embryonic development & GO:0060324 & 50 & 3 & 0.1 & 30.51 & $1.5 \mathrm{E}-04$ & $3.9 \mathrm{E}-02$ \\
\hline Embryonic development & GO:0009790 & 1003 & 9 & 1.97 & 4.56 & 1.2E-04 & $3.2 \mathrm{E}-02$ \\
\hline Embryonic development & GO:0010720 & 568 & 7 & 1.12 & 6.27 & $1.2 \mathrm{E}-04$ & $3.1 \mathrm{E}-02$ \\
\hline Embryonic development & GO:0051130 & 1229 & 10 & 2.42 & 4.14 & 1.1E-04 & $2.9 \mathrm{E}-02$ \\
\hline Embryonic development & GO:0040016 & 5 & 2 & 0.01 & $>100$ & $7.8 \mathrm{E}-05$ & $2.4 \mathrm{E}-02$ \\
\hline Embryonic development & GO:0048856 & 5489 & 23 & 10.79 & 2.13 & 5.7E-05 & $2.0 \mathrm{E}-02$ \\
\hline Embryonic development & GO:0045595 & 1884 & 13 & 3.7 & 3.51 & 4.2E-05 & $1.8 \mathrm{E}-02$ \\
\hline Embryonic development & GO:0048639 & 180 & 5 & 0.35 & 14.13 & 3.0E-05 & $1.4 \mathrm{E}-02$ \\
\hline Embryonic development & GO:2000026 & 2107 & 14 & 4.14 & 3.38 & $3.0 \mathrm{E}-05$ & $1.4 \mathrm{E}-02$ \\
\hline Embryonic development & GO:0007275 & 5106 & 23 & 10.04 & 2.29 & $2.5 \mathrm{E}-05$ & $1.2 \mathrm{E}-02$ \\
\hline Embryonic development & GO:0051128 & 2436 & 16 & 4.79 & 3.34 & 7.1E-06 & $5.4 \mathrm{E}-03$ \\
\hline Embryonic development & GO:0060322 & 820 & 11 & 1.61 & 6.82 & 3.9E-07 & $5.7 \mathrm{E}-04$ \\
\hline Embryonic development & GO:0060284 & 978 & 12 & 1.92 & 6.24 & 2.7E-07 & 4.2E-04 \\
\hline CNS development & GO:0051960 & 959 & 12 & 1.89 & 6.36 & 2.2E-07 & 4.3E-04 \\
\hline CNS development & GO:0050767 & 847 & 11 & 1.67 & 6.6 & 5.4E-07 & 7.1E-04 \\
\hline CNS development & GO:0007420 & 775 & 10 & 1.52 & 6.56 & 2.1E-06 & $2.1 \mathrm{E}-03$ \\
\hline CNS development & GO:0007399 & 2437 & 16 & 4.79 & 3.34 & 7.1E-06 & $5.2 \mathrm{E}-03$ \\
\hline CNS development & GO:0048699 & 1599 & 13 & 3.14 & 4.13 & 7.4E-06 & $5.1 \mathrm{E}-03$ \\
\hline CNS development & GO:0022008 & 1703 & 13 & 3.35 & 3.88 & $1.5 \mathrm{E}-05$ & 8.6E-03 \\
\hline CNS development & GO:0007417 & 1025 & 10 & 2.02 & 4.96 & $2.4 \mathrm{E}-05$ & $1.2 \mathrm{E}-02$ \\
\hline CNS development & GO:0050769 & 490 & 7 & 0.96 & 7.27 & 4.6E-05 & $1.8 \mathrm{E}-02$ \\
\hline CNS development & GO:0045664 & 680 & 8 & 1.34 & 5.98 & 4.9E-05 & $1.7 \mathrm{E}-02$ \\
\hline CNS development & GO:0051962 & 558 & 7 & 1.1 & 6.38 & 1.0E-04 & $3.0 \mathrm{E}-02$ \\
\hline CNS development & GO:0045773 & 44 & 3 & 0.09 & 34.67 & 1.1E-04 & $3.0 \mathrm{E}-02$ \\
\hline CNS development & GO:0048731 & 4525 & 20 & 8.9 & 2.25 & $1.6 \mathrm{E}-04$ & 4.1E-02 \\
\hline CNS development & GO:0021537 & 265 & 5 & 0.52 & 9.6 & $1.8 \mathrm{E}-04$ & 4.4E-02 \\
\hline
\end{tabular}


Table S7 continued

\begin{tabular}{|c|c|c|c|c|c|c|c|}
\hline 2h UP & & & & & & & \\
\hline General term & GO \# & $\begin{array}{l}\text { in } \\
\text { Ref }\end{array}$ & $\begin{array}{l}\text { in } \\
\text { data }\end{array}$ & $\begin{array}{l}\text { exp- } \\
\text { ected }\end{array}$ & $\begin{array}{l}\text { fold } \\
\text { Enr. }\end{array}$ & $\begin{array}{l}\text { raw } \\
\text { P-value }\end{array}$ & FDR \\
\hline Cell cycle and cell division & GO:0022402 & 1069 & 15 & 2.1 & 7.14 & 8.6E-10 & $1.4 \mathrm{E}-05$ \\
\hline Cell cycle and cell division & GO:0051301 & 501 & 11 & 0.99 & 11.17 & $2.8 \mathrm{E}-09$ & 2.2E-05 \\
\hline Cell cycle and cell division & GO:0007049 & 1390 & 16 & 2.73 & 5.85 & 3.4E-09 & $1.8 \mathrm{E}-05$ \\
\hline Cell cycle and cell division & GO:0007346 & 640 & 11 & 1.26 & 8.74 & 3.3E-08 & $1.3 \mathrm{E}-04$ \\
\hline Cell cycle and cell division & GO:0051726 & 1210 & 14 & 2.38 & 5.88 & $4.1 \mathrm{E}-08$ & $1.3 \mathrm{E}-04$ \\
\hline Cell cycle and cell division & GO:0051276 & 1062 & 13 & 2.09 & 6.23 & 7.6E-08 & 2.0E-04 \\
\hline Cell cycle and cell division & GO:1903047 & 695 & 11 & 1.37 & 8.05 & 7.6E-08 & 1.7E-04 \\
\hline Cell cycle and cell division & GO:0000278 & 772 & 11 & 1.52 & 7.25 & $2.2 \mathrm{E}-07$ & $3.8 \mathrm{E}-04$ \\
\hline Cell cycle and cell division & GO:0006996 & 3576 & 21 & 7.03 & 2.99 & $6.6 \mathrm{E}-07$ & 8.1E-04 \\
\hline Cell cycle and cell division & GO:0007059 & 278 & 7 & 0.55 & 12.81 & $1.2 \mathrm{E}-06$ & $1.4 \mathrm{E}-03$ \\
\hline Cell cycle and cell division & GO:0010564 & 770 & 10 & 1.51 & 6.6 & 2.0E-06 & 2.1E-03 \\
\hline Cell cycle and cell division & GO:0071103 & 309 & 7 & 0.61 & 11.52 & $2.5 \mathrm{E}-06$ & 2.3E-03 \\
\hline Cell cycle and cell division & GO:0007017 & 806 & 10 & 1.58 & 6.31 & $2.9 \mathrm{E}-06$ & 2.6E-03 \\
\hline Cell cycle and cell division & GO:0098813 & 220 & 6 & 0.43 & 13.87 & 4.9E-06 & 4.1E-03 \\
\hline Cell cycle and cell division & GO:0033043 & 1332 & 12 & 2.62 & 4.58 & $6.6 \mathrm{E}-06$ & $5.2 \mathrm{E}-03$ \\
\hline Cell cycle and cell division & GO:0016043 & 5699 & 25 & 11.21 & 2.23 & $8.8 \mathrm{E}-06$ & $5.8 \mathrm{E}-03$ \\
\hline Cell cycle and cell division & GO:0000819 & 140 & 5 & 0.28 & 18.16 & $9.2 \mathrm{E}-06$ & $5.9 \mathrm{E}-03$ \\
\hline Cell cycle and cell division & GO:0051383 & 19 & 3 & 0.04 & 80.3 & $1.1 \mathrm{E}-05$ & $6.4 \mathrm{E}-03$ \\
\hline Cell cycle and cell division & GO:0071840 & 5919 & 25 & 11.64 & 2.15 & $1.5 \mathrm{E}-05$ & 8.6E-03 \\
\hline Cell cycle and cell division & GO:1901990 & 420 & 7 & 0.83 & 8.48 & $1.8 \mathrm{E}-05$ & $9.6 \mathrm{E}-03$ \\
\hline Cell cycle and cell division & GO:0007088 & 168 & 5 & 0.33 & 15.14 & $2.2 \mathrm{E}-05$ & $1.2 \mathrm{E}-02$ \\
\hline Cell cycle and cell division & GO:0000280 & 302 & 6 & 0.59 & 10.1 & 2.9E-05 & $1.4 \mathrm{E}-02$ \\
\hline Cell cycle and cell division & GO:1901987 & 458 & 7 & 0.9 & 7.77 & $3.0 \mathrm{E}-05$ & $1.3 \mathrm{E}-02$ \\
\hline Cell cycle and cell division & GO:0051783 & 195 & 5 & 0.38 & 13.04 & 4.3E-05 & $1.8 \mathrm{E}-02$ \\
\hline Cell cycle and cell division & GO:0051651 & 96 & 4 & 0.19 & 21.19 & 4.4E-05 & $1.8 \mathrm{E}-02$ \\
\hline Cell cycle and cell division & GO:0051642 & 32 & 3 & 0.06 & 47.68 & 4.4E-05 & $1.8 \mathrm{E}-02$ \\
\hline Cell cycle and cell division & GO:0010389 & 198 & 5 & 0.39 & 12.84 & 4.7E-05 & $1.8 \mathrm{E}-02$ \\
\hline Cell cycle and cell division & GO:0048285 & 332 & 6 & 0.65 & 9.19 & $4.8 \mathrm{E}-05$ & $1.8 \mathrm{E}-02$ \\
\hline Cell cycle and cell division & GO:0061842 & 33 & 3 & 0.06 & 46.23 & $4.8 \mathrm{E}-05$ & 1.7E-02 \\
\hline Cell cycle and cell division & GO:0006325 & 701 & 8 & 1.38 & 5.8 & $6.0 \mathrm{E}-05$ & 2.0E-02 \\
\hline Cell cycle and cell division & GO:0007018 & 346 & 6 & 0.68 & 8.82 & $6.0 \mathrm{E}-05$ & 2.0E-02 \\
\hline Cell cycle and cell division & GO:0051983 & 108 & 4 & 0.21 & 18.84 & $6.8 \mathrm{E}-05$ & $2.2 \mathrm{E}-02$ \\
\hline Cell cycle and cell division & GO:1902749 & 215 & 5 & 0.42 & 11.83 & $6.8 \mathrm{E}-05$ & $2.2 \mathrm{E}-02$ \\
\hline Cell cycle and cell division & GO:0000070 & 111 & 4 & 0.22 & 18.33 & 7.5E-05 & $2.4 \mathrm{E}-02$ \\
\hline
\end{tabular}


Table S7 continued

\begin{tabular}{|c|c|c|c|c|c|c|c|}
\hline 2h UP & & & & & & & \\
\hline General term & GO \# & $\begin{array}{l}\text { in } \\
\text { Ref }\end{array}$ & $\begin{array}{l}\text { in } \\
\text { data }\end{array}$ & $\begin{array}{l}\text { exp- } \\
\text { ected }\end{array}$ & $\begin{array}{l}\text { fold } \\
\text { Enr. }\end{array}$ & $\begin{array}{l}\text { raw } \\
\text { P-value }\end{array}$ & FDR \\
\hline Cell cycle and cell division & GO:0033044 & 367 & 6 & 0.72 & 8.31 & 8.3E-05 & $2.5 \mathrm{E}-02$ \\
\hline Cell cycle and cell division & GO:0051661 & 6 & 2 & 0.01 & $>100$ & $1.0 \mathrm{E}-04$ & 3.0E-02 \\
\hline Cell cycle and cell division & GO:0030261 & 44 & 3 & 0.09 & 34.67 & $1.1 \mathrm{E}-04$ & 3.0E-02 \\
\hline Cell cycle and cell division & GO:0045786 & 579 & 7 & 1.14 & 6.15 & $1.3 \mathrm{E}-04$ & $3.4 \mathrm{E}-02$ \\
\hline Cell cycle and cell division & GO:0051640 & 605 & 7 & 1.19 & 5.88 & 1.7E-04 & 4.2E-02 \\
\hline Cell cycle and cell division & GO:0044848 & 270 & 5 & 0.53 & 9.42 & 1.9E-04 & 4.7E-02 \\
\hline Cell cycle and cell division & GO:0022403 & 270 & 5 & 0.53 & 9.42 & $1.9 \mathrm{E}-04$ & 4.5E-02 \\
\hline Cell cycle and cell division & GO:0098763 & 270 & 5 & 0.53 & 9.42 & $1.9 \mathrm{E}-04$ & 4.6E-02 \\
\hline Cell cycle and cell division & GO:0006928 & 1599 & 11 & 3.14 & 3.5 & $2.0 \mathrm{E}-04$ & 4.5E-02 \\
\hline Cell cycle and cell division & GO:0051310 & 55 & 3 & 0.11 & 27.74 & 2.0E-04 & 4.5E-02 \\
\hline Cell cycle and cell division & GO:0006265 & 9 & 2 & 0.02 & $>100$ & $2.0 \mathrm{E}-04$ & 4.5E-02 \\
\hline Cell cycle and cell division & GO:0007050 & 145 & 4 & 0.29 & 14.03 & 2.1E-04 & 4.5E-02 \\
\hline
\end{tabular}

\begin{tabular}{|c|c|c|c|c|c|c|c|}
\hline \multirow{2}{*}{$\begin{array}{l}\text { 2h DOWN } \\
\text { General term }\end{array}$} & \multirow[b]{2}{*}{ GO \# } & \multirow[b]{2}{*}{$\begin{array}{l}\text { in } \\
\text { Ref }\end{array}$} & \multirow[b]{2}{*}{$\begin{array}{l}\text { in } \\
\text { data }\end{array}$} & \multirow[b]{2}{*}{$\begin{array}{l}\text { exp- } \\
\text { ected }\end{array}$} & \multirow[b]{2}{*}{$\begin{array}{l}\text { fold } \\
\text { Enr. }\end{array}$} & \multirow[b]{2}{*}{$\begin{array}{l}\text { raw } \\
\text { P-value }\end{array}$} & \multirow[b]{2}{*}{ FDR } \\
\hline & & & & & & & \\
\hline Protein production and regulation & GO:0072657 & 528 & 4 & 0.2 & 19.75 & 2.7E-05 & 4.3E-02 \\
\hline Protein production and regulation & GO:0006413 & 145 & 3 & 0.06 & 53.92 & $1.9 \mathrm{E}-05$ & $3.8 \mathrm{E}-02$ \\
\hline Protein production and regulation & GO:0070972 & 142 & 3 & 0.05 & 55.06 & $1.8 \mathrm{E}-05$ & 4.1E-02 \\
\hline Protein production and regulation & GO:0072599 & 115 & 3 & 0.04 & 67.99 & 9.7E-06 & $3.1 \mathrm{E}-02$ \\
\hline Protein production and regulation & GO:0045047 & 111 & 3 & 0.04 & 70.44 & 8.7E-06 & 4.6E-02 \\
\hline RNA productio and processing & GO:0000184 & 121 & 3 & 0.05 & 64.62 & $1.1 \mathrm{E}-05$ & $3.0 \mathrm{E}-02$ \\
\hline Viral processes & GO:0019080 & 154 & 3 & 0.06 & 50.77 & $2.3 \mathrm{E}-05$ & 4.0E-02 \\
\hline Viral processes & GO:0019083 & 115 & 3 & 0.04 & 67.99 & $9.7 \mathrm{E}-06$ & 3.9E-02 \\
\hline
\end{tabular}

\begin{tabular}{|c|c|c|c|c|c|c|c|}
\hline \multirow{2}{*}{$\begin{array}{l}\text { 3h UP } \\
\text { General term }\end{array}$} & & & & & & & \\
\hline & GO \# & $\begin{array}{l}\text { in } \\
\text { Ref }\end{array}$ & $\begin{array}{l}\text { in } \\
\text { data }\end{array}$ & $\begin{array}{l}\text { exp- } \\
\text { ected }\end{array}$ & $\begin{array}{l}\text { fold } \\
\text { Enr. }\end{array}$ & $\begin{array}{l}\text { raw } \\
\text { P-value }\end{array}$ & FDR \\
\hline Embryonic development & GO:0060322 & 820 & 11 & 2.87 & 3.83 & $1.4 \mathrm{E}-04$ & $4.8 \mathrm{E}-02$ \\
\hline Embryonic development & GO:0060284 & 978 & 13 & 3.42 & 3.8 & $3.4 \mathrm{E}-05$ & $2.2 \mathrm{E}-02$ \\
\hline CNS development & GO:0051960 & 959 & 13 & 3.36 & 3.87 & $2.8 \mathrm{E}-05$ & $2.0 \mathrm{E}-02$ \\
\hline CNS development & GO:0050767 & 847 & 12 & 2.97 & 4.05 & $3.9 \mathrm{E}-05$ & $2.2 \mathrm{E}-02$ \\
\hline CNS development & GO:0045664 & 680 & 10 & 2.38 & 4.2 & $1.4 \mathrm{E}-04$ & $4.9 \mathrm{E}-02$ \\
\hline Cell cycle and cell division & GO:0051276 & 1062 & 17 & 3.72 & 4.57 & $1.3 \mathrm{E}-07$ & $1.0 \mathrm{E}-03$ \\
\hline Cell cycle and cell division & GO:0022402 & 1069 & 17 & 3.74 & 4.54 & $1.4 \mathrm{E}-07$ & 7.6E-04 \\
\hline
\end{tabular}


Table S7 continued

\begin{tabular}{|c|c|c|c|c|c|c|c|}
\hline \multirow{2}{*}{$\begin{array}{l}\text { 3h UP } \\
\text { General term }\end{array}$} & \multirow[b]{2}{*}{ GO \# } & \multirow[b]{2}{*}{$\begin{array}{l}\text { in } \\
\text { Ref }\end{array}$} & \multirow[b]{2}{*}{$\begin{array}{l}\text { in } \\
\text { data }\end{array}$} & \multirow[b]{2}{*}{$\begin{array}{l}\text { exp- } \\
\text { ected }\end{array}$} & \multirow[b]{2}{*}{$\begin{array}{l}\text { fold } \\
\text { Enr. }\end{array}$} & \multirow[b]{2}{*}{$\begin{array}{l}\text { raw } \\
\text { P-value }\end{array}$} & \multirow[b]{2}{*}{ FDR } \\
\hline & & & & & & & \\
\hline Cell cycle and cell division & GO:0007049 & 1390 & 19 & 4.87 & 3.9 & $2.3 \mathrm{E}-07$ & $9.2 \mathrm{E}-04$ \\
\hline Cell cycle and cell division & GO:1903047 & 695 & 13 & 2.43 & 5.34 & $9.1 \mathrm{E}-07$ & 2.9E-03 \\
\hline Cell cycle and cell division & GO:0006325 & 701 & 13 & 2.45 & 5.3 & 1.0E-06 & $2.6 \mathrm{E}-03$ \\
\hline Cell cycle and cell division & GO:0006260 & 223 & 8 & 0.78 & 10.25 & $1.4 \mathrm{E}-06$ & $2.8 \mathrm{E}-03$ \\
\hline Cell cycle and cell division & GO:0051301 & 501 & 11 & 1.75 & 6.27 & 1.5E-06 & 2.7E-03 \\
\hline Cell cycle and cell division & GO:0000278 & 772 & 13 & 2.7 & 4.81 & $2.9 \mathrm{E}-06$ & 4.1E-03 \\
\hline Cell cycle and cell division & GO:0007017 & 806 & 13 & 2.82 & 4.61 & 4.5E-06 & 5.1E-03 \\
\hline Cell cycle and cell division & GO:0006996 & 3576 & 29 & 12.52 & 2.32 & 5.5E-06 & $5.8 \mathrm{E}-03$ \\
\hline Cell cycle and cell division & GO:0033047 & 73 & 5 & 0.26 & 19.56 & $7.8 \mathrm{E}-06$ & 7.7E-03 \\
\hline Cell cycle and cell division & GO:0071103 & 309 & 8 & 1.08 & 7.39 & $1.5 \mathrm{E}-05$ & $1.3 \mathrm{E}-02$ \\
\hline Cell cycle and cell division & GO:0033045 & 85 & 5 & 0.3 & 16.8 & 1.6E-05 & $1.3 \mathrm{E}-02$ \\
\hline Cell cycle and cell division & GO:0000226 & 568 & 10 & 1.99 & 5.03 & 3.1E-05 & $2.2 \mathrm{E}-02$ \\
\hline Cell cycle and cell division & GO:0051983 & 108 & 5 & 0.38 & 13.22 & 4.7E-05 & 2.6E-02 \\
\hline Cell cycle and cell division & GO:0032508 & 110 & 5 & 0.39 & 12.98 & 5.1E-05 & 2.7E-02 \\
\hline Cell cycle and cell division & GO:0051383 & 19 & 3 & 0.07 & 45.1 & $6.0 \mathrm{E}-05$ & 3.0E-02 \\
\hline Cell cycle and cell division & GO:0032392 & 118 & 5 & 0.41 & 12.1 & 7.1E-05 & $3.3 \mathrm{E}-02$ \\
\hline Cell cycle and cell division & GO:0051726 & 1210 & 14 & 4.24 & 3.3 & 7.3E-05 & 3.3E-02 \\
\hline Cell cycle and cell division & GO:0010564 & 770 & 11 & 2.7 & 4.08 & 7.9E-05 & $3.5 \mathrm{E}-02$ \\
\hline Cell cycle and cell division & GO:1905269 & 122 & 5 & 0.43 & 11.71 & $8.2 \mathrm{E}-05$ & $3.5 \mathrm{E}-02$ \\
\hline Cell cycle and cell division & GO:0007346 & 640 & 10 & 2.24 & 4.46 & 8.3E-05 & $3.5 \mathrm{E}-02$ \\
\hline Cell cycle and cell division & GO:1901990 & 420 & 8 & 1.47 & 5.44 & $1.2 \mathrm{E}-04$ & 4.5E-02 \\
\hline RNA production and processing & GO:0010467 & 2109 & 19 & 7.38 & 2.57 & $9.2 \mathrm{E}-05$ & 3.7E-02 \\
\hline RNA production and processing & GO:1901360 & 3218 & 25 & 11.27 & 2.22 & $6.4 \mathrm{E}-05$ & 3.1E-02 \\
\hline RNA production and processing & GO:0032239 & 19 & 3 & 0.07 & 45.1 & $6.0 \mathrm{E}-05$ & $3.1 \mathrm{E}-02$ \\
\hline RNA production and processing & GO:0046831 & 16 & 3 & 0.06 & 53.56 & $3.8 \mathrm{E}-05$ & $2.2 \mathrm{E}-02$ \\
\hline RNA production and processing & GO:0016070 & 1621 & 17 & 5.68 & 3 & 3.7E-05 & 2.3E-02 \\
\hline RNA production and processing & GO:0016071 & 701 & 11 & 2.45 & 4.48 & $3.4 \mathrm{E}-05$ & 2.3E-02 \\
\hline RNA production and processing & GO:0006725 & 2986 & 25 & 10.45 & 2.39 & 1.7E-05 & 1.3E-02 \\
\hline RNA production and processing & GO:0046483 & 2936 & 25 & 10.28 & 2.43 & $1.2 \mathrm{E}-05$ & $1.2 \mathrm{E}-02$ \\
\hline RNA production and processing & GO:0006396 & 929 & 14 & 3.25 & 4.3 & 4.0E-06 & 4.9E-03 \\
\hline RNA production and processing & GO:0006139 & 2740 & 25 & 9.59 & 2.61 & $3.6 \mathrm{E}-06$ & 4.8E-03 \\
\hline RNA production and processing & GO:0008380 & 410 & 10 & 1.44 & 6.97 & $1.9 \mathrm{E}-06$ & 3.1E-03 \\
\hline RNA production and processing & GO:0006397 & 489 & 11 & 1.71 & 6.43 & $1.2 \mathrm{E}-06$ & $2.8 \mathrm{E}-03$ \\
\hline RNA production and processing & GO:0090304 & 2237 & 25 & 7.83 & 3.19 & 8.0E-08 & 1.3E-03 \\
\hline
\end{tabular}


Table S7 continued

\begin{tabular}{|c|c|c|c|c|c|c|c|}
\hline \multirow{2}{*}{$\begin{array}{l}\text { 3h UP } \\
\text { General term }\end{array}$} & \multirow[b]{2}{*}{ GO \# } & \multirow[b]{2}{*}{$\begin{array}{l}\text { in } \\
\text { Ref }\end{array}$} & \multirow[b]{2}{*}{$\begin{array}{l}\text { in } \\
\text { data }\end{array}$} & \multirow[b]{2}{*}{$\begin{array}{l}\text { exp- } \\
\text { ected }\end{array}$} & \multirow[b]{2}{*}{$\begin{array}{l}\text { fold } \\
\text { Enr. }\end{array}$} & \multirow[b]{2}{*}{$\begin{array}{l}\text { raw } \\
\text { P-value }\end{array}$} & \multirow[b]{2}{*}{ FDR } \\
\hline & & & & & & & \\
\hline General cellular processes & GO:0043170 & 6337 & 40 & 22.19 & 1.8 & 1.7E-05 & $1.3 \mathrm{E}-02$ \\
\hline General cellular processes & GO:0010558 & 1566 & 16 & 5.48 & 2.92 & 9.0E-05 & 3.7E-02 \\
\hline General cellular processes & GO:0019222 & 7060 & 41 & 24.72 & 1.66 & $1.0 \mathrm{E}-04$ & $3.9 \mathrm{E}-02$ \\
\hline General cellular processes & GO:0031327 & 1626 & 16 & 5.69 & 2.81 & $1.4 \mathrm{E}-04$ & 4.8E-02 \\
\hline Response to DNA damage & GO:0006974 & 785 & 11 & 2.75 & 4 & $9.3 \mathrm{E}-05$ & 3.6E-02 \\
\hline
\end{tabular}

\begin{tabular}{|c|c|c|c|c|c|c|c|}
\hline \multirow{2}{*}{$\begin{array}{l}\text { 3h DOWN } \\
\text { General term }\end{array}$} & \multirow[b]{2}{*}{ GO \# } & \multirow[b]{2}{*}{$\begin{array}{l}\text { in } \\
\text { Ref }\end{array}$} & \multirow[b]{2}{*}{$\begin{array}{l}\text { in } \\
\text { data }\end{array}$} & \multirow[b]{2}{*}{$\begin{array}{l}\text { exp- } \\
\text { ected }\end{array}$} & \multirow[b]{2}{*}{$\begin{array}{l}\text { fold } \\
\text { Enr. }\end{array}$} & \multirow[b]{2}{*}{$\begin{array}{l}\text { raw } \\
\text { P-value }\end{array}$} & \multirow[b]{2}{*}{ FDR } \\
\hline & & & & & & & \\
\hline Protein production and regulation & GO:0006614 & 96 & 7 & 0.16 & 43.44 & $3.5 \mathrm{E}-10$ & $1.8 \mathrm{E}-06$ \\
\hline Protein production and regulation & GO:0006613 & 101 & 7 & 0.17 & 41.29 & $4.9 \mathrm{E}-10$ & $1.9 \mathrm{E}-06$ \\
\hline Protein production and regulation & GO:0045047 & 111 & 7 & 0.19 & 37.57 & $9.1 \mathrm{E}-10$ & 2.9E-06 \\
\hline Protein production and regulation & GO:0072599 & 115 & 7 & 0.19 & 36.26 & $1.1 \mathrm{E}-09$ & 2.6E-06 \\
\hline Protein production and regulation & GO:0070972 & 142 & 7 & 0.24 & 29.37 & 4.6E-09 & $6.2 \mathrm{E}-06$ \\
\hline Protein production and regulation & GO:0006413 & 145 & 7 & 0.24 & 28.76 & $5.3 \mathrm{E}-09$ & $6.1 \mathrm{E}-06$ \\
\hline Protein production and regulation & GO:0006612 & 184 & 7 & 0.31 & 22.66 & $2.6 \mathrm{E}-08$ & 2.3E-05 \\
\hline Protein production and regulation & GO:0090150 & 294 & 8 & 0.49 & 16.21 & $2.9 \mathrm{E}-08$ & 2.4E-05 \\
\hline Protein production and regulation & GO:0046907 & 1528 & 14 & 2.56 & 5.46 & 7.1E-08 & 4.9E-05 \\
\hline Protein production and regulation & GO:0006605 & 374 & 8 & 0.63 & 12.74 & $1.8 \mathrm{E}-07$ & $1.1 \mathrm{E}-04$ \\
\hline Protein production and regulation & GO:0072657 & 528 & 9 & 0.89 & 10.15 & $1.8 \mathrm{E}-07$ & $1.1 \mathrm{E}-04$ \\
\hline Protein production and regulation & GO:0051641 & 3007 & 18 & 5.05 & 3.57 & $2.8 \mathrm{E}-07$ & $1.4 \mathrm{E}-04$ \\
\hline Protein production and regulation & GO:0033365 & 776 & 10 & 1.3 & 7.68 & 4.2E-07 & $1.9 \mathrm{E}-04$ \\
\hline Protein production and regulation & GO:0072594 & 453 & 8 & 0.76 & 10.52 & $7.4 \mathrm{E}-07$ & 3.0E-04 \\
\hline Protein production and regulation & GO:0034613 & 1646 & 13 & 2.76 & 4.71 & $1.3 \mathrm{E}-06$ & 5.0E-04 \\
\hline Protein production and regulation & GO:0070727 & 1655 & 13 & 2.78 & 4.68 & $1.4 \mathrm{E}-06$ & 4.9E-04 \\
\hline Protein production and regulation & GO:0051649 & 2378 & 15 & 3.99 & 3.76 & $2.5 \mathrm{E}-06$ & $8.4 \mathrm{E}-04$ \\
\hline Protein production and regulation & GO:0006412 & 394 & 7 & 0.66 & 10.58 & $3.9 \mathrm{E}-06$ & $1.3 \mathrm{E}-03$ \\
\hline Protein production and regulation & GO:0043043 & 419 & 7 & 0.7 & 9.95 & 5.7E-06 & $1.8 \mathrm{E}-03$ \\
\hline Protein production and regulation & GO:0034645 & 1639 & 12 & 2.75 & 4.36 & $8.6 \mathrm{E}-06$ & $2.5 \mathrm{E}-03$ \\
\hline Protein production and regulation & GO:0002181 & 75 & 4 & 0.13 & 31.77 & $9.1 \mathrm{E}-06$ & 2.6E-03 \\
\hline Protein production and regulation & GO:0009059 & 1689 & 12 & 2.84 & 4.23 & $1.2 \mathrm{E}-05$ & $3.2 \mathrm{E}-03$ \\
\hline Protein production and regulation & GO:1901576 & 2802 & 15 & 4.7 & 3.19 & $1.9 \mathrm{E}-05$ & 4.8E-03 \\
\hline Protein production and regulation & GO:0009058 & 2861 & 15 & 4.8 & 3.12 & $2.5 \mathrm{E}-05$ & $6.0 \mathrm{E}-03$ \\
\hline Protein production and regulation & GO:0006518 & 539 & 7 & 0.9 & 7.74 & $2.9 \mathrm{E}-05$ & $6.8 \mathrm{E}-03$ \\
\hline
\end{tabular}


Table S7 continued

\begin{tabular}{|c|c|c|c|c|c|c|c|}
\hline \multirow{2}{*}{$\begin{array}{l}\text { 3h DOWN } \\
\text { General term }\end{array}$} & \multirow[b]{2}{*}{ GO \# } & \multirow[b]{2}{*}{$\begin{array}{l}\text { in } \\
\text { Ref }\end{array}$} & \multirow[b]{2}{*}{$\begin{array}{l}\text { in } \\
\text { data }\end{array}$} & \multirow[b]{2}{*}{$\begin{array}{l}\text { exp- } \\
\text { ected }\end{array}$} & \multirow[b]{2}{*}{$\begin{array}{l}\text { fold } \\
\text { Enr. }\end{array}$} & \multirow[b]{2}{*}{$\begin{array}{l}\text { raw } \\
\text { P-value }\end{array}$} & \multirow[b]{2}{*}{ FDR } \\
\hline & & & & & & & \\
\hline Protein production and regulation & GO:0006886 & 992 & 9 & 1.67 & 5.4 & $2.9 \mathrm{E}-05$ & $6.9 \mathrm{E}-03$ \\
\hline Protein production and regulation & GO:0006810 & 4572 & 19 & 7.67 & 2.48 & $3.0 \mathrm{E}-05$ & 7.0E-03 \\
\hline Protein production and regulation & GO:0043604 & 547 & 7 & 0.92 & 7.62 & 3.1E-05 & 7.1E-03 \\
\hline Protein production and regulation & GO:0008104 & 2200 & 13 & 3.69 & 3.52 & $3.2 \mathrm{E}-05$ & 7.1E-03 \\
\hline Protein production and regulation & GO:0051234 & 4704 & 19 & 7.9 & 2.41 & 4.6E-05 & $9.8 \mathrm{E}-03$ \\
\hline Protein production and regulation & GO:0034622 & 823 & 8 & 1.38 & 5.79 & $5.5 \mathrm{E}-05$ & 1.1E-02 \\
\hline Protein production and regulation & GO:1901566 & 1407 & 10 & 2.36 & 4.23 & 7.7E-05 & $1.6 \mathrm{E}-02$ \\
\hline Protein production and regulation & GO:0051179 & 5862 & 21 & 9.84 & 2.13 & $9.1 \mathrm{E}-05$ & $1.8 \mathrm{E}-02$ \\
\hline Protein production and regulation & GO:0033036 & 2564 & 13 & 4.3 & 3.02 & $1.6 \mathrm{E}-04$ & $2.8 \mathrm{E}-02$ \\
\hline Protein production and regulation & GO:0045184 & 1594 & 10 & 2.68 & 3.74 & $2.1 \mathrm{E}-04$ & 3.7E-02 \\
\hline Protein production and regulation & GO:0065003 & 1304 & 9 & 2.19 & 4.11 & $2.4 \mathrm{E}-04$ & 4.0E-02 \\
\hline RNA productio and processing & GO:0000375 & 306 & 5 & 0.51 & 9.73 & $1.6 \mathrm{E}-04$ & $2.8 \mathrm{E}-02$ \\
\hline RNA productio and processing & GO:0006397 & 489 & 6 & 0.82 & 7.31 & $1.6 \mathrm{E}-04$ & $2.8 \mathrm{E}-02$ \\
\hline RNA productio and processing & GO:0000398 & 303 & 5 & 0.51 & 9.83 & $1.5 \mathrm{E}-04$ & $2.8 \mathrm{E}-02$ \\
\hline RNA productio and processing & GO:0000377 & 303 & 5 & 0.51 & 9.83 & $1.5 \mathrm{E}-04$ & $2.8 \mathrm{E}-02$ \\
\hline RNA productio and processing & GO:0009057 & 1058 & 9 & 1.78 & 5.07 & $4.9 \mathrm{E}-05$ & 1.0E-02 \\
\hline RNA productio and processing & GO:0071840 & 5919 & 22 & 9.94 & 2.21 & $3.1 \mathrm{E}-05$ & 7.0E-03 \\
\hline RNA productio and processing & GO:0034660 & 525 & 7 & 0.88 & 7.94 & $2.4 \mathrm{E}-05$ & 5.9E-03 \\
\hline RNA productio and processing & GO:0034470 & 433 & 7 & 0.73 & 9.63 & 7.1E-06 & 2.1E-03 \\
\hline RNA productio and processing & GO:0042254 & 336 & 7 & 0.56 & 12.41 & $1.4 \mathrm{E}-06$ & 4.9E-04 \\
\hline RNA productio and processing & GO:1901361 & 491 & 8 & 0.82 & 9.71 & $1.3 \mathrm{E}-06$ & $4.8 \mathrm{E}-04$ \\
\hline RNA productio and processing & GO:0019439 & 458 & 8 & 0.77 & 10.41 & 8.0E-07 & 3.0E-04 \\
\hline RNA productio and processing & GO:1901360 & 3218 & 18 & 5.4 & 3.33 & 8.0E-07 & $3.1 \mathrm{E}-04$ \\
\hline RNA productio and processing & GO:0046700 & 440 & 8 & 0.74 & 10.83 & $6.0 \mathrm{E}-07$ & $2.6 \mathrm{E}-04$ \\
\hline RNA productio and processing & GO:0010467 & 2109 & 15 & 3.54 & 4.24 & $5.5 \mathrm{E}-07$ & 2.4E-04 \\
\hline RNA productio and processing & GO:0016072 & 270 & 7 & 0.45 & 15.45 & $3.3 \mathrm{E}-07$ & $1.5 \mathrm{E}-04$ \\
\hline RNA productio and processing & GO:0006725 & 2986 & 18 & 5.01 & 3.59 & $2.6 \mathrm{E}-07$ & $1.3 \mathrm{E}-04$ \\
\hline RNA productio and processing & GO:0006364 & 260 & 7 & 0.44 & 16.04 & $2.6 \mathrm{E}-07$ & 1.4E-04 \\
\hline RNA productio and processing & GO:0034655 & 383 & 8 & 0.64 & 12.44 & $2.1 \mathrm{E}-07$ & $1.2 \mathrm{E}-04$ \\
\hline RNA productio and processing & GO:0046483 & 2936 & 18 & 4.93 & 3.65 & $2.0 \mathrm{E}-07$ & 1.1E-04 \\
\hline RNA productio and processing & GO:0090304 & 2237 & 16 & 3.75 & 4.26 & 1.7E-07 & $1.1 \mathrm{E}-04$ \\
\hline RNA productio and processing & GO:0016070 & 1621 & 14 & 2.72 & 5.15 & $1.5 \mathrm{E}-07$ & $9.8 \mathrm{E}-05$ \\
\hline RNA productio and processing & GO:0006139 & 2740 & 18 & 4.6 & 3.91 & $6.8 \mathrm{E}-08$ & 4.9E-05 \\
\hline RNA productio and processing & GO:0022613 & 469 & 9 & 0.79 & 11.43 & 6.7E-08 & 5.0E-05 \\
\hline
\end{tabular}


Table S7 continued

\begin{tabular}{|c|c|c|c|c|c|c|c|}
\hline \multirow{2}{*}{$\begin{array}{l}\text { 3h DOWN } \\
\text { General term }\end{array}$} & \multirow[b]{2}{*}{ GO \# } & \multirow[b]{2}{*}{$\begin{array}{l}\text { in } \\
\text { Ref }\end{array}$} & \multirow[b]{2}{*}{$\begin{array}{l}\text { in } \\
\text { data }\end{array}$} & \multirow[b]{2}{*}{$\begin{array}{l}\text { exp- } \\
\text { ected }\end{array}$} & \multirow[b]{2}{*}{$\begin{array}{l}\text { fold } \\
\text { Enr. }\end{array}$} & \multirow[b]{2}{*}{$\begin{array}{l}\text { raw } \\
\text { P-value }\end{array}$} & \multirow[b]{2}{*}{ FDR } \\
\hline & & & & & & & \\
\hline RNA productio and processing & GO:0006401 & 251 & 8 & 0.42 & 18.99 & $8.8 \mathrm{E}-09$ & 8.3E-06 \\
\hline RNA production and processing & GO:0006402 & 220 & 8 & 0.37 & 21.66 & 3.3E-09 & 4.7E-06 \\
\hline RNA productio and processing & GO:0006396 & 929 & 13 & 1.56 & 8.34 & 1.7E-09 & 2.7E-06 \\
\hline RNA productio and processing & GO:0000956 & 200 & 8 & 0.34 & 23.83 & $1.6 \mathrm{E}-09$ & 3.1E-06 \\
\hline RNA productio and processing & GO:0016071 & 701 & 13 & 1.18 & 11.05 & $5.8 \mathrm{E}-11$ & 4.6E-07 \\
\hline RNA productio and processing & GO:0000184 & 121 & 8 & 0.2 & 39.39 & $3.5 \mathrm{E}-11$ & $5.5 \mathrm{E}-07$ \\
\hline Cellular respirarion and energy production & GO:0006119 & 121 & 7 & 0.2 & 34.46 & $1.6 \mathrm{E}-09$ & $2.8 \mathrm{E}-06$ \\
\hline Cellular respirarion and energy production & GO:0046034 & 210 & 7 & 0.35 & 19.86 & $6.2 \mathrm{E}-08$ & 5.0E-05 \\
\hline Cellular respirarion and energy production & GO:0006091 & 414 & 7 & 0.69 & 10.07 & $5.3 \mathrm{E}-06$ & 1.7E-03 \\
\hline Cellular respirarion and energy production & GO:0016310 & 1304 & 11 & 2.19 & 5.03 & $6.1 \mathrm{E}-06$ & $1.9 \mathrm{E}-03$ \\
\hline Cellular respirarion and energy production & GO:0022900 & 178 & 5 & 0.3 & 16.73 & $1.3 \mathrm{E}-05$ & $3.5 \mathrm{E}-03$ \\
\hline Cellular respirarion and energy production & GO:0042775 & 89 & 4 & 0.15 & 26.77 & 1.7E-05 & 4.5E-03 \\
\hline Cellular respirarion and energy production & GO:0042773 & 90 & 4 & 0.15 & 26.48 & $1.8 \mathrm{E}-05$ & 4.6E-03 \\
\hline Cellular respirarion and energy production & GO:0022904 & 110 & 4 & 0.18 & 21.66 & $3.9 \mathrm{E}-05$ & $8.4 \mathrm{E}-03$ \\
\hline Cellular respirarion and energy production & GO:1902600 & 138 & 4 & 0.23 & 17.27 & $9.1 \mathrm{E}-05$ & $1.8 \mathrm{E}-02$ \\
\hline Cellular respirarion and energy production & GO:0007005 & 467 & 6 & 0.78 & 7.65 & $1.2 \mathrm{E}-04$ & 2.4E-02 \\
\hline Cellular respirarion and energy production & GO:0045333 & 160 & 4 & 0.27 & 14.89 & 1.6E-04 & $2.8 \mathrm{E}-02$ \\
\hline Antibacterial response & GO:0019731 & 56 & 3 & 0.09 & 31.91 & $1.3 \mathrm{E}-04$ & $2.5 \mathrm{E}-02$ \\
\hline Antibacterial response & GO:0044419 & 2110 & 12 & 3.54 & 3.39 & $1.1 \mathrm{E}-04$ & 2.1E-02 \\
\hline Viral processes & GO:0044403 & 912 & 9 & 1.53 & 5.88 & $1.5 \mathrm{E}-05$ & 4.0E-03 \\
\hline Viral processes & GO:0016032 & 820 & 9 & 1.38 & 6.54 & $6.5 \mathrm{E}-06$ & $2.0 \mathrm{E}-03$ \\
\hline Viral processes & GO:0019080 & 154 & 7 & 0.26 & 27.08 & 8.0E-09 & $8.4 \mathrm{E}-06$ \\
\hline Viral processes & GO:0019083 & 115 & 7 & 0.19 & 36.26 & 1.1E-09 & 3.0E-06 \\
\hline Viral processes & GO:0006807 & 7090 & 23 & 11.9 & 1.93 & $2.1 \mathrm{E}-04$ & 3.7E-02 \\
\hline General cellular processes & GO:0044238 & 7570 & 24 & 12.71 & 1.89 & $1.4 \mathrm{E}-04$ & $2.6 \mathrm{E}-02$ \\
\hline General cellular processes & GO:0044248 & 1825 & 11 & 3.06 & 3.59 & $1.3 \mathrm{E}-04$ & $2.6 \mathrm{E}-02$ \\
\hline General cellular processes & GO:0044260 & 5145 & 20 & 8.64 & 2.32 & 4.3E-05 & $9.2 \mathrm{E}-03$ \\
\hline General cellular processes & GO:0044265 & 924 & 9 & 1.55 & 5.8 & 1.7E-05 & 4.4E-03 \\
\hline General cellular processes & GO:0044249 & 2699 & 15 & 4.53 & 3.31 & $1.2 \mathrm{E}-05$ & $3.4 \mathrm{E}-03$ \\
\hline General cellular processes & GO:0034641 & 3401 & 18 & 5.71 & 3.15 & $1.8 \mathrm{E}-06$ & $6.2 \mathrm{E}-04$ \\
\hline General cellular processes & GO:0044271 & 1571 & 13 & 2.64 & 4.93 & $7.9 \mathrm{E}-07$ & $3.2 \mathrm{E}-04$ \\
\hline General cellular processes & GO:0044270 & 441 & 8 & 0.74 & 10.81 & $6.1 \mathrm{E}-07$ & $2.5 \mathrm{E}-04$ \\
\hline General cellular processes & GO:0044085 & 2656 & 17 & 4.46 & 3.81 & $2.9 \mathrm{E}-07$ & $1.4 \mathrm{E}-04$ \\
\hline General cellular processes & GO:0008152 & 8585 & 31 & 14.41 & 2.15 & 8.0E-09 & 7.9E-06 \\
\hline
\end{tabular}


Table S7 continued

\begin{tabular}{|c|c|c|c|c|c|c|c|}
\hline \multirow{2}{*}{$\begin{array}{l}\text { 3h DOWN } \\
\text { General term }\end{array}$} & & & & & & & \\
\hline & GO \# & $\begin{array}{l}\text { in } \\
\text { Ref }\end{array}$ & $\begin{array}{l}\text { in } \\
\text { data }\end{array}$ & $\begin{array}{l}\text { exp- } \\
\text { ected }\end{array}$ & $\begin{array}{l}\text { fold } \\
\text { Enr. }\end{array}$ & $\begin{array}{l}\text { raw } \\
\text { P-value }\end{array}$ & FDR \\
\hline General cellular processes & GO:0044237 & 7782 & 30 & 13.06 & 2.3 & $5.2 \mathrm{E}-09$ & $6.3 \mathrm{E}-06$ \\
\hline
\end{tabular}

\begin{tabular}{|c|c|c|c|c|c|c|c|}
\hline 4h UP & & & & & & & \\
\hline General term & GO \# & $\begin{array}{l}\text { in } \\
\text { Ref }\end{array}$ & $\begin{array}{l}\text { in } \\
\text { data }\end{array}$ & $\begin{array}{l}\text { exp- } \\
\text { ected }\end{array}$ & $\begin{array}{l}\text { fold } \\
\text { Enr. }\end{array}$ & $\begin{array}{l}\text { raw } \\
\text { P-value }\end{array}$ & FDR \\
\hline CNS development & GO:0045664 & 680 & 14 & 4.27 & 3.28 & $1.1 \mathrm{E}-04$ & $3.8 \mathrm{E}-02$ \\
\hline Cell cycle and cell division & GO:0051276 & 1062 & 21 & 6.67 & 3.15 & $3.5 \mathrm{E}-06$ & 2.3E-03 \\
\hline Cell cycle and cell division & GO:0006260 & 223 & 9 & 1.4 & 6.42 & $1.6 \mathrm{E}-05$ & 6.7E-03 \\
\hline Cell cycle and cell division & GO:0006338 & 175 & 8 & 1.1 & 7.28 & $2.0 \mathrm{E}-05$ & $8.4 \mathrm{E}-03$ \\
\hline Cell cycle and cell division & GO:0071103 & 309 & 10 & 1.94 & 5.15 & 3.3E-05 & $1.3 \mathrm{E}-02$ \\
\hline Cell cycle and cell division & GO:0032508 & 110 & 6 & 0.69 & 8.68 & $8.9 \mathrm{E}-05$ & $3.2 \mathrm{E}-02$ \\
\hline Cell cycle and cell division & GO:0051130 & 1229 & 20 & 7.72 & 2.59 & $9.5 \mathrm{E}-05$ & $3.3 \mathrm{E}-02$ \\
\hline Cell cycle and cell division & GO:0051128 & 2436 & 31 & 15.3 & 2.03 & $1.1 \mathrm{E}-04$ & $3.8 \mathrm{E}-02$ \\
\hline Cell cycle and cell division & GO:0032392 & 118 & 6 & 0.74 & 8.09 & $1.3 \mathrm{E}-04$ & 4.2E-02 \\
\hline Cell cycle and cell division & GO:0033044 & 367 & 10 & 2.31 & 4.34 & $1.3 \mathrm{E}-04$ & 4.2E-02 \\
\hline Cell cycle and cell division & GO:1905269 & 122 & 6 & 0.77 & 7.83 & $1.5 \mathrm{E}-04$ & 4.8E-02 \\
\hline RNA production and processing & GO:0008380 & 410 & 17 & 2.58 & 6.6 & $1.4 \mathrm{E}-09$ & 2.3E-05 \\
\hline RNA production and processing & GO:0019219 & 4078 & 54 & 25.62 & 2.11 & $1.4 \mathrm{E}-08$ & 1.1E-04 \\
\hline RNA production and processing & GO:0006397 & 489 & 17 & 3.07 & 5.53 & $1.8 \mathrm{E}-08$ & $9.5 \mathrm{E}-05$ \\
\hline RNA production and processing & GO:0051252 & 3809 & 51 & 23.93 & 2.13 & $4.0 \mathrm{E}-08$ & 1.6E-04 \\
\hline RNA production and processing & GO:0090304 & 2237 & 36 & 14.05 & 2.56 & $9.4 \mathrm{E}-08$ & 3.0E-04 \\
\hline RNA production and processing & GO:0045934 & 1572 & 29 & 9.88 & 2.94 & $1.5 \mathrm{E}-07$ & $2.9 \mathrm{E}-04$ \\
\hline RNA production and processing & GO:0010468 & 4913 & 58 & 30.87 & 1.88 & $2.4 \mathrm{E}-07$ & $4.2 \mathrm{E}-04$ \\
\hline RNA production and processing & GO:0006396 & 929 & 21 & 5.84 & 3.6 & $4.4 \mathrm{E}-07$ & $5.4 \mathrm{E}-04$ \\
\hline RNA production and processing & GO:0016071 & 701 & 18 & 4.4 & 4.09 & $5.5 \mathrm{E}-07$ & $6.2 \mathrm{E}-04$ \\
\hline RNA production and processing & GO:0006139 & 2740 & 38 & 17.21 & 2.21 & 1.7E-06 & 1.4E-03 \\
\hline RNA production and processing & GO:0006355 & 3462 & 44 & 21.75 & 2.02 & $2.6 \mathrm{E}-06$ & $1.8 \mathrm{E}-03$ \\
\hline RNA production and processing & GO:1903506 & 3531 & 44 & 22.18 & 1.98 & $3.6 \mathrm{E}-06$ & $2.2 \mathrm{E}-03$ \\
\hline RNA production and processing & GO:2001141 & 3536 & 44 & 22.22 & 1.98 & 3.7E-06 & $2.2 \mathrm{E}-03$ \\
\hline RNA production and processing & GO:0000398 & 303 & 11 & 1.9 & 5.78 & 4.6E-06 & 2.6E-03 \\
\hline RNA production and processing & GO:0000377 & 303 & 11 & 1.9 & 5.78 & 4.6E-06 & $2.5 \mathrm{E}-03$ \\
\hline RNA production and processing & GO:0000375 & 306 & 11 & 1.92 & 5.72 & $5.0 \mathrm{E}-06$ & 2.7E-03 \\
\hline RNA production and processing & GO:0046483 & 2936 & 38 & 18.45 & 2.06 & $1.2 \mathrm{E}-05$ & $5.9 \mathrm{E}-03$ \\
\hline RNA production and processing & GO:1903507 & 1351 & 23 & 8.49 & 2.71 & $1.3 \mathrm{E}-05$ & $6.3 \mathrm{E}-03$ \\
\hline
\end{tabular}


Table S7 continued

\begin{tabular}{|c|c|c|c|c|c|c|c|}
\hline 4h UP & & & & & & & \\
\hline General term & GO \# & $\begin{array}{l}\text { in } \\
\text { Ref }\end{array}$ & $\begin{array}{l}\text { in } \\
\text { data }\end{array}$ & $\begin{array}{l}\text { exp- } \\
\text { ected }\end{array}$ & $\begin{array}{l}\text { fold } \\
\text { Enr. }\end{array}$ & $\begin{array}{l}\text { raw } \\
\text { P-value }\end{array}$ & FDR \\
\hline RNA production and processing & GO:1902679 & 1353 & 23 & 8.5 & 2.71 & $1.3 \mathrm{E}-05$ & $6.2 \mathrm{E}-03$ \\
\hline RNA production and processing & GO:0051253 & 1455 & 24 & 9.14 & 2.63 & $1.4 \mathrm{E}-05$ & $6.2 \mathrm{E}-03$ \\
\hline RNA production and processing & GO:0006725 & 2986 & 38 & 18.76 & 2.03 & $1.5 \mathrm{E}-05$ & $6.5 \mathrm{E}-03$ \\
\hline RNA production and processing & GO:1901360 & 3218 & 39 & 20.22 & 1.93 & 4.6E-05 & $1.8 \mathrm{E}-02$ \\
\hline RNA production and processing & GO:0045892 & 1310 & 21 & 8.23 & 2.55 & 7.7E-05 & $2.8 \mathrm{E}-02$ \\
\hline General cellular processes & GO:0034641 & 3401 & 40 & 21.37 & 1.87 & 7.0E-05 & $2.6 \mathrm{E}-02$ \\
\hline General cellular processes & GO:0031324 & 2700 & 34 & 16.96 & 2 & $6.6 \mathrm{E}-05$ & $2.5 \mathrm{E}-02$ \\
\hline General cellular processes & GO:0051053 & 140 & 7 & 0.88 & 7.96 & $3.9 \mathrm{E}-05$ & $1.5 \mathrm{E}-02$ \\
\hline General cellular processes & GO:0051172 & 2497 & 34 & 15.69 & 2.17 & $1.1 \mathrm{E}-05$ & $5.6 \mathrm{E}-03$ \\
\hline General cellular processes & GO:0080090 & 6118 & 64 & 38.44 & 1.67 & $3.0 \mathrm{E}-06$ & 2.0E-03 \\
\hline General cellular processes & GO:0051171 & 5920 & 63 & 37.19 & 1.69 & $2.2 \mathrm{E}-06$ & $1.6 \mathrm{E}-03$ \\
\hline General cellular processes & GO:0009889 & 4270 & 51 & 26.83 & 1.9 & $1.9 \mathrm{E}-06$ & $1.5 \mathrm{E}-03$ \\
\hline General cellular processes & GO:0019222 & 7060 & 72 & 44.36 & 1.62 & $1.0 \mathrm{E}-06$ & 8.5E-04 \\
\hline General cellular processes & GO:0031326 & 4184 & 51 & 26.29 & 1.94 & $8.8 \mathrm{E}-07$ & 7.7E-04 \\
\hline General cellular processes & GO:0031323 & 6329 & 67 & 39.76 & 1.68 & $8.2 \mathrm{E}-07$ & 7.7E-04 \\
\hline General cellular processes & GO:2000113 & 1512 & 27 & 9.5 & 2.84 & $8.1 \mathrm{E}-07$ & 8.0E-04 \\
\hline General cellular processes & GO:0060255 & 6510 & 69 & 40.9 & 1.69 & 5.7E-07 & $6.1 \mathrm{E}-04$ \\
\hline General cellular processes & GO:0009890 & 1657 & 29 & 10.41 & 2.79 & 4.3E-07 & 5.7E-04 \\
\hline General cellular processes & GO:0010556 & 4032 & 51 & 25.33 & 2.01 & $3.2 \mathrm{E}-07$ & 4.6E-04 \\
\hline General cellular processes & GO:0031327 & 1626 & 29 & 10.22 & 2.84 & $2.9 \mathrm{E}-07$ & 4.7E-04 \\
\hline General cellular processes & GO:0010558 & 1566 & 29 & 9.84 & 2.95 & $1.4 \mathrm{E}-07$ & 3.1E-04 \\
\hline General cellular processes & GO:2000112 & 3924 & 51 & 24.65 & 2.07 & $1.2 \mathrm{E}-07$ & $3.1 \mathrm{E}-04$ \\
\hline
\end{tabular}

\begin{tabular}{|c|c|c|c|c|c|c|c|}
\hline 4h DOWN & & & & & & & \\
\hline General term & GO \# & $\begin{array}{l}\text { in } \\
\text { Ref }\end{array}$ & $\begin{array}{l}\text { in } \\
\text { data }\end{array}$ & $\begin{array}{l}\text { exp- } \\
\text { ected }\end{array}$ & $\begin{array}{l}\text { fold } \\
\text { Enr. }\end{array}$ & $\begin{array}{l}\text { raw } \\
\text { P-value }\end{array}$ & FDR \\
\hline Osteoblast differentation & GO:0045668 & 48 & 4 & 0.09 & 43.44 & 2.9E-06 & 4.6E-02 \\
\hline RNA production and processing & GO:0000184 & 121 & 5 & 0.23 & 21.54 & 4.1E-06 & 2.2E-02 \\
\hline Osteoblast differentation & GO:0045667 & 121 & 5 & 0.23 & 21.54 & 4.1E-06 & 3.3E-02 \\
\hline
\end{tabular}

\begin{tabular}{|c|c|c|c|c|c|c|c|}
\hline $6 h$ UP & & & & & & & \\
\hline General term & GO \# & $\begin{array}{l}\text { in } \\
\text { Ref }\end{array}$ & $\begin{array}{l}\text { in } \\
\text { data }\end{array}$ & $\begin{array}{l}\text { exp- } \\
\text { ected }\end{array}$ & $\begin{array}{l}\text { fold } \\
\text { Enr. }\end{array}$ & $\begin{array}{l}\text { raw } \\
\text { P-value }\end{array}$ & FDR \\
\hline RNA production and processing & GO:0010629 & 2065 & 42 & 23.47 & 1.79 & 2.7E-04 & 4.4E-02 \\
\hline RNA production and processing & GO:1903311 & 351 & 13 & 3.99 & 3.26 & $2.6 \mathrm{E}-04$ & 4.3E-02 \\
\hline
\end{tabular}




\begin{tabular}{|c|c|c|c|c|c|c|c|}
\hline 6h UP & & & & & & & \\
\hline General term & GO \# & $\begin{array}{l}\text { in } \\
\text { Ref }\end{array}$ & $\begin{array}{l}\text { in } \\
\text { data }\end{array}$ & $\begin{array}{l}\text { exp- } \\
\text { ected }\end{array}$ & $\begin{array}{l}\text { fold } \\
\text { Enr. }\end{array}$ & $\begin{array}{l}\text { raw } \\
\text { P-value }\end{array}$ & FDR \\
\hline RNA production and processing & GO:0050684 & 154 & 9 & 1.75 & 5.14 & $9.8 \mathrm{E}-05$ & $1.9 \mathrm{E}-02$ \\
\hline RNA production and processing & GO:0010467 & 2109 & 44 & 23.97 & 1.84 & 8.5E-05 & 1.7E-02 \\
\hline RNA production and processing & GO:0000375 & 306 & 13 & 3.48 & 3.74 & 7.0E-05 & $1.4 \mathrm{E}-02$ \\
\hline RNA production and processing & GO:0045892 & 1310 & 32 & 14.89 & 2.15 & 7.0E-05 & $1.4 \mathrm{E}-02$ \\
\hline RNA production and processing & GO:0000398 & 303 & 13 & 3.44 & 3.77 & $6.4 \mathrm{E}-05$ & $1.4 \mathrm{E}-02$ \\
\hline RNA production and processing & GO:0000377 & 303 & 13 & 3.44 & 3.77 & $6.4 \mathrm{E}-05$ & $1.4 \mathrm{E}-02$ \\
\hline RNA production and processing & GO:0045893 & 1601 & 37 & 18.2 & 2.03 & 4.2E-05 & $1.1 \mathrm{E}-02$ \\
\hline RNA production and processing & GO:0016071 & 701 & 22 & 7.97 & 2.76 & $2.4 \mathrm{E}-05$ & $6.4 \mathrm{E}-03$ \\
\hline RNA production and processing & GO:1902679 & 1353 & 34 & 15.38 & 2.21 & 1.7E-05 & 4.9E-03 \\
\hline RNA production and processing & GO:1903507 & 1351 & 34 & 15.36 & 2.21 & $1.6 \mathrm{E}-05$ & $4.8 \mathrm{E}-03$ \\
\hline RNA production and processing & GO:0051253 & 1455 & 36 & 16.54 & 2.18 & $1.6 \mathrm{E}-05$ & $4.9 \mathrm{E}-03$ \\
\hline RNA production and processing & GO:0045935 & 1953 & 44 & 22.2 & 1.98 & 1.6E-05 & $4.9 \mathrm{E}-03$ \\
\hline RNA production and processing & GO:0016070 & 1621 & 39 & 18.42 & 2.12 & $1.4 \mathrm{E}-05$ & 4.5E-03 \\
\hline RNA production and processing & GO:1901360 & 3218 & 63 & 36.58 & 1.72 & $1.2 \mathrm{E}-05$ & $3.9 \mathrm{E}-03$ \\
\hline RNA production and processing & GO:1902680 & 1687 & 40 & 19.18 & 2.09 & $1.2 \mathrm{E}-05$ & $3.9 \mathrm{E}-03$ \\
\hline RNA production and processing & GO:1903508 & 1686 & 40 & 19.16 & 2.09 & $1.2 \mathrm{E}-05$ & 4.0E-03 \\
\hline RNA production and processing & GO:0006357 & 2628 & 55 & 29.87 & 1.84 & $7.8 \mathrm{E}-06$ & 2.7E-03 \\
\hline RNA production and processing & GO:0006397 & 489 & 19 & 5.56 & 3.42 & $5.2 \mathrm{E}-06$ & 2.0E-03 \\
\hline RNA production and processing & GO:0006725 & 2986 & 61 & 33.94 & 1.8 & 4.1E-06 & $1.6 \mathrm{E}-03$ \\
\hline RNA production and processing & GO:0051254 & 1780 & 43 & 20.23 & 2.13 & $3.4 \mathrm{E}-06$ & $1.4 \mathrm{E}-03$ \\
\hline RNA production and processing & GO:0046483 & 2936 & 61 & 33.37 & 1.83 & $2.3 \mathrm{E}-06$ & $1.0 \mathrm{E}-03$ \\
\hline RNA production and processing & GO:0008380 & 410 & 18 & 4.66 & 3.86 & $1.8 \mathrm{E}-06$ & $9.1 \mathrm{E}-04$ \\
\hline RNA production and processing & GO:0006396 & 929 & 30 & 10.56 & 2.84 & 4.1E-07 & 2.6E-04 \\
\hline RNA production and processing & GO:0045934 & 1572 & 42 & 17.87 & 2.35 & $3.8 \mathrm{E}-07$ & 2.5E-04 \\
\hline RNA production and processing & GO:0006139 & 2740 & 61 & 31.14 & 1.96 & $2.2 \mathrm{E}-07$ & 1.7E-04 \\
\hline RNA production and processing & GO:0090304 & 2237 & 57 & 25.43 & 2.24 & $6.1 \mathrm{E}-09$ & $5.4 \mathrm{E}-06$ \\
\hline RNA production and processing & GO:0006355 & 3462 & 78 & 39.35 & 1.98 & $1.2 \mathrm{E}-09$ & 1.6E-06 \\
\hline RNA production and processing & GO:2001141 & 3536 & 79 & 40.19 & 1.97 & $1.1 \mathrm{E}-09$ & 1.6E-06 \\
\hline RNA production and processing & GO:1903506 & 3531 & 79 & 40.13 & 1.97 & 1.0E-09 & 1.7E-06 \\
\hline RNA production and processing & GO:0010468 & 4913 & 100 & 55.84 & 1.79 & 4.1E-10 & $8.1 \mathrm{E}-07$ \\
\hline RNA production and processing & GO:0051252 & 3809 & 89 & 43.29 & 2.06 & 4.7E-12 & 3.7E-08 \\
\hline RNA production and processing & GO:0019219 & 4078 & 94 & 46.35 & 2.03 & $1.5 \mathrm{E}-12$ & 2.4E-08 \\
\hline CNS development & GO:0003008 & 2079 & 7 & 23.63 & 0.3 & $6.6 \mathrm{E}-05$ & $1.4 \mathrm{E}-02$ \\
\hline CNS development & GO:0050877 & 1413 & 3 & 16.06 & 0.19 & $1.2 \mathrm{E}-04$ & $2.2 \mathrm{E}-02$ \\
\hline
\end{tabular}


Table S7 continued

\begin{tabular}{|c|c|c|c|c|c|c|c|}
\hline 6h UP & & & & & & & \\
\hline General term & GO \# & $\begin{array}{l}\text { in } \\
\text { Ref }\end{array}$ & $\begin{array}{l}\text { in } \\
\text { data }\end{array}$ & $\begin{array}{l}\text { exp- } \\
\text { ected }\end{array}$ & $\begin{array}{l}\text { fold } \\
\text { Enr. }\end{array}$ & $\begin{array}{l}\text { raw } \\
\text { P-value }\end{array}$ & FDR \\
\hline CNS development & GO:0007600 & 987 & 1 & 11.22 & 0.09 & 2.7E-04 & 4.3E-02 \\
\hline Stemness and stemcell maintenance & GO:0098727 & 143 & 9 & 1.63 & 5.54 & 5.7E-05 & $1.3 \mathrm{E}-02$ \\
\hline Stemness and stemcell maintenance & GO:0019827 & 141 & 9 & 1.6 & 5.62 & $5.2 \mathrm{E}-05$ & $1.2 \mathrm{E}-02$ \\
\hline Embryonic development & GO:0001701 & 360 & 15 & 4.09 & 3.67 & 2.4E-05 & $6.3 \mathrm{E}-03$ \\
\hline Embryonic development & GO:0048608 & 433 & 16 & 4.92 & 3.25 & $5.3 \mathrm{E}-05$ & $1.2 \mathrm{E}-02$ \\
\hline Embryonic development & GO:0061458 & 437 & 16 & 4.97 & 3.22 & $5.8 \mathrm{E}-05$ & $1.3 \mathrm{E}-02$ \\
\hline Embryonic development & GO:0009792 & 643 & 20 & 7.31 & 2.74 & $6.5 \mathrm{E}-05$ & $1.4 \mathrm{E}-02$ \\
\hline Embryonic development & GO:0009790 & 1003 & 26 & 11.4 & 2.28 & $1.1 \mathrm{E}-04$ & 2.0E-02 \\
\hline Embryonic development & GO:0043009 & 623 & 19 & 7.08 & 2.68 & $1.3 \mathrm{E}-04$ & 2.3E-02 \\
\hline Embryonic development & GO:0010171 & 46 & 5 & 0.52 & 9.56 & 2.7E-04 & 4.3E-02 \\
\hline Cell cycle and cell division & GO:0071103 & 309 & 12 & 3.51 & 3.42 & 3.0E-04 & 4.7E-02 \\
\hline Cell cycle and cell division & GO:0031023 & 98 & 7 & 1.11 & 6.28 & $1.9 \mathrm{E}-04$ & $3.2 \mathrm{E}-02$ \\
\hline Cell cycle and cell division & GO:0033043 & 1332 & 31 & 15.14 & 2.05 & $1.6 \mathrm{E}-04$ & 2.9E-02 \\
\hline Cell cycle and cell division & GO:0022402 & 1069 & 27 & 12.15 & 2.22 & $1.5 \mathrm{E}-04$ & $2.8 \mathrm{E}-02$ \\
\hline Cell cycle and cell division & GO:0007098 & 86 & 7 & 0.98 & 7.16 & $8.6 \mathrm{E}-05$ & 1.7E-02 \\
\hline Cell cycle and cell division & GO:0006338 & 175 & 10 & 1.99 & 5.03 & 4.8E-05 & $1.2 \mathrm{E}-02$ \\
\hline Cell cycle and cell division & GO:1905269 & 122 & 9 & 1.39 & 6.49 & $1.8 \mathrm{E}-05$ & $5.1 \mathrm{E}-03$ \\
\hline Cell cycle and cell division & GO:0016043 & 5699 & 97 & 64.78 & 1.5 & 7.1E-06 & 2.6E-03 \\
\hline Cell cycle and cell division & GO:2001251 & 138 & 10 & 1.57 & 6.38 & 7.0E-06 & 2.6E-03 \\
\hline Cell cycle and cell division & GO:0071840 & 5919 & 101 & 67.28 & 1.5 & $3.2 \mathrm{E}-06$ & $1.4 \mathrm{E}-03$ \\
\hline Cell cycle and cell division & GO:1902275 & 213 & 13 & 2.42 & 5.37 & $1.8 \mathrm{E}-06$ & $9.2 \mathrm{E}-04$ \\
\hline Cell cycle and cell division & GO:0007049 & 1390 & 38 & 15.8 & 2.41 & $6.6 \mathrm{E}-07$ & $3.9 \mathrm{E}-04$ \\
\hline Cell cycle and cell division & GO:0006996 & 3576 & 73 & 40.65 & 1.8 & 3.7E-07 & 2.6E-04 \\
\hline Cell cycle and cell division & GO:0033044 & 367 & 19 & 4.17 & 4.55 & 8.5E-08 & 7.1E-05 \\
\hline Cell cycle and cell division & GO:0006325 & 701 & 29 & 7.97 & 3.64 & 3.9E-09 & $3.9 \mathrm{E}-06$ \\
\hline Cell cycle and cell division & GO:0051276 & 1062 & 40 & 12.07 & 3.31 & 4.7E-11 & 2.5E-07 \\
\hline Protein production and regulation & GO:0010557 & 1930 & 42 & 21.94 & 1.91 & $6.6 \mathrm{E}-05$ & $1.4 \mathrm{E}-02$ \\
\hline Protein production and regulation & GO:0010604 & 3630 & 67 & 41.26 & 1.62 & 4.6E-05 & $1.1 \mathrm{E}-02$ \\
\hline General cellular processes & GO:0009890 & 1657 & 41 & 18.83 & 2.18 & $3.0 \mathrm{E}-06$ & $1.3 \mathrm{E}-03$ \\
\hline General cellular processes & GO:0031327 & 1626 & 41 & 18.48 & 2.22 & 2.3E-06 & $1.1 \mathrm{E}-03$ \\
\hline General cellular processes & GO:2000113 & 1512 & 39 & 17.19 & 2.27 & 2.0E-06 & $9.5 \mathrm{E}-04$ \\
\hline General cellular processes & GO:0010558 & 1566 & 41 & 17.8 & 2.3 & $7.4 \mathrm{E}-07$ & 4.2E-04 \\
\hline General cellular processes & GO:0010605 & 2951 & 62 & 33.54 & 1.85 & $1.4 \mathrm{E}-06$ & 7.5E-04 \\
\hline General cellular processes & GO:0009892 & 3205 & 64 & 36.43 & 1.76 & 5.0E-06 & $1.9 \mathrm{E}-03$ \\
\hline
\end{tabular}


Table S7 continued

\begin{tabular}{|c|c|c|c|c|c|c|c|}
\hline 6h UP & & & & & & & \\
\hline General term & GO \# & $\begin{array}{l}\text { in } \\
\text { Ref }\end{array}$ & $\begin{array}{l}\text { in } \\
\text { data }\end{array}$ & $\begin{array}{l}\text { exp- } \\
\text { ected }\end{array}$ & $\begin{array}{l}\text { fold } \\
\text { Enr. }\end{array}$ & $\begin{array}{l}\text { raw } \\
\text { P-value }\end{array}$ & FDR \\
\hline General cellular processes & GO:0009889 & 4270 & 88 & 48.53 & 1.81 & 5.6E-09 & $5.3 \mathrm{E}-06$ \\
\hline General cellular processes & GO:0031326 & 4184 & 88 & 47.56 & 1.85 & 1.6E-09 & $1.9 \mathrm{E}-06$ \\
\hline General cellular processes & GO:2000112 & 3924 & 86 & 44.6 & 1.93 & $3.4 \mathrm{E}-10$ & 7.7E-07 \\
\hline General cellular processes & GO:0010556 & 4032 & 88 & 45.83 & 1.92 & $2.8 \mathrm{E}-10$ & 7.3E-07 \\
\hline General cellular processes & GO:0019222 & 7060 & 126 & 80.25 & 1.57 & 1.9E-09 & $2.0 \mathrm{E}-06$ \\
\hline General cellular processes & GO:0034641 & 3401 & 65 & 38.66 & 1.68 & $1.9 \mathrm{E}-05$ & $5.3 \mathrm{E}-03$ \\
\hline General cellular processes & GO:0043170 & 6337 & 109 & 72.03 & 1.51 & $5.6 \mathrm{E}-07$ & $3.4 \mathrm{E}-04$ \\
\hline General cellular processes & GO:0051172 & 2497 & 57 & 28.38 & 2.01 & $3.2 \mathrm{E}-07$ & 2.3E-04 \\
\hline General cellular processes & GO:0006807 & 7090 & 114 & 80.59 & 1.41 & $9.2 \mathrm{E}-06$ & $3.2 \mathrm{E}-03$ \\
\hline General cellular processes & GO:0009893 & 3937 & 69 & 44.75 & 1.54 & $1.6 \mathrm{E}-04$ & $2.9 \mathrm{E}-02$ \\
\hline General cellular processes & GO:0051173 & 3267 & 61 & 37.13 & 1.64 & 7.1E-05 & $1.4 \mathrm{E}-02$ \\
\hline General cellular processes & GO:0051171 & 5920 & 113 & 67.29 & 1.68 & $4.6 \mathrm{E}-10$ & 8.0E-07 \\
\hline General cellular processes & GO:0031324 & 2700 & 60 & 30.69 & 1.96 & $3.1 \mathrm{E}-07$ & 2.3E-04 \\
\hline General cellular processes & GO:0048523 & 4981 & 89 & 56.62 & 1.57 & 3.7E-06 & $1.5 \mathrm{E}-03$ \\
\hline General cellular processes & GO:0051053 & 140 & 9 & 1.59 & 5.66 & 4.9E-05 & $1.2 \mathrm{E}-02$ \\
\hline General cellular processes & GO:0031323 & 6329 & 119 & 71.94 & 1.65 & $2.5 \mathrm{E}-10$ & $7.8 \mathrm{E}-07$ \\
\hline General cellular processes & GO:0060255 & 6510 & 123 & 74 & 1.66 & 7.6E-11 & $3.0 \mathrm{E}-07$ \\
\hline General cellular processes & GO:0080090 & 6118 & 114 & 69.54 & 1.64 & 1.7E-09 & $1.9 \mathrm{E}-06$ \\
\hline General cellular processes & GO:0009891 & 2066 & 42 & 23.48 & 1.79 & 2.7E-04 & $4.4 \mathrm{E}-02$ \\
\hline General cellular processes & GO:0031328 & 2031 & 42 & 23.09 & 1.82 & $1.6 \mathrm{E}-04$ & $2.8 \mathrm{E}-02$ \\
\hline General cellular processes & GO:0065007 & 12629 & 179 & 143.55 & 1.25 & 1.7E-06 & $9.1 \mathrm{E}-04$ \\
\hline General cellular processes & GO:0048519 & 5643 & 95 & 64.14 & 1.48 & $1.8 \mathrm{E}-05$ & $5.1 \mathrm{E}-03$ \\
\hline General cellular processes & GO:0031325 & 3454 & 63 & 39.26 & 1.6 & 1.0E-04 & 2.0E-02 \\
\hline General cellular processes & GO:0048522 & 5742 & 96 & 65.27 & 1.47 & 2.0E-05 & $5.5 \mathrm{E}-03$ \\
\hline General cellular processes & GO:0050789 & 11955 & 167 & 135.88 & 1.23 & $3.9 \mathrm{E}-05$ & $9.8 \mathrm{E}-03$ \\
\hline General cellular processes & GO:0050794 & 11390 & 162 & 129.46 & 1.25 & $2.4 \mathrm{E}-05$ & $6.5 \mathrm{E}-03$ \\
\hline Epigenetic regulation & GO:0031062 & 43 & 5 & 0.49 & 10.23 & $2.0 \mathrm{E}-04$ & $3.4 \mathrm{E}-02$ \\
\hline Epigenetic regulation & GO:0031060 & 72 & 7 & 0.82 & 8.55 & 3.0E-05 & $7.8 \mathrm{E}-03$ \\
\hline Cell death & GO:0006915 & 918 & 25 & 10.43 & 2.4 & 8.3E-05 & 1.7E-02 \\
\hline Cell death & GO:0008219 & 1087 & 27 & 12.36 & 2.19 & $1.8 \mathrm{E}-04$ & $3.1 \mathrm{E}-02$ \\
\hline Cell death & GO:0012501 & 1049 & 26 & 11.92 & 2.18 & 2.6E-04 & 4.3E-02 \\
\hline
\end{tabular}


Table S7 continued

\begin{tabular}{|c|c|c|c|c|c|c|c|}
\hline 6h DOWN & & & & & & & \\
\hline General term & GO \# & $\begin{array}{l}\text { in } \\
\text { Ref }\end{array}$ & $\begin{array}{l}\text { in } \\
\text { data }\end{array}$ & $\begin{array}{l}\text { exp- } \\
\text { ected }\end{array}$ & $\begin{array}{l}\text { fold } \\
\text { Enr. }\end{array}$ & $\begin{array}{l}\text { raw } \\
\text { P-value }\end{array}$ & FDR \\
\hline Embryonic development & GO:0048638 & 342 & 7 & 1.13 & 6.19 & $1.50 \mathrm{E}-04$ & $4.2 \mathrm{E}-02$ \\
\hline Embryonic development & GO:0042127 & 1677 & 17 & 5.55 & 3.06 & $2.62 \mathrm{E}-05$ & $1.2 \mathrm{E}-02$ \\
\hline Embryonic development & GO:0040008 & 685 & 11 & 2.27 & 4.85 & $1.61 \mathrm{E}-05$ & $9.1 \mathrm{E}-03$ \\
\hline RNA production and processing & GO:2001141 & 3536 & 25 & 11.7 & 2.14 & 1.37E-04 & $3.9 \mathrm{E}-02$ \\
\hline RNA production and processing & GO:1903506 & 3531 & 25 & 11.68 & 2.14 & $1.35 \mathrm{E}-04$ & $3.9 \mathrm{E}-02$ \\
\hline RNA production and processing & GO:0006355 & 3462 & 25 & 11.46 & 2.18 & 1.19E-04 & 3.7E-02 \\
\hline RNA production and processing & GO:0010468 & 4913 & 31 & 16.26 & 1.91 & 9.57E-05 & $3.1 \mathrm{E}-02$ \\
\hline RNA production and processing & GO:0051252 & 3809 & 27 & 12.6 & 2.14 & $5.56 \mathrm{E}-05$ & 2.0E-02 \\
\hline RNA production and processing & GO:0019219 & 4078 & 28 & 13.49 & 2.07 & $5.49 E-05$ & 2.0E-02 \\
\hline RNA production and processing & GO:1902679 & 1353 & 17 & 4.48 & 3.8 & $1.56 \mathrm{E}-06$ & $1.6 \mathrm{E}-03$ \\
\hline RNA production and processing & GO:1903507 & 1351 & 17 & 4.47 & 3.8 & $1.53 \mathrm{E}-06$ & $1.6 \mathrm{E}-03$ \\
\hline RNA production and processing & GO:0045892 & 1310 & 17 & 4.34 & 3.92 & $1.00 \mathrm{E}-06$ & $1.3 \mathrm{E}-03$ \\
\hline RNA production and processing & GO:0051253 & 1455 & 18 & 4.81 & 3.74 & 8.86E-07 & $1.3 \mathrm{E}-03$ \\
\hline RNA production and processing & GO:0000122 & 970 & 15 & 3.21 & 4.67 & $5.80 \mathrm{E}-07$ & $9.2 \mathrm{E}-04$ \\
\hline RNA production and processing & GO:0045934 & 1572 & 19 & 5.2 & 3.65 & $5.76 \mathrm{E}-07$ & $1.0 \mathrm{E}-03$ \\
\hline RNA production and processing & GO:0010629 & 2065 & 23 & 6.83 & 3.37 & $1.08 \mathrm{E}-07$ & 1.7E-03 \\
\hline General cellular processes & GO:0009892 & 3205 & 29 & 10.61 & 2.73 & $1.09 \mathrm{E}-07$ & 8.7E-04 \\
\hline General cellular processes & GO:0010605 & 2951 & 27 & 9.77 & 2.76 & 3.06E-07 & 8.1E-04 \\
\hline General cellular processes & GO:0048519 & 5643 & 39 & 18.67 & 2.09 & 3.67E-07 & 8.3E-04 \\
\hline General cellular processes & GO:0019222 & 7060 & 44 & 23.36 & 1.88 & 5.64E-07 & $1.1 \mathrm{E}-03$ \\
\hline General cellular processes & GO:0031324 & 2700 & 24 & 8.93 & 2.69 & 3.17E-06 & $2.5 \mathrm{E}-03$ \\
\hline General cellular processes & GO:0048523 & 4981 & 34 & 16.48 & 2.06 & 5.85E-06 & 4.4E-03 \\
\hline General cellular processes & GO:2000113 & 1512 & 17 & 5 & 3.4 & $6.85 \mathrm{E}-06$ & $5.0 \mathrm{E}-03$ \\
\hline General cellular processes & GO:0031323 & 6329 & 39 & 20.94 & 1.86 & $8.78 \mathrm{E}-06$ & $6.0 \mathrm{E}-03$ \\
\hline General cellular processes & GO:0010558 & 1566 & 17 & 5.18 & 3.28 & $1.08 \mathrm{E}-05$ & $6.9 \mathrm{E}-03$ \\
\hline General cellular processes & GO:0051172 & 2497 & 22 & 8.26 & 2.66 & $1.11 \mathrm{E}-05$ & $6.8 \mathrm{E}-03$ \\
\hline General cellular processes & GO:0031327 & 1626 & 17 & 5.38 & 3.16 & $1.76 \mathrm{E}-05$ & 9.7E-03 \\
\hline General cellular processes & GO:0010604 & 3630 & 27 & 12.01 & 2.25 & $1.90 \mathrm{E}-05$ & $9.8 \mathrm{E}-03$ \\
\hline General cellular processes & GO:0060255 & 6510 & 39 & 21.54 & 1.81 & $1.98 \mathrm{E}-05$ & $9.9 \mathrm{E}-03$ \\
\hline General cellular processes & GO:0009890 & 1657 & 17 & 5.48 & 3.1 & $2.25 \mathrm{E}-05$ & 1.0E-02 \\
\hline General cellular processes & GO:0080090 & 6118 & 37 & 20.25 & 1.83 & $2.79 \mathrm{E}-05$ & $1.2 \mathrm{E}-02$ \\
\hline General cellular processes & GO:0009893 & 3937 & 28 & 13.03 & 2.15 & $3.43 \mathrm{E}-05$ & 1.4E-02 \\
\hline General cellular processes & GO:0065009 & 3078 & 23 & 10.19 & 2.26 & 1.07E-04 & $3.4 \mathrm{E}-02$ \\
\hline General cellular processes & GO:0051171 & 5920 & 35 & 19.59 & 1.79 & $1.32 \mathrm{E}-04$ & $3.9 \mathrm{E}-02$ \\
\hline
\end{tabular}


Table S7 continued

\begin{tabular}{|c|c|c|c|c|c|c|c|}
\hline 6h DOWN & & & & & & & \\
\hline General term & GO \# & $\begin{array}{l}\text { in } \\
\text { Ref }\end{array}$ & $\begin{array}{l}\text { in } \\
\text { data }\end{array}$ & $\begin{array}{l}\text { exp- } \\
\text { ected }\end{array}$ & $\begin{array}{l}\text { fold } \\
\text { Enr. }\end{array}$ & $\begin{array}{l}\text { raw } \\
\text { P-value }\end{array}$ & FDR \\
\hline General cellular processes & GO:0010556 & 4032 & 27 & 13.34 & 2.02 & $1.67 \mathrm{E}-04$ & 4.6E-02 \\
\hline Kinase activity and regulation & GO:0010563 & 577 & 9 & 1.91 & 4.71 & 1.27E-04 & $3.8 \mathrm{E}-02$ \\
\hline Kinase activity and regulation & GO:0045936 & 576 & 9 & 1.91 & 4.72 & $1.25 \mathrm{E}-04$ & $3.8 \mathrm{E}-02$ \\
\hline Kinase activity and regulation & GO:0050790 & 2397 & 20 & 7.93 & 2.52 & $6.98 \mathrm{E}-05$ & 2.3E-02 \\
\hline Kinase activity and regulation & GO:0033674 & 616 & 10 & 2.04 & 4.91 & $3.71 E-05$ & 1.4E-02 \\
\hline Kinase activity and regulation & GO:0031399 & 1891 & 18 & 6.26 & 2.88 & $3.29 \mathrm{E}-05$ & $1.3 \mathrm{E}-02$ \\
\hline Kinase activity and regulation & GO:0042326 & 456 & 9 & 1.51 & 5.96 & 2.13E-05 & 10.0E-03 \\
\hline Kinase activity and regulation & GO:0001932 & 1469 & 16 & 4.86 & 3.29 & $2.01 \mathrm{E}-05$ & 9.7E-03 \\
\hline Kinase activity and regulation & GO:0051347 & 696 & 11 & 2.3 & 4.78 & $1.86 \mathrm{E}-05$ & 9.9E-03 \\
\hline Kinase activity and regulation & GO:0045860 & 539 & 10 & 1.78 & 5.61 & $1.21 \mathrm{E}-05$ & 7.1E-03 \\
\hline Kinase activity and regulation & GO:0045859 & 805 & 13 & 2.66 & 4.88 & $2.31 \mathrm{E}-06$ & 2.2E-03 \\
\hline Kinase activity and regulation & GO:0051174 & 1842 & 20 & 6.1 & 3.28 & 1.43E-06 & $1.6 \mathrm{E}-03$ \\
\hline Kinase activity and regulation & GO:0019220 & 1841 & 20 & 6.09 & 3.28 & $1.41 \mathrm{E}-06$ & 1.7E-03 \\
\hline Kinase activity and regulation & GO:0043549 & 915 & 15 & 3.03 & 4.95 & $2.79 \mathrm{E}-07$ & 8.9E-04 \\
\hline Kinase activity and regulation & GO:0042325 & 1642 & 20 & 5.43 & 3.68 & $2.35 \mathrm{E}-07$ & 9.3E-04 \\
\hline Kinase activity and regulation & GO:0051338 & 1032 & 16 & 3.42 & 4.69 & 2.19E-07 & $1.2 \mathrm{E}-03$ \\
\hline Osteoblast differentation & GO:0045667 & 121 & 5 & 0.4 & 12.49 & $6.04 \mathrm{E}-05$ & 2.1E-02 \\
\hline Osteoblast differentation & GO:0030278 & 205 & 6 & 0.68 & 8.84 & $6.92 \mathrm{E}-05$ & 2.3E-02 \\
\hline Osteoblast differentation & GO:0030279 & 80 & 4 & 0.26 & 15.11 & $1.71 \mathrm{E}-04$ & 4.6E-02 \\
\hline Cell death & GO:0042981 & 1566 & 18 & 5.18 & 3.47 & $2.51 \mathrm{E}-06$ & 2.2E-03 \\
\hline Cell death & GO:0043067 & 1588 & 18 & 5.25 & 3.43 & $3.06 \mathrm{E}-06$ & 2.6E-03 \\
\hline Cell death & GO:0010941 & 1722 & 18 & 5.7 & 3.16 & $9.37 \mathrm{E}-06$ & $6.2 \mathrm{E}-03$ \\
\hline Cell death & GO:0060548 & 1027 & 13 & 3.4 & 3.83 & 3.03E-05 & $1.3 \mathrm{E}-02$ \\
\hline Cell death & GO:0043066 & 915 & 12 & 3.03 & 3.96 & 4.57E-05 & 1.7E-02 \\
\hline Cell death & GO:0043069 & 933 & 12 & 3.09 & 3.89 & 5.50E-05 & 2.0E-02 \\
\hline
\end{tabular}




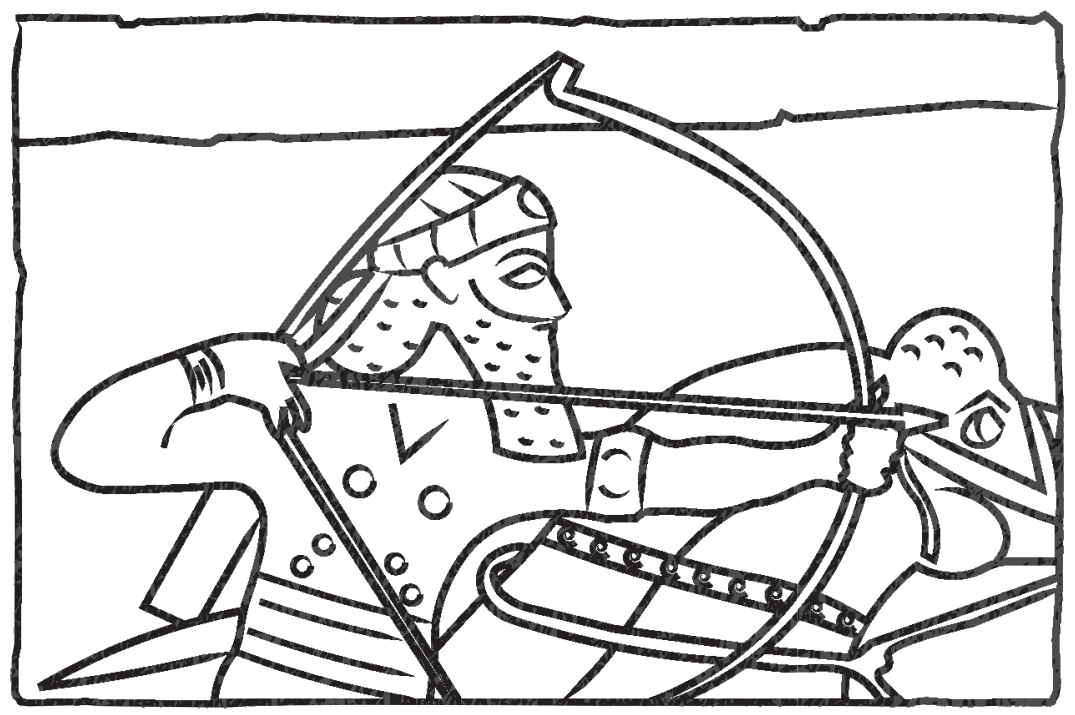

Illustration based on a stone carving on display at the British museum 


\section{Chapter 4 \\ A genome-wide CRISPR/Cas \\ phenotypic screen for modulators \\ of DUX4 cytotoxicity reveals screen \\ complications}

Ator Ashoti ${ }^{1 *}$, Francesco Limone ${ }^{2,5^{*}}$, Melissa van Kranenburg ${ }^{1}$, Anna Alemany ${ }^{1}$, Mirna Baak' ${ }^{1}$, Judith Vivié ${ }^{1,3}$, Federica Piccioni ${ }^{7}$, Menno Creyghton $^{1,6}$, Kevin Eggan ${ }^{2,5^{* * *}}$, Niels Geijsen ${ }^{1,4^{* *}}$

${ }^{1}$ Hubrecht Institute, Developmental Biology and Stem Cell Research, Utrecht, Netherlands

${ }^{2}$ Department of Stem Cell and Regenerative Biology, Harvard University Cambridge, MA, USA

${ }^{3}$ Single Cell Discoveries, Utrecht, Netherlands

${ }^{4}$ Leiden University Medical Center, Leiden, The Netherlands

${ }^{5}$ Stanley Center for Psychiatric Research, Broad Institute of MIT and Harvard, Cambridge, MA, USA

${ }^{6}$ Erasmus University Medical Centre, Rotterdam, The Netherlands

${ }^{7}$ Broad institute, Cambridge, MA, USA

* Equal contribution

${ }^{*}$ Correspondence: n.geijsen@lumc.nl, eggan@mcb.harvard.edu

This chapter is available on bioRxiv

Ashoti, A. et al. A genome-wide CRISPR/Cas phenotypic screen for modulators of DUX4 cytotoxicity reveals screen complications. bioRxiv (2020). doi:10.1101/2020.07.27.223420 


\section{Abstract}

Facioscapulohumeral muscular dystrophy (FHSD), a fundamentally complex muscle disorder that thus far remains untreatable. As the name implies, FSHD starts in the muscles of the face and shoulder gridle. The main perturbator of the disease is the pioneer transcription factor DUX4, which is misexpressed in affected tissues due to a failure in epigenetic repressive mechanisms. In pursuit of unraveling the underlying mechanism of FSHD and finding potential therapeutic targets or treatment options, we performed an exhaustive genomewide CRISPR/Cas9 phenotypic rescue screen to identify modulators of DUX4 cytotoxicity. We found no key effectors other than DUX4 itself, suggesting treatment efforts in FSHD should be directed towards its direct modulation.

The screen did however reveal some rare and unexpected Cas9-induced genomic events, that may provide important considerations for planning future CRISPR/Cas9 knock-out screens. 


\section{Introduction}

Facioscapulohumeral muscular dystrophy (FSHD) is an autosomal dominant degenerative muscle disease. It's one of the most prevalent neuromuscular disorder ${ }^{1}$, characterized by progressive and asymmetric muscle weakness which generally starts in facial muscles, and then slowly progresses to muscles of the shoulders, upper limbs and eventually the lower extremities $^{2}$. Age of onset is highly variable, but calculations based on a 122 case study demonstrates that the mean age of onset is in the early twenties (21-23) ${ }^{3}$. The primary cause of the disease is the misexpression of the double homeobox 4 (DUX4) transcription factor, due to failure in epigenetic silencing ${ }^{3-6}$. DUX4 is normally expressed early in development in the cleavage stage embryo ${ }^{7,8}$, in the adult testis ${ }^{6}$ and in the thymus ${ }^{9}$. De-repression of DUX4 in muscle activates a large cascade of events, triggering the activation of many pathways $\mathrm{s}^{8,10-19}$, with target genes being involved in biological processes such as RNA splicing and processing (DBR1 $^{10,20-22}, \mathrm{CWC}^{15^{10,20,22}}, \mathrm{PNN}^{10,21}, \mathrm{CLP} 1^{10,21,22}$, TFIP11 $^{10,20-22}$ ), spermatogenesis $\left(C C N A 1^{10,20-}\right.$ 22, ZNF296 ${ }^{10,20-22}$, TESK2 $2^{10,20,21}$ ), early embryonic development (ZSCAN4 ${ }^{10,20-22}$, LEUTX ${ }^{20-22}$,

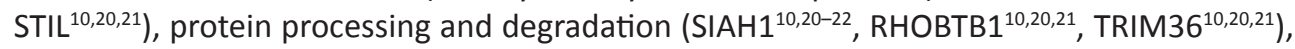
and cell motility and migration (CXCR4 $4^{10,20,21}$, ROCK1 ${ }^{10,21}$, SNAI1 ${ }^{10,20-22}$ ).

We hypothesized that of one or more factors downstream of DUX4 expression are responsible for the rapid apoptotic response that follows DUX4 induction. Knowing if there are key downstream targets of DUX4 can have important clinical applications as they could direct intelligent therapy design. We tested this hypothesis by performing a genome wide CRISPR/Cas9 knockout screen.

CRISPR/Cas9, which is now a highly popular and widely used genome editing technique, was initially discovered as the adaptive immune system of bacteria, to protect against viral infection ${ }^{23,24}$. Although not the first genome editing method, CRISPR/Cas9 has proven to be much more user friendly due to its easy manipulability, and being more cost-, labor- and time-efficient compared to its predecessors: transcription activator-like effector nucleases $(\text { TALENs) })^{25-28}$ and ZINC-fingers nucleases (ZFNs) ${ }^{29-33}$. Its ability to knock-out any gene by creating a double stranded break ${ }^{34-36}$ in such an easy manner, makes this technique very suitable for genome-wide loss off function studies. The advantages and ease-of-use of the CRISPR/Cas9 technology inspired us to perform a genome wide screen on a FSHD in-vitro model, to find potential modulators that contribute or aggravate the FSHD pathophysiology. Successful performance of a FSHD genome-wide screen will critically depend on the cell system being used. The cells should be highly proliferative, easily transfected and display a robust DUX4-induced phenotype. These parameters make human primary myoblasts a less suitable basis for a genome-wide CRISPR Knockout screen. Fortunately, DUX4 is a so-called pioneer factor ${ }^{37,38}$, capable of regulating its target genes independent of their chromatinstate. The network of genes activated by pioneer factors is therefore less affected by cellular identity. Indeed, Jones and colleagues have demonstrated that DUX4 activates the same downstream target genes in B-lymphocytes as previously identified in skeletal muscle myoblasts ${ }^{39,40}$. Using an adherent leukemic cell line that is frequently used for genomewide screening purposes $\left(K B M 7^{41,42}\right)$, we performed an exhaustive CRISPR knockout screen to identify factors that could mitigate DUX4-induced cytotoxicity. We had inserted a doxycycline-inducible DUX4 transgene into the adherent KBM7 cells ${ }^{41,42}$ to generate DUX4 inducible expression (DIE) cells. Using the Brunello CRISPR/Cas9 library ${ }^{43}$, we screened for modulators of DUX4 cytotoxicity. Our results suggest that no single gene knockout is capable of rescuing DUX4-triggered apoptosis in our transgene model system. 
This study does however, provide some interesting insight into critical parameters that need to be considered when executing a genome-wide CRISPR screen.

\section{Results}

\section{Genome-wide CRISPR Screen reveals large chromosomal truncations}

Using our DIE cell system, we sought out to identify modulators of DUX4 cytotoxicity by performing a genome wide CRISPR/Cas9 knockout screen. The Brunello human CRISPR knockout pooled library was used for this purpose ${ }^{43}$. This library contains 77.441 gRNAs targeting all protein coding genes, with an average of 4 gRNAs per gene as well as 1000 nontargeting control gRNAs. To optimize the signal-to-noise ratio of the experimental system, we titrated the timing and dose of the doxycycline-mediated DUX4 induction and selected two conditions, low $(250 \mathrm{ng} / \mathrm{ml})$ and high $(1000 \mathrm{ng} / \mathrm{ml})$ doxycycline with an exposure time of $24 \mathrm{~h}$ (Fig. 1A). At these concentrations 95 to $99 \%$ of the cells die, respectively. Figure 1B and S1 outline the setup of the screen. In addition to the high and low doxycycline concentrations, cells were harvested at two timepoints after doxycycline exposure to allow recovery, early $(24 \mathrm{~h})$ and late $(72 \mathrm{~h})$, ultimately resulting in 4 separate 4 screens; low doxycycline/early harvest, low doxycycline/late harvest, high doxycycline/early harvest, and high doxycycline/ late harvest.

Upon doxycycline administration and induction of DUX4 expression, cells from the surviving populations were harvested, genomic DNA was extracted and the gRNA sequence was amplified and sent for sequencing. Sequencing results of the treated samples revealed a large number of significantly enriched hits (Fig. 1C and S2). This included DUX4 itself and some other hits performing as well as the DUX4 gRNAs. However, upon closer examination it became clear that the majority of these enriched guides were located on the $q$ arm of chromosome 5, suggesting an FSHD unrelated experimental artefact. Since the rtTA transgene responsible for DUX4 induction is located on the $5 q$ arm, it is likely that when Cas9 is being targeted to the q-arm of chromosome 5 it leads to the removal the rtTA transgene, potentially through generation of a large deletion, chromosomal truncation or chromosomal rearrangement. It appears that as the rtTA integration site is located at the end of chromosome $5 q$, each target upstream of this site (towards the centromere) can cause a Cas9-mediated truncation, thereby removing the rtTA. (Fig. 1D, for phenograms of all 4 screens see Fig. S3). The correlation between the significance of a hit and its position along chromosome 5 highlights the strong association of these unexpected chromosomal rearrangements and the integration of rtTA at the end of chromosome 5, where the most significant hits reside in all four screens (Supplementary Figure S4).

Some of these $5 q$ locating guides (Fig. 1E) were tested individually in DIE cells containing a constitutively expressing Cas9 in its genome (DIE-Cas9), and without selecting for the rtTA and DUX4 transgene. No increased survival was detected compared to the background surviving cells that are seen in the control situation (Fig. 1F). This suggests that the Cas9induced truncation of a chromosomal arm and subsequent removal of rtTA activity is a rare event that was only identified due to the high sensitivity of our screen.

Data shown here was analyzed one-sidedly, and only truly represent enrichment. When analyzing the screen data double sided, one can again notice a clear enrichment of gRNA sequences, however no real depletion is seen (Fig. S5). 
A

$100 \quad \mathrm{ng} / \mathrm{ml}$ doxy

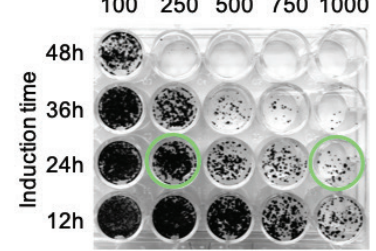

C

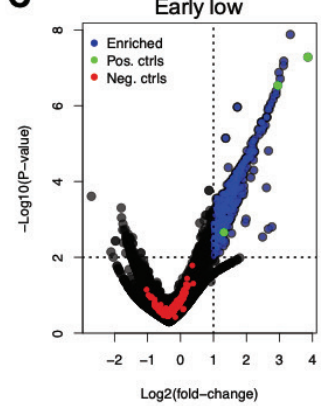

$\mathbf{E}$

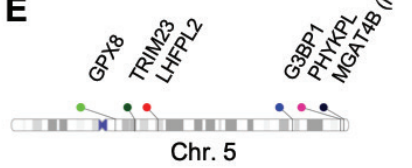

B

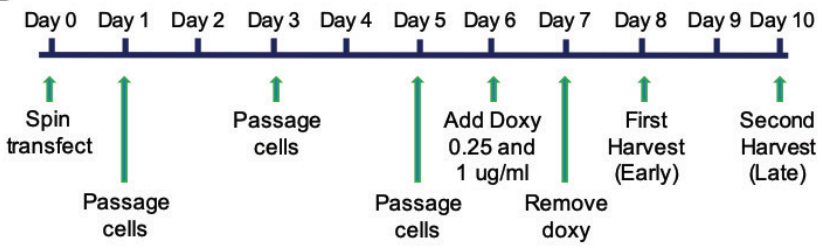

D
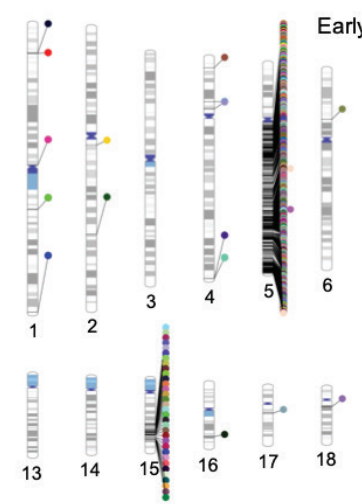

Early low
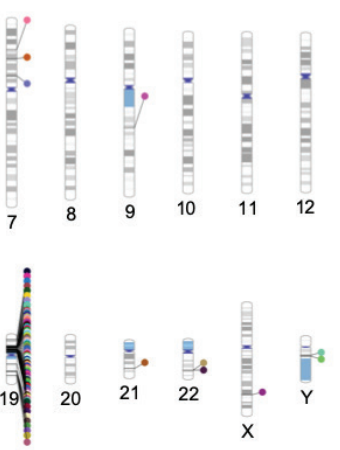

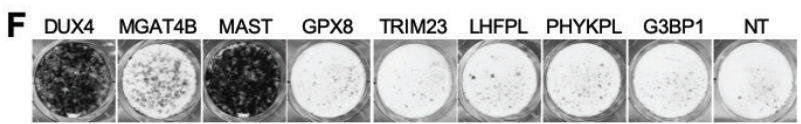

Figure 1. CRISPR Screen set up and discovery of a Cas9 artefact. (A) Viability staining of DIE cells treated with a doxycycline titration curve to determine which concentration and exposure time to use to induce sufficient cell death rates in DIE cells. Green circles indicate which conditions were used for the genome wide CRIPSR/Cas9 screen. (B) The CRISPR/Cas9 screen timeline from the moment of library transfection (Day 0) to the final harvest of surviving DIE cells (Day 10). (C) Volcano plot showing the enrichment of sets of guides of the low doxycycline/ early harvest screen. For a two-sided analysis see supplementary figure S5. Data shown here shows the average $\log 2$ (foldchange) and - $\log 10$ (p-value) of each guide set (set: 4 guides per gene). The log2(foldchange) is plotted on the $x$-axis and the $-\log 10$ ( $p$-value) on the $y$-axis. Blue points represent guide sets that are significantly enriched ( $P$-value $\leq 0.01$ ), LFC $\geq 1$ ), green point are the positive controls (DUX4, MAST1, MGAT4B), red points represent the Non-Target/negative control guides. (D) Chromosomal ideogram indicating the location of enriched hits in the human genome, of the low doxycycline/early harvest screen. (E) Schematic representation of the location of a small number of false positive hits on chromosome 5. (F) Viability staining demonstrating surviving DIE-Cas9 cells, containing knock-outs of the same genes mentioned in (E), after 250ng/ml doxycycline exposure. Media did not contain any selection markers. NT: Non-Target controls.

\section{Filtered Genome wide CRISPR screen results reveal no single targetable gene}

Since potential hits were likely obscured by the large number of false-positive hits that resulted from Cas9-mediated elimination of either the DUX4 or the rtTA transgenes, we filtered the screen results to remove all hits located on the q-arm of chromosome 5, or the p-arm of chromosome 19 (Figure 2A). After analyzing individual guides for their apparent effectiveness in the genome wide screen (instead of the group average), a list of potential hits emerged ( $p$-value $\leq 0.05$, Log2(foldchange) $\geq 1$ ) for each of the 4 screens. Figure $2 B$ shows the number of potential hits that met these criteria for each screen and how many of these hits are shared between them (See Table S1 to S4 for the lists of potential hits). We further focused on hits that emerged in at least 3 out of the 4 screens. Hits were validated 
by performing individual knock outs in the DIE-Cas9 cells, now also containing an inducible eGFP in its genome (DIE -ieGFP-Cas9). The tetracycline response element (TRE) controlling eGFP expression is identical to the TRE controlling DUX4 expression. If there is a true target that can mitigate the apoptotic phenotype without interfering with the inducible system, these positively targeted cells should not only survive but also emit an eGFP signal upon doxycycline admission (Fig. S6). Results show that MED25 increased cell survival when knocked-out (Fig. 2C). MED25 is a subunit of Mediator, a large complex that functions as a bridge between transcription factors and the transcriptional machinery. This includes RNA polymerase II, needed for the transcription of all protein coding genes in eukaryotes (reviewed by Soutourina ${ }^{44}$ ). The rescue seen in doxycycline induced DIE cells after MED25 knock-out diminishes upon higher doxycycline exposure, suggesting that loss of MED25 provides a partial rescue. Other genes belonging to the same mediator complex, that initially didn't meet our criteria, were reevaluated by lowering the parameters $(P \leq 0.05$, foldchange of $\geq 1.5$ ), identifying a number of other subunits. When individual knock-outs of these genes were performed, two more subunits of the Mediator-complex showed partial rescue (Fig. 2D). Finally, the individually tested $\mathrm{KO}$ cells were analyzed by flow cytometry, for the detection of eGFP. FACS analysis reveals that Mediator-complex components have a general effect on the inducible transcription of DUX4, since the knock-out of Mediator genes did not induce eGFP expression in surviving DIE cells (Fig. 2E). This suggest that their survival was due to a generally reduced ability of rtTA to mediate transgene activation.

In a recent study by Shadle and colleagues, a siRNA screen was performed targeting the "druggable" genome to identify pathways of DUX4 toxicity. The study revealed the MYCmediated apoptotic pathway and the viral dsRNA-mediated innate immune response pathway to be involved in DUX4 induced apoptosis ${ }^{45}$. We examined our data for enrichment of gRNA sequences that target the genes identified in the Shadle study, but did not observe significant enrichment in our CRIPSR screen data of these sequences. Figure 3A shows data plots that display the enrichment (Log2(foldchange)) and significance (-Log10(P-value)) of DUX4 and 3 other genes that were initially considered hits. However, subsequent single knock-outs validations demonstrated them to either have a generally effect on transcription (MED25), or upon their knockout did not exhibit any additional survival in induced DIE cells (RPS25 and CISD). Genes were only considered if a minimum of one gRNA showed significant enrichment in at least 3 out of 4 screens. Genes involved in the pathways identified by Shadle et all. did not meet these criteria (Fig. 3B). Furthermore, knocking out these genes in the DIE cells did not show an increased survival compared to background noise (Fig. 3C, top panel), as is noticeable in some of the false positives identified during this CRIPSR screen (Fig. 3C, lower panel). It should be mentioned that the two screens have major technical differences, such as the screening method, the complete or partial loss of function of genes, the scale of the screens (druggable genome vs whole genome) and the different cellular backgrounds, which most likely all attributed to the little correlation seen between the two studies. 
A

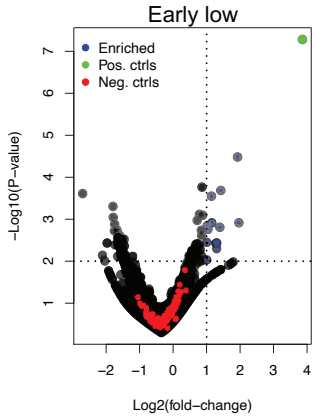

B

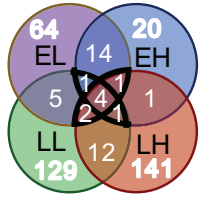

C

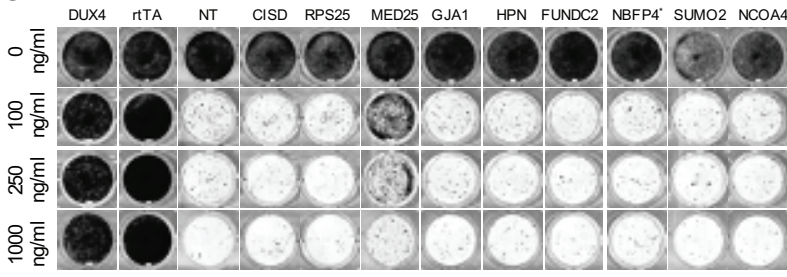

D $250 \mathrm{ng} / \mathrm{ml}$ doxy

DUX4 MED25 NT CCNC TRIP4 MED7 MED12 MED15 MED16 MED18 MED19 MED24 MED29 MED31
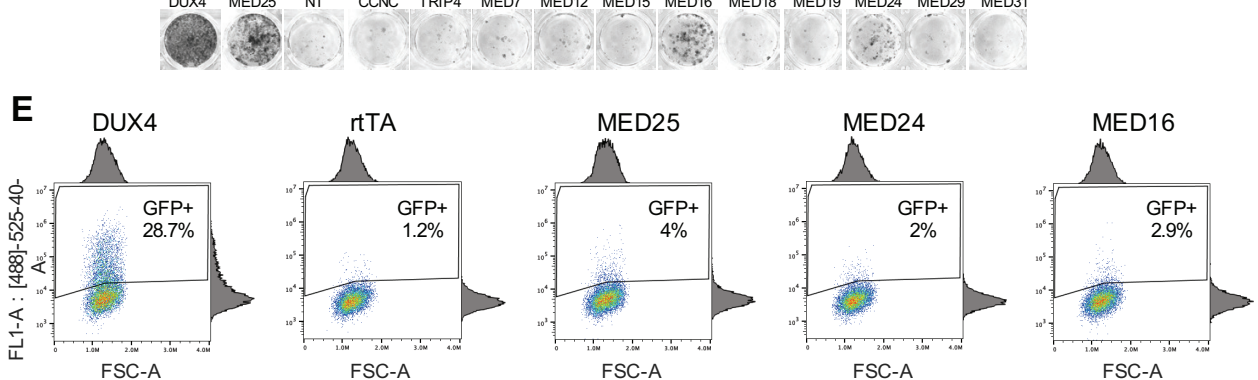

Figure 2. Filtered CRISPR screen data and validation of potential hits. (A) Adjusted volcano plot of low doxycycline/ early harvest screen data showing the enrichment of sets of guides targeting genes not located on chromosome $5 q$ or chromosome $19 p$. Blue points represent guide sets that are significantly enriched ( $P$-value $\leq 0.01)$, Log2(foldchange) $\geq 1$ ), the green point is the positive control (DUX4), red points represent the Non-Target control guides. (B) Venn diagram showing the overlap of 5q-filtered hits between the four screens (EL: Early harvest/Low doxy, LL: Late harvest/Low doxy, EH: Early harvest/High doxy, LH: Late harvest/High doxy). (C) Viability staining showing surviving DIE cells containing single knockouts of potentials hits identified in the CRISPR screen, after exposure to 3 different concentrations of doxycycline. (D) Viability staining showing the surviving DIE-ieGFP-Cas9 cells containing single knockouts of mediator complex subunits, after exposure to $250 \mathrm{ng} / \mathrm{ml}$ doxycycline. (E) FACs data showing GFP positive cells in surviving populations of DIE-ieGFP-Cas9 cells containing single knock-outs. DIEieGFP-Cas9 cells comprise of $42 \%$ of eGFP positive cells after DUX4 knock out. rtTA, MED25, MED24 and MED16 knock-outs show little eGFP expressing cells, comprising between 1.2-4\% of eGFP expressing cells. 
A
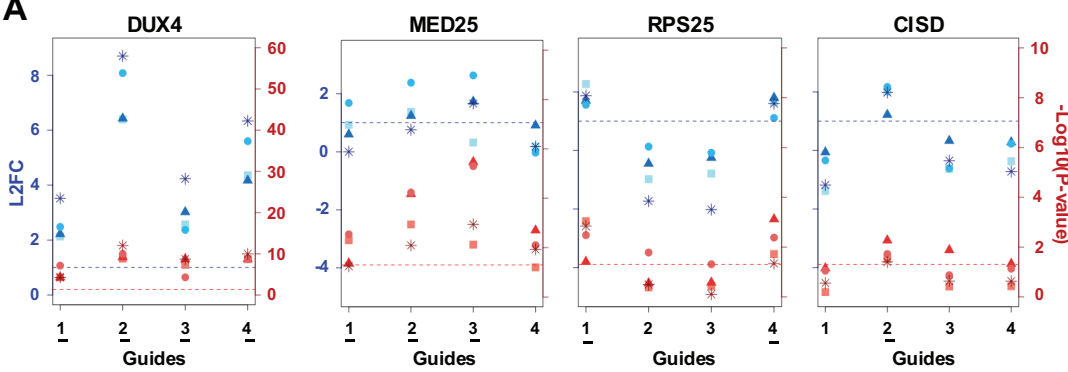

- Early low

4 Early high

- Late low

* Late high
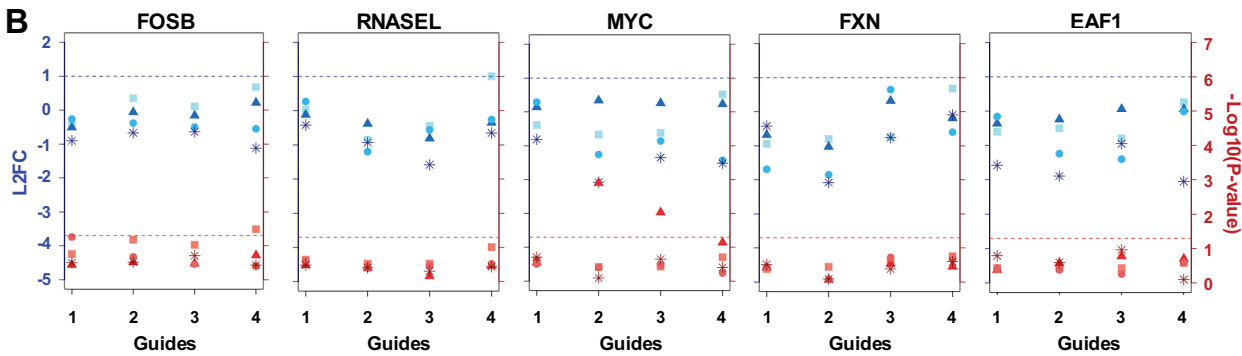

C
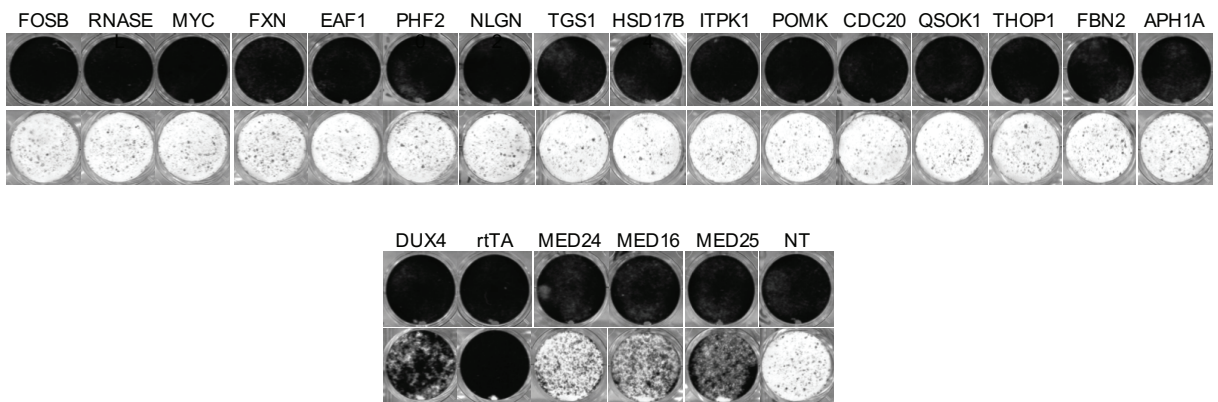

Figure 3. Validation of genes involved in the MYC-mediated apoptotic pathway and the viral dsRNA-mediated innate immune response pathway. (A) Data plots showing the significance and enrichment of gRNAs targeting DUX4, MED25, RPS25 and CISD, in all 4 screens. The Log2(fold-change) (L2FC) of each individual guide is plotted on the left $y$-axis indicated in blue, and the -Log10(P-value) is plotted on the right $y$-axis, in red. When guides fall above the blue and red intermitted ablines, they are considered significant (Log2(foldchange) $>1$, - Log10(P-value) $>1.3$ ). The gRNAs that are significantly enriched in all 4 screens are underlined. All 4 gRNAs targeting DUX4 are significantly enriched. 3 out of 4 gRNA's targeting MED25 are significantly enriched (guides 1, 2 and 3). Guides 1 and 4 targeting PRS25 are significantly enriched, and CISD has one guide that is significantly enriched in all 4 screens. (B) Data plots showing the enrichment of gRNA targeting FOSB, RNASEL, MYC, FXN and EAF1. None of the 4 guides show significant enrichment in any of the 4 screens. (C) Viability staining showing surviving DIE-Cas 9 cells containing single knockouts of genes involved in the MYC-mediated apoptotic pathway and the dsRNA-mediated immune response pathway (Top panel). Controls can be found in the bottom panel and are as followed, positive controls: DUX4, rtTA, MED24, MED16 and MED25; Negative control: NT 


\section{Discussion}

At present there are no effective pharmacological treatment options that can improve muscle strength or slow down disease progression in FSHD patients ${ }^{46}$. Unravelling the underlying mechanism of DUX4 cytotoxicity would help identify therapeutic targets. We hypothesized that inhibition of key downstream DUX4 effectors would slow or abrogate the cytotoxic process, and set out to identify such genes by performing an exhaustive genome wide CRISPR/Cas9 screen. We know the screen was exhaustive because we picked up rare rearrangements disabling the DUX4 transgene or the rTTA inducer. The goal of the screen was to identify targets that can mitigate DUX4 induced toxicity. While the screen's technical execution went very well and displayed high sensitivity, specificity toward candidate editing events that indeed mitigated cytotoxicity in our transgene model, none of the obtained hits had a direct effect on DUX4 its downstream transcriptional network. Rather, screen hits seemed to specifically affect the experimental system itself, either by affecting the tetracycline-inducible system responsible for DUX4 transgene induction, or by mutations of the DUX4 transgene. The main contributor is likely a rare Cas9-induced chromosomal truncation event, that removes the transgenes when targeted to the chromosomal arm to which they have integrated. Although these events appear to be rare, nearly all guides that targeted genes located on the chromosomal arm to which rtTA had integrated (5q) were robustly enriched, underwriting the sensitivity of this screening method. Most remaining hits did not appear to effect DIE cell survival upon individual validation, but members of the Mediator complex did show a positive effect on survival. Unfortunately, these mediator subunit genes seemed to generally suppress rtTA-mediated transcription so their mitigating effect was not mediated by specifically altering DUX4 cytotoxicity. We therefore concluded, that based on the conditions used in this study, there to be no individual target (other than DUX4 itself) that upon knockout can provide a strong inhibition of DUX4 induced cytotoxicity. Efforts should therefore be redirected to the direct modulation of DUX4.

While our library only targeted protein-coding genes, we believe we would have pickedup any mitigating non-coding RNAs as well, had they provided a strong rescue from the DUX4 cytotoxic effects. In that case, one would have expected to see a similar hotspot of gRNAs on and around the true target sites, as we observed for, MED16, where a hotspot of gRNAs was observed on the p-arm of chromosome 19, corresponding to the location of MED16. Another hotspot can be seen on the $q$ arm of chromosome 19, corresponding to the location of MED25. The hotspot on chromosome 15 can be explained by the genetic makeup of the KBM7 cell line. KBM7 cells not only have the Philadelphia chromosome, but also an integration event where a region of chromosome 15 integrated on chromosome $19 \mathrm{p}^{47}$. The hotspot on chromosome 15 correlates to the region that has integrated on chromosome $19 p$, close to the MED25 site.

Our screen results shown here do not corroborate previous findings of Shadle et $\mathrm{al}^{45}$. However, their siRNA screen differs in many aspects to the performed genome wide CRIPSR/ Cas9 screen we executed. Their screen was knocking-down the druggable genome, using Lipofectamine RNAiMAX to deliver the siRNA library, in Rhabdomyosarcoma derived cells; whereas our screen was knocking-out protein coding genes genome wide, using a viral library, in chronic myeloid leukemia derived cells. These differences could explain why results between the two screens are not correlating with one another. Furthermore, A side by side comparison study of CRISPR/Cas9 and a next generation RNAi screens reveals that the screening methods seem to effect different biological aspect of the cells, therefore finding little correlations between results. The authors also in part attribute these differences to the 
technical differences between the two techniques ${ }^{48}$.

Another recently published genome wide CRISPR/Cas9 study, where a similar methodology was used in a DUX4 inducible immortalized myoblast line, identified the HIF1 oxidative stress pathway as a modulator of DUX4-induced apoptosis ${ }^{49}$. This study, as well as previous reports, clearly demonstrate the role of the HIF1 hypoxia pathway in DUX4-mediated cytotoxicity ${ }^{49-51}$. The HIF1 pathways did not come up in our screen (Fig. S7). This demonstrates that changes in this pathway are likely not the only DUX4 induced cellular changes that push cells towards apoptosis. The fact that the HIF1 pathway did not come up in our screen could also indicate differences in sensitivity to oxidative stress between cellular systems. Different cell types experience and respond differently to oxidative stress, with differences in culturing conditions as further attributing factors, for example the concentration of 2-mercapto ethanol to cell culture media.

While our screen did not identify target genes that can mitigate DUX4 cytotoxicity, it does illustrate some important aspects that need to be considered when performing phenotypic CRISPR/Cas9 screens. One being the large chromosomal truncations that can be induced by Cas9, a phenomena also recently reported by Cullot et $\mathrm{al}^{52}$. While these are rare events in a cell population, our results demonstrate that in a sufficiently sensitive screening system, they are robustly identified and can crowd potential positive hits. Sufficient selection should at least help in this aspect by removing cells that had their resistance marker (linked to the transgene) deleted. Another aspect that needs consideration are the endogenous genes that have a general effect on transcription and translation, in this case effecting the inducible system, like subunits of the mediator complex identified in this study. Potential hits will always need to be validated individually in such a way that can exclude this possibility, like shown here, or by Shadle et al. where some of the same genes were identified effecting their inducible Tet-On system ${ }^{45}$.

This study started out with the aim of trying to contribute to the understanding of the underlying molecular mechanism of FSHD, by performing a genome wide CRISPR-Cas9 phenotypic screen. However, with no significant hits that can explain their contribution to the apoptotic phenotype, this story also tells a cautionary tale for knockout screens through the use CRISPR-Cas9, which will benefit future groups planning to execute similar screens.

\section{Methods}

\section{Cell culture}

HAP1 cells were cultured in IMDM media (Fischer Scientific) supplemented with $10 \%$ FBS. DIE cells were cultured in IMDM media supplemented with $10 \%$ Tet system approved FBS (Clontech), $5 \mu \mathrm{g} / \mathrm{ml}$ Puromycin, $6 \mu \mathrm{g} / \mathrm{ml}$ Blasticidin, and $100 \mu \mathrm{M}$ Beta-mercaptoethanol.

\section{Cloning p2T-Cas9, p2T-ieGFP and sgRNA constructs, and generating DIE-Cas9 and DIE-}

\section{Cas9-ieGFP cell lines}

The p2T-CAG-spCas9-NeoR mammalian expression plasmid was created by replacing the Blasticidin resistance gene (BlastR) in the p2T-CAG-spCas9-BlastR (Addgene: 107190) ${ }^{53}$ with a Neomycin resistance gene (NeoR). The p2T-CAG-spCas9-BlastR plasmid is contained in a p2Tol2 backbone ${ }^{54}$. The BlastR gene was removed using restriction digestion, using Mfel and 
Aflll (NEB). Cloning the NeoR DNA fragment into the p2T-CAG-spCas9 backbone was done in similar fashion as described above. The p2T-CAG-SpCas9-BlastR was a gift from Richard Sherwood. The p2T-TetO-eGFP-HygroR plasmid was generated in a similar way as the p2TCAG-spCas9-NeoR. In short, all sequences between transposable elements of a p2T plasmid were removed by restriction digestion using Alel and EcoRI (NEB). The TetO-eGFP-HygroR cassette was created by amplifying each subunit individually, and thereafter cloned into the empty $\mathrm{p} 2 \mathrm{~T}$ backbone, using in-fusion cloning.

Both p2T-CAG-spCas9-NeoR and p2T-TetO-eGFP-HygroR were introduced in the DIE cell line by using Transposase. The P2T-CAG-spCas9-NeoR was introduced into DIE cells together with a plasmid encoding for transposase, using Polyethylenimine (PEI) transfection reagent (4ug PEI per 1ug DNA). The DIE cells were exposed to the transfection mixture for 14-16h, after which the transfection media was replaced with growth media. Geneticin g418 selection was started two days post transfection, generating the DIE-Cas9 line. The DIE-Cas9-ieGFP cell line was created by adding Transposase andp2T-TetO-eGFP-HygroR the DIE-Cas9 line, described as above.

spCas9-sgRNA constructs were cloned using a plasmid containing a U6 promotor, 2 BsmBI sites with directly adjacent the tracrRNA sequence, and a Hygromycin resistance gene (made in house). This U6-2xBsmBI-Tracr-HygroR plasmid was digested with the BsmBI restriction enzymes (NEB), after which the CRISPR inserts were ligated in using T4 DNA ligase (NEB). CRISPR inserts were generated by annealing two complementary oligos containing a $4 \mathrm{bp}$ adapter serving as the BsmBI sticky end.

All plasmids mentioned in this study were transformed in chemically competent Stbl3 Escherichia coli (E.coli), and prepped using a HiPure plasmid Midi or Maxi kit (Invitrogen).

\section{Doxycycline titration curve}

200.000 cells were seeded into wells of a 24-wells plate and incubated overnight at $5 \% \mathrm{CO}_{2}$, and $37^{\circ} \mathrm{C}$. When cells reached a density of $90-100 \%$ confluency, different concentrations of doxycycline were added to the vertical lanes $(100 \mathrm{ng} / \mathrm{ml}, 250 \mathrm{ng} / \mathrm{ml}, 500 \mathrm{ng} / \mathrm{ml}, 750 \mathrm{ng} /$ $\mathrm{ml}, 1000 \mathrm{ng} / \mathrm{ml}$ ), with the horizontal lanes experiencing different exposure times (48h, $36 \mathrm{~h}, 24 \mathrm{~h}, 12 \mathrm{~h}$ ). After a recovery period of $96 \mathrm{~h}$ (after doxy exposure was ended), cells were washed with DPBS, and fixed with Methanol for 10 minutes. Giemsa stain, modified solution (Sigma) was subsequently added for 45 minutes, after which it was removed and the wells were washed with demineralized water.

\section{Genome-wide CRISPR screen}

The screen on the DIE line was performed as previously described by Doench et al. ${ }^{43}$. Due to a shared selection marker between the DIE line and the Brunello lentiviral library, transfected cells could not be selected for, thus the total number of cells was raised to 1500 cells per guide, when considering an average transfection efficiency of $30-50 \%$ in all cell lines tested by Doench et $\mathrm{al}^{43}$. The transfection efficiency was determined and calculated using the DIE parental line, the rediplodized HAP1 cells. With 1500 cells per guide (total of 77.441guides), each of the technical three replicates contained $120 * 10 \mathrm{E} 6$ cells, that were spin transfected for $2 \mathrm{~h}$ at $1000 \mathrm{~g}$ with $82 * 10 \mathrm{E} 6$ Brunello virus particles (LentiCRISPRv2, Addgene 73179-LV). With a multiplicity of infection (MOI) of 0.65 , transfection efficiency reached $60 \%$ upon testing the viral library on the diploid HAP1 parental line. After transfection the $120 * 10 \mathrm{E} 6$ transfected cells (contained in 40 wells of 12 -well tissue culturing plates) were trypsinized and passaged to $60145 \mathrm{~mm}$ TC plates. Mutagenized cells were maintained for 6 days, 
before inducing a set of 24 plates with either a low or high doxycycline concentration (low: $250 \mathrm{ng} / \mathrm{ml}$, high: $1000 \mathrm{ng} / \mathrm{ml}$ ). The remaining 12 plates were harvested for cryofreezing (7 plates) and for determining library coverage (5 plates). After a $24 \mathrm{~h}$ doxycycline induction period, 12 plates were given a $24 \mathrm{~h}$ recovery period (early harvest) of both the low and high doxycycline exposed sets. The remaining 24 plates received an additional $48 \mathrm{~h}$ of recovery time (late harvest), before harvesting the surviving cells for sequencing (Fig. S1). Cell Pellets were stored at $-80^{\circ} \mathrm{C}$ until further processing. The Human Brunello CRISPR knockout pooled lentiviral prep library was a gift from David Root and John Doench.

\section{Library prep, sequencing and analysis}

Genomic DNA (gDNA) was isolated using NucleoSpin Blood Mini (less than 5 million cells), Midi (L) (5-20 million cells) and Maxi (XL) (more than 20 million cells) kits, depending on the size of cell pellet. Libraries were prepared and sequenced on a HiSeq2000 (Illumina) as described by Doench et al. Analysis was conducted using "STARS", gene-ranking method to generate FDR values developed by Doench et al. that was used to generate $p$-values and FDR rates. ${ }^{43}$ Chromosomal ideogram were generated by using the PhenoGram webtool from the Ritchie lab from the university of Pennsylvania ${ }^{57}$.

\section{Individual knock outs in DIE cells}

DIE-Cas9 and DIE-Cas9-ieGFP were seeded in a 24-well setting. Next day, when the cells had reached $70-90 \%$ confluency, cells were transfected with 500 ng guide plasmid per well using 4ug PEI per 1ug DNA. During the overnight transfection no selection markers were presents in the media, however growth media was supplemented with $100 \mathrm{U} / \mathrm{ml}$ pen-strep. Cells were passaged with or without selection markers during a period of 6-7, after which doxycycline was added $(100,250$ or $1000 \mathrm{ng} / \mathrm{ml})$ for a $24 \mathrm{~h}$ period. Wells were washed with DPBS to remove dead cells and debris. Remaining cells were given the opportunity to grow out, or to perish (if they had already entered the apoptotic pathway) for an additional 4896 hours. The wells were stained using Giemsa modified solution, as described previously.

\section{Flowcytometry sorting (FACS) and analysis}

DIE-Cas9-ieGFP cells were induced with $250 \mathrm{ng} / \mathrm{ml}$ doxycycline $24 \mathrm{~h}$ prior to FACS analysis. After the $24 \mathrm{~h}$ doxycycline exposure, cells were trypsinized using $0.25 \%$ Trypsin-EDTA, resuspended in iMDM media supplemented with Tet approved FBS and DAPI nuclear staining, and strained using a Cell-strainer capped tubes (Falcon). Cells were analyzed using the Beckman coulter Cytoflex S flow cytometer.

\section{Data Resources}

Data containing the Genome wide CRISPR/Cas9 samples in triplicate are available from the GEO data base, accession number: GSE155034.

\section{Acknowledgements}

This study was supported by Stichting FSHD nd the SingelSwim Utrecht. The authors like to thank Nune Schelling, Peng Shang, Stefan van der Elst, en Reinier van der Linden for excellent technical assistance. 


\section{References}

1. Deenen, J. C. W. et al. Population-based incidence and prevalence of facioscapulohumeral dystrophy. Neurology 83, 1056-1059 (2014).

2. Tawil, R. \& Van Der Maarel, S. M. Facioscapulohumeral muscular dystrophy. Muscle and Nerve 34, 1-15 (2006).

3. Pastorello, E., Cao, M. \& Trevisan, C. P. Atypical onset in a series of 122 cases with FacioScapuloHumeral Muscular Dystrophy. Clin. Neurol. Neurosurg. 114, 230-234 (2012).

4. Dixit, M. et al. DUX4, a candidate gene of facioscapulohumeral muscular dystrophy, encodes a transcriptional activator of PITX1. Proc. Natl. Acad. Sci. U. S. A. 104, 18157-18162 (2007).

5. Lemmers, R. J. L. F. et al. A unifying genetic model for facioscapulohumeral muscular dystrophy. Science 329, 1650-1653 (2010).

6. Snider, L. et al. Facioscapulohumeral dystrophy: Incomplete suppression of a retrotransposed gene. PLoS Genet. 6, 1-14 (2010).

7. Whiddon, J. L., Langford, A. T., Wong, C. J., Zhong, J. W. \& Tapscott, S. J. Conservation and innovation in the DUX4-family gene network. Nat. Genet. 49, 935-940 (2017).

8. Hendrickson, P. G. et al. Conserved roles of mouse DUX and human DUX4 in activating cleavage-stage genes and MERVL/HERVL retrotransposons. Nat. Genet. 49, 925-934 (2017).

9. Das, S. \& Chadwick, B. P. Influence of repressive histone and DNA methylation upon D4Z4 transcription in non-myogenic cells. PLoS One 11, 1-26 (2016).

10. Geng, L. N. et al. DUX4 Activates Germline Genes, Retroelements, and Immune Mediators: Implications for Facioscapulohumeral Dystrophy. Dev. Cell 22, 38-51 (2012).

11. Dmitriev, P. et al. DUX4-induced constitutive DNA damage and oxidative stress contribute to aberrant differentiation of myoblasts from FSHD patients. Free Radic. Biol. Med. 99, 244258 (2016).

12. Iaco, A. De et al. A family of double-homeodomain transcription factors regulates zygotic genome activation in placental mammals. Nat. Genet. 49, 941-945 (2017).

13. Knopp, P. et al. DUX4 induces a transcriptome more characteristic of a less-differentiated cell state and inhibits myogenesis. J. Cell Sci. 129, 3816-3831 (2016).

14. Bosnakovski, D. et al. High-throughput screening identifies inhibitors of DUX4-induced myoblast toxicity. Skelet. Muscle 4, 1-11 (2014).

15. Bosnakovski, D. et al. DUX4c, an FSHD candidate gene, interferes with myogenic regulators and abolishes myoblast differentiation. Exp. Neurol. 214, 87-96 (2008).

16. Winokur, S. T. et al. Expression profiling of FSHD muscle supports a defect in specific stages of myogenic differentiation. Hum. Mol. Genet. 12, 2895-2907 (2003).

17. Bosnakovski, D. et al. An isogenetic myoblast expression screen identifies DUX4-mediated FSHD-associated molecular pathologies. EMBO J. 27, 2766-2779 (2008).

18. Bosnakovski, D. et al. p53-independent DUX4 pathology in cell and animal models of facioscapulohumeral muscular dystrophy. DMM Dis. Model. Mech. 10, 1211-1216 (2017).

19. Feng, Q. et al. A feedback loop between nonsense-mediated decay and the retrogene DUX4 in facioscapulohumeral muscular dystrophy. Elife 2015, 1-13 (2015).

20. Rickard, A. M., Petek, L. M. \& Miller, D. G. Endogenous DUX4 expression in FSHD myotubes is sufficient to cause cell death and disrupts RNA splicing and cell migration pathways. Hum. Mol. Genet. 24, 5901-5914 (2015).

21. Jagannathan, S. et al. Model systems of DUX4 expression recapitulate the transcriptional profile of FSHD cells. Hum. Mol. Genet. 25, ddw271 (2016).

22. Van Den Heuvel, A. et al. Single-cell RNA sequencing in facioscapulohumeral muscular dystrophy disease etiology and development. Hum. Mol. Genet. 28, 1064-1075 (2019).

23. Barrangou, R. et al. CRISPR provides acquired resistance against viruses in prokaryotes. Science 315, 1709-1712 (2007).

24. Brouns, S. J. et al. Small CRISPR RNAs guide antiviral defense in prokaryotes. Science 321, 960-964 (2008).

25. Christian, M. et al. Targeting DNA double-strand breaks with TAL effector nucleases. Genetics 186, 756-761 (2010).

26. Li, T. et al. TAL nucleases (TALNs): Hybrid proteins composed of TAL effectors and FokI DNAcleavage domain. Nucleic Acids Res. 39, 359-372 (2011).

27. Mussolino, C. et al. A novel TALE nuclease scaffold enables high genome editing activity in 
combination with low toxicity. Nucleic Acids Res. 39, 9283-9293 (2011).

28. Miller, J. C. et al. A TALE nuclease architecture for efficient genome editing. Nat. Biotechnol. 29, 143-150 (2011).

29. Kim, Y. G., Cha, J. \& Chandrasegaran, S. Hybrid restriction enzymes: Zinc finger fusions to Fok I cleavage domain. Proc. Natl. Acad. Sci. U. S. A. 93, 1156-1160 (1996).

30. Kim, Y. G., Kim, P. S., Herbert, A. \& Rich, A. Construction of a Z-DNA-specific restriction endonuclease. Proc. Natl. Acad. Sci. U. S. A. 94, 12875-12879 (1997).

31. Smith, J. et al. Requirements for double-strand cleavage by chimeric restriction enzymes with zinc finger DNA-recognition domains. Nucleic Acids Res. 28, 3361-3369 (2000).

32. Bibikova, M. et al. Stimulation of Homologous Recombination through Targeted Cleavage by Chimeric Nucleases. Mol. Cell. Biol. 21, 289-297 (2001).

33. Bibikova, M., Golic, M., Golic, K. G. \& Carroll, D. Targeted Chromosomal Cleavage and Mutagenesis in Drosophila Using Zinc-Finger Nucleases. Genetics 161, 1169-1175 (2002).

34. Gasiunas, G., Barrangou, R., Horvath, P. \& Siksnys, V. Cas9-crRNA ribonucleoprotein complex mediates specific DNA cleavage for adaptive immunity in bacteria. Proc. Natl. Acad. Sci. U. S. A. 109, 2579-2586 (2012).

35. Jinek, M. et al. RNA-programmed genome editing in human cells. Elife 2013, 1-9 (2013).

36. Cong, L. et al. Multiplex Genome Engineering Using CRISPR/Cas Systems. Science 339, 819823 (2013).

37. Choi, S. H. et al. DUX4 recruits p300/CBP through its C-terminus and induces global H3K27 acetylation changes. Nucleic Acids Res. 44, 5161-5173 (2016).

38. Vuoristo, S. et al. DUX4 regulates oocyte to embryo transition in human. Biorxiv (2019) doi:http://dx.doi.org/10.1101/732289.

39. Jones, T. I., Himeda, C. L., Perez, D. P. \& Jones, P. L. Large family cohorts of lymphoblastoid cells provide a new cellular model for investigating facioscapulohumeral muscular dystrophy. Neuromuscul. Disord. 27, 221-238 (2017).

40. Banerji, C. R. S., Zammit, P. S. \& Panamarova, M. Lymphocytes contribute to DUX4 target genes in 1 FSHD muscle biopsies. Biorxiv (2019) doi:http://dx.doi.org/10.1101/717652.

41. Andersson, B. S. et al. Ph-positive chronic myeloid leukemia with near-haploid conversion in vivo and establishment of a continuously growing cell line with similar cytogenetic pattern. Cancer Genet. Cytogenet. 24, 335-343 (1987).

42. Mulherkar, N. et al. Ebola Virus entry requires the cholesterol transporter Niemann-Pick C1. Nature 477, 340-343 (2012).

43. Doench, J. G. et al. Optimized sgRNA design to maximize activity and minimize off-target effects of CRISPR-Cas9. Nat. Biotechnol. 34, 184-191 (2016).

44. Soutourina, J. Transcription regulation by the Mediator complex. Nat. Rev. Mol. Cell Biol. 19, 262-274 (2018).

45. Shadle, S. C. et al. DUX4-induced dsRNA and MYC mRNA stabilization activate apoptotic pathways in human cell models of facioscapulohumeral dystrophy. PLoS Genet. 13, 1-25 (2017).

46. Tawil, R. et al. Evidence-based guideline summary: Evaluation, diagnosis, and management of facioscapulohumeral muscular dystrophy. Neurology 85, 357-364 (2015).

47. Essletzbichler, P. et al. Megabase-scale deletion using CRISPR/Cas9 to generate a fully haploid human cell line. Genome Res. 24, 2059-2065 (2014).

48. Morgens, D. W., Deans, R. M., Li, A. \& Bassik, M. C. Systematic comparison of CRISPR/Cas9 and RNAi screens for essential genes. Nat. Biotechnol. 34, 634-636 (2016).

49. Lek, A. et al. Applying genome-wide CRISPR-Cas9 screens for therapeutic discovery in facioscapulohumeral muscular dystrophy. Sci. Transl. Med. 12, 9-11 (2020).

50. Banerji, C. R. S. et al. $\beta$-catenin is central to DUX4-driven network rewiring in facioscapulohumeral muscular dystrophy. J. R. Soc. Interface 12, (2015).

51. Tsumagari, K. et al. Gene expression during normal and FSHD myogenesis. BMC Med. Genomics 4, (2011).

52. Cullot, G. et al. CRISPR-Cas9 genome editing induces megabase-scale chromosomal truncations. Nat. Commun. 10, 1-14 (2019).

53. Shen, M. W. et al. Predictable and precise template-free CRISPR editing of pathogenic variants. Nature 563, 646-651 (2018).

54. Urasaki, A., Morvan, G. \& Kawakami, K. Functional dissection of the Tol2 transposable 
element identified the minimal cis-sequence and a highly repetitive sequence in the subterminal region essential for transposition. Genetics 174, 639-649 (2006).

55. Ferreboeuf, M. et al. DUX4 and DUX4 downstream target genes are expressed in fetal FSHD muscles. Hum. Mol. Genet. 23, 171-181 (2014).

56. Borgström, A. et al. A novel multiplex qPCR targeting 23S rDNA for diagnosis of swine dysentery and porcine intestinal spirochaetosis. BMC Vet. Res. 13, 1-8 (2017).

57. Wolfe, D., Dudek, S., Ritchie, M. D. \& Pendergrass, S. A. Visualizing genomic information across chromosomes with PhenoGram. BioData Min. 6, 1-12 (2013).

\section{Supplementary figures}

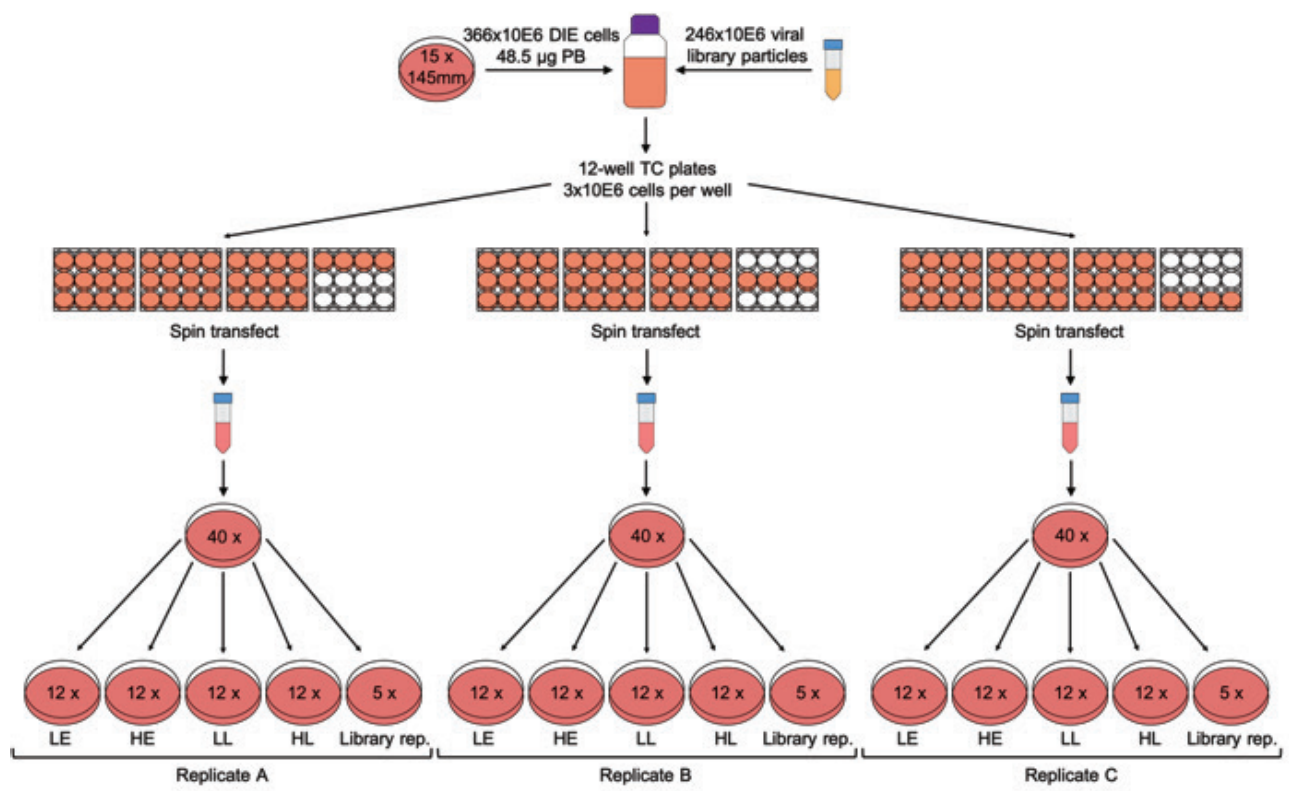

Figure S1. Execution of the CRIPSR/Cas9 genome wide screens. A schematic representation of the execution of the CRISPR/Cas9 screens. PB: Polybrene, TC: Tissue culture, LE: Low doxycycline/Early harvest, HE: High doxycycline/Early harvest, LL: Low doxycycline/Late harvest, HL: High doxycycline/Late harvest, Library rep: Library representation. 


\section{Early low}

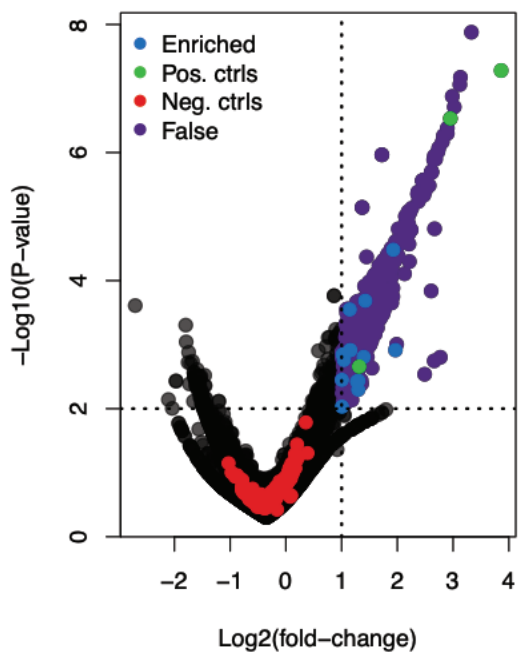

Late low

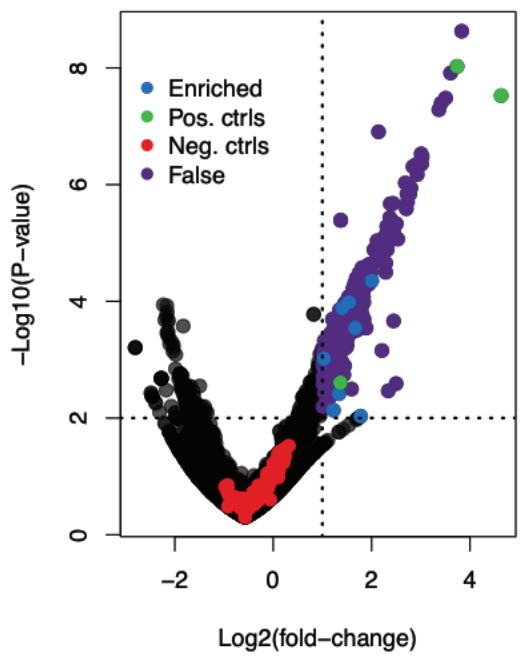

Early high

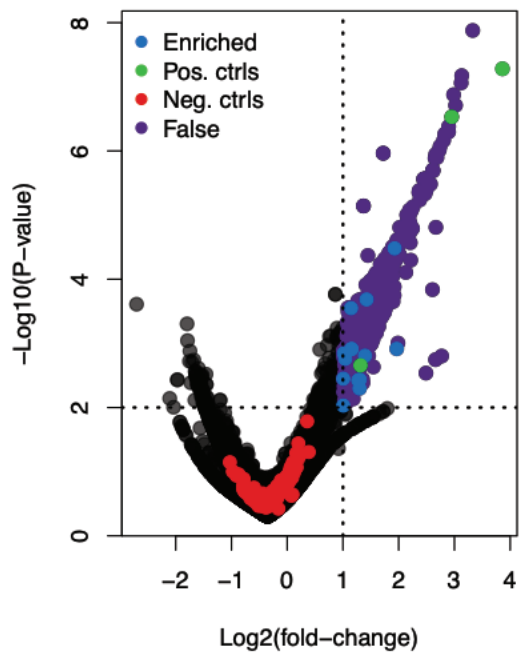

Late high

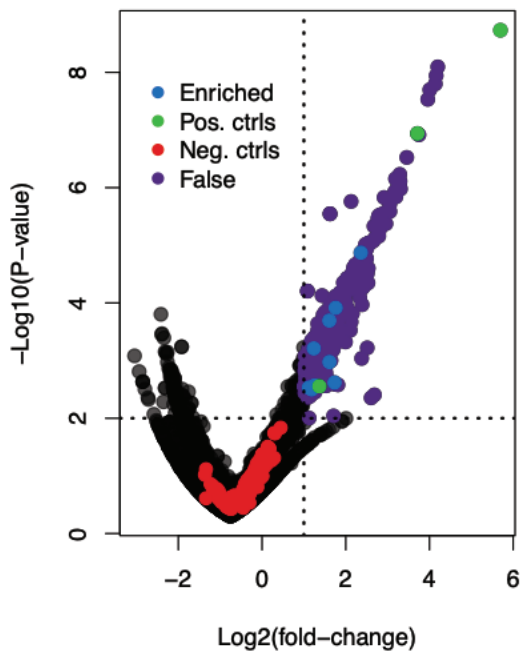

Figure S2. gRNAs enrichment from screen data processed with a one-sided analysis. Volcano plots illustrating enrichment of gRNAs in the surviving population of DIE cells of all 4 screens. Due to the one-sided analysis, depletion data should not be taken into consideration. For a two-sided analysis see supplementary figure S3. The Log2(foldchange) is plotted on the $x$-axis and the -Log10(p-value), is plotted on the $y$-axis. Data shown here shows the average $\log 2$ (foldchange) and $-\log 10$ (P-value) of each guide set (set: 4 guides per gene). Blue points represent guide sets that are significantly enriched (Log2(foldchange) $\geq 1,-\log 10$ (P-value) $\geq 2$ ), purple points represent the false positive hits on chromosome $5 q$ and chromosome $19 p$, green point are the positive controls (DUX4, MAST1, MGAT4B), red points represent the Non-Target control guides. 

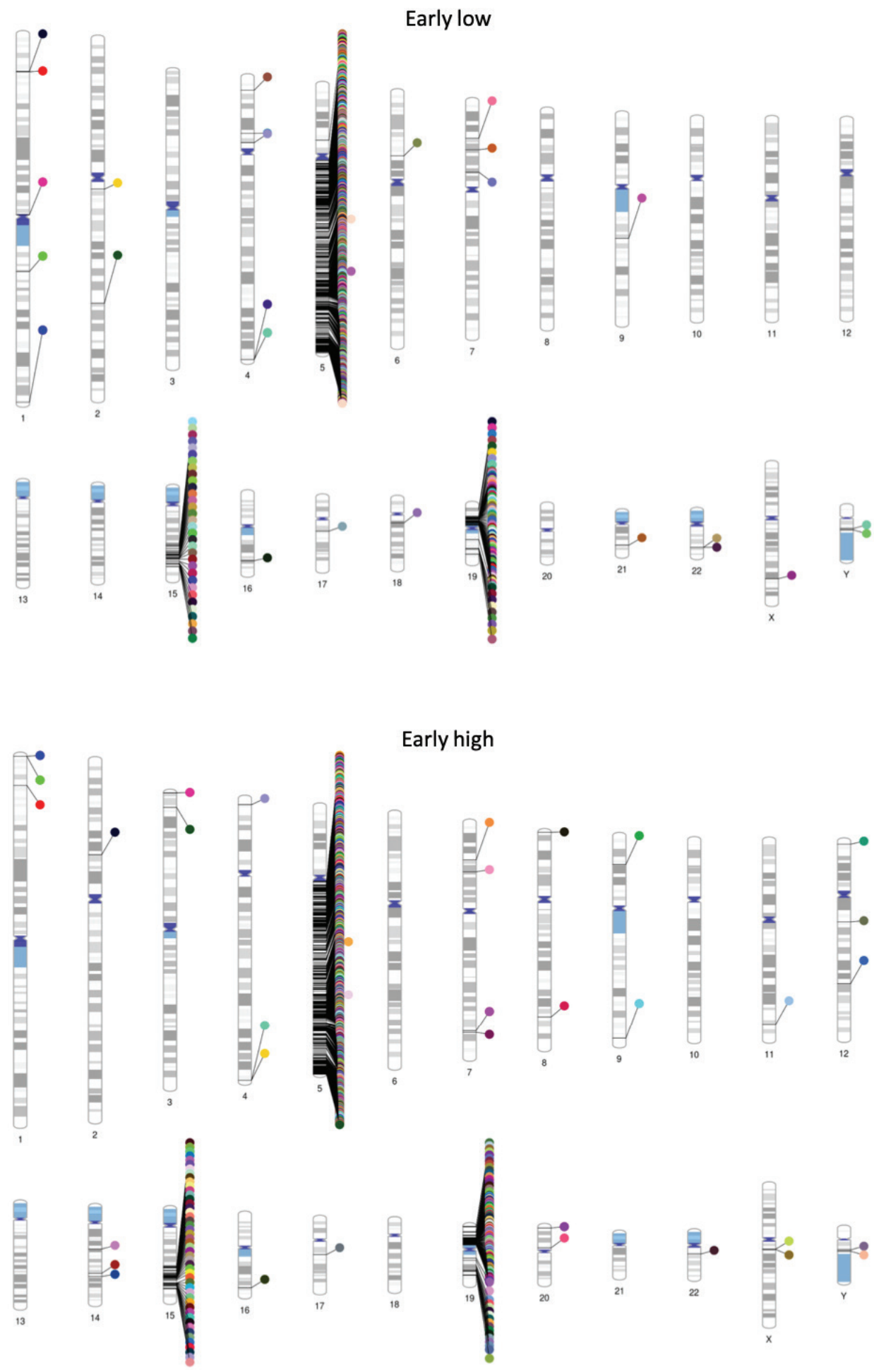


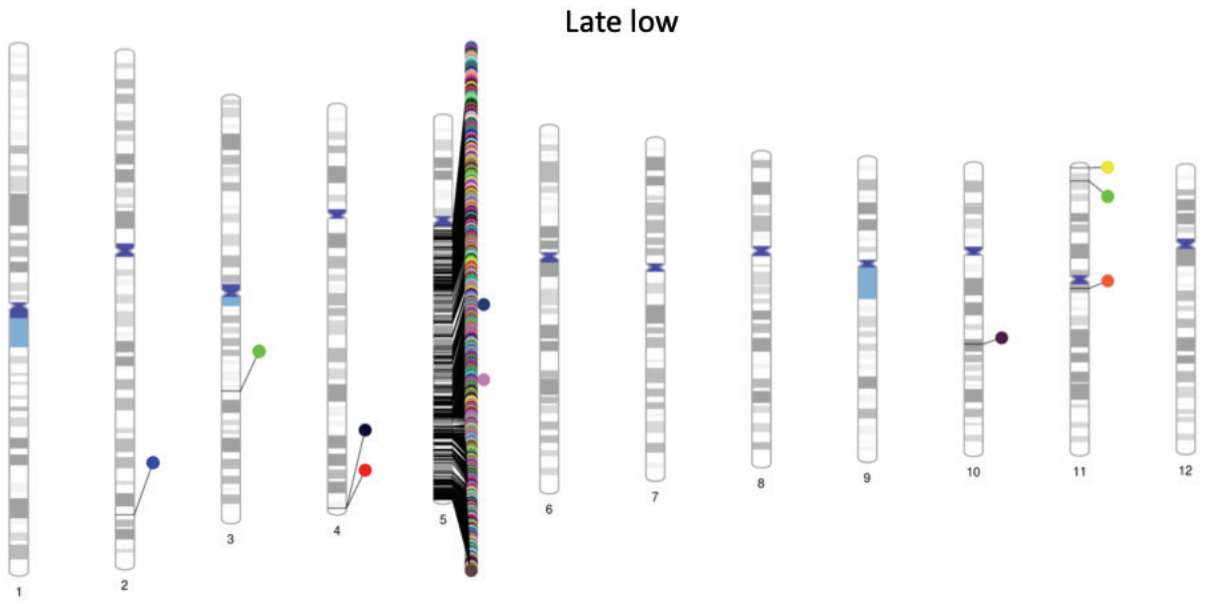

I

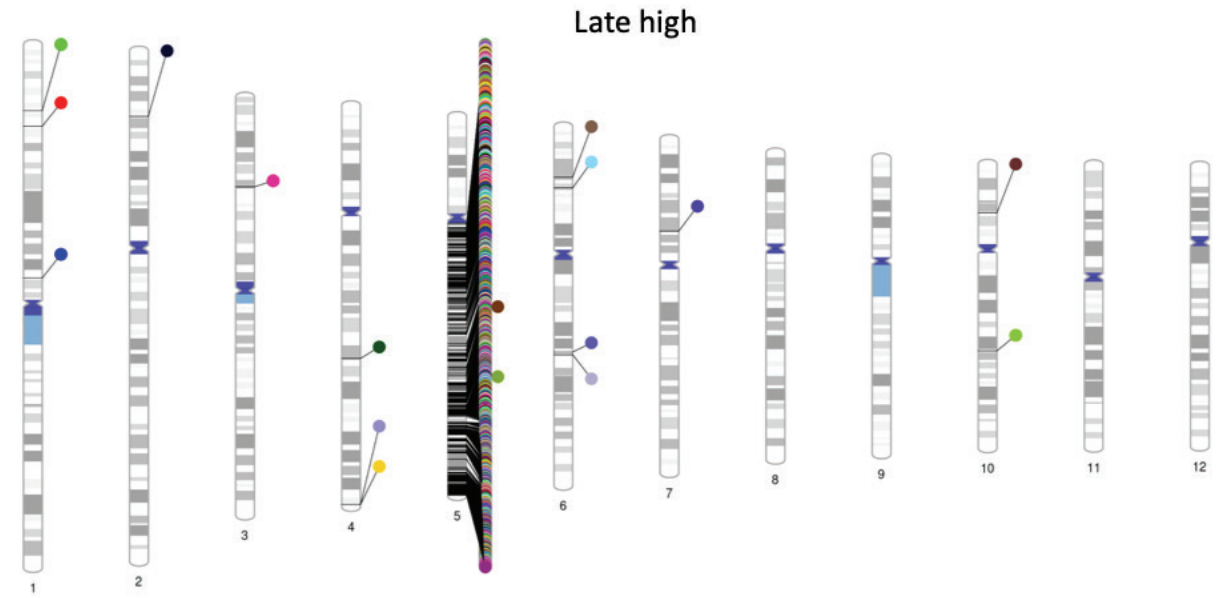

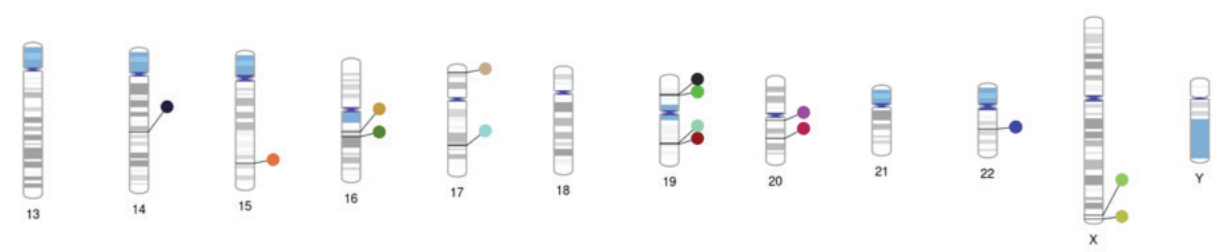


Figure S3. PhenoGrams showing enriched hits in the human genome. Chromosomal ideogram indicating the location of enriched hits in the human genome, for each of the 4 screens. PhenoGram is a software created by the Ritchie lab from the university of Pennsylvania ${ }^{57}$.
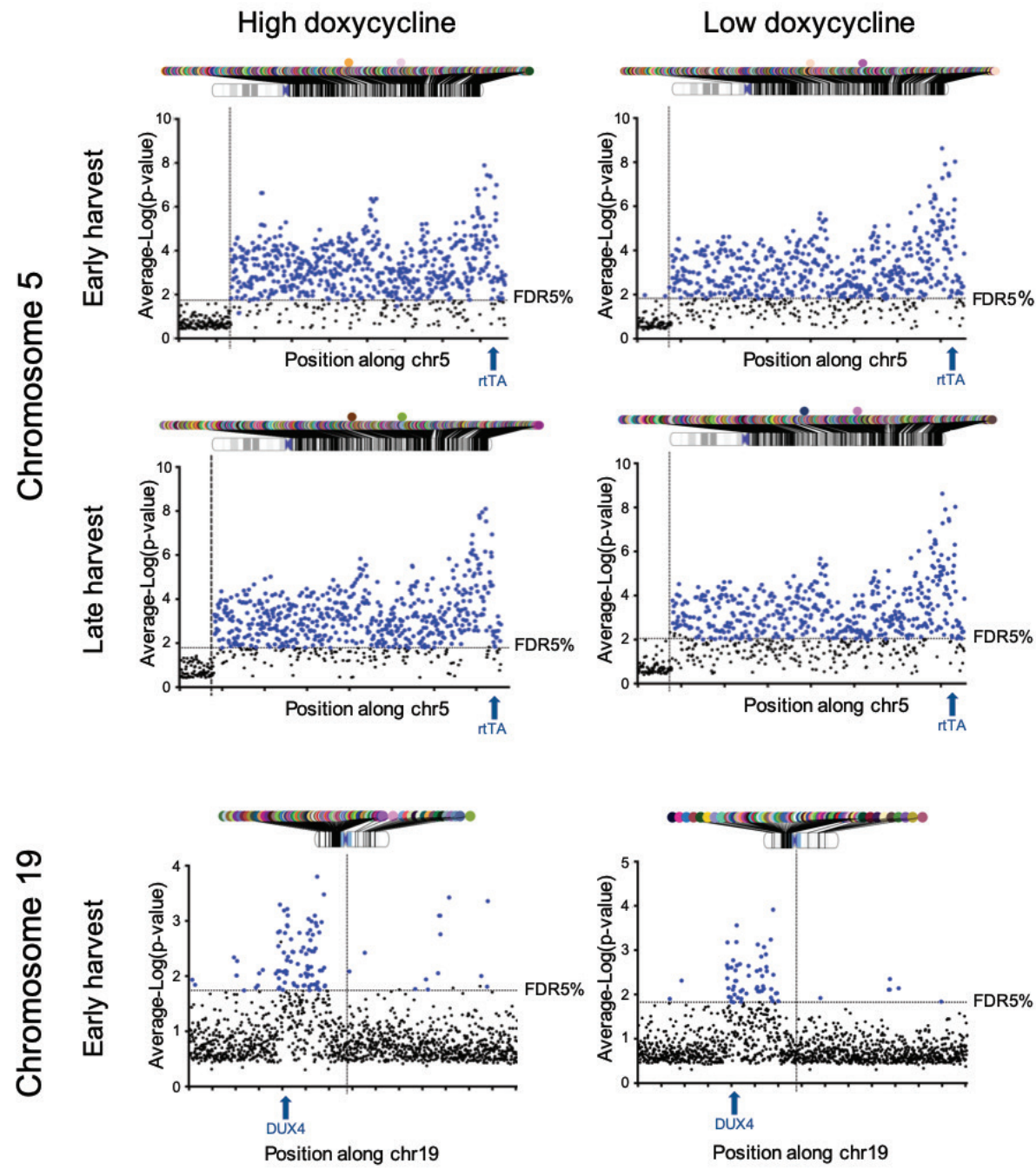

Figure S4. Data plot displaying enriched hits on chromosome $5 q$ and chromosome 19. The average - $\log (p-v a l u e)$ is plotted on the $y$-axis, and the $x$-axis is displaying the position on the chromosome. The vertical abline indicates the position of the centromere. All points above the horizontal abline (in blue) indicate significantly enriched hits that fall below the 5\% False Discovery Rate (FDR) threshold. The location of the transgene is annotated with a blue arrow on the $\mathrm{x}$-axis. 

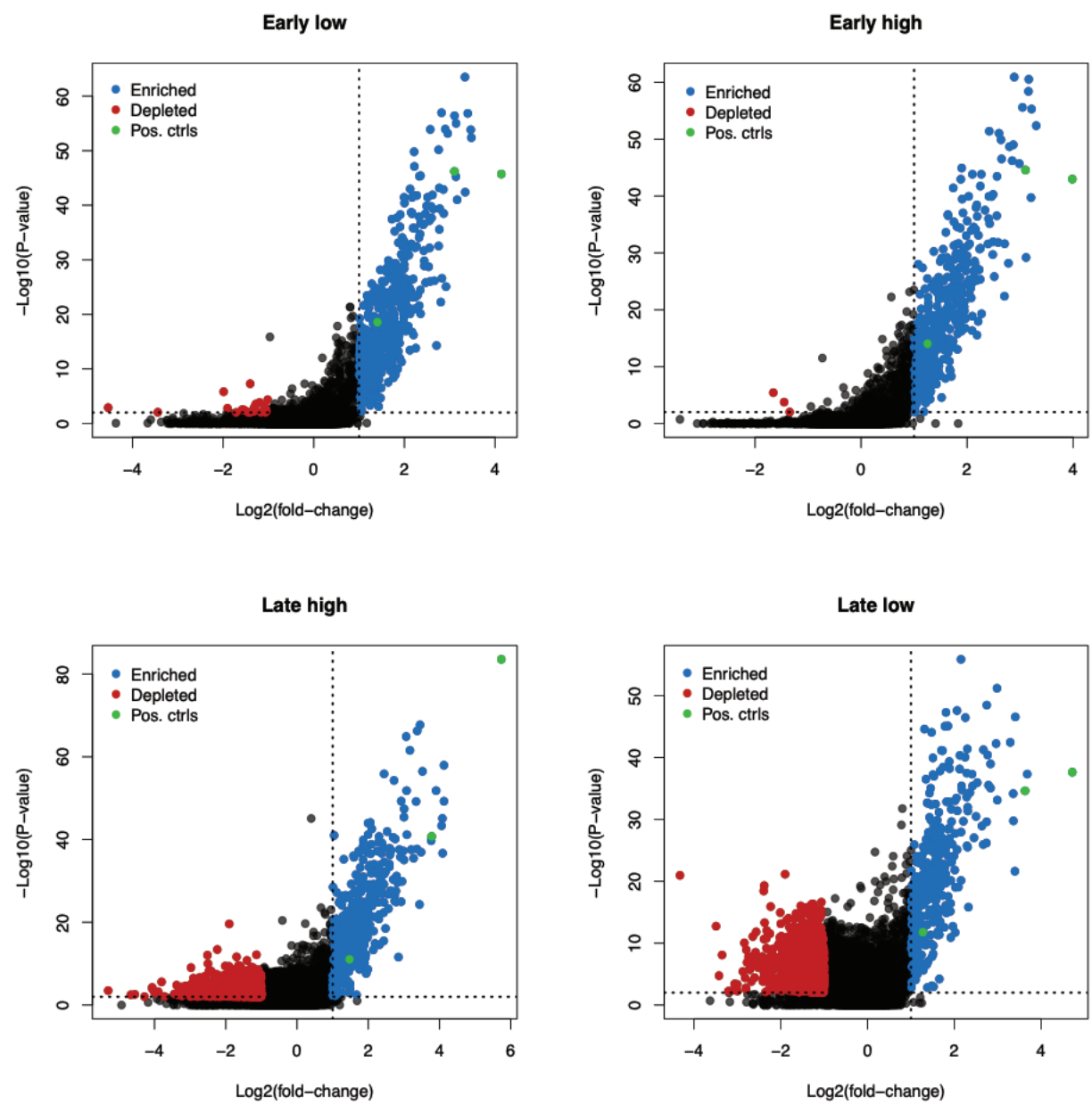

Figure S5. Analysis of enrichment and depletion of gRNAs from screen data analyzed with a two-sided analysis. Volcano plots illustrating enrichment and depletion of gRNAs in all 4 screens. The Log2(foldchange) is plotted on the $x$-axis and the -Log10(P-value) is plotted on the $y$-axis. Blue points represent significantly enriched gRNA sequences (Log2(foldchange) $\geq 1,-\log 10$ (P-value) $\geq 2$ ). Green dots represent positive controls (DUX4, MAST1, MGAT4B), and red dots represent the depleted targets. 

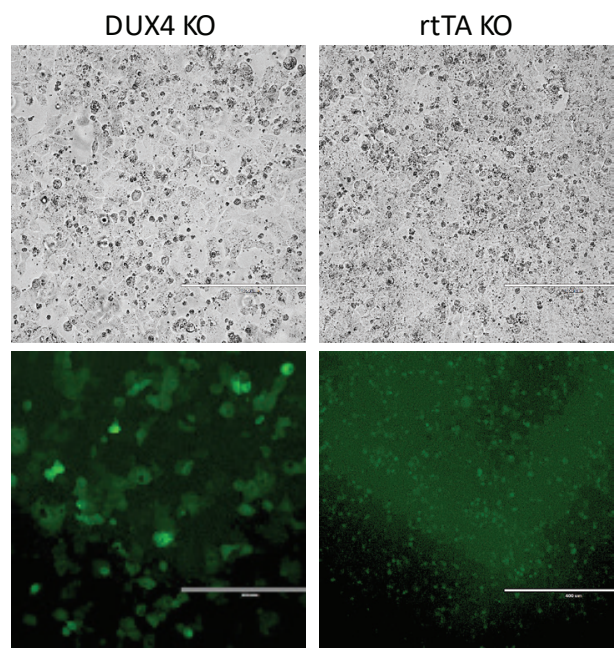

Figure S6. Individual knock-outs in DIE-ieGFP-Cas9 cells demonstrating eGFP activation in cells with a functional TetO inducible system. Phase contrast (top panel) and fluorescent images (bottom panel) of DIE cells containing a DUX4 KO (left panel), and rtTA KO (right panel) induced with $250 \mathrm{ng} / \mathrm{ml}$ doxycycline. DIE cells had reached over $100 \%$ confluency.
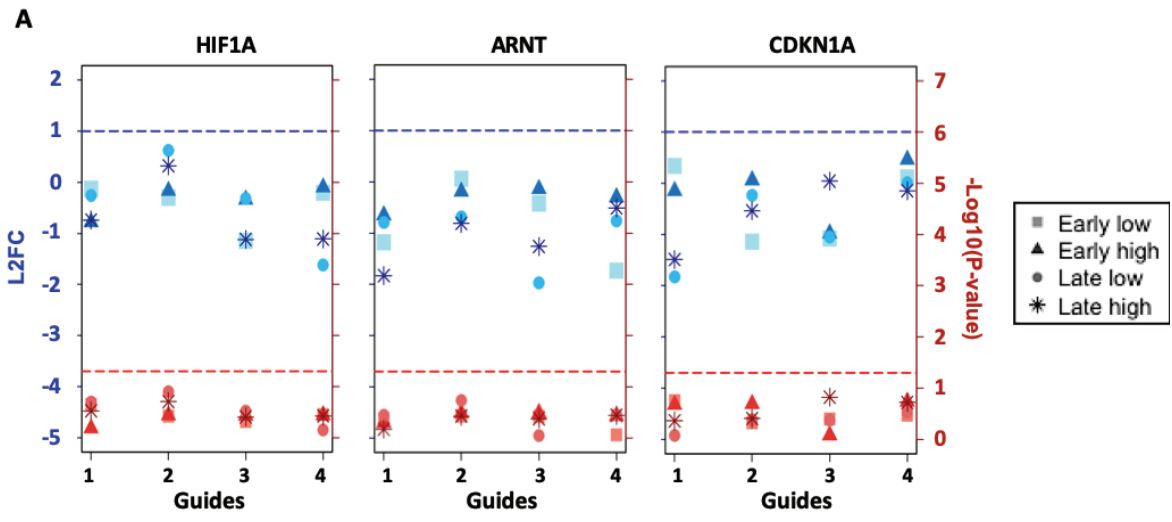

B

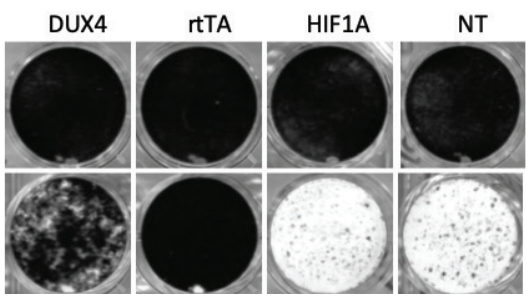

Figure S7. Validation of genes involved in the HIF1 hypoxia pathway (A) Data plots showing the enrichment of gRNA's targeting HIF1A, HIF1B/ARNT, and CDKN1A. The Log2(foldchange) value of each individual guide is plotted on the left $y$-axis, indicated in blue, and the -Log10(P-value) is plotted on the right $y$-axis, in red. Guides located above the blue and red intermitted ablines (blue: Log2(foldchange) > 1, red: -Log10(P-value) > 1.3) are considered to be significantly enriched. (B) Viability staining of DIE-Cas9 uninduced cells (top panel) and DIE-Cas9 100ng/ml doxycycline induced cells (lower panel), transfected with DUX4, rtTA, HIF1A and non-targeting (NT) sgRNA coding plasmids. 
Table S1: Enriched sgRNA's and their corresponding genes. Screen: Low doxycyline-early harvest

\begin{tabular}{|c|c|c|c|c|c|c|c|c|c|}
\hline Target gene & chr & LFC 1 & LFC 2 & LFC 3 & LFC 4 & $\begin{array}{l}-\log P \\
1\end{array}$ & $\begin{array}{l}-\log P \\
2\end{array}$ & $\begin{array}{l}-\log P \\
3\end{array}$ & $\begin{array}{l}-\log P \\
4\end{array}$ \\
\hline PAFAH1B3 & chr19q & 0.32 & 1.30 & -0.37 & 0.15 & 1.16 & 1.59 & 1.13 & 1.15 \\
\hline RINL & chr19q & -0.38 & -0.75 & 1.56 & 0.71 & 0.62 & 0.57 & 1.93 & 1.28 \\
\hline LGALS7B & chr19q & -0.10 & -0.33 & 1.26 & 0.99 & 0.69 & 0.59 & 1.88 & 0.93 \\
\hline MED25 & chr19q & 0.93 & 1.38 & 0.32 & 0.18 & 2.30 & 2.94 & 2.12 & 1.20 \\
\hline CD177 & chr19q & 0.55 & -0.11 & 0.35 & 1.00 & 1.85 & 1.01 & 1.59 & 2.21 \\
\hline $\mathrm{CDH} 10$ & $\operatorname{chr} 5 p$ & -0.69 & 1.34 & 0.86 & -1.25 & 0.40 & 2.19 & 1.18 & 0.26 \\
\hline USH2A & chr1 & 1.01 & -1.44 & 0.23 & 1.05 & 1.84 & 0.17 & 1.04 & 2.40 \\
\hline TMEM57 & chr1 & 0.04 & 1.41 & 0.14 & 1.02 & 1.21 & 2.42 & 1.57 & 2.37 \\
\hline NBPF4/6 & chr1 & 1.66 & 0.27 & 0.12 & 0.68 & 2.55 & 1.82 & 1.28 & 2.29 \\
\hline S100A7L2 & chr1 & 1.01 & 0.44 & 0.37 & 0.03 & 2.31 & 2.28 & 1.35 & 1.01 \\
\hline UBE2D1 & chr10 & -0.07 & 0.14 & 1.80 & 0.70 & 1.40 & 1.57 & 1.97 & 1.89 \\
\hline TBATA & chr10 & -1.22 & 1.20 & -1.01 & 0.85 & 0.28 & 2.18 & 0.38 & 1.12 \\
\hline NCOA4 & chr10 & 0.30 & 1.64 & -0.41 & -0.05 & 1.09 & 1.31 & 1.08 & 1.08 \\
\hline CISD1 & chr10 & -1.38 & 2.01 & -0.63 & -0.37 & 0.19 & 1.53 & 0.42 & 0.43 \\
\hline METTL10 & chr10 & 1.32 & -0.42 & 1.02 & -0.98 & 2.41 & 0.54 & 1.17 & 0.42 \\
\hline OR5M9 & chr11 & -1.83 & -0.27 & 0.77 & 1.59 & 0.05 & 0.70 & 1.29 & 2.04 \\
\hline RPS25 & chr11 & 2.27 & -0.98 & -0.79 & 1.66 & 3.04 & 0.38 & 0.42 & 1.72 \\
\hline BUD13 & chr11 & -0.31 & 1.11 & 0.54 & 0.59 & 1.07 & 2.85 & 1.28 & 1.66 \\
\hline OR5B3 & chr11 & 0.47 & -0.16 & 1.55 & -0.47 & 1.27 & 0.99 & 1.42 & 0.87 \\
\hline RAB21 & chr12 & 1.24 & -0.79 & -1.93 & 1.82 & 1.41 & 0.38 & 0.03 & 2.66 \\
\hline GTSF1 & chr12 & -0.91 & 1.06 & 1.10 & 0.56 & 0.48 & 2.46 & 2.92 & 1.07 \\
\hline STYK1 & chr12 & 0.28 & 1.51 & -1.51 & 0.06 & 1.26 & 1.35 & 0.14 & 1.04 \\
\hline ZNF10 & chr12 & 1.09 & 0.96 & -0.48 & -0.40 & 2.34 & 1.06 & 0.56 & 0.97 \\
\hline NFE2 & chr12 & -1.25 & 1.80 & 0.18 & 0.10 & 0.26 & 1.45 & 1.40 & 0.86 \\
\hline NAA25 & chr12 & 1.66 & -0.84 & -0.30 & -1.61 & 1.32 & 0.38 & 0.43 & 0.10 \\
\hline VCPKMT & chr14 & 0.24 & 1.10 & 0.15 & 0.31 & 1.09 & 2.79 & 1.07 & 1.85 \\
\hline GOLGA6L4 & chr15 & 1.13 & 1.08 & 1.62 & NA & 2.16 & 1.56 & 6.83 & NA \\
\hline GOLGA6L10 & chr15 & -0.53 & 1.19 & 1.26 & 1.42 & 2.16 & 3.54 & 5.10 & 5.89 \\
\hline CHRNA5 & chr15 & 0.37 & 0.39 & 0.29 & 1.03 & 1.25 & 2.28 & 1.03 & 3.42 \\
\hline GOLGA6C & chr15 & 0.15 & 0.89 & 0.71 & 1.27 & 1.15 & 2.79 & 2.23 & 3.39 \\
\hline GOLGA6L9 & chr15 & -0.76 & 0.82 & -0.25 & 1.27 & 0.76 & 1.06 & 0.98 & 3.34 \\
\hline ST20-MTHFS & chr15 & 0.68 & 1.03 & 0.19 & 0.75 & 2.23 & 2.63 & 1.96 & 2.51 \\
\hline ANKDD1A & chr15 & 0.41 & 0.41 & -1.35 & 1.09 & 1.06 & 1.30 & 0.21 & 2.41 \\
\hline
\end{tabular}


Table S1 continued

\begin{tabular}{|c|c|c|c|c|c|c|c|c|c|}
\hline Target gene & chr & LFC 1 & LFC 2 & LFC 3 & LFC 4 & $\begin{array}{l}-\log P \\
1\end{array}$ & $\begin{array}{l}-\log P \\
2\end{array}$ & $\begin{array}{l}-\log P \\
3\end{array}$ & $\begin{array}{l}-\log P \\
4\end{array}$ \\
\hline VWA9 & chr15 & 0.70 & 0.05 & 1.21 & 0.75 & 1.98 & 1.12 & 3.38 & 2.38 \\
\hline CYP1A2 & chr15 & 0.92 & 1.06 & 0.15 & 0.64 & 2.80 & 3.16 & 1.04 & 2.27 \\
\hline CCDC33 & chr15 & -0.23 & 0.89 & -0.09 & 1.29 & 1.00 & 1.48 & 1.16 & 2.24 \\
\hline CYP1A1 & chr15 & -0.03 & 1.43 & 0.30 & 0.51 & 1.22 & 2.11 & 1.50 & 2.06 \\
\hline SMAD3 & chr15 & 0.27 & 0.06 & 1.10 & 0.48 & 1.45 & 1.07 & 2.44 & 1.95 \\
\hline EMC7 & chr15 & 1.16 & 0.35 & -0.59 & 0.72 & 2.23 & 1.10 & 0.80 & 1.95 \\
\hline EFTUD1 & chr15 & -0.71 & 0.11 & -0.46 & 1.99 & 0.51 & 0.76 & 0.66 & 1.51 \\
\hline RASL12 & chr15 & 0.56 & 1.31 & -0.07 & 0.01 & 1.60 & 1.97 & 1.17 & 1.22 \\
\hline UBE2Q2L & chr15 & 1.12 & 1.35 & 1.20 & 0.91 & 2.62 & 6.04 & 4.36 & 1.18 \\
\hline ADAMTS7 & chr15 & 0.52 & 0.79 & 1.07 & 0.36 & 2.06 & 2.79 & 3.72 & 1.05 \\
\hline GOLGA8R & chr15 & -0.30 & 2.25 & -0.39 & -0.56 & 0.87 & 1.41 & 0.84 & 0.70 \\
\hline ODF3L1 & chr15 & -0.02 & 0.92 & 1.40 & -1.23 & 1.16 & 1.21 & 2.27 & 0.27 \\
\hline RPL4 & chr15 & 0.24 & 1.05 & 1.30 & -1.43 & 1.16 & 1.87 & 2.45 & 0.17 \\
\hline HSD3B7 & chr16 & 0.11 & -0.24 & 0.67 & 1.89 & 1.46 & 1.45 & 1.49 & 1.83 \\
\hline PDZD9 & chr16 & -0.87 & 1.69 & -0.21 & -1.20 & 0.38 & 1.33 & 0.45 & 0.28 \\
\hline CCL8 & chr17 & 1.62 & -0.95 & -0.66 & 0.07 & 1.30 & 0.41 & 0.44 & 0.70 \\
\hline SUMO2 & chr17 & -0.51 & -0.03 & 1.66 & -1.28 & 0.48 & 0.58 & 1.32 & 0.24 \\
\hline MALT1 & chr18 & 0.25 & 1.32 & 0.43 & 0.81 & 1.07 & 1.91 & 1.61 & 1.67 \\
\hline SEPT10 & chr2 & 1.19 & -0.93 & 0.41 & 0.93 & 2.41 & 0.46 & 1.11 & 2.29 \\
\hline CGREF1 & chr2 & -0.21 & 0.57 & -0.05 & 1.50 & 1.07 & 1.53 & 1.25 & 1.63 \\
\hline KANSL1L & chr2 & 0.35 & 0.12 & -0.45 & 1.67 & 1.32 & 1.18 & 1.01 & 1.49 \\
\hline AMER3 & chr2 & -0.28 & 1.78 & -0.76 & 0.48 & 0.69 & 1.45 & 0.61 & 1.39 \\
\hline SPAG16 & chr2 & 0.10 & -0.52 & 1.62 & -0.08 & 1.02 & 0.74 & 1.31 & 0.90 \\
\hline C2orf80 & chr2 & -1.92 & 1.68 & 0.33 & -1.29 & 0.03 & 1.33 & 1.14 & 0.47 \\
\hline MGME1 & chr20 & 1.90 & 0.24 & -1.62 & 0.76 & 2.02 & 1.46 & 0.10 & 1.86 \\
\hline GNAS & chr20 & 0.07 & 1.05 & 0.11 & 0.21 & 0.92 & 2.46 & 1.03 & 1.47 \\
\hline NSFL1C & chr20 & 0.21 & -1.10 & 0.07 & 1.11 & 1.07 & 0.35 & 0.92 & 1.37 \\
\hline TTC3 & chr21 & 0.35 & 0.41 & 0.33 & 1.03 & 1.30 & 2.22 & 1.03 & 3.59 \\
\hline BAGE2 & chr21 & 0.34 & 1.13 & 0.07 & 0.23 & 1.82 & 2.48 & 1.08 & 1.15 \\
\hline DOPEY2 & chr21 & 1.69 & -0.59 & -0.46 & 0.00 & 1.33 & 0.51 & 0.61 & 0.80 \\
\hline SNRPD3 & chr22 & -1.13 & 0.29 & 0.85 & 1.28 & 0.33 & 1.16 & 2.03 & 2.18 \\
\hline SREBF2 & chr22 & -1.08 & -0.02 & -1.66 & 1.83 & 0.40 & 0.59 & 0.08 & 1.42 \\
\hline L3MBTL2 & chr22 & -0.26 & 1.80 & 0.30 & -1.27 & 0.72 & 1.40 & 1.09 & 0.25 \\
\hline C22orf46 & chr22 & 0.18 & 0.29 & 1.25 & -1.35 & 1.05 & 1.14 & 1.69 & 0.21 \\
\hline OR5K1 & chr3 & 0.18 & 1.53 & 0.38 & 0.51 & 1.18 & 2.33 & 1.31 & 1.97 \\
\hline
\end{tabular}


Table S1 continued

\begin{tabular}{|c|c|c|c|c|c|c|c|c|c|}
\hline Target gene & chr & LFC 1 & LFC 2 & LFC 3 & LFC 4 & $\begin{array}{l}-\log P \\
1\end{array}$ & $\begin{array}{l}-\log P \\
2\end{array}$ & $\begin{array}{l}-\log P \\
3\end{array}$ & $\begin{array}{l}-\log P \\
4\end{array}$ \\
\hline MRAS & chr3 & -1.21 & -1.12 & 1.76 & -1.57 & 0.44 & 0.90 & 1.37 & 0.11 \\
\hline RPSA & chr3 & 0.48 & 1.56 & NA & -1.86 & 1.40 & 1.70 & NA & 0.03 \\
\hline PTTG2 & chr4 & 1.10 & 0.41 & 0.51 & 1.40 & 2.75 & 1.21 & 2.51 & 3.96 \\
\hline PRSS48 & chr4 & -0.73 & 0.59 & 0.60 & 1.15 & 0.64 & 1.09 & 1.70 & 3.01 \\
\hline PDE6B & chr4 & 0.30 & -1.74 & -1.05 & 2.15 & 1.07 & 0.06 & 0.39 & 1.62 \\
\hline MRPL2 & chr6 & 0.06 & 0.73 & 0.54 & 1.03 & 1.02 & 2.44 & 1.95 & 2.85 \\
\hline RPF2 & chr6 & 0.19 & 0.82 & -0.78 & 1.03 & 1.02 & 1.70 & 0.59 & 2.13 \\
\hline NR2E1 & chr6 & -1.19 & -1.27 & 1.17 & 0.47 & 0.43 & 0.25 & 1.43 & 1.10 \\
\hline IGF2R & chr6 & 1.46 & 1.25 & -1.48 & -0.88 & 2.68 & 1.24 & 0.15 & 0.38 \\
\hline HECA & chr6 & -0.97 & 0.29 & 1.68 & -1.50 & 0.38 & 1.06 & 1.33 & 0.14 \\
\hline GJA1 & chr6 & -0.04 & 1.73 & 0.30 & -1.93 & 1.08 & 1.36 & 1.11 & 0.03 \\
\hline CYCS & chr7 & 1.81 & 1.41 & 0.43 & 0.51 & 4.05 & 2.82 & 1.41 & 2.76 \\
\hline NUB1 & chr7 & 0.11 & -1.30 & -0.20 & 1.79 & 0.80 & 0.23 & 0.76 & 1.39 \\
\hline ZNF/626/680 & chr7 & -0.13 & 1.56 & -0.43 & 0.97 & 0.61 & 1.98 & 0.56 & 1.11 \\
\hline PDAP1 & chr7 & 0.44 & -1.38 & 1.37 & -0.48 & 1.20 & 0.19 & 1.35 & 0.49 \\
\hline ISCA1 & chr9 & 1.72 & 0.87 & -0.35 & -0.24 & 2.20 & 1.35 & 0.73 & 1.21 \\
\hline DMRT2 & chr9 & 0.65 & -1.18 & 1.35 & 0.29 & 1.79 & 0.30 & 2.01 & 1.19 \\
\hline GPR21 & chr9 & -0.83 & 1.16 & 1.01 & 0.15 & 0.55 & 2.41 & 1.60 & 1.09 \\
\hline UXT & $\operatorname{chrX}$ & 0.85 & -0.66 & -0.13 & 1.23 & 1.13 & 0.71 & 0.93 & 2.18 \\
\hline PLCXD1 & $\operatorname{chrX}$ & 0.60 & -0.29 & -0.12 & 1.72 & 1.35 & 0.95 & 1.34 & 1.70 \\
\hline UPRT & $\operatorname{chrX}$ & 0.67 & 1.23 & 0.02 & 0.05 & 1.83 & 2.30 & 1.13 & 1.32 \\
\hline BMX & $\operatorname{chrX}$ & 0.57 & 1.52 & -1.13 & 0.38 & 1.62 & 2.31 & 0.33 & 1.26 \\
\hline FUNDC2 & $\operatorname{chrX}$ & 1.23 & 0.44 & 0.22 & 0.10 & 2.58 & 1.79 & 1.37 & 1.13 \\
\hline RNF113A & chrX & 0.29 & 1.02 & 0.83 & -0.23 & 1.48 & 2.14 & 2.01 & 1.02 \\
\hline CXorf36 & chrX & 0.42 & 1.22 & -0.24 & -0.41 & 1.12 & 1.31 & 1.08 & 0.74 \\
\hline
\end{tabular}

Table S2: Enriched sgRNA's and their corresponding genes. Screen: High doxycyline-early harvest

\begin{tabular}{|llccccccccc|}
\hline & & & & & & & \multicolumn{1}{l}{-LogP } & \multicolumn{1}{l}{ LogP } & \multicolumn{1}{l}{-LogP } & \multicolumn{1}{l}{-LogP } \\
Gene & chr & LFC 1 & LFC 2 & LFC 3 & LFC 4 & $\mathbf{1}$ & $\mathbf{2}$ & $\mathbf{3}$ & $\mathbf{4}$ \\
PLAUR & chr19q & 1.09 & -0.10 & 0.51 & 0.33 & 2.46 & 1.09 & 1.79 & 1.67 \\
HPN & chr19q & 1.21 & -0.12 & -0.17 & 0.29 & 1.38 & 1.14 & 0.81 & 1.23 \\
SNRPD2 & chr19q & 0.34 & 0.43 & -0.09 & 1.43 & 1.60 & 1.74 & 1.23 & 2.50 \\
MED25 & chr19q & 0.60 & 1.24 & 1.73 & 0.91 & 1.37 & 4.17 & 5.47 & 2.70
\end{tabular}


Table S2 continued

\begin{tabular}{|c|c|c|c|c|c|c|c|c|c|}
\hline Target gene & chr & LFC 1 & LFC 2 & LFC 3 & LFC 4 & $\begin{array}{l}-\log P \\
1\end{array}$ & $\begin{array}{l}-\log P \\
2\end{array}$ & $\begin{array}{l}-\log P \\
3\end{array}$ & $\begin{array}{l}-\log P \\
4\end{array}$ \\
\hline NBPF4/6 & chr1 & 1.39 & 0.40 & 0.60 & 0.86 & 2.73 & 0.94 & 1.74 & 2.44 \\
\hline OR4F29 & chr1 & 1.04 & 0.10 & 0.46 & 0.49 & 2.97 & 1.73 & 2.26 & 2.38 \\
\hline CISD1 & chr10 & -0.06 & 1.22 & 0.33 & 0.28 & 1.15 & 2.27 & 1.88 & 1.33 \\
\hline NCOA4 & chr10 & -0.72 & 1.76 & -0.21 & 0.11 & 0.23 & 1.39 & 0.61 & 0.80 \\
\hline RPS25 & chr11 & 1.70 & -0.45 & -0.25 & 1.80 & 1.41 & 0.55 & 0.58 & 3.12 \\
\hline MTRNR2L2/8 & chr11 & -0.09 & -0.87 & 1.27 & 2.09 & 0.68 & 0.23 & 1.37 & 2.20 \\
\hline OR5B3 & chr11 & 0.30 & 0.25 & 1.05 & -0.30 & 1.26 & 1.08 & 2.11 & 0.96 \\
\hline CYB561A3 & chr11 & -0.06 & 1.26 & 0.17 & -0.08 & 0.99 & 1.77 & 1.16 & 0.94 \\
\hline SNRPF & chr12 & 0.21 & 0.28 & 0.53 & 1.11 & 1.10 & 1.83 & 2.25 & 3.35 \\
\hline PRMT8 & chr12 & 0.59 & -1.15 & -0.65 & 1.03 & 1.07 & 0.04 & 0.43 & 1.96 \\
\hline LRP6 & chr12 & 1.01 & 0.38 & -0.06 & 0.24 & 2.08 & 1.85 & 1.06 & 1.47 \\
\hline SLC2A13 & chr12 & 1.12 & 0.76 & -0.44 & -0.08 & 2.25 & 1.10 & 0.61 & 0.92 \\
\hline GOLGA6L4 & chr15 & 1.40 & 0.63 & 0.98 & NA & 8.62 & 2.25 & 4.99 & NA \\
\hline GOLGA6L10 & chr15 & 0.75 & 0.49 & 1.34 & 0.91 & 2.25 & 0.99 & 6.18 & 3.51 \\
\hline PPCDC & chr15 & 0.80 & 0.55 & -0.12 & 1.14 & 2.30 & 1.58 & 1.11 & 3.32 \\
\hline GOLGA6C & chr15 & 0.03 & 0.96 & 0.36 & 1.33 & 1.19 & 2.47 & 2.29 & 2.59 \\
\hline GOLGA6L9 & chr15 & 0.32 & -0.77 & 1.11 & 0.77 & 1.12 & 0.24 & 3.36 & 2.37 \\
\hline THAP10 & chr15 & 1.06 & 0.19 & 0.06 & 0.57 & 2.49 & 1.88 & 1.08 & 1.93 \\
\hline PEAK1 & chr15 & 0.54 & 1.02 & 0.16 & 0.17 & 1.85 & 3.01 & 1.07 & 1.78 \\
\hline ADAMTSL3 & chr15 & -0.11 & 1.21 & 0.81 & 0.05 & 1.14 & 2.30 & 1.62 & 1.33 \\
\hline RASL12 & chr15 & 1.01 & 0.52 & -0.19 & 0.41 & 2.83 & 1.81 & 1.06 & 1.31 \\
\hline UBE2Q2L & chr15 & 0.97 & 0.78 & 1.43 & 0.57 & 3.93 & 2.48 & 5.29 & 1.23 \\
\hline HERC1 & chr15 & 0.17 & 0.02 & 1.31 & 0.06 & 1.36 & 0.94 & 2.25 & 1.17 \\
\hline OAZ2 & chr15 & 0.46 & 0.89 & 1.18 & 0.25 & 2.40 & 3.02 & 3.58 & 1.13 \\
\hline ODF3L1 & chr15 & 0.14 & 0.52 & 1.02 & -0.40 & 1.07 & 1.67 & 1.82 & 0.69 \\
\hline RPL4 & chr15 & 0.12 & 1.18 & 0.90 & -0.48 & 1.13 & 2.41 & 1.59 & 0.52 \\
\hline SUMO2 & chr17 & -0.28 & 0.81 & 1.04 & -1.55 & 0.51 & 1.08 & 2.31 & 0.01 \\
\hline MYEOV2 & chr2 & -0.60 & 0.97 & 0.17 & 1.05 & 0.35 & 1.78 & 1.08 & 2.49 \\
\hline C22orf46 & chr22 & -0.89 & 0.15 & 1.11 & 0.09 & 0.12 & 1.10 & 1.49 & 0.87 \\
\hline RHOA & chr3 & 0.30 & -0.39 & 0.62 & 1.12 & 1.10 & 0.72 & 2.02 & 2.36 \\
\hline GJA1 & chr6 & -0.33 & 1.70 & 0.31 & 0.11 & 0.85 & 1.54 & 1.36 & 1.29 \\
\hline HECA & chr6 & 0.08 & -0.94 & 2.10 & -1.51 & 0.73 & 0.73 & 1.61 & 0.01 \\
\hline CYCS & chr7 & 1.83 & 1.85 & 0.40 & 0.93 & 4.20 & 4.48 & 1.44 & 3.26 \\
\hline NDUFA5 & chr7 & -0.26 & -0.34 & 0.01 & 1.65 & 0.61 & 0.54 & 0.84 & 1.33 \\
\hline DNAJB9 & chr7 & -0.33 & 1.40 & -0.98 & 0.76 & 0.46 & 2.24 & 0.08 & 1.21 \\
\hline
\end{tabular}


Table S2 continued

\begin{tabular}{|c|c|c|c|c|c|c|c|c|c|}
\hline Target gene & chr & LFC 1 & LFC 2 & LFC 3 & LFC 4 & $\begin{array}{l}-\log P \\
1\end{array}$ & $\begin{array}{l}-\log P \\
2\end{array}$ & $\begin{array}{l}-\log P \\
3\end{array}$ & $\begin{array}{l}-\log P \\
4\end{array}$ \\
\hline SLC12A9 & chr7 & 1.00 & 0.36 & 0.18 & 0.03 & 2.31 & 1.85 & 1.43 & 1.06 \\
\hline PDAP1 & chr7 & 0.36 & -0.61 & 1.28 & -0.49 & 1.16 & 0.34 & 1.41 & 0.38 \\
\hline ACER2 & chr9 & 0.21 & 0.01 & 0.66 & 1.02 & 1.98 & 1.07 & 2.09 & 2.20 \\
\hline TOMM5 & chr9 & -0.10 & 0.20 & 1.20 & 0.27 & 1.14 & 1.17 & 1.93 & 1.68 \\
\hline ISCA1 & chr9 & 1.20 & 0.13 & 0.19 & -0.58 & 1.65 & 0.98 & 1.14 & 0.37 \\
\hline RBMY1J & chrY & 0.34 & 0.07 & 0.14 & 1.00 & 1.69 & 1.06 & 1.36 & 2.54 \\
\hline
\end{tabular}

Table S3: Enriched sgRNA's and their corresponding genes. Screen: Low doxycyline-late harvest

\begin{tabular}{|c|c|c|c|c|c|c|c|c|c|}
\hline Target gene & chr & LFC 1 & LFC 2 & LFC 3 & LFC 4 & $\begin{array}{l}-\log P \\
1\end{array}$ & $\begin{array}{l}-\log P \\
2\end{array}$ & $\begin{array}{l}-\log P \\
3\end{array}$ & $\begin{array}{l}-\log P \\
4\end{array}$ \\
\hline HPN & chr19q & 2.07 & 0.14 & -1.13 & 0.32 & 1.80 & 1.21 & 0.45 & 1.65 \\
\hline ZNF599 & chr19q & 0.42 & 0.24 & -0.01 & 1.00 & 2.04 & 1.37 & 0.98 & 2.60 \\
\hline PPP1R15A & chr19q & 0.27 & -0.98 & -1.04 & 1.66 & 1.11 & 0.54 & 0.38 & 1.36 \\
\hline MED25 & chr19q & 1.68 & 2.38 & 2.63 & -0.03 & 2.54 & 4.23 & 5.30 & 2.10 \\
\hline CEACAM 20 & chr19q & -1.29 & 1.21 & 2.20 & -0.64 & 0.33 & 1.75 & 2.61 & 0.55 \\
\hline MARK4 & chr19q & -1.12 & 1.54 & -0.56 & -0.78 & 0.43 & 1.30 & 0.46 & 0.45 \\
\hline ZNF534 & chr19q & 1.01 & 1.10 & -0.96 & 0.20 & 1.95 & 2.34 & 0.64 & 1.04 \\
\hline DAB2 & chr5p & -0.76 & 0.96 & 1.89 & 0.03 & 0.89 & 1.53 & 2.26 & 1.51 \\
\hline C1QTNF3 & chr5p & 0.04 & 1.02 & 0.44 & 0.52 & 1.35 & 2.23 & 1.87 & 2.15 \\
\hline GDNF & chr5p & -0.44 & 1.57 & -1.31 & -1.11 & 0.44 & 1.31 & 0.32 & 0.38 \\
\hline OR2L2 & chr1 & 0.64 & 1.24 & 0.34 & 1.00 & 2.11 & 3.15 & 1.04 & 2.78 \\
\hline PTGER3 & chr1 & 0.16 & 0.28 & -0.04 & 1.28 & 1.15 & 1.83 & 1.13 & 2.51 \\
\hline RBM15 & chr1 & -0.10 & -0.18 & 0.75 & 1.11 & 1.27 & 1.05 & 1.93 & 2.08 \\
\hline PMVK & chr1 & -0.49 & -0.10 & 0.79 & 1.04 & 1.00 & 1.27 & 1.36 & 2.00 \\
\hline NLRP3 & chr1 & 1.22 & 0.32 & -0.36 & 0.77 & 2.24 & 1.64 & 1.12 & 1.96 \\
\hline SYNC & chr1 & 0.25 & -0.58 & -0.59 & 2.18 & 1.18 & 1.08 & 0.60 & 1.73 \\
\hline B3GALT6 & chr1 & 0.48 & 1.83 & -0.42 & -0.23 & 1.47 & 1.52 & 1.03 & 1.47 \\
\hline RSG1 & chr1 & -0.06 & 1.06 & -0.07 & -0.06 & 1.01 & 2.41 & 0.69 & 1.35 \\
\hline $\mathrm{TCHH}$ & chr1 & 1.80 & -0.08 & -0.98 & 0.27 & 1.46 & 1.12 & 0.61 & 1.30 \\
\hline CASQ1 & chr1 & -1.50 & -0.78 & -0.86 & 1.54 & 0.20 & 0.47 & 0.42 & 1.30 \\
\hline CRTC2 & chr1 & 0.28 & -0.27 & -1.47 & 1.55 & 1.13 & 0.98 & 0.22 & 1.30 \\
\hline RWDD3 & chr1 & -1.35 & 1.59 & -0.12 & 0.10 & 0.29 & 1.33 & 0.87 & 1.24 \\
\hline CDC14A & chr1 & -0.20 & 1.05 & -0.61 & 1.03 & 1.09 & 2.36 & 1.00 & 1.13 \\
\hline
\end{tabular}


Table S3 continued

\begin{tabular}{|c|c|c|c|c|c|c|c|c|c|}
\hline Target gene & chr & LFC 1 & LFC 2 & LFC 3 & LFC 4 & $\begin{array}{l}-\log P \\
1\end{array}$ & $\begin{array}{l}-\log P \\
2\end{array}$ & $\begin{array}{l}-\log P \\
3\end{array}$ & $\begin{array}{l}-\log P \\
4\end{array}$ \\
\hline MCOLN2 & chr1 & 1.06 & -1.11 & 1.08 & -0.17 & 1.14 & 0.47 & 2.41 & 1.03 \\
\hline UBE4B & chr1 & -0.19 & 0.08 & 1.61 & -0.44 & 1.10 & 1.34 & 1.47 & 0.85 \\
\hline CD34 & chr1 & 0.50 & 1.61 & -0.40 & -0.54 & 1.34 & 1.50 & 1.26 & 0.80 \\
\hline MR1 & chr1 & -1.79 & 1.60 & -0.69 & -0.57 & 0.09 & 1.33 & 0.43 & 0.50 \\
\hline RNF115 & chr1 & 1.57 & 0.31 & -0.07 & -1.52 & 1.33 & 1.31 & 1.17 & 0.19 \\
\hline FAM213B & chr1 & -0.69 & 0.07 & 1.57 & -2.14 & 0.50 & 0.83 & 1.31 & 0.03 \\
\hline RBBP5 & chr1 & -0.76 & 1.62 & 0.54 & -2.29 & 0.46 & 1.57 & 1.35 & 0.02 \\
\hline NCOA4 & chr10 & 0.06 & 1.07 & -0.62 & 0.72 & 1.12 & 1.88 & 1.02 & 1.59 \\
\hline LIPK & chr10 & -1.32 & -1.09 & -0.22 & 1.99 & 0.31 & 0.38 & 0.55 & 1.58 \\
\hline РРР3СВ & chr10 & 1.02 & -1.34 & 0.32 & 0.33 & 2.26 & 0.30 & 0.99 & 1.21 \\
\hline CISD1 & chr10 & -0.34 & 2.17 & -0.61 & 0.23 & 1.05 & 1.72 & 0.87 & 1.13 \\
\hline CFAP43 & chr10 & 1.54 & -1.36 & -0.84 & -0.82 & 1.30 & 0.28 & 0.43 & 0.48 \\
\hline ARFIP2 & chr11 & 0.49 & 0.49 & 0.65 & 1.05 & 1.77 & 1.01 & 2.73 & 4.37 \\
\hline RPS25 & chr11 & 1.56 & 0.13 & -0.08 & 1.11 & 2.48 & 1.78 & 1.31 & 2.38 \\
\hline TRIM68 & chr11 & 1.00 & -0.85 & -0.43 & 1.58 & 1.32 & 0.76 & 0.76 & 2.32 \\
\hline RIC8A & chr11 & 0.49 & -0.15 & 1.82 & 0.91 & 2.17 & 1.47 & 2.73 & 2.19 \\
\hline FAM76B & chr11 & -0.70 & -1.23 & 0.81 & 1.05 & 0.50 & 0.37 & 1.00 & 2.04 \\
\hline DCPS & chr11 & -0.12 & 0.71 & -0.53 & 1.70 & 1.29 & 1.39 & 1.23 & 1.87 \\
\hline MTRNR2L2/8 & chr11 & -0.39 & 0.32 & 0.65 & 1.86 & 0.86 & 1.30 & 1.47 & 1.75 \\
\hline MICALCL & chr11 & -1.15 & -1.74 & -2.24 & 1.55 & 0.74 & 0.71 & 0.02 & 1.30 \\
\hline PAFAH1B2 & chr11 & 1.35 & 0.24 & -0.04 & -0.20 & 2.04 & 1.38 & 1.19 & 1.07 \\
\hline OR5B12 & chr11 & 1.14 & -1.49 & -1.15 & 1.01 & 2.33 & 0.21 & 0.38 & 1.06 \\
\hline DHCR7 & chr11 & 0.74 & 1.34 & 0.43 & -0.84 & 1.91 & 2.54 & 1.18 & 0.79 \\
\hline KMT2A & chr11 & -0.72 & 1.26 & 1.43 & -1.10 & 0.49 & 1.24 & 2.67 & 0.49 \\
\hline SLC29A2 & chr11 & -2.21 & 1.68 & -0.66 & -1.60 & 0.02 & 1.38 & 0.58 & 0.43 \\
\hline C11orf88 & chr11 & -1.20 & 0.04 & 1.77 & -0.97 & 0.39 & 0.80 & 1.44 & 0.39 \\
\hline TRIM29 & chr11 & 0.92 & 1.20 & -1.23 & -1.12 & 1.10 & 2.19 & 0.37 & 0.38 \\
\hline KRT83 & chr12 & 0.38 & -0.77 & 0.53 & 1.32 & 1.17 & 0.88 & 1.57 & 2.43 \\
\hline LOH12CR1 & chr12 & -0.96 & 0.29 & 0.34 & 1.35 & 0.64 & 1.19 & 1.24 & 2.18 \\
\hline PRB1 & chr12 & 0.49 & 1.29 & -0.30 & 0.71 & 1.66 & 2.37 & 1.27 & 1.66 \\
\hline LIMA1 & chr12 & -0.50 & -0.20 & -0.20 & 1.68 & 0.56 & 1.34 & 1.09 & 1.38 \\
\hline ATF7 & chr12 & -0.37 & -0.11 & 1.57 & -0.39 & 0.83 & 1.31 & 1.58 & 0.64 \\
\hline C12orf65 & chr12 & 1.85 & -0.85 & -1.44 & -0.32 & 1.49 & 0.42 & 0.24 & 0.49 \\
\hline RERG & chr12 & -0.61 & -1.48 & 2.40 & -0.74 & 0.48 & 0.21 & 1.91 & 0.43 \\
\hline NR4A1 & chr12 & 1.31 & 1.12 & -1.85 & -0.85 & 2.49 & 1.17 & 0.08 & 0.42 \\
\hline
\end{tabular}


Table S3 continued

\begin{tabular}{|c|c|c|c|c|c|c|c|c|c|}
\hline Target gene & chr & LFC 1 & LFC 2 & LFC 3 & LFC 4 & $\begin{array}{l}-\log P \\
1\end{array}$ & $\begin{array}{l}-\log P \\
2\end{array}$ & $\begin{array}{l}-\log P \\
3\end{array}$ & $\begin{array}{l}-\log P \\
4\end{array}$ \\
\hline GLT8D2 & chr12 & 0.30 & 1.66 & 0.44 & -1.19 & 1.36 & 2.20 & 1.39 & 0.40 \\
\hline RASSF8 & chr12 & 1.76 & -1.16 & -0.70 & -1.67 & 1.43 & 0.38 & 0.44 & 0.13 \\
\hline MTMR6 & chr13 & -1.67 & -0.02 & 2.01 & -0.41 & 0.13 & 0.78 & 1.60 & 0.73 \\
\hline ATG2B & chr14 & -1.45 & -1.09 & -1.05 & 1.70 & 0.23 & 0.38 & 0.65 & 1.39 \\
\hline PNP & chr14 & 0.97 & -0.48 & 1.52 & -0.16 & 1.38 & 1.17 & 2.27 & 1.29 \\
\hline RNASE1 & chr14 & 0.19 & 2.10 & 0.98 & -0.79 & 1.67 & 2.29 & 1.91 & 0.85 \\
\hline KCNH5 & chr14 & 0.43 & 1.69 & -1.11 & -0.66 & 1.38 & 1.38 & 0.47 & 0.53 \\
\hline TECPR2 & chr14 & -0.11 & -0.33 & 1.59 & -1.48 & 0.89 & 0.64 & 1.33 & 0.21 \\
\hline GOLGA6L4 & chr15 & -1.82 & -0.22 & -0.85 & NA & 0.14 & 1.62 & 1.30 & NA \\
\hline CRTC3 & chr15 & -0.12 & 1.28 & 0.03 & 0.31 & 1.15 & 2.26 & 1.18 & 1.53 \\
\hline NR2F2 & chr15 & -0.02 & 1.58 & -0.53 & 0.08 & 1.28 & 1.43 & 0.85 & 1.32 \\
\hline CHRNA5 & chr15 & 0.57 & 1.14 & 0.28 & -0.74 & 1.62 & 2.15 & 1.07 & 0.93 \\
\hline ESRP2 & chr16 & 0.37 & 0.95 & -1.60 & 1.09 & 1.03 & 2.24 & 0.16 & 2.38 \\
\hline ZNF720 & chr16 & -0.54 & -0.11 & -1.60 & 2.15 & 0.64 & 0.64 & 0.16 & 1.70 \\
\hline PSMB10 & chr16 & -0.02 & -0.85 & 0.07 & 1.07 & 0.83 & 0.77 & 1.02 & 1.44 \\
\hline FOXL1 & chr16 & -1.08 & 0.82 & 1.04 & -0.05 & 0.50 & 1.38 & 2.05 & 1.00 \\
\hline PLA2G15 & chr16 & 1.25 & -0.05 & 1.17 & -1.67 & 2.57 & 1.13 & 1.37 & 0.13 \\
\hline MED31 & chr17 & 0.10 & -1.19 & -0.98 & 1.59 & 0.87 & 0.38 & 0.41 & 1.33 \\
\hline ZZEF1 & chr17 & 0.15 & 0.06 & 1.02 & -0.14 & 1.59 & 0.99 & 2.21 & 0.94 \\
\hline HOXB8 & chr17 & 1.70 & -0.68 & -0.96 & -0.51 & 1.39 & 0.51 & 0.43 & 0.63 \\
\hline SUMO2 & chr17 & 0.24 & 0.04 & 1.06 & -1.64 & 1.07 & 1.01 & 1.55 & 0.14 \\
\hline ALPK2 & chr18 & -1.40 & -0.37 & 0.97 & 1.01 & 0.25 & 0.83 & 0.98 & 2.27 \\
\hline FBXO15 & chr18 & 1.03 & -0.38 & 0.51 & 0.36 & 2.36 & 0.99 & 1.60 & 1.52 \\
\hline MBD2 & chr18 & -0.83 & 1.01 & 0.98 & -0.45 & 0.73 & 2.28 & 0.98 & 0.79 \\
\hline PARD6G & chr18 & -0.75 & 1.58 & -1.27 & -1.67 & 0.46 & 1.32 & 0.40 & 0.13 \\
\hline UGT1A1 & chr2 & 0.43 & 0.27 & -1.63 & 1.09 & 1.37 & 1.03 & 0.15 & 2.13 \\
\hline CCNT2 & chr2 & 0.59 & -0.21 & -0.55 & 1.58 & 1.32 & 1.24 & 1.06 & 1.66 \\
\hline DTNB & chr2 & 1.63 & -0.14 & -0.33 & -0.09 & 1.71 & 1.20 & 0.65 & 1.35 \\
\hline LPIN1 & chr2 & 1.18 & 0.58 & -0.56 & 0.23 & 2.02 & 1.65 & 1.09 & 1.22 \\
\hline GPR35 & chr2 & 0.32 & 1.01 & 0.29 & 0.19 & 2.19 & 3.27 & 1.20 & 0.98 \\
\hline HSPA12B & chr20 & 1.10 & 0.62 & -1.67 & 0.68 & 3.09 & 1.03 & 0.13 & 1.82 \\
\hline CST1 & chr20 & -1.59 & -1.86 & 0.19 & 2.21 & 0.57 & 0.07 & 1.00 & 1.77 \\
\hline GNAS & chr20 & 1.03 & 0.94 & 0.59 & -0.24 & 2.99 & 2.23 & 1.94 & 1.00 \\
\hline NSFL1C & chr20 & 0.75 & 1.20 & -1.63 & -0.84 & 1.11 & 1.93 & 0.15 & 0.43 \\
\hline CYP24A1 & chr20 & 1.62 & -0.82 & 0.50 & -1.37 & 1.50 & 0.43 & 1.35 & 0.28 \\
\hline
\end{tabular}


Table S3 continued

\begin{tabular}{|c|c|c|c|c|c|c|c|c|c|}
\hline Target gene & chr & LFC 1 & LFC 2 & LFC 3 & LFC 4 & $\begin{array}{l}-\log P \\
1\end{array}$ & $\begin{array}{l}-\log P \\
2\end{array}$ & $\begin{array}{l}-\log P \\
3\end{array}$ & $\begin{array}{l}-\log P \\
4\end{array}$ \\
\hline JAM2 & chr21 & -0.14 & -1.37 & 0.89 & 1.19 & 1.09 & 0.28 & 1.19 & 2.15 \\
\hline KCNE2 & chr21 & 0.29 & -0.23 & 1.48 & 0.68 & 1.82 & 1.26 & 2.18 & 1.95 \\
\hline KCNE2 & chr21 & 0.29 & -0.23 & 1.48 & 0.68 & 1.82 & 1.26 & 2.18 & 1.95 \\
\hline PLAC4 & chr21 & 0.11 & -0.72 & 1.73 & 0.50 & 1.41 & 0.95 & 1.72 & 1.50 \\
\hline COL18A1 & chr21 & 0.14 & 1.52 & -0.55 & -0.02 & 1.28 & 1.43 & 0.92 & 1.24 \\
\hline EMID1 & chr22 & -0.40 & -1.00 & 1.69 & -2.17 & 0.46 & 0.38 & 1.38 & 0.02 \\
\hline TSC22D2 & chr3 & 0.53 & 0.35 & 0.09 & 1.15 & 2.33 & 1.56 & 1.07 & 2.90 \\
\hline ADPRH & chr3 & 0.20 & 0.84 & 0.01 & 1.05 & 1.95 & 2.07 & 1.00 & 2.65 \\
\hline TUSC2 & chr3 & -0.06 & 1.16 & -0.63 & 1.43 & 1.23 & 1.35 & 1.10 & 2.55 \\
\hline RAB5A & chr3 & -1.31 & 0.03 & 0.16 & 1.86 & 0.32 & 0.95 & 1.49 & 1.53 \\
\hline PRR23B & chr3 & 1.06 & 0.58 & 0.59 & -0.16 & 2.99 & 1.67 & 2.15 & 1.02 \\
\hline ZNF501 & chr3 & 0.64 & 1.77 & -0.63 & -1.15 & 1.43 & 1.76 & 0.55 & 0.43 \\
\hline RSRC1 & chr3 & 1.35 & -0.42 & 1.22 & -1.31 & 2.62 & 0.77 & 1.19 & 0.31 \\
\hline SNRK & chr3 & -0.10 & -0.37 & 1.57 & -1.42 & 0.83 & 0.64 & 1.31 & 0.24 \\
\hline ERC2 & chr3 & 1.15 & -0.65 & 1.07 & -1.51 & 2.42 & 0.54 & 1.07 & 0.20 \\
\hline NGLY1 & chr3 & 1.61 & -0.45 & -0.81 & -1.51 & 1.34 & 0.44 & 0.44 & 0.20 \\
\hline FAM47E-STBD1 & chr4 & 0.30 & 0.31 & 0.28 & 1.02 & 2.03 & 2.75 & 1.99 & 2.79 \\
\hline PPA2 & chr4 & 1.26 & 0.06 & -0.19 & 0.04 & 2.06 & 1.55 & 0.81 & 1.14 \\
\hline G3BP2 & chr4 & 1.66 & -0.27 & -0.59 & -0.49 & 1.37 & 1.16 & 0.52 & 0.68 \\
\hline TMEM155 & chr4 & 0.99 & -1.15 & 1.28 & -0.50 & 1.15 & 0.44 & 2.32 & 0.67 \\
\hline EPGN & chr4 & -0.13 & 1.54 & -0.21 & -0.59 & 1.17 & 1.30 & 1.07 & 0.62 \\
\hline WHSC1 & chr4 & -0.13 & -0.77 & 1.97 & -0.82 & 0.81 & 0.62 & 1.57 & 0.46 \\
\hline GUCY1A3 & chr4 & -0.65 & -0.76 & 1.59 & -0.76 & 0.89 & 0.47 & 1.33 & 0.43 \\
\hline FBXW7 & chr4 & -0.80 & 1.89 & -0.17 & -1.21 & 0.45 & 1.51 & 0.58 & 0.39 \\
\hline FBXO30 & chr6 & 0.69 & -0.38 & 0.59 & 1.13 & 1.84 & 1.06 & 1.61 & 3.00 \\
\hline ULBP2 & chr6 & 0.74 & -0.32 & -0.01 & 1.98 & 1.48 & 1.17 & 1.23 & 1.72 \\
\hline $\mathrm{CCNC}$ & chr6 & -0.67 & 0.58 & 1.08 & 0.60 & 1.03 & 1.04 & 2.98 & 1.67 \\
\hline CCDC170 & chr6 & -0.82 & -0.93 & -1.95 & 1.80 & 0.49 & 0.39 & 0.06 & 1.46 \\
\hline CLVS2 & chr6 & -1.05 & -1.30 & -2.05 & 1.72 & 0.65 & 0.41 & 0.04 & 1.40 \\
\hline AGPAT4 & chr6 & 1.16 & 0.48 & 0.25 & -0.15 & 2.18 & 2.07 & 1.47 & 1.08 \\
\hline GJA1 & chr6 & 0.37 & 1.28 & 0.33 & -0.80 & 1.28 & 2.29 & 1.15 & 0.84 \\
\hline SUMO4 & chr6 & 2.06 & -0.20 & -1.04 & -0.60 & 1.64 & 0.57 & 0.54 & 0.56 \\
\hline B3GAT2 & chr6 & -1.45 & -0.06 & 1.62 & -0.81 & 0.23 & 0.69 & 1.34 & 0.44 \\
\hline ZPBP & chr7 & 0.92 & 0.23 & -1.45 & 1.40 & 2.01 & 1.22 & 0.23 & 2.20 \\
\hline NUB1 & chr7 & -0.50 & 0.41 & -0.03 & 1.04 & 1.00 & 1.35 & 1.34 & 1.42 \\
\hline
\end{tabular}


Table S3 continued

\begin{tabular}{|c|c|c|c|c|c|c|c|c|c|}
\hline Target gene & chr & LFC 1 & LFC 2 & LFC 3 & LFC 4 & $\begin{array}{l}-\log P \\
1\end{array}$ & $\begin{array}{l}-\log P \\
2\end{array}$ & $\begin{array}{l}-\log P \\
3\end{array}$ & $\begin{array}{l}-\log P \\
4\end{array}$ \\
\hline RADIL & chr7 & 0.35 & -0.95 & 1.82 & 0.29 & 1.47 & 0.64 & 2.19 & 1.25 \\
\hline HIPK2 & chr7 & -0.58 & 1.57 & -1.27 & 0.19 & 0.59 & 1.32 & 0.35 & 0.99 \\
\hline LMBR1 & chr7 & 0.06 & 1.07 & 1.11 & -0.91 & 1.05 & 1.61 & 2.42 & 0.69 \\
\hline HUS1 & chr7 & -1.17 & 1.56 & -0.99 & -0.97 & 0.38 & 1.31 & 0.42 & 0.58 \\
\hline C1GALT1 & chr7 & -0.96 & 0.71 & 1.40 & -1.77 & 0.39 & 1.22 & 1.87 & 0.10 \\
\hline LRRD1 & chr7 & 1.66 & -1.26 & -0.56 & -2.12 & 1.37 & 0.40 & 0.43 & 0.03 \\
\hline RALYL & chr8 & 0.99 & -1.04 & -0.47 & 1.09 & 1.03 & 0.55 & 0.70 & 2.30 \\
\hline STAR & chr8 & -0.72 & -0.06 & -0.36 & 1.54 & 0.68 & 0.95 & 0.84 & 1.30 \\
\hline PXDNL & chr8 & 1.25 & 0.56 & 0.14 & -0.10 & 2.32 & 1.79 & 1.62 & 1.13 \\
\hline ZNF707 & chr8 & -0.28 & -0.42 & 1.56 & -0.62 & 1.12 & 0.77 & 1.31 & 0.51 \\
\hline QSOX2 & chr9 & 0.01 & 2.03 & -0.49 & 0.17 & 1.36 & 1.62 & 0.97 & 1.49 \\
\hline PTPRD & chr9 & -1.50 & -0.15 & 0.42 & 1.51 & 0.20 & 1.18 & 1.28 & 1.36 \\
\hline TOMM5 & chr9 & -0.53 & -0.41 & 1.83 & -0.08 & 0.67 & 0.78 & 1.48 & 1.29 \\
\hline LURAP1L & chr9 & 1.59 & 0.26 & 0.42 & -0.55 & 2.09 & 1.33 & 1.36 & 1.25 \\
\hline IFNE & chr9 & 0.92 & -0.88 & 1.09 & -0.82 & 1.03 & 0.43 & 2.20 & 0.73 \\
\hline CXorf51A/B & $\operatorname{chrX}$ & 1.57 & -0.43 & 0.46 & 0.82 & 2.28 & 1.45 & 1.73 & 1.77 \\
\hline MED12 & chrX & 0.05 & 1.30 & 0.01 & 0.07 & 1.16 & 2.65 & 0.83 & 1.57 \\
\hline KCND1 & $\operatorname{chrX}$ & 0.25 & 0.37 & 1.60 & -0.08 & 1.33 & 2.08 & 2.40 & 1.29 \\
\hline OGT & $\operatorname{chrX}$ & -0.05 & 0.37 & 1.43 & 0.33 & 1.24 & 2.28 & 2.47 & 1.28 \\
\hline HSFX1/2 & $\operatorname{chrX}$ & 0.33 & 1.62 & 0.56 & -0.07 & 1.23 & 1.42 & 1.35 & 1.08 \\
\hline FUNDC2 & $\operatorname{chrX}$ & 1.69 & -0.82 & -0.66 & -0.19 & 1.38 & 0.53 & 0.57 & 0.81 \\
\hline CACNA1F & chrX & -0.97 & 1.85 & -0.68 & -0.09 & 0.51 & 1.49 & 0.62 & 0.66 \\
\hline ARAF & $\operatorname{chrX}$ & 0.21 & 1.26 & 1.32 & -1.03 & 1.17 & 1.97 & 2.68 & 0.55 \\
\hline CXorf36 & chrX & -0.76 & 1.55 & 0.17 & -0.85 & 0.76 & 1.30 & 0.97 & 0.46 \\
\hline KDM5D & chrY & -1.37 & 1.93 & -0.06 & -1.56 & 0.44 & 1.54 & 0.69 & 0.17 \\
\hline RPS4Y2 & chrY & 1.58 & -1.19 & 0.07 & -1.70 & 1.32 & 0.38 & 0.84 & 0.12 \\
\hline
\end{tabular}

Table S4: Enriched sgRNA's and their corresponding genes. Screen: HIgh doxycyline-late harvest

\begin{tabular}{|llcrrrrrrrr|}
\hline & & & & & & -LogP & \multicolumn{1}{l}{-LogP } & \multicolumn{1}{l}{-LogP } & \multicolumn{1}{l}{-LogP } \\
Target gene & chr & LFC 1 & LFC 2 & LFC 3 & LFC 4 & $\mathbf{1}$ & $\mathbf{2}$ & $\mathbf{3}$ & $\mathbf{4}$ \\
PAFAH1B3 & chr19q & -0.23 & 1.22 & 0.43 & -0.36 & 1.27 & 2.00 & 1.44 & 1.06 \\
GPATCH1 & chr19q & 0.20 & 0.99 & -0.86 & 1.10 & 1.04 & 2.12 & 0.99 & 2.24 \\
HPN & chr19q & 2.56 & -1.27 & -0.17 & -0.79 & 1.73 & 0.55 & 0.70 & 0.58
\end{tabular}


Table S4 continued

\begin{tabular}{|c|c|c|c|c|c|c|c|c|c|}
\hline Target gene & chr & LFC 1 & LFC 2 & LFC 3 & LFC 4 & $\begin{array}{l}-\log P \\
1\end{array}$ & $\begin{array}{l}-\log P \\
2\end{array}$ & $\begin{array}{l}-\log P \\
3\end{array}$ & $\begin{array}{l}-\log P \\
4\end{array}$ \\
\hline ZNF793 & chr19q & 0.14 & 1.58 & -0.13 & -0.17 & 1.46 & 2.49 & 1.21 & 1.04 \\
\hline EGLN2 & chr19q & -1.98 & -0.90 & 1.90 & -0.43 & 0.12 & 0.50 & 1.36 & 0.51 \\
\hline MED25 & chr19q & 0.00 & 0.76 & 1.66 & 0.18 & 1.25 & 2.09 & 2.94 & 1.93 \\
\hline ZNF221 & chr19q & 0.78 & -0.45 & -1.36 & 1.78 & 1.30 & 0.93 & 0.46 & 1.96 \\
\hline LIN7B & chr19q & -1.23 & 2.31 & 0.02 & -0.11 & 0.59 & 1.57 & 1.49 & 0.90 \\
\hline SIGLEC6 & chr19q & -0.71 & 1.37 & 0.75 & 0.49 & 1.12 & 2.81 & 1.92 & 1.28 \\
\hline FPR3 & chr19q & -0.83 & 1.00 & 0.69 & 0.67 & 0.94 & 3.21 & 1.83 & 1.08 \\
\hline KLK13 & chr19q & 1.09 & 0.35 & 0.24 & 0.15 & 3.39 & 2.22 & 1.33 & 0.98 \\
\hline IGLON5 & chr19q & -1.57 & 0.51 & 1.50 & -1.44 & 0.31 & 1.18 & 1.57 & 0.39 \\
\hline BIRC8 & chr19q & 0.29 & -0.36 & 1.68 & -0.31 & 1.26 & 1.14 & 2.01 & 1.25 \\
\hline NLRP4 & chr19q & 0.26 & -0.32 & 1.61 & -0.45 & 1.22 & 1.20 & 1.78 & 1.12 \\
\hline C1QTNF3 & chr5p & -0.62 & -0.58 & 0.63 & 1.86 & 0.98 & 1.25 & 1.49 & 1.55 \\
\hline C5orf22 & chr5p & -1.15 & 1.02 & -0.09 & 1.09 & 0.67 & 1.51 & 0.99 & 2.26 \\
\hline C5orf49 & chr5p & 2.37 & -0.95 & -0.46 & -1.94 & 1.61 & 0.47 & 0.50 & 0.13 \\
\hline IRX1 & chr5p & -0.04 & 1.20 & -2.20 & 1.03 & 1.05 & 2.28 & 0.06 & 1.63 \\
\hline CD53 & chr1 & 0.66 & 0.06 & -0.05 & 1.30 & 1.83 & 1.79 & 1.09 & 2.80 \\
\hline GSTM3 & chr1 & -0.42 & 0.66 & 0.27 & 1.10 & 0.99 & 1.85 & 1.79 & 2.29 \\
\hline RCOR3 & chr1 & -1.63 & -0.71 & 0.99 & 1.33 & 0.27 & 0.64 & 1.10 & 2.24 \\
\hline RRAGC & chr1 & 0.84 & 0.20 & -0.40 & 1.37 & 2.03 & 1.91 & 1.12 & 2.13 \\
\hline USH $2 \mathrm{~A}$ & chr1 & 0.15 & -1.30 & 0.62 & 1.84 & 1.33 & 0.52 & 1.73 & 2.02 \\
\hline OR2T35 & chr1 & -0.18 & 0.47 & -0.23 & 1.60 & 1.06 & 1.18 & 1.00 & 1.79 \\
\hline LCE2C/D & chr1 & -0.70 & 0.91 & -0.33 & 1.78 & 0.72 & 1.13 & 0.79 & 1.77 \\
\hline NBPF4/6 & chr1 & 1.82 & 0.42 & -0.71 & 1.52 & 2.13 & 1.04 & 0.81 & 1.65 \\
\hline B4GALT3 & chr1 & -0.39 & -0.65 & -0.63 & 1.56 & 1.20 & 0.54 & 0.72 & 1.39 \\
\hline CASQ1 & chr1 & -1.58 & -1.33 & 0.36 & 1.34 & 0.31 & 0.38 & 1.11 & 1.35 \\
\hline SMCP & chr1 & 1.18 & -0.23 & -0.20 & 0.00 & 2.34 & 0.88 & 1.04 & 1.33 \\
\hline WDR47 & chr1 & -0.94 & -1.32 & 1.61 & 0.54 & 0.51 & 0.48 & 1.61 & 1.22 \\
\hline TMEM167B & chr1 & 0.96 & 1.20 & -1.78 & -0.12 & 1.47 & 2.20 & 0.20 & 1.05 \\
\hline LCE3C & chr1 & 1.79 & -1.01 & -1.11 & 0.14 & 1.31 & 0.72 & 0.45 & 1.04 \\
\hline PPM1J & chr1 & 0.33 & 0.34 & 1.12 & -0.42 & 1.32 & 1.85 & 2.41 & 1.00 \\
\hline SORT1 & chr1 & -1.42 & 0.97 & 1.10 & -0.60 & 0.42 & 0.99 & 2.21 & 0.75 \\
\hline HPCA & chr1 & 0.48 & 1.04 & 0.59 & -1.29 & 0.96 & 2.78 & 1.69 & 0.53 \\
\hline CAMK1G & chr1 & -2.42 & 2.44 & -0.87 & -1.88 & 0.03 & 1.65 & 0.59 & 0.44 \\
\hline NES & chr1 & 1.94 & -0.33 & -0.93 & -1.44 & 1.38 & 0.57 & 0.48 & 0.40 \\
\hline RNF115 & chr1 & 1.76 & 0.24 & 0.08 & -1.58 & 1.87 & 1.29 & 1.18 & 0.30 \\
\hline
\end{tabular}


Table S4 continued

\begin{tabular}{|c|c|c|c|c|c|c|c|c|c|}
\hline Target gene & chr & LFC 1 & LFC 2 & LFC 3 & LFC 4 & $\begin{array}{l}-\log P \\
1\end{array}$ & $\begin{array}{l}-\log P \\
2\end{array}$ & $\begin{array}{l}-\log P \\
3\end{array}$ & $\begin{array}{l}-\log P \\
4\end{array}$ \\
\hline CFHR4 & chr1 & 0.80 & 1.24 & 0.09 & -1.92 & 1.88 & 2.00 & 1.07 & 0.14 \\
\hline HPS1 & chr10 & 1.17 & 0.47 & -1.81 & 1.34 & 2.44 & 1.11 & 0.18 & 2.74 \\
\hline WNT8B & chr10 & -0.30 & 0.55 & 0.27 & 1.15 & 1.02 & 2.16 & 1.63 & 2.29 \\
\hline MSMB & chr10 & -1.51 & 1.00 & 0.06 & 1.72 & 0.35 & 1.84 & 1.28 & 2.24 \\
\hline DHX32 & chr10 & -0.04 & 1.21 & 0.05 & 0.12 & 1.02 & 2.83 & 1.05 & 1.81 \\
\hline LIPN & chr10 & -0.02 & 1.85 & 0.63 & 0.16 & 1.34 & 2.90 & 2.03 & 1.74 \\
\hline ZNF485 & chr10 & -1.33 & -1.46 & 1.75 & 0.54 & 0.39 & 0.38 & 1.60 & 1.29 \\
\hline SLC18A2 & chr10 & 0.10 & 1.57 & 0.19 & -0.19 & 1.21 & 2.43 & 1.90 & 1.10 \\
\hline AKR1C2 & chr10 & -0.68 & 1.53 & -1.00 & 0.72 & 0.51 & 1.52 & 0.50 & 1.03 \\
\hline CISD1 & chr10 & -1.18 & 1.98 & -0.35 & -0.72 & 0.56 & 1.40 & 0.64 & 0.63 \\
\hline MSRB2 & chr10 & 1.63 & 1.75 & 0.90 & -1.87 & 2.87 & 3.71 & 1.29 & 0.16 \\
\hline RPS25 & chr11 & 1.87 & -1.73 & -2.02 & 1.60 & 2.84 & 0.49 & 0.10 & 1.34 \\
\hline CNTN5 & chr11 & -1.02 & 1.80 & -2.08 & 0.69 & 0.44 & 1.83 & 0.09 & 1.31 \\
\hline OR6T1 & chr11 & 0.44 & 1.58 & 0.76 & -0.82 & 1.21 & 2.67 & 1.94 & 1.11 \\
\hline MMP1 & chr11 & -0.90 & 1.92 & 0.42 & -0.34 & 0.99 & 1.43 & 1.37 & 1.10 \\
\hline ATG16L2 & chr11 & 0.96 & -2.15 & 1.02 & -0.57 & 0.95 & 0.07 & 2.20 & 0.78 \\
\hline DUSP8 & chr11 & -1.66 & -2.34 & 1.85 & -0.30 & 0.45 & 0.04 & 1.34 & 0.60 \\
\hline ANKRD13D & chr11 & 0.76 & 1.91 & -0.37 & -1.26 & 1.36 & 1.93 & 1.04 & 0.56 \\
\hline OR52L1 & chr11 & -0.51 & 2.16 & -0.99 & -1.40 & 0.47 & 1.49 & 0.45 & 0.43 \\
\hline COLCA2 & chr11 & 0.93 & 0.10 & 1.12 & -1.58 & 1.91 & 1.00 & 2.15 & 0.30 \\
\hline PUS7L & chr12 & -1.63 & 0.83 & -0.31 & 1.09 & 0.27 & 1.15 & 0.98 & 2.03 \\
\hline CLEC4A & chr12 & 0.55 & 1.22 & -0.34 & -0.22 & 1.63 & 2.04 & 1.06 & 1.29 \\
\hline LRCOL1 & chr12 & -0.94 & 0.52 & 1.61 & 0.11 & 0.92 & 1.58 & 1.93 & 1.23 \\
\hline PRMT8 & chr12 & 2.24 & -1.21 & -1.52 & 0.08 & 1.53 & 0.38 & 0.35 & 0.97 \\
\hline STAC3 & chr12 & -2.07 & 1.31 & 0.86 & -0.88 & 0.09 & 2.07 & 1.10 & 0.51 \\
\hline THSD1 & chr13 & -0.65 & -0.05 & 0.18 & 1.66 & 1.09 & 1.25 & 1.39 & 1.59 \\
\hline CDC42BPB & chr14 & -1.63 & -1.39 & 0.37 & 1.78 & 0.27 & 0.38 & 1.30 & 1.36 \\
\hline ITPK1 & chr14 & 1.01 & -0.72 & 0.14 & 0.16 & 2.00 & 0.94 & 1.07 & 1.26 \\
\hline GPR33 & chr14 & -0.83 & 1.38 & -0.88 & 1.38 & 1.01 & 2.65 & 0.54 & 1.13 \\
\hline RNASE1 & chr14 & 1.04 & 0.66 & 0.06 & -1.64 & 1.84 & 1.79 & 0.96 & 0.27 \\
\hline EXD1 & chr15 & 1.47 & -0.58 & -0.64 & 1.36 & 2.62 & 1.17 & 0.77 & 1.41 \\
\hline SNRPN & chr15 & -0.33 & 0.87 & 1.02 & -0.27 & 0.95 & 2.07 & 2.08 & 1.20 \\
\hline LRRK1 & chr15 & 1.93 & 0.17 & -0.50 & -0.87 & 1.37 & 1.08 & 1.02 & 0.87 \\
\hline PKM & chr15 & 2.37 & -1.73 & -1.97 & -0.89 & 1.61 & 0.44 & 0.12 & 0.49 \\
\hline ADGRG1 & chr16 & 0.75 & -0.75 & 0.89 & 1.14 & 1.22 & 1.01 & 2.11 & 3.40 \\
\hline
\end{tabular}


Table S4 continued

\begin{tabular}{|c|c|c|c|c|c|c|c|c|c|}
\hline Target gene & chr & LFC 1 & LFC 2 & LFC 3 & LFC 4 & $\begin{array}{l}-\log P \\
1\end{array}$ & $\begin{array}{l}-\log P \\
2\end{array}$ & $\begin{array}{l}-\log P \\
3\end{array}$ & $\begin{array}{l}-\log P \\
4\end{array}$ \\
\hline FTO & chr16 & 1.04 & 0.19 & -0.01 & 0.39 & 2.93 & 1.39 & 0.96 & 2.11 \\
\hline ST3GAL2 & chr16 & -0.61 & 0.34 & 1.46 & 0.52 & 1.16 & 1.46 & 2.46 & 1.58 \\
\hline VPS4A & chr16 & -1.43 & 0.29 & -0.21 & 1.51 & 0.41 & 1.24 & 1.18 & 1.31 \\
\hline MT1X & chr16 & -1.15 & 1.66 & -0.22 & 0.50 & 0.57 & 1.36 & 1.02 & 1.16 \\
\hline PSMB10 & chr16 & 0.23 & 1.43 & -0.23 & -0.28 & 1.27 & 2.19 & 1.16 & 1.15 \\
\hline NOMO3 & chr16 & 1.62 & -0.28 & 0.40 & -1.07 & 1.34 & 0.89 & 0.96 & 0.81 \\
\hline OR1D2 & chr17 & 0.61 & 0.42 & 1.21 & 0.64 & 1.75 & 1.05 & 4.25 & 3.10 \\
\hline АРPBP 2 & chr17 & -0.13 & 0.12 & 1.13 & 0.94 & 1.01 & 1.95 & 2.60 & 2.17 \\
\hline KRT33A & chr17 & 0.17 & -1.27 & 0.24 & 1.17 & 1.03 & 0.55 & 1.17 & 2.05 \\
\hline MED24 & chr17 & 1.28 & 0.24 & -0.34 & 0.25 & 2.22 & 1.18 & 1.09 & 2.05 \\
\hline UNK & chr17 & -1.02 & -1.67 & -0.03 & 2.23 & 0.44 & 0.25 & 0.84 & 1.53 \\
\hline $\mathrm{CCL} 2$ & chr17 & -0.73 & 1.12 & -0.25 & 0.98 & 1.00 & 2.23 & 1.23 & 1.24 \\
\hline RECQL5 & chr17 & -1.08 & 0.18 & 2.04 & -0.26 & 0.75 & 1.22 & 1.43 & 1.10 \\
\hline KRTAP4-11 & chr17 & -2.28 & 1.38 & -1.58 & -2.25 & 0.92 & 2.08 & 1.54 & 1.04 \\
\hline CYB5A & chr18 & 0.73 & -0.01 & -0.19 & 1.12 & 1.89 & 1.69 & 1.00 & 2.44 \\
\hline RAB31 & chr18 & 0.10 & -1.02 & 0.90 & 1.13 & 1.01 & 0.82 & 1.91 & 2.12 \\
\hline GALNT1 & chr18 & -0.29 & -1.14 & -1.82 & 1.83 & 0.60 & 0.40 & 0.18 & 1.32 \\
\hline DYM & chr18 & 0.22 & 2.57 & -1.57 & -0.23 & 1.27 & 1.74 & 0.31 & 1.14 \\
\hline TEX261 & chr2 & 1.02 & -1.95 & -0.17 & 1.34 & 1.39 & 0.13 & 1.11 & 2.27 \\
\hline CAPN14 & chr2 & -0.29 & 1.42 & 0.25 & 0.88 & 1.15 & 2.24 & 2.10 & 2.16 \\
\hline SUPT7L & chr2 & -0.62 & -0.62 & 0.20 & 1.69 & 0.73 & 1.12 & 1.26 & 1.44 \\
\hline KCNK12 & chr2 & 1.26 & -0.87 & 0.31 & 0.40 & 2.37 & 1.02 & 1.07 & 1.39 \\
\hline SPC25 & chr2 & 2.28 & -1.04 & -0.64 & 0.27 & 1.55 & 0.71 & 0.80 & 1.22 \\
\hline CALCRL & chr2 & -0.74 & 1.05 & -0.75 & 0.93 & 0.97 & 2.15 & 0.62 & 1.21 \\
\hline ORC4 & chr2 & 1.28 & 0.91 & -0.43 & -0.37 & 2.14 & 1.83 & 1.04 & 1.09 \\
\hline INHBB & chr2 & 1.23 & 0.04 & 1.18 & -0.53 & 2.45 & 1.61 & 1.79 & 1.06 \\
\hline LPIN1 & chr2 & 1.05 & 1.22 & -1.03 & -0.09 & 1.52 & 2.31 & 0.81 & 1.06 \\
\hline ATG16L1 & chr2 & 2.08 & -0.62 & -1.03 & -0.09 & 1.46 & 0.78 & 0.73 & 0.81 \\
\hline ALLC & chr2 & -0.11 & -1.39 & 1.59 & -0.16 & 1.22 & 0.44 & 1.40 & 0.75 \\
\hline MAL & chr2 & -0.85 & 1.87 & -0.99 & -1.78 & 0.45 & 1.34 & 0.43 & 0.20 \\
\hline DEFB119 & chr20 & 0.59 & 1.58 & -0.73 & 0.62 & 1.25 & 3.03 & 1.21 & 1.74 \\
\hline LKAAEAR1 & chr20 & 0.24 & 1.12 & -0.94 & 0.27 & 1.00 & 2.21 & 0.92 & 1.22 \\
\hline TMEM230 & chr20 & -0.03 & 0.17 & 1.20 & -0.28 & 1.08 & 1.64 & 2.20 & 1.05 \\
\hline SH3BGR & chr21 & 0.74 & 0.94 & -1.77 & 1.68 & 1.26 & 2.18 & 0.20 & 3.37 \\
\hline RSPH1 & chr21 & 1.65 & 0.55 & -1.27 & -0.28 & 1.62 & 1.25 & 0.55 & 1.19 \\
\hline
\end{tabular}


Table S4 continued

\begin{tabular}{|c|c|c|c|c|c|c|c|c|c|}
\hline Target gene & chr & LFC 1 & LFC 2 & LFC 3 & LFC 4 & $\begin{array}{l}-\log P \\
1\end{array}$ & $\begin{array}{l}-\log P \\
2\end{array}$ & $\begin{array}{l}-\log P \\
3\end{array}$ & $\begin{array}{l}-\log P \\
4\end{array}$ \\
\hline KRTAP11-1 & chr21 & -1.50 & -0.75 & 2.18 & 0.24 & 0.36 & 0.61 & 1.50 & 1.18 \\
\hline PDXK & chr21 & 0.45 & -1.17 & 1.37 & 0.19 & 1.48 & 0.65 & 2.10 & 1.12 \\
\hline $\mathrm{ABCC5}$ & chr3 & -0.78 & 1.98 & -0.21 & 0.45 & 1.17 & 1.47 & 1.31 & 1.40 \\
\hline P2RY1 & chr3 & -1.14 & 1.58 & -0.85 & 0.35 & 0.53 & 1.32 & 0.68 & 1.21 \\
\hline SKIL & chr3 & 1.30 & -0.85 & 0.55 & 0.17 & 2.05 & 1.05 & 1.63 & 1.09 \\
\hline GRIP2 & chr3 & -0.23 & 0.10 & 1.22 & -0.06 & 1.00 & 1.58 & 2.32 & 1.06 \\
\hline FAM19A1 & chr3 & -0.23 & 1.81 & 0.14 & -1.09 & 1.05 & 1.31 & 1.27 & 0.74 \\
\hline OR5H14 & chr3 & 0.44 & 0.72 & 1.08 & -0.87 & 0.83 & 1.52 & 2.06 & 0.69 \\
\hline LSMEM2 & chr3 & -0.44 & 1.12 & 0.88 & -2.24 & 0.94 & 2.09 & 1.00 & 0.05 \\
\hline HPGD & chr4 & -0.49 & -1.19 & 0.95 & 1.04 & 0.87 & 0.62 & 0.96 & 2.18 \\
\hline MAD2L1 & chr4 & 0.91 & -0.27 & 1.38 & 0.07 & 2.13 & 1.12 & 2.23 & 1.84 \\
\hline PSORS1C1 & chr6 & -2.44 & -1.27 & -1.60 & 2.03 & 0.03 & 0.63 & 0.43 & 1.42 \\
\hline GUCA1A & chr6 & -1.79 & 2.23 & -1.74 & 0.35 & 0.19 & 1.52 & 0.49 & 1.32 \\
\hline CD24 & chr6 & 1.64 & 0.12 & 1.30 & -0.53 & 2.58 & 1.63 & 1.96 & 1.24 \\
\hline NR2E1 & chr6 & 1.60 & 1.17 & 0.63 & -0.52 & 3.14 & 2.45 & 1.64 & 1.22 \\
\hline SMLR1 & chr6 & 1.86 & -0.37 & 0.39 & -0.71 & 1.39 & 1.29 & 1.34 & 1.04 \\
\hline ID4 & chr6 & -1.39 & 0.63 & 1.52 & -0.58 & 0.44 & 1.18 & 1.75 & 0.78 \\
\hline GJA1 & chr6 & -1.86 & 1.52 & 0.33 & -0.59 & 0.16 & 1.30 & 1.18 & 0.76 \\
\hline EEF1E1 & chr6 & 1.13 & 0.86 & 0.78 & -0.20 & 2.40 & 1.47 & 1.06 & 0.75 \\
\hline LTV1 & chr6 & 1.36 & -0.66 & 0.40 & -1.21 & 1.40 & 0.69 & 1.12 & 0.61 \\
\hline COA1 & chr7 & 1.09 & 0.27 & -0.33 & 0.90 & 2.29 & 2.07 & 0.98 & 2.13 \\
\hline PEX1 & chr7 & -3.15 & 1.18 & 0.23 & 0.63 & 0.00 & 2.20 & 1.04 & 1.75 \\
\hline $\mathrm{CHCHD} 2$ & chr7 & 1.97 & -0.58 & -0.77 & -0.46 & 1.39 & 0.77 & 0.50 & 1.18 \\
\hline NOS3 & chr7 & 0.07 & -0.63 & 1.67 & -0.29 & 1.25 & 0.96 & 1.42 & 1.17 \\
\hline RADIL & chr7 & -1.02 & 1.32 & 0.70 & -0.06 & 0.83 & 1.85 & 1.57 & 1.10 \\
\hline C1GALT1 & chr7 & -2.64 & 1.04 & 0.23 & -0.11 & 0.01 & 1.49 & 1.16 & 0.96 \\
\hline TMEM140 & chr7 & 0.19 & 0.97 & 1.51 & -1.00 & 1.18 & 2.10 & 2.21 & 0.85 \\
\hline AMPH & chr7 & 1.16 & 1.58 & -0.59 & -0.66 & 1.36 & 2.43 & 1.21 & 0.77 \\
\hline FAM71F1 & chr7 & 1.03 & -0.31 & 0.75 & -1.16 & 1.92 & 0.95 & 1.13 & 0.66 \\
\hline ZPBP & chr7 & 2.14 & -0.80 & -0.68 & -1.01 & 1.48 & 0.57 & 0.84 & 0.43 \\
\hline DUSP4 & chr8 & 0.43 & 0.41 & -1.58 & 1.06 & 1.44 & 0.97 & 0.30 & 2.60 \\
\hline SLA & chr8 & 0.38 & -0.72 & 0.27 & 1.22 & 1.37 & 1.06 & 1.26 & 2.28 \\
\hline TDRP & chr8 & 0.25 & 1.18 & 0.23 & -0.37 & 1.96 & 2.19 & 1.18 & 1.04 \\
\hline EXT1 & chr8 & 0.40 & 1.69 & -0.43 & -0.87 & 1.26 & 1.40 & 1.02 & 0.96 \\
\hline EXD3 & chr9 & 1.11 & -1.58 & -1.41 & 1.15 & 1.02 & 0.30 & 0.38 & 2.37 \\
\hline
\end{tabular}


Table S4 continued

\begin{tabular}{|c|c|c|c|c|c|c|c|c|c|}
\hline Target gene & chr & LFC 1 & LFC 2 & LFC 3 & LFC 4 & $\begin{array}{l}-\log P \\
1\end{array}$ & $\begin{array}{l}-\log P \\
2\end{array}$ & $\begin{array}{l}-\log P \\
3\end{array}$ & $\begin{array}{l}-\log P \\
4\end{array}$ \\
\hline OR1B1 & chr9 & 0.32 & 0.26 & -1.04 & 1.03 & 1.28 & 0.96 & 0.80 & 2.26 \\
\hline COL5A1 & chr9 & 0.20 & 0.17 & -0.41 & 1.14 & 1.88 & 1.11 & 1.02 & 2.06 \\
\hline GTF3C4 & chr9 & 0.41 & 1.17 & -0.21 & 0.20 & 2.13 & 2.38 & 1.03 & 1.42 \\
\hline DNAJB5 & chr9 & 0.24 & 1.76 & -0.84 & 0.29 & 1.24 & 2.23 & 1.06 & 1.29 \\
\hline C9orf62 & chr9 & 0.05 & 1.39 & 0.52 & -0.21 & 1.57 & 2.39 & 1.80 & 1.14 \\
\hline TLN1 & chr9 & 1.50 & -2.37 & 0.51 & -0.54 & 1.56 & 0.04 & 1.18 & 0.81 \\
\hline TMEM8B & chr9 & -1.60 & 1.80 & -0.59 & -1.73 & 0.43 & 1.31 & 0.45 & 0.22 \\
\hline CXorf51A/B & chrX & 2.13 & 0.35 & -0.84 & 0.30 & 1.72 & 1.53 & 0.84 & 1.41 \\
\hline FAM127A & $\operatorname{chrX}$ & 0.93 & -0.68 & 1.06 & 0.13 & 1.97 & 0.97 & 2.15 & 1.33 \\
\hline FUNDC2 & chrX & 1.70 & 0.34 & 1.39 & 0.13 & 3.35 & 2.45 & 2.67 & 1.27 \\
\hline NLGN3 & $\operatorname{chrX}$ & -1.24 & 1.32 & -1.47 & 0.89 & 0.38 & 2.11 & 0.38 & 1.10 \\
\hline RPA4 & $\operatorname{chrX}$ & 2.12 & -0.39 & 0.20 & -0.54 & 1.61 & 1.13 & 1.47 & 1.01 \\
\hline SPANXN2 & chrX & 1.83 & -0.88 & 0.17 & -1.08 & 1.33 & 0.75 & 1.07 & 0.51 \\
\hline ARAF & $\operatorname{chrX}$ & 0.02 & -1.35 & 1.81 & -1.63 & 0.90 & 0.38 & 1.31 & 0.28 \\
\hline Non-Target & unknown & 1.21 & 0.14 & 0.50 & -0.07 & 2.76 & 1.55 & 1.99 & 1.05 \\
\hline Non-Target & unknown & -0.31 & 1.82 & 0.07 & -0.43 & 1.14 & 1.82 & 1.32 & 0.96 \\
\hline
\end{tabular}




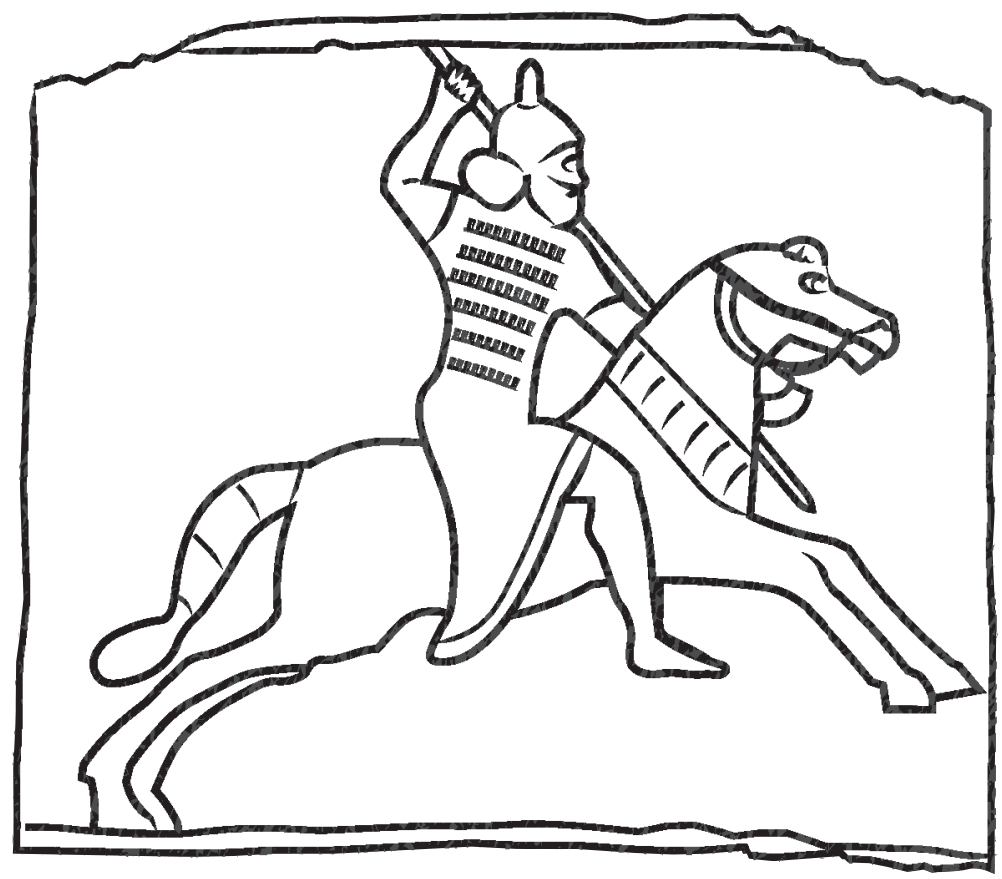

Illustration based on a stone carving on display at the British museum 


\section{Chapter 5 \\ CRISPR-mediated functional silencing of DUX4}

Ator Ashoti, Richard Lemmers, Silvère van der Maarel and Niels Geijsen 


\section{Abstract}

Facioscapulohumeral muscular dystrophy (FSHD) is a genetic muscle disorder that leads to progressive muscle weakness predominantly of the face, shoulders and upper arms. The origin of FSHD lies in the D4Z4 repeat in the subtelomeric region of chromosome $4 q$, which contains the DUX4 gene. Ectopic expression of DUX4 is cytotoxic to muscle cells. The open reading frame of DUX4 is present in all D4Z4 sequences within the human genome, which hampers the application of conventional CRISPR/Cas9 genome-editing strategies to try and block DUX4 expression. Here we report an alternative targeting strategy that does not target the coding sequence of the DUX4 gene itself, but instead makes use of a relative unique region in the $3^{\prime}$ UTR, upstream of the polyadenylation signal. This could potentially destabilize the DUX4 transcript, and thus minimize the possibility of DUX4 translation. Our DUX4-inducible cell line contains the genomic DUX4 sequence, and we were able to rescue cells from DUX4-induced apoptosis. This demonstrates the feasibility that these types of targeting strategies may abrogate DUX4 expression, and the potential for FSHD treatment in the future. 


\section{Introduction}

Double homeobox 4 (DUX4), a pioneer transcription factor ${ }^{1,2}$, is the main cause for the development of facioscapulohumeral muscular dystrophy (FSHD) due to its inappropriate expression in muscle ${ }^{3-5}$. The open reading frame (ORF) of the DUX4 gene can be found in D4Z4 repeats, which are present at several distinct loci in the human genome and in a tandem repeat sequence on chromosomes 4 and $10^{6-8}$. The pathological expression of DUX4 is caused by multiple genetic and epigenetic events that initiate the epigenetic derepression of DUX4 at the subtelomeric region of chromosome 4. Contraction of the D4Z4 tandem repeat array on chromosome 4 to less than 10 D4Z4 repeats removes chromatin features needed for effective repression of this locus $^{9}$ (FHSD1). Alternatively, loss-of-function mutations in chromatin modifier genes, such as structural maintenance of chromosome hinge domain 1 (SMCHD1) or DNA-methyltransferase 3 beta (DNMT3B) ${ }^{10-12}$ can also cause de-repression of the D4Z4 repeat array and its embedded DUX4 gene (FSHD2). These two modifier genes collaboratively establish and maintain the hypomethylated state of their target genes, including DUX4, thereby repressing their expression ${ }^{13-18}$. Insufficient epigenetic repression of the D4Z4 repeats (due to a contracted D4Z4 repeat array, and/or the loss of epigenitic modifier genes) results in chromatin relaxation, which in itself is not enough to cause FSHD, but does render the DUX4 gene permissive for transcription. Only when derepression occurs in a $4 \mathrm{qA}$ genetic background can it lead to the development of FSHD. The DUX4 transcript is stabilized by a polyA sequence that is present in exon 3 on the 4qA allele. This stabilized DUX4 transcript can then be translated into DUX4 protein and lead to the development of FSHD ${ }^{4}$. The $4 \mathrm{qB}$ variant on the other hand that does not possess this polyA sequence, diminishing pathological DUX4 expression, and is therefore generally classified as non-pathogenic ${ }^{19,20}$.

In general, silencing disease-causing genes in gain-of-function disorders is a relatively straightforward approach with new genome-editing techniques such as CRISPR/Cas9 ${ }^{21-23}$. Although this may be an option for many genetic disorders, FSHD has a much more complex genetic and epigenetic structure, which complicates a simple targeting approach. The presence of multiple copies of DUX4 throughout the human genome complicates the use of genome-editing techniques to silence the gene. Targeting DUX4 with CRISPR/Cas9 can lead to shortening of the D4Z4 repeat sequence, and possibly aggravate the pathophysiology of both FHSD1 and FSHD2. In FSHD1, it can shorten an already contracted sequence, which results in further loss of repressive chromatin. In FSHD2, it can shorten a normal-sized D4Z4 allele to a contracted D4Z4 allele, in addition to the mutation in the SMCHD1/DNMT3B chromatin-modifier gene. Together with the above risks of using CRISPR/Cas9 to target the DUX4 locus, the occurrence of D4Z4 repeats throughout the genome will result in Cas9induced double-strand breaks at multiple places in the host's genome, which can have unpredictable and unwanted outcomes including off-target insertions and deletions (indels) or translocation events. Recent attempts at targeting DUX4 directly include systems that do not lead to DNA damage, such as the use of antisense morpholino oligonucleotides to target and knock-down the DUX4 transcript ${ }^{24}$. In another study, a catalytically disabled Cas9 fused to a Krüppel-associated box (dCas9-KRAB) was used to target the promotor of DUX4, inducing epigenetic repression of DUX $4^{25}$. Both studies show the ability to successfully diminish DUX4 expression in patient-derived cells. While these studies show promising results, unless a gene-therapy approach is taken to introduce these systems in vivo (which has other practical and ethical issues), the fact that these approaches have transient effects makes them less ideal for the long-term treatment of FSHD. 
We therefore explored options to target DUX4 directly, in order to permanently disable expression of this gene. One promising approach is to use CRISPR/Cas9 to target a region of DUX4 that is not in the ORF of the gene and that does not frequently occur in other regions of the human genome. Lemmers et al. recently described a relative unique sequence present in the most distal copy of DUX4, termed the E3 sequence ${ }^{4,26}$. The E3 sequence is located downstream of the DUX4 stop codon and upstream from the polyA signal. Here, we explored if CRISPR/Cas9-mediated targeting of the E3 sequence can abrogate DUX4 expression in our DUX4-inducible in vitro model.

\section{Results}

\section{Direct targeting of DUX4-E3 with CRISPR/Cas9 modified systems}

The E3 sequence upstream of the polyadenylation signal (PAS) is found in a subpopulation of patients with the $4 \mathrm{qA}$ allele ${ }^{26}$. The $\mathrm{E} 3$ sequence is a promising region to directly target the DUX4 gene. CRISPR/Cas9-generated indels at this region can potentially disrupt regulatory function and destabilize the DUX4 transcript. To explore the possibility of abrogating DUX4 expression by targeting the E3 sequence, we designed several guide RNA (gRNA) sequences targeting this relatively unique region using the online WU-CRISPR gRNA design algorithm ${ }^{27,28}$. Of the possible gRNA sequences, two were selected for further analysis (Fig. $1 \mathrm{~A}$ ) based on their predicted effectiveness as well as the low predicted chance of off-target editing events at other genomic loci29.

Using our DUX4 inducible expression (DIE) cell model system (described in detail in chapter 2), in which DUX4 expression is induced in a doxycycline-dependent manner, we tested the ability of these two gRNAs to inhibit DUX4 expression. DIE cells were transduced with recombinant CRISPR/Cas9 ribonucleoprotein complex using the iTOP transduction method ${ }^{30}$. DUX4 was induced 48-96 hours (h) after CRISPR/Cas9 transduction. 24h after doxycyclinemediated induction of DUX4 expression, survival of DIE cells was measured by fluorescenceactivated cell sorting (FACS) analysis. As shown in Figure 1B, CRISPR/Cas9 targeting of the E3 sequence significantly increased cell survival post-DUX4 induction compared to the control (Cas9 protein, no guide) (Fig. 1B; gRNA1: $24.3 \% \pm 3.1 \%$, $\mathrm{p}$-value $=7 \mathrm{E}-05$; gRNA2: $18.2 \% \pm$ $1.7 \%, \mathrm{p}$-value $=2 \mathrm{E}-05)$. gRNA1 was significantly more efficient at promoting cell survival after doxycycline induction of DUX4 expression, compared to gRNA2 ( $p$-value $=0.008$ ) (Fig. 1B). To further increase knock-out efficiency, synthetic single guide RNA (sgRNA) were used from Synthego (California, USA) that carry prime-end thiol modifications to increase RNA stability. Furthermore, spCas9 was optimized to contain four SV40 nuclear localization signals (NLSs) at the protein's N-terminal and two SV40 NLSs at its C-terminal, to improve its nuclear import ability ${ }^{31}$. These modifications further increased editing efficiency and significantly increased DIE cell survival to around 51.6\% ( $\pm 1.56 \%, p$-value $=0.004$ ) (Fig. $1 \mathrm{C}$ ). Unexpectedly, repeated targeting of the E3 sequence either by the same gRNA or by using different gRNAs in each round of targeting, only incrementally enhanced DIE cell survival (Fig. 1D). However, these data could be an overestimation of the DIE cell rescue due to proliferation of the positively-targeted cells between the time of doxycycline administration and FACS analysis (16-18 h). To examine this, we analyzed CRISPR/Cas9-mediated disruption of DUX4 at the clonal level. DUX4 targeted DIE cells were single-cell sorted into a 96-well plate $48 \mathrm{~h}$ after CRISPR/Cas9 transduction and allowed to expand. Clones were then induced with doxycycline and scored (live or dead) $48 \mathrm{~h}$ after doxycycline administration. Survival of 
individual clones was similar to the survival of the heterogeneous cell population (Fig. 1E), indicating that proliferation and selection of targeted cells did not significantly contribute to the overall rescue effect.

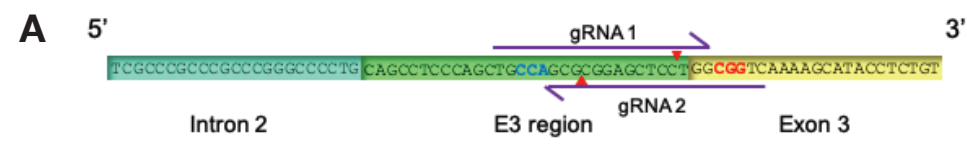

B
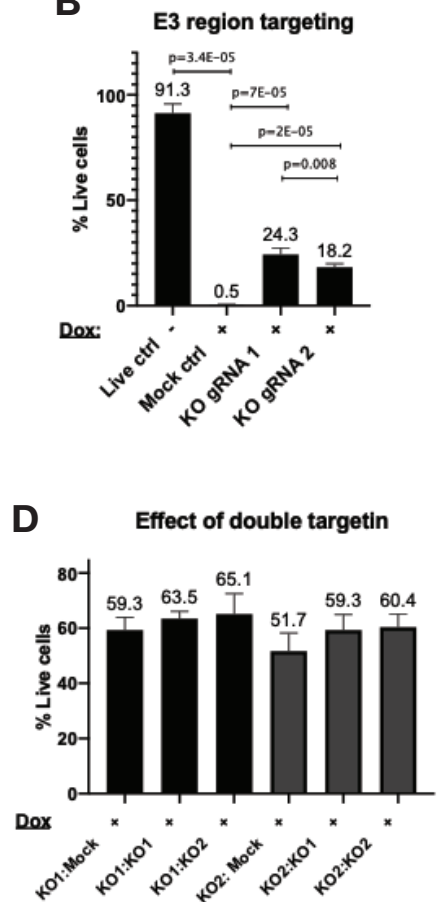

C
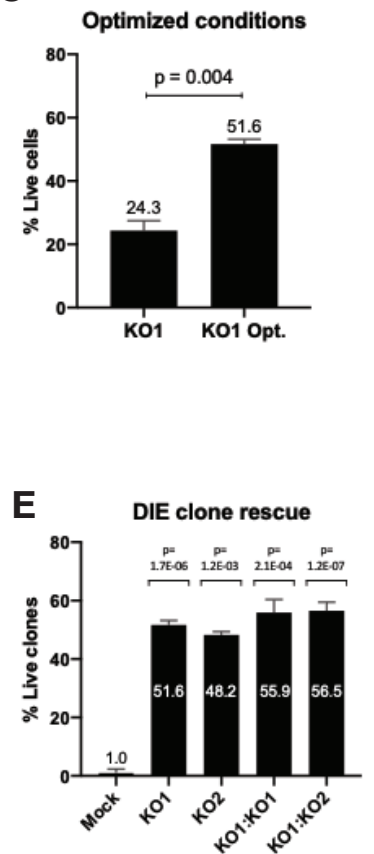

Figure 1. Silencing DUX4 with CRISPR/Cas9. (A) Schematic representation of the E3 region and context sequences. Purple half arrows indicate gRNA sequences, their location and orientation at the E3 site, with the red triangles representing the Cas9 cut sites. Red lettering is the PAM sequence of gRNA1, and blue lettering is the complementary sequence of the gRNA2 PAM, located on the anti-sense strand not shown in this figure. (B) FACS analysis of doxycycline uninduced (-) and induced (+) DIE cells. Live ctrl: DIE cells that have not been transduced with CRIPSR/ CAS9 or exposed to doxycycline. Mock ctrl: DIE cells transduced with only spCas9 protein. KO gRNA1/2: DIE cells transduced with spCas9 protein and DUX4 gRNA1 or gRNA2. Statistical significance of FACS data was determined by a two-tailed Student t-test. (C) Rescue efficiency of DIE cells with conventional targeting (KO1), and optimized targeting (KO opt.). Statistical significance of FACS data was determined by a one-tailed Student t-test. (D) FACS analyzed data including double targeted E3 sequence using optimized conditions. Mock: DIE cells were transduced with 4xSV40 NLS-spCas9-2xSV40 NLS protein only. KO1: DIE cells were transduced with 4xSV40 NLS-spCas9-2xSV40 NLS protein and gRNA1. KO2 DIE cells were transduced with 4xSV40 NLS-spCas9-2xSV40 NLS protein and gRNA2. (E) Rescue percentage single and double targeted DIE clones. All surviving clones were counted after $48 \mathrm{~h}$ of doxycycline exposure. A significant increase in survival can be seen when DIE cells were transduced with $4 \times \mathrm{SV} 40$ NLS-spCas9-2xSV40 NLS and a DUX4 specific gRNA (KO1, KO2, KO1:KO1, KO2:KO2), compared to cells that were only transduced with 4xSV40 NLS-spCas9-2xSV40 NLS protein (Mock). Statistical significance was determined by a two-tailed Student t-test. gRNA: guide RNA, DIE: DUX4 induced expression, E3: exon3 antecedent sequence, FACS: fluorescence-activated cell sorting, KO: knock-out, NLS: nuclear localization signal, PAM: protospacer adjacent motif. 
Next, we examined the type of indel that was able to eliminate DUX4 expression using TIDE analysis (Tracking of Indels by Decomposition) ${ }^{32}$. Targeting the E3 sequence with gRNA1 primarily causes an insertion of one nucleotide $(40.1 \% \pm 10 \%$, p-value $=0)$. Insertions of more than one nucleotide were not detected. The wildtype sequence was found at a frequency of $35.8 \% \pm 10.4 \%$. The remaining $3.7-44.5 \%$ consisted of deletions of different sizes, however, none reached a frequency higher than $5 \%$ (Fig. 2A). To discern which indels are responsible for the rescue, the same population of targeted DIE cells were exposed to doxycycline for a period of $24 \mathrm{~h}$ to obtain an enriched population of rescued cells that were subsequently also analyzed for their indel frequency. All indels previously detected were still visible in these rescued cells (Fig. 2B), suggesting that all indels presented in these graphs can contribute to silencing the DUX4 gene. Sequencing data further showed that the inserted nucleotide consisted of a cytosine in 83.4-88.2\% of all gRNA1 targeted samples (Fig. 2C). This cytosine insertion can be found directly to the left side of the break (Fig. 2D, bottom panel).

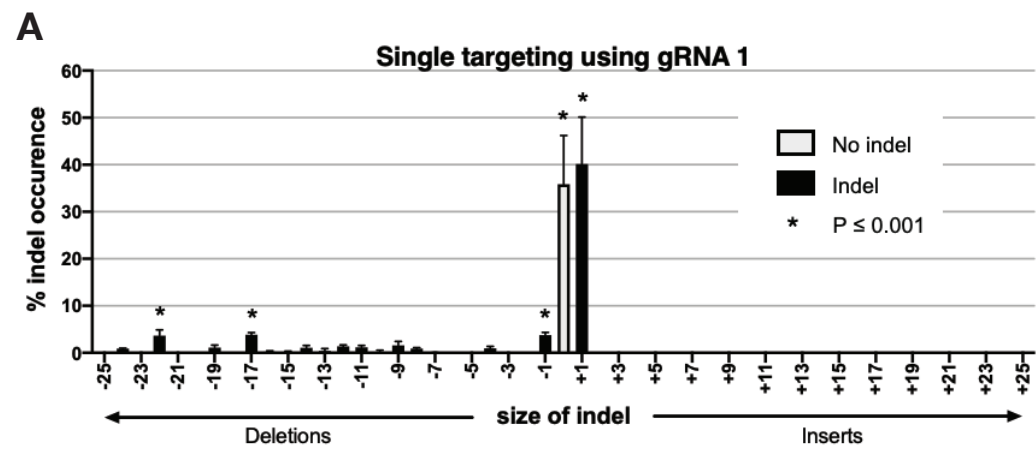

B

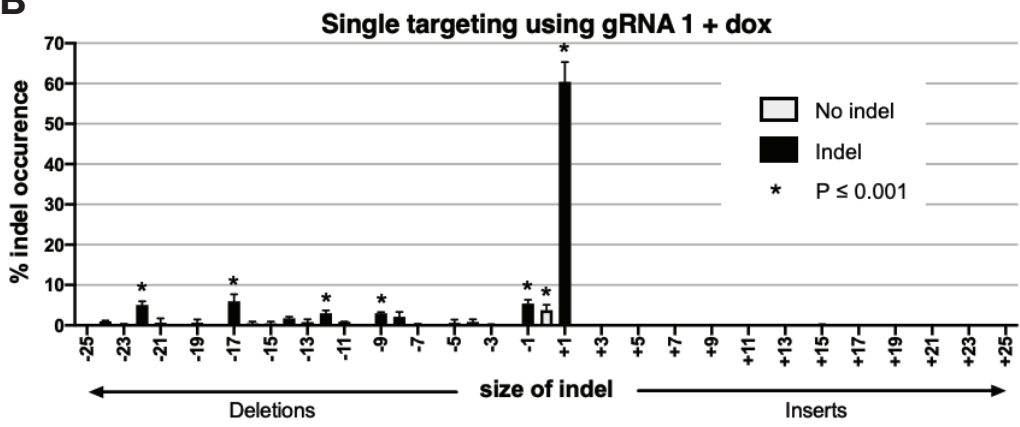

D

C

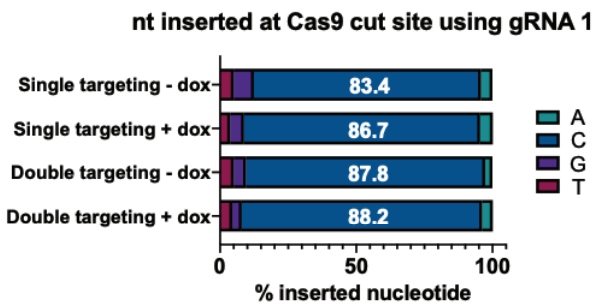

\section{Targeted sequence}
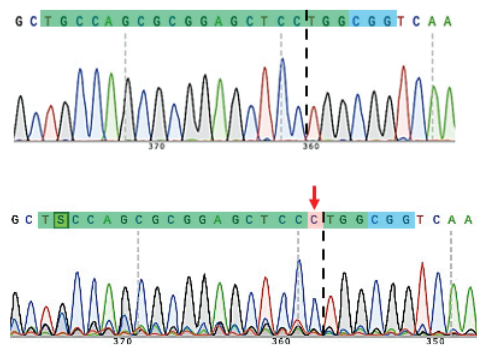
Figure 2. Type and frequency of CRIPSR/Cas9-induced indel at the E3 site when targeted with gRNA1. (A \& B) The percentage of inserted or deleted nucleotides found at the cut site of the gRNA1 targeted E3 region. (A) In DIE cells that were not exposed to doxycycline, gRNA1 demonstrates a high tendency of a one nucleotide insertion ( $40.1 \%$ $\pm 10 \%, p$-value $=0)$. The wildtype sequence can be found at a frequency of $35.8 \% \pm 10.4 \%(p$-value $=0)$. Deletions of 1,17 and 22 nucleotides were also detected in a significant amount (-1nt: $3.8 \% \pm 0.6 \%$, p-value $=4.3 \mathrm{E}-08 ;-17 \mathrm{nt}$ : $3.8 \% \pm 0.5 \%$, p-value $=9.1 \mathrm{E}-07 ;-22 \mathrm{nt}: 3.6 \% \pm 1.3 \%$, $\mathrm{p}$-value $=0.0002)$. (B) sequencing data of gRNA1 targeted DIE cells that were exposed to doxycycline (+1nt: $60.4 \% \pm 4.9 \%$, p-value $=0$; Ont: $3.7 \% \pm 1.3 \%$, p-value $=1.8 \mathrm{E}-04 ;-1 \mathrm{nt}$ : $5.4 \% \pm 1 \%$, p-value $=1.7 \mathrm{E}-10 ;-9 \mathrm{nt}: 3 \% \pm 0.3 \%$, p-value $=3.7 \mathrm{E}-05 ;-12 \mathrm{nt}: 3 \% \pm 0.7 \%$, p-value $=1.2 \mathrm{E}-04 ;-17 \mathrm{nt}: 5.9 \%$ $\pm 1.8 \%$, p-value $=3.7 \mathrm{E}-10 ;-22 \mathrm{nt}: 5 \% \pm 0.9 \%, \mathrm{p}$-value $=6.9 \mathrm{E}-12)$. (C) The nucleotide inserted when the E3 sequence is targeted with gRNA1 is predominantly a cytosine (Single targeting - dox: $83.4 \% \pm 2.7 \%$, p-value $=1.7 E-05$; Single targeting + dox: $86.7 \% \pm 2.6 \%, p$-value $=4 \mathrm{E}-05$; Double targeting - dox: $87.8 \% \pm 7.7 \%, p$-value $=0.001 ;$ Double targeting + dox: $88.2 \% \pm 2.4 \%, p$-value $=1.5 \mathrm{E}-05)$. (D) Sanger sequencing data demonstrating the cytosine insertion at the cleavage site. Upper panel shows the wild type situation. Cut site is indicated with a black intermitted line. The spacer sequence is highlighted in green, and the PAM is highlighted in blue. The one nucleotide cytosine insertion when targeting the E3 site with gRNA1 is highlighted in red and can be found directly to the left of the cut site. gRNA: guide RNA, DIE: DUX4 induced expression, E3: exon3 antecedent sequence, PAM: protospacer adjacent motif.

Targeting E3 with gRNA2 on the other hand, mainly resulted in deletions, the most frequent being two, seven and fourteen nucleotides (Fig 3A and 3B). These deletions can be seen to the right side of the double-stranded break (Fig. 3C, right panel). The wildtype sequence can be found at a frequency of $58.6 \% \pm 1.8 \%$ (Fig. $3 \mathrm{~A}$ ), suggesting that gRNA2 is less efficient than gRNA1 in its genome-editing capacity. These results corroborate previous results (Fig. $1 B)$ that gRNA2 is less effective in rescuing DUX4-induced apoptosis than gRNA1.

Results shown here also affirm that DNA repair is not random. The tendency to produce a specific type of indel at a particular target region has previously been shown to be highly reproducible, non-random and dependent on local sequence context at the break site ${ }^{33-35}$. The type of indels that were generated by the gRNAs were the same in several independent experiments and are thus likely target region dependent.

Upon closer examination of the E3 region by RBPmap ${ }^{36}$, motifs of different RNA binding proteins can be found (Fig. 4). These RNA binding proteins are known to play a role in RNA splicing and mRNA processing ${ }^{37-41}$, which suggests the importance of this region in regulating the stability of the DUX4 transcript. 
A

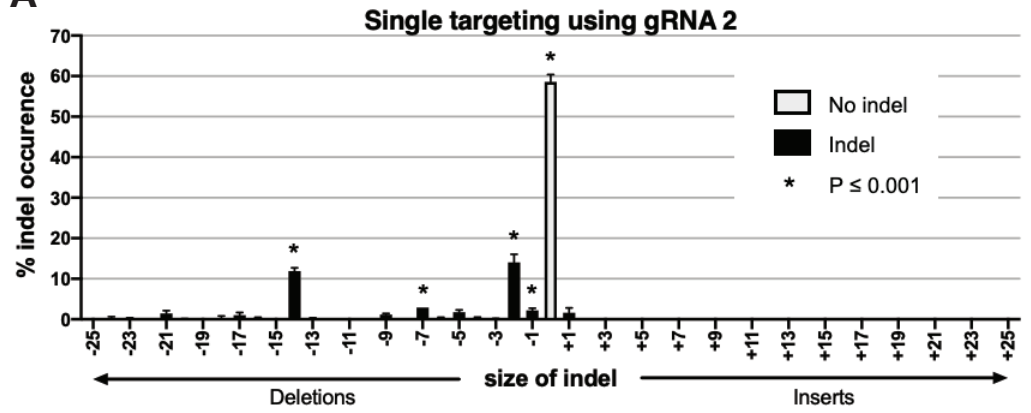

B

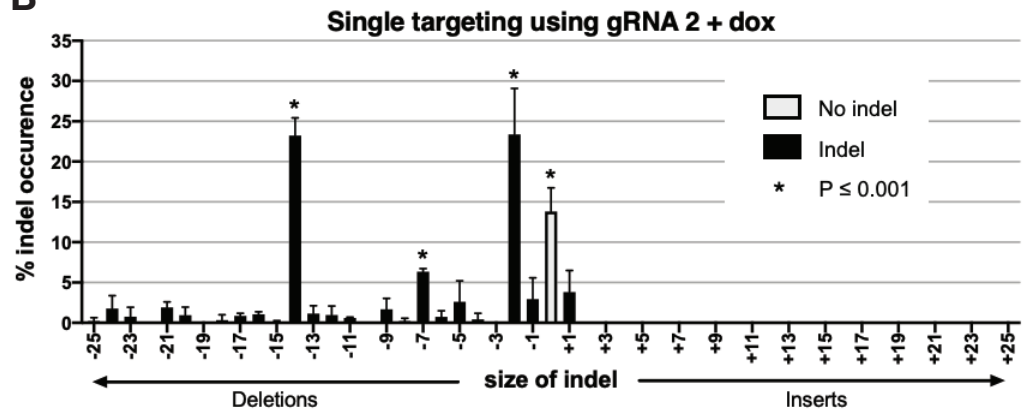

C

Target sequence gRNA 2
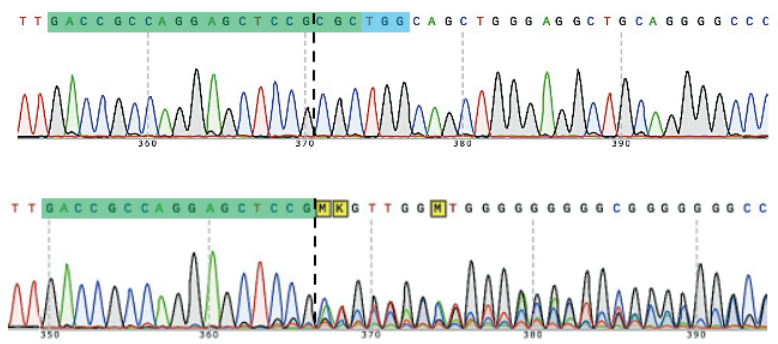

Figure 3. Type and frequency of CRIPSR/Cas9-induced indel at the E3 site when targeted with gRNA2. (A) Nondoxycycline exposed DIE cells targeted with gRNA2 show a higher tendency towards deletion. The most frequent deletions are deletions of two and fourteen nucleotides ( $2 \mathrm{nt}: 14 \% \pm 2 \%$, p-value $=1.5 \mathrm{E}-83 ; 14 \mathrm{nt}$ : $11.9 \% \pm 0.8 \%$, $\mathrm{p}$-value $=1.1 \mathrm{E}-70)$. Two other deletions were found that are less frequent, but still significantly detected among the population (1nt: $2.2 \% \pm 0.5 \%$, p-value $=0.0003 ; 7 n t: 2.9 \% \pm 0 \%$, p-value $=2 \mathrm{E}-06$ ). The wildtype sequence can be found at a frequency of $58.6 \% \pm 1.8 \%$ (p-value $=0)$. (B) Sequencing results of doxycycline treated DIE cells targeted with gRNA2 (0nt: $13.8 \% \pm 3 \%$, p-value $=9.7 \mathrm{E}-36 ;-2 \mathrm{nt}: 23.4 \% \pm 5.7 \%$, p-value $=3.3 \mathrm{E}-49 ;-7 \mathrm{nt}: 6.3 \% \pm 0.4 \%$, p-value $=5.3 \mathrm{E}-08 ;-14 \mathrm{nt}: 23.2 \% \pm 2.2 \%$, p-value $=1.5 \mathrm{E}-69)$. (C) The deletions when targeting the E3 site with gRNA2 can be seen in the lower panel, where a deletion has occurred directly to the right of the cut site, thereby also removing the last three nucleotides of the gRNA sequence and the PAM sequence. DIE: DUX4 induced expression, nt: nucleotide, E3: exon3 antecedent sequence, gRNA: guide RNA, PAM: protospacer adjacent motif. 


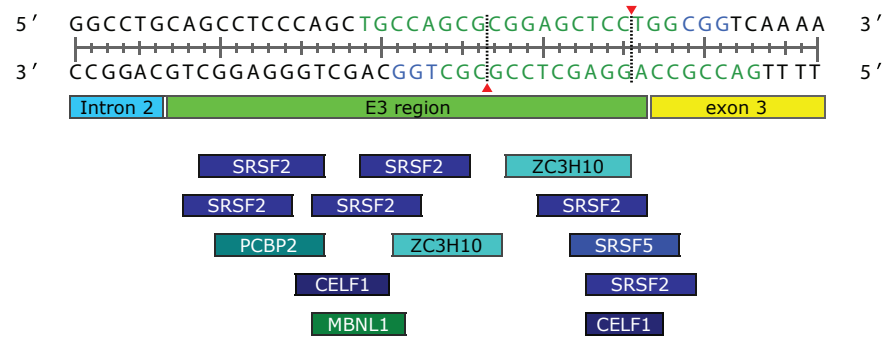

Figure 4. RNA binding protein motifs at the E3 region. Target sequence is indicated in green lettering, and the PAM sequence in blue lettering. gRNA1 targets the green sequence on the leading strand (top), and gRNA2 targets the lagging strand (bottom). Red triangle and intermitted line indicate cleavage sites from the Cas9 protein. RNA binding proteins are annotated below the DNA sequence, their location corresponding to their binding motif on the DNA. E3: exon3 antecedent sequence, gRNA: guide RNA, PAM: protospacer adjacent motif.

\section{Discussion}

In the search for a treatment for FSHD, recently developed genome-editing technologies offer interesting new possibilities to permanently shut down DUX4 expression in affected tissues. However, the repetitive nature of DUX4, the disease-causing gene in FSHD, makes it challenging to identify suitable gRNAs that specifically target the disease causing DUX4 open reading frame (ORF). We therefore designed a CRIPSR/Cas9 approach to silence DUX4 without targeting the ORF. Because pathological DUX4 expression needs a a stable transcript, we decided to target a site upstream of the polyadelylation sequence that is relatively 'unique', and could be important for the stabilization or processing of the DUX4 transcript. This site differs in two nucleotides from sequences found in the preceding repeats and from D4Z4 repeats at other places in the genome ${ }^{26}$. Any indel created in this region could potentially destabilize the DUX4 transcript, by interfering with regulatory functions. Targeting the $\mathrm{E} 3$ sequence with an in vitro transcription (IVT)-generated guide and traditional spCas9 protein indeed showed some rescue in DIE cells upon doxycycline exposure, with an efficiency of approximately $24 \%$. Because skeletal muscle fibers are multinucleated, it takes only a few DUX4-expressing nuclei to deteriorate the entire muscle fiber ${ }^{5,42}$. Therefore, a high knock-out efficiency would be required to provide therapeutic efficacy. By using optimized recombinant CRIPSR/Cas9 components (synthetic guides, adding additional NLSs to Cas9), the knock-out efficiency significantly increased from $\sim 24 \%$ to $~ 51 \%$. Multiple consecutive targeting's only marginally increased cell survival, despite the fact that these double-targeted DIE cells demonstrate near-90\% Indel formation at the CRISPR/Cas9 target site (Fig. S1). One explanation could be that the mutations introduced at the $\mathrm{E} 3$ region are not as potent in functionally disrupting DUX4 expression as, for example, disruptions of the DUX4 ORF itself. Indels at the E3 site likely destabilize the DUX4 transcript, but this can also depend on other factors such as cell cycle state ${ }^{43-46}$ and cellular stress ${ }^{47,48}$. RNA destabilization is therefore not a black or white event and the disruption of the DUX4 (pre-)mRNA stability by targeting the E3 locus is thus not sufficient for full elimination of all DUX4 transcript from cells, as depicted by the 'rescue-cap' illustrated in Figure 5. Destabilizing the DUX4 transcripts by targeting the 3'UTR may therefore not be enough to provide therapeutic benefit. 


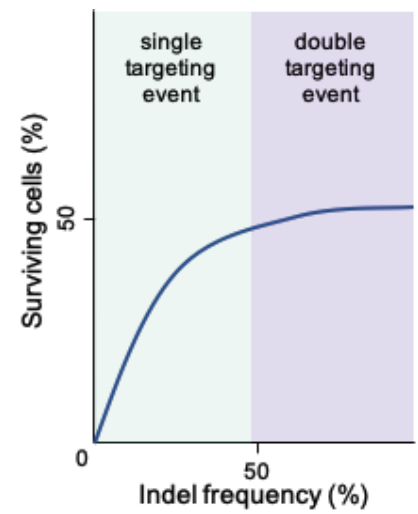

Figure 5. Schematic representation of the 'rescue cap' seen in DIE cells targeted at the E3 region, upon DUX4 induction. When targeting the $\mathrm{E} 3$ region, the frequency of indels at this region, that functionally disrupts DUX4 stability, determines the likelihood of cell survival upon DUX4 induction. A higher indel frequency beyond a certain point results in minimal effect on cell survival upon DUX4 induction. This suggests that the ability to disrupt the E3 region to destabilize DUX4 expression is limited, and maximal destabilization is almost achieved after a single round of targeting.

Analyzing the target sequence in single-targeted and double-targeted DIE cells revealed the type and frequency of indels that were generated at the E3 site. It is know that the indels generated by CRISPR/Cas9 targeting are non-random ${ }^{33-35}$. Based on local sequence context of the genomic ends flanking the cleavage site, the type of indel is highly reproducible and predictable. This has led to the development of various algorithms that can predict, to a degree of certainty, the type of indel that will be produced at a specific cleavage site $^{49-51}$. In this particular project, targeting with gRNA1 introduced a single cytosine directly to the left of the cleavage site at the highest frequency. Interestingly, the InDelphi algorithm ${ }^{49}$ correctly predicted that no more than one nucleotide would be inserted at this site when targeting with this gRNA. The InDelphi model also correctly predicted that this insertion would most likely be a cytosine. However, the algorithm predicted that a deletion of 12 nucleotides would be the most likely mutation to occur upon targeting, which was not the case. When targeting with the other gRNA (gRNA2), the most commonly found indels were two, seven and fourteen nucleotide deletions, which were indeed also the top three predictions with InDelphi. Although these algorithms can be extremely useful when generating a shortlist of guides for when a specific indel is required, experimental testing of gRNAs should not be omitted. In particular, since InDel size does not seem to be a good predictor of the functional effect on cell survival when targeted to other genomic regions than ORFs. Without additional context, it is perhaps surprising that a single cytosine addition in the $3^{\prime} U T R$ of the DUX4 transcript, achieved by sgRNA1 targeting, is sufficient to achieve $50 \%$ cell survival as compared to control.

Taken together, we demonstrated here that a single base insertion at a specific intronic site can disrupt DUX4 expression greatly, despite the fact that this manipulation did not occur in the ORF of the DUX4 gene, nor a splice site for RNA spicing events. This data suggest that the E3 is important for stabilization of the pre-mRNA. This region can potentially function as a site for RNA binding proteins and thus stabilize pre-mRNA. It can also be important for the secondary or tertiary structure of the RNA, necessary for correct splicing events. Potentially it can be important for both, as structure of the RNA, and binding of RNA binding proteins are interconnected ${ }^{52,53}$. Thus, by modifying this region, this extra layer of RNA stability can be lost, reducing the probability of translation, but not completely abolishing it. 


\section{Methods}

\section{Cell culturing and seeding}

DUX4 inducible expression (DIE) cells were cultured in IMDM media with $10 \%$ Tet system approved FBS (Clonetech), $100 \mu \mathrm{M}$ 2-mercapto-ethanol, $5 \mu \mathrm{g} / \mathrm{ml}$ Puromycin and $6 \mu \mathrm{g} / \mathrm{ml}$ Blasticidin. Cells were kept at $5 \% \mathrm{CO}_{2}$ and $37^{\circ} \mathrm{C}$.

\section{Producing IV-RT guide}

For the production of single guide (sgRNA), a single stranded DNA template (supplied by IDT, California, USA) was amplified with Taq DNA polymerase by PCR. The template encodes the T7 promotor with an additional guanine at the end, a 20 nt variable spacer sequence, and the spCas9 tracr sequence with a polyT signal: 5'-TAATACGACTCACTATAGG20nt-GTTTTAGAGCTAGAAATAGCAAGTTAAAATAAGGCTAGTCCGTTATCAACTTGAAAAAGTG GCACCGAGTCGGTGCTTTTTT-3'. The following primer set was used to amplify the doublestranded DNA template and add small adapter sequences (underlined) for improved binding of DNA and RNA polymerases: 5'-ggcactcTAATACGACTCACTATAGG -3' and 5'-cggagcgAAAAAAGCACCGACTC-3'. The PCR product was purified using a PCR purification kit (Qiagen), and diluted to $250 \mathrm{ng} / \mu \mathrm{l}$. sgRNA was produced by reverse transcription (RT) using an IV-RT kit from NTRANS Technologies (Utrecht, The Netherlands), according to manufacturer instructions. Remaining DNA was removed by the addition of $2 \mathrm{U}$ of Turbo DNAse to each $20 \mu \mathrm{I}$ IV-RT reaction. Six IV-RT reactions were pooled and sgRNA was purified by phenol-chloroform extraction. The dried RNA pellet was dissolved in nuclease-free water and diluted to $10 \mu \mathrm{g} / \mu \mathrm{l}$. sgRNA samples were used immediately or stored at $-80^{\circ} \mathrm{C}$ for a maximum of 30 days.

\section{Producing spCas 9 and modified spCas 9}

Recombinant proteins were expressed in BL21 (DE3) E. coli and soluble fractions were extracted as previously described by D'Astolfo et al. ${ }^{30}$. A $20^{\circ} \mathrm{C}$ induction temperature was used for overnight induction. The soluble fraction containing the recombinant protein was degassed and filtered through a $22 \mu \mathrm{m}$ filter before loading it onto a HisTrap high performance column (GE Healthcare), using an AKTA Pure FPLC ${ }_{\mathrm{v} 2.0}$ system (GE Healthcare,). The soluble fraction was loaded at a speed of $0.6 \mathrm{ml} / \mathrm{min}$ for a $1 \mathrm{ml}$ column, or $3 \mathrm{ml} / \mathrm{min}$ for a $5 \mathrm{ml}$ column. Protein was eluted from the HisTrap column by using increasing amounts of imidazole, and fractions of each measured peak were collected. Correct proteins factions were confirmed by loading a sample of each fraction onto an SDS PAGE gel and staining with Coomassie blue. The fraction that contained protein of the right size was than purified and the buffer was exchanged (into $5 x$ transduction buffer ${ }^{30}$ ) using HiLoad Superdex 200 pg preparative SEC columns. Protein was concentrated to $75 \mu \mathrm{M}$ using an Amicon ultra centrifugal filter unit with a $100 \mathrm{kDA}$ cutoff (Merck). Protein aliquots were used immediately or snap frozen in liquid nitrogen and stored at $-80^{\circ} \mathrm{C}$.

\section{Transduction CRISPR/Cas9 into DIE cells}

96-well tissue culture plates were coated with a Matrigel (Matrigel in PBS, 1:250). Subsequently, 15,000 DIE cells were seeded on top of Matrigel-coated wells and incubated overnight at $5 \% \mathrm{CO}_{2}$ and $37^{\circ} \mathrm{C}$, until $70-80 \%$ confluency was reached. The cells were transduced with spCas 9 or $4 \times$ SV40-spCas9-2xSV40 protein and sgRNAs targeting the E3 
sequence using the iTOP transduction method ${ }^{30}$. After a recovery period of a minimum of $24 \mathrm{~h}$, cells were exposed to a high concentration of doxycycline $(1000 \mathrm{ng} / \mathrm{ml})$ for $16-48 \mathrm{~h}$, depending of the type of experiment. DIE cells (dead and alive) were collected and stained with Annexin-5 FITC and DAPI and subsequently analyzed by FACS.

\section{Flowcytometry sorting (FACS) and analysis and dead live staining}

DIE cells were treated with doxycycline for $16 \mathrm{~h}$ prior to FACS analysis. After doxycycline exposure, the culture media was collected, as was the DBPS wash that followed, to collect all dying and detached cells. The remaining cells were trypsinized using 0.25\% Trypsin-EDTA and resuspended in culture media. The trypsinized cells were added to the previously collected sample of detached cells and pelleted by centrifugation (500 g for $10 \mathrm{~min}$ ). The supernatant was removed and the cell pellet resuspended in DPBS with 5\% FBS, supplemented with annexin-V FITC. Cells were left to incubated for $15-20 \mathrm{~min}$ at $4{ }^{\circ} \mathrm{C}$ in a dark environment. iMDM media with $10 \%$ Tet approved FBS and DAPI nuclear staining was subsequently added and cells were strained using Cell-strainer capped tubes (Falcon) and analyzed using the BD FACSCanto II flow cytometer.

\section{Sample preparation and indel analysis}

Transduced DIE cells were harvested, pelleted and frozen at $-20^{\circ} \mathrm{C}$. Genomic DNA was extracted and purified using the DNeasy Blood \& Tissue kit (Qiagen). A 558 basepair fragment was amplified from genomic DNA samples using high fidelity Phusion polymerase (ThermoFisher), the Phusion GC $5 x$ buffer and the following primers flanking the E3 cleavage site: 5' - AAACGCGTCGTCCCCTG-3' and 5'- GCCAGAGGCCACTTGTGTAG-3'. A PCR program of 35 cycles consisted of denaturation at $98^{\circ} \mathrm{C}, 60$ seconds; annealing at $68^{\circ} \mathrm{C}, 20$ seconds; and elongation at $72{ }^{\circ} \mathrm{C}, 20$ seconds, before visualizing on a $1 \%$ TAE agarose gel. The amplified products were gel purified and send for Sanger sequencing (BaseClear). Indel frequency was determined using tracking of indels by decomposition analysis ${ }^{32}$. Data from three biological replicates were combined and average values (per sample) are displayed.

\section{Reference}

1. Choi, S. H. et al. DUX4 recruits p300/CBP through its C-terminus and induces global H3K27 acetylation changes. Nucleic Acids Res. 44, 5161-5173 (2016).

2. Vuoristo, S. et al. DUX4 regulates oocyte to embryo transition in human. Biorxiv (2019) doi:http://dx.doi.org/10.1101/732289.

3. Dixit, M. et al. DUX4, a candidate gene of facioscapulohumeral muscular dystrophy, encodes a transcriptional activator of PITX1. Proc. Natl. Acad. Sci. U. S. A. 104, 18157-18162 (2007).

4. Lemmers, R. J. L. F. et al. A unifying genetic model for facioscapulohumeral muscular dystrophy. Science (80-. ). 329, 1650-1653 (2010).

5. Snider, L. et al. Facioscapulohumeral dystrophy: Incomplete suppression of a retrotransposed gene. PLoS Genet. 6, 1-14 (2010).

6. Hewitt, J. E. et al. Analysis of the tandem repeat locus D4Z4 associated with facioscapulohumeral muscular dystropothhy. Hum. Mol. Genet. 3, 1287-1295 (1994).

7. Winokur, S. T. et al. The DNA rearrangement associated with facioscapulohumeral muscular dystrophy involves a heterochromatin-associated repetitive element: Implications for a role of chromatin structure in the pathogenesis of the disease. Chromosom. Res. 2, 225-234 (1994).

8. Deidda, G. et al. Physical mapping evidence for a duplicated region on chromosome 10qter showing high homology with the facioscapulohumeral muscular dystrophy locus on chromosome 4qter. European Journal of Human Genetics vol. 3 155-167 (1995). 
9. Van Der Maarel, S. M. \& Frants, R. R. The D4Z4 repeat-mediated pathogenesis of facioscapulohumeral muscular dystrophy. Am. J. Hum. Genet. 76, 375-386 (2005).

10. Lemmers, R. J. L. F. et al. Digenic inheritance of an SMCHD1 mutation and an FSHDpermissive D4Z4 allele causes facioscapulohumeral muscular dystrophy type 2. Nat. Genet. 44, 1370-1374 (2012).

11. Larsen, M. et al. Diagnostic approach for FSHD revisited: SMCHD1 mutations cause FSHD2 and act as modifiers of disease severity in FSHD1. Eur. J. Hum. Genet. 23, 808-816 (2015).

12. Van Den Boogaard, M. L. et al. Mutations in DNMT3B Modify Epigenetic Repression of the D4Z4 Repeat and the Penetrance of Facioscapulohumeral Dystrophy. Am. J. Hum. Genet. 98, 1020-1029 (2016).

13. Okano, M., Xie, S. \& Li, E. Cloning and characterization of a family of novel mammalian DNA ( cytosine-5) methyltransferases Non-invasive sexing of preimplantation stage mammalian embryos. Nat. Am. Inc. 19, 219-220 (1998).

14. Okano, M., Bell, D. W., Haber, D. A. \& Li, E. DNA methyltransferases Dnmt3a and Dnmt3b are essential for de novo methylation and mammalian development. Cell 99, 247-257 (1999).

15. Blewitt, M. E. et al. An N-ethyl-N-nitrosourea screen for genes involved in variegation in the mouse. Proc. Natl. Acad. Sci. U. S. A. 102, 7629-7634 (2005).

16. Blewitt, M. E. et al. SmcHD1, containing a structural-maintenance-of-chromosomes hinge domain, has a critical role in X inactivation. Nat. Genet. 40, 663-669 (2008).

17. Gendrel, A. V. et al. Smchd1-Dependent and-Independent Pathways Determine Developmental Dynamics of CpG Island Methylation on the Inactive X Chromosome. Dev. Cell 23, 265-279 (2012).

18. Greenberg, M. V. C. \& Bourc'his, D. The diverse roles of DNA methylation in mammalian development and disease. Nat. Rev. Mol. Cell Biol. 20, 590-607 (2019).

19. Lemmers, R. J. F. L. et al. Contractions of D4Z4 on 4qB subtelomeres do not cause facioscapulohumeral muscular dystrophy. Am. J. Hum. Genet. 75, 1124-1130 (2004).

20. Wang, Z. Q., Wang, N., Van Der Maarel, S., Murong, S. X. \& Wu, Z. Y. Distinguishing the 4qA and $4 \mathrm{qB}$ variants is essential for the diagnosis of facioscapulohumeral muscular dystrophy in the Chinese population. Eur. J. Hum. Genet. 19, 64-69 (2011).

21. Jinek, M. et al. A programmable dual-RNA-guided DNA endonuclease in adaptive bacterial immunity. Science (80-. ). 337, 816-821 (2012).

22. Cong, L. et al. Multiplex Genome Engineering Using CRISPR/Cas Systems. Science (80-. ). 339, 819-823 (2013).

23. Gasiunas, G., Barrangou, R., Horvath, P. \& Siksnys, V. Cas9-crRNA ribonucleoprotein complex mediates specific DNA cleavage for adaptive immunity in bacteria. Proc. Natl. Acad. Sci. U. S. A. 109, 2579-2586 (2012).

24. Ansseau, E. et al. Antisense oligonucleotides used to target the DUX4 mRNA as therapeutic approaches in faciosscapulohumeral muscular dystrophy (FSHD). Genes (Basel). 8, (2017).

25. Himeda, C. L., Jones, T. I. \& Jones, P. L. CRISPR/dCas9-mediated transcriptional inhibition ameliorates the epigenetic dysregulation at D4Z4 and represses DUX4-fl in FSH muscular dystrophy. Mol. Ther. 24, 527-535 (2016).

26. Lemmers, R. J. et al. Deep characterization of a common D4Z4 variant identifies biallelic DUX4 expression as a modifier for disease penetrance in FSHD2. Eur. J. Hum. Genet. 26, 94-106 (2018).

27. Wong, N., Liu, W. \& Wang, X. WU-CRISPR: Characteristics of functional guide RNAs for the CRISPR/Cas9 system. Genome Biol. 16, 1-8 (2015).

28. Hiranniramol, K. et al. Generalizable sgRNA design for improved CRISPR/Cas9 editing efficiency. Bioinformatics 36, 2684-2689 (2020).

29. Hodgkins, A. et al. WGE: A CRISPR database for genome engineering. Bioinformatics 31, 3078-3080 (2015).

30. D'Astolfo, D. S. et al. Efficient intracellular delivery of native proteins. Cell $161,674-690$ (2015).

31. Staahl, B. T. et al. Efficient genome editing in the mouse brain by local delivery of engineered Cas9 ribonucleoprotein complexes. Nat. Biotechnol. 35, 431-434 (2017).

32. Brinkman, E. K., Chen, T., Amendola, M. \& Van Steensel, B. Easy quantitative assessment of genome editing by sequence trace decomposition. Nucleic Acids Res. 42, (2014).

33. van Overbeek, M. et al. DNA Repair Profiling Reveals Nonrandom Outcomes at Cas9- 
Mediated Breaks. Mol. Cell 63, 633-646 (2016).

34. Lemos, B. R. et al. CRISPR/Cas9 cleavages in budding yeast reveal templated insertions and strand-specific insertion/deletion profiles. Proc. Natl. Acad. Sci. U. S. A. 115, E2010-E2047 (2018).

35. Shou, J., Li, J., Liu, Y. \& Wu, Q. Precise and Predictable CRISPR Chromosomal Rearrangements Reveal Principles of Cas9-Mediated Nucleotide Insertion. Mol. Cell 71, 498509.e4 (2018).

36. Paz, I., Kosti, I., Ares, M., Cline, M. \& Mandel-Gutfreund, Y. RBPmap: A web server for mapping binding sites of RNA-binding proteins. Nucleic Acids Res. 42, 361-367 (2014).

37. Sapra, A. K. et al. SR Protein Family Members Display Diverse Activities in the Formation of Nascent and Mature mRNPs In Vivo. Mol. Cell 34, 179-190 (2009).

38. Xia, H. et al. CELF1 preferentially binds to exon-intron boundary and regulates alternative splicing in HeLa cells. BBA - Gene Regul. Mech. 1860, 911-921 (2017).

39. Tripathi, V. et al. Direct Regulation of Alternative Splicing by SMAD3 through PCBP1 Is Essential to the Tumor-Promoting Role of TGF- $\beta$. Mol. Cell 64, 549-564 (2016).

40. Ho, T. H. et al. Muscleblind proteins regulate alternative splicing. EMBO J. 23, 3103-3112 (2004).

41. Gaudet, P., Livstone, M. S., Lewis, S. E. \& Thomas, P. D. Phylogenetic-based propagation of functional annotations within the Gene Ontology consortium. Brief. Bioinform. 12, 449-462 (2011).

42. Jones, T. I. et al. Facioscapulohumeral muscular dystrophy family studies of DUX4 expression: Evidence for disease modifiers and a quantitative model of pathogenesis. Hum. Mol. Genet. 21, 4419-4430 (2012).

43. Trcek, T., Larson, D. R., Moldón, A., Query, C. C. \& Singer, R. H. Single-molecule mRNA decay measurements reveal promoter-regulated mRNA stability in yeast. Cell 147, 1484-1497 (2011).

44. Penelova, A., Richman, L., Neupert, B., Simanis, V. \& Kühn, L. C. Analysis of the contribution of changes in mRNA stability to the changes in steady-state levels of cyclin mRNA in the mammalian cell cycle. FEBS J. 272, 5217-5229 (2005).

45. Choudhury, A. D., Xu, H. \& Baer, R. Ubiquitination and proteasomal degradation of the BRCA1 tumor suppressor is regulated during cell cycle progression. J. Biol. Chem. 279, 33909-33918 (2004).

46. Saunus, J. M., Edwards, S. L., French, J. D., Smart, C. E. \& Brown, M. A. Regulation of BRCA1 messenger RNA stability in human epithelial cell lines and during cell cycle progression. FEBS Lett. 581, 3435-3442 (2007).

47. Fan, J. et al. Global analysis of stress-regulated mRNA turnover by using cDNA arrays. Proc. Natl. Acad. Sci. U. S. A. 99, 10611-10616 (2002).

48. Lü, X., De La Peña, L., Barker, C., Camphausen, K. \& Tofilon, P. J. Radiation-induced changes in gene expression involve recruitment of existing messenger RNAs to and away from polysomes. Cancer Res. 66, 1052-1061 (2006).

49. Shen, M. W. et al. Predictable and precise template-free CRISPR editing of pathogenic variants. Nature 563, 646-651 (2018).

50. Allen, F. et al. Predicting the mutations generated by repair of Cas9-induced double-strand breaks. Nat. Biotechnol. 37, 64-82 (2019).

51. Chen, W. et al. Massively parallel profiling and predictive modeling of the outcomes of CRISPR/Cas9-mediated double-strand break repair. Nucleic Acids Res. 47, 7989-8003 (2019).

52. Soemedia, R. et al. The Effects of Structure on pre-mRNA Processing and Stability. Methods 125, 36-44 (2017).

53. Boo, S. H. \& Kim, Y. K. The emerging role of RNA modifications in the regulation of mRNA stability. Exp. Mol. Med. 52, 400-408 (2020). 


\section{Supplementary material}

A

Double targeting using gRNA 1

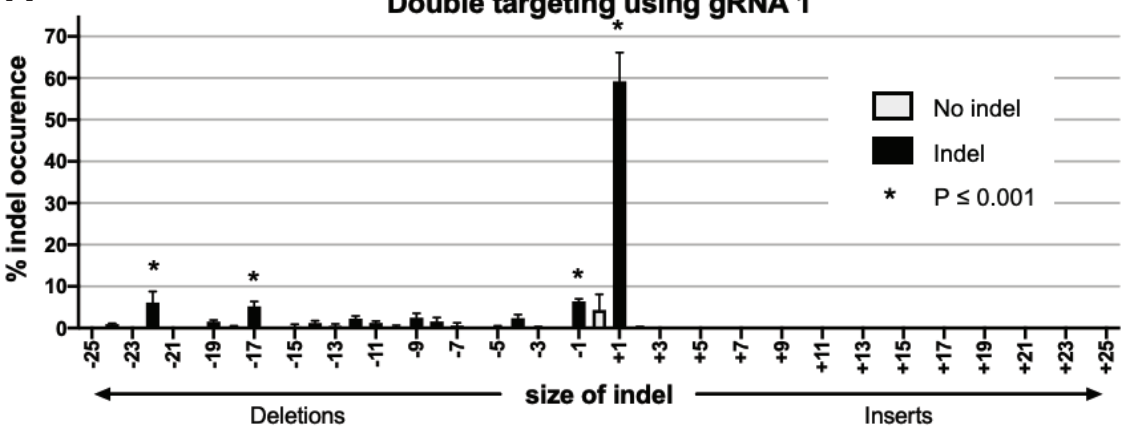

B

Double targeting using gRNA 2

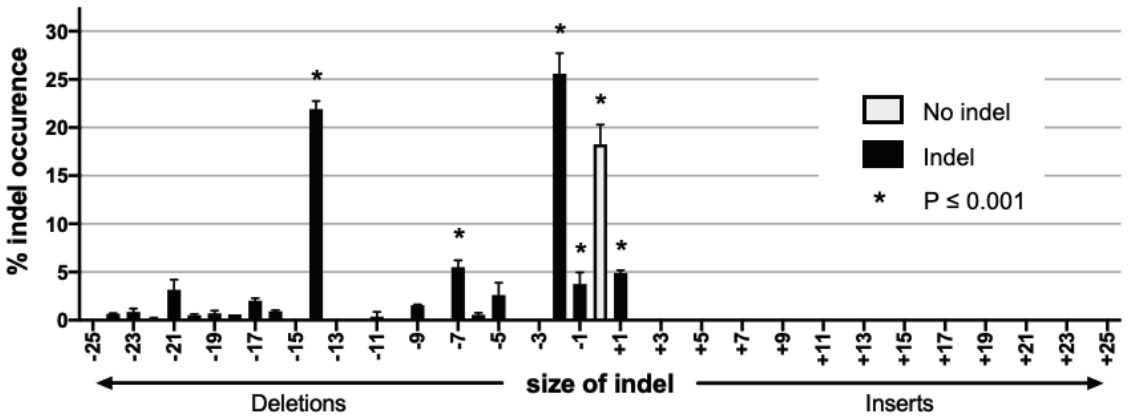

Figure S1. Indel frequency of double-targeted DIE cells not exposed to doxycycline. (A) Double targeting of the E3 region with gRNA1. The wildtype sequence can be found at a frequency of $4.3 \% \pm 3.8 \%(p$-value $=0.007)$. A one nucleotide insertion has a frequency of $59.2 \% \pm 6.9 \%(p$-value $=0)$. Three deletions of one, seventeen and twentytwo nucleotides are significantly present at a frequency of $6.4 \% \pm 0.6 \%$ ( $p$-value $=5.7 E-17), 5.2 \% \pm 1.2 \%$ ( $p$-value $=5.7 \mathrm{E}-06)$, and $6.1 \% \pm 2.7 \%$ ( $\mathrm{p}$-value $=4.3 \mathrm{E}-11$ ), respectively. $(\mathrm{B})$ Double targeting of the E3 region with gRNA2. The wildtype sequence is present at a frequency of $18.3 \% \pm 2.1 \%$ ( $p$-value $=9 \mathrm{E}-09$ ). The one nucleotide insertion is present at a frequency of $4.9 \% \pm 0.3 \%$ ( $p$-value $=3.6 \mathrm{E}-08$ ). Deletions of $1,3,7$ and 14 nucleotides were significantly present in the DIE cells at frequencies of $3.8 \% \pm 1.2 \%$ ( $p$-value $=4.6 \mathrm{E}-05), 25.6 \pm 2.1 \%(p$-value $=2.9 \mathrm{E}-212), 5.5 \% \pm$ $0.7 \%$ (p-value $3.4 \mathrm{E}-12$ ), and $21.9 \% \pm 0.8 \%$ (p-value $=6.5 \mathrm{E}-134)$, respectively. 


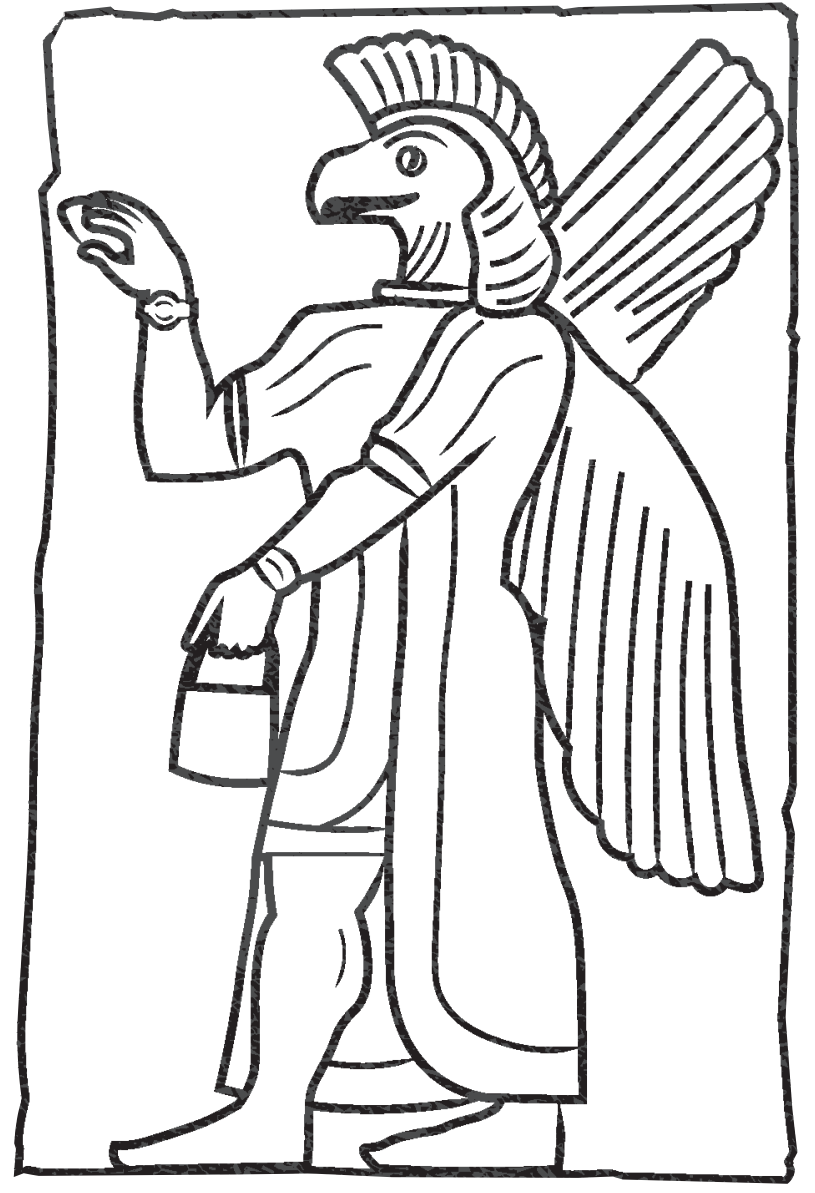

Illustration based on a stone carving on display at the MET 


\section{Chapter 6 \\ General discussion}


It has been over a 136 years since Facioscapulohumeral muscular dystrophy (FSHD) was first described ${ }^{1}$ and still we continue to search for treatment options for this disorder. As we unravel its underlying mechanisms, we move closer to solving more pieces of this very large and complex puzzle. For the past few decades, researchers have narrowed their focus to the transcription factor DUX4, as its misexpression lies at the center of the pathophysiology seen in FSHD patients ${ }^{2-4}$. DUX4 is part of a repeat sequence which can be found at multiple loci in the human genome. This complicates the more obvious and widely used targeting strategy, CRISPR/Cas9-mediated knock out. CRISPR/Cas9 is a genome editing technique, derived from the prokaryotic adaptive immune response ${ }^{5-8}$. CRISPR/Cas9 uses a guide RNA to navigate the Cas9 endonuclease to a specific site in the host its DNA, where it induces a double stranded break. Upon repair, errors can be introduced at this cleavage site, which can render a gene non-functional ${ }^{9,10}$. This new genome editing strategy has revolutionized the genome editing field, and has quickly become the most widely used strategy to knockout genes. As DUX4 is part of a repeated sequence, gRNAs that target the body of the DUX4 gene will cause multiple double stranded breaks throughout the human genome, risking further contraction and or translocation events. Some have therefore focused their efforts on modulating the expression or activity of DUX4-linked genes. These include genes that play a role in the expression of DUX4 itself such as p38 MAPK ${ }^{11,12}$, epigenetic regulators ${ }^{13}$, and potential transcription factors ${ }^{14,15}$; and genes and pathways that are regulated by DUX4 and contribute to its cytotoxic effect such as the MYC-mediated apoptotic pathway, the dsRNA innate immune response pathways ${ }^{16}$, and genes involved in the hypoxia-related HIF1 pathway ${ }^{17,18}$. However, other new promising avenues for future therapeutic intervention do aim to target DUX4 expression directly, without introducing any DNA double stranded breaks. For example, the strategy of using a nuclease-dead Cas9 fused to a Krüppel-associated box (dCas9-KRAB) inhibits DUX4 transcription ${ }^{19}$, or the anti-sense oligonucleotides strategy that inhibits translation ${ }^{20-22}$. Direct intervention at the source will naturally be an efficient and promising strategy that may save both the cells and their biological function, but these approaches will likely be transient unless a permanent approach is used, such as a gene therapy. Inhibiting epigenetic regulators, such as chromatin remodelers, epigenetic readers, methyltransferases that add activation marks, and demethylases that remove repressive marks, may also reduce expression of $\mathrm{DUX} 4^{13}$, and can therefore also be explored as a potential therapeutic treatment for FSHD.

Recently, an increase in hyaluronic acid (HA) has been associated with FHSD. Accumulation of HA occurs after DUX4 expression and the inhibition of HA biosynthesis prevented FSHD-related pathologies, such as RNA granule formation, FUS (fused in sarcoma) protein aggregation, DNA damage, caspase activation, and apoptosis. The exact role of HA in FSHD pathology is unclear, but the involvement of Complement component $1 \mathrm{Q}$ subcomponentbinding protein (C1QBP) and mitochondria is considered ${ }^{23}$. Interestingly, HA inhibition has limited effect on DUX4 expression and a partial effect on the DUX4 induced transcriptional program. This could suggest that HA works relatively independent of the DUX4-induced transcriptional program, or at least it effects only a part and specific aspect of this program. Our data demonstrates that DUX4 activates the expression of a network of downstream transcriptional regulators, which seem to reprogram cells into a more stem-cell like state before pushing the cells into apoptosis (Chapters $\mathbf{2}$ and 3). While HA inhibition can rescue cells from DUX4-induced apoptosis, the continued dysregulation of the transcriptional program may still trigger a loss of cell identity, which may prove similarly detrimental to the 
muscle fiber and or its function.

Currently, a single drug losmapimod, a p38/MAPK inhibitor, has entered phase 2 clinical trials for the treatment of FHSD. The p38 pathway has been identified as an activator of DUX4 expression, and inhibition of the p38/MAPK pathway interfered with DUX4 expression itself and prevented DUX4-induced cell loss ${ }^{11,12}$. The exact molecular mechanism involved in the regulation of DUX4 by p38 is as of yet unknown, but p38/MAPK inhibitors have been shown to lower DUX4 and DUX4 target gene expression, both in vitro in FSHD patientderived myoblasts ${ }^{11,12}$ as well as in vivo in a humanized mouse xenograft model ${ }^{11}$.

Although all these advancements in the treatment of FSHD are very promising, it should be noted that systemic administration of drugs targeting multipurpose factors such as kinases, transcription factors and epigenetic regulators can result in undesirable side effects. These side effects can outweigh the potential benefit of the FHSD treatment, rendering it unsuitable. The unraveling of the underlying FHSD pathophysiology and the search for specific FSHD treatment options should therefore continue until safe and perhaps universal treatment options have been developed.

This thesis describes several ways that we explored the possibilities of mitigating the DUX4 cytotoxic effect. To achieve this goal, we built a human in-vitro cell model system in which we could induce and regulate the expression of DUX4, and which demonstrated a clear apoptotic phenotype upon induction. This model furthermore contains the endogenous DUX4 coding sequence, so specific targeting strategies could also be tested. Together with the cell line's highly proliferative nature, simple maintenance requirements, high transfectability/transduceability, and its robust induction phenotype made this cell model system versatile and multipurpose. The DUX4 inducible expression (DIE) model validated the pioneer qualities of DUX4, after performing RNA sequencing to explore the transcriptional events that follow DUX4 induction. With the DIE cell line, we aimed to uncover players in the DUX4-induced signaling cascade. DUX4 induction in our DIE cell model shows a high degree of similarity with its transcriptome to other FSHD models and FSHD-affected muscle cells ${ }^{24-27}$ (Chapter 2 ). The DIE cell system was therefore used to find genes involved in the DUX4-induced pathways that mediate the toxicity of DUX4. Initial bulk sequencing demonstrated that many of the early differentially upregulated genes found after only $4.5 \mathrm{~h}$ hours of doxycycline induction are germline or stemness genes, or genes related to early embryonic development. These results confirm the developmental role of DUX4 $4^{28-30}$, which have also been found by others that have studied the DUX4-induced transcriptome ${ }^{24-27}$. One assumption was that the induction of an early developmental stage in mature somatic cells would create contradictory signals within the cells, that might cause the cell to enter apoptosis. As these genes were differentially expressed after only 4.5 hours of DUX4 induction, we hypothesized that these genes are targets of DUX4 that are activated very early on in the toxic process. Logic dictates that intervening early in this toxic cascade would show a greater impact on reducing the toxic effects, rather than intervening later in the process when this cascade has already triggered the activation of many downstream pathways. The contribution of these early DUX4 targets to the cytotoxic cascade was therefore tested by individual knock out experiments. These yielded no viable hits, as none of the tested genes rescued or even slowed down the apoptotic phenotype upon their elimination and DUX4 induction. 
We thus continued our search of finding major players in FSHD. To explore the dynamics of DUX4-induced cytotoxicity in more detail, we performed single cell RNA sequencing (SCS) on DIE cells induced for short and multiple consecutive time periods of 2, 3, 4 and 6 hours (Chapter 3 ). Performing dimensionality reduction on the single cell data revealed one large cell population, in which the cells orientated themselves on the $y$-axis of a t-SNE map, based on their induction status. The lack of well-defined clusters suggests that the induced transcriptomes in these cells are very similar, and that DUX4 activates the same program in most, if not all, induced cells. Differential expression analysis between the induced clusters and the uninduced clusters reveals lists of differentially expressed genes, many of which are shared between induction states. This indeed corroborating the notion that DUX4 activates the same cascade of events in most cells. This cascade of events starts with the activation of early developmental processes, quickly followed by a large variety of other cellular processes, and eventually leading to the activation of apoptotic processes. Significant changes in the cell's transcriptome can be seen as early as $2 \mathrm{~h}$ post DUX4 induction, with most of the genes (94\%) remaining differentially expressed at later timepoints. Approximately $33 \%$ of the differentially upregulated genes were transcription factors, some of which left an obvious signature expression profile. The 'footprint' expression profiles of transcription factors that were themselves not identified in the single-cell sequencing data were also identified. This suggests the involvement of "elusive" transcription factors, comparable with DUX4 itself, who's expression was to low and/or transient following DUX4 induction to be detected with SCS. These elusive transcription factors could potentially be of importance in the DUX4induced cytotoxic cascade and could therefore be of interest to be studied more in depth in the context of FSHD. SCS analysis furthermore revealed a number of different expression profiles, some demonstrating an oscillating pattern during the course of induction, suggesting that DUX4 activates a complex and dynamic process. Further investigation into this dynamic process could potentially give more insight into the molecular workings of DUX4-induced cytotoxicity and apoptosis.

With both RNA sequencing experiments (bulk and SCS), it was extraordinary to see such robust and reproducible transcriptional changes in cells that had been induced for a relative short period of time (2-6 hours). This is, to the best of our knowledge, the earliest timepoints in which changes in the transcriptome of DUX4 affected cells were studies, at such a high resolution. This revealed a list of potential early target genes of DUX4 that hadn't been identified previous, or had been but were not necessarily classified as early target genes.

Next, we used our DIE cell system to try and identify modulators of DUX4 cytotoxicity by performing a genome-wide CRISPR/Cas9 knockout screen. The goal was to identify factors that could mitigate DUX4-induced toxicity. We were able to screen for such modulators based on their ability to rescue the apoptotic phenotype upon their knockout (Chapter 4). If any of the differentially upregulated genes induced by DUX4 are indeed playing a role in FSHD pathophysiology, or any other genes play an active role in the DUX4 induced cytotoxicity, we would expect to find them back in the genome-wide screen data. However, we did not find such modulators in this particular screen. This suggests that no single gene, other than DUX4 itself, when knocked out, can rescue DUX4 cytotoxicity.

A more direct and permanent approach of reducing DUX4 expression is to knock out the DUX4 gene directly, but since DUX4 is part of a repeated sequence, this approach is challenging. We set up a DUX4 knock out strategy that targets a relatively unique intronic sequence directly adjacent to exon three $(E 3)^{31}$ (Chapter 5 ). We hypothesized that targeting 
this E3 sequence could lead to the disruption of a regulatory region needed for pre-mRNA stabilization or processing, thereby destabilizing the DUX4 mRNA transcript. Indeed, with the use of optimized CRISPR/Cas9 tools, a functional knockout efficiency of $\sim 50 \%$ was reached. However, due to the multinucleated nature of muscle fibers, and the stochastic burst-like expression of DUX4 in myonuclei, a 50\% efficiency in functional depletion of DUX4 expression is likely not enough to show a significant effect on relieving FSHD symptoms. The study did show that the editing efficiency is likely much higher than $50 \%$, as the wildtype sequence falls below $20 \%$ upon a second targeting experiment. This would suggest that not all edits at this site resulted in an efficient knockout of the gene. If so, a targeting strategy that does not rely on indel occurrence in an intron region might be more beneficial and could therefore result in a higher knockout efficiency.

New evolutions of the CRISPR/Cas9 gene editing system offer hope of a genome editing therapy that is both safe and effective. Himeda at all. has demonstrated that targeting a dCas9-KRAB epigenetic silencer to the DUX4 repeat sequence can effectively inhibit DUX4 expression without the danger of introducing multiple double-strand breaks ${ }^{19}$. However, as this approach relies on the temporary binding of the dCas9-KRAB to the regulatory region of DUX4, and not on permanently altering the coding sequence, this inhibition of DUX4 will be of a transient nature.

The recent development of CRISPR/Cas9-based base editors ${ }^{32,33}$ and the prime editing system ${ }^{34}$ does allow the introduction of subtle changes to the DUX4 coding sequence, which can introduce non-sense mutations, thus disrupting the translational reading frame. Baseediting technologies employ a Cas9 nickase (nCas9) that is fused to nucleobase deaminase enzyme $^{32,33}$. These base-editing fusion proteins can facilitate the conversion of a $C \bullet G$ to $T \bullet A$ bases or $T \bullet A$ to $G \bullet C$ bases, depending on the deaminase enzyme. Since this technology does not introduce double-strand breaks, it can be targeted toward the DUX4 coding region, introducing a nonsense mutation. This would disrupt the DUX4 translational reading frame by introducing a premature translational stop (Fig. 1), resulting in a severely truncated protein that would lack both its homeodomains and its functional domain. This truncated protein will most likely render DUX4 non-functional. Furthermore, as base editing uses a nCas9, it does not introduce double stranded breaks like Cas9 does, and the risk of further contracting the D4Z4 repeat array, or cause translocation events is therefore limited.

In conclusion, we have found that DUX4 homogeneously induces a network of transcription factors that quickly triggers a cascade of transcriptional events, which are ultimately detrimental for cells.

Knocking out individual downstream target genes of DUX4 or even performing a genomewide knockout screen did not identify individual factors that can mitigate DUX4 cytotoxicity, suggesting that either a multifactorial approach is needed or, more likely, that the only way to effectively eliminate DUX4 cytotoxicity is by eliminating DUX4 activity itself. After thoroughly studying the effects of pioneer transcription factor DUX4 in human cells, and concluding that no other individual factors plays a large enough role in the DUX4 induced cytotoxic cascade, we have come to the conclusion that the best way forward in finding treatments options for FSHD lies in targeting DUX4 directly. Our efforts of doing so by targeting the E3 sequence in the DUX4 3'UTR has shown that specific targeting of DUX4 with CRISPR/Cas9 is possible. However, due to the multinucleated nature of skeletal muscle fibers, targeting DUX4 would require a near $100 \%$ knockout efficiency to be clinically relevant, which is with 
current technologies unfeasible. However, with the fast-evolving genome editing field, new strategies to target the DUX4 gene in an efficient and safe manner may come up, strategies like the base-editing strategy mentioned above, which will be a way forward to find better treatment options for FSHD.

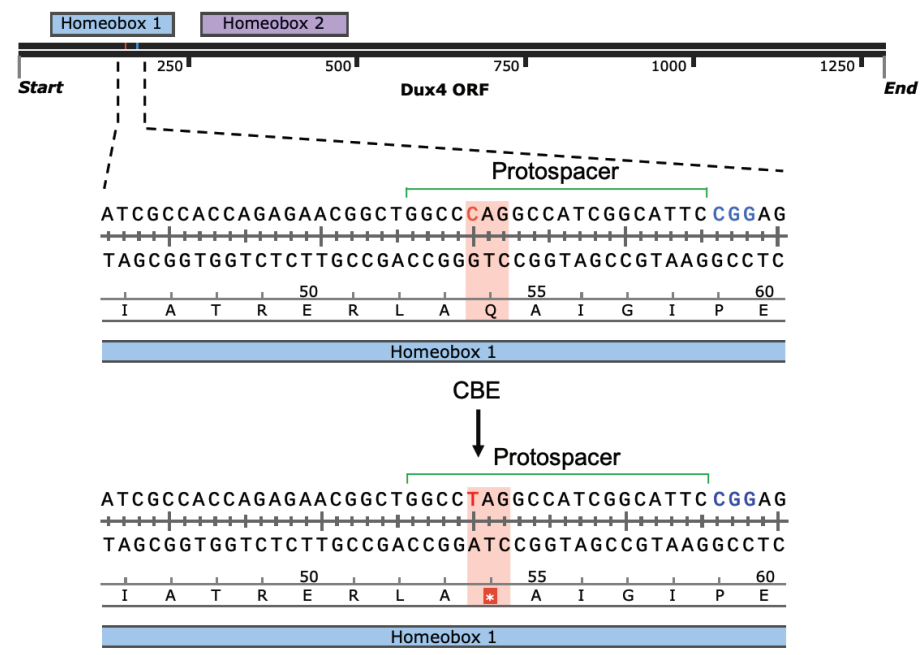

Figure 1. Base editing approach in knocking out DUX4. A schematic representation of a genome editing approach using the a Cas9 nickase fused to a nucleobase deaminase enzyme. The targeted region falls within the first homeobox of the DUX4 open reading frame, 155 nucleotides from the start codon. The PAM sequence is annotated in blue. The nucleotide that is mutated (cytosine to a thymine) is annotated in red, and the amino acid that changes from a glutamine $(\mathrm{Q})$ to a stop codon $\left({ }^{*}\right)$ is highlighted in red.

\section{References}

1. Landouzy, L. \& Dejerine, J. De la myopathie atrophique progressive (Myopathie héréditaire débutant, dans l'enfance, par la face, sans altération du système nerveux). C R Acad Sci 98, 53-55 (1884).

2. Kowaljow, V. et al. The DUX4 gene at the FSHD1A locus encodes a pro-apoptotic protein. Neuromuscul. Disord. 17, 611-623 (2007).

3. Dixit, M. et al. DUX4, a candidate gene of facioscapulohumeral muscular dystrophy, encodes a transcriptional activator of PITX1. Proc. Natl. Acad. Sci. U. S. A. 104, 18157-18162 (2007).

4. Snider, L. et al. RNA transcripts, miRNA-sized fragments and proteins produced from D4Z4 units: New candidates for the pathophysiology of facioscapulohumeral dystrophy. Hum. Mol. Genet. 18, 2414-2430 (2009).

5. Barrangou, R. et al. CRISPR provides acquired resistance against viruses in prokaryotes. Sciencee 315, 1709-1712 (2007).

6. Hale, C. R. et al. RNA-Guided RNA Cleavage by a CRISPR RNA-Cas Protein Complex. Cell 139, 945-956 (2009).

7. Deltcheva, E. et al. CRISPR RNA maturation by trans-encoded small RNA and host factor RNase III. Nature 471, 602-607 (2011).

8. Jinek, M. et al. A programmable dual-RNA-guided DNA endonuclease in adaptive bacterial immunity. Science 337, 816-821 (2012).

9. Endo, M., Mikami, M. \& Toki, S. Multigene knockout utilizing off-target mutations of the CRISPR/cas9 system in rice. Plant Cell Physiol. 56, 41-47 (2015).

10. Hsu, P. D., Lander, E. S. \& Zhang, F. Development and applications of CRISPR-Cas9 for genome engineering. Cell 157, 1262-1278 (2014). 
11. Oliva, J. et al. Clinically advanced p38 inhibitors suppress DUX4 expression in cellular and animal models of facioscapulohumeral muscular dystrophys. J. Pharmacol. Exp. Ther. 370, 219-230 (2019).

12. Rojas, L. et al. P38 $\alpha$ Regulates Expression of DUX4 in Facioscapulohumeral Muscular Dystrophy. Biorxiv 1-19 (2019) doi:https://doi.org/10.1101/700195.

13. Himeda, C. L. et al. Identification of Epigenetic Regulators of DUX4-fl for Targeted Therapy of Facioscapulohumeral Muscular Dystrophy. Mol. Ther. 26, 1797-1807 (2018).

14. Himeda, C. L. et al. Myogenic Enhancers Regulate Expression of the Facioscapulohumeral Muscular Dystrophy-Associated DUX4 Gene. Mol. Cell. Biol. 34, 1942-1955 (2014).

15. Campbell, A. E. et al. BET bromodomain inhibitors and agonists of the beta-2 adrenergic receptor identified in screens for compounds that inhibit DUX4 expression in FSHD muscle cells. Skelet. Muscle 7, 1-18 (2017).

16. Shadle, S. C. et al. DUX4-induced dsRNA and MYC mRNA stabilization activate apoptotic pathways in human cell models of facioscapulohumeral dystrophy. PLoS Genet. 13, 1-25 (2017).

17. Banerji, C. R. S. et al. $\beta$-catenin is central to DUX4-driven network rewiring in facioscapulohumeral muscular dystrophy. J. R. Soc. Interface 12, (2015).

18. Lek, A. et al. Applying genome-wide CRISPR-Cas9 screens for therapeutic discovery in facioscapulohumeral muscular dystrophy. Sci. Transl. Med. 12, 9-11 (2020).

19. Himeda, C. L., Jones, T. I. \& Jones, P. L. CRISPR/dCas9-mediated transcriptional inhibition ameliorates the epigenetic dysregulation at D4Z4 and represses DUX4-fl in FSH muscular dystrophy. Mol. Ther. 24, 527-535 (2016).

20. Vanderplanck, C. et al. The FSHD atrophic myotube phenotype is caused by DUX4 expression. PLoS One 6, e26820 (2011).

21. Marsollier, A. C. et al. Antisense targeting of $3^{\prime}$ end elements involved in DUX4 mRNA processing is an efficient therapeutic strategy for facioscapulohumeral dystrophy: A new gene-silencing approach. Hum. Mol. Genet. 25, 1468-1478 (2016).

22. Chen, J. C. J. et al. Morpholino-mediated Knockdown of DUX4 Toward Facioscapulohumeral Muscular Dystrophy Therapeutics. Mol. Ther. 24, 1405-1411 (2016).

23. DeSimone, A. M., Leszyk, J., Wagner, K. \& Emerson, C. P. Identification of the hyaluronic acid pathway as a therapeutic target for facioscapulohumeral muscular dystrophy. Sci. Adv. 5, (2019).

24. Geng, L. N. et al. DUX4 Activates Germline Genes, Retroelements, and Immune Mediators: Implications for Facioscapulohumeral Dystrophy. Dev. Cell 22, 38-51 (2012).

25. Rickard, A. M., Petek, L. M. \& Miller, D. G. Endogenous DUX4 expression in FSHD myotubes is sufficient to cause cell death and disrupts RNA splicing and cell migration pathways. Hum. Mol. Genet. 24, 5901-5914 (2015).

26. Jagannathan, S. et al. Model systems of DUX4 expression recapitulate the transcriptional profile of FSHD cells. Hum. Mol. Genet. 25, ddw271 (2016).

27. Van Den Heuvel, A. et al. Single-cell RNA sequencing in facioscapulohumeral muscular dystrophy disease etiology and development. Hum. Mol. Genet. 28, 1064-1075 (2019).

28. Whiddon, J. L., Langford, A. T., Wong, C. J., Zhong, J. W. \& Tapscott, S. J. Conservation and innovation in the DUX4-family gene network. Nat. Genet. 49, 935-940 (2017).

29. Hendrickson, P. G. et al. Conserved roles of mouse DUX and human DUX4 in activating cleavage-stage genes and MERVL/HERVL retrotransposons. Nat. Genet. 49, 925-934 (2017).

30. Vuoristo, S. et al. DUX4 regulates oocyte to embryo transition in human. Biorxiv (2019) doi:http://dx.doi.org/10.1101/732289.

31. Lemmers, R. J. et al. Deep characterization of a common D4Z4 variant identifies biallelic DUX4 expression as a modifier for disease penetrance in FSHD2. Eur. J. Hum. Genet. 26, 94-106 (2018).

32. Komor, A. C., Kim, Y. B., Packer, M. S., Zuris, J. A. \& Liu, D. R. Programmable editing of a target base in genomic DNA without double-stranded DNA cleavage. Nature 533, 420-424 (2016).

33. Gaudelli, N. M. et al. Programmable base editing of $\mathrm{T}$ to $\mathrm{G} C$ in genomic DNA without DNA cleavage. Nature 551, 464-471 (2017).

34. Anzalone, A. V. et al. Search-and-replace genome editing without double-strand breaks or donor DNA. Nature 576, 149-157 (2019). 


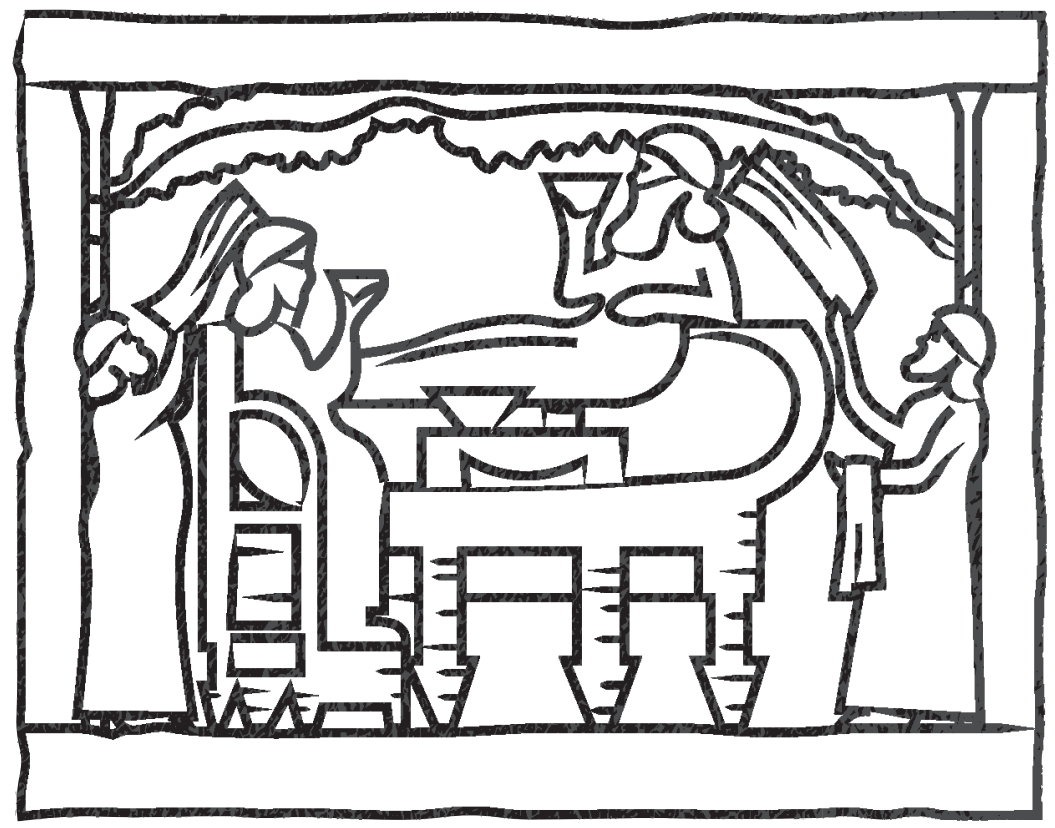

Illustration based on a stone carving on display at the British museum 


\section{Addendum}

Nederlandse samenvatting

Acknowledgements

Curriculum Vitae

List of publications 


\section{Nederlandse samenvatting}

Facioscapulohumerale spierdystrofie (FSHD) is één van de meest voorkomende spierziekte wereldwijd. Zoals vele andere spierziektes heeft FSHD een genetische component. De ziekte kan daarom worden geërfd, of kan zich ontwikkelen tijdens de embryonale ontwikkeling. De meeste patiënten ontwikkelen symptomen in de tweede decennia van hun leven. De ziekte openbaart zichzelf beginnend met het verzwakken van de spieren in het gelaat en de schoudergordel, wat zich vervolgens langzaam verspreid naar de boven armen, de romp en in sommige gevallen de onderbenen. FSHD wordt veroorzaakt door veel samenkomende factoren op een moleculair niveau, die vervolgens tot de ongepaste activatie van of Double homeobox 4 (DUX4) leidt. Een gen wat normaal gesproken streng wordt gecontroleerd, en alleen actief is in erg specifieke weefsels en cellen (4-cell embryo, de thymus en de testis). Het exacte moleculaire mechanisme van deze ziekte is erg complex en nog niet helemaal bekent of begrepen. Dit heeft de ontwikkeling van effectieve behandel methodes voor FSHD in weg gestaan. Momenteel worden FSHD-patiënten behandeld met ontstekingsremmers en bewegingsactiviteiten, die een erg beperkt effect hebben op het verloop van de ziekte. Hoofdstuk 1 geeft een gedetailleerd verslag van wat er bekend is over FSHD tot op heden, en hoe we hier zijn beland na 136 jaar research. Er is nog steeds onderzoek gaande naar het in kaart brengen van het moleculaire mechanisme van de ziekte, om zo de ziekte als geheel beter te kunnen begrijpen. Als dit kan worden gerealiseerd, kan er worden bepaald waar we moeten ingrijpen tijdens dit pathologische proces om zo effectievere behandelingsmethodes te ontwikkelen voor FSHD. In dit proefschrift wordt beschreven hoe wij hebben bijgedragen aan het veld, via verschillende routes. Om de ziekte beter te bestuderen hebben we een veelzijdig FSHD-cel model opgezet, waarbij het DUX4 gen naar eigen willen kan worden geactiveerd door het toevoegen van een component genaamd doxycycline. Hoofdstuk 2 legt het door ons ontwikkelde FSHD-cel model in meer detail uit, en beschrijft wat voor effect DUX4 activatie heeft op de cellen. Net als spiercellen gaan de cellen in ons FSHD model dood na de activatie van DUX4. Het effect van DUX4 op deze cellen werd ook bestudeerd op een moleculair niveau, met behulp van RNA-sequencing. Met deze techniek kunnen wij achterhalen welke gene worden beïnvloed door DUX4 activatie. Zo hebben wij vast kunnen stellen dat ons FSHD-model op moleculair niveau in veel opzichten lijkt op FSHD. Om de vroege effecten van DUX4 activatie te kunnen bestuderen is er RNA-sequencing uitgevoerd op afzonderlijke cellen waarin het DUX4 gen slechts enkele uren was geactiveerd (hoofdstuk 3). Als wij in staat zijn deze zeer vroege gebeurtenissen na DUX4 activatie in kaart te brengen, kan er geprobeerd worden in deze vroege stadia in te grijpen. Vroeg ingrijpen in het pathologisch proces zou de kans waarschijnlijk vergroten op het vertragen van het ziekte verloop. De data liet interessante veranderingen zien in de activiteit status van een specifieke set genen, bekend als transcriptiefactoren. Net als DUX4 kunnen deze transcriptiefactoren de moleculaire werking van de cellen beïnvloeden wanneer ze worden geactiveerd of onderdrukt. Sommige van deze transcriptiefactoren waren duidelijk detecteerbaar, terwijl andere alleen tekenen van hun aanwezigheid of afwezigheid vertoonden. Deze factoren zouden van groot belang kunnen zijn in het moleculaire mechanisme van FSHD, en ze zouden daarom interessant kunnen zijn voor verdere onderzoek.

Hoofdstuk 4 richt zich op het vinden van sleutelfiguren in het pathologische proces die DUX4 activeert, door het uitvoeren van een knock-out screen. Dit werd uitgevoerd met behulp van de veelgebruikte genoom bewerkings-techniek, bekend als CRIPSR/Cas9. Een knock- 
out betekent dat een gen volledig wordt gedeactiveerd. Met CRIPSR/Cas9 wordt het gen gedeactiveerd door op de plaats van het gen in het DNA te knippen. De cel zal proberen zijn DNA te repareren en daardoor fouten introduceren die kunnen leiden tot gen deactivatie. Een deel van de cellen zullen op deze manier een enkele knock-out van een gen bevatten. Door gebruik te maken van een groot aantal cellen, kunnen we er zeker van zijn dat er van elk gene in het humane genoom een klein groepje cellen zal bestaan waarvan dit gen is gedeactiveerd. Als het deactiveren van een bepaald gen het pathologische proces kan vertragen of stoppen, zouden deze cellen DUX4 activatie betere moeten overleven. Wanneer dit gebeurt, kunnen deze overlevende cellen geanalyseerd worden voor het type knock-out die zij bevatten. Zo kan er worden vastgesteld welk gen belangrijk is in pathologische proces. Zo'n gen werd niet gevonden in deze screen. Dit suggereert dat er na DUX4 activatie niet een ander gen op zichzelf een grote invloed heeft op het pathologische proces van FSHD. We kwamen daarom tot de conclusie dat de beste manier om FSHD af te remmen is om te onderzoeken hoe DUX4 efficiënt kan worden gedeactiveerd. Hoofdstuk 5 demonstreert een manier om DUX4 te deactiveren door gebruik te maken van CRISPR/Cas9. Bij het gebruik maken van CRISPR/Cas9 als een mogelijke therapeutische interventie, moet men altijd rekening houden met de risico's. Omdat CRISPR/Cas9 niet $100 \%$ accuraat is, bestaat er een kans dat er op andere plaatsen in het genoom geknipt wordt in het DNA. Dit kan ongewenste en onvoorspelbare bijwerkingen veroorzaken. Omdat er op veel plaatsen in het menselijk genoom kopieën van het DUX4 gen aanwezig zijn, is het een uitdaging om de juiste kopie te deactiveren zonder al te veel DNA-schade in te veroorzaken. De knock-out-methode in hoofdstuk 5 is opgezet om de kans te verkleinen dat andere kopieën van DUX4 worden aangetast, door zich te richten op een relatief unieke sequentie in de ziekteverwekkende kopie van DUX4. Met deze strategie waren we in staat DUX4 in ongeveer de helft van de cellen te deactiveren. Ten slotte zal hoofdstuk 6 een overzicht geven van alle onderzoekshoofdstukken en eventuele problemen bediscussiëren.

Alles bij elkaar heeft aangetoond hoe gecompliceerd FSHD is, en dat het vinden van een behandelmethode nog erg lastig zou kunnen zijn. Wat wel duidelijk naar voren is gekomen is dat DUX4 inderdaad een grote rol speelt in FSHD, en dat alleen de deactivatie van dit gen het ziekteverloop effectief kan vertragen of zelfs stoppen. Wij zijn daarom van mening dat er meer aandacht moet komen voor het vinden van behandelingsmethoden die zich richten op het verminderen of stoppen van DUX4 activatie. 


\section{Acknowledgements}

First and foremost, I would like thank Niels Geijsen for accepting me in his lab, and giving me to opportunity to work on such an interesting research field. Your almost eternal optimism and passion for science is truly inspiring and (thankfully) contagious. Your trust and support have helped raised my confidence and encouraged me to keep going forward, even in the tougher times, and I'm therefore incredibly grateful to have had you as my promotor.

I would like to thank Wouter de Laat for taking me in when Niels moved his lab to the LUMC to become head of Department of Anatomy and Embryology. You were kind enough to become my promotor so I could finish my work and remain at the Hubrecht institute, giving me the opportunity to finish my PhD the way I had envisioned. Thank you so very much for all the help.

Thank you to all the members from the Geijsen group that I had the pleasure of sharing a lab with, and who had to endure my occasional (or quite often) and somewhat-crazy rants about real or fictional scenarios. You really made my time in the Geijsen group unforgettable and so much fun. Starting with Nune. The person who I sat next to the first two years of my $\mathrm{PhD}$, and who has always been so supporting and went above and beyond to help out with anything at any time. I really miss our incomprehensive dialogs, that only we thought were tremendously funny (VUUR!). I was so lucky to have you there when I started, and have learned so much from you. Fanny, who I could always go to with any question, be it science related or not. You introduced me to the good wines, and have turned me a bit into a wine snob (no more $\mathrm{AH}$ wine for me!). You are one of the nicest people I have ever met, with an immense amount of patience and endurance. You have taught me so much during my time there, and I will be forever grateful. Peng, who has taught me almost everything I know about cloning. You have always been such a big help, and a general fun person to be around, saying peculiar things at unexpected times which always cracked me up. The PhD bromance trio that was there when I started, and that I luckily had to share an office with: Javier, Axel and PJ. I loved eavesdropping in your often times strange conversations, sometimes attempting to join in, and eventually succeeding without making an absolute fool myself (right?). Thank you guys so much for being so welcoming, and making me laugh all the time. Sonja, the very first student I supervised (halfsies). I always admired you for wanting to eat healthy. Even bringing in plain boiled chicken and rice, pretending wholeheartedly you enjoyed it, while simultaneously gobbling down all my sweets (in stark contrast to the other person sitting next to me). Because we started in the Geijsen group at the same time, we were stuck with each other, and I (thankfully) haven't able to get rid of you since. Nicolas, not only an exceptional scientist but also highly skilled at foraging snacks. No food was safe when you were around. Snacks that were left unsupervised mysteriously went missing. But then again, so did the snacks that were supervised. You were so much fun to be around, and I will never 
forget one of our first interactions, which was trying to figure out if the oliebollen Nune left on her desk were there to torment us. We eventually justified eating them anyways, seeing that we both feel very strongly about not wasting food. Pascale Dijkers, whom without I wouldn't have ended up in the Geijsen group. Thank you for connecting me to Niels, and always helping out anyway you could, even before starting my PhD during my masters where you had supervised me during one of my internships. You also provided me with the much-needed brain food (loads of chocolate) during the final years of my PhD, when my main treats provider (Nune) had already left. Melissa, who I'm convinced woke up every morning thinking: which snack shall I take with me today that will make the most noise while eating (healthy veggie things). But all jokes aside, you are also the person who was always asking me if you could help out in any way. Which you have done in a major way, by not in the least going to Boston during winter, excellently performing a major experiment in my stead, all the while trying not to freeze your behind of. Thank you so much for all your help, and for tricking me into taking a scuba diving crash course. Lin, my gym buddy, kickboxing sparring partner, and fellow victim of Melissa's scuba diving crash course scheme. You were a great person to have around in the lab, because you weren't shy to give your upfront and unsalted opinion about anything. I genuinely appreciate all the advice and comments you have given me during my time as a $\mathrm{PhD}$, and will take them along in my following endeavors. Ouafa, for being the other strong Dutch women with an immigration background (as we say in PC Dutch) in the group. We come from such similar backgrounds, and I loved cracking jokes about all the similar things we had to go through during our upbringing. You were always so uplifting, trying to keep me positives when I was at my limit. Thank you so much for that. Jorik and Darnell for always entertaining my crazy end of the world, but mainly zombie apocalypse, scenarios. Clara, for being such a grounded and hardworking person, yet so much fun on our nights out. Ada, for being an absolutely lovely person, with whom I swapped many stories about culture, music and fun TV shows. A big thank you to Anna, for helping analyze most of my large data sets, and always answering all my questions, despite being incredibly busy yourself. Although you officially started in the Geijsen group at the end of my PhD, you had already been helping me out with things long before that. I am so thankful that you made time for me in such a busy period in your career, where you had so many different things going on, and even taken the time to actually explains the analysis to me, step by step. Thanks to all the other members in the Geijsen group and the Ntrans team, Stefan, Zeliha, Chen, Manda, Ive, Julie, Marco, Ruud, Marieke, Kim, Anna, Kelly, Jan, and all the students that have come and gone in the time that I was there, who all contributed in making my time there so happy.

I have to thank everybody who made the Hubrecht institute such great environment to work at. I can truly say that I have never before experienced such a sociable institute, performing at such a high level. My time working at the Hubrecht was absolutely wonderful, meeting so many amazing people. Ilia, my cell culture companion. I loved our complaining session in the cell culture lab, complaining about all things in live and PhD. You were a great person 
to have around on eventful, or even less eventful days. Other members of the Creyghton group, Caroline, Bas, and Mirna, for our fun and informative chats at the coffee machine, and for answering many of my questions about coding, with a special thanks to Mirna who kindly helped me analyze my first large dataset. Thank you for your patience and all the help. My PV buddies! Jimmy, Ajit, Stijn, Brian, Laura, and Lotte. My time in the PV was a blast, and it had everything to do with all the people in it. We got along amazingly well, and meetings were never boring. My TGIF drinking buddies, Jimmy and Arjan. Jimmy, I consider you one of the first friends I made at the Hubrecht institute. Together with Arjan we spend many Friday afternoons and evenings drinking beers, chatting and joking away. Playing rocket league together on some of those famous Hubrecht TGIFs are some of my fondest memories of my time at the Hubrecht. Thank you guys for being there for me at the end of many long, and though weeks, and always managing to cheer me up when needed.

My PMC writing buddy Evelien, for keeping me motivated during those first few weeks of writing. Our super-efficient writing sessions were unfortunately cut short due to the pandemic, but kickstarted the writing part of my PhD in such a nice way

I would like to thank all the people that have helped me out to get me here and all the people I have collaborated with in and outside of the Hubrecht. All the co-authors that helped putting this work together, Menno Creyghton, Kevin Eggan, Francesco Limone, Federica Piccioni, Judith Vivé, Silvere van der Maarel, Richard Lemmers, Anna Alemany, Mirna Baak, Fanny Sage, and Melissa van Kranenburg. Thanks to Richard Sherwood for advising me about performing and setting up genome wide screens. Frank van Steenbeek for being part of my PhD committee, these past 5 years. All my thesis committee members, Roos Masereeuw, Eva van Rooij, Joost Sluiter, Marie-José Goumans, Bart Spee, and Richard Lemmers, for taking the time to go through my thesis, and for being there on the day of my defense.

A special thank you to my game night buddies! Sonja, Javi, Axel, for all the fun times we had outside of the lab. You guys were and are an essential part of my almost non-existing social life. I am so happy we have kept up with these awesome get togethers, albeit many of them having to be virtual due to the pandemic. I'm always looking forward to seeing all of you again, together with Rel, Eldebar, Fonkin and Nin.

Thank you Massimiliano and Enrico for giving me the oppertunity to start a new position in your department and group, while finishing up my $\mathrm{PhD}$, and to all the people of the pharmaceutics department of the David de Wied buidling for making me feel so welcome and helping me get started in the new lab.

To all the uncles, aunts and cousins that are spread around the world. Thank you for the love and support, and taking care of me during my studies/rotations abroad. 
Mijn twee goeie vriendinnen en paranimfen, Sevgim Ugurlu en Chiwan Chiang. Wie had gedacht dat een vriendschap die 14 jaar geleden zo terughoudend is begonnen, nu zo sterk zou zijn? En hoewel we allemaal een ongelooflijk druk leven leidden nadat we de universiteit hadden verlaten, en elkaar niet zo vaak zagen als we gewend waren, slaagden we er altijd in om tijd voor elkaar te maken wanneer het er echt toe deed. Ik vind het geweldig dat onze vriendschap onverminderd sterk is gebleven, ook al kunnen er maanden voorbijgaan zonder dat we elkaar hebben gesproken of gezien. Bedankt voor alle emotionele steun door de jaren heen en alle geweldige afleidingen tijdens mijn hectische PhD-leven.

Maar het meest dankbaar ben ik mijn geweldige familie. De mensen waarvan ik weet dat ze alles voor me over hebben, en ik voor hun. Ik had dit natuurlijk nooit gekund zonder jullie steun en liefde. Mijn zussen, Batul en Shamiran, de twee personen waarbij ik werkelijk alles kwijt kan. Mijn grote zussen die ook meteen mijn beste vriendinnen zijn, en die mij altijd hebben gesteund en hun trots voor mij hebben laten blijken. Sham, dank je wel dat je altijd je nonchalante zelf bent, altijd klaar om op je meest enthousiast "ik ben blij voor $j e^{\prime \prime}$ te zeggen. Betta, dank je wel dat je het 5 jaar lang met mij hebt uitgehouden hier in Utrecht, en natuurlijk voor het geweldige design van dit boekje. Mijn grote broer Daniel, die mij als kind zijnde altijd heeft bescherm, op de eerste paar maanden van mijn leven na waar hij aanvankelijk er alles aan deed om van mij af te komen. Ik aapte hem altijd na, en volgde hem waar hij ook heen ging, of hij dat wilde of niet. Dank je wel dat jij de meest evenwichtige persoon bent in de familie, die mij altijd kan geruststellen wanneer mijn hoofd weer op standje onrealistisch rampscenario staat. Mijn schoonzusje Marloes, en de drie schattigste super boefjes die er bestaan, mijn neefjes en nichtje, Jeshua, James en Amara. Ik kijk er altijd zo naar uit om weer thuis te komen om jullie allemaal te zien, met de kinderen te spelen, en me vol te proppen met het eten wat jullie hebben bereid (Marloes en mams, ook wel bekend als oma Bia). Het doet me altijd zo veel goed! En dan als laats mijn ouders, Rafael en Harbia. Geboren en opgegroeid als minderheden in een land dat altijd in oorlog was, hebben jullie besloten alles wat jullie kenden, alles wat jullie bezaten, en iedereen die jullie liefhadden achter te laten om jullie kinderen een beter leven te gunnen. Jullie zijn met helemaal niks, behalve jullie 4 kleine kinderen, naar een land gekomen waar jullie niemand kenden, niets hadden, en op dat moment nog niks van af wisten. Jullie hebben jezelf vervolgens kapot gewerkt om jullie gezin alle mogelijkheden te kunnen bieden die jullie als kind nooit hebben gehad. Door alles wat jullie hebben opgeofferd, ben ik nu zover gekomen. Met het volste vertrouwen hebben jullie mij vrijgelaten mijn eigen beslissingen te nemen, en me ook altijd gesteund in die beslissingen, met alleen de boodschap om voorzichtig te zijn en goed op mezelf te passen. Mijn dankbaarheid is immense, en niet in woorden uit te drukken, en ik zeg dit bij lange na niet genoeg: paps, mams, ik hou ontzettend veel van jullie. 


\section{Curriculum Vitae}

Ator Rafael Odisho Ashoti was born in 1989, on the $25^{\text {th }}$ of November in Dihok, Irak. With her parents and 3 siblings, she moved to the Netherlands in 1993 at the age of 31/2. She grew up in Assen, where she attended primary school de Driemaster, and high school Dr. Nassau college Quintus. She started her bachelor of applied science at the Hanze Univeristy in Groningen in 2007. In the last year of her bachelor's program, she obtained an Erasmus scholarship for an internship at the University of Oxford, with the Childhood Cancer Research group, in Oxford (UK). With the supervision of Dr. Kate O'Neill, she tried to uncover a potential link between contracting an Adenovirus or Epstein-Barr virus infection during development or infancy, and childhood leukemia. She graduated from her bachelor's program in 2011, and entered into a pre-master program, with the University of Groningen. She started the Master's program Biomedical Sciences at the University of Groningen in 2012, during which she completed two internships. The first was with the group of prof. Ody Sibon, in the department of Cell Biology, at the University Medical Center Groningen, and under the direct supervision of Dr. Pascale Dijkers. During this 6-month internship Ator studied the role of the enzyme Vanin in immunity and ageing of Drosophila melanogaster. Her second internship was with the Children's Medical Research Institute in Sydney Australia, within the group of prof. Patrick Tam in the department of Embryology, and under the direct supervision of Dr. David Loebel. Here Ator studied downstream targets of Twist1 in murine cranial mesoderm. Ator graduated Cum Laude from the Biomedical Sciences Master's program in 2014. In April 2015, she was awarded a 4-year PhD fellowship in Regenerative Medicine from the Netherlands Organization for Scientific Research (NWO). She moved to Utrecht and started her PhD in September 2015 under the supervision of prof. Niels Geijsen, at the Hubrecht institute in the Netherlands. The result of her PhD is described in this thesis. 


\section{List of publications}

Ashoti, A.*, Limone, F.*, van Kranenburg, M., Alemany, A., Baak, M., Vivie, J., Piccioni, F., Creyghton, M., Eggan, K., \& Geijsen, N. Considerations and practical implications of performing a phenotypic CRISPR/Cas survival screen.

Submitted

Available in and adapted form on bioRixv (2020).

https://doi.org/10.1101/2020.07.27.223420

Ashoti, A., Alemany, A., Sage, F. \& Geijsen, N. DUX4 induces a homogeneous sequence of molecular changes, culminating in the activation of a stem-cell-like transcriptional network and induction of apoptosis in somatic cells. bioRxiv (2021). https://doi.org/10.1101/2021.05.04.442407.

Submitted

Publication before PhD

Bildsoe, H., Fan, X., Wilkie, E. E., Ashoti, A., Jones, V. J., Power, M., Qin, J., Wang, J., Tam P. P. L., \& Loebel, D. A. F. (2016). Transcriptional targets of TWIST1 in the cranial mesoderm regulate cellmatrix interactions and mesenchyme maintenance. Developmental Biology, 418(1), 189-203. https://doi.org/10.1016/j.ydbio.2016.08.016

* Equal contribution 
